\title{
Panorama de la santé 2007
}

LES INDICATEURS DE L'OCDE

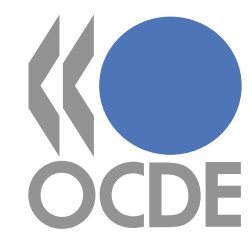





\title{
Panorama de la santé 2007
}

\author{
LES INDICATEURS DE L'OCDE
}




\section{ORGANISATION DE COOPÉRATION ET DE DÉVELOPPEMENT ÉCONOMIQUES}

L'OCDE est un forum unique en son genre où les gouvernements de 30 démocraties œuvrent ensemble pour relever les défis économiques, sociaux et environnementaux que pose la mondialisation. L'OCDE est aussi à l'avant-garde des efforts entrepris pour comprendre les évolutions du monde actuel et les préoccupations qu'elles font naître. Elle aide les gouvernements à faire face à des situations nouvelles en examinant des thèmes tels que le gouvernement d'entreprise, l'économie de l'information et les défis posés par le vieillissement de la population. L'Organisation offre aux gouvernements un cadre leur permettant de comparer leurs expériences en matière de politiques, de chercher des réponses à des problèmes communs, d'identifier les bonnes pratiques et de travailler à la coordination des politiques nationales et internationales.

Les pays membres de l'OCDE sont : l'Allemagne, l'Australie, l'Autriche, la Belgique, le Canada, la Corée, le Danemark, l'Espagne, les États-Unis, la Finlande, la France, la Grèce, la Hongrie, l'Irlande, l'Islande, l'Italie, le Japon, le Luxembourg, le Mexique, la Norvège, la Nouvelle-Zélande, les Pays-Bas, la Pologne, le Portugal, la République slovaque, la République tchèque, le Royaume-Uni, la Suède, la Suisse et la Turquie. La Commission des Communautés européennes participe aux travaux de l'OCDE.

Les Éditions OCDE assurent une large diffusion aux travaux de l'Organisation. Ces derniers comprennent les résultats de l'activité de collecte de statistiques, les travaux de recherche menés sur des questions économiques, sociales et environnementales, ainsi que les conventions, les principes directeurs et les modèles développés par les pays membres.

Cet ouvrage est publié sous la responsabilité du Secrétaire général de l’OCDE. Les opinions et les interprétations exprimées ne reflètent pas nécessairement les vues de l'OCDE ou des gouvernements de ses pays membres.

\footnotetext{
Publié en anglais sous le titre:

Health at a Glance 2007

OECD INDICATORS
}

Photo Credit: COMSTOCK Images/Jupiterimages, CREATAS/Jupiterimages, () Vincent Hazat/PhotoAlto and @) Inmagine ltd. Les corrigenda des publications de l'OCDE sont disponibles sur : www.oecd.org/editions/corrigenda. (C) OCDE 2007

\footnotetext{
Toute reproduction, copie, transmission ou traduction de cette publication doit faire l'objet d'une autorisation écrite. Les demandes doivent être adressées aux Éditions OCDE rights@oecd.org ou par fax 3314524 99 30. Les demandes d'autorisation de photocopie partielle doivent être adressées au Centre français d'exploitation du droit de copie (CFC), 20, rue des Grands-Augustins, 75006 Paris, France, fax 331463467 19, contact@cfcopies.com ou (pour les États-Unis exclusivement) au Copyright Clearance Center (CCC), 222 Rosewood Drive, Danvers, MA 01923, USA, fax 19786468600 , info@copyright.com.
} 


\section{Avant-propos}

C ette nouvelle édition du Panorama de la santé témoigne des progrès accomplis en matière d'évaluation des performances des systèmes de santé depuis la toute première réunion des ministres de la Santé des pays de l'OCDE, en mai 2004. Lors de cette réunion, les ministres de la Santé avaient clairement donné mandat à l'OCDE de travailler de concert avec les administrations nationales à l'amélioration de la base d'éléments de comparaison des performances des systèmes de santé :1) en garantissant l'actualité et l'exactitude de la base de données Eco-Santé OCDE ; 2) en poursuivant la mise en œuvre des comptes de la santé afin d'améliorer la disponibilité et la comparabilité des données sur les dépenses de santé et leur financement; et 3) en élaborant, en collaboration avec des experts nationaux, des indicateurs de la qualité des soins. Des progrès significatifs ont été réalisés dans tous ces domaines depuis 2004, comme le montre l'éventail plus large d'indicateurs des intrants, des extrants et des résultats des systèmes de santé, présentés dans cette publication.

La production du Panorama de la santé n'aurait pu voir le jour sans le concours des correspondants nationaux d'Eco-Santé OCDE, des spécialistes des comptes de la santé et des experts participant au projet sur les indicateurs de la qualité des soins de santé dans les 30 pays de l'OCDE. L'OCDE les remercie chaleureusement pour leurs efforts, consciente qu'elle leur doit la plupart des données et des informations qualitatives contenues dans cette publication. L'OCDE exprime également sa gratitude à d'autres organisations internationales, en particulier l'Organisation mondiale de la santé et Eurostat, dont certaines données sont reprises dans ce rapport.

Cette publication a été préparée par la Division de la santé de l'OCDE et coordonnée par Gaétan Lafortune. Le chapitre 1 a été préparé par David Morgan; le chapitre 2 par Gaétan Lafortune et Michael de Looper (de l'Australian Institute of Health and Welfare); le chapitre 3 par Franco Sassi; le chapitre 4 par Jeremy Hurst, Francesca Colombo, Rie Fujisawa, Maria Hofmarcher, Pierre Moïse, Valérie Paris et Gaëlle Balestat; le chapitre 5 par David Morgan et Sandra Hopkins; et le chapitre 6 par Sandra Garcia-Armesto, Niek Klazinga et Soeren Mattke (de RAND). Les graphiques et tableaux des cinq premiers chapitres ont été préparés par Gaëlle Balestat, Caroline Berchet et David Morgan; ceux du chapitre 6 ont été préparés par Maria Luisa Gil Lapetra et Lihan Wei. Enfin, cette publication a bénéficié des nombreux commentaires et suggestions de Elizabeth Docteur et Peter Scherer. 



\section{Table des matières}

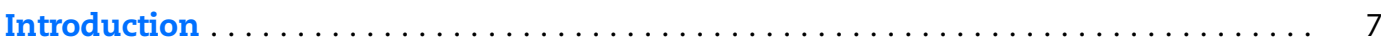

Chapitre 1. Contexte démographique et économique $\ldots \ldots \ldots \ldots \ldots \ldots \ldots \ldots \ldots$

1.1. Population totale et structure de la population $\ldots \ldots \ldots \ldots \ldots \ldots \ldots \ldots \ldots$

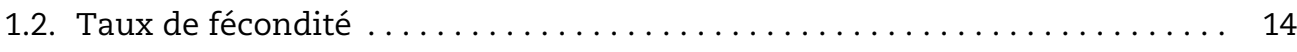

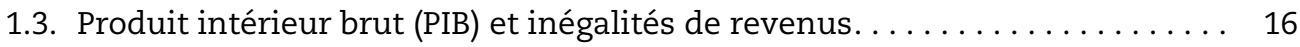

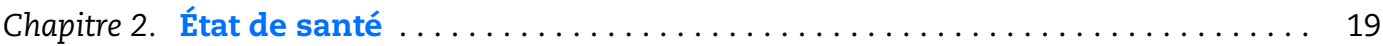

2.1. Espérance de vie à la naissance........................ 20

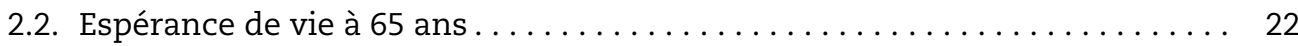

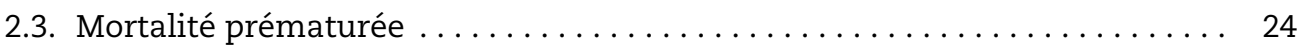

2.4. Mortalité due aux maladies cardiaques et aux accidents

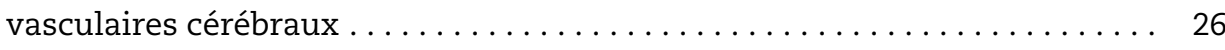

2.5. Mortalité due au cancer ............................. 28

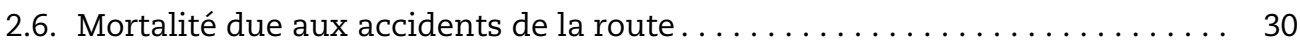

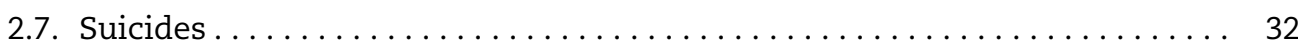

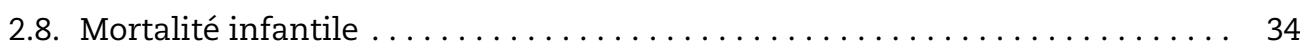

2.9. Santé du nourrisson : faible poids à la naissance $\ldots \ldots \ldots \ldots \ldots \ldots \ldots . \ldots \ldots$

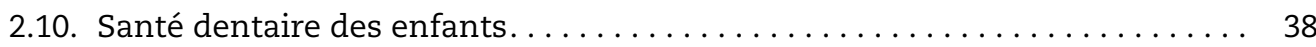

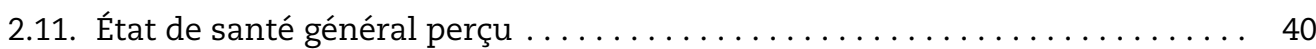

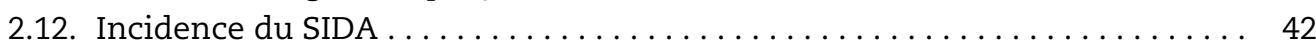

Chapitre 3. Déterminants non médicaux de la santé............... 45

3.1. Consommation de tabac. . . . . . . . . . . . . . . . . . . . . . 46

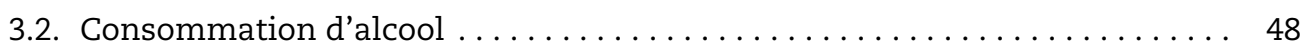

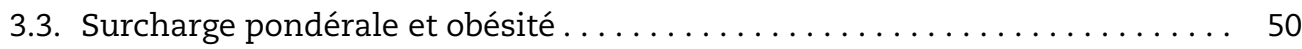

Chapitre 4. Ressources en santé et leur utilisation.................. 53

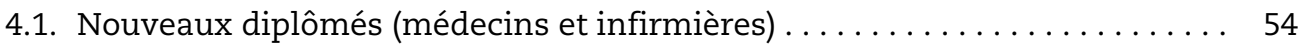

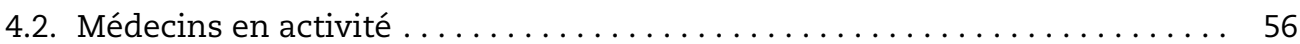

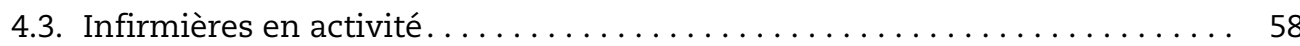

4.4. Rémunération des professionnels de santé (médecins et infirmières) . . . . 60

4.5. Lits de soins aigus, disponibilité et taux d'occupation. . . . . . . . . . 62

4.6. Lits de soins de longue durée dans les hôpitaux et les établissements

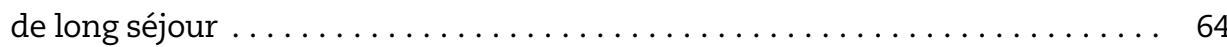

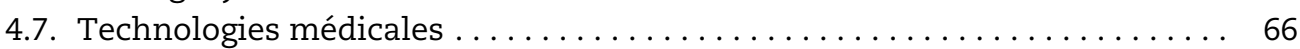

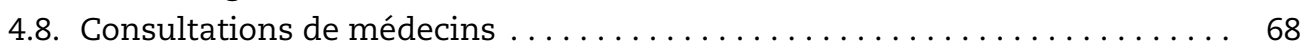

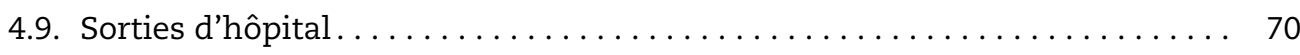

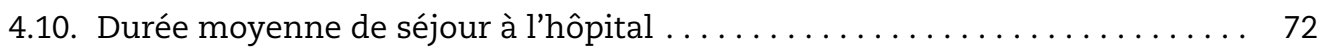

4.11. Chirurgies cardio-vasculaires (pontages et angioplasties) $\ldots \ldots \ldots \ldots \ldots$ 
4.12. Traitement des insuffisances rénales

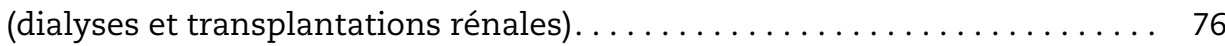

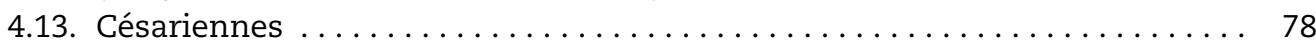

4.14. Opérations de la cataracte, en ambulatoire et avec hospitalisation ...... 80

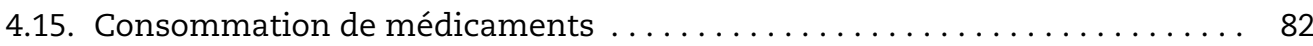

Chapitre 5. Dépenses de santé et financement $\ldots \ldots \ldots \ldots \ldots \ldots \ldots \ldots \ldots \ldots \ldots$

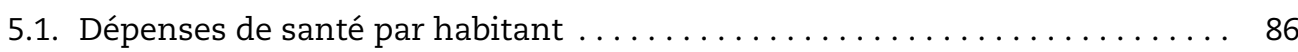

5.2. Dépenses de santé par rapport au produit intérieur brut (PIB) . . . . . . 88

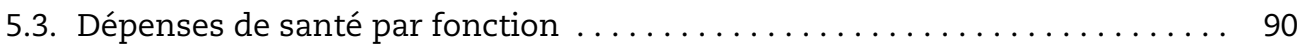

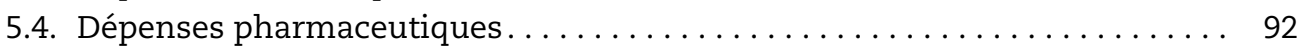

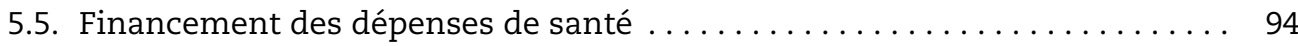

5.6. Couverture de l'assurance maladie (publique et privée) . . . . . . . . . . 96

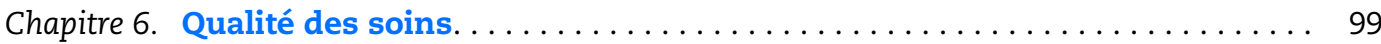

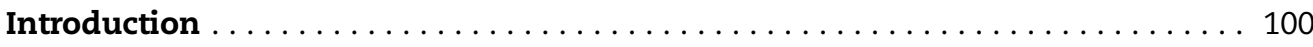

\section{Les affections aiguës}

6.1. Mortalité à l'hôpital suite à un infarctus aigu du myocarde . . . . . . . . . . . 104

6.2. Mortalité à l'hôpital suite à un accident vasculaire cérébral . . . . . . . . 106

\section{Le cancer}

6.3. Survie au cancer colorectal . . . . . . . . . . . . . . . . . . . . . . . . . . . . . 108

6.4. Survie au cancer du sein et dépistage . . . . . . . . . . . . . . . . . 110

6.5. Survie au cancer du col de l'utérus et dépistage. . . . . . . . . . . 112

Les affections chroniques

6.6. Admissions à l'hôpital et mortalité évitables dues à l'asthme . . . . . . . . 114

6.7. Examens annuels de la rétine pour les diabétiques . . . . . . . . . 116

\section{Les maladies transmissibles}

6.8. Vaccination des personnes âgées contre la grippe . . . . . . . . . . . . 118

6.9. Programmes de vaccination des enfants . . . . . . . . . . . . . . 120

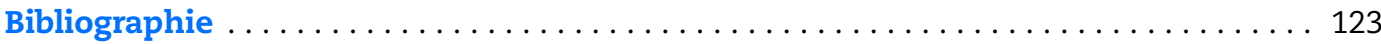

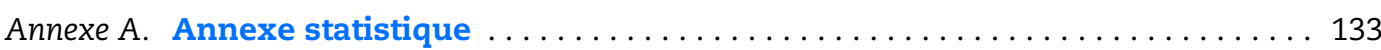

Annexe B. Définition des dépenses de santé et notes méthodologiques

sur la comparabilité des données. . . . . . . . . . . . . . . 192

Annexe C. Liste des variables contenues dans Eco-Santé OCDE 2007 . . . . . . . . . . . 194

Annexe D. Catégories de maladies et blessures et codes CIM . . . . . . . . . . . . . 195

\section{Ce livre contient des...}

En bas à droite des tableaux ou graphiques de cet ouvrage, vous trouverez des StatLinks. Pour télécharger le fichier Excel囚 correspondant, il vous suffit de retranscrire dans votre navigateur Internet le lien commençant par : $h t t p: / / d x$.doi.org.

Si vous lisez la version PDF de l'ouvrage, et que votre ordinateur est connecté à Internet, il vous suffit de cliquer sur le lien.

Les StatLinks sont de plus en plus répandus dans les publications de l'OCDE. 


\section{Introduction}

L

a publication Panorama de la santé 2007 permet aux lecteurs de comparer les systèmes de santé et leurs performances pour un certain nombre de dimensions clés, à l'aide d'un ensemble d'indicateurs choisis pour leur pertinence, mais aussi sur la base de la disponibilité et la comparabilité des données.

Depuis longtemps, l'OCDE est l'un des chefs de file internationaux de l'élaboration d'outils et de la collecte de données pour l'évaluation des performances des systèmes de santé. Au cours des quinze dernières années, la base Eco-Santé OCDE a été une source de données de premier plan sur la santé et les systèmes de santé des pays de l'OCDE. À l'exception du nouveau chapitre sur les indicateurs de la qualité des soins de santé, toutes les données et métadonnées présentées dans cette publication sont tirées de Eco-Santé OCDE 2007.

\section{Contexte politique}

Les dépenses de santé qui, à l'époque de la création de l'OCDE en 1960, représentaient environ $4 \%$ du PIB des pays de l'OCDE, représentent aujourd'hui $9 \%$ en moyenne, et près de $11 \%$ du PIB, voire plus dans plusieurs grandes économies nationales. Au fil des ans, l'importance du secteur de la santé dans les économies développées s'est accrue de façon importante; pourtant, de grandes disparités subsistent entre les pays, et pas seulement en matière de dépenses.

Bien que les systèmes de santé diffèrent considérablement par leur conception, les moyens mis en œuvre et les résultats obtenus, les décideurs des pays de l'OCDE ont un objectif commun qui est de se doter de systèmes hautement performants (OCDE, 2004a). Les objectifs des politiques de santé sont notamment:

- d'améliorer l'état de santé de la population et les résultats en termes de santé des interventions médicales ;

- de promouvoir un accès aux soins adéquat et équitable ;

- d'accroître la réactivité des systèmes de santé ;

- d'améliorer l'efficience des systèmes de santé; et

- de veiller à ce que les coûts et leur financement soient viables.

Panorama de la santé 2007 fournit des informations sur les performances des systèmes de santé au regard de plusieurs de ces objectifs, mais aussi des informations contextuelles nécessaires pour comprendre les disparités de performances entre les pays et l'évolution de ces performances dans le temps. L'OCDE s'emploie, avec le concours des experts nationaux des pays membres, à améliorer les informations disponibles sur les systèmes de santé et la capacité technique à évaluer et comparer les performances de ces systèmes. Signalons notamment que cette édition du Panorama de la santé comporte, pour la première fois, un chapitre sur la qualité des soins de santé qui répond à l'intérêt grandissant des décideurs concernant l'évaluation, la comparaison et l'amélioration de la qualité des soins dispensés aux patients et, ce faisant, optimiser le rapport coût/efficacité des dépenses de santé. 


\section{Structure de la publication}

L'édition 2007 de Panorama de la santé est organisée de la manière suivante :

Le chapitre 1 fournit quelques indicateurs du contexte démographique et économique dans lequel les systèmes de santé opèrent dans les différents pays de l'OCDE.

Le chapitre 2 État de santé - met en évidence les grandes disparités entre les pays en termes d'espérance de vie et autres mesures de l'état de santé de la population.

Le chapitre 3 Déterminants non médicaux de la santé - se concentre sur une sélection de facteurs de risque en rapport avec des modes de vie et des comportements modifiables.

Le chapitre 4 Ressources en santé et leur utilisation-compare notamment le nombre de médecins et d'infirmières en activité ainsi que le nombre de nouveaux diplômés, dans un contexte de crainte grandissante que les pénuries actuelles et futures de professionnels de santé n'entrave l'accès aux soins. Il présente également des indicateurs qui fournissent des mesures au moins partielles de l'efficience de l'offre de services de santé, comme le nombre annuel de consultations par médecin, la durée moyenne de séjour à l'hôpital pour différentes pathologies, et la proportion des interventions chirurgicales, comme les opérations de la cataracte, effectuées désormais en ambulatoire dans les différents pays.

Le chapitre 5 - Dépenses de santé et financement - évalue les montants dépensés par les pays de l'OCDE au titre de la santé, globalement et pour les différents types de produits et services de santé, ainsi que leur mode de financement (public, assurance privée et paiements pris en charge directement par les patients). Il contient également de nouvelles informations sur le degré de couverture maladie des populations (assurance publique et privée), qui est un déterminant important de l'accès aux soins et de la protection financière.

Le chapitre 6 - Qualité des soins - présente un premier ensemble d'indicateurs de la qualité des soins. Reflétant les progrès accomplis en matière d'élaboration d'indicateurs à utiliser dans les comparaisons internationales, il porte notamment sur la qualité des soins suite à une crise cardiaque et un accident vasculaire cérébral (AVC), des traitements des cancers, des soins liés aux maladies chroniques, ainsi que la prévention des maladies transmissibles. Ce nouveau chapitre inclut notamment un certain nombre d'indicateurs de résultats, tels que les taux de survie après une crise cardiaque, un AVC ou un cancer.

\section{Présentation des indicateurs}

\section{Texte et graphiques}

Chacun des sujets abordés dans les différents chapitres de cette publication fait l'objet d'une présentation sur deux pages. Sur la première page, le lecteur trouvera un texte succinct qui résume les principales observations émanant des données, définit les indicateurs et indique les principales divergences nationales par rapport à cette définition susceptibles d'affecter la comparabilité des données. La seconde page reproduit un ensemble de graphiques qui montrent généralement les niveaux actuels de l'indicateur et, dans la mesure du possible, ses évolutions dans le temps. Dans certains cas, un graphique supplémentaire présente la relation existant entre l'indicateur concerné et une autre variable. Lorsqu'un graphique comporte une moyenne de l'OCDE, il s'agit d'une moyenne non pondérée des pays présentés, sauf indication contraire des notes accompagnant le graphique.

\section{Tableaux}

Des données complémentaires sont présentées dans l'annexe statistique (annexe A) à la fin de cette publication. Dans les cas où les données de certains pays ne sont pas disponibles pour les années retenues, les tableaux présentent les données les plus près, qui portent normalement sur l'une des trois années précédant ou suivant l'année concernée.

Les tableaux contiennent un maximum de deux statistiques sommaires. La moyenne cohérente désigne la moyenne non pondérée des pays pour lesquels des données sont disponibles pour toute la période considérée, l'objectif étant de présenter des informations pour un groupe de pays constant dans le temps. Les pays non pris en compte dans cette 
moyenne (en raison de données manquantes) sont indiqués sous le tableau. Dans la plupart des cas, la moyenne cohérente s'accompagne de la moyenne la plus récente, qui porte uniquement sur la dernière année disponible et couvre le plus grand nombre de pays possible.

Sauf indication contraire, les données sur les dépenses sont présentées en dollars américains et corrigées des différences de pouvoir d'achat des monnaies nationales de manière à neutraliser l'effet des différences de prix entre pays. Pour les taux de croissance, les dépenses nominales sont déflatées à l'aide des indices de prix. En l'absence d'indice des prix de la santé fiable et disponible pour un grand nombre de pays, c'est l'indice des prix de l'économie entière (PIB) qui est utilisé dans cette publication (voir l'annexe B pour des informations complémentaires sur l'utilisation des parités de pouvoir d'achat et le calcul des taux de croissance réels).

Les données manquantes, sans objet ou non disponibles sont signalées par le signe « . . » et les ruptures dans les séries par un signe "|» entre les colonnes. Les autres remarques méthodologiques pertinentes apparaissent directement sous le tableau concerné.

\section{Limites des données}

Les limites de comparabilité des données sont indiquées dans le texte (dans un encadré intitulé "Définition et écarts") ainsi que dans les notes sous les graphiques et tableaux. Il convient de noter que les données tendancielles concernant l'Allemagne doivent être interprétées avec une prudence particulière. En effet, les données recueillies pour ce pays se rapportent généralement à l'ex-Allemagne de l'Ouest jusqu'en 1990 et à l'Allemagne réunifiée à partir de 1991.

Les lecteurs qui souhaiteraient utiliser les données présentées dans cette publication pour des analyses et des recherches plus approfondies sont invités à consulter la documentation complète sur les définitions, les sources et les méthodes contenues dans Eco-Santé OCDE 2007. Eco-Santé OCDE 2007 peut être commandé en ligne auprès de SourceOCDE (www.sourceOCDE.org) ou de la librairie en ligne de l'OCDE (www.oecd.org/bookshop).

Concernant le nouveau chapitre sur les indicateurs de la qualité des soins de santé, on trouvera plus d'informations sur les définitions, sources et méthodes à l'adresse www.oecd.org/health/hcqi.

\section{Les chiffres de population}

Les chiffres de population, présentés au chapitre 1 et utilisés pour calculer les taux par habitant dans l'ensemble de la publication, sont tirés de la base de données de l'OCDE sur la population active (en date de mai 2007). Ces chiffres se rapportent à la population au milieu de l'année. Ils ne sont pas nécessairement exactement les mêmes que les chiffres les plus récents publiés par les instituts statistiques nationaux des pays membres de l'OCDE.

Il convient aussi de noter que pour certains pays comme la France, le Royaume-Uni et les États-Unis, qui ont des colonies, des protectorats et des territoires outre-mer, ces populations ne sont généralement pas prises en compte. Toutefois, la population utilisée pour le calcul du PIB par habitant et d'autres mesures économiques pour ces pays peut varier.

\section{Abbréviation des pays (codes ISO)}

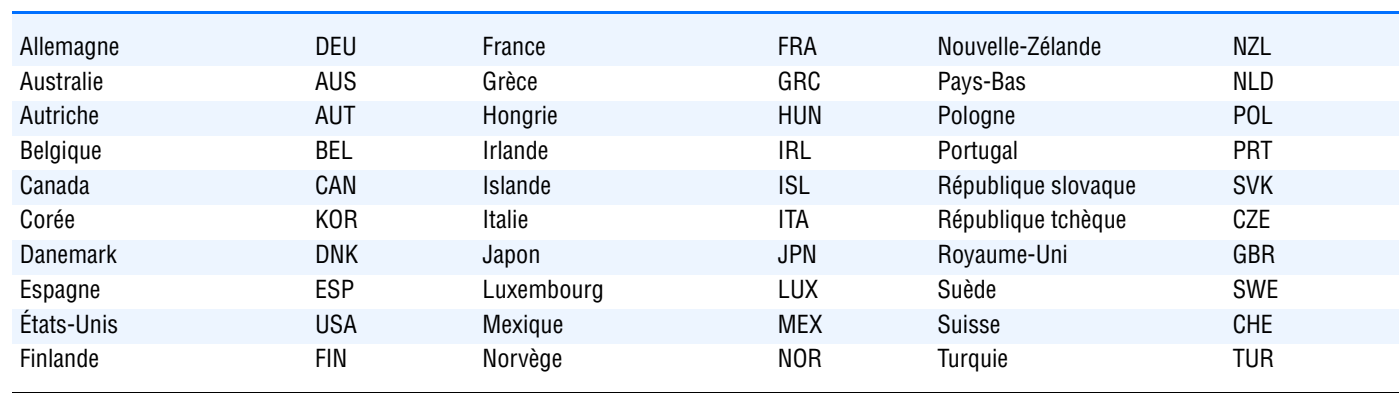




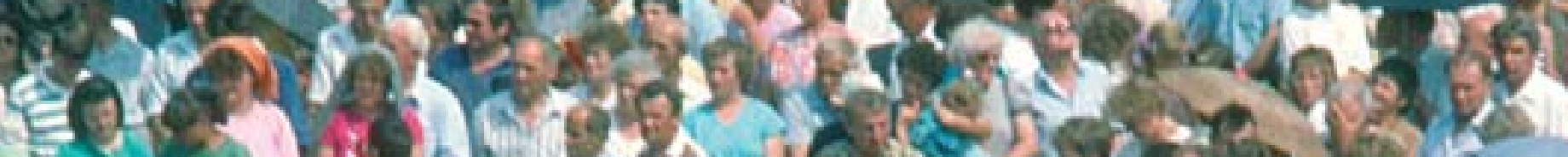

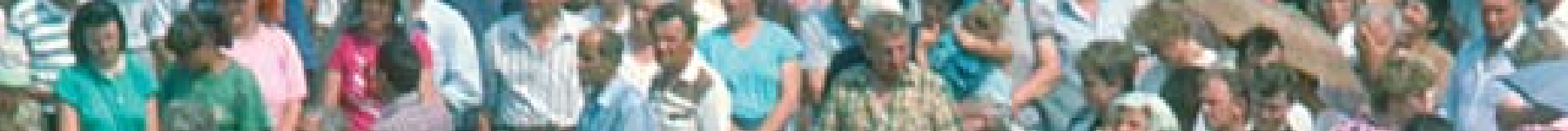

(1) - 2 a $^{2}(1+1$

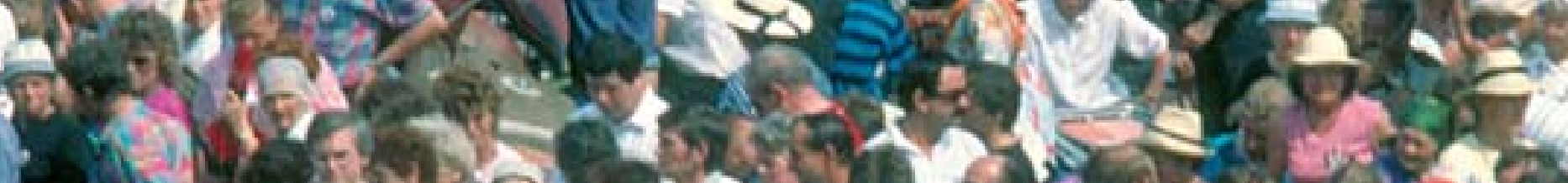
1)

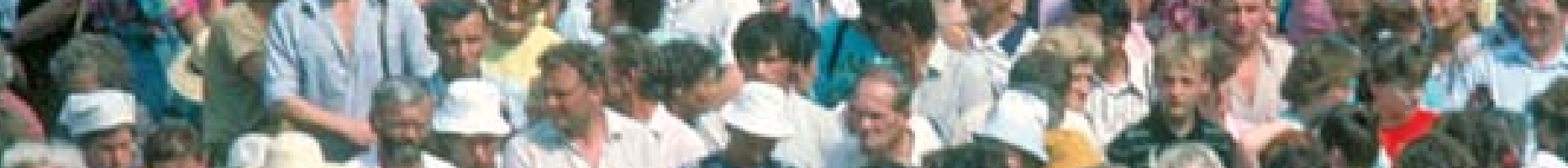

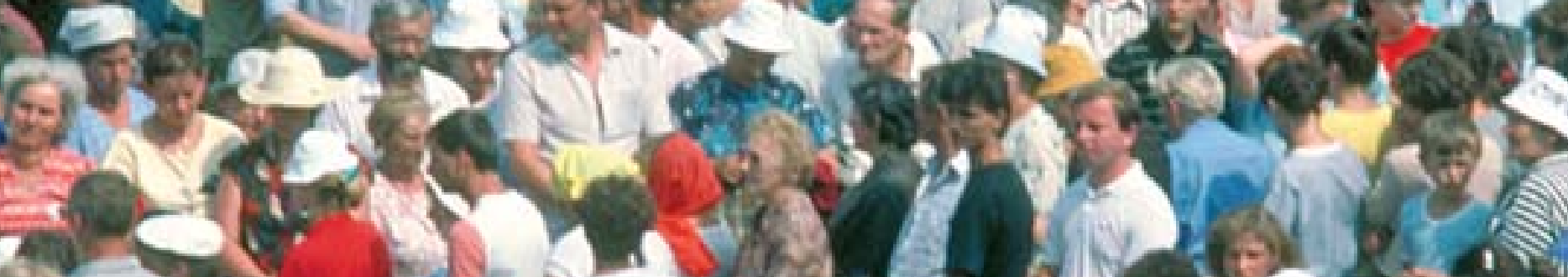

Qu 25 is

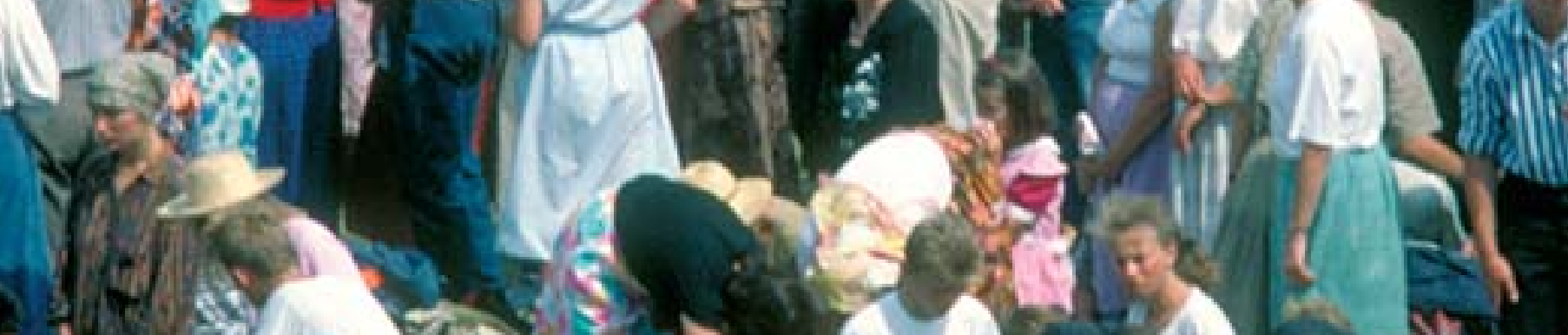

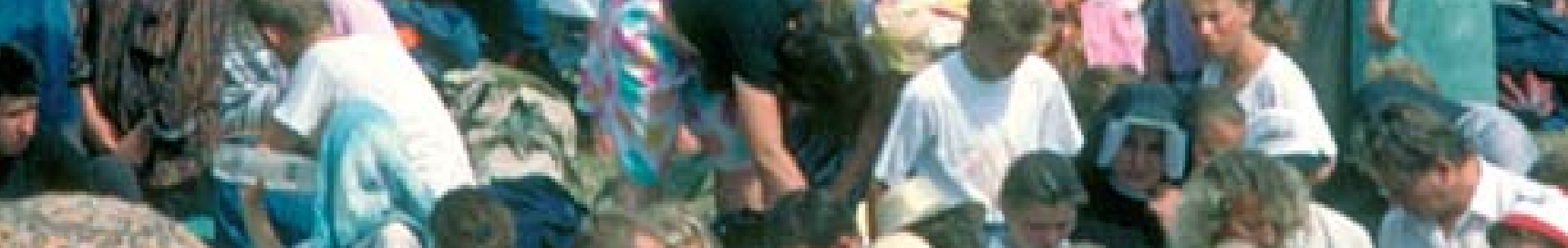

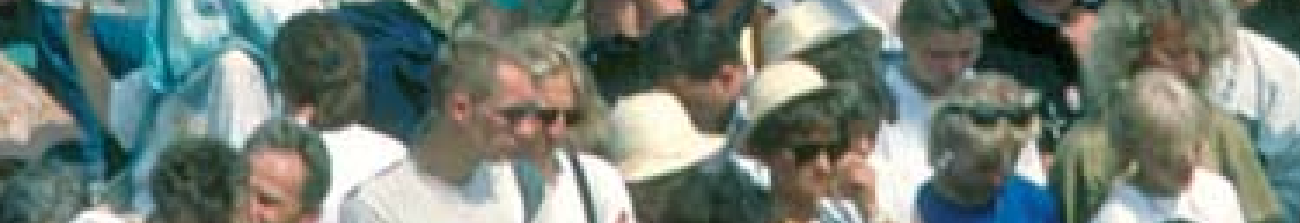

(a)

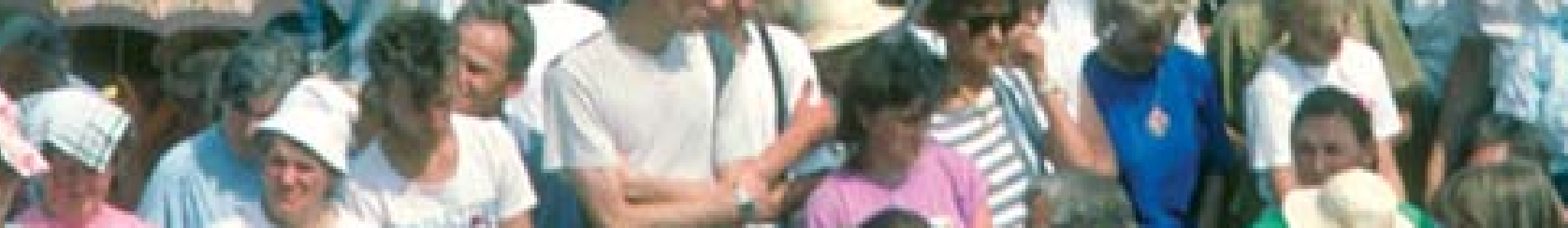

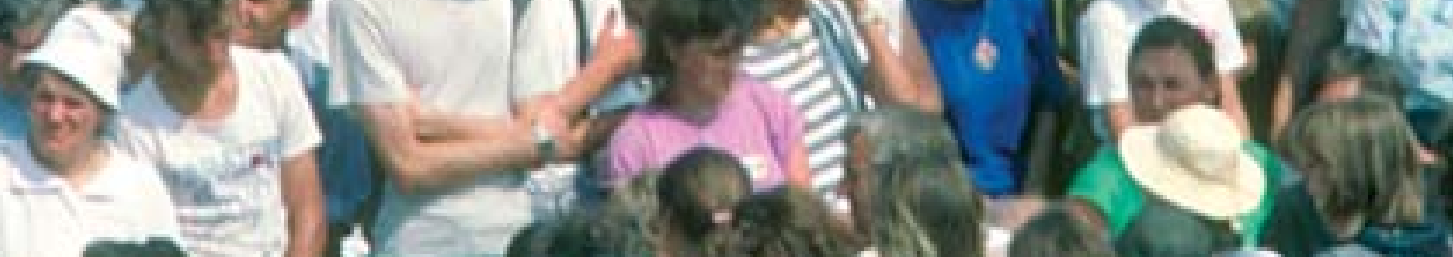

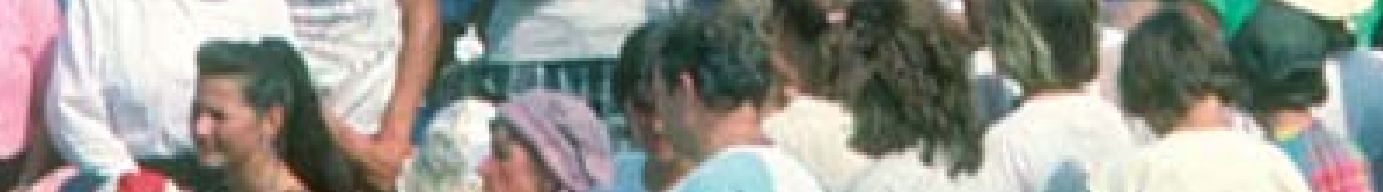
(e) $A . \mathrm{A}_{2}$ 


\section{CONTEXTE DÉMOGRAPHIQUE ET ÉCONOMIQUE}

1.1. POPULATION TOTALE ET STRUCTURE DE LA POPULATION . . . 12

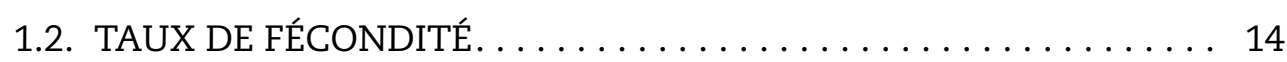

1.3. PRODUIT INTÉRIEUR BRUT (PIB) ET INÉGALITÉS DE REVENUS . . 16 


\subsection{POPULATION TOTALE ET STRUCTURE DE LA POPULATION}

La croissance et la composition de la population d'un pays peuvent avoir des implications importantes en termes de dépenses actuelles et futures de santé et de soins de longue durée. Au cours des dernières décennies, l'accroissement naturel (naissances moins décès) de la population s'est généralement ralenti dans tous les pays de l'OCDE, conduisant à une élévation de l'âge moyen de la population. Les phénomènes migratoires ont également affecté l'évolution de la population.

En 2005, les pays de l'OCDE représentaient environ $18 \%$ de la population mondiale qui était alors de 6.5 milliards de personnes. À l'intérieur de la zone OCDE, les États-Unis demeurent le pays le plus peuplé avec près de 300 millions de personnes. Le Japon et le Mexique sont les deux seuls autres pays de l'OCDE dont la population dépasse les 100 millions d'habitants. À l'autre extrémité de l'échelle, l'Islande et le Luxembourg ont chacun une population de moins de 500000 personnes (graphique 1.1.1 et tableaux A.1.1a-A.1.1b)

Depuis 1960, la population totale des pays de l'OCDE a augmenté de plus de $50 \%$. C'est entre 1960 et 1980 que la progression a été la plus forte en raison de taux de fécondité relativement élevés et d'une chute rapide du taux de mortalité. Depuis lors, l'accroissement de la population s'est considérablement ralenti dans de nombreux pays de l'OCDE, sous l'effet d'une chute des taux de fécondité (voir l'indicateur 1.2 " Taux de fécondité ") et d'une modification des schémas migratoires. Entre 1990 et 2005, le taux d'accroissement de la population pour tous les pays de l'OCDE a été divisé par deux par rapport aux années 60 et 70, s'établissant en moyenne à un peu plus de $0.6 \%$ par an (graphique 1.1.2). Mais les disparités sont grandes. Le Mexique et la Turquie ont continué à afficher la plus forte progression de leur population mais nettement inférieure aux taux observés dans les années 70. Dans des pays comme l'Australie, le Canada, la Nouvelle-Zélande et les États-Unis, la croissance de la population est restée relativement forte, s'établissant à un peu plus de $1 \%$ par an, du fait d'un taux de fécondité plus élevé que dans d'autres pays mais également d'une migration nette à destination de ces pays. En revanche, la Hongrie, la République tchèque, la Pologne et la République slovaque ont enregistré, ces dernières années, des taux d'accroissement de leur population faibles, voire même négatifs, du fait de taux de fécondité bas (et, dans le cas de la Pologne, d'un solde migratoire négatif).

La demande de soins de santé et de soins de longue durée et leur financement, mais aussi les retraites et autres avantages sociaux, dépendent pour partie de l'évolution de la structure démographique d'un pays. Le pourcentage de la population des 65 ans et plus a augmenté dans tous les pays de l'OCDE et ce phénomène devrait se poursuivre au cours des prochaines décennies. Dans les pays de l'OCDE, en moyenne près de $15 \%$ de la population a plus de 65 ans (graphique 1.1.3). Au Japon, en Italie et en Allemagne, ce chiffre avoisine les $20 \%$. Les pays les "plus jeunes " (la Turquie, le Mexique et la Corée) ont encore moins de $10 \%$ de leur population âgée de plus de 65 ans, bien que la Corée ait enregistré l'un des plus forts taux de progression depuis 1960.

Le ratio de dépendance des personnes âgées, qui compare le nombre de personnes âgées à la population en âge de travailler, est un autre moyen utile d'évaluation de l'impact du vieillissement de la population sur le financement des soins de santé et des retraites. En 2005, ce ratio variait de moins de $10 \%$ dans le cas de la Turquie et du Mexique, à près de $30 \%$ au Japon, en Italie et en Allemagne. Dans une multitude d'autres pays européens, dont le Royaume-Uni et la France, il s'est établi à environ $25 \%$. La moyenne de l'OCDE, qui actuellement dépasse les $20 \%$, devrait plus que doubler d'ici à 2050 ; le ratio serait alors de l'ordre d'environ une personne âgée pour deux actifs. Dans la mesure où les personnes âgées tendent à être en moins bonne santé et donc à avoir davantage besoin de soins médicaux et de soins de longue durée, on peut penser que le vieillissement de la population va accroître les dépenses publiques dans ces domaines. De plus, l'accroissement de la population inactive sur le marché du travail et la diminution de l'offre de travail devraient ralentir la croissance du PIB par habitant dans de nombreux pays de l'OCDE au cours des prochaines décennies (Oliveira et al., 2005).

\section{Définition et écarts}

On entend par population totale l'ensemble des ressortissants présents dans le pays ou absents de façon temporaire, et des étrangers installés dans le pays de façon permanente. Pour la plupart des pays de l'OCDE, les estimations de population se fondent sur les résultats des recensements effectués à intervalles réguliers de dix ans, corrigés des données administratives pour les années intermédiaires. Les chiffres sur la population sont tirés de la base de données de l'OCDE sur la population active (en date de mai 2007) et se rapportent à la population au milieu de l'année. Ces chiffres ne sont pas nécessairement exactement les mêmes que les chiffres les plus récents publiés par les instituts statistiques nationaux des pays de l'OCDE. Il convient aussi de noter que pour certains pays comme la France, le Royaume-Uni et les États-Unis, qui ont des colonies, des protectorats et des territoires outre-mer, ces populations ne sont généralement pas prises en compte. Toutefois, la population utilisée pour le calcul du PIB par habitant et d'autres mesures économiques pour ces pays peut varier.

Le ratio de dépendance des personnes âgées compare la population des 65 ans et plus à la population en âge de travailler que l'on considère couvrir toute la tranche d'âge comprise entre 15 et 64 ans. L'inclusion de la classe d'âge des 15-19 ans repose sur l'hypothèse selon laquelle la fraction des adolescents de moins de 20 ans déjà dans la vie active est égale à la fraction de la population des personnes de 65 ans et plus encore en activité sur le marché du travail.

Il convient de noter que, pour les années antérieures à 1991, les chiffres de population indiqués pour l'Allemagne correspondent à l'ex-Allemagne de l'Ouest. 


\section{CONTEXTE DÉMOGRAPHIQUE ET ÉCONOMIQUE}

\subsection{POPULATION TOTALE ET STRUCTURE DE LA POPULATION}

1.1.1. Population totale des pays de l'OCDE, en millions, 2005

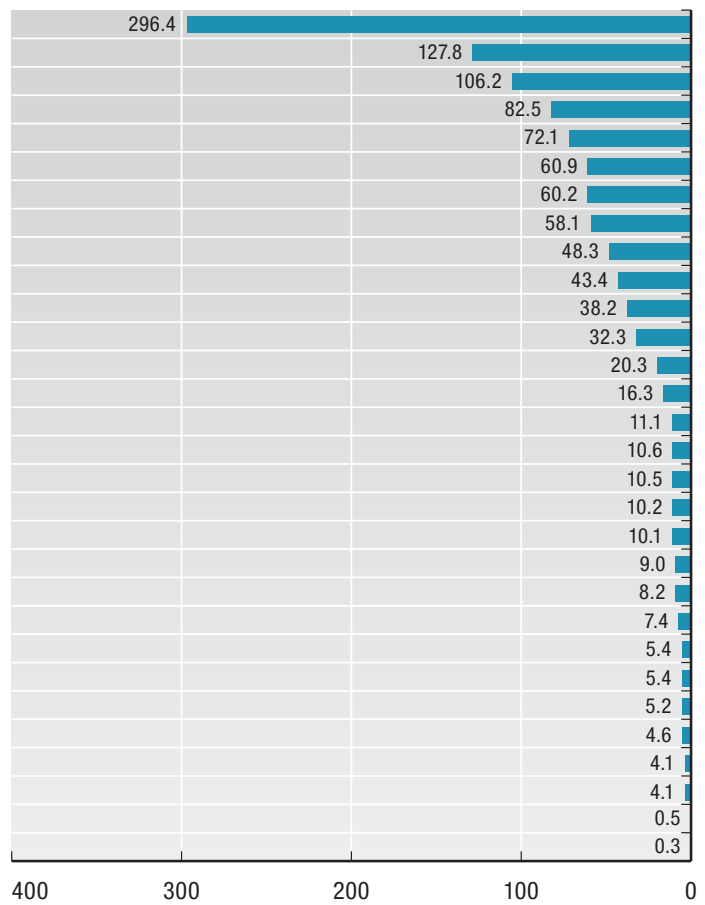

Population en millions

1. Le taux de croissance annuel moyen se réfère à 1991-2005

\subsubsection{Croissance annuelle moyenne de la population, 1990-2005}

États-Unis
Japon
Mexique
Allemagne ${ }^{1}$
Turquie
France
Royaume-Uni
Italie
Corée
Espagne
Pologne
Canada
Australie
Pays-Bas
Grèce
Portugal
Belgique
République tchèque
Hongrie
Suède
Autriche
Suisse
Danemark
République slovaque
Finlande
Norvège
Irlande
Nouvelle-Zélande
Luxembourg
Islande

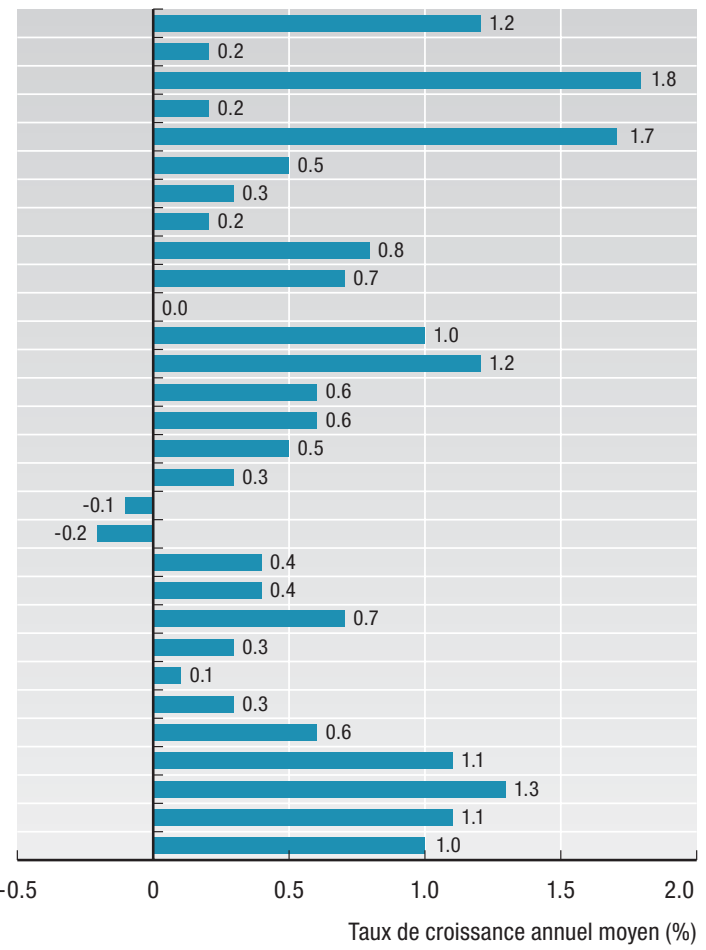

\subsubsection{Pourcentage de la population âgée de 65 ans et plus, 1960 et 2005}

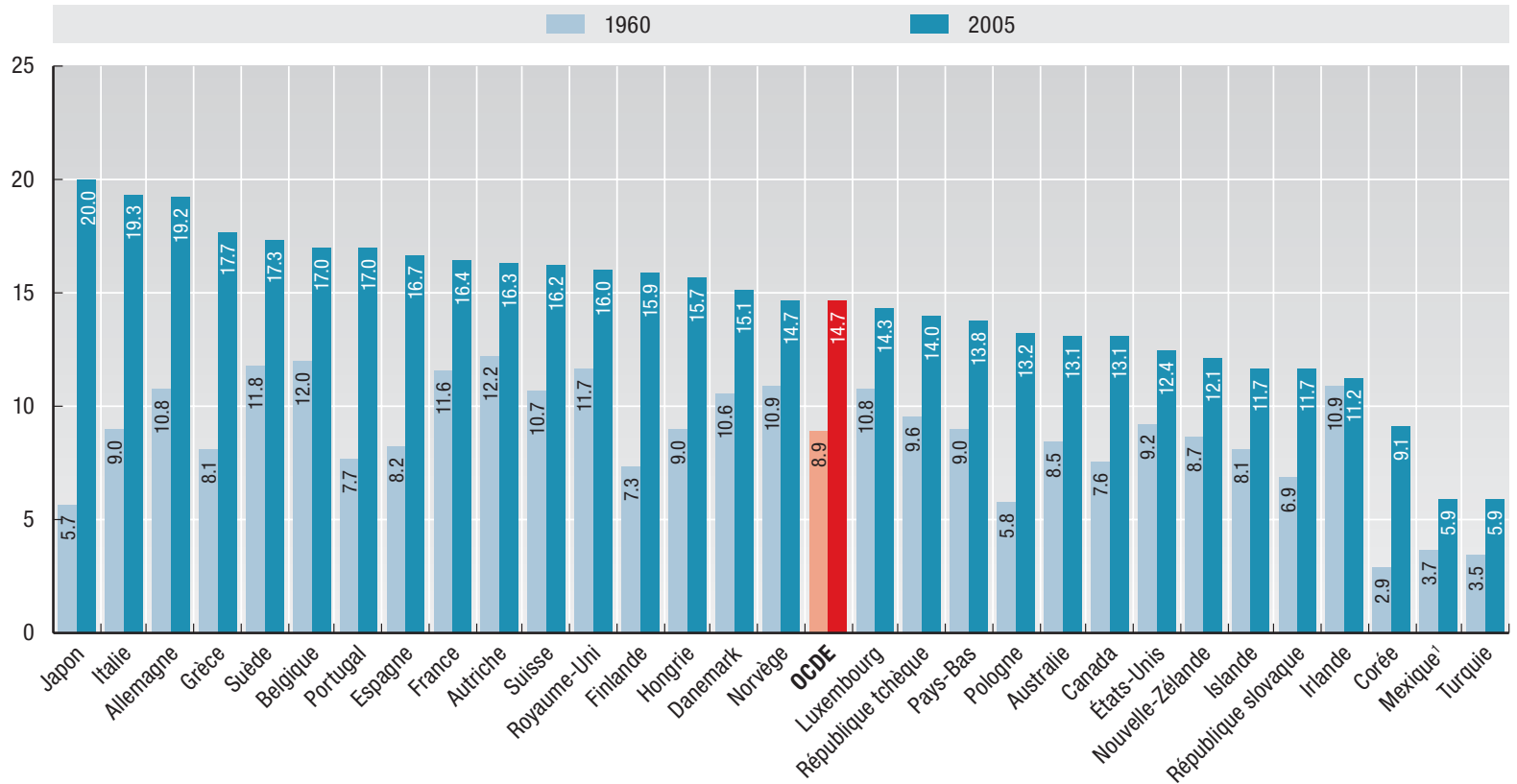

1. 1970

Source : Eco-Santé OCDE 2007. 


\subsection{TAUX DE FÉCONDITÉ}

Avec le déclin du taux de mortalité, la baisse spectaculaire des taux de fécondité dans les pays de l'OCDE au cours des dernières décennies a entraîné un vieillissement démographique (voir l'indicateur 1.1 " Population totale et structure de la population »). Les pays peuvent être affectés par une baisse durable des taux de natalité due à une perte de potentiel reproductif suite à une diminution du nombre de femmes en âge d'avoir un enfant. Plusieurs pays examinent dans quelle mesure leurs politiques affectent, directement ou indirectement, le taux de natalité.

Tous les pays de l'OCDE ont enregistré, au cours des dernières décennies, une chute de leur taux de fécondité qui est tombé de 3.2 enfants par femme en âge de procréer, en 1960, à moins de deux enfants au début des années 80 et s'établissait à un peu plus de 1.6 enfant en 2005 (graphique 1.2.1). Actuellement, le Mexique et la Turquie sont les deux seuls pays de l'OCDE dont le taux de fécondité demeure supérieur au seuil de renouvellement des générations (2.1 enfants), c'est-àdire au niveau requis pour assurer la stabilité de la population sans immigration nette et sans évolution du taux de mortalité. Mais même ces deux pays ont enregistré une baisse spectaculaire de leur taux de fécondité depuis les années 60 et 70 ; à l'époque, ce taux était de l'ordre de six, voire sept enfants.

Le rythme de cette baisse a varié d'un pays à l'autre. Alors qu'au Japon et en Corée les taux de fécondité ont fortement chuté et continuent de baisser - en Corée, la baisse a été spectaculaire, le taux passant de six enfants en 1960 à 1.08 en 2005, soit le taux le plus bas de tous les pays de l'OCDE -, une certaine inversion de tendance a été observée aux États-Unis et au Danemark dans les années 80 mais aussi en France au milieu des années 90 (graphique 1.2.2). Un grand nombre d'autres pays semblent s'engager sur la même voie avec, ces dernières années, une stabilisation voire une légère inversion de la tendance à la baisse.

Un grand nombre de facteurs liés entre eux et reflétant à la fois les préférences individuelles en matière de mode de vie et les influences sociales et historiques de chaque pays affectent les taux de fécondité. La progression rapide de la mise à disposition de méthodes contraceptives dans la deuxième moitié $d u x^{e}$ siècle a joué un rôle majeur dans la chute historique de la fécondité dans de nombreux pays. Une autre raison importante de l'évolution observée a été, dans de nombreux pays, le recul de l'âge auquel les femmes ont leur premier enfant. L'âge moyen des mères à la naissance de leur premier enfant a augmenté, en moyenne, d'un an par décennie depuis 1970 (graphique 1.2.3) pour s'établir à 27.6 ans en 2004. Le cas le plus extrême est celui de l'Allemagne où cet âge est passé de 24 ans en 1970 à 29 en 2004. Le fait que les femmes retardent l'âge auquel elles décident d'avoir un enfant accroît également la probabilité pour qu'elles n'en aient pas ou qu'elles en aient moins. Ce recul peut être lié à différents facteurs d'ordre individuel et sociétal tels que l'évolution du rôle des femmes dans la société et l'évolution du lien entre mariage et maternité (D'Addio et Mira d'Ercole, 2005).

Étant donné l'impact sur la structure de la population des taux de fécondité passés et futurs, les pays doivent examiner avec soin les politiques susceptibles d'avoir une incidence sur la taille de la famille. Des politiques de la famille permettant de concilier études ou carrière et vie de famille (par le biais d'un système de garde d'enfants à un prix abordable et de congé parental, par exemple) et l'impact des avantages fiscaux et des prestations familiales sont autant d'éléments qui peuvent influer sur l'évolution du taux de fécondité (OCDE, 2005d).

\section{Définition et écarts}

Le taux de fécondité pour une année spécifique est une estimation du nombre moyen d'enfants par femme en âge de procréer (entre 15 et 49 ans), en supposant que les taux de fécondité actuels par âge demeurent constants.

On considère qu'un taux de fécondité de 2.1 enfants par femme assure globalement la stabilité de la population (en supposant l'absence de flux migratoires nets et d'une modification du taux de mortalité). 


\section{CONTEXTE DÉMOGRAPHIQUE ET ÉCONOMIQUE}

1.2. TAUX DE FÉCONDITÉ

\subsubsection{Taux de fécondité, $1960, \mathbf{1 9 8 0}$ et 2005}

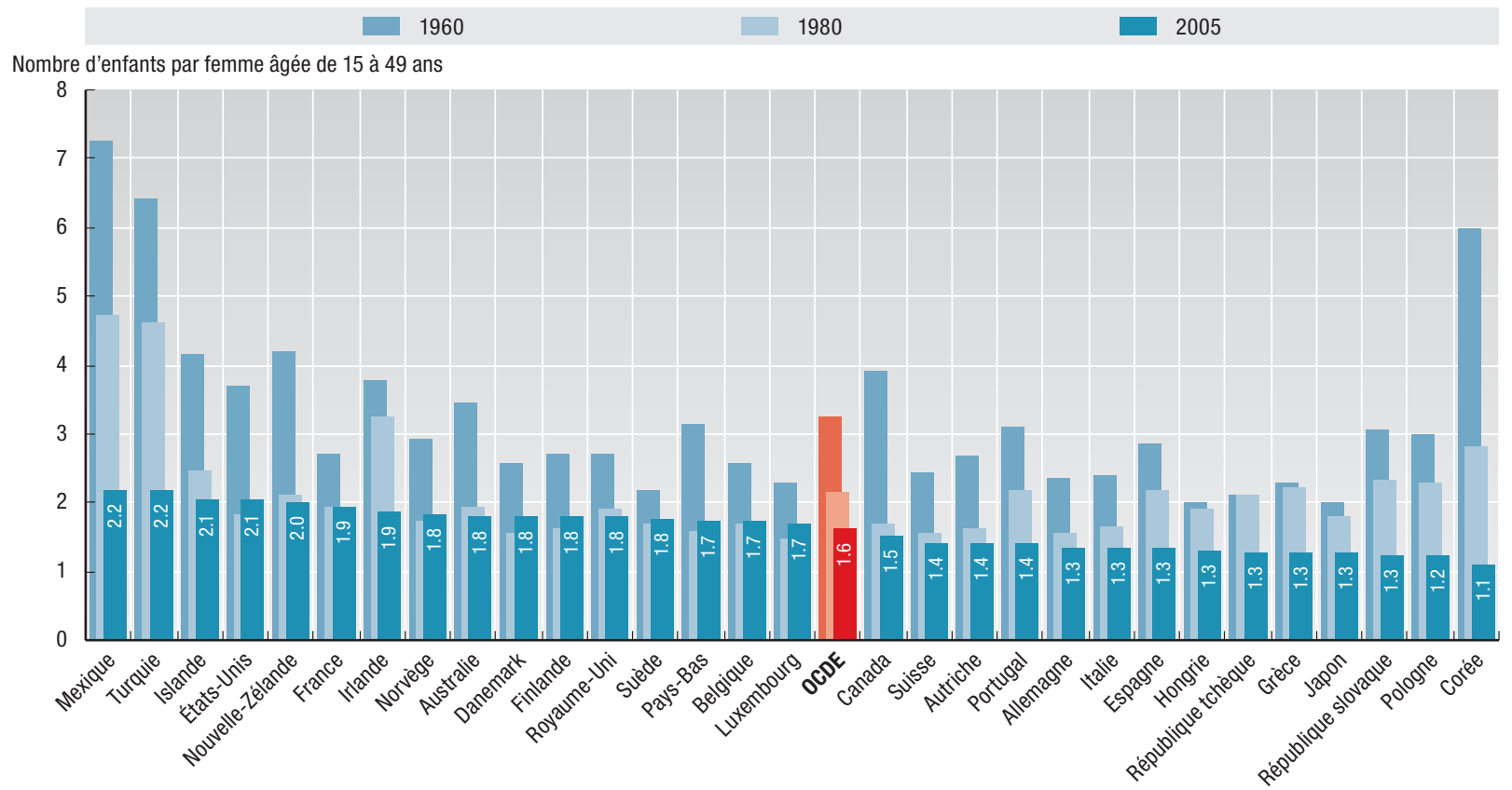

\subsubsection{Taux de fécondité, 1970 à 2005}

$$
\begin{aligned}
& \text { - - - France - - - Corée - ..- E Espagne } \\
& \text { - - États-Unis — OCDE }
\end{aligned}
$$

Nombre d'enfants par femme âgée de 15 à 49 ans

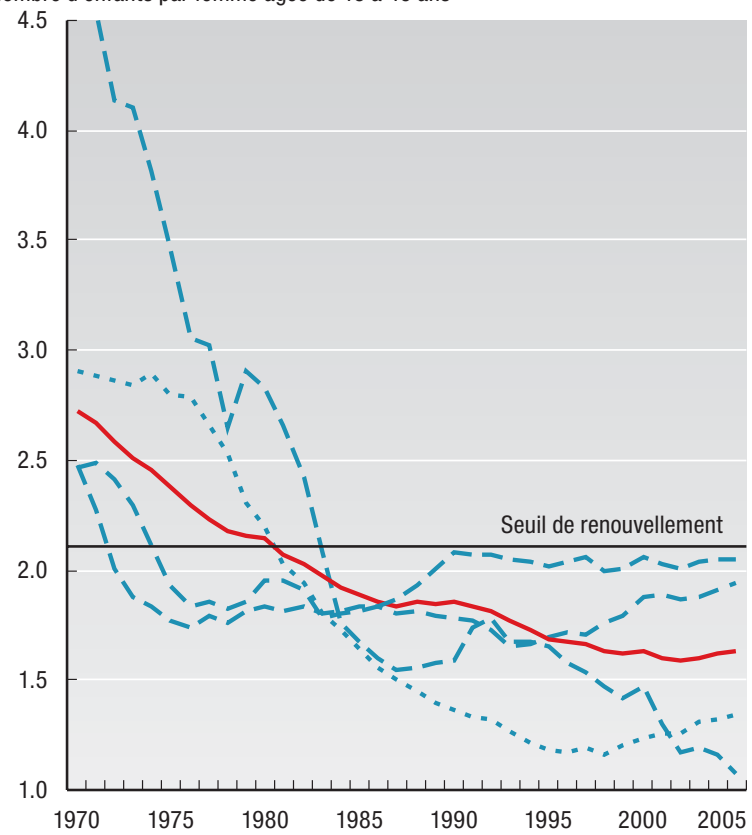

Source : Eco-Santé OCDE 2007.

\subsection{3. Âge moyen des mères à la première naissance}

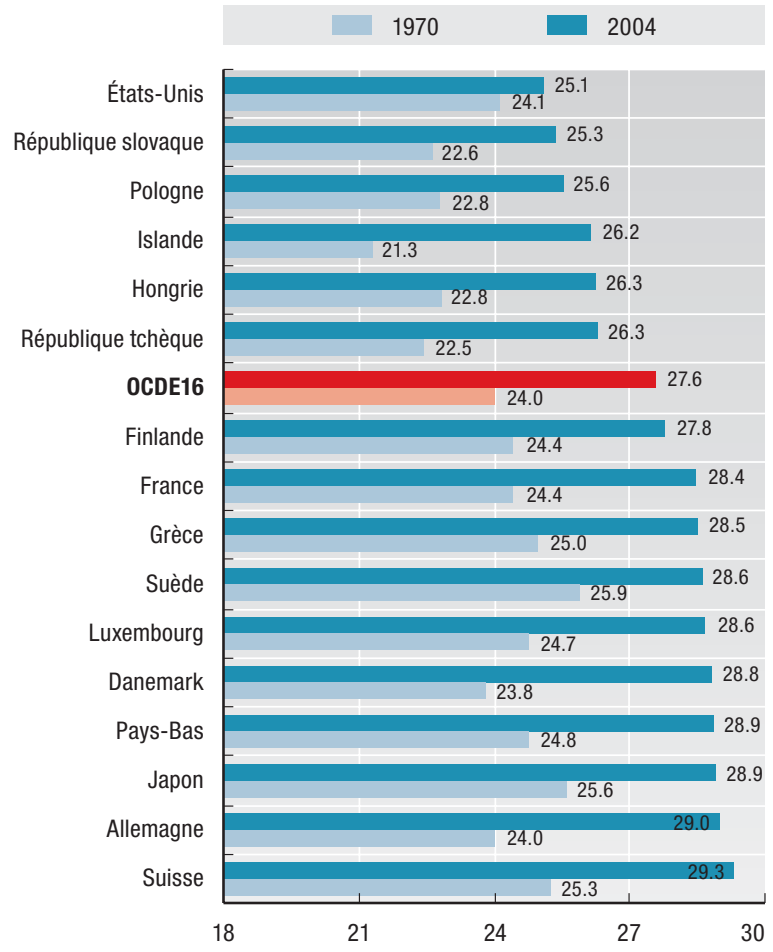

Source : D'Addio et Mira d'Ercole (2005), sauf pour le Danemark (Danish Registry of Birth) et la Grèce (National Statistical Service). StatLink ants http://dx.doi.org/10.1787/128071207166 


\subsection{PRODUIT INTÉRIEUR BRUT (PIB) ET INÉGALITÉS DE REVENUS}

Alors que le PIB permet de mesurer le niveau de développement économique d'un pays en additionnant la valeur de tous les biens et services produits au cours d'une période, le PIB par habitant fournit une indication du niveau de vie de sa population.

En 2005, le PIB moyen par habitant de la zone OCDE, exprimé en dollars PPA (parité de pouvoir d'achat) était légèrement supérieur à 30000 USD, mais l'écart entre le pays ayant le PIB le plus élevé et celui ayant le PIB le moins élevé était de un à neuf (graphique 1.3.1). En dehors du Luxembourg (voir l'encadré "Définition et écarts " cidessous), ce sont la Norvège et les États-Unis qui ont le PIB par habitant le plus élevé. À l'autre extrémité de l'échelle, la Turquie et le Mexique ont le PIB par habitant le plus bas ( $26 \%$ et $35 \%$ respectivement de la moyenne de l'OCDE). Plus de la moitié des pays de l'OCDE se situent dans une fourchette comprise entre 25000 et 35000 USD PPA.

Au cours des quinze dernières années, la progression réelle du PIB par habitant a été en moyenne de $2.2 \%$ par an. L'Irlande et la Corée ont fait nettement mieux que la moyenne avec un taux annuel de croissance de $5.3 \%$ et $4.7 \%$ respectivement. Après avoir, dans un premier temps, enregistré une baisse de leur PIB réel dans les premières années de leur transition vers une économie de marché, la Pologne, la République slovaque et la Hongrie ont, elles aussi, affiché un taux de croissance de leur PIB par habitant supérieur à $3 \%$ par an en moyenne depuis 1990. En revanche, quatre grandes puissances économiques (le Japon, l'Italie, l'Allemagne et la France) ont connu une croissance lente avec un taux égal à environ la moitié du taux moyen de la zone OCDE (graphique 1.3.2).
Alors qu'un PIB par habitant plus élevé est généralement associé à un meilleur état de santé de la population, la relation est moins nette à des niveaux de revenu national plus élevés et il existe des différences d'état de santé importantes entre des pays de l'OCDE ayant des revenus par habitant analogues (voir l'indicateur 2.1 "Espérance de vie à la naissance "). En soi, le PIB par habitant ne fournit qu'un niveau moyen du revenu national et ne mesure pas la répartition des revenus dans la population. Or, la répartition du revenu national peut également avoir un impact sur l'état de santé de la population d'un pays. Certains analystes ont trouvé une certaine relation entre l'espérance de vie et les inégalités de revenus, l'espérance de vie étant plus grande dans les pays où les inégalités de revenus sont moindres (Wilkinson, 1996, 2000). Le graphique 1.3.3 présente une mesure des inégalités de revenus dans les pays de l'OCDE, exprimée par le coefficient de Gini, et les changements observés depuis le milieu des années 80 . Alors que dans la plupart des pays les inégalités de revenu n'ont guère (voire pas) varié sur la période, dans d'autres, en revanche, elles ont fortement varié. En Espagne et en Irlande, la répartition des revenus est devenue sensiblement plus égalitaire alors que c'est l'inverse qui s'est produit en Nouvelle-Zélande, en Finlande ou en Suède même si cette dernière reste l'un des pays de l'OCDE où les inégalités de revenus sont les plus faibles. En 2000, les pays qui affichaient les plus fortes inégalités de revenus étaient le Mexique et la Turquie, suivis de la Pologne et des États-Unis.

\section{Définition et écarts}

Le produit intérieur brut (PIB) est l'indicateur habituellement utilisé pour apprécier la valeur des biens et services produits par un pays au cours d'une période. Trois méthodes différentes peuvent être utilisées pour mesurer le PIB. On peut : 1) prendre la différence entre la production brute et les consommations intermédiaires ; 2) prendre la somme des revenus du travail, des profits nets et des amortissements; ou 3) prendre la somme des dépenses de consommation, des investissements, des variations de stocks et des exportations nettes (OCDE, 2007b).

Chaque pays calcule son PIB dans sa propre monnaie et peut l'exprimer en prix courants (PIB nominal) ou en prix constants (PIB réel). Le PIB réel, qui est mesuré en déflatant les composantes des dépenses par les indices de prix appropriés, convient mieux pour des comparaisons dans le temps.

Les comparaisons du PIB entre les pays doivent s'effectuer uniquement sur la base des parités de pouvoir d'achat (PPA) (voir également l'annexe B, p. 192). En raison des marges d'erreur statistiques que comportent le PIB et les PPA, on considère généralement que des différences de PIB par habitant de $5 \%$ ou moins entre les pays ne sont pas significatives.

Désormais, pratiquement tous les pays de l'OCDE suivent le système des comptes nationaux de 1993. Le Luxembourg et, dans une moindre mesure, la Suisse, qui ont un nombre relativement important de travailleurs frontaliers, ont un PIB par habitant surestimé par rapport à celui des autres pays. L'Irlande se trouve dans une situation analogue du fait de l'influence des sociétés étrangères opérant dans le pays.

La répartition des revenus est mesurée par le coefficient de Gini. Le coefficient de Gini est défini par la zone située entre la courbe de Lorenz (qui montre les proportions cumulées de la population, des groupes les plus pauvres aux groupes les plus riches, par rapport à la part cumulée des revenus qu'ils perçoivent) et la ligne de $45^{\circ}$ (" ligne de l'égalité parfaite »). Les valeurs s'échelonnent entre 0 dans le cas de «l'égalité parfaite » et 100 dans le cas de «l'inégalité parfaite ». Une augmentation du coefficient de Gini représente donc une augmentation des inégalités. 


\section{CONTEXTE DÉMOGRAPHIQUE ET ÉCONOMIQUE}

\subsection{PRODUIT INTÉRIEUR BRUT (PIB) ET INÉGALITÉS DE REVENUS}

\subsubsection{Produit intérieur brut par habitant, 2005}

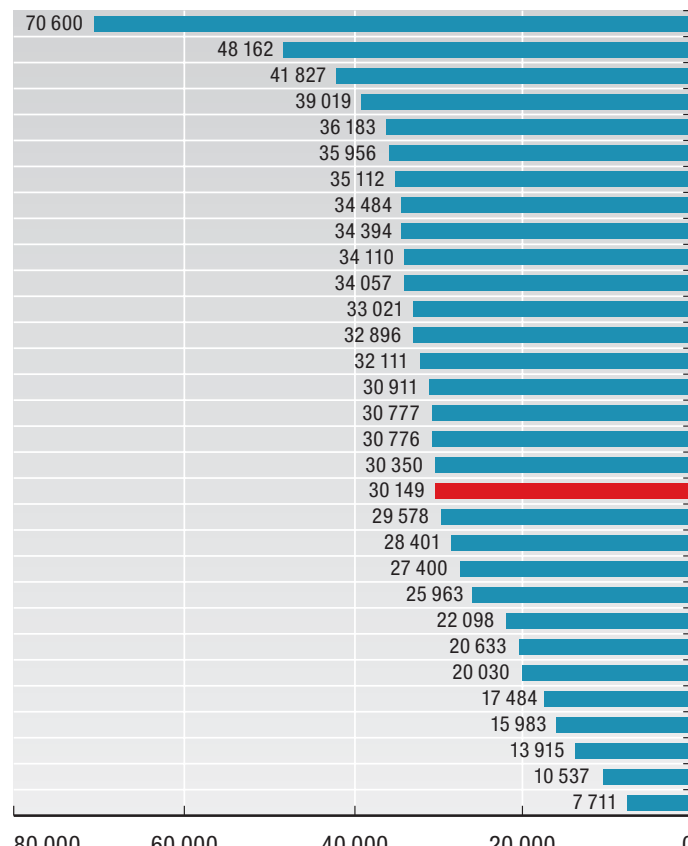

USD PPA

1. Taux de croissance annuel moyen 1992-2005.

2. Taux de croissance annuel moyen 1991-2005.
1.3.2. Taux de croissance annuel du PIB par habitant, en termes réels, 1990 à 2005

Luxembourg
Norvège
États-Unis
Irlande
Islande
Suisse
Pays-Bas
Australie
Autriche
Danemark
Canada
Belgique
Royaume-Uni
Suède
Finlande
Japon
Allemagne
France
OCDE
Grèce
Italie
Espagne
Nouvelle-Zélande
Corée
République tchèque
Portugal
Hongrie
République slovaque
Pologne
Mexique
Turquie

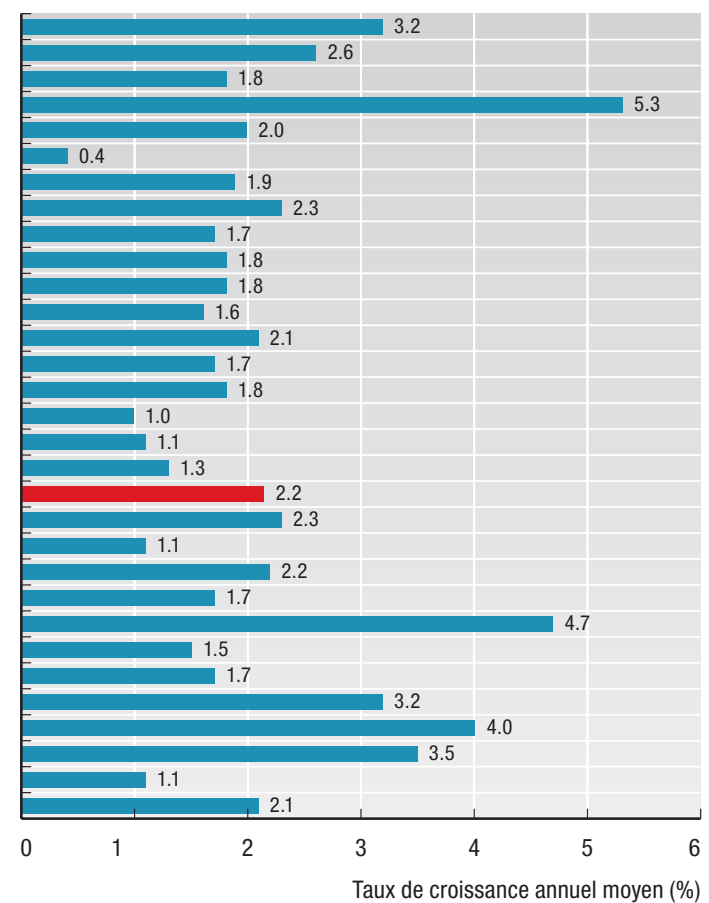

\subsubsection{Inégalité des revenus, coefficient de Gini, milieu des années 80 et 90 , et 2000}

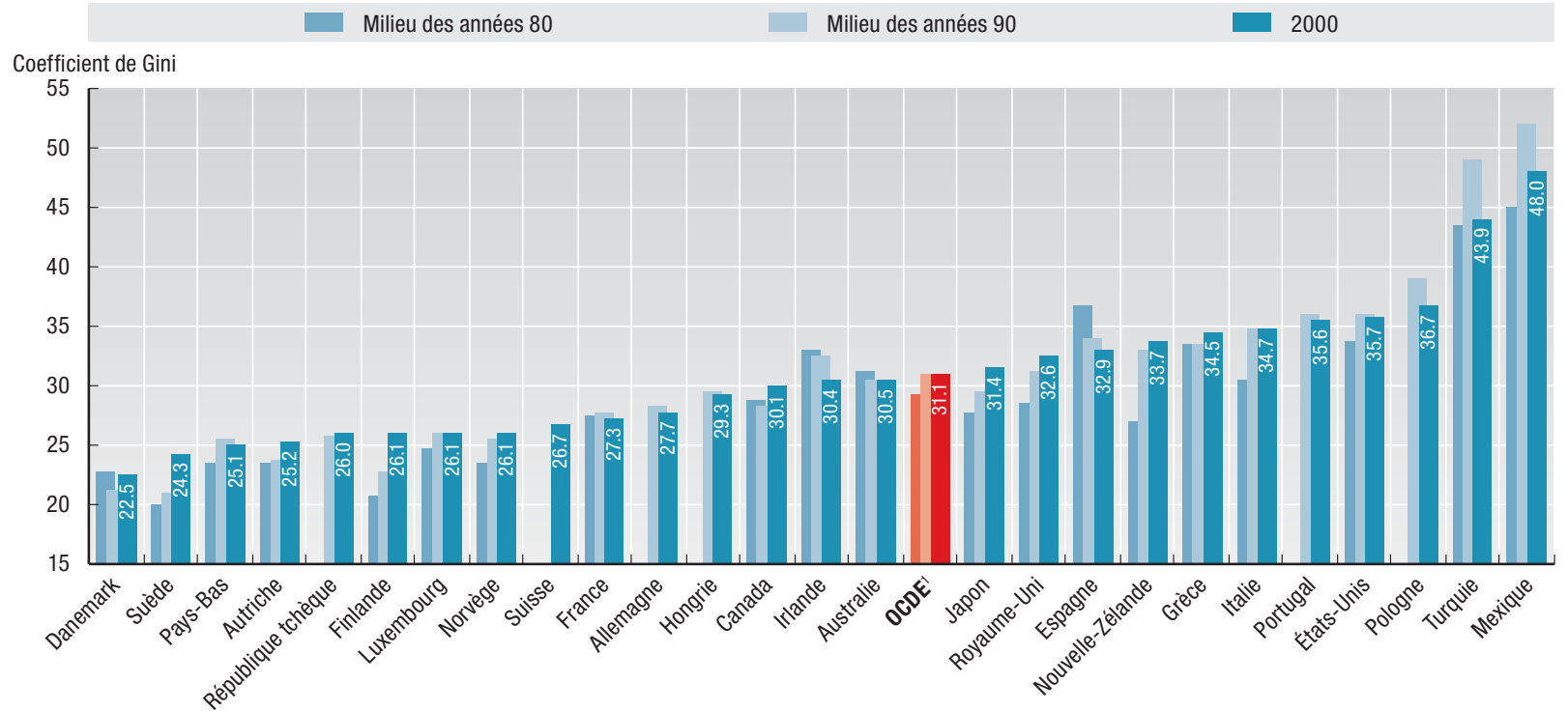

Note: Un coefficient de Gini plus élevé reflète une plus grande inégalité des revenus.

1. La moyenne OCDE comprend les pays pour lesquels les données sont disponibles sur l'ensemble de la période.

Source : Förster et Mira d'Ercole (2005). 


\section{2. ÉTAT DE SANTÉ}

2.1. ESPÉRANCE DE VIE À LA NAISSANCE . . . . . . . . . . . 20

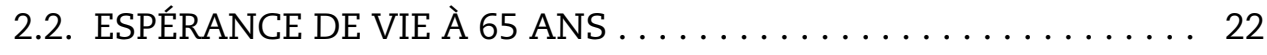

2.3. MORTALITÉ PRÉMATURÉE ...................... 24

2.4. MORTALITÉ DUE AUX MALADIES CARDIAQUES

ET AUX ACCIDENTS VASCULAIRES CÉRÉBRAUX ........ 26

2.5. MORTALITÉ DUE AU CANCER $\ldots \ldots \ldots \ldots \ldots \ldots \ldots \ldots \ldots$

2.6. MORTALITÉ DUE AUX ACCIDENTS DE LA ROUTE. . . . . . . 30

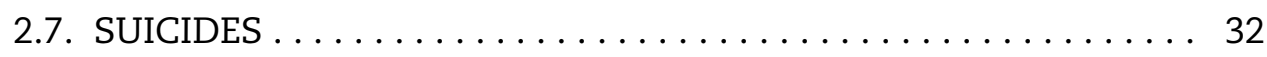

2.8. MORTALITÉ INFANTILE. . . . . . . . . . . . . . . 34

2.9. SANTÉ DU NOURRISSON : FAIBLE POIDS À LA NAISSANCE . . . 36

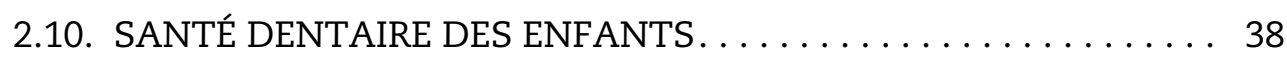

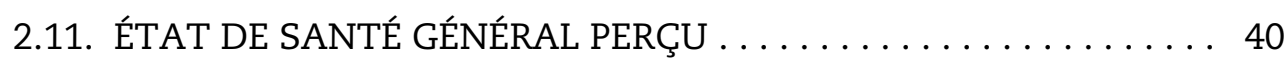

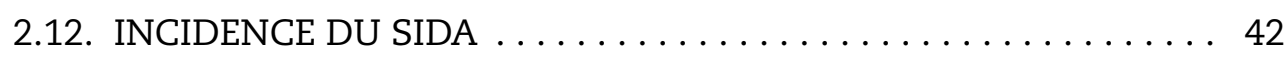




\subsection{ESPÉRANCE DE VIE À LA NAISSANCE}

L'espérance de vie à la naissance a considérablement augmenté dans les pays de l'OCDE au cours des dernières décennies, reflétant une forte baisse des taux de mortalité à tous les âges. Ces gains de longévité peuvent être attribués à un certain nombre de facteurs, notamment à l'élévation des niveaux de vie, à l'amélioration de l'hygiène de vie et du niveau d'éducation mais aussi à un meilleur accès à des services de santé de qualité. D'autres facteurs, tels que l'amélioration de l'alimentation, des conditions sanitaires et du logement, ont également joué un rôle, en particulier dans les pays dont l'économie était en phase de développement (OCDE, 2004a).

Dans la zone OCDE, l'espérance de vie à la naissance pour l'ensemble de la population était en moyenne de 78.6 ans en 2005 , soit un gain de dix années par rapport à 1960 (graphique 2.1.1). Dans un tiers des pays de l'OCDE, elle était de plus de 80 ans en 2005 . Le pays qui affichait l'espérance de vie la plus élevée était le Japon avec une espérance de vie de 82.1 ans pour l'ensemble de sa population (hommes et femmes). À l'autre extrémité de l'échelle, c'est en Turquie (suivie de la Hongrie) qu'elle était la plus faible. Mais si en Hongrie l'espérance de vie n'a augmenté que dans des proportions modestes depuis 1960, en Turquie elle a fortement progressé permettant au pays de rattraper rapidement la moyenne de l'OCDE.

Dans la zone OCDE, l'écart d'espérance de vie entre les sexes était de 5.7 ans en moyenne en 2005, avec une espérance de vie de 75.7 ans pour les hommes et de 81.4 ans pour les femmes (graphique 2.1.2). Entre 1960 et 2005, cet écart a augmenté légèrement en moyenne dans l'ensemble des pays (tableaux A.2.1b et A.2.1c). Ce résultat masque cependant des évolutions différentes entre les premières décennies et les décennies ultérieures. Dans de nombreux pays, l'écart d'espérance de vie entre les sexes s'est fortement creusé dans les années 60 et 70, pour se resserrer au cours des vingtcinq dernières années, reflétant une progression plus rapide de l'espérance de vie des hommes dans la plupart des pays de l'OCDE. Ce phénomène de convergence observé sur les vingt-cinq dernières années peut être attribué, du moins en partie, à la diminution des écarts entre les sexes au niveau des comportements à risque (tels que la consommation de tabac), ainsi qu'à une forte baisse des taux de mortalité liés aux maladies cardiovasculaires chez les hommes.

Il est difficile d'estimer la contribution relative des nombreux facteurs médicaux et non médicaux aux variations de l'espérance de vie au fil du temps et entre les pays. Un revenu national (mesuré par le PIB par habitant) supérieur est généralement associé à une espérance de vie à la naissance plus grande, même si la relation est moins étroite à des niveaux de revenu national plus élevés (graphique 2.1.3). On note également des différences d'espérance de vie notoires entre des pays de l'OCDE qui ont le même revenu par habitant. Si le Japon et l'Espagne ont des espérances de vie plus élevées que prévu sur la seule base de leur PIB par habitant, aux États-Unis, au Danemark et en Hongrie, en revanche, c'est l'inverse.

Le graphique 2.1.4 montre la relation existant entre l'espérance de vie à la naissance et les dépenses de santé par habitant dans les pays de l'OCDE. Comme pour le PIB par habitant, des dépenses de santé par habitant plus élevées sont généralement associées à une espérance de vie à la naissance plus grande, même si cette relation tend à être moins nette dans les pays où les dépenses de santé par habitant sont plus élevées. Ici encore, le Japon et l'Espagne affichent des espérances de vie relativement élevées par rapport à leur niveau de dépenses de santé, et les États-Unis, le Danemark et la Hongrie des espérances de vie relativement basses.

Ces corrélations simples sont intéressantes mais une analyse plus approfondie s'impose. Les variations du PIB par habitant peuvent influencer à la fois l'espérance de vie et les dépenses de santé par habitant. Pour expliquer les variations de l'espérance de vie entre pays, il faut également prendre en compte de nombreux autres facteurs en plus du revenu national et des dépenses de santé totales.

\section{Définition et écarts}

L'espérance de vie mesure le nombre moyen d'années qu'une personne peut espérer vivre, sur la base d'un ensemble donné de taux de mortalité par âge. Cependant, les taux de mortalité par âge effectifs d'une cohorte de naissance donnée ne peuvent pas être connus à l'avance. Si les taux de mortalité par âge diminuent (comme cela a été le cas au cours des dernières décennies dans les pays de l'OCDE), la durée de vie effective des individus sera supérieure à l'espérance de vie calculée avec les taux de mortalité actuels.

Chaque pays calcule l'espérance de vie de sa population selon des méthodologies qui peuvent varier quelque peu d'un pays à l'autre. Ces différences de méthodologie peuvent affecter la comparabilité des estimations notifiées par les pays car, en fonction de la méthode, l'espérance de vie calculée d'un pays peut varier d'une fraction d'année. Pour l'ensemble de la population, l'espérance de vie à la naissance est calculée par le Secrétariat de l'OCDE pour tous les pays, sur la base de la moyenne non pondérée de l'espérance de vie des hommes et des femmes. 


\subsubsection{Espérance de vie à la naissance, population totale, 1960 et 2005}

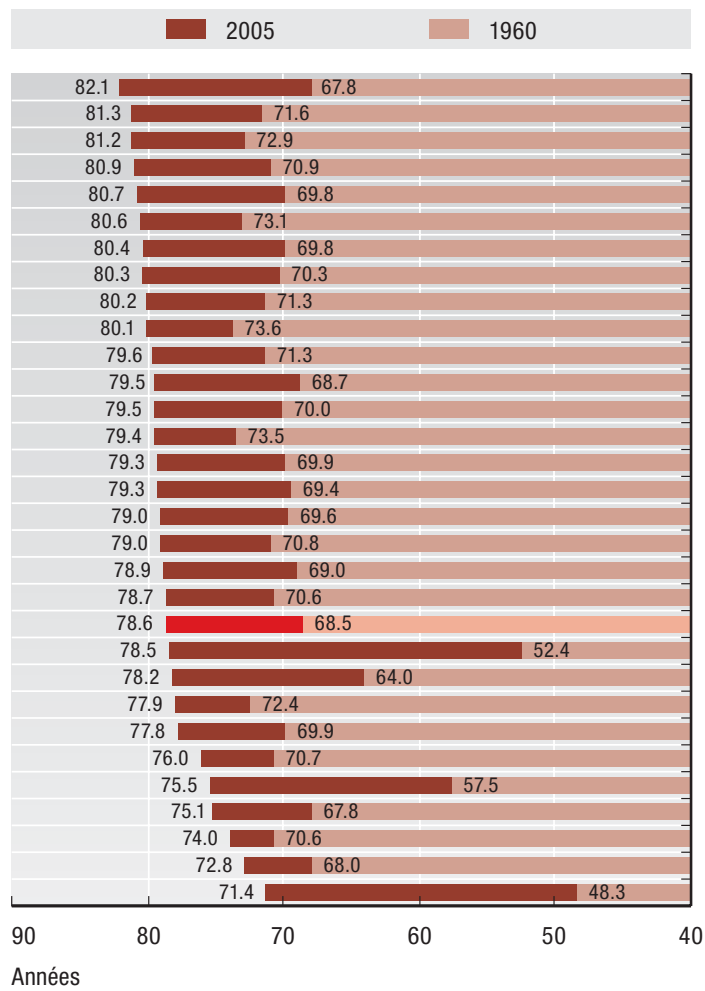

1. 2004 .

\subsubsection{Espérance de vie à la naissance et PIB par habitant, 2005}

Espérance de vie en années

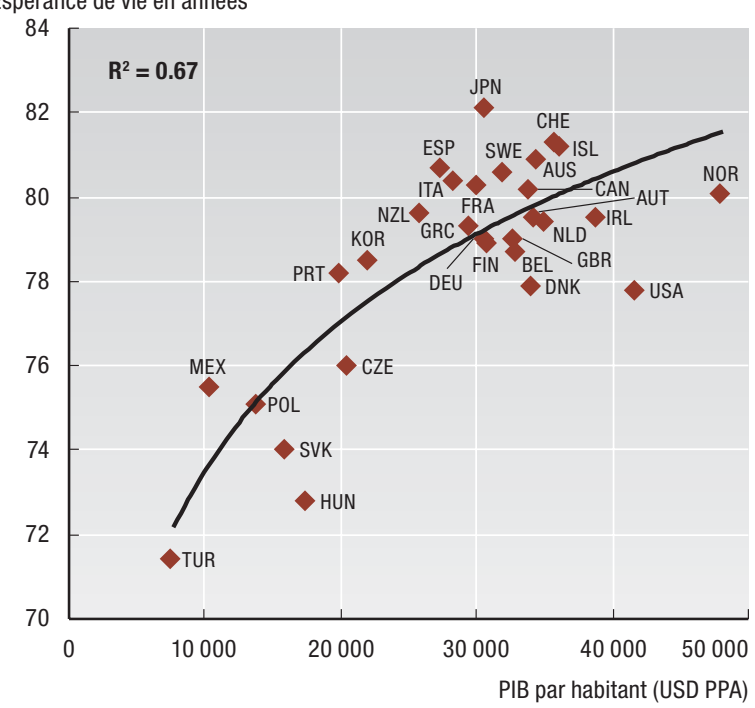

Source : Eco-Santé OCDE 2007.

\subsection{ESPÉRANCE DE VIE À LA NAISSANCE}

\subsubsection{Espérance de vie à la naissance,} par sexe, 2005
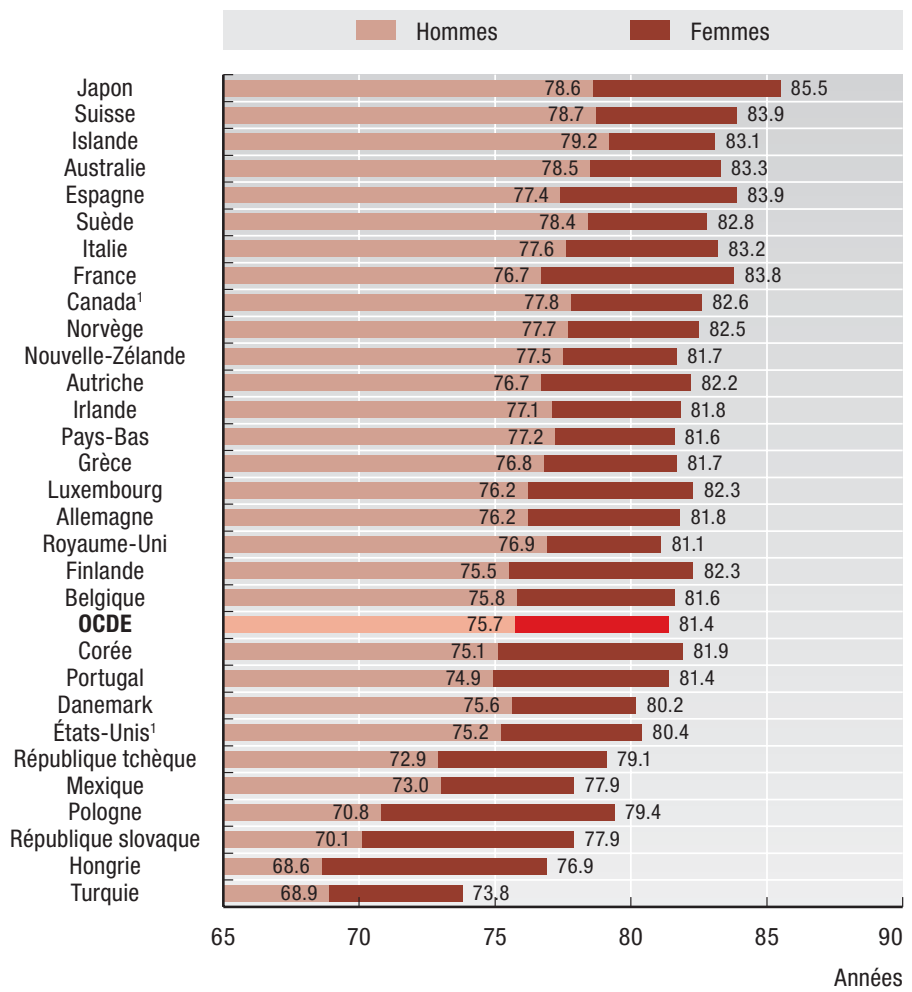

\subsubsection{Espérance de vie à la naissance et dépenses} de santé par habitant, 2005

Espérance de vie en années

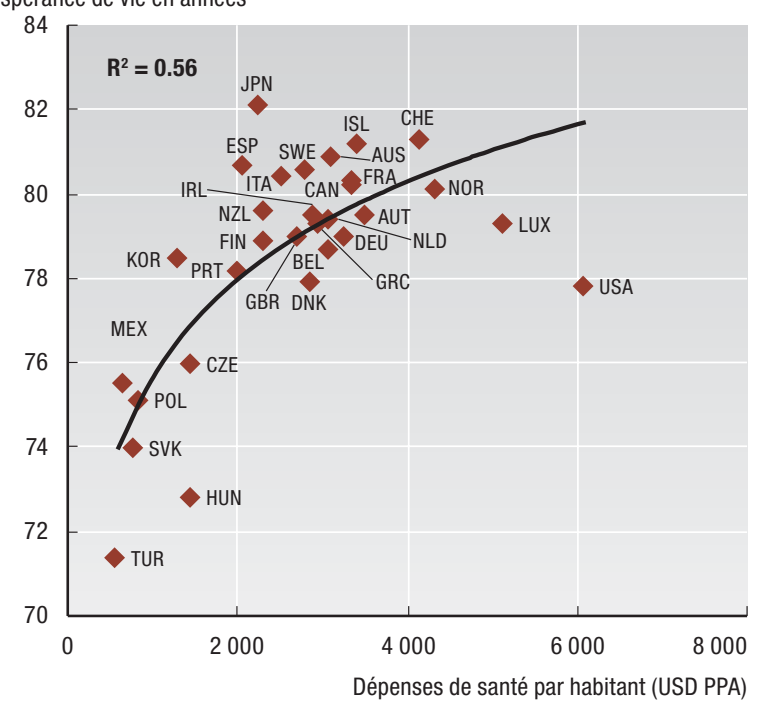

StatLink कils http://dx.doi.org/10.1787/128134483043 


\subsection{ESPÉRANCE DE VIE À 65 ANS}

$\mathrm{Au}$ cours des dernières décennies, l'espérance de vie à 65 ans a considérablement augmenté, pour les hommes comme pour les femmes, dans tous les pays de l'OCDE. Parmi les facteurs qui expliquent les gains d'espérance de vie à 65 ans figurent les progrès des soins médicaux, combinés à l'amélioration de l'accès aux soins de santé, de l'hygiène de vie et des conditions de vie avant et après 65 ans.

En 2005, l'espérance de vie à 65 ans dans les pays de l'OCDE était en moyenne de près de 20 ans pour les femmes et de plus de 16 ans pour les hommes (graphique 2.2.1; tableaux A.2.2a et A.2.2b). Cela représente un gain moyen de quatre ans pour les femmes et de 3.5 ans pour les hommes depuis 1970. L'écart d'espérance de vie à 65 ans entre hommes et femmes s'est donc légèrement creusé entre 1970 et 2005 dans de nombreux pays.

De même, l'espérance de vie à 80 ans dans les pays de l'OCDE a augmenté en moyenne plus vite pour les femmes que pour les hommes au cours des trente-cing dernières années (graphique 2.2.2). En 2005, l'espérance de vie à 80 ans était en moyenne de 8.8 ans (contre 6.5 ans en 1970) pour les femmes et de 7.3 ans (contre 5.7 ans en 1970) pour les hommes.

$\mathrm{Au}$ cours des dernières décennies, le Japon a enregistré des gains d'espérance de vie à 65 ans particulièrement élevés : près de huit ans pour les femmes et 5.6 ans pour les hommes entre 1970 et 2005. En conséquence, le Japon était en 2005 le pays de l'OCDE où les femmes et les hommes avaient l'espérance de vie à 65 ans la plus longue : 23.2 et 18.1 ans respectivement (l'Australie affichait un chiffre identique pour les hommes). Ces gains au Japon peuvent s'expliquer, du moins en partie, par une forte baisse de la mortalité liée aux maladies cardio-vasculaires et aux accidents vasculaires cérébraux chez les personnes âgées au cours des dernières décennies (OCDE, 2003a ; Moon et al., 2003).

Les gains de longévité enregistrés par les personnes âgées dans tous les pays de l'OCDE au cours des dernières décennies, combinés au déclin tendanciel des taux de fécondité, concourent à une hausse régulière de la proportion de personnes âgées dans les pays de l'OCDE (voir les indicateurs 1.1 "Population totale et structure de la population » et 1.2 « Taux de fécondité »).

L'espérance de vie à 65 ans devrait continuer à progresser dans les décennies à venir. Sur la base des données démographique des Nations unies et de la Banque mondiale on a estimé que l'espérance de vie à 65 ans dans les pays de l'OCDE devrait être de 21.6 ans pour les femmes et de 18.1 ans pour les hommes en moyenne en 2040 (OCDE, 2007e).

La question de savoir si les populations vieillissantes conservent un état de santé et fonctionnel satisfaisant à mesure que leur espérance de vie s'allonge a d'importantes implications pour les systèmes de santé et de soins de longue durée. De récents travaux de l'OCDE ont montré que si dans certains pays (par exemple les États-Unis, l'Italie et les Pays-Bas) les incapacités sévères tendent à diminuer parmi les populations âgées, le constat n'est pas universel (graphique 2.2.3). Dans d'autres pays (par exemple l'Australie et le Canada), le taux des incapacités sévères est stable et dans d'autres encore (par exemple, la Suède et le Japon), il paraît avoir augmenté au cours des cinq à dix dernières années. S'ajoutant au vieillissement de la population, ces tendances donnent à penser que dans les prochaines décennies une offre croissante de soins de longue durée sera nécessaire dans la plupart, sinon tous les pays de l'OCDE (Lafortune et al., 2007).

\section{Définition et écarts}

L'espérance de vie est le nombre moyen d'années qu'une personne ayant atteint un âge donné peut espérer vivre, calculé sur la base d'un ensemble donné de taux de mortalité par âge. Cependant, les taux de mortalité par âge effectifs d'une cohorte de naissance particulière ne peuvent pas être connus à l'avance. Si les taux de mortalité par âge diminuent (comme cela a été le cas dans les pays de l'OCDE au cours des dernières décennies), la durée de vie effective des individus sera plus longue que l'espérance de vie calculée avec les taux de mortalité actuels.

Chaque pays calcule l'espérance de vie de sa population selon des méthodologies qui peuvent varier quelque peu d'un pays à l'autre. Ces différences de méthodologie peuvent affecter la comparabilité des estimations notifiées par les pays car, en fonction de la méthode, l'espérance de vie estimée d'un pays peut varier d'une fraction d'année. 


\section{2. ÉTAT DE SANTÉ}

2.2. ESPÉRANCE DE VIE À 65 ANS

2.2.1. Espérance de vie à 65 ans, par sexe, 1970 et 2005
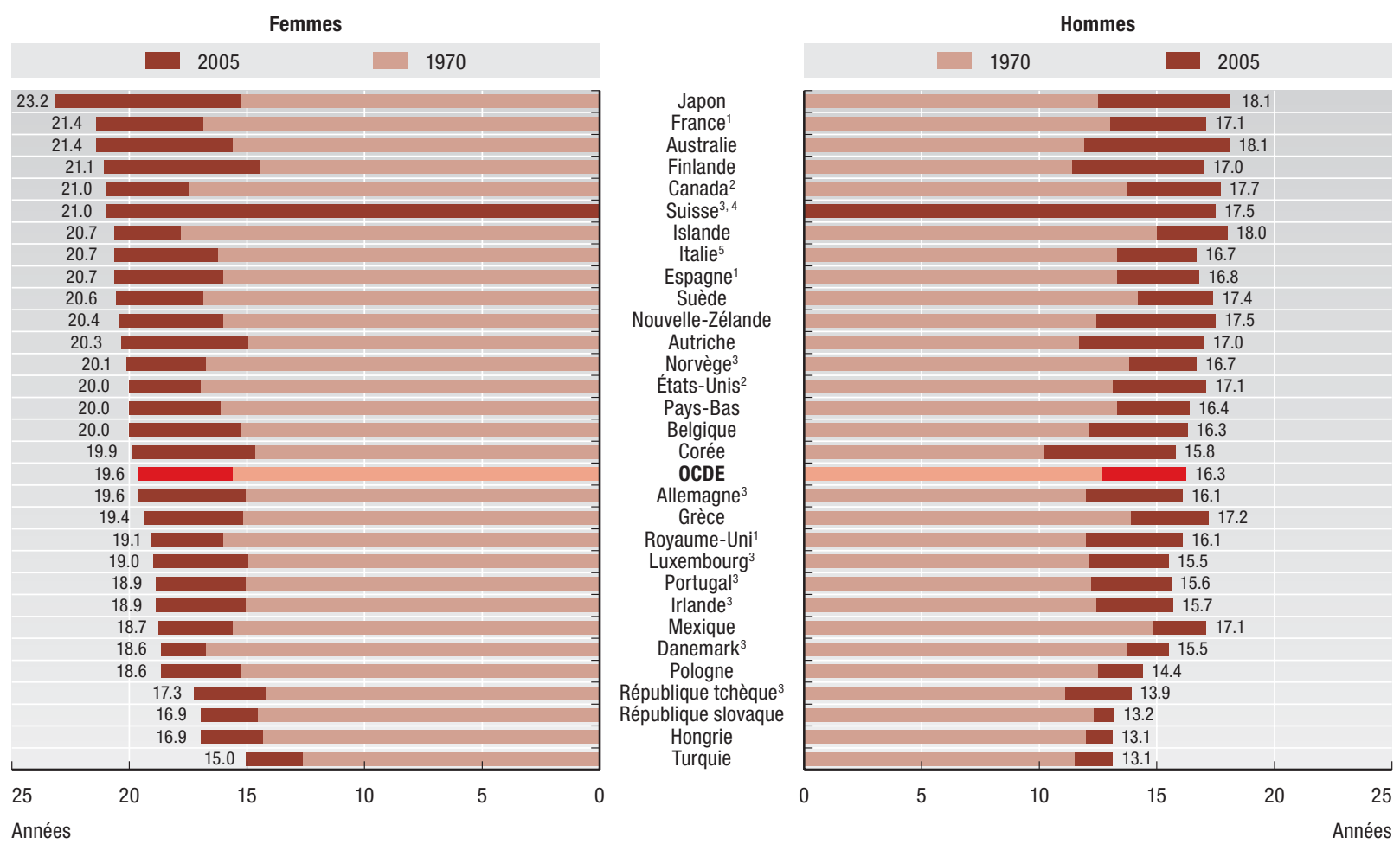

1. 2002. 2. 2004. 3. 2003.

4. Données non disponibles pour 1970.

5. 2001.

2.2.2. Évolution de l'espérance de vie à 65 ans et 80 ans, hommes et femmes, moyenne OCDE, 1970-2005

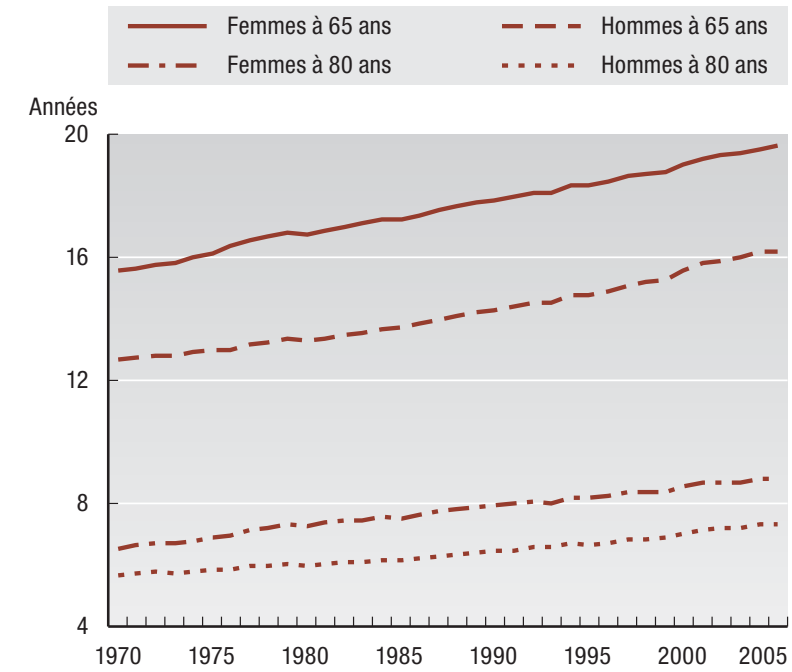

Source : Eco-Santé OCDE 2007.

\subsection{3. Évolution des taux d'incapacité sévère parmi la population âgée de 65 ans et plus dans quelques pays de l'OCDE, 1980-2005}

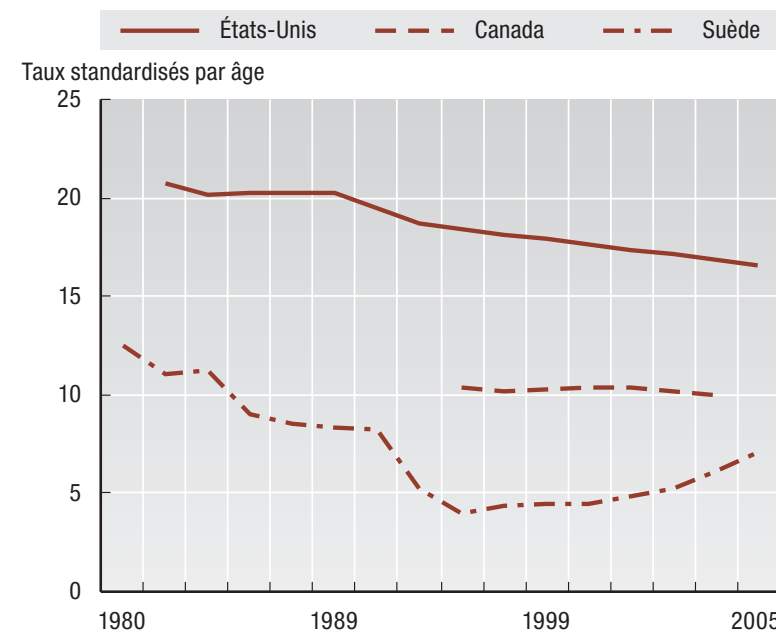

Note : Pour la Suède, les données se rapportent à la population âgée de 65 à 84 ans.

Source: Lafortune, Balestat et al. (2007).

StatLink ला15स http://dx.doi.org/10.1787/128142583121 


\section{2. ÉTAT DE SANTÉ}

\subsection{MORTALITÉ PRÉMATURÉE}

La mortalité prématurée, mesurée en termes d'années potentielles de vie perdues, met l'accent sur les décès parmi les groupes les plus jeunes de la population. La mortalité infantile et les décès par suite de maladies et de blessures affectant des enfants et de jeunes adultes influent fortement sur les valeurs de cet indicateur.

Dans les pays de l'OCDE, la mortalité prématurée a été, en moyenne, réduite de plus de moitié depuis 1970 (graphique 2.3.1). Dans les premières années, l'évolution à la baisse de la mortalité infantile a été un facteur majeur de la baisse de la mortalité prématurée (voir l'indicateur 2.8 " Mortalité infantile »). Plus récemment, la baisse parmi les adultes des décès dus à des maladies cardiaques a contribué à la baisse globale de la mortalité prématurée dans de nombreux pays (voir l'indicateur 2.4 " Mortalité due aux maladies cardiaques et aux accidents vasculaires cérébraux »).

Le Portugal a enregistré depuis 1970 une baisse particulièrement rapide de sa mortalité prématurée pour les hommes comme pour les femmes. La forte diminution de la mortalité infantile a fortement contribué à cette baisse. En Hongrie, en revanche, la mortalité prématurée a diminué plus lentement, en particulier chez les hommes, ceci en raison de la persistance de niveaux élevés de mortalité due aux maladies cardiaques (qui sont actuellement plus de deux fois supérieures à la moyenne de l'OCDE) et aux cirrhoses et autres maladies du foie (près de cinq fois supérieures à la moyenne de l'OCDE). Ces phénomènes reflètent des modes de vie nocifs pour la santé chez les hommes en Hongrie, notamment en termes de consommation de tabac et d'alcool. Des taux de suicides élevés chez les hommes en Hongrie contribuent également à ces niveaux élevés de mortalité prématurée.

Les États-Unis déclarent également des taux de mortalité prématurée supérieurs à la moyenne de l'OCDE, de $28 \%$ pour les hommes et de $42 \%$ pour les femmes (graphique 2.3.2). Cette mortalité prématurée peut être attribuée pour moitié dans le cas des hommes (et pour près d'un tiers dans le cas des femmes) à des décès résultant de causes externes incluant les accidents, les suicides et les homicides. Aux États-Unis, les décès prématurés par homicide sont plus de cinq fois supérieurs à la moyenne de l'OCDE.

En moyenne dans les pays de l'OCDE, les principales causes d'années de vie potentielles perdues avant 70 ans pour les hommes sont des causes externes incluant les accidents et la violence $(29 \%)$; viennent ensuite le cancer $(21 \%)$ et les maladies du système circulatoire $(18 \%)$. Pour les femmes, les principales causes sont le cancer (31\%), des causes externes (17\%) et les maladies du système circulatoire $(13 \%)$.

\section{Définition et écarts}

Les années potentielles de vie perdues (APVP) est un indicateur synthétique de la mortalité prématurée qui fournit un moyen explicite de pondérer les décès survenant à des âges relativement jeunes. Le calcul des APVP consiste à additionner les décès par âge se produisant à chaque âge et pondérés par le nombre d'années restant à vivre jusqu'à une limite d'âge donnée, établie ici à 70 ans. Par exemple, un décès survenant à l'âge de cinq ans est comptabilisé comme un nombre d'APVP de 65 ans. L'indicateur est exprimé pour 100000 femmes et hommes. 
2.3. MORTALITÉ PRÉMATURÉE

\subsubsection{Diminution des années potentielles de vie perdues (APVP), population totale, $\mathbf{1 9 7 0 - 2 0 0 4}$}

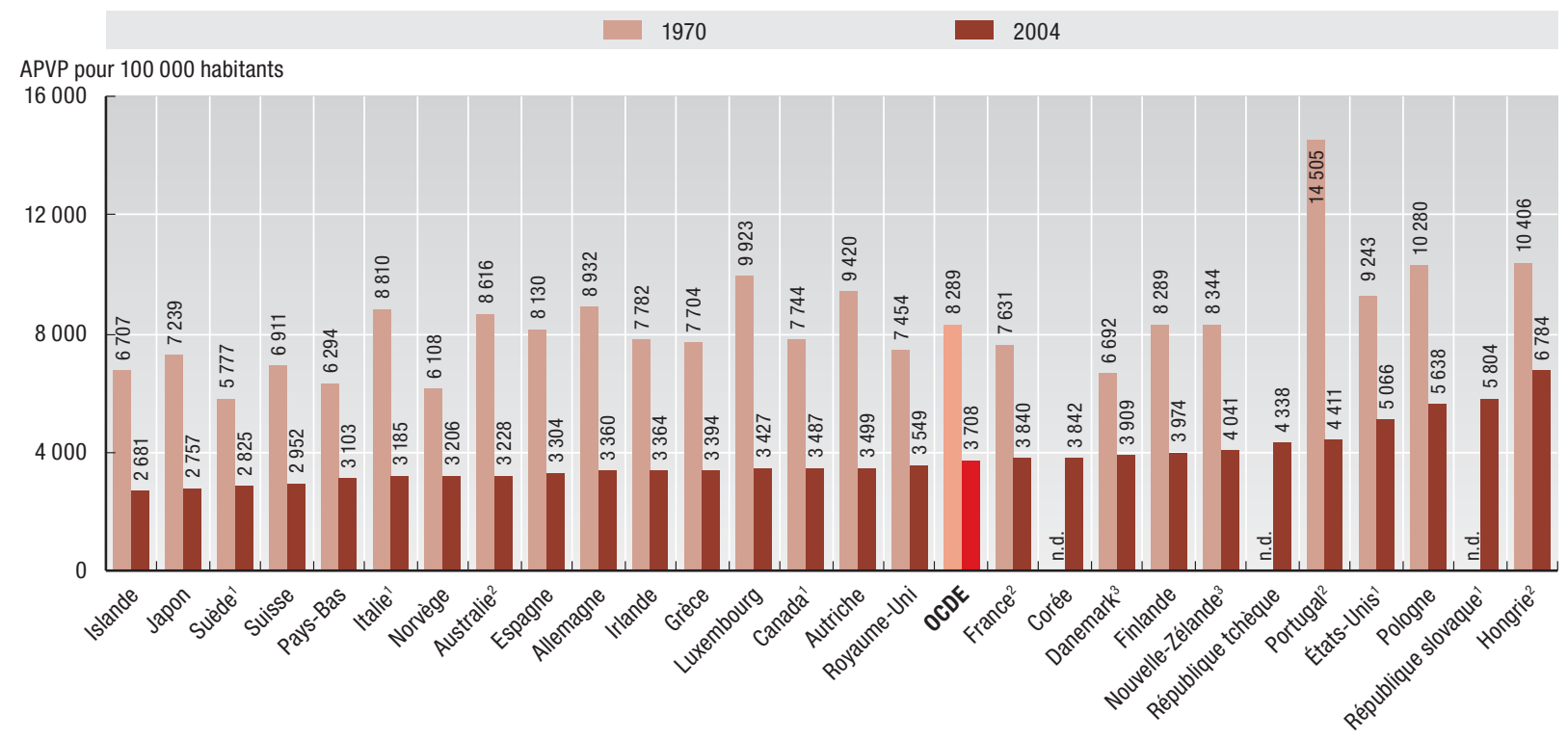

1. 2002. 2. 2003. 3. 2001.

\subsubsection{Années potentielles de vie perdues (APVP) des femmes et des hommes, 2004}

Pays ordonnés des taux les plus faibles aux plus élevés pour les femmes

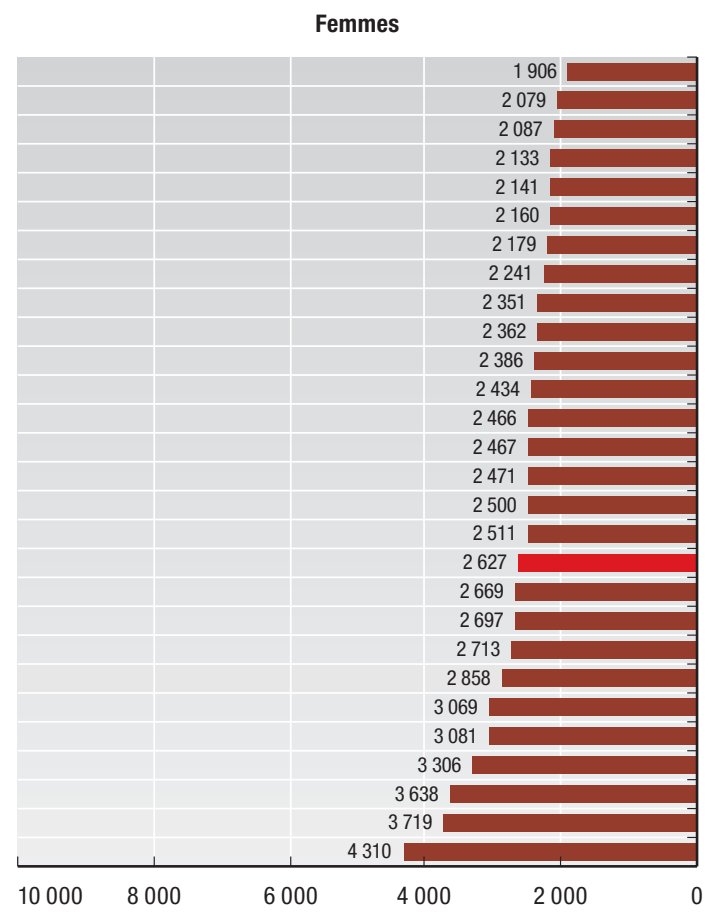

APVP pour 100000 femmes

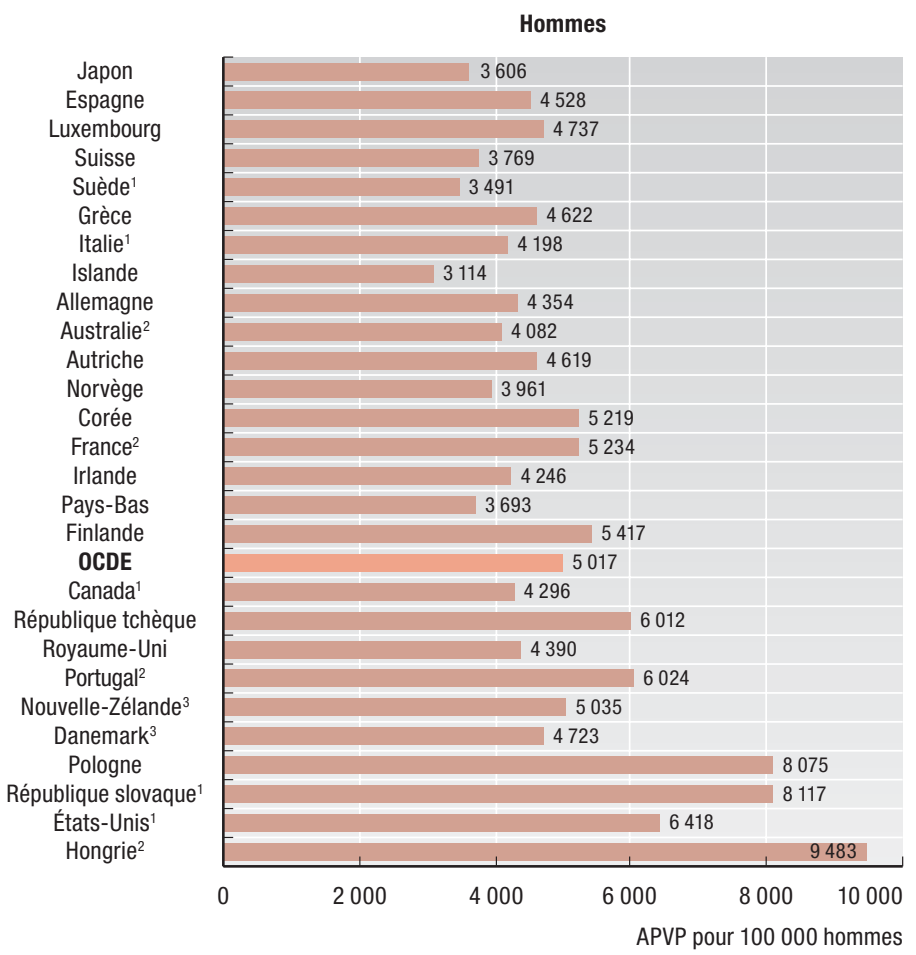

1. 2002. 2. 2003. 3. 2001.

Source: Eco-Santé OCDE 2007. Les statistiques de mortalité sont extraites de la base de données sur la mortalité de l'OMS.

StatLink तीजा http://dx.doi.org/10.1787/128187367417 


\subsection{MORTALITÉ DUE AUX MALADIES CARDIAQUES ET AUX ACCIDENTS VASCULAIRES CÉRÉBRAUX}

En 2004, les crises cardiaques et les accidents vasculaires cérébraux, considérés ensemble, ont été responsables ensemble d'un quart de tous les décès enregistrés dans les pays de l'OCDE.

Les crises cardiaques sont provoquées par l'accumulation de dépôts graisseux sur la paroi interne d'une artère coronaire, qui restreint le flux sanguin vers le cœur. En 2004, ces maladies ont causé à elles seules $16 \%$ de l'ensemble des décès dans les pays de l'OCDE. Cependant, la mortalité due aux crises cardiaques varie considérablement entre les pays (graphique 2.4.1). Le pays qui affiche le taux de mortalité dû aux crises cardiaques le plus élevé (pour les hommes et les femmes) est la République slovaque, suivie de la Hongrie et de la République tchèque. Les taux de mortalité par crise cardiaque sont aussi relativement élevés en Finlande, en Nouvelle-Zélande et aux États-Unis, avec des valeurs plusieurs fois supérieures à celles du Japon et de la Corée, les deux pays aux taux de mortalité les plus bas. La variabilité des taux de mortalité dus aux crises cardiaques se conforme clairement à un modèle régional. Les deux pays asiatiques de l'OCDE qui affichent la plus faible mortalité par crise cardiaque sont suivis de quatre pays situés dans le sud de l'Europe (la France, l'Espagne, le Portugal et l'Italie) et des Pays-Bas. Cela renforce l'hypothèse selon laquelle les écarts de mortalité par crise cardiaque entre les pays s'expliquent en partie par des facteurs de risque sous-jacents tels que le régime alimentaire.

On observe un écart significatif entre les sexes, la mortalité par crise cardiaque étant beaucoup plus élevée chez les hommes que chez les femmes dans tous les pays (graphique 2.4.1). Dans les pays de l'OCDE, en moyenne, les taux de mortalité par crise cardiaque étaient en 2004 près de deux fois plus élevés chez les hommes que chez les femmes.
Depuis 1980, les taux de mortalité par crise cardiaque ont diminué dans presque tous les pays de l'OCDE (tableau A.2.4). Le déclin a été particulièrement remarquable au Danemark, en Suède, en Australie, aux Pays-Bas et au Canada, où ces taux ont diminué de plus de moitié. Un certain nombre de facteurs expliquent cette baisse. La diminution de la consommation de tabac a contribué à la baisse de l'incidence des crises cardiaques et, par voie de conséquence, du taux de mortalité par crise cardiaque. Les progrès significatifs des traitements médicaux des crises cardiaques ont également contribué à la baisse des taux de mortalité (voir l'indicateur 4.11 "Chirurgies cardio-vasculaires » et l'indicateur 6.1 « Mortalité à l'hôpital suite à un infarctus aigu du myocarde »).

L'accident vasculaire cérébral (AVC), qui résulte d'une interruption de l'irrigation sanguine du cerveau, constitue une autre cause de mortalité importante dans les pays de l'OCDE. En plus des nombreux décès qu'il provoque, il entraîne souvent chez les malades de lourds handicaps (Moon et al., 2003). Le taux de mortalité par AVC varie fortement entre les pays (graphique 2.4.2). La Hongrie, le Portugal, la Grèce et la République tchèque affichent les taux les plus élevés. La Suisse, la France et le Canada affichent les taux les plus bas.

Si l'on examine les tendances dans le temps, la mortalité par AVC a diminué dans tous les pays de l'OCDE (à l'exception de la Pologne) depuis 1980 (tableau A.2.4). Comme dans le cas des crises cardiaques, cette réduction peut être attribuée du moins pour partie à la diminution des facteurs de risque. La consommation de tabac et l'hypertension sont les principaux facteurs de risque modifiables pour les AVC (Stegmayr et al., 1997). Les progrès réalisés en matière de traitement médical de l'AVC ont également accru les taux de survie (voir l'indicateur 6.2 « Mortalité à l'hôpital suite à un accident vasculaire cérébral »).

\section{Définition et écarts}

Les taux de mortalité sont estimés à partir du nombre brut de décès dus à une sélection de causes, tel qu'il figure dans la base de données sur la mortalité de l'OMS. L'OMS a publié une évaluation générale de la couverture, du degré d'exhaustivité et de la fiabilité des données sur les causes de décès (Mathers et al., 2005). Les taux de mortalité ont été normalisés par rapport à l'âge sur la base de la structure démographique des pays de l'OCDE en 1980, de manière à éliminer les variations résultant des différences de structures d'âge entre pays ou de l'évolution de ces structures dans chaque pays au fil du temps. 


\subsection{MORTALITÉ DUE AUX MALADIES CARDIAQUES ET AUX ACCIDENTS VASCULAIRES CÉRÉBRAUX}

\subsubsection{Taux de mortalité due à une crise cardiaque, 2004}

Femmes

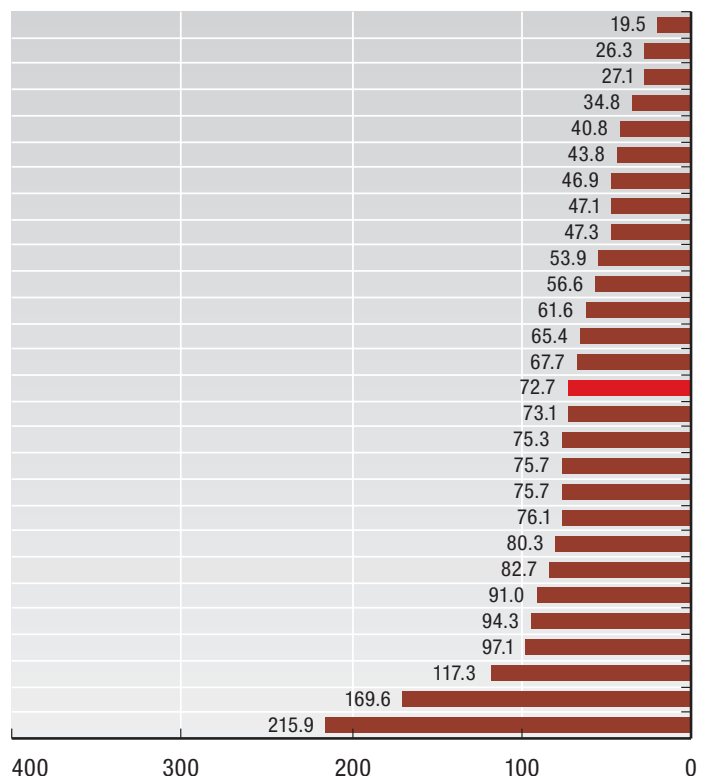

Taux normalisés par rapport à l'âge pour 100000 femmes

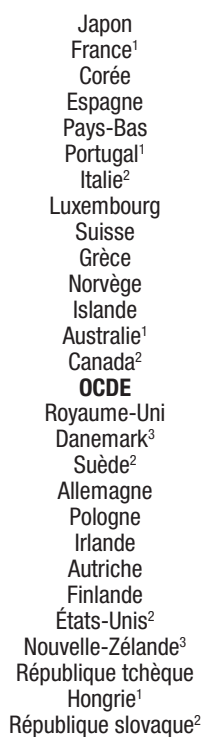

1. 2003. 2. 2002. 3. 2001.

\subsubsection{Taux de mortalité due à un accident vasculaire cérébral, 2004}

Femmes

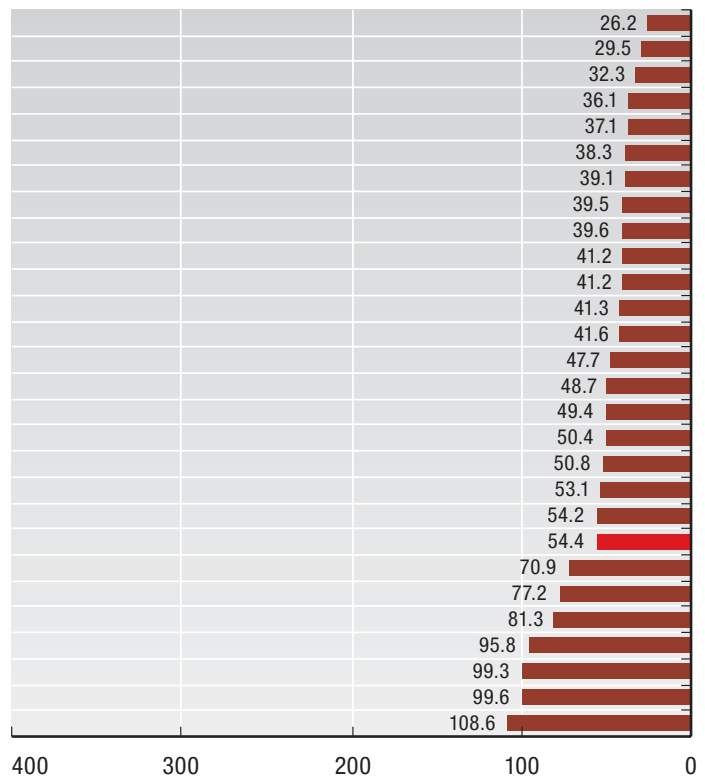

Taux normalisés par rapport à l'âge pour 100000 femmes
Hommes

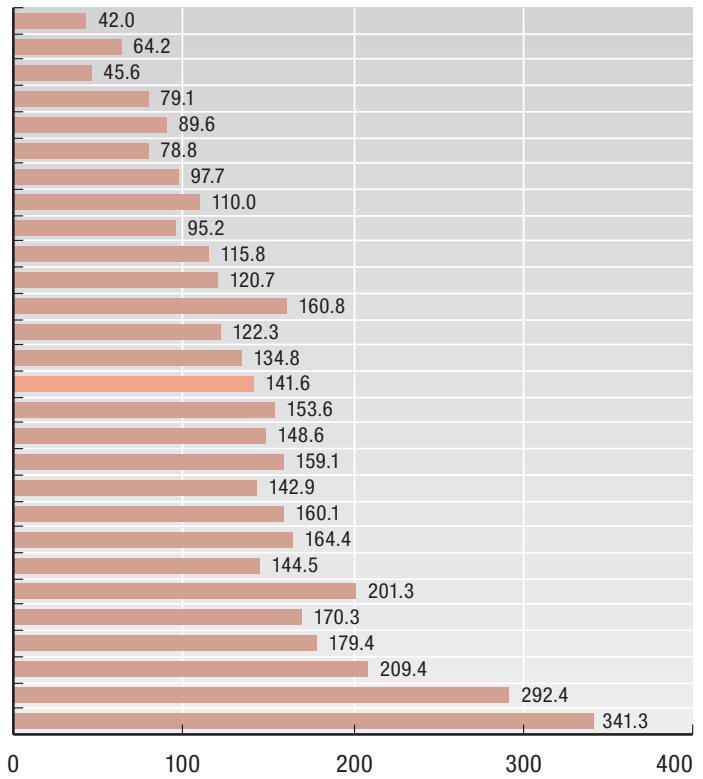

Taux normalisés par rapport à l'âge pour 100000 hommes

1. 2003. 2. 2002. 3. 2001.

Source : Eco-Santé OCDE 2007. Les statistiques de mortalité sont extraites de la base de données sur la mortalité de l'OMS, et sont normalisées par rapport à l'âge en fonction de la structure de la population des pays de l'OCDE en 1980. 


\subsection{MORTALITÉ DUE AU CANCER}

Dans les pays de l'OCDE, le cancer est la deuxième grande cause de mortalité après les maladies du système circulatoire. En 2004, il a provoqué en moyenne $27 \%$ de l'ensemble des décès.

En 2004, les pays affichant les taux de mortalité par cancer (hommes et femmes confondus) les plus bas étaient les pays nordiques (à l'exception du Danemark), la Suisse et le Japon. La Hongrie, la République tchèque, la République slovaque et la Pologne affichaient pour leur part les taux les plus élevés (graphique 2.5.1 et tableau A.2.5a). Le Danemark déclare également des taux de mortalité par cancer relativement élevés pour les hommes et pour les femmes. Les disparités entre pays peuvent s'expliquer à la fois par des facteurs non médicaux, tels que l'exposition de la population aux facteurs de risque (comme la consommation de tabac), et des facteurs médicaux, notamment la précocité du diagnostic et l'efficacité du traitement de différents types de cancer (voir la section sur le traitement du cancer au chapitre 6 traitant de la qualité des soins).

Les taux de mortalité par cancer sont plus élevés pour les hommes que pour les femmes dans tous les pays de l'OCDE (graphique 2.5.1). En 2004, l'écart de mortalité entre les sexes était particulièrement marqué au Japon, en Corée, en France, au Luxembourg, en Espagne et en République slovaque : dans tous ces pays, la mortalité due au cancer est plus de deux fois plus élevée chez les hommes que chez les femmes. Cette disparité entre les sexes s'explique au moins en partie par la prévalence plus élevée des facteurs de risque chez les hommes et par la disponibilité ou l'utilisation moindre des programmes de dépistage pour différents types de cancer affectant les hommes, ce qui se traduit par des taux de survie moindres après le diagnostic.

Si l'on concentre l'analyse sur des types spécifiques de cancer, le cancer du poumon reste le plus mortel chez les hommes dans tous les pays de l'OCDE (à l'exception de la Suède) et demeure l'une des principales causes de décès par cancer chez les femmes. La consommation de tabac est le facteur de risque le plus important pour le cancer du poumon. En 2004, les pays qui affichaient les taux les plus élevés de décès par cancer du poumon étaient les pays d'Europe centrale et orientale (la Hongrie, la Pologne, la Républiques tchèque et slovaque), les Pays-Bas, la Grèce et la Corée (graphique 2.5.2). Dans tous ces pays, la proportion de fumeurs chez les hommes a été de tout temps (et reste) relativement élevée. C'est en Suède que le taux de décès par cancer du poumon est le plus faible; ce pays est également l'un de ceux dans lesquels les hommes fument le moins (voir l'indicateur 3.1 « Consommation de tabac »).

Le cancer du sein est le cancer le plus fréquent chez les femmes dans tous les pays de l'OCDE (CIRC, 2004). Dans de nombreux pays, il représente $30 \%$ ou plus de l'incidence de cancers chez les femmes et 15 à $20 \%$ des décès dus au cancer. Si les taux d'incidence mesuré du cancer du sein ont augmenté dans la plupart des pays au cours de la dernière décennie, les taux de décès ont diminué ou sont restés stables dans la plupart des pays, indiquant une augmentation des taux de survie due à un dépistage plus précoce du cancer et/ou à une amélioration des traitements (voir la section sur le traitement des cancers au chapitre 6). En 2004, les taux de mortalité par cancer du sein étaient très variables d'un pays à l'autre (graphique 2.5.3). Les taux de mortalité les plus bas sont observés en Corée et au Japon; les taux les plus élevés sont observés au Danemark, en Hongrie, en Irlande et aux Pays-Bas.

Dans de nombreux pays de l'OCDE, le cancer de la prostate est devenu le cancer le plus courant chez les hommes, en particulier chez les hommes de plus de 65 ans. Cependant, le cancer de la prostate reste moins mortel que celui du poumon dans tous les pays excepté la Suède. L'augmentation de l'incidence du cancer de la prostate dans de nombreux pays pendant les années 90 est due, dans une large mesure, à l'utilisation plus répandue des tests de diagnostic par l'antigène spécifique de la prostate (PSA). En 2004, le taux de décès par cancer de la prostate était très variable, allant de moins de 10 pour 100000 hommes en Corée et au Japon à plus de 34 pour 100000 hommes en Norvège, au Danemark et en Suède (graphique 2.5.4). Les causes du cancer de la prostate sont encore mal connues. Il se pourrait que des facteurs liés à l'environnement et à l'alimentation influent sur le risque de cancer de la prostate (Institute of Cancer Research, 2003).

Globalement, les taux de décès dus à l'ensemble des types de cancers chez les hommes et les femmes ont diminué au moins légèrement dans la plupart des pays de l'OCDE depuis 1980, même si cette baisse a été plus modeste que celle de la mortalité due aux maladies du système circulatoire (ce qui explique que le cancer soit maintenant responsable d'une plus grande proportion des décès). Les exceptions à cette tendance sont la Grèce, la Hongrie, la Pologne et l'Espagne; dans ces pays, le taux de décès par cancer a augmenté entre 1980 et 2004 (tableau A.2.5a).

\section{Définition et écarts}

Les taux de mortalité par cancer sont estimés à partir du nombre brut des décès dus à une sélection de causes, tel qu'il figure dans la base de données sur la mortalité de l'OMS. L'OMS a publié une évaluation générale de la couverture, du degré d'exhaustivité et de la fiabilité des données sur les causes de décès (Mathers et al., 2005). La comparabilité internationale des données sur la mortalité par cancer peut être affectée par des différences entre les pays en termes de formation des médecins et de pratiques médicales mais aussi de procédures d'enregistrement des décès. Les taux de mortalité ont été normalisés par rapport à l'âge sur la base de la structure démographique des pays de l'OCDE en 1980, de manière à éliminer les variations résultant des différences de structures d'âge entre pays ou de l'évolution de ces structures dans chaque pays au fil du temps. 
2.5. MORTALITÉ DUE AU CANCER

\subsubsection{Taux de mortalité due au cancer, hommes} et femmes, 2004

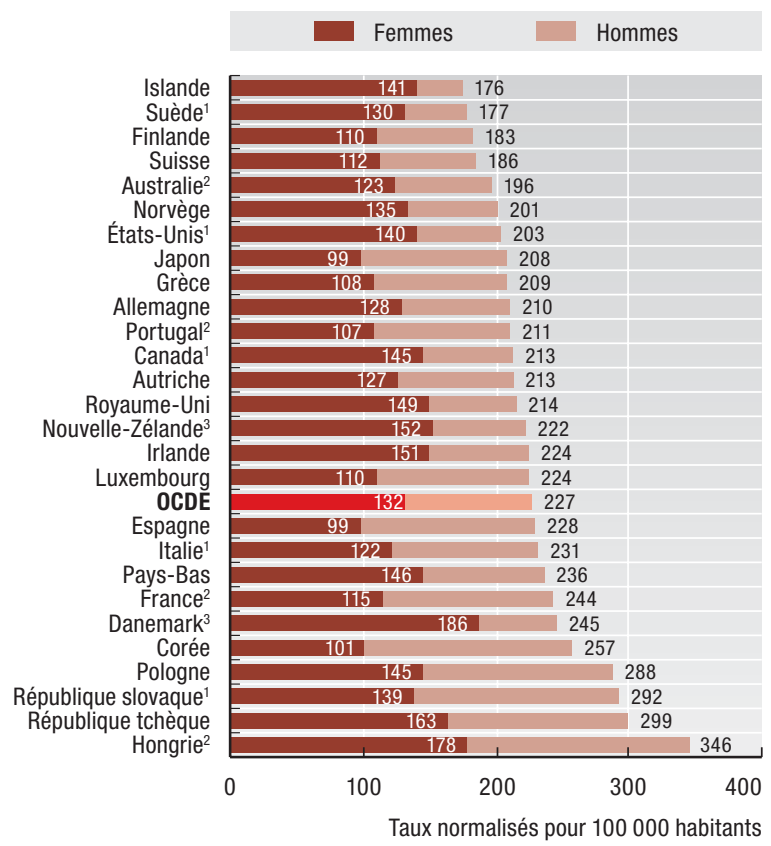

\subsubsection{Taux de mortalité due au cancer du poumon,} hommes et femmes, 2004

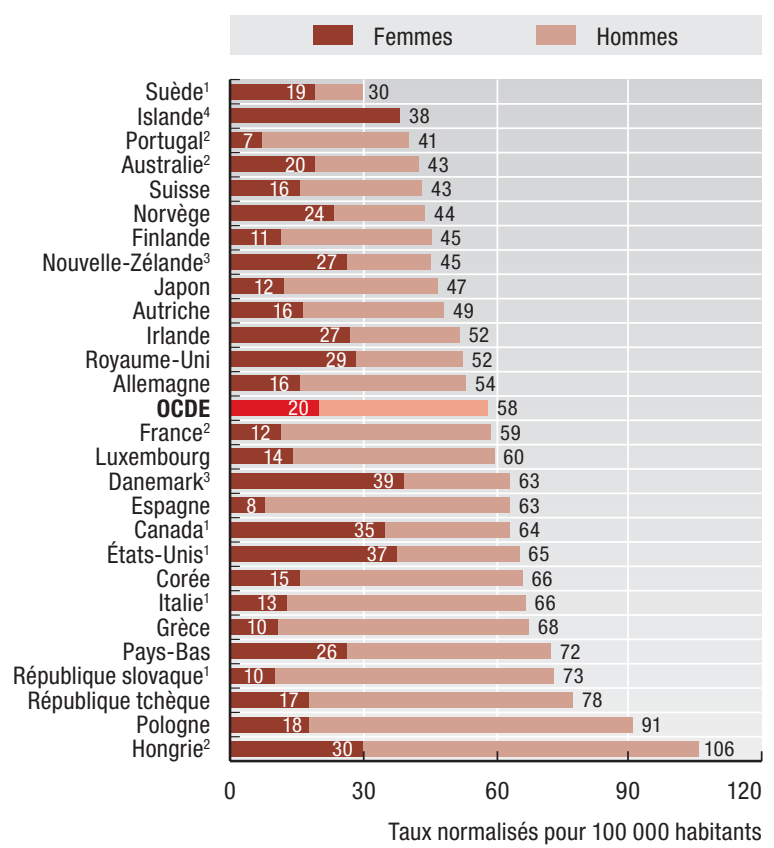

1. 2002. 2. 2003. 3. 2001.

4. En Islande, le taux de mortalité due au cancer du poumon est similaire pour les femmes et les hommes.

\subsubsection{Taux de mortalité due au cancer du sein, femmes, 2004}

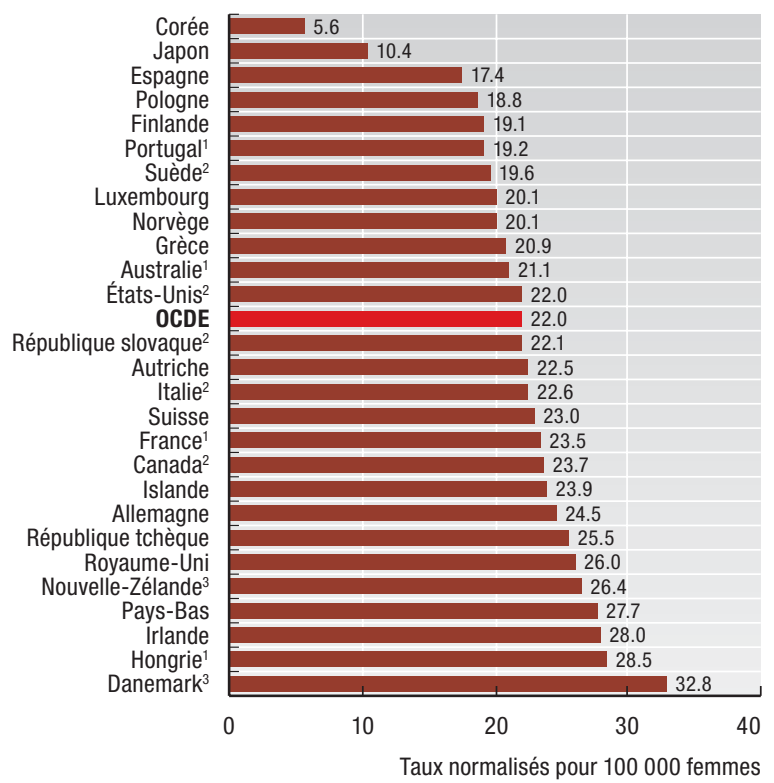

1. 2003. 2. 2002. 3. 2001.

Source: Eco-Santé OCDE 2007. Les statistiques de mortalité sont extraites de la base de données sur la mortalité de l'OMS, et sont normalisées par rapport à l'âge en fonction de la structure de la population des pays de l'OCDE en 1980.

StatLink AाIsस http://dx.doi.org/10.1787/128205126101 


\section{2. ÉTAT DE SANTÉ}

\subsection{MORTALITÉ DUE AUX ACCIDENTS DE LA ROUTE}

Au niveau mondial, on estime à 1.2 million le nombre des personnes tuées chaque année dans des accidents de la route et à 50 millions le nombre blessés. Dans les seuls pays de l'OCDE, les accidents de la route ont causé plus de 120000 décès en 2004. La mortalité due aux accidents de la route est la principale cause de décès parmi les jeunes hommes dans de nombreux pays. Le nombre des blessés et des morts dans des accidents de la route demeure un grave problème de santé publique dans de nombreux pays.

Les taux de décès par accident de la route varient largement d'un pays à l'autre. En 2004, les taux de décès par accident de la route (pour les deux sexes confondus) les plus élevés ont été ceux de la Corée et du Portugal, suivis par la Grèce, les États-Unis, la Pologne et la Hongrie (graphique 2.6.1). Les plus bas ont été ceux des Pays-Bas, de la Suède et du Royaume-Uni. Dans tous les pays de l'OCDE, les accidents de la route font beaucoup plus de victimes chez les hommes que chez les femmes; l'écart est de 2.2 fois plus de morts chez les hommes que chez les femmes en Islande et jusqu'à 4.2 fois plus en Suisse (graphique 2.6.2).

Un grand nombre de ces accidents pourraient être évités. La sécurité routière s'est améliorée dans de nombreux pays au cours des dernières décennies, notamment au travers de l'amélioration des systèmes routiers, de campagnes d'éducation et de prévention, de l'adoption de nouvelles lois et réglementations et de la multiplication des contrôles routiers pour faire respecter ces lois. En conséquence, depuis 1970, les taux de décès dus aux accidents de la route ont diminué en moyenne de plus de moitié dans les pays de l'OCDE (graphique 2.6.3). C'est en Allemagne, aux Pays-Bas et en Suisse que la baisse des taux de décès a été la plus forte, avec une diminution d'environ $75 \%$ depuis 1970, bien que le nombre de kilomètres parcourus sur la même période dans les pays d'Europe de l'Ouest ait été multiplié en moyenne par 2.6 (ECMT, 2007). Les taux de décès ont également diminué aux États-Unis mais à un rythme moindre et restent donc supérieurs à la moyenne de l'OCDE. En Grèce et en Pologne, les taux de décès dus aux accidents de la route ont fortement augmenté depuis 1970 (graphique 2.6.4).

Si l'on extrapole les tendances passées, des projections de la Banque mondiale indiquent qu'entre 2000 et 2020 le nombre des décès dus aux accidents de la route va probablement diminuer encore d'environ $30 \%$ dans les pays à hauts revenus, mais qu'il va probablement augmenter de manière sensible dans les pays à revenus faibles ou moyens si des mesures supplémentaires ne sont pas mises en place pour améliorer la sécurité routière (Peden et al., 2004).

\section{Définition et écarts}

Les taux de mortalité sont estimés à partir du nombre brut de décès dus à une sélection de causes, tel qu'il figure dans la base de données sur la mortalité de l'OMS. L'OMS a publié une évaluation générale de la couverture, du degré d'exhaustivité et de la fiabilité des données sur les causes de décès (Mathers et al., 2005). Les taux de mortalité ont été normalisés par rapport à l'âge sur la base de la structure démographique des pays de l'OCDE en 1980, de manière à éliminer les variations résultant des différences de structures d'âge entre pays ou de l'évolution de ces structures dans chaque pays au fil du temps.

Les taux de mortalité dus aux accidents de la route au Luxembourg sont surestimés en raison de l'importance du transit routier, qui se traduit par une forte proportion de décès de non-résidents sur les routes luxembourgeoises. 
2.6. MORTALITÉ DUE AUX ACCIDENTS DE LA ROUTE

2.6.1. Taux de mortalité suite à un accident de la route, population totale, 2004

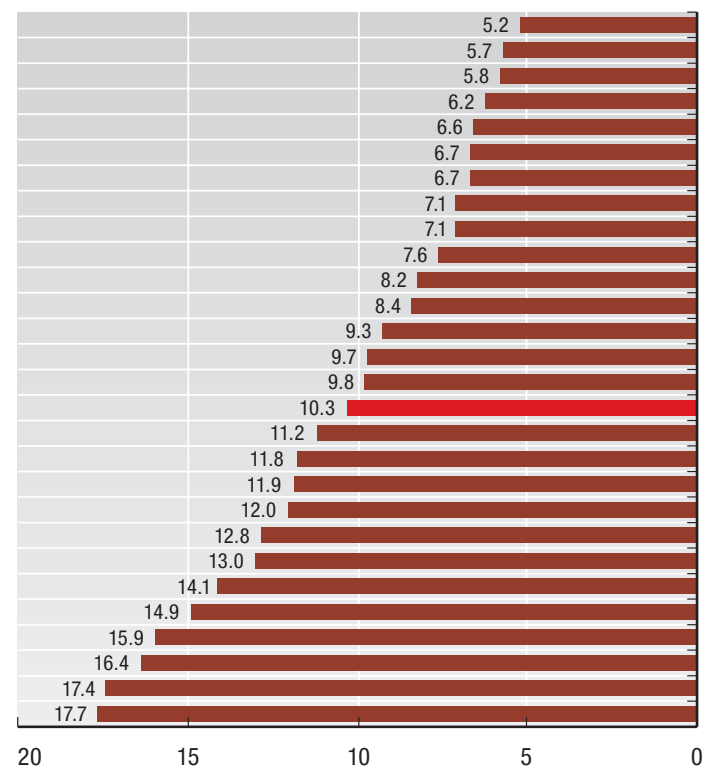

Taux normalisés par rapport à l'âge pour 100000 habitants

1. 2002. 2. 2001. 3. 2003.

\subsubsection{Taux de mortalité suite à un accident} de la route, hommes et femmes, 2004

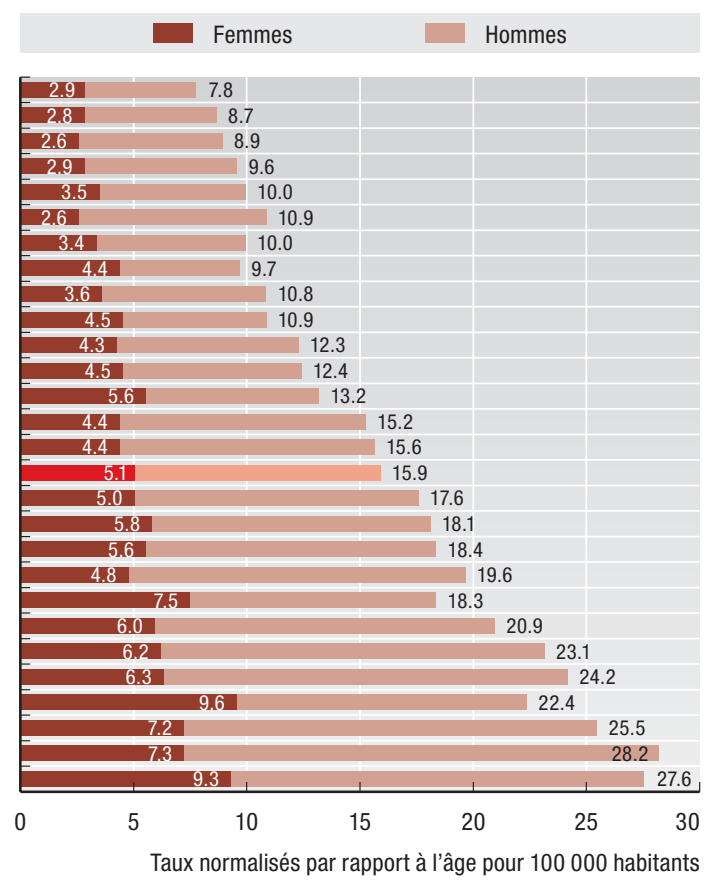

\subsubsection{Variation des taux de mortalité suite à un accident de la route, 1970-2004 \\ (ou année la plus proche)}

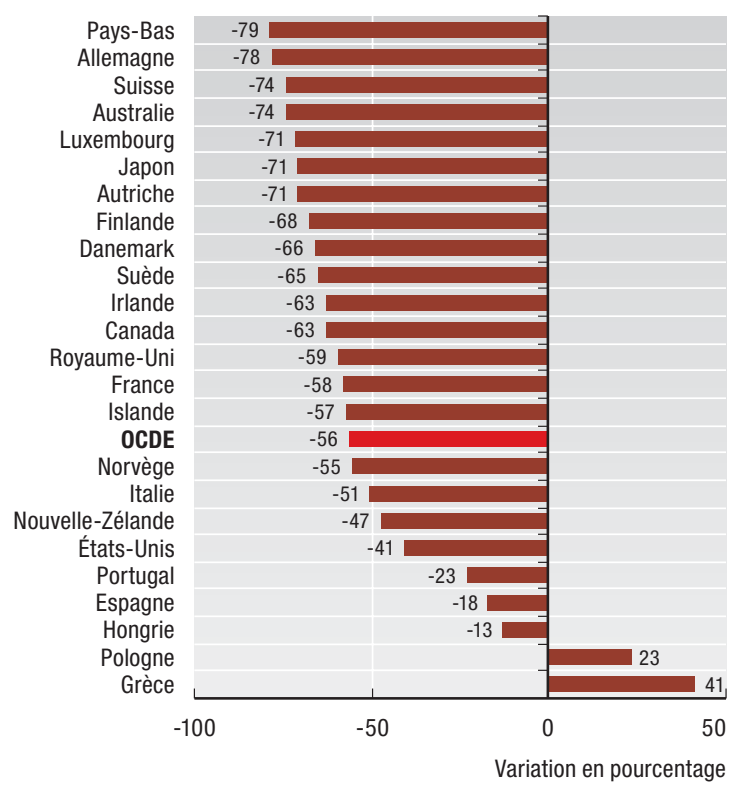

Source : Eco-Santé OCDE 2007. Les statistiques de mortalité sont extraites de la base de données sur la mortalité de l'OMS, et sont normalisées par rapport à l'âge en fonction de la structure de la population des pays de l'OCDE en 1980. 


\section{2. ÉTAT DE SANTÉ}

\subsection{SUICIDES}

Le fait de se donner délibérément la mort témoigne certes de graves problèmes personnels mais aussi d'une dégradation du climat social dans lequel vit un individu. Bien qu'un certain nombre de facteurs différents peuvent conduire à mettre fin à ses jours, un suicide est plus susceptible d'intervenir pendant une période de crise associée à un divorce, à la consommation abusive d'alcool et de drogues, au chômage, à une dépression nerveuse ou à différentes formes de maladie mentale. C'est pourquoi le suicide est souvent utilisé comme un indicateur indirect de la santé mentale d'une population. Il est possible toutefois que, dans certains pays, le nombre des suicides soit sous-estimé en raison du tabou dont cet acte continue de faire l'objet.

Les suicides ont provoqué plus de 130000 décès en 2004 dans l'ensemble des pays de l'OCDE. Les taux de suicide varient considérablement entre les pays (graphique 2.7.1). En 2004, les taux les plus bas ont été enregistrés dans les pays d'Europe méridionale (la Grèce, l'Italie et l'Espagne) et au Royaume-Uni (sept décès au plus pour 100000 personnes) et les taux les plus élevés ont été enregistrés en Corée, en Hongrie, au Japon et en Finlande avec un taux de 18 décès et plus pour 100000 personnes.

Depuis 1980, les taux de suicide ont diminué dans de nombreux pays de l'OCDE; ils ont fortement baissé (40\% et plus) au Danemark, en Hongrie, en Allemagne et en Suisse (graphique 2.7.3). En dépit de ces progrès, la Hongrie a toujours un taux de suicide parmi les plus élevés de la zone OCDE. À l'inverse, c'est en Espagne et en Irlande que les taux de décès par suicide ont le plus augmenté depuis 1980, même s'ils demeurent relativement faibles. En Corée et au Japon, ces taux ont augmenté depuis 1990 et sont désormais bien supérieurs à la moyenne de l'OCDE (graphique 2.7.4). En Corée, les taux de suicide parmi la population masculine ont été multipliés par trois, passant de 12 pour 100000 en 1990 à 36 pour 100000 en 2004, et parmi la population féminine ces taux sont les plus élevés de la zone OCDE (14 pour 100 000). Le stress d'une modernisation rapide et l'érosion du socle familial traditionnel ont contribué à l'augmentation récente des taux de suicide dans ce pays (Park et al., 2003; Ra et al., 2006).

En général, les taux de décès par suicide sont entre trois et quatre fois plus élevés chez les hommes que chez les femmes (graphique 2.7.2) et cet écart entre les sexes a été relativement stable dans le temps. L'écart entre hommes et femmes est moindre pour les tentatives de suicide, ce qui reflète le fait que les femmes ont recours à des méthodes moins radicales que les hommes.

Le suicide est également lié à l'âge, les moins de 25 ans et les personnes âgées étant des sujets particulièrement à risque. Si le taux de suicide parmi la population âgée a, de manière générale, baissé au cours des vingt dernières années, pratiquement aucun progrès n’a été observé parmi les jeunes.

La prévention du suicide n'est pas chose facile. Dans la mesure où les suicides sont, dans la grande majorité des cas, liés à un état dépressif ou à la consommation abusive d'alcool et autres substances toxiques, la détection précoce de ces problèmes psychosociaux par les familles, les travailleurs sociaux et les professionnels de santé, est importante, et doit s'accompagner d'un soutien et d'un traitement efficaces. En Finlande et en Islande, les programmes de prévention du suicide ont reposé sur des efforts visant à promouvoir une collaboration multisectorielle entre différents intervenants (NOMESCO, 2007).

\section{Définition et écarts}

L'Organisation mondiale de la santé définit le suicide comme un acte délibéré initié et commis par une personne connaissant parfaitement, voire escomptant, son issue fatale.

Les taux de mortalité sont estimés à partir du nombre brut de décès dus à une sélection de causes, tel qu'il figure dans la base de données sur la mortalité de l'OMS. L'OMS a publié une évaluation générale de la couverture, du degré d'exhaustivité et de la fiabilité des données sur les causes de décès (Mathers et al., 2005). Les taux de mortalité ont été normalisés par rapport à l'âge sur la base de la structure démographique des pays de l'OCDE en 1980, de manière à éliminer les variations résultant des différences de structures d'âge entre pays ou de l'évolution de ces structures dans chaque pays au fil du temps.

La comparabilité internationale des taux de décès par suicide est affectée par un certain nombre de critères de notification, notamment par la manière dont l'intention de se donner la mort est établie, par le fait qu'une enquête de médecine légale est ou non effectuée et par les dispositions relatives au caractère confidentiel de la cause du décès. Une certaine prudence s'impose donc dans l'interprétation des disparités d'un pays à l'autre. 


\section{2. ÉTAT DE SANTÉ}

\subsection{SUICIDES}

\subsubsection{Taux de mortalité due à un suicide, population totale, 2004}

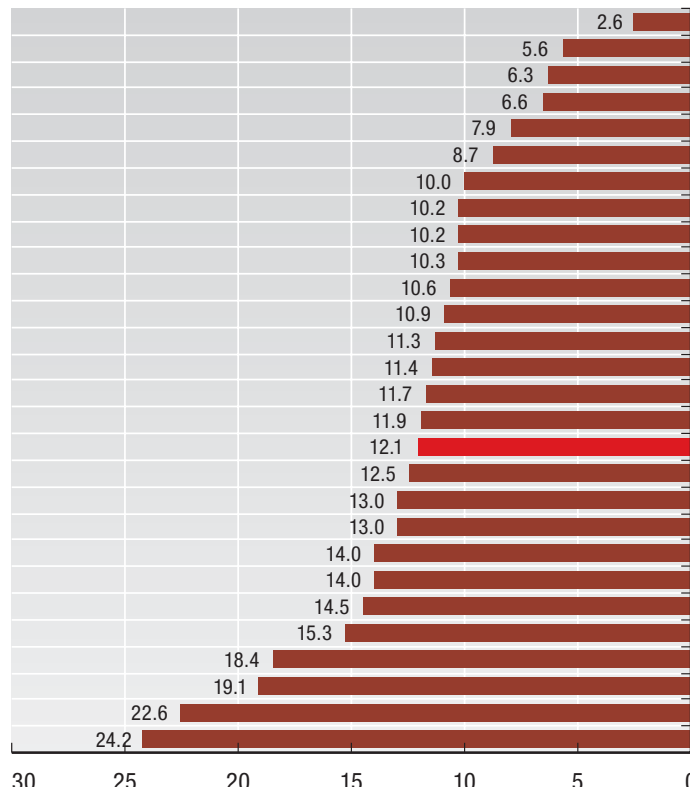

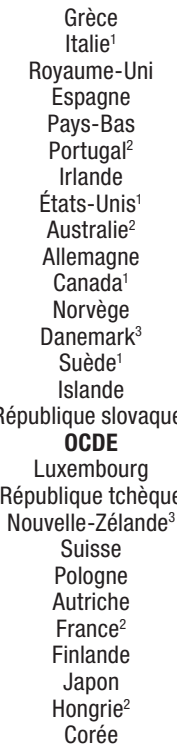

Taux normalisés par rapport à l'âge pour 100000 habitants

1. 2002. 2. 2003. 3. 2001.

\subsubsection{Taux de mortalité due à un suicide, hommes et femmes, 2004}

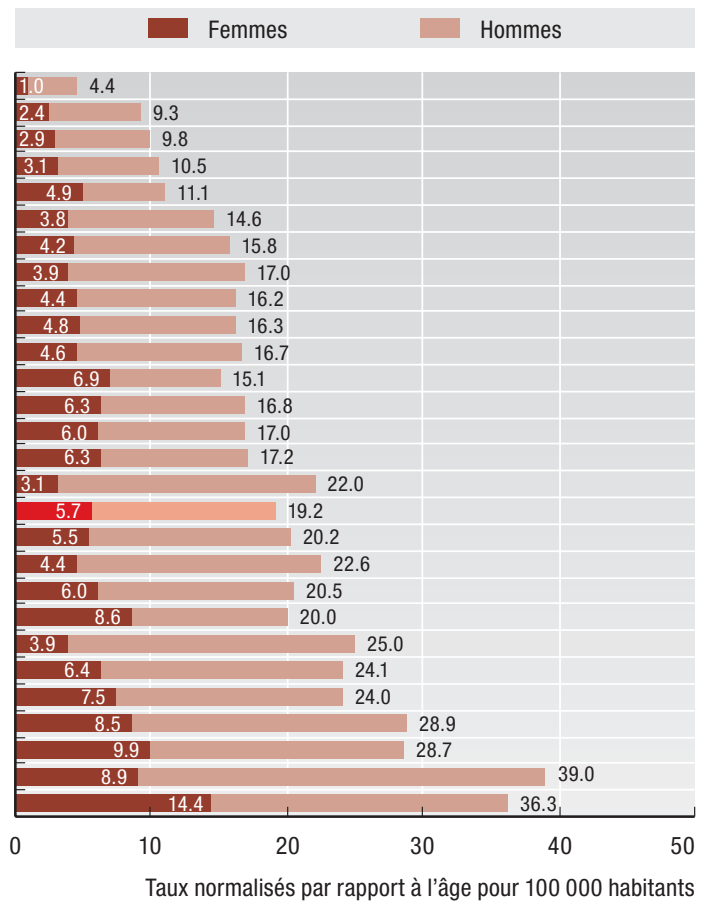

\subsubsection{Variation des taux de suicide, 1980-2004 (ou année la plus proche)}

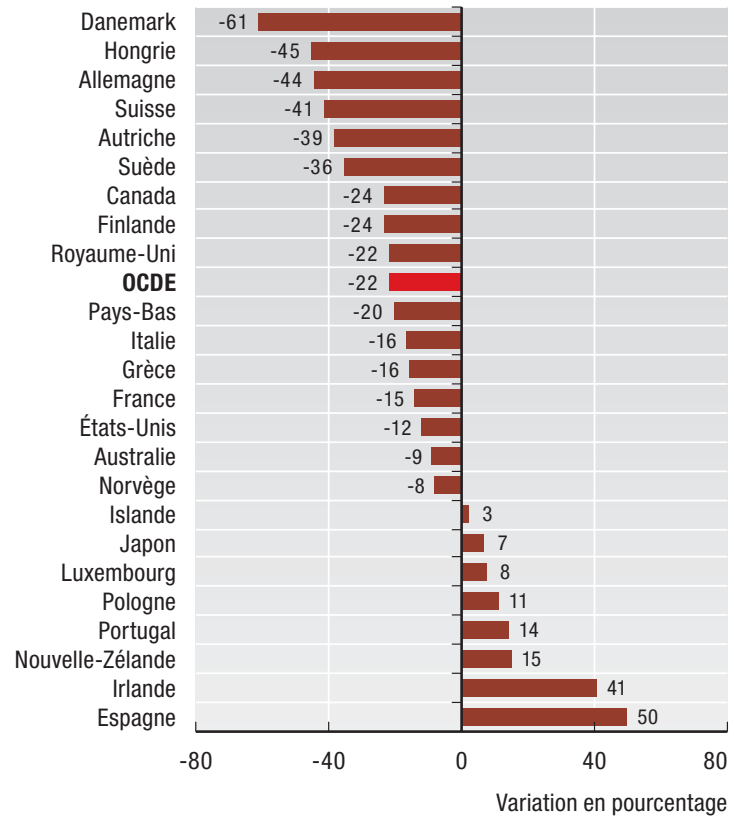

\subsection{4. Évolution des taux de suicide dans quelques pays de l'OCDE, 1980-2004

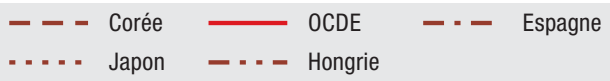

Taux normalisés de décès pour 100000 habitants

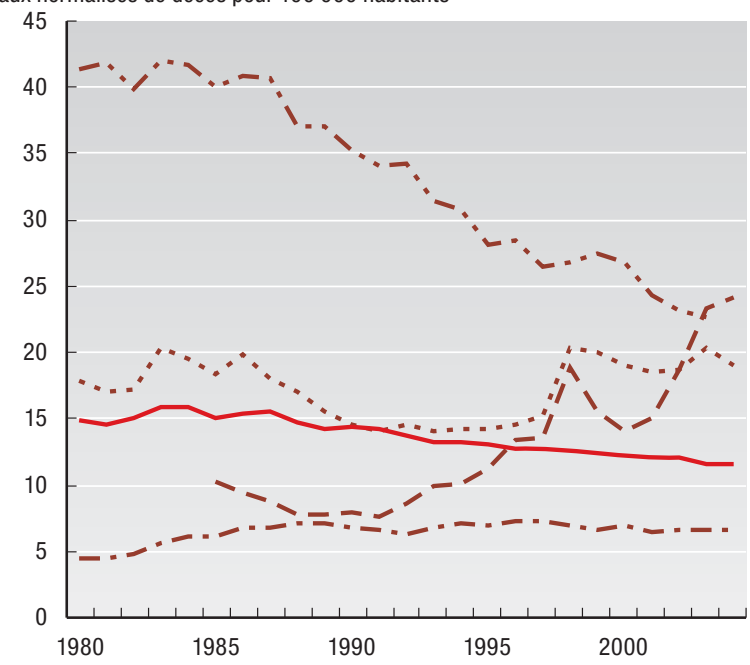

Source: Eco-Santé OCDE 2007. Les statistiques de mortalité sont extraites de la base de données sur la mortalité de l'OMS, et sont normalisées par rapport à l'âge en fonction de la structure de la population des pays de l'OCDE en 1980. 


\subsection{MORTALITÉ INFANTILE}

Le taux de mortalité infantile, qui mesure la proportion de décès chez les nourrissons de moins d'un an, reflète non seulement l'effet des conditions économiques et sociales sur la santé des mères et des nouveaux-nés mais aussi l'efficacité des systèmes de santé.

En 2005, les taux de mortalité infantile dans les pays de l'OCDE oscillaient entre deux à trois décès pour 1000 naissances vivantes au Japon, dans les pays nordiques (Danemark excepté) et au Luxembourg, jusqu'à 19 et 24 décès respectivement pour 1000 naissances vivantes au Mexique et en Turquie (graphique 2.8.1). Ces taux étaient également relativement élevés (plus de six décès pour 1000 naissances vivantes) aux États-Unis et dans certains pays d'Europe centrale et orientale. Le taux moyen dans les pays de l'OCDE était de 5.4 en 2005.

Les deux tiers environ des décès qui surviennent au cours de la première année de vie sont des décès néonataux (intervenant entre la naissance et le $28^{\mathrm{e}}$ jour). Dans les pays développés, les principales causes de mortalité néonatale sont les malformations congénitales, un poids de naissance insuffisant (enfants prématurés) et d'autres problèmes survenant pendant la grossesse. Les femmes ayant leurs enfants de plus en plus tard et le nombre des grossesses multiples augmentant du fait des traitements contre la stérilité, les naissances prématurées tendent à être de plus en plus nombreuses (voir l'indicateur 2.9 "Faible poids de naissance »). Dans un certain nombre de pays à hauts revenus, ce phénomène a contribué à enrayer la tendance à la baisse des taux de mortalité infantile au cours des dernières années. De fait, l'augmentation des naissances de bébés de très faible poids a été citée comme étant la principale cause de la première hausse (depuis les années 50) du taux de mortalité infantile aux États-Unis entre 2001 et 2002 (CDC, 2003). Pour les décès de nourrissons de plus d'un mois (mortalité post-néonatale), les causes possibles tendent à être plus variées, les plus courantes étant le syndrome de mort subite du nourrisson, les anomalies congénitales, les infections et les accidents.

Dans tous les pays de l'OCDE, les taux de mortalité infantile ont considérablement diminué par rapport à leurs niveaux de 1970, époque à laquelle la moyenne avoisinait les 30 décès pour 1000 naissances vivantes (graphique 2.8.3). Cela équivaut à une réduction cumulée de plus de $80 \%$ depuis 1970. Le Portugal a vu son taux de mortalité infantile diminuer en moyenne de près de $8 \%$ par an depuis 1970. Après avoir été le pays européen à la mortalité infantile la plus élevée, il est devenu en 2005 l'un des pays de l'OCDE où ce taux est le plus bas (graphique 2.8.2). Des baisses importantes du taux de mortalité infantile ont été également observées en Corée. En revanche, la diminution de ce taux a été plus lente aux Pays-Bas et aux États-Unis. Dans ce pays où le taux de mortalité infantile était habituellement inférieur à la moyenne (et à la valeur médiane) de l'OCDE, il est désormais supérieur à la moyenne (graphique 2.8.3).

De nombreuses études ont utilisé le taux de mortalité infantile comme indicateur sanitaire pour examiner l'effet de divers déterminants médicaux et non médicaux de la santé. Bien que la plupart des analyses montrent l'existence d'une relation globalement négative entre la mortalité infantile et les dépenses de santé, le fait que certains pays ayant un niveau élevé de dépenses de santé n'affichent pas nécessairement de faibles taux de mortalité infantile a conduit certains chercheurs à en conclure que l'amélioration des résultats ne passe pas nécessairement par une augmentation des dépenses de santé (Retzlaff-Roberts et al., 2004). Un vaste corpus de recherches donne également à penser que de nombreux facteurs autres que la qualité et l'efficience du système de santé, tels que les inégalités de revenus, l'environnement social, les modes de vie et les comportements individuels influent sur les taux de mortalité infantile (Kiely et al., 1995).

\section{Définition et écarts}

Le taux de mortalité infantile est le nombre de décès d'enfants de moins de un an pour 1000 naissances vivantes, au cours d'une année. La mortalité néonatale est le nombre de décès d'enfants de moins de 28 jours.

Les variations internationales des taux de mortalité infantile et néonatale peuvent s'expliquer en partie par les différentes manières d'enregistrer les prématurés (déclarés en naissances vivantes ou en morts fœtales). Dans plusieurs pays, comme les États-Unis, le Canada, le Japon et les pays nordiques, les grands prématurés (ayant relativement peu de chances de survie) sont enregistrés comme naissances vivantes, ce qui accroît le taux de mortalité, alors que dans d'autres, ils ne le sont pas (Sachs et al., 1995). 


\section{2. ÉTAT DE SANTÉ}

\subsubsection{Taux de mortalité infantile,} 2005

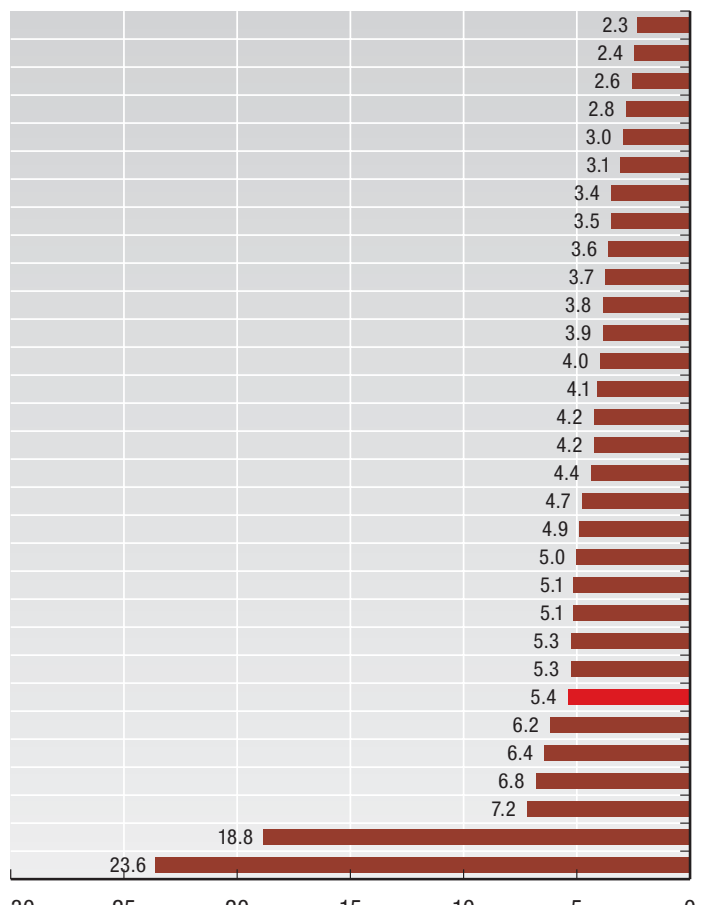

Décès pour 1000 naissances vivantes
2.8. MORTALITÉ INFANTILE

\subsubsection{Déclin des taux de mortalité infantile,} 1970-2005

Islande
Suède
Luxembourg
Japon
Finlande
Norvège
République tchèque
Portugal
France
Belgique
Grèce
Allemagne
Irlande
Espagne
Suisse
Autriche
Danemark
Italie
Pays-Bas
Australie
Royaume-Uni
Nouvelle-Zélande
Corée ${ }^{1}$
Canada
OCDE
Hongrie
Pologne
États-Unisª
République slovaque
Mexique
Turquie
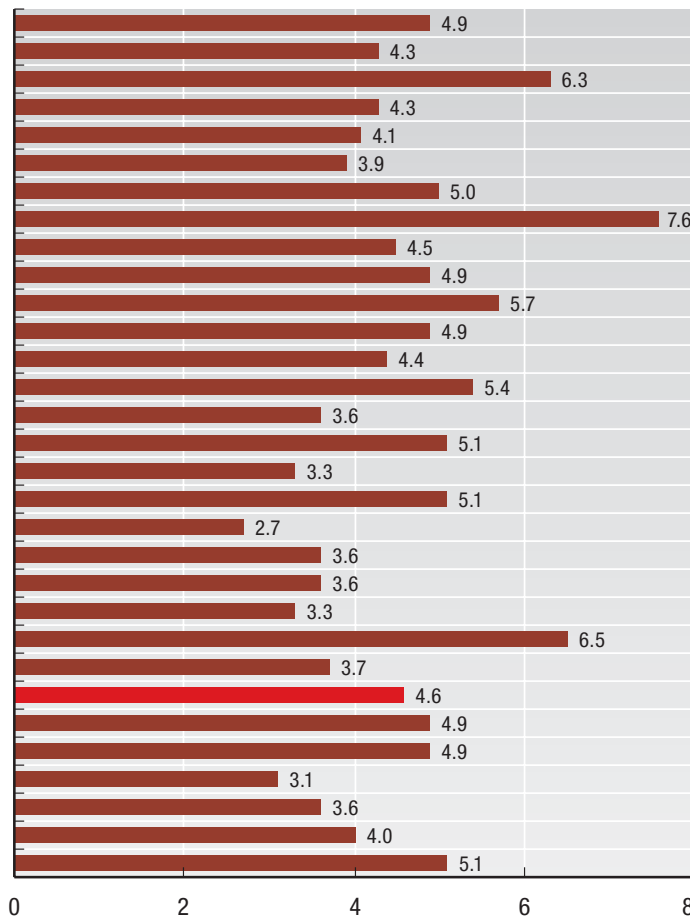

Taux annuel de décroissance $(\%)$

Note : Au Canada, aux États-Unis, au Japon et dans les pays nordiques, les grands prématurés avec une faible chance de survie sont enregistrés comme naissances vivantes, ce qui se traduit par des taux de mortalité infantile plus élevés que dans les autres pays.

1. 2002. 2. 2004

\subsubsection{Taux de mortalité infantile, $1970-2005$}

Canada, États-Unis, Suède et moyenne OCDE

$$
\text { - - - Canada }
$$$$
-- \text { Suède }
$$

$$
\text { .... États-Unis }
$$

\section{Décès pour 1000 naissances vivantes}

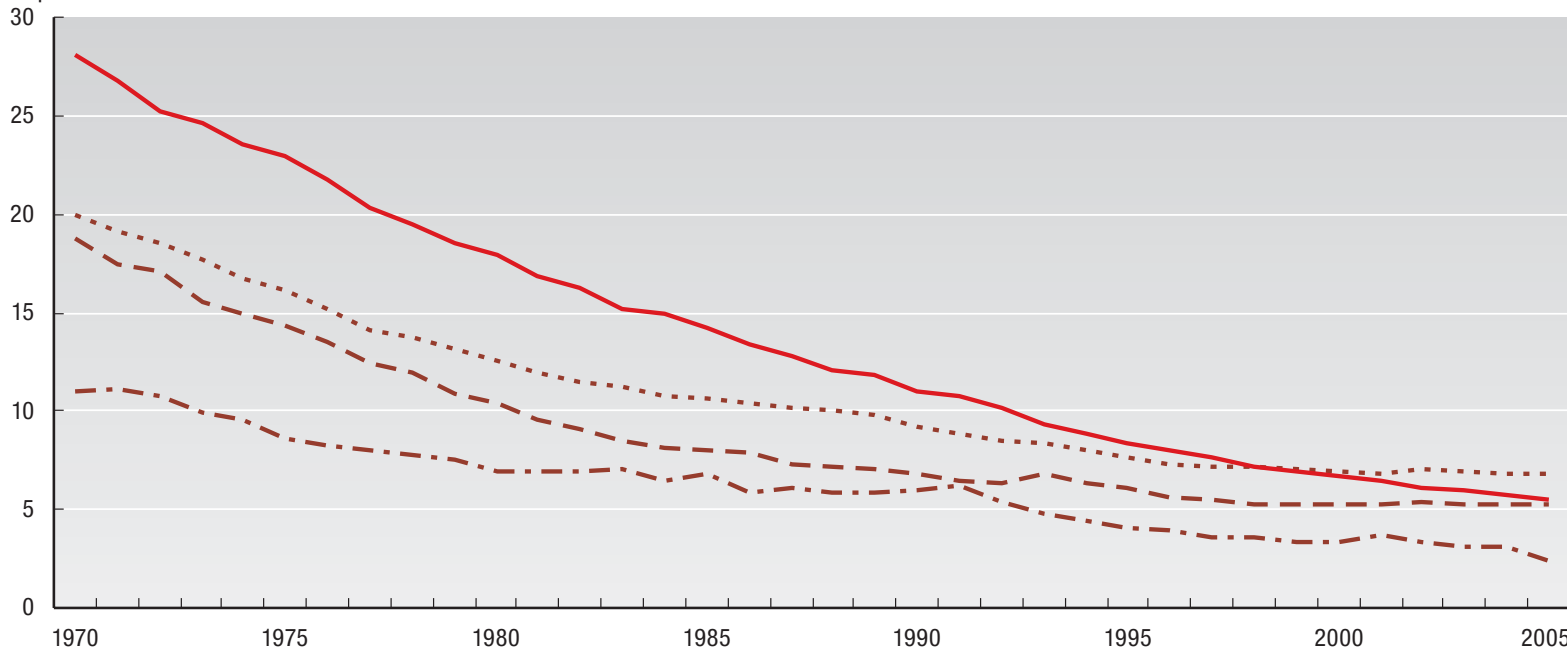

Source : Eco-Santé OCDE 2007. 


\subsection{SANTÉ DU NOURRISSON : FAIBLE POIDS À LA NAISSANCE}

Le faible poids à la naissance (défini ici comme inférieur à 2.5 kilos) est un indicateur important de la santé du nourrisson en raison de la relation qui existe entre le poids de naissance d'une part, et la mortalité et la morbidité infantiles d'autre part. Un faible poids de naissance peut être dû à deux facteurs : un retard de croissance intra-utérin ou une naissance prématurée. Les nourrissons de faible poids ont plus de risque de décéder ou d'être en mauvaise santé; ils nécessitent souvent une longue période d'hospitalisation après la naissance et sont plus susceptibles de développer des incapacités importantes (UNICEF et OMS, 2004). Les facteurs de risque prédisposant à un faible poids de naissance peuvent être d'ordre socio-économique (âge avancé de la mère et grossesses multiples) ou comportemental (tabac, consommation excessive d'alcool et malnutrition); ils peuvent également être liés à une faible qualité des soins prénataux.

En 2005, les pays nordiques (l'Islande, la Finlande, la Suède, la Norvège et le Danemark) mais aussi la Corée, le Luxembourg et l'Irlande étaient les pays qui déclaraient le plus faible pourcentage de faibles poids de naissance (moins de 2.5 kilos), à savoir $5 \%$ au plus des naissances vivantes. À l'autre extrémité, on trouve la Turquie, le Japon, la Grèce, le Mexique, la Hongrie et les États-Unis avec une proportion d'enfants de faible poids de naissance de plus de $8 \%$ (graphique 2.9.1). La moyenne dans les pays de l'OCDE s'établissait à $6.6 \%$.

Plusieurs facteurs peuvent expliquer la prévalence accrue du nombre de naissances de faible poids observée dans de nombreux pays de l'OCDE depuis 1980 (graphique 2.9.2 et tableau A.2.9). Premièrement, le nombre des naissances multiples, qui ont pour corollaire un risque accrû de naissance prématurée et de faible poids de naissance, a augmenté régulièrement, en partie du fait de l'augmentation des traitements contre la stérilité. Deuxièmement, dans de nombreux pays de l'OCDE, les femmes ont de plus en plus tendance à avoir leurs enfants à un âge plus avancé, ce qui accroît le risque de faible poids de naissance. Enfin, l'utilisation croissante de différentes techniques d'accouchement, telles que la provocation d'accouchement et les césariennes, accroissent les chances de naissance de bébés de faible poids.

Le Japon et l'Espagne, qui figurait dans le passé, parmi les pays affichant une proportion peu élevée de bébés de faible poids de naissance, ont connu une augmentation rapide de cette proportion au cours des vingt-cinq dernières années. En conséquence, dans ces deux pays, la proportion de bébés ayant un faible poids de naissance est désormais supérieure à la moyenne de l'OCDE (graphique 2.9.3). Dans le cas du Japon, cette augmentation a été attribuée à un certain nombre de facteurs de risque, en particulier à la prévalence croissante du tabagisme chez les jeunes Japonaises depuis les années 70, ainsi qu'à une tendance marquée aux maternités plus tardives (Jeong et Hurst, 2001; et Ohmi et al., 2001). En dépit d'une augmentation du nombre des bébés de faible poids de naissance, les soins médicaux aux nouveaux-nés se sont révélés particulièrement efficaces au Japon pour réduire la mortalité infantile.

Le graphique 2.9.4 montre qu'il existe une certaine corrélation entre le pourcentage d'enfants à faible poids de naissance et le taux de mortalité infantile. En général, les pays dans lesquels la proportion d'enfants ayant un faible poids de naissance est faible affichent également des taux de mortalité infantile relativement bas. C'est le cas, par exemple, des pays nordiques. Le Japon fait toutefois exception à cette règle puisqu'il affiche la plus forte proportion d'enfants ayant un faible poids de naissance et l'un des taux de mortalité infantile les plus bas.

Au sein d'un pays, la proportion d'enfants à faible poids de naissance peut être influencée par des différences de niveaux d'instruction, de revenu et de conditions de vie. Aux États-Unis, on observe des différences importantes entre les groupes ethniques pour ce qui est de la proportion d'enfants ayant un faible poids de naissance, la proportion étant pratiquement deux fois plus élevée parmi la population noire que parmi la population blanche (CDC, 2003). Des différences similaires ont été observées entre les populations indigènes et non indigènes en Australie (Laws et al., 2006) et au Mexique; elles reflètent les conditions de vie défavorisées de nombreuses mères indigènes.

\section{Définition et écarts}

Selon l'Organisation mondiale de la santé (OMS), un enfant ayant un faible poids de naissance est un enfant qui pèse à la naissance moins de 2.5 kilos, ceci indépendamment de l'âge gestationnel de l'enfant. Cette définition se fonde sur des observations épidémiologiques concernant le risque accru de décès de l'enfant; elle est utilisée pour les comparaisons internationales de statistiques de santé. Le nombre des naissances d'enfants de faible poids est ensuite exprimé en pourcentage du total des naissances vivantes.

Les données proviennent en majorité des registres d'état-civil, sauf dans le cas des Pays-Bas où elles proviennent d'entretiens dans le cadre d'une enquête nationale de santé. 
2.9. SANTÉ DU NOURRISSON : FAIBLE POIDS À LA NAISSANCE

2.9.1. Faible poids des nourrissons à la naissance, 2005

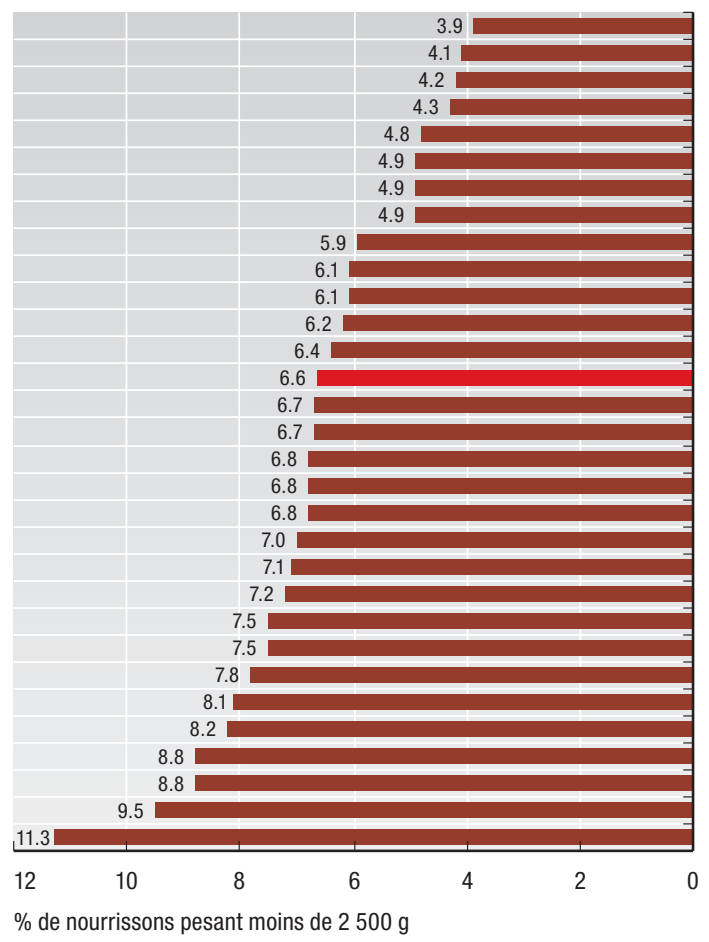

1. 2003. 2. 2004.
2.9.2. Variation de la proportion des nourrissons ayant un faible poids à la naissance, 1980 à 2005

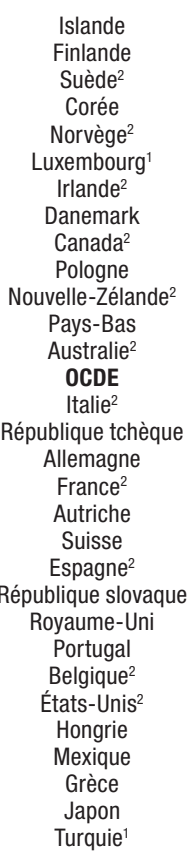

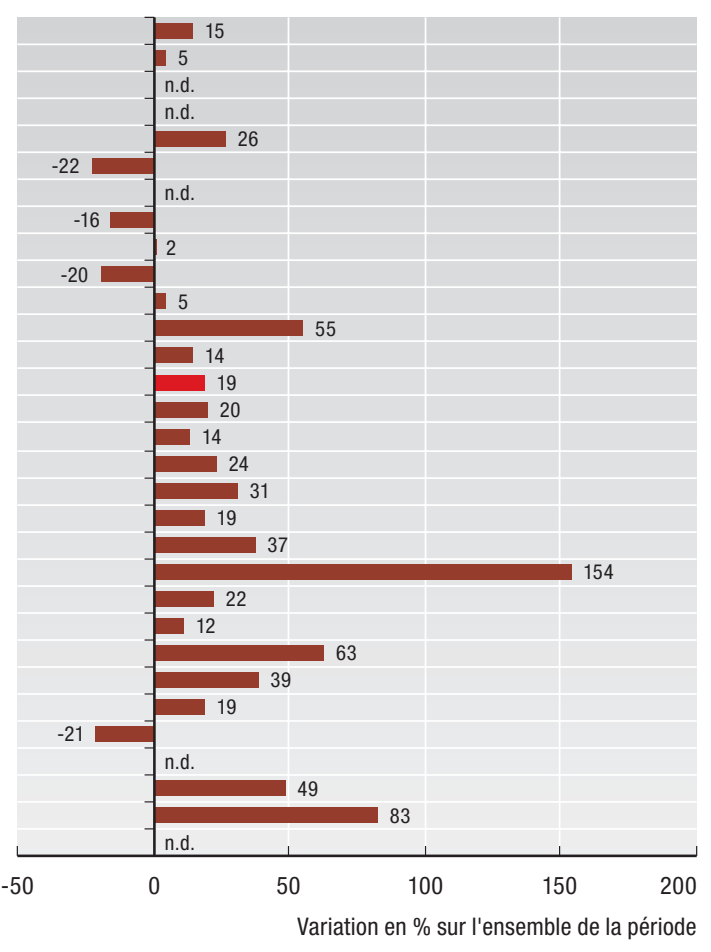

2.9.4. Faible poids à la naissance et mortalité infantile, 2005
2.9.3. Tendances dans la proportion de nourrissons ayant un faible poids à la naissance dans certains pays de l'OCDE, 1980-2005

$$
\begin{array}{lll}
\text { - - - - Japon } & \text {-. Suède } & \text { Espagne } \\
& &
\end{array}
$$

$\%$ de nourrissons pesant moins de $2500 \mathrm{~g}$

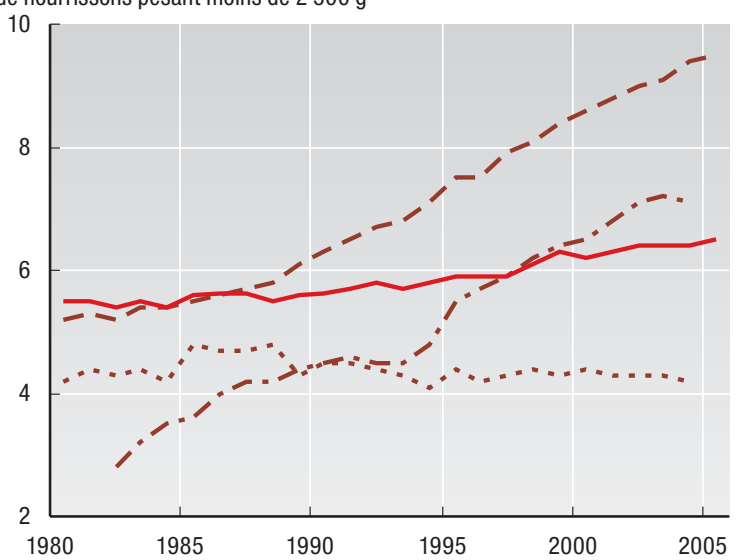

Source : Eco-Santé OCDE 2007.

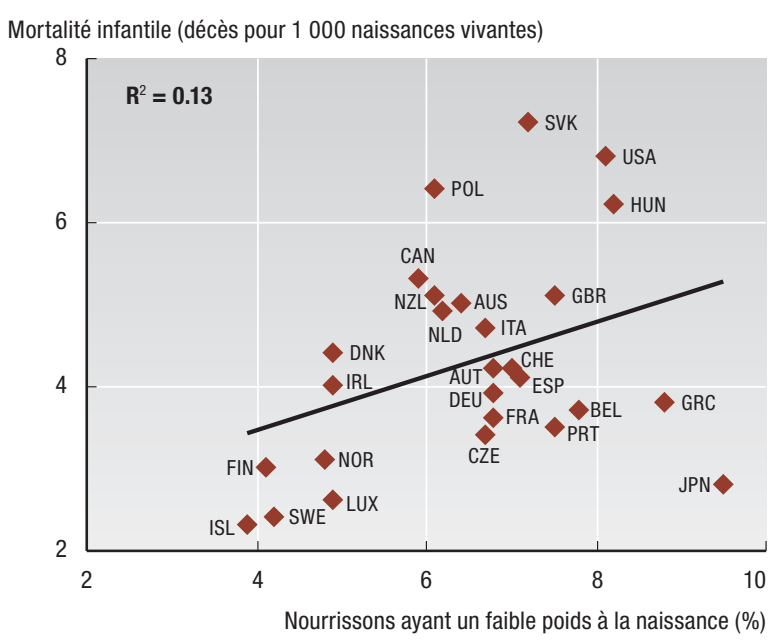

StatLink काग $h$ ttp://dx.doi.org/10.1787/128288765250 


\subsection{SANTÉ DENTAIRE DES ENFANTS}

Dans les pays développés, les problèmes dentaires, qui recouvrent principalement les caries et les affections gingivales, sont courants puisqu'ils affectent entre 60 et $90 \%$ des enfants scolarisés et l'immense majorité des adultes (OMS, 2003). À ce titre, les maladies bucco-dentaires représentent un problème de santé publique majeur. Elles sont étroitement liées à l'hygiène de vie, notamment à une alimentation riche en sucres, mais reflètent également la présence ou l'absence de mesures de protection telles l'exposition au fluorure et la qualité de l'hygiène dentaire. Les problèmes dentaires peuvent s'accompagner chez les personnes qui en souffrent, de sensations de douleur et de gêne, de déficiences fonctionnelles, d'une perte d'estime de soi et d'un sentiment d'insatisfaction lié à des considérations esthétiques. Les maladies dentaires affectent de manière disproportionnée les catégories défavorisées et socialement marginalisées (OMS, 2003). Dans les pays développés, le traitement des problèmes dentaires est souvent coûteux.

En 2003 (ou pour l'année disponible la plus proche), les enfants de 12 ans vivant en Allemagne, au Royaume-Uni, aux Pays-Bas, en Suisse, au Luxembourg et au Danemark avaient en moyenne moins d'une dent permanente cariée, absente ou obturée (DCAO) (graphique 2.10.1). En revanche, en Pologne, en Hongrie, en République tchèque et au Portugal, les enfants de 12 ans avaient un nombre de DCAO de trois ou plus. Dans la plupart des pays de l'OCDE, les enfants de 12 ans avaient entre une et trois DCAO.
Au cours des vingt-cinq dernières années, le nombre de DCAO a fortement diminué dans les pays de l'OCDE. Dans un groupe de pays pour lesquels on dispose de séries temporelles longues, il est passé d'une moyenne de 4.5 en 1980 à 2.6 en 1990 puis à 1.4 en 2003 (tableau A.2.1. et graphique 2.10.3). Sur cette période, 16 des 19 pays de l'OCDE pour lesquels des données sont disponibles ont vu leur indice DCAO diminuer de $50 \%$ ou plus (graphique 2.10.2). Il s'agit là d'un progrès considérable en termes de santé publique. Une grande majorité de pays a atteint l'objectif fixé par l'Organisation mondiale de la santé (pas plus de trois DCAO) pour l'an 2000 (OMS, 2003).

La diminution des problèmes de caries et autres problèmes dentaires est à mettre au compte de nombreuses mesures de santé publique comme les programmes communautaires de fluoruration de l'eau, mais aussi de l'évolution des conditions de vie et d'une meilleure hygiène bucco-dentaire.

Le graphique 2.10.4 montre une faible corrélation entre le nombre de DCAO chez les enfants et le nombre de dentistes par habitant. Des pays qui ont le même nombre de dentistes par habitant présentent des écarts substantiels au niveau de l'indice DCAO, ce qui signifie que des facteurs autres que la disponibilité des dentistes affectent la santé dentaire.

Certains pays s'inquiètent de constater, ces dernières années, un ralentissement du déclin voire une reprise de l'augmentation du nombre de DCAO parmi les enfants (tableau A.2.10).

\section{Définition et écarts}

L'un des indicateurs de la santé dentaire couramment utilisés est l'indice DCAO, qui mesure le nombre de caries dentaires d'une personne en termes de dents permanentes cariées (C), absentes (A) ou obturées (O). La somme de ces trois chiffres donne l'indice DCAO. Les données concernent ici les enfants de 12 ans. Un indice DCAO est jugé très faible lorsqu'il est inférieur à 1.2, faible lorsqu'il est compris entre 1.2 et 2.6, modéré dans la fourchette 2.7-4.4, et élevé lorsqu'il s'établit à 4.4 et plus.

La Norvège calcule un indice DAO, qui ne prend pas en compte les dents cariées, et la Suède un indice DCO, qui n'inclut pas les dents absentes. En Nouvelle-Zélande, il est possible que l'âge de référence moyen soit légèrement supérieur à 12 ans, car l'enquête porte sur des enfants dans leur huitième année de scolarité. 
2.10. SANTÉ DENTAIRE DES ENFANTS

2.10.1. Nombre moyen de dents cariées, absentes ou obturées chez les enfants de 12 ans, 2003 (ou année la plus proche)

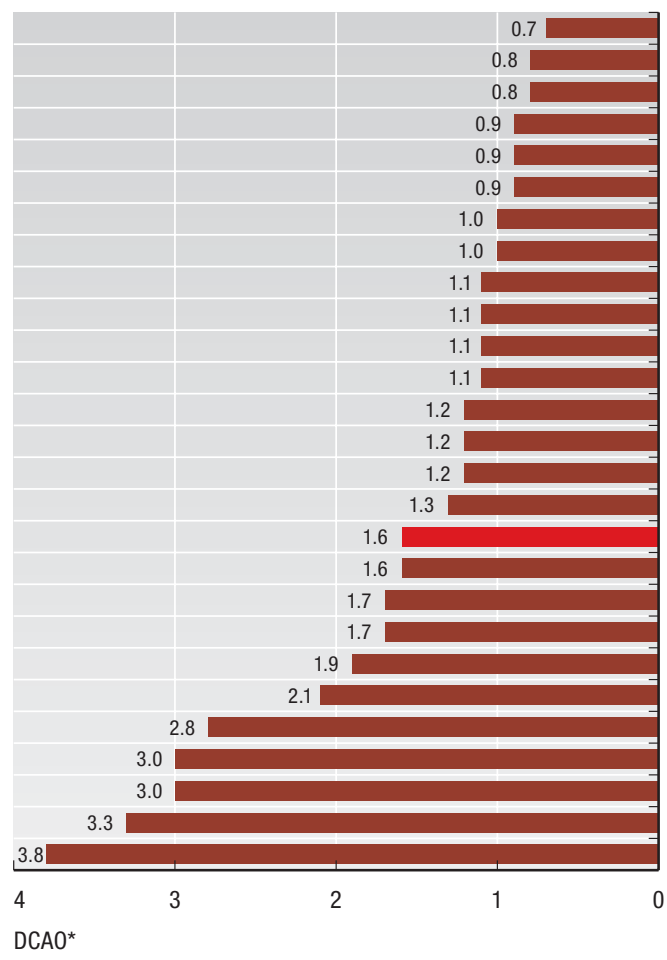

Allemagne (2003) Royaume-Uni (2003)

Pays-Bas (2002)

Suisse (2005)

Luxembourg (2003)

Danemark (2003)

Autriche (2002)

Australie (2001)

Suède (2002)

Irlande (2002)

Belgique(2001)

Espagne (2000)

Italie (2003)

France (2006)

Finlande (2003)

États-Unis (2004) OCDE

Nouvelle-Zélande (2003)

Norvège (2003)

Japon (2005)

Turquie (2004)

Islande (2005)

République slovaque (2003)

Portugal (2000)

République tchèque (2003)

Hongrie (2001)

Pologne (2000)
2.10.2. Déclin du nombre moyen de dents cariées, absentes ou obturées chez les enfants de 12 ans, 1980 à 2003

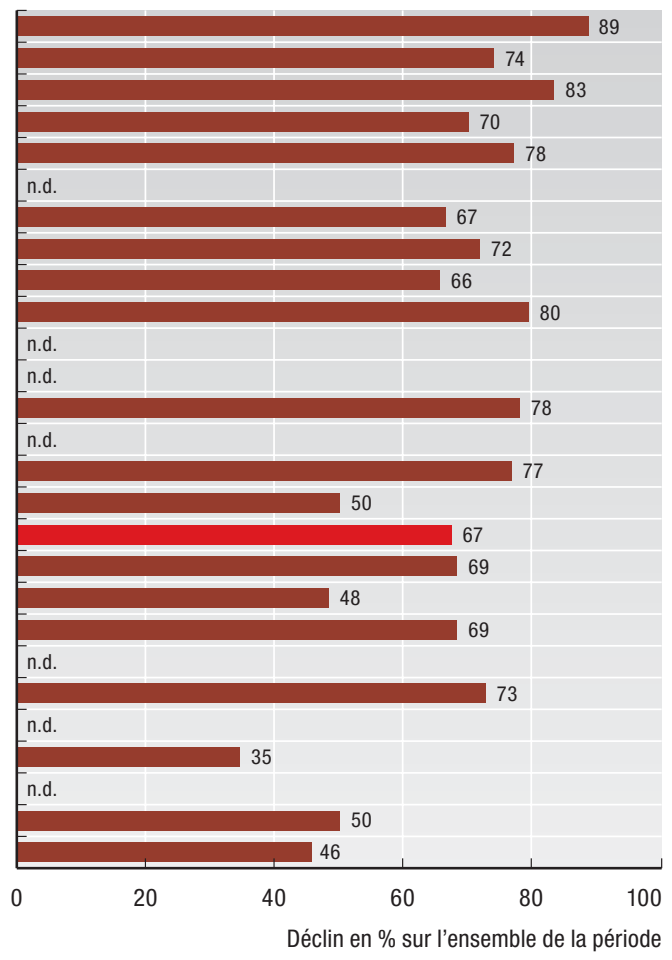

2.10.3. Nombre moyen de dents cariées, absentes ou obturées chez les enfants de 12 ans dans certains pays de l'OCDE, 1980-2005

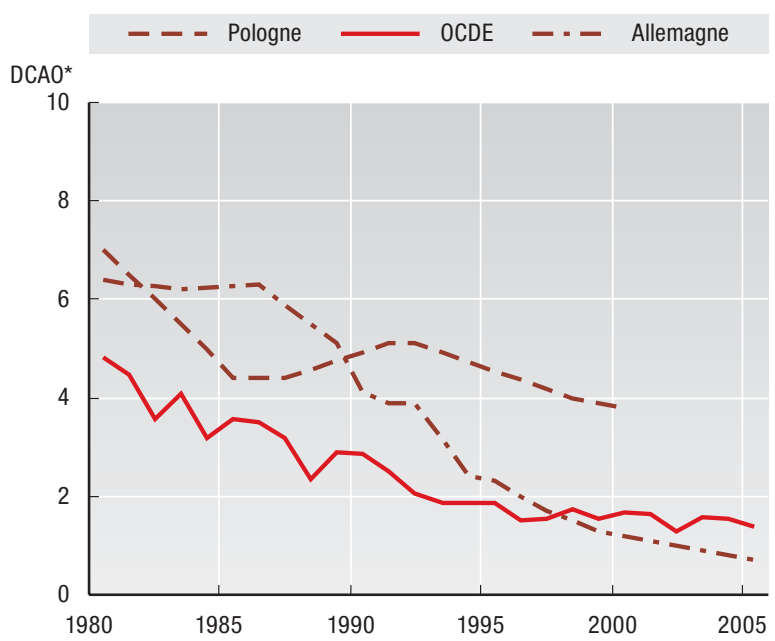

2.10.4. Nombre moyen de dents cariées, absentes ou obturées chez les enfants de 12 ans et nombre de dentistes pour 1000 habitants, 2003

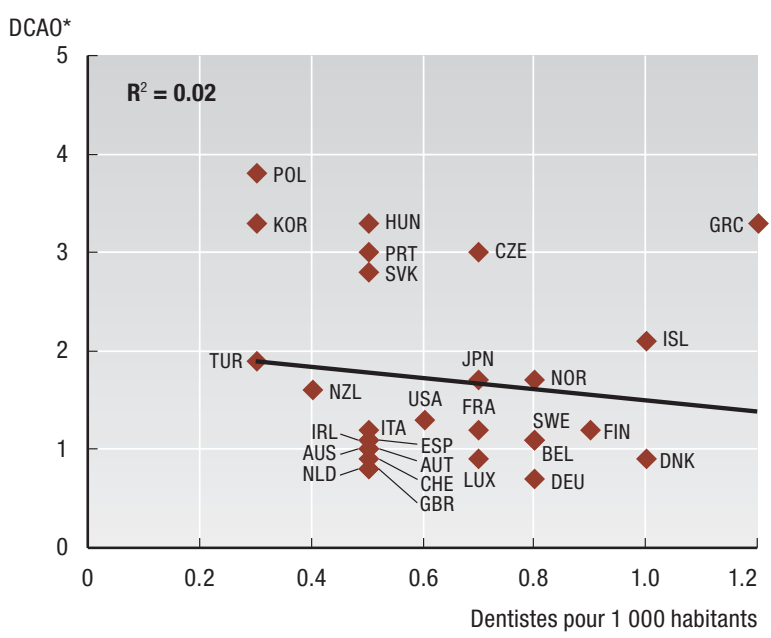

* DCAO: Dents cariées, absentes ou obstruées.

Source: Eco-Santé OCDE 2007. 


\subsection{1. ÉTAT DE SANTÉ GÉNÉRAL PERÇU}

La plupart des pays de l'OCDE réalisent des enquêtes de santé régulières qui permettent aux répondants d'évaluer différents aspects de leur état de santé. L'une des questions fréquemment posée concernent leur état de santé en général tel qu'ils le perçoivent. En dépit du caractère général et subjectif de cette question, on s'est aperçu que les indicateurs de perception de l'état de santé permettaient de faire de bonnes prévisions de l'évolution future de la consommation de soins de santé et de la mortalité (voir, par exemple, Miilunpalo et al., 1997). Dans le cadre des comparaisons internationales, cependant, les différences entre pays de la perception de l'état de santé général sont souvent difficiles à interpréter car les réponses peuvent être affectées non seulement par des différences dans la formulation des questions et réponses mais aussi par des facteurs culturels.

Tout en gardant présentes à l'esprit ces limites, dans la moitié des pays de l'OCDE, $75 \%$ ou plus de la population adulte déclare être en bonne, très bonne, voire excellente santé (graphique 2.11.1). Avec environ neuf personnes sur dix qui se déclarent en bonne santé, les États-Unis, le Canada et la Nouvelle-Zélande sont les trois pays qui ont la plus forte proportion de personnes jugeant leur santé bonne ou meilleure. Mais les catégories de réponses proposées aux répondants dans ces trois pays sont différentes de celles utilisées dans les pays européens et asiatiques de l'OCDE, ce qui introduit un biais vers le haut dans les résultats (voir ci-dessous l'encadré « Définition et écarts ").

En Espagne et en Finlande, les deux tiers environ de la population adulte considèrent leur santé comme bonne ou très bonne. À l'autre extrémité de l'échelle, moins de la moitié de la population adulte déclare être en bonne ou très bonne santé en République slovaque, en Hongrie, au Portugal, au Japon et en Corée.

Pour ce qui est des différences à l'intérieur d'un même pays, les hommes sont plus enclins que les femmes à qualifier leur santé de bonne ou très bonne dans la majorité des pays (graphique 2.11.2). Logiquement, le degré d'appréciation tend à décliner avec l'âge. Dans de nombreux pays, l'évaluation positive de l'état de santé diminue très nettement après 45 ans, puis connaît une nouvelle baisse après 65 ans. Dans tous les pays de l'OCDE, les personnes ayant un niveau d'instruction ou un niveau de revenus moindre n'ont pas une appréciation aussi positive de leur état de santé que les personnes à niveau d'instruction ou niveau de revenus plus élevé.

Le pourcentage de la population adulte se considérant en bonne ou très bonne santé est resté généralement stable sur les vingt-cinq dernières années dans les pays pour lesquels on dispose de séries temporelles longues (graphique 2.11.2). Il en est de même, en général, pour les personnes âgées de 65 ans et plus. Une interprétation possible de la coexistence de taux relativement stables de l'état de santé général autodéclaré parmi la population adulte et d'une progression régulière de l'espérance de vie sur les vingt-cinq dernières années peut être que les populations de ces pays vivent désormais plus longtemps mais que leur état de santé n'est peut-être pas meilleur. Une autre interprétation possible de la relative stabilité de l'indicateur de l'état de santé autodéclaré peut être liée à la manière spécifique dont il est apprécié, laquelle se fonde sur une variable bornée (il est demandé aux répondants de noter leur état de santé sur une échelle de cinq points qui reste inchangée dans le temps), alors que l'espérance de vie est évaluée sans aucune limite de ce type.

\section{Définition et écarts}

L'état de santé général perçu reflète la perception globale que les individus ont de leur propre santé, et englobe possiblement des dimensions physiques et psychologiques. Dans les enquêtes, la question est généralement formulée de la manière suivante : "Diriez-vous de votre état de santé général qu'il est : très bon, bon, moyen, mauvais ou très mauvais? ». La base Eco-Santé OCDE présente des chiffres correspondant à la proportion de la population qui déclare être en « bonne ou en très bonne santé ».

Il convient cependant d'être prudent dans les comparaisons internationales de l'état de santé perçu, pour au moins deux raisons. La première est liée à la subjectivité de l'appréciation générale que les personnes portent sur leur santé, laquelle peut être influencée par plusieurs facteurs en sus de leur état de santé « réel » tels que le contexte culturel et les spécificités nationales. La deuxième raison vient de ce que la formulation de la question et les catégories de réponses proposées ne sont pas identiques d'une enquête ou d'un pays à l'autre. En particulier, l'échelle de réponses utilisée aux États-Unis, au Canada, en Nouvelle-Zélande et en Australie est asymétrique (elle est biaisée vers les réponses positives) et propose les catégories suivantes: « excellente, très bonne, bonne, moyenne, mauvaise ». Les résultats présentés dans EcoSanté OCDE correspondent à l'une des trois réponses positives (" excellente, très bonne ou bonne "). En revanche, dans la plupart des autres pays de l'OCDE, l'échelle de réponses est symétrique, les catégories proposées étant " très bonne, bonne, moyenne, mauvaise, très mauvaise ". Les données ne renvoient alors qu'aux deux premières catégories (" très bonne, bonne »). Les résultats sont donc biaisés vers le haut dans les pays utilisant une échelle asymétrique par rapport aux pays utilisant une échelle symétrique. 


\section{2. ÉTAT DE SANTÉ}

\subsubsection{Pourcentage des adultes déclarant être en bonne santé, population totale, 2005 \\ (ou dernière année disponible)}

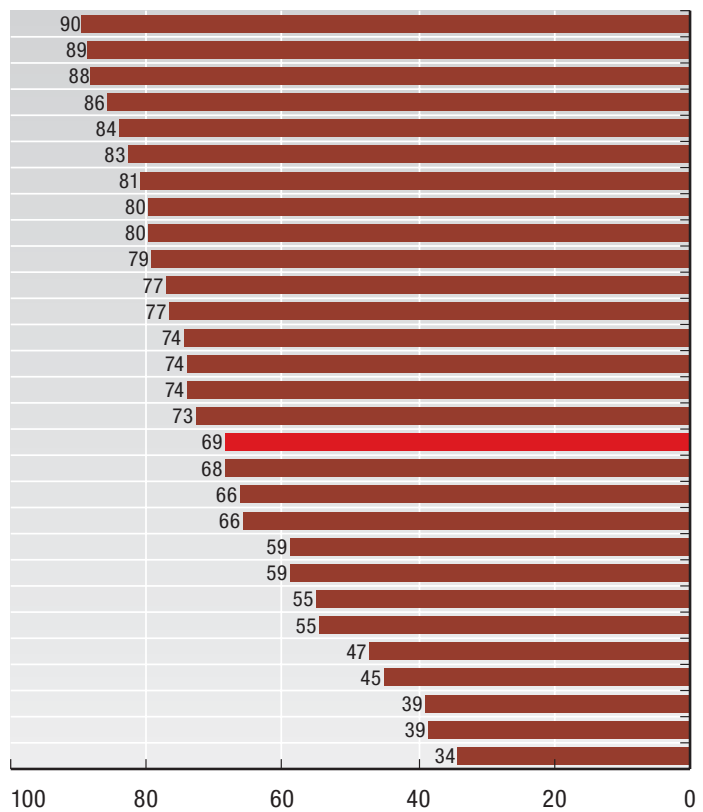

$\%$ de la population âgée de 15 ans et plus

\subsection{1. ÉTAT DE SANTÉ GÉNÉRAL PERÇU}

\subsubsection{Pourcentage des adultes déclarant} être en bonne santé, par sexe, 2005

(ou dernière année disponible)

Nouvelle-Zélande ${ }^{1}$
États-Unis $^{1}$
Canada $^{1}$
Suisse
Australie
Irlande
Norvège
Islande
France
Danemark
Belgique
Pays-Bas
Suède
Luxembourg
Royaume-Uni
Allemagne
OCDE
Espagne
Finlande
Mexique
République tchèque
Italie
Turquie
Pologne
Corée
Hongrie
Portugal
Japon
République slovaque

1. Les données de ces pays ne sont pas directement comparables à celles des autres pays, en raison de différences méthodologiques dans les questionnaires d'enquête (entraînant un biais à la hausse).

\subsection{3. Évolution du pourcentage d'adultes déclarant être en bonne santé dans quelques pays de l'OCDE, 1980 à 2005}

— États-Unis

- - - Pays-Bas

-- Suède

..... Finlande

$\%$ de la population âgée de 15 ans et plus

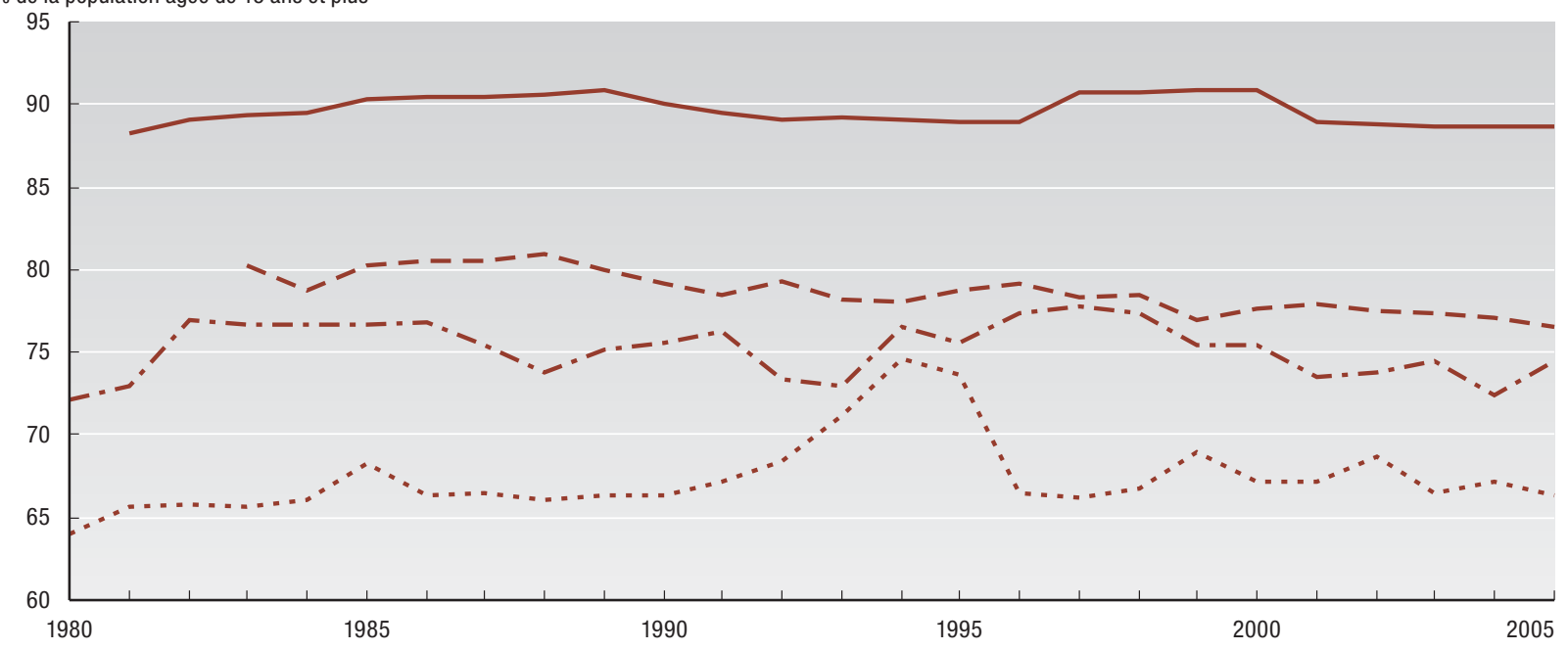

Source : Eco-Santé OCDE 2007. 


\subsection{INCIDENCE DU SIDA}

Les premiers cas de syndrome immunodéficitaire acquis (SIDA) ont été diagnostiqués il y a 25 ans. Le SIDA est normalement déclenché par une infection par le VIH (virus de l'immunodéficience humaine) et peut se manifester par de nombreuses maladies différentes, telles que la pneumonie et la tuberculose, car le système immunitaire n'est plus en mesure de défendre l'organisme. L'infection par le VIH, le diagnostic du SIDA et le décès provoqué par une infection peuvent être séparés par un nombre d'années très variable selon le traitement administré. En dépit d'une recherche à l'échelle mondiale, il n'existe pas actuellement de traitement permettant de guérir la maladie.

En 2005, le nombre de nouveaux cas déclarés de SIDA s'élevait à environ 55000 pour toute la zone OCDE, soit un taux d'incidence moyen non pondéré de 18.8 par million d'habitants (graphique 2.12.1 et tableau A.2.12). Depuis les premiers cas de SIDA signalés au début des années 80, leur nombre a rapidement grimpé pour culminer à plus de 44 nouveaux cas par million d'habitants en moyenne dans les pays de l'OCDE dans la première moitié des années 90 , soit plus du double des taux d'incidence actuels (graphique 2.12.2). Des campagnes de sensibilisation du public ont contribué à une baisse régulière du nombre de cas déclarés pendant la deuxième moitié des années 90. De plus, le développement des antirétroviraux, qui réduisent ou ralentissent le développement de la maladie, et un accès plus large à ces thérapies ont conduit à une forte diminution de l'incidence entre 1996 et 1997.

Les États-Unis ont constamment présenté les taux d'incidence du SIDA les plus élevés des pays de l'OCDE, même s'il est important de noter que les définitions régissant l'enregistrement des cas ont été élargies en 1993 et diffèrent par conséquent de celle utilisée en Europe et dans les autres pays de l'OCDE. La modification de la définition explique également la forte augmentation des cas aux États-Unis en 1993 (graphique 2.12.2). En Europe, l'Espagne a régulièrement affiché les taux d'incidence les plus élevés dans la première décennie qui a suivi l'apparition de la maladie, avec toutefois un brusque déclin depuis 1994, laissant le Portugal afficher le taux le plus élevé d'Europe. Les pays d'Europe centrale comme la République tchèque, la République slovaque et la Hongrie, mais aussi la Corée et le Japon déclarent les taux d'incidence du SIDA les plus faibles des pays de l'OCDE.

Aux États-Unis, les minorités raciales et ethniques continuent d'être touchées par l'épidémie de façon disproportionnée. Au Canada, les populations aborigènes sont surreprésentées parmi les catégories touchées. Dans la plupart des pays de l'OCDE, les rapports homosexuels non protégés demeurent le principal facteur de risque d'infection par le VIH (ONUSIDA, 2006). Parallèlement, c'est parmi les immigrants et les migrants que l'on trouve environ $75 \%$ des infections par le VIH acquises par des hétérosexuels en Europe occidentale et centrale.

Ces dernières années, le déclin général du nombre de cas de SIDA s'est ralenti. Cette inversion de tendance s'est accompagnée d'indications d'une résurgence de nouveaux taux d'infection par le VIH. Ce phénomène peut être attribué à une complaisance à l'égard de l'efficacité des traitements et à une moindre sensibilisation du public aux risques que présente la consommation de drogues et certaines pratiques sexuelles. Pour faire à nouveau reculer les taux d'incidence du SIDA, il faudra mettre en œuvre des programmes plus intensifs de prévention du VIH ciblés sur les sujets les plus à risque d'être infectés par le VIH.

\section{Définition et écarts}

Le taux d'incidence du SIDA est le nombre de nouveaux cas par million d'habitants dans l'année du diagnostic. Il convient de noter que les données portant sur les années récentes sont provisoires en raison des délais de notification qui peuvent parfois être de plusieurs années selon le pays.

En 1993, les États-Unis ont étendu leur définition des cas de SIDA à des fins de surveillance pour y inclure le critère de numération des lymphocytes $\mathrm{T}$. Cet élargissement de la définition a abouti à une forte augmentation du nombre de nouveaux cas aux États-Unis en 1993 et explique en partie les variations actuelles de l'incidence du SIDA observées entre les États-Unis et les autres pays de l'OCDE. 


\section{2. ÉTAT DE SANTÉ}

\subsection{INCIDENCE DU SIDA}

\subsubsection{Taux d'incidence du SIDA, 2005}

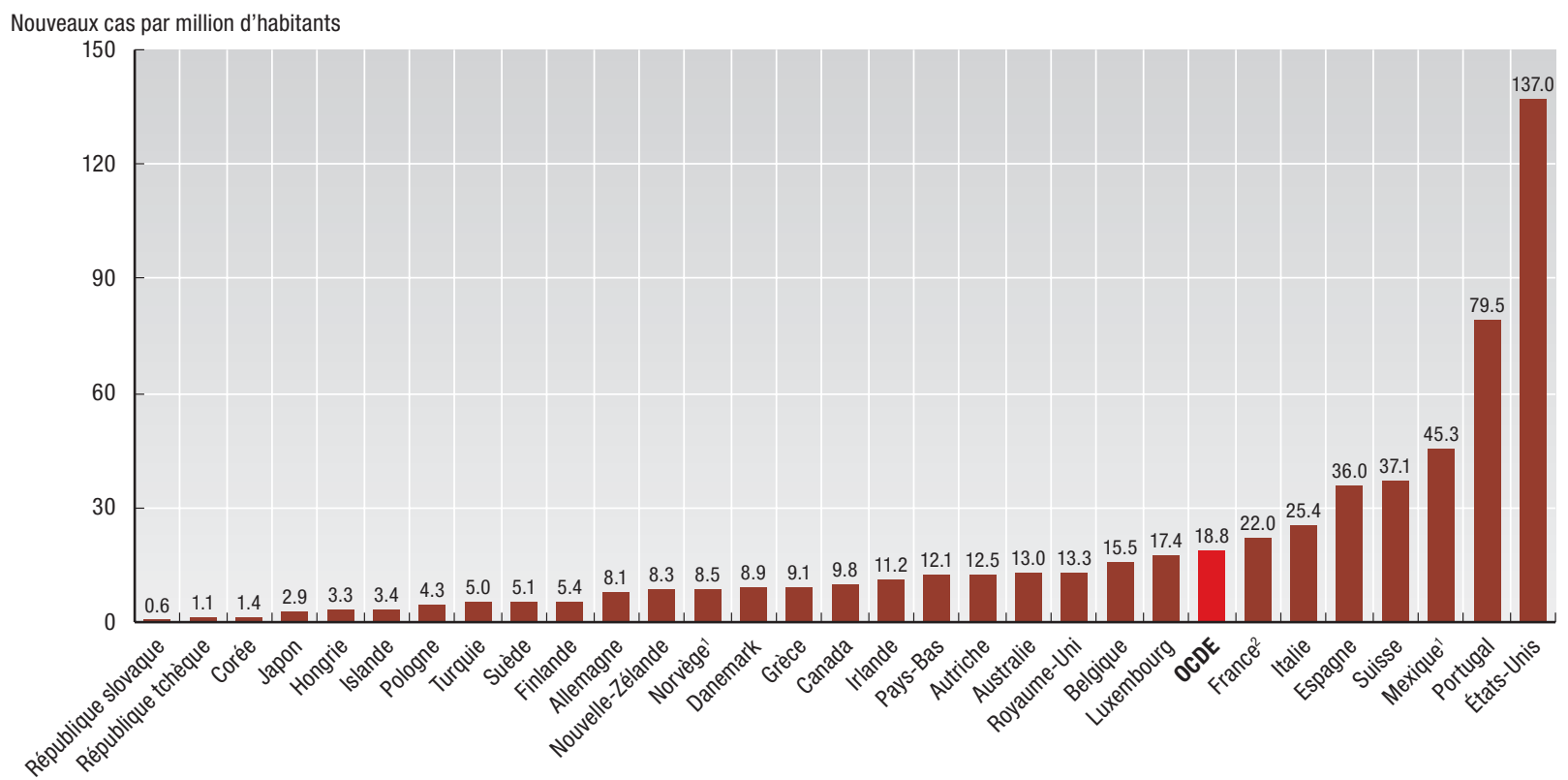

1. 2003. 2. 2004

2.12.2. Tendances de l'incidence du SIDA, 1980-2005

- - - Canada $\quad$ - - Portugal .... États-Unis _ OCDE

Nouveaux cas par million d'habitants

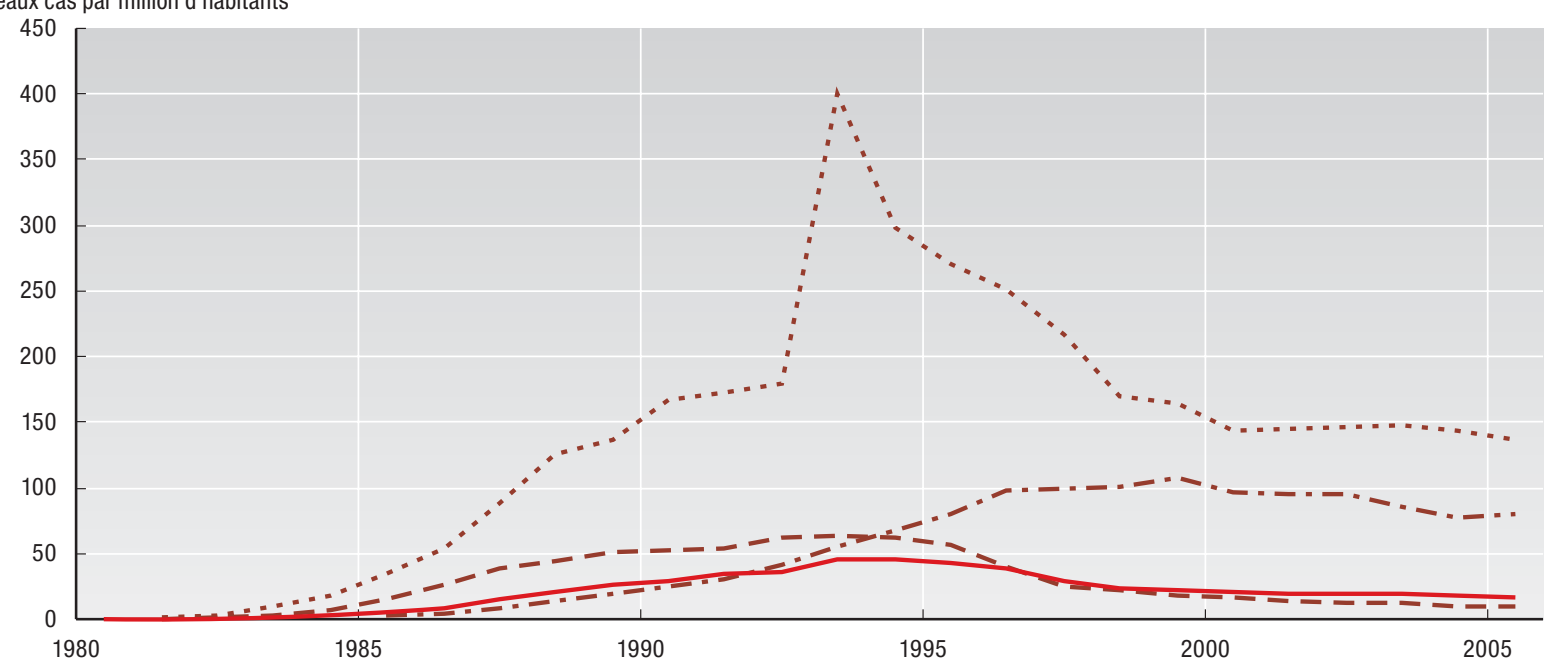

Note : Les États-Unis ont étendu leur définition des cas avérés de SIDA en 1993.

Les données pour les pays européens sont extraites du Centre européen pour la surveillance épidémiologique du SIDA.

Source: Eco-Santé OCDE 2007. 


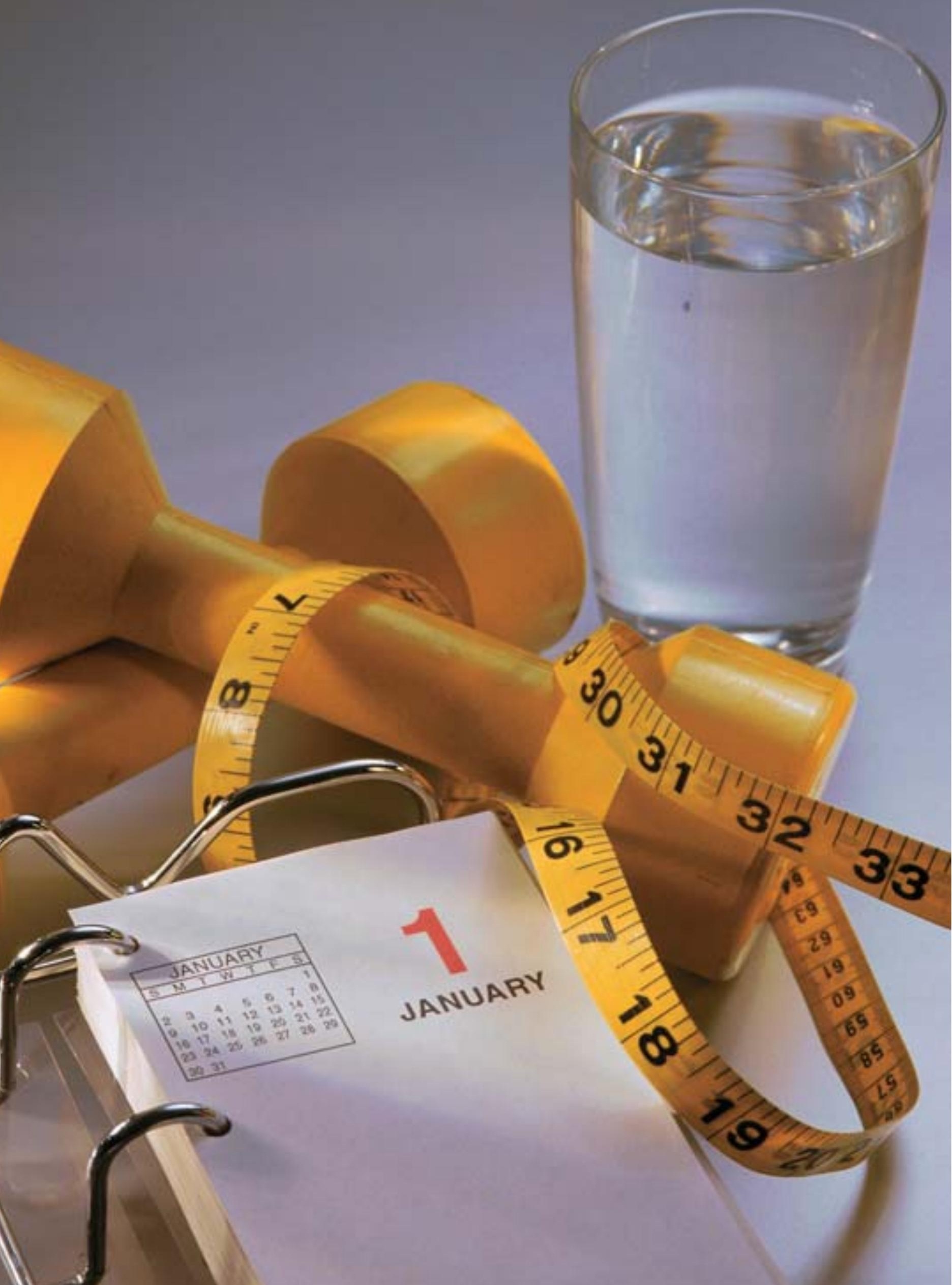




\section{DÉTERMINANTS NON MÉDICAUX DE LA SANTÉ}

3.1. CONSOMMATION DE TABAC ................. 46

3.2. CONSOMMATION D'ALCOOL ................... 48

3.3. SURCHARGE PONDÉRALE ET OBÉSITÉ $\ldots \ldots \ldots \ldots \ldots \ldots \ldots$ 


\subsection{CONSOMMATION DE TABAC}

Selon l'Organisation mondiale de la santé, le tabac est la deuxième grande cause de décès dans le monde, étant directement responsable d'environ un décès sur dix chez les adultes, soit environ 5 millions de décès chaque année (OMS, 2002). Il est un facteur de risque important pour au moins deux des principales causes de mortalité prématurée: les maladies du système circulatoire et toute une série de cancers. En outre, le tabac contribue de manière importante à l'apparition de maladies respiratoires tandis que sa consommation pendant la grossesse peut avoir pour conséquence des bébés dont le poids de naissance est faible et souffrant de maladies. Le tabac demeure le plus gros risque évitable pour la santé dans les pays de l'OCDE.

La proportion de fumeurs quotidiens parmi la population adulte varie considérablement d'un pays de l'OCDE à l'autre, même entre pays voisins (graphique 3.1.1). L'Amérique du Nord, l'Australie et des pays aussi différents que la Suède et le Portugal affichent les pourcentages de fumeurs les plus faibles. Dans les pays de l'OCDE, la proportion des fumeurs a diminué de près de 3 points de pourcentage en moyenne depuis 2000 , chez les hommes comme chez les femmes. Les principaux pays ayant contribué à cette baisse sont la Belgique (31\% à $20 \%)$, le Canada (22\% à $17 \%$ ), le Danemark (30\% à $26 \%$ ), la Corée (30\% à $25 \%$ ) et le Luxembourg (30\% à $23 \%$ ). La Grèce, qui conserve la plus forte proportion de fumeurs, est le seul pays de l'OCDE dans lequel la consommation semble augmenter (35\% à $39 \%$ ) chez les hommes comme chez les femmes.

Dans les années d'après-guerre, la plupart des pays de l'OCDE ont eu tendance à suivre un schéma général marqué par une très forte proportion de fumeurs parmi les hommes (environ $50 \%$, voire davantage) jusque dans les années 60 et 70, alors que les années 80 et 90 se sont caractérisées par une nette diminution de la consommation de tabac. Cette baisse peut être attribuée, pour une large part, aux politiques mises en œuvre, face à l'accroissement des maladies liées au tabac, pour réduire sa consommation par des campagnes de sensibilisation du public, une interdiction de la publicité sur le tabac et une augmentation des taxes (Banque mondiale, 1999). À ces politiques gouvernementales sont venues s'ajouter les actions très efficaces des lobbies anti-tabac, en particulier en Amérique du Nord, qui sont parvenus à faire changer les mentalités quant aux effets du tabac pour la santé (Cutler et Glaeser, 2006).

Bien que de grandes disparités subsistent, la consommation de tabac a nettement diminué au cours des dernières décennies dans la plupart des pays de l'OCDE (graphique 3.1.3). La prévalence de fumeurs demeure plus élevée parmi les hommes que parmi les femmes dans tous les pays de l'OCDE à l'exception de la Suède. La proportion de fumeurs chez les femmes continue de baisser dans la plupart des pays de l'OCDE, dans certains cas plus vite même que chez les hommes. Dans quatre pays seulement (en Grèce, en Allemagne, au Mexique et en Espagne), la proportion de fumeurs chez les femmes semble avoir augmenté au cours des quinze dernières années mais, dans ces pays, la proportion de fumeurs parmi les femmes reste bien inférieure à celle observée chez les hommes. En 2005, l'écart entre les hommes et les femmes était particulièrement important en Corée, au Japon et en Turquie mais aussi, dans une moindre mesure, au Mexique, au Portugal, en Grèce et en Pologne (graphique 3.1.2).

Le graphique 3.1.4 montre la corrélation existant entre la consommation de tabac (mesurée en grammes par habitant) et l'incidence des cancers du poumon dans les pays de l'OCDE, une vingtaine d'années plus tard. À une consommation de tabac plus élevée au niveau national sont aussi généralement associés des taux plus élevés de mortalité par cancer du poumon une ou deux décennies plus tard dans les pays de l'OCDE.

\section{Définition et écarts}

La proportion de fumeurs quotidiens se définit par le pourcentage de la population de 15 ans et plus déclarant fumer tous les jours.

La comparabilité internationale est limitée en raison de l'absence de normalisation de la mesure des habitudes de fumer dans les enquêtes sur la santé menées dans les pays de l'OCDE. Des disparités subsistent dans la formulation des questions, les catégories de réponses et les méthodes d'enquête. 


\section{DÉTERMINANTS NON MÉDICAUX DE LA SANTÉ}

\subsubsection{Pourcentage de la population adulte déclarant fumer quotidiennement, 2005}

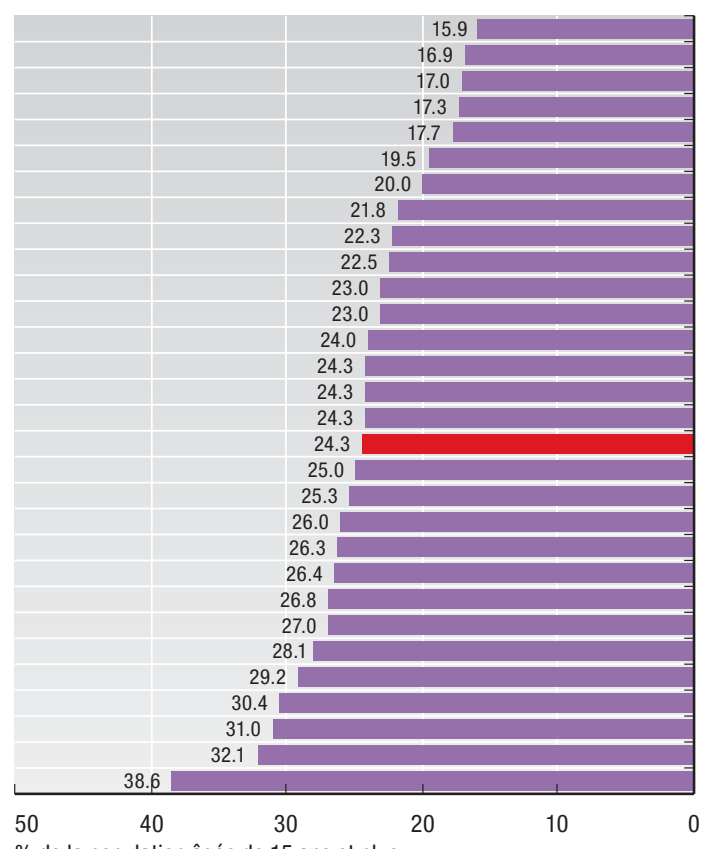

$\%$ de la population âgée de 15 ans et plus

1. 2004. 2. 2003. 3. 2002.

\subsection{CONSOMMATION DE TABAC}

\subsubsection{Pourcentage de femmes et d'hommes} déclarant fumer quotidiennement, 2005

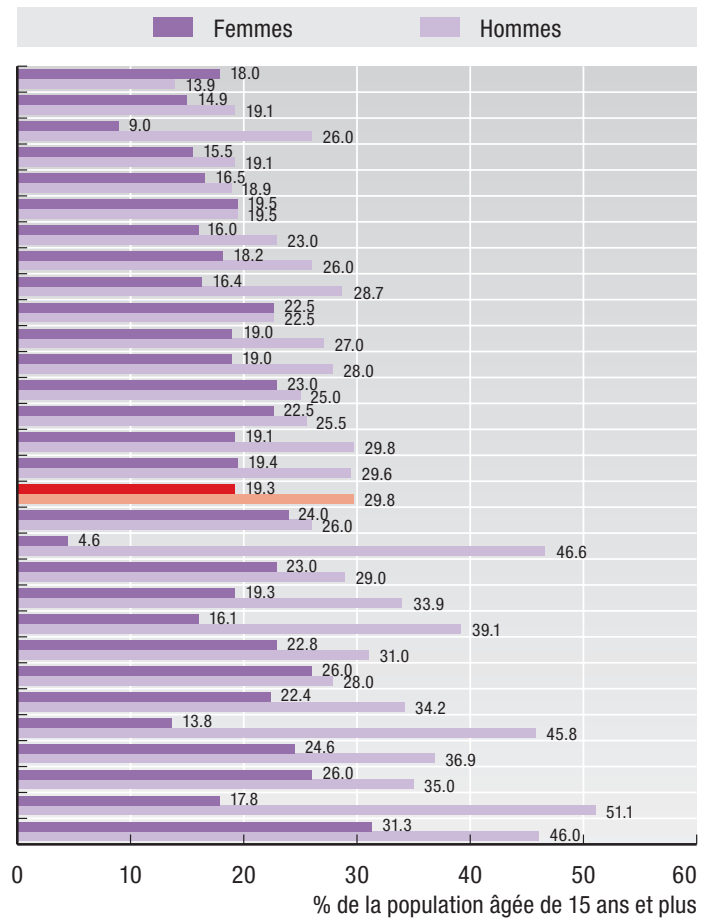

\subsubsection{Variation des taux de tabagisme par sexe,} 1990 à 2005 (ou année la plus proche)

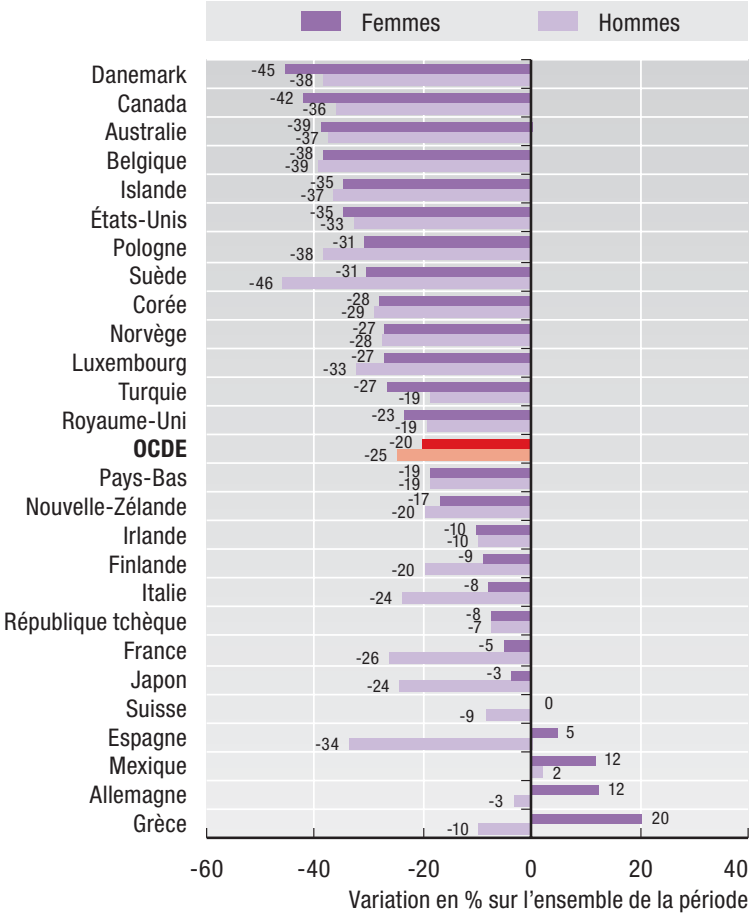

Source : Eco-Santé OCDE 2007.

\subsubsection{Consommation de tabac, 1980 et incidence du cancer du poumon, 2002}

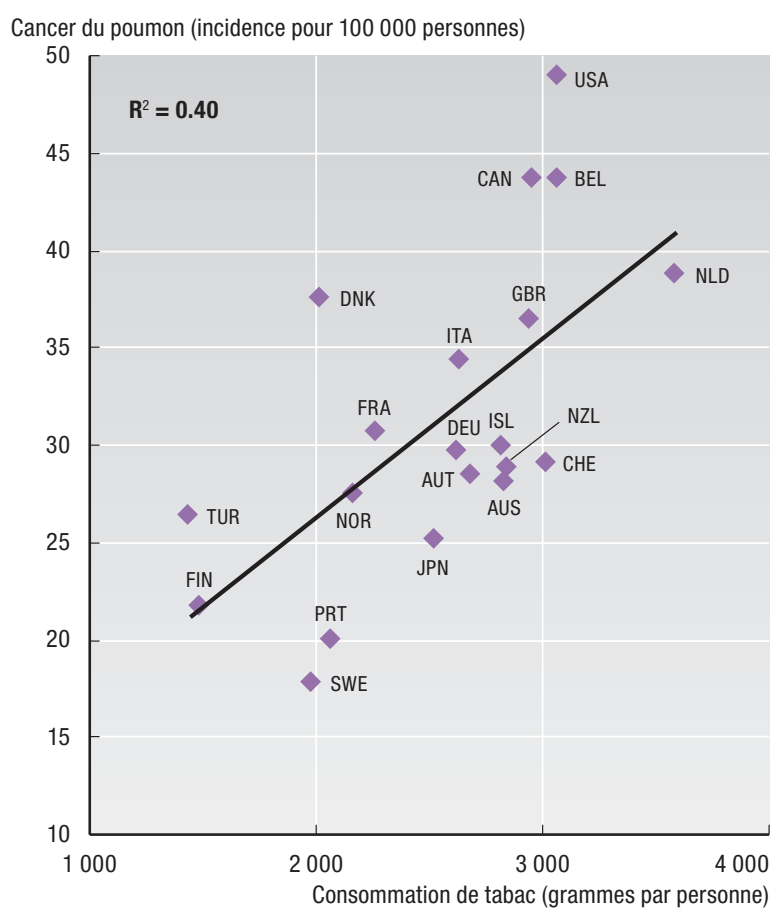




\subsection{CONSOMMATION D'ALCOOL}

Une consommation excessive d'alcool engendre un grand nombre d'effets préjudiciables pour la santé. Une forte absorption d'alcool accroît le risque d'accidents vasculaires cérébraux et de maladies cardio-vasculaires mais aussi de cirrhose du foie et de certains cancers. L'exposition du fœtus à l'alcool accroît les risques de malformations congénitales et de retard mental. L'alcool est également une cause de décès et d'incapacités par accidents et blessures, agression, homicides et suicides.

Dans les pays de l'OCDE, la consommation d'alcool, mesurée par les ventes annuelles d'alcool, s'établit en moyenne à 9.5 litres par adulte, selon les données disponibles les plus récentes. Toutefois, la situation varie beaucoup d'un pays à l'autre. Si on laisse de côté le Luxembourg en raison du volume important d'achats effectués dans ce pays par des non-résidents, ce sont l'Irlande, la Hongrie, la France et la République tchèque qui font état de la plus forte consommation d'alcool, avec 12 litres ou plus par adulte et par an en 2005 (ou 2004). À l'autre extrémité de l'échelle, la Turquie, le Mexique et certains pays nordiques (la Norvège, la Suède et l'Islande) ont fait état de niveaux relativement faibles de consommation d'alcool, s'échelonnant entre 1.3 et 7.1 litres par adulte (graphique 3.2.1).

Bien que la consommation moyenne d'alcool ait progressivement diminué dans de nombreux pays de l'OCDE au cours des vingt dernières années, elle a augmenté dans d'autres (graphique 3.2.2). On a observé une certaine convergence dans les habitudes de consommation des différents pays de l'OCDE, avec une augmentation de la consommation de vin dans des pays traditionnellement buveurs de bière et vice versa. Les producteurs traditionnels de vin, comme l'Italie et la France, ont vu leur consommation d'alcool par habitant chuter considérablement depuis 1980 (graphiques 3.2.2 et 3.2.3). En revanche, en Islande, en Irlande et au Mexique, la consommation d'alcool par habitant a augmenté dans des proportions de $40 \%$ et plus entre 1980 et 2005 (bien que, dans le cas de l'Islande et du Mexique, elle soit partie d'un niveau très bas et demeure relativement faible).
Les variations de la consommation d'alcool dans le temps et d'un pays à l'autre reflètent non seulement une modification des habitudes de consommation mais également les politiques mises en œuvre pour lutter contre l'abus d'alcool. La limitation de la publicité, les restrictions de ventes et la taxation ont été autant de mesures efficaces pour réduire la consommation d'alcool (Bennett, 2003). Un strict contrôle des ventes et une taxation plus lourde se sont traduits par une diminution globale de la consommation dans la plupart des pays nordiques tandis que la chute de la consommation en France, en Italie et en Espagne a été associée à une réglementation plus stricte de la publicité, en partie après l'adoption de la directive européenne de 1989.

Bien que la consommation d'alcool par habitant des adultes fournisse des indications utiles sur les tendances à long terme, elle n'identifie pas les sous-populations à risque du fait d'habitudes de consommation nocives pour la santé. La consommation occasionnelle de grosses quantités d'alcool ("cuite d'un soir ") est un schéma de consommation particulièrement dangereux (Institute of Alcohol Studies, 2007) qui est en augmentation dans certains pays et certains groupes sociaux (en particulier chez les jeunes hommes). Malheureusement, cette information sur les schémas de consommation ne peut généralement être obtenue qu'à partir d'enquêtes détaillées sur les habitudes de consommation et non pas à partir de grandes enquêtes sur la santé.

Le graphique 3.2.4 montre la relation existant entre la consommation d'alcool en 1990 et le nombre des décès par cirrhose du foie en 2004. En général, les pays ayant un niveau élevé de consommation d'alcool ont tendance à enregistrer, 10 à 15 ans plus tard, des taux de décès par cirrhose du foie supérieurs à ceux de pays ayant des niveaux de consommation moindres. Dans la plupart des pays de l'OCDE, le taux des décès par cirrhose du foie a chuté au cours des vingt dernières années, suivant étroitement la diminution globale de la consommation d'alcool.

\section{Définition et écarts}

La consommation d'alcool est mesurée par les ventes annuelles d'alcool pur, en nombre de litres par personne âgée de 15 ans et plus. La méthode utilisée pour convertir les boissons alcoolisées en alcool pur peut varier d'un pays à l'autre.

Dans certains pays comme le Luxembourg, les ventes nationales ne reflètent pas avec exactitude la consommation réelle des résidents, dans la mesure où les achats des non-résidents peuvent créer un décalage important entre les ventes nationales et la consommation. 


\section{DÉTERMINANTS NON MÉDICAUX DE LA SANTÉ}

\subsubsection{Consommation d'alcool en litres par habitant, population} âgée de 15 ans et plus, 2005

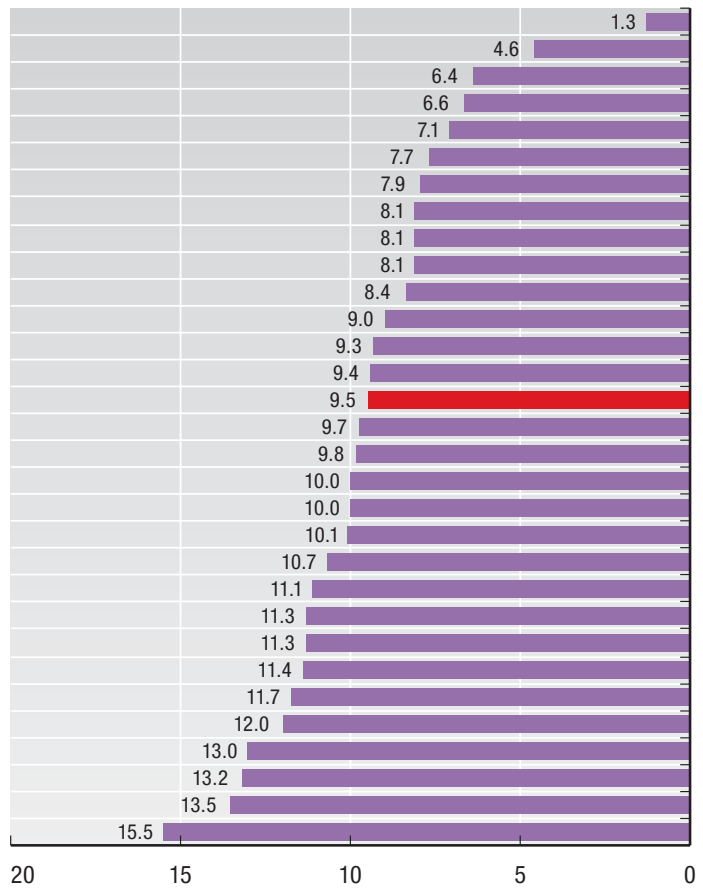

Litres par habitant âgé de 15 ans et plus

1. 2003. 2. 2004.

3.2.3. Tendances de la consommation d'alcool dans quelques pays de l’OCDE, 1980 à 2005

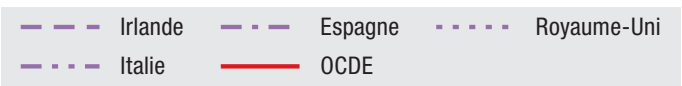

Litres par habitant âgé de 15 ans et plus

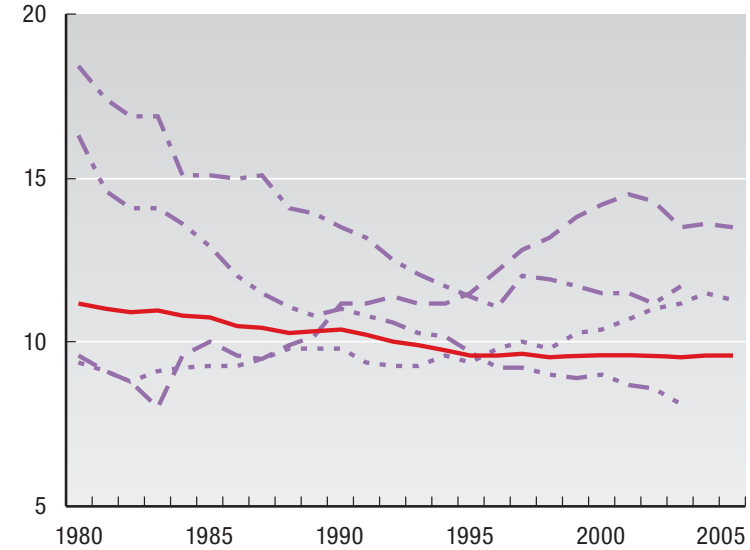

Source : Eco-Santé OCDE 2007.

\subsection{CONSOMMATION D'ALCOOL}

3.2.2. Variation de la consommation d'alcool par habitant, population

âgée de 15 ans et plus, 1980 à 2005

Turquie
Mexique $^{1}$
Norvège
Suède
Islande
Japon $^{2}$
Canada $^{2}$
Pologne
Corée
Italie $^{1}$
États-Unis $^{2}$
Grèce $^{1}$

République slovaque

Nouvelle-Zélande

OCDE

Pays-Bas ${ }^{1}$

Australie $^{2}$

Allemagne

Finlande

Suisse

Belgique $^{1}$

Autriche $^{1}$

Royaume-Uni

Danemark

Portugal ${ }^{1}$

Espagne

République tchèque

France $^{2}$

Hongrie $^{2}$

Irlande

Luxembourg $^{1}$

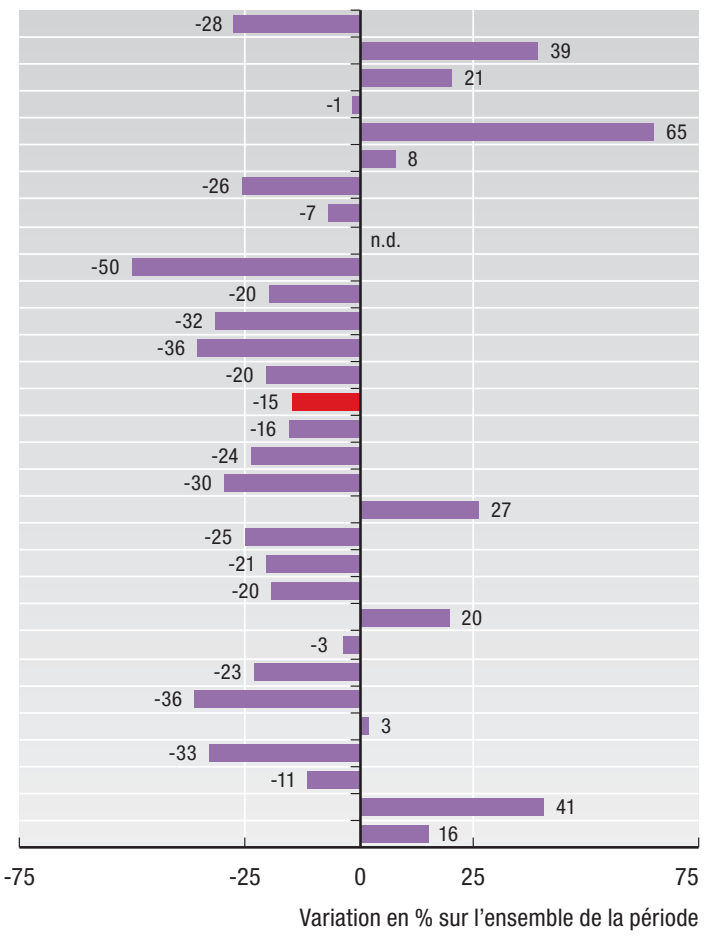

3.2.4. Mortalité par cirrhose du foie, 2004 et consommation d'alcool, 1990
Cirrhose du foie (décès pour 100000 personnes)

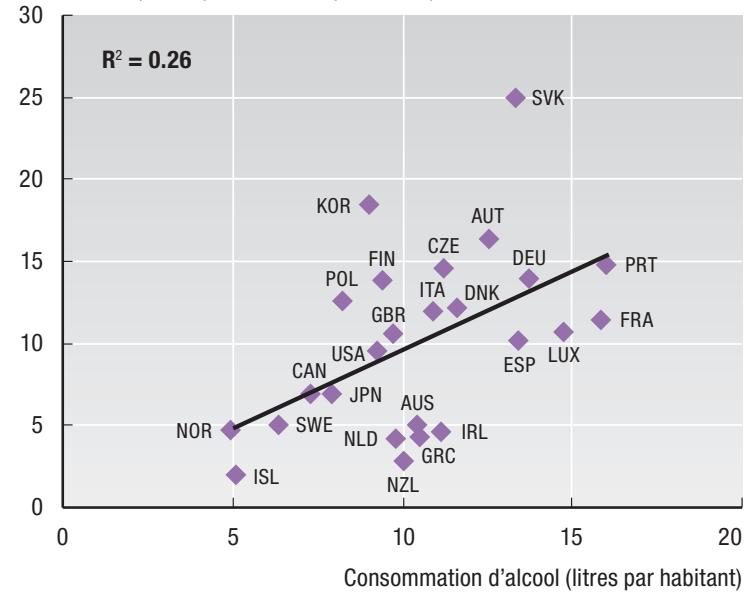




\subsection{SURCHARGE PONDÉRALE ET OBÉSITÉ}

Dans de nombreux pays de l'OCDE, l'augmentation des cas de surcharge pondérale et d'obésité parmi les enfants et les adultes est en train de devenir rapidement un grave problème de santé publique. L'obésité est un facteur de risque connu pour plusieurs problèmes de santé, dont l'hypertension, le cholestérol, le diabète, les maladies cardio-vasculaires, les problèmes respiratoires (l'asthme), l'arthrite et certaines formes de cancer. Aux États-Unis, où désormais plus de trois adultes sur dix sont obèses, une étude a estimé que le coût lié à l'obésité est désormais supérieur au coût lié au tabac et à l'abus d'alcool combinés pour un ensemble de problèmes de santé chroniques (Sturm, 2002).

Environ la moitié ou plus de la population adulte est désormais définie comme étant en situation de surcharge pondérale ou d'obésité dans 15 pays de l'OCDE : le Mexique, les États-Unis, le Royaume-Uni, l'Australie, la Grèce, la Nouvelle-Zélande, le Luxembourg, la Hongrie, la République tchèque, le Canada, l'Allemagne, le Portugal, la Finlande, l'Espagne et l'Islande (tableau A.3.3). À l'inverse, les cas de surcharge pondérale et d'obésité sont beaucoup moins nombreux dans les deux pays asiatiques de l'OCDE (le Japon et la Corée) et dans certains pays européens (la France et la Suisse), bien qu'ils soient également en augmentation dans ces pays. Si l'on se concentre sur la seule obésité (qui présente des risques plus grands pour la santé que la surcharge pondérale), la prévalence de l'obésité parmi les adultes varie de $3 \%$ au Japon et en Corée à plus de $30 \%$ aux États-Unis et au Mexique (graphiques 3.3.1 et 3.3.2).

Sur la base de mesures de l'obésité cohérentes dans le temps, la proportion d'obèses a plus que doublé sur les vingt dernières années aux États-Unis, alors qu'elle a presque triplé en Australie et plus que triplé au Royaume-Uni (graphique 3.3.3). Quelque 21 à $23 \%$ des adultes au Royaume-Uni, en Grèce, en Australie et en Nouvelle-Zélande sont désormais définis comme obèses, soit un pourcentage à peu près identique à celui des États-Unis au début des années 90. Dans de nombreux pays d'Europe occidentale, la proportion d'obèses a également fortement augmenté au cours de la dernière décennie.

Dans de nombreux pays, la progression de l'obésité a touché toutes les catégories de population, sans distinction de sexe, d'âge, de race, de revenu ou de niveau d'instruction. Des données émanant des États-Unis, du Canada et du Royaume-Uni indiquent toutefois que l'obésité tend à être plus répandue parmi les catégories socio-économiques défavorisées que parmi les catégories plus aisées (Statistique Canada et CDC, 2004). Cela est particulièrement vrai chez les femmes alors que la tendance est absente ou beaucoup moins prononcée chez les hommes.

Parce que l'obésité est associée à des risques plus élevés de maladies chroniques, elle est liée à une augmentation importante des dépenses de santé. Au niveau macroéconomique, on estime que les coûts de santé pouvant être attribués à l'obésité représentaient environ 5 à $7 \%$ des dépenses totales de santé des États-Unis à la fin des années 90 et entre 2 et $3.5 \%$ des dépenses de santé d'autres pays comme le Canada, l'Australie et la Nouvelle-Zélande (Thompson et Wolf, 2001). Au niveau microéconomique, des estimations en provenance des États-Unis indiquent que le coût des services de santé et le coût des médicaments pour les personnes obèses sont respectivement supérieurs de $36 \%$ et de $77 \%$ par rapport à ces mêmes coûts pour des personnes d'un poids normal (Sturm, 2002). On observe un décalage de plusieurs années entre l'apparition de l'obésité et les problèmes de santé connexes, ce qui donne à penser que l'accroissement de l'obésité observé sur les vingt dernières années dans la plupart des pays de l'OCDE va générer dans les années à venir des coûts de santé plus élevés.

Un certain nombre de facteurs comportementaux et environnementaux ont contribué à l'augmentation des cas de surcharge pondérale et d'obésité dans les pays industrialisés ; ces facteurs sont notamment la baisse du prix réel des aliments et l'augmentation du temps d'inactivité physique. La surcharge pondérale et l'obésité progressent également vite chez les enfants, atteignant des niveaux à deux chiffres dans la plupart des pays de l'OCDE : un tiers des enfants de 13 à 14 ans en Espagne (2000-02) ; $29 \%$ des enfants de 5 à 17 ans en Angleterre (2004); et environ un quart des enfants de 5 à 17 ans en Italie (1993-2001) et de 5 à 15 ans en Belgique (1998-99) (International Association for the Study of Obesity, 2007).

\section{Définition et écarts}

La surcharge pondérale et l'obésité se définissent comme un poids excessif présentant des risques pour la santé en raison d'une proportion élevée d'adipométrie. La mesure la plus fréquemment utilisée de la surcharge pondérale et de l'obésité se fonde sur l'indice de masse corporelle (IMC) qui est un chiffre évaluant le rapport entre le poids et la taille ${ }^{2}$ d'un individu (le poids étant exprimé en kilos et la taille en mètres). Sur la base de la classification actuelle de l'OMS (OMS, 1997), des adultes ayant un IMC compris entre 25 et 30 sont définis comme étant en état de surcharge pondérale et des adultes ayant un IMC supérieur à 30 sont définis comme obèses. Toutefois, cette classification risque de n'être pas appropriée pour tous les groupes ethniques, qui peuvent avoir des niveaux de risque équivalents pour un IMC inférieur (les asiatiques, par exemple) ou supérieur (AIHW, 2004). Les seuils fixés pour les adultes ne sont pas non plus appropriés pour mesurer la surcharge pondérale et l'obésité chez les enfants.

Pour la plupart des pays, les estimations de la surcharge pondérale et de l'obésité sont autodéclarées dans le cadre d'enquêtes par entretien effectuées auprès de la population. Ce n'est pas le cas pour l'Australie, les États-Unis, le Luxembourg, la Nouvelle-Zélande, la République tchèque et le Royaume-Uni où les estimations sont basées sur des mesures précises de la taille et du poids des personnes. Ces différences de méthode de collecte des données limitent sérieusement leur comparabilité. Les estimations obtenues à partir de mesures de la taille et du poids des personnes sont généralement plus élevées que celles provenant d'autodéclarations. Aux États-Unis, par exemple, le taux d'obésité des adultes obtenu sur la base d'enquêtes par entretien était de $22 \%$ en 1999 contre un taux de $31 \%$ la même année sur la base de mesures précises. 


\section{DÉTERMINANTS NON MÉDICAUX DE LA SANTÉ}

3.3.1. Pourcentage de la population adulte ayant un IMC* $>30$ (population obèse), 2005 ou année la plus proche

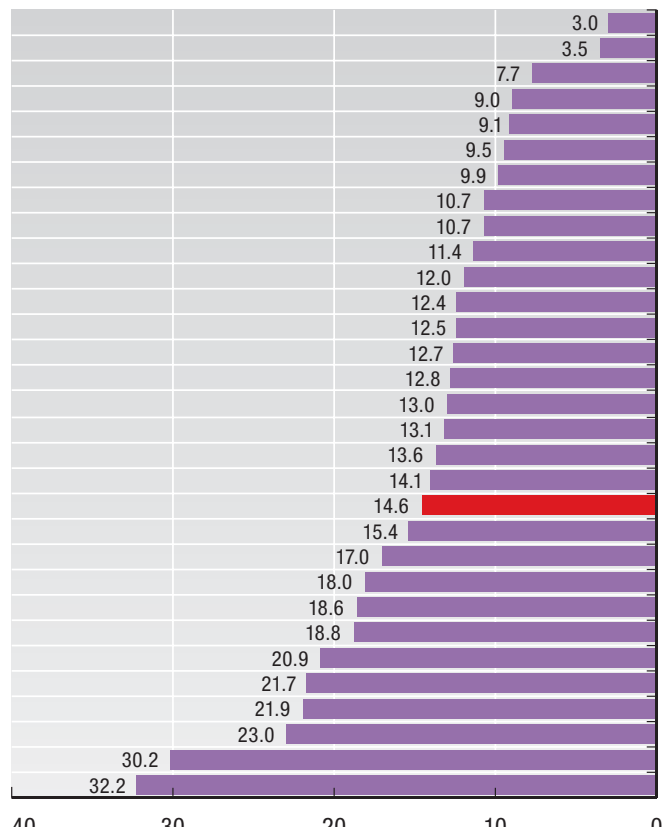

$\%$ de la population adulte
3.3. SURCHARGE PONDÉRALE ET OBÉSITÉ

\subsubsection{Pourcentage de femmes et d'hommes ayant un IMC* $>30$ (population obèse), 2005 ou année la plus proche}

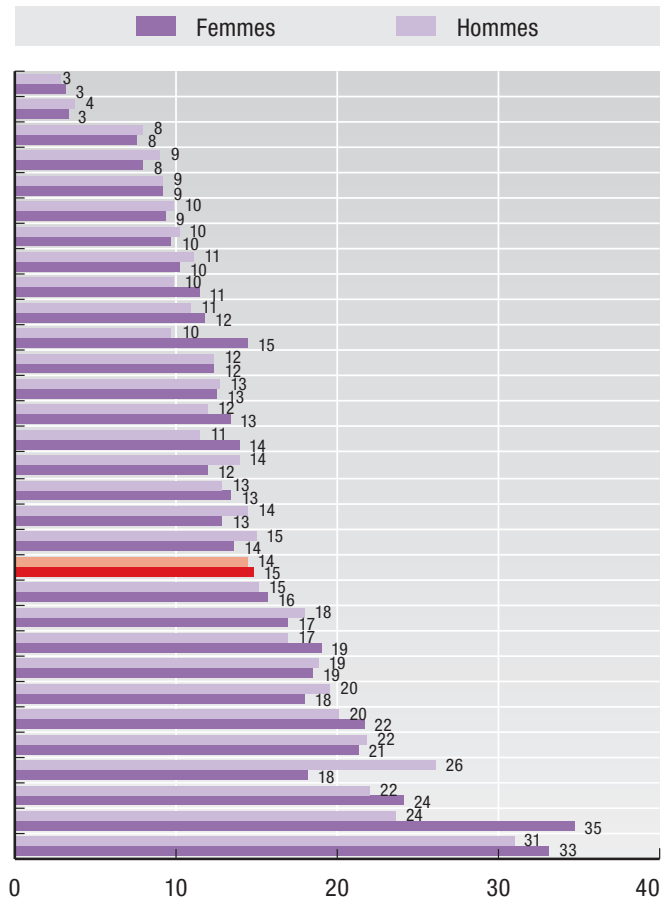

Japon (2004) Corée (2005) Suisse (2002)

Norvège (2005)

Autriche (1999)

France (2004)

Italie (2005)

Suède (2005)

Pays-Bas (2005)

Danemark (2005)

Turquie (2003)

Islande (2002)

Pologne (2004)

Belgique (2004)

Portugal (1999)

Irlande (2002)

Espagne (2003)

Allemagne (2005)

Finlande (2005) OCDE

République slovaque (2003) République tchèque ${ }^{1}$ (2005) Canada (2005)

Luxembourg ${ }^{1}$ (2005 Hongrie (2003)

Nouvelle-Zélande ${ }^{1}$ (2003)

Australie $^{1}$ (1999)

Grèce (2003)

Royaume-Uni' (2005)

Mexique (2005)

États-Unis ${ }^{1}$ (2004)

0

$\%$

3.3.3. Augmentation de la prévalence de l'obésité parmi la population adulte des pays de l'OCDE

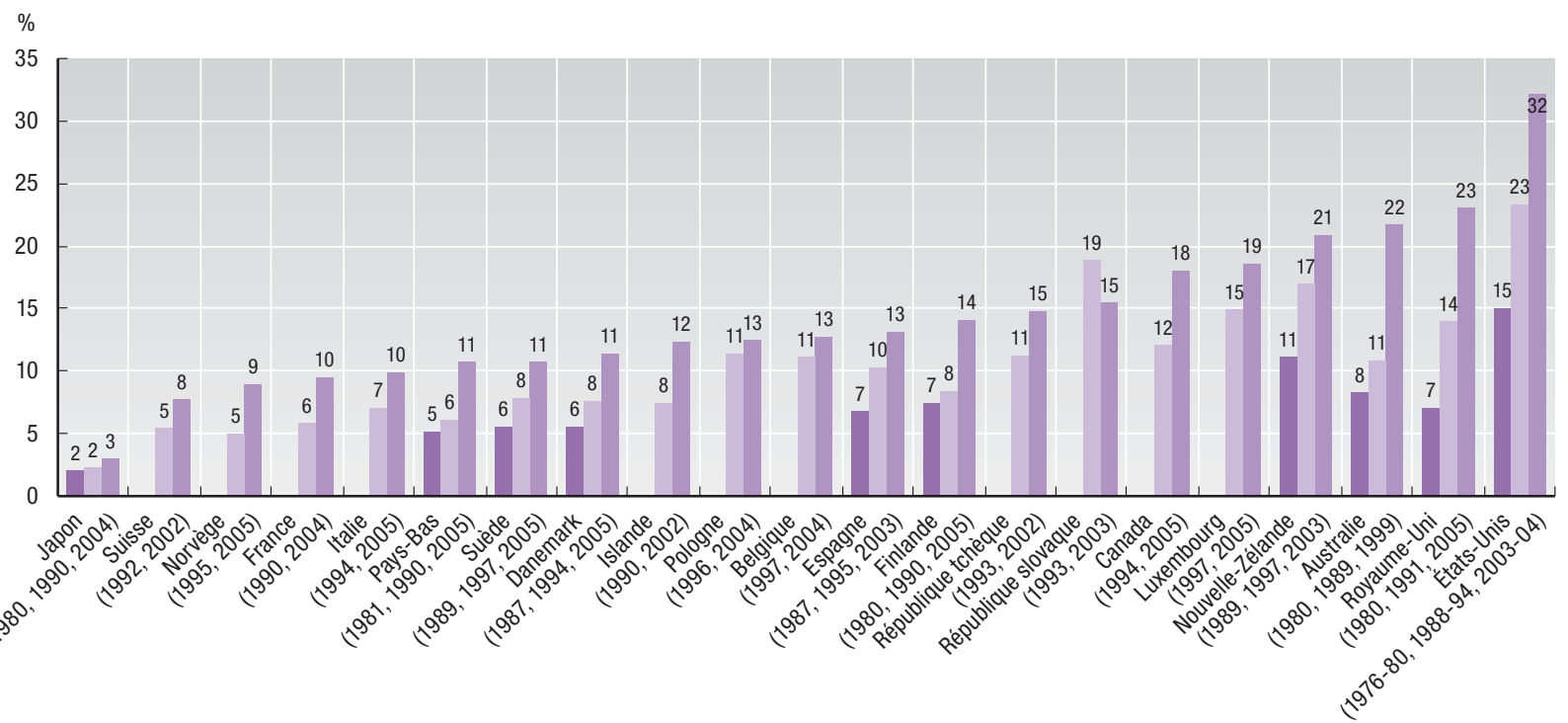

* IMC : Indice de masse corporelle.

1. Pour l'Australie, les États-Unis, le Luxembourg, la Nouvelle-Zélande, la République tchèque (2005) et le Royaume-Uni, les données sont basées sur des enquêtes de santé avec examen, et non pas sur des enquêtes de santé avec entretien.

Source : Eco-Santé OCDE 2007. 



\section{RESSOURCES EN SANTÉ ET LEUR UTILISATION}

4.1. NOUVEAUX DIPLÔMÉS (MÉDECINS ET INFIRMIÈRES) . . . . . . 54

4.2. MÉDECINS EN ACTIVITÉ ..................... 56

4.3. INFIRMIÈRES EN ACTIVITÉ $\ldots \ldots \ldots \ldots \ldots \ldots \ldots \ldots \ldots$

4.4. RÉMUNÉRATION DES PROFESSIONNELS DE SANTÉ (MÉDECINS ET INFIRMIÈRES) $\ldots \ldots \ldots \ldots \ldots \ldots \ldots \ldots \ldots, 60$

4.5. LITS DE SOINS AIGUS, DISPONIBILITÉ

ET TAUX D'OCCUPATION . . . . . . . . . . . . . . . 62

4.6. LITS DE SOINS DE LONGUE DURÉE DANS LES HÔPITAUX

ET LES ÉTABLISSEMENTS DE LONG SÉJOUR . . . . . . . . . . . . . 64

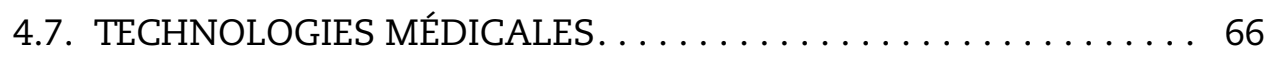

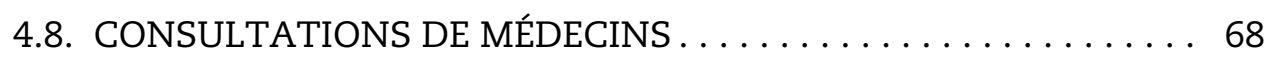

4.9. SORTIES D'HÔPITAL. ....................... 70

4.10. DURÉE MOYENNE DE SÉJOUR À L'HÔPITAL ............ 72

4.11. CHIRURGIES CARDIO-VASCULAIRES

(PONTAGES ET ANGIOPLASTIES) . ............... 74

4.12. TRAITEMENT DES INSUFFISANCES RÉNALES

(DIALYSES ET TRANSPLANTATIONS RÉNALES) . ........ 76

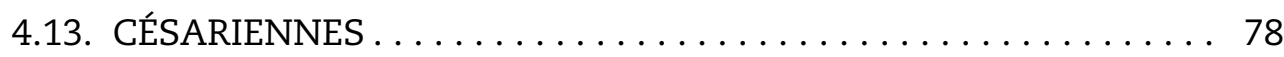

4.14. OPÉRATIONS DE LA CATARACTE, EN AMBULATOIRE

ET AVEC HOSPITALISATION . . . . . . . . . . . . . . 80

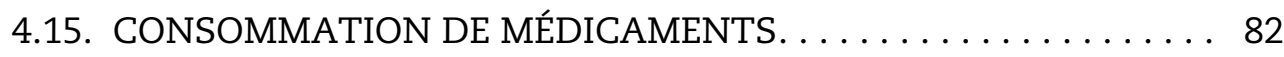




\subsection{NOUVEAUX DIPLÔMÉS (MÉDECINS ET INFIRMIÈRES)}

Pour maintenir ou étoffer les effectifs de professionnels de santé, il faut investir dans la formation de nouveaux personnels ou recruter à l'étranger des professionnels déjà formés, en particulier dans un contexte où la génération de médecins et d'infirmières du baby-boom approche l'âge de la retraite.

Trouver l'équilibre entre l'offre et la demande n'est pas chose facile étant donné le temps très long de formation des professionnels de santé. S'il faut environ dix ans pour former un médecin, cela signifie que, sauf à avoir des médecins au chômage, on ne pourra répondre à une augmentation soudaine et imprévue de la demande qu'en recrutant à l'étranger des médecins qualifiés. À l'inverse, en cas de baisse soudaine de la demande, les nouveaux diplômés auront des difficultés à trouver des postes vacants dans leur pays.

Les mécanismes institutionnels mis en place pour la formation du personnel médical et infirmier diffèrent grandement d'un pays de l'OCDE à l'autre. Dans certains pays, c'est l'administration centrale qui décide des effectifs à former et la formation est principalement financée sur les deniers publics et dispensée dans des établissements publics. Dans d'autres, le processus de décision est décentralisé et la formation est principalement financée par des sources privées et dispensée dans des établissements privés.

Les graphiques 4.1.1 et 4.1.2 indiquent respectivement les taux d'obtention de diplômes de médecins et d'infirmières (par rapport aux nombres de médecins et d'infirmières en activité) dans les pays de l'OCDE en 2005. La variation entre pays est considérable. Les pays qui, en 2005, affichaient des taux élevés d'obtention de diplômes (comme la Corée et l'Autriche, pour les médecins, la Finlande et le Portugal pour les infirmières) avaient enregistré une progression rapide de la densité de médecins et d'infirmières sur la période 1990-2005 (voir graphique 4.2.2 de l'indicateur 4.2 " Médecins en activité » et graphique 4.3.2 de l'indicateur 4.3 "Infirmières en activité »). Un grand nombre de pays qui affichaient de faibles taux d'obtention de diplômes, comme la France, le Portugal et le Canada pour les médecins, l'Australie et le Canada pour les infirmières, se trouvaient dans la situation inverse. Il est probable que les taux actuels de formation sont, dans une certaine mesure, sensibles aux fluctuations passées et prévues de la demande. Toutefois, il est également probable que dans de nombreux pays les taux de formation actuels et passés et l'augmentation récente de la densité de personnel ont été pour partie décidés du côté de l'offre. La Corée et l'Autriche, qui avaient le plus grand nombre de diplômés en médecine par médecin en exercice, n'exercent aucun contrôle centralisé à l'entrée dans les écoles de médecine. En revanche, les pays dans lesquels les taux d'obtention de diplômes de médecine étaient inférieurs à la moyenne de l'OCDE en 2005, contrôlent l'entrée dans ces écoles (Simoens et Hurst, 2006).

En 2005, le taux moyen d'obtention de diplômes a été d'environ 35 pour 1000 médecins en activité et d'environ 46 pour 1000 infirmières en activité dans la zone OCDE. Cette différence entre les deux professions n'est pas étonnante dans la mesure où la durée d'activité moyenne des infirmières tend à être bien inférieure à celle des médecins, du fait en partie d'une répartition hommes-femmes différente entre les deux professions.

Les graphiques 4.1.3 et 4.1.4 montrent respectivement l'évolution des taux d'obtention de diplômes de médecins et d'infirmières pour une sélection de pays de l'OCDE sur la période 1985-2005. La baisse du taux moyen d'obtention de diplômes dans la zone OCDE et dans les pays pris individuellement est nette; dans la deuxième moitié de la période, un petit nombre de pays ont montré, toutefois, des signes de reprise modeste de la progression de ce taux. Cette baisse a été associée à un ralentissement du rythme de croissance de la densité de médecins sur la période 1990-2005 par rapport à la période 1975-90 (voir indicateur 4.2 "Médecins en activité "). Il est difficile de dire dans quelle mesure cette baisse a été entraînée par des fluctuations de la demande et dans quelle mesure cette baisse comme celle du taux de croissance de la densité de médecins ont été déterminées du côté de l'offre, par l'adoption ou le durcissement du contrôle à l'entrée des écoles de médecine, dans certains pays.

Pour ce qui est des infirmières, les signes d'une baisse tendancielle des taux d'obtention de diplômes dans la sélection de pays analysés sont moindres. Mais il est frappant d'observer les signes de fluctuations périodiques des taux d'obtention de diplômes d'infirmières sur une période d'une dizaine d'années dans plusieurs pays. Ce phénomène est probablement dû à des délais d'ajustement de l'offre aux changements de la demande (Simoens et al., 2005).

\section{Définition et écarts}

Les titulaires de diplômes de médecine se réfèrent au nombre d'étudiants ayant obtenu, dans une année donnée, un diplôme d'une école de médecine ou d'un établissement assimilé. Ce chiffre n'englobe pas les titulaires de diplômes de dentisterie, de santé publique et d'épidémiologie. Les titulaires de diplômes d'infirmières se définissent comme le nombre d'étudiant(e)s ayant obtenu, dans une année donnée, une qualification reconnue exigée pour devenir infirmières autorisées. Ce chiffre englobe les sages-femmes autorisées.

S'agissant des titulaires de diplômes de médecine, la République tchèque et le Royaume-Uni ne prennent pas en compte les diplômés d'origine étrangère. Il en va de même au Royaume-Uni pour les titulaires de diplômes d'infirmières. 


\section{RESSOURCES EN SANTÉ ET LEUR UTILISATION}

4.1. NOUVEAUX DIPLÔMÉS (MÉDECINS ET INFIRMIÈRES)

\subsubsection{Nombre de diplômés en médecine pour 1000 médecins, 2005 (ou dernière année disponible)}

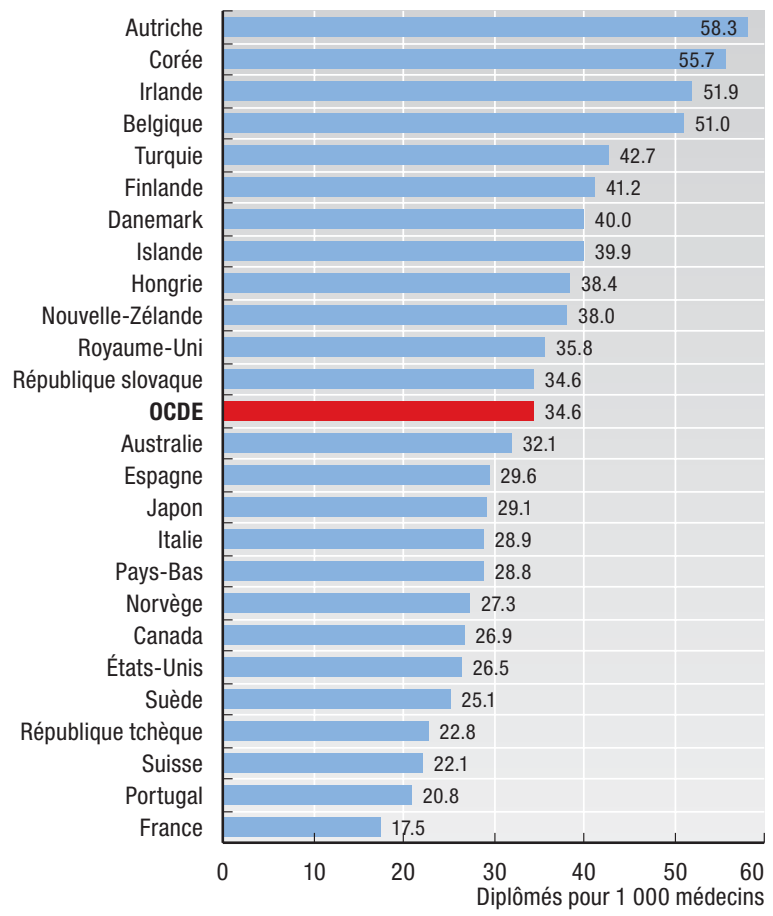

\subsubsection{Nombre de diplômés en médecine pour 1000 médecins} dans quelques pays de l'OCDE, 1985 à 2005

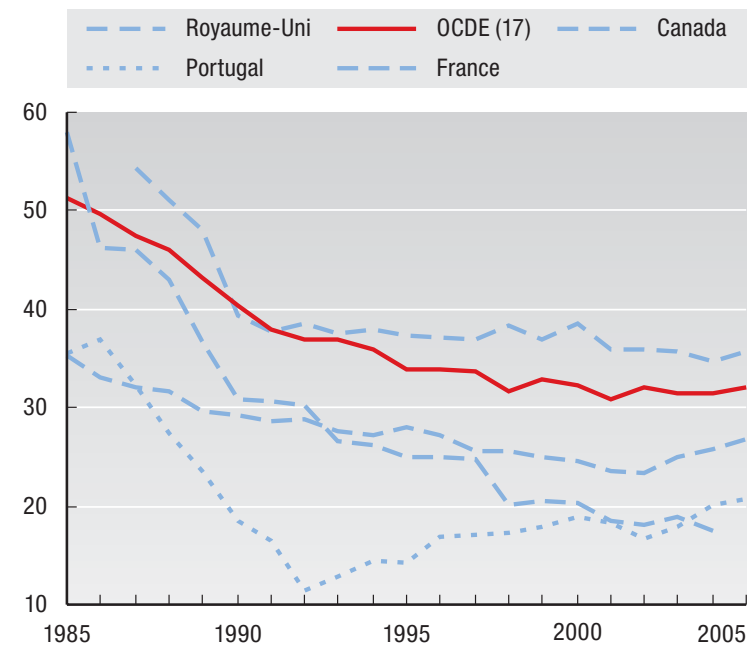

Source : Eco-Santé OCDE 2007.
4.1.2. Nombre de diplômés en études d'infirmière pour 1000 infirmières, 2005 (ou dernière année disponible)

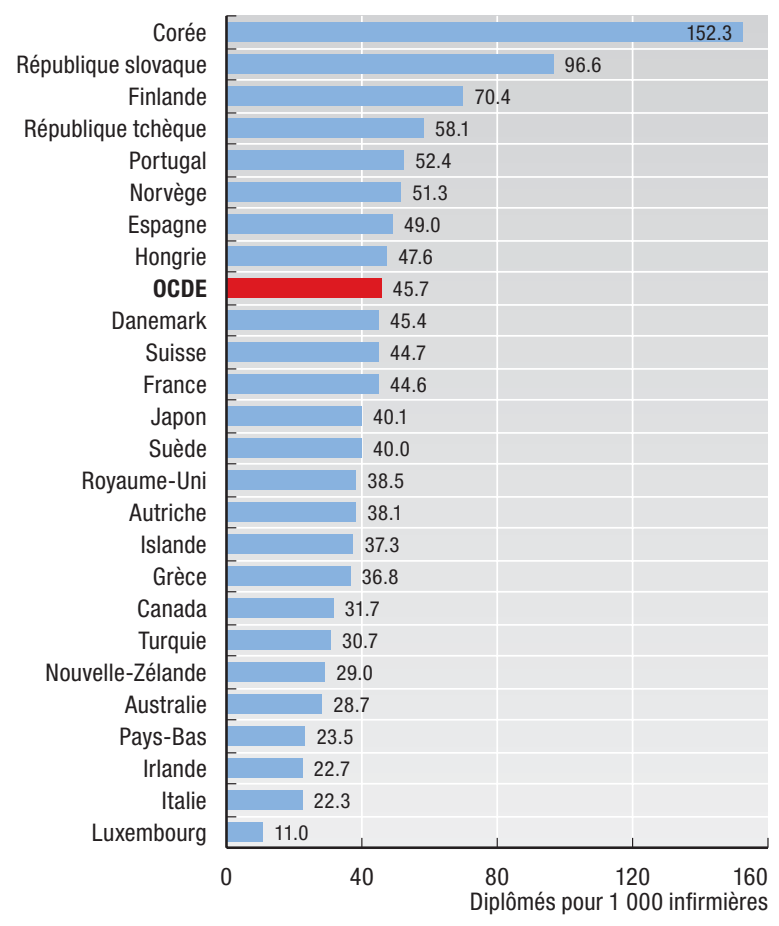

4.1.4. Nombre de diplômés en études d'infirmière pour 1000 infirmières

dans quelques pays de l'OCDE, 1985 à 2005

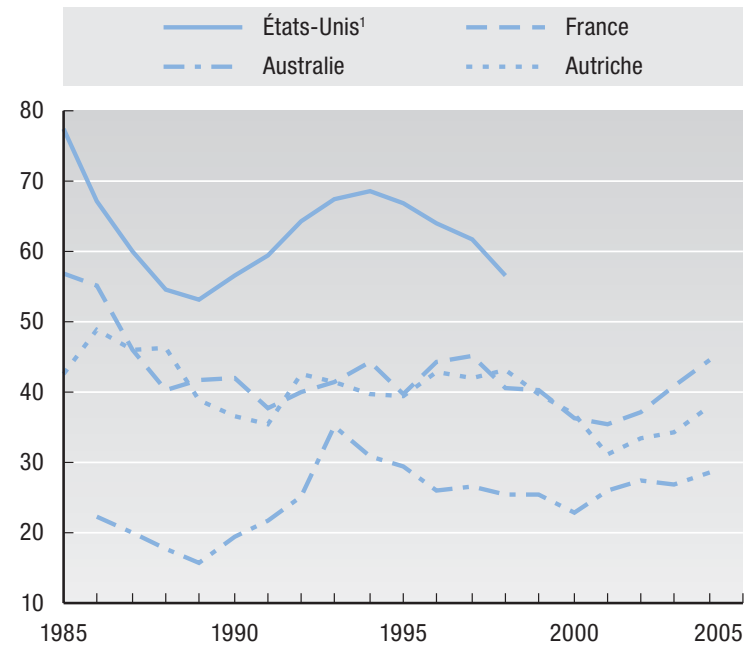

Note : La disponibilité des données étant limitée, la moyenne de l'OCDE n'est pas disponible.

1. Pour les États-Unis, les données sont disponibles jusqu'en 1998 seulement, car la collecte des données concernant certaines catégories d'infirmières diplômées s'est arrêtée par la suite. 


\subsection{MÉDECINS EN ACTIVITÉ}

Une offre adéquate de médecins bien formés et bien répartis sur le territoire est indispensable pour assurer à l'ensemble des patients l'accès à des soins médicaux de qualité. Les médecins prennent aussi un grand nombre de décisions importantes concernant le diagnostic et le traitement des patients et orchestrent la demande de moyens (humains et autres) pour les soins de santé, demande dont le coût est bien supérieur à la rémunération du temps des médecins telle qu'elle se reflète dans les honoraires et les salaires des médecins.

En 2005, la « densité » de médecins, c'est-à-dire le nombre de médecins en activité par habitant, variait fortement d'un pays de l'OCDE à l'autre, oscillant entre quatre médecins et plus pour 1000 habitants en Belgique et en Grèce et moins de deux pour 1000 en Corée, au Mexique et en Turquie (graphique 4.2.1). La moyenne de l'OCDE était de trois médecins pour 1000 habitants.

$\mathrm{Au}$ cours des trente dernières années, la densité de médecins a considérablement augmenté dans tous les pays de l'OCDE, mais à un rythme qui se ralentit dans pratiquement tous les pays. Ainsi, elle a augmenté à un rythme moyen de $3 \%$ par an entre 1975 et 1990 dans les pays de l'OCDE pour lesquels on dispose de séries temporelles longues mais, entre 1990 et 2005, elle a augmenté dans ces mêmes pays à un rythme moyen de $1.6 \%$ par an seulement (graphique 4.2.2). Seuls l'Autriche et le Royaume-Uni ont enregistré sur cette dernière période des taux de croissance supérieurs à ceux de la première période. Dans de nombreux pays, les efforts de compression des coûts ont contribué à faire baisser le taux d'augmentation de la densité de médecins. On pense communément que les médecins peuvent induire la demande de soins médicaux, en particulier lorsqu'ils sont rémunérés à l'acte. Dans les années 80 et 90 , un certain nombre de pays ont adopté un système de numerus clausus (pour limiter les admissions dans les écoles de médecine) ou renforcé les contrôles existants à l'entrée des écoles de médecine (OCDE, 2006a).

Le graphique 4.2.3 fournit des données sur la densité de généralistes et de spécialistes dans les pays de l'OCDE. Il convient de noter que, dans certains pays, ces deux catégories ne regroupent pas la totalité des médecins en activité. Dans les pays de l'OCDE, les spécialistes sont en moyenne beaucoup plus nombreux que les généralistes, la densité de spécialistes s'établissant, en moyenne, à 1.7 contre 0.8 pour la densité de médecins en médecine générale. Toutefois, l'Australie et la Belgique déclarent avoir plus de généralistes que de spécialistes et la France, le Portugal, la Nouvelle-Zélande et la Turquie déclarent en avoir le même nombre. Bien que les politiques de santé et la recherche médicale tendent à souligner l'importance et le rapport coût/efficacité des soins primaires dispensés par les généralistes (Starfield et al., 2002 et 2005), les progrès des technologies médicales semblent conduire à une spécialisation toujours plus grande de la médecine. En moyenne, le rapport spécialistes/généralistes est passé de 1.5 en 1990 à 2.0 en 2005 dans les pays de l'OCDE pour lesquels on dispose de données.

Les conséquences indirectes, en termes de coûts, des décisions de diagnostic et de traitement semblent avoir augmenté régulièrement dans la plupart des pays de l'OCDE. Le graphique 4.2.4 indique le montant des dépenses de santé en termes réels (en millions de dollars américains et ajustées sur la base de la parité des pouvoirs d'achat) par médecin en activité dans une sélection de pays de l'OCDE et pour une moyenne de l'OCDE entre 1990 et 2005. Les dépenses de santé par médecin aux États-Unis ont été, en 2005, plus de six fois supérieures aux dépenses de santé par médecin en Pologne. Elles ont augmenté sur la période dans pratiquement tous les pays pour lesquels on dispose de données, affichant une progression moyenne sur la période de l'ordre d'un tiers. Une étude récente de l'OCDE donne à penser que l'accroissement du revenu national et les progrès des technologies médicales ont plus largement contribué à la hausse des dépenses de santé dans les vingt dernières années que le vieillissement des populations (OCDE, 2006b). Une augmentation du prix des soins médicaux par rapport au PIB a également joué un rôle.

L'augmentation de la densité de médecins, la spécialisation grandissante et l'augmentation du montant de dépenses par médecin semblent avoir coïncidé avec une amélioration de la qualité technique des soins de santé pour une sélection de pathologies dans certains pays (voir le chapitre 6 sur la qualité des soins).

\section{Définition et écarts}

Le nombre de médecins en activité est défini comme le nombre de médecins qui exercent de façon active la médecine dans des établissements publics et privés. Plusieurs pays, mais pas tous, incluent les internes et résidents (médecins en formation). Le nombre de médecins est établi sur la base des effectifs, sauf en Norvège qui communiquait avant 2002 des équivalents plein-temps. L'Irlande, la Nouvelle-Zélande, les Pays-Bas et le Portugal indiquent le nombre de médecins autorisés à exercer (d'où une surestimation). L'Espagne inclut les dentistes et stomatologistes dans le nombre de médecins. Par ailleurs, tous les médecins en activité ne sont pas inclus dans les nombres de spécialistes et de généralistes communiqués par certains pays (notamment parce qu'on ne dispose pas des chiffres par spécialité pour les médecins en formation dans certains pays ou pour les médecins travaillant en libéral dans d'autres pays). 


\section{RESSOURCES EN SANTÉ ET LEUR UTILISATION}

\subsubsection{Médecins en activité, 2005 (ou dernière année disponible)}

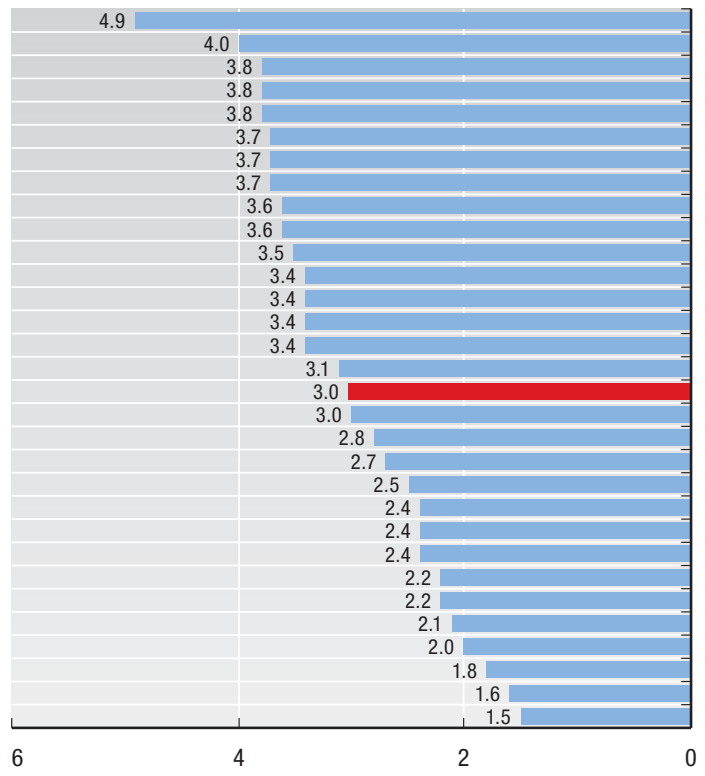

Pour 1000 habitants

\subsection{MÉDECINS EN ACTIVITÉ}

\subsubsection{Croissance de la densité des médecins en activité, $1975-90$ et 1990-2005}

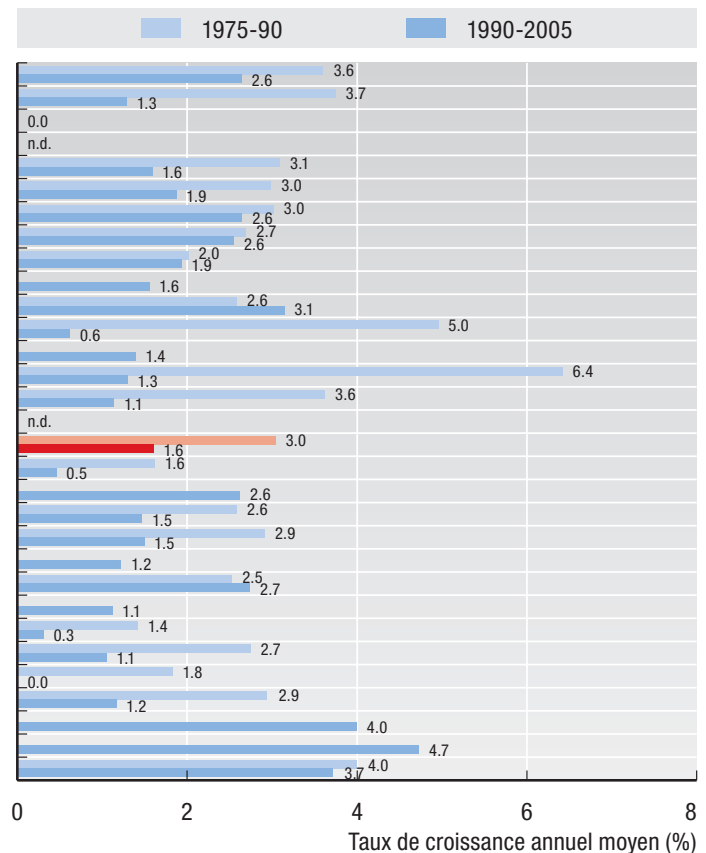

1. L'Espagne inclut les dentistes et stomatologistes dans le nombre de médecins. 2. L'Irlande, la Nouvelle-Zélande, les Pays-Bas et le Portugal communiquent le nombre de tous les médecins autorisés à exercer plutôt que celui des médecins effectivement en activité.

\subsubsection{Généralistes et spécialistes, 2005 (ou dernière année disponible)}

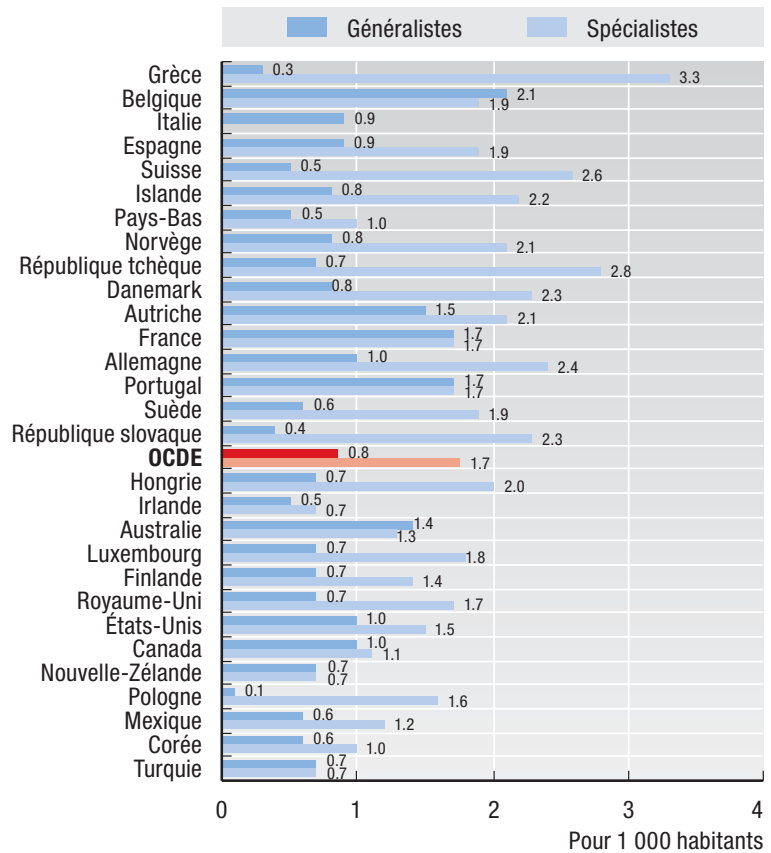

Note: Certains pays ne font pas état de tous leurs médecins selon ces deux catégories de généralistes et spécialistes.

Source : Eco-Santé OCDE 2007.

\subsubsection{Dépenses de santé réelles par médecin en activité, 1990 à 2005}

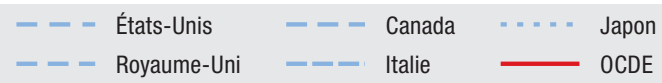

Millions de USD, aux taux de PPA et prix du PIB constants

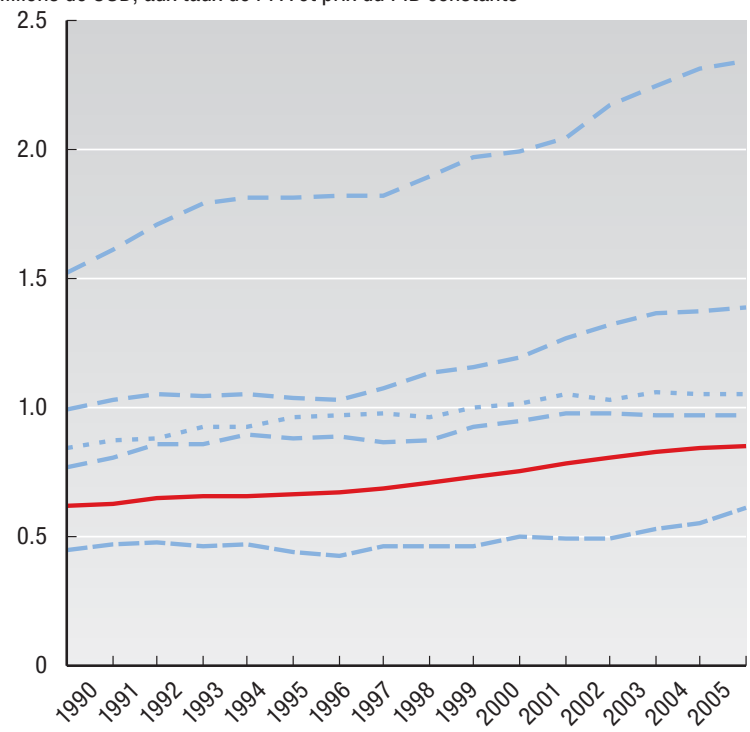

Note: La moyenne OCDE comprend aussi les pays faisant état de ruptures dans les séries de dépenses de santé. 


\subsection{INFIRMIÈRES EN ACTIVITÉ}

Dans les pays de l'OCDE, les infirmières constituent habituellement la catégorie la plus nombreuse de professionnels de santé; on en recense environ trois fois plus que de médecins. Les infirmières jouent un rôle essentiel dans la dispensation de soins de santé, dans les structures traditionnelles comme les hôpitaux et les établissements de soins de longue durée mais aussi, de plus en plus, dans les centres de soins primaires (en particulier pour les soins aux personnes souffrant de maladies chroniques) et au domicile des patients. Un grand nombre de pays s'inquiètent d'une pénurie de personnel infirmier et cette inquiétude pourrait fort bien s'intensifier dans les années à venir, car la demande d'infirmières va continuer à s'accroître alors que le vieillissement de la génération des « baby-boomers » va générer une vague de départs à la retraite parmi le personnel infirmier.

En 2005, on continuait d'observer des disparités importantes entre pays de l'OCDE dans le nombre d'infirmières pour 1000 habitants (" densité "). Alors que des pays comme l'Irlande et la Norvège avaient plus de 15 infirmières pour 1000 habitants, la Corée et la Turquie en avaient moins de deux (graphique 4.3.1). La moyenne de l'OCDE était de 8.9 infirmières pour 1000 habitants.

Si l'on examine les tendances dans le temps, la densité d'infirmières a progressé à un taux d'un peu plus de $1.1 \%$ par an entre 1990 et 2005 en moyenne dans les pays de l'OCDE pour lesquels des données sont disponibles (graphique 4.3.2). Là encore, les disparités entre pays sont considérables. Trois pays (l'Australie, le Canada et la Pologne) font état d'une baisse du nombre d'infirmières par habitant sur les quinze dernières années.

On observe également une grande disparité du ratio infirmières en activité/médecins en activité d'un pays de l'OCDE à l'autre (graphique 4.3.3), en 1990 comme en 2005. En 2005, le ratio infirmières/médecins oscillait entre plus de cinq infirmières pour un médecin en Irlande et au Luxembourg et moins d'une infirmière pour un médecin en Grèce.

Il est intéressant de noter qu'entre 1990 et 2005 (graphique 4.3.3), davantage de pays font état de ratios infirmières/médecins en baisse que de ratios infirmières/ médecins en hausse. En moyenne, ce ratio a légèrement diminué, passant de 3.1 à 2.9 dans les pays de l'OCDE, ce qui donne à penser que l'équilibre des compétences ainsi défini tend vers le haut. Une explication possible est que les progrès des technologies médicales et l'accroissement des taux d'activité ont continué à pousser la demande de médecins et une partie de la demande d'infirmières à la hausse alors que, dans le même temps, ils ont infléchi à la baisse une autre partie de la demande d'infirmières car des techniques chirurgicales moins invasives et les progrès réalisés en termes de médicaments et de produits d'anesthésie ont accru la pratique ambulatoire, réduit les temps d'hospitalisation et permettent de soigner dans des centres de soins primaires un nombre croissant de patients souffrant de maladies chroniques.

\section{Définition et écarts}

Le nombre d'infirmières en activité prend en compte le nombre d'infirmières employées dans tous les établissements publics et privés, y compris les infirmières exerçant en libéral. Il inclut les infirmières diplômées (ayant suivi une formation postsecondaire d'infirmières) et les infirmières auxiliaires qualifiées (qui ont un niveau moindre de compétences mais qui sont généralement agréées). Les sages-femmes, les aides-soignantes et les infirmières travaillant à des postes administratifs sont, en théorie, exclues. Toutefois, la moitié environ des pays de l'OCDE englobent les sages-femmes dans leurs chiffres et un certain nombre de pays incluent les infirmières qui ne sont pas en activité, d'où une surestimation. En revanche, l'Autriche ne prend en compte que les infirmières travaillant dans les hôpitaux, d'où une sous-estimation. 


\section{RESSOURCES EN SANTÉ ET LEUR UTILISATION}

4.3.1. Personnel infirmier en activité pour 1000 habitants, 2005 (ou dernière année disponible)

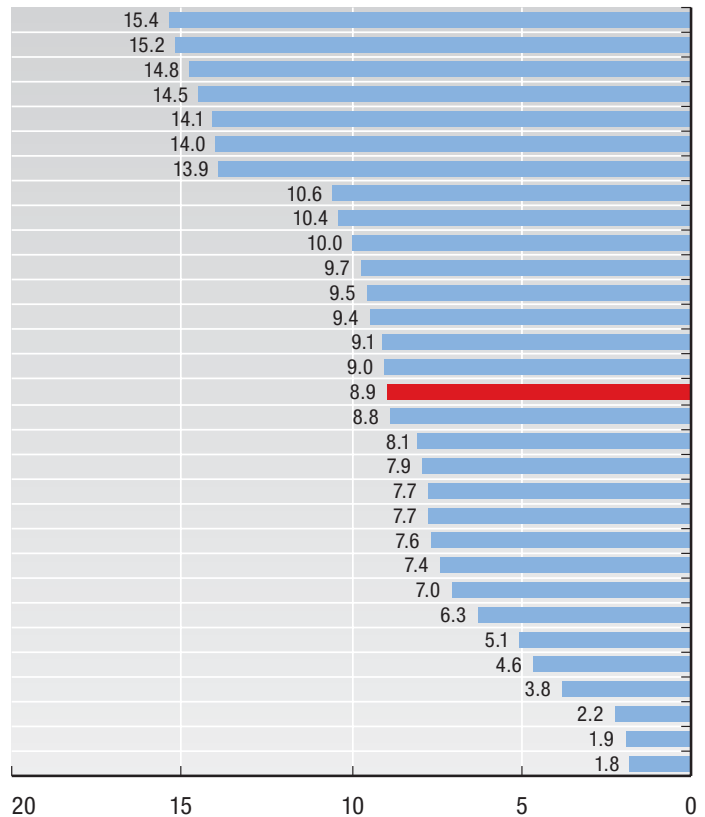

Pour 1000 habitants

\subsection{INFIRMIÈRES EN ACTIVITÉ}

\subsubsection{Variation du nombre d'infirmières en activité pour 1000 habitants, 1990 à 2005}

Norvège
Irlande
Belgique
Pays-Bas ${ }^{1}$
Suisse
Islande
Luxembourg
Suède
Australie
Canada
Allemagne
Nouvelle-Zélande
Autriche
Royaume-Uni
Japon
OCDE
Hongrie
République tchèque
États-Unis
Danemark
France
Finlande
Espagne
Italie
République slovaque
Pologne
Portugal
Grèce
Mexique
Corée
Turquie

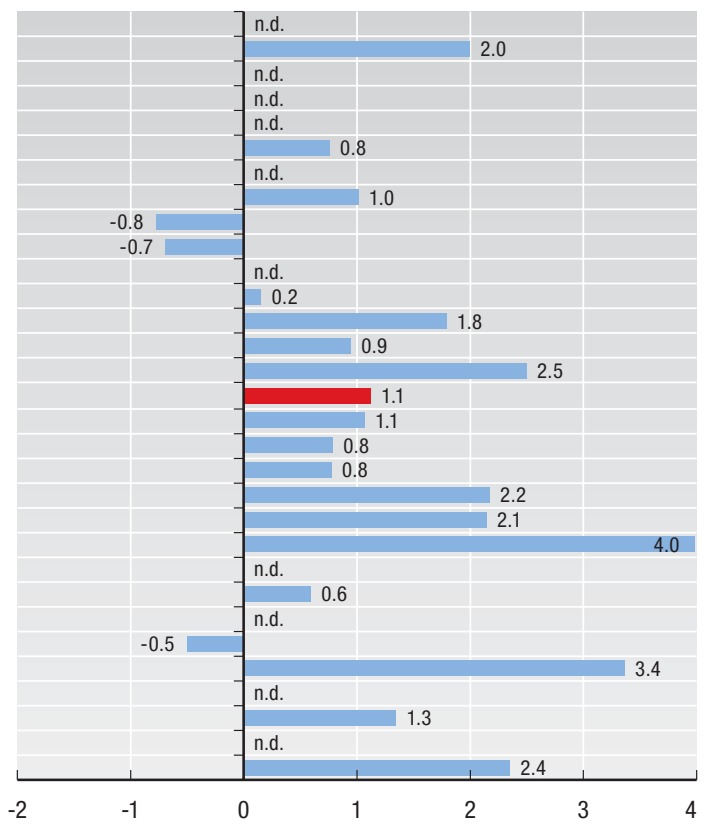

Taux de croissance annuel moyen (\%)

1. Les Pays-Bas communiquent le nombre de toutes les infirmières autorisées à exercer plutôt que celui des infirmières effectivement en activité.

2. Le Luxembourg inclut les aides-soignantes.

3. L'Autriche ne prend en compte que le personnel infirmier employé dans les hôpitaux.

4. Le calcul du taux de croissance annuel moyen pour le Japon et l'Italie est basé sur une période de temps légèrement différente, afin d'éviter des ruptures de séries résultant de modifications méthodologiques.

\subsubsection{Ratio du personnel infirmier par rapport au nombre de médecins, 1990 à 2005 (ou année la plus proche)}

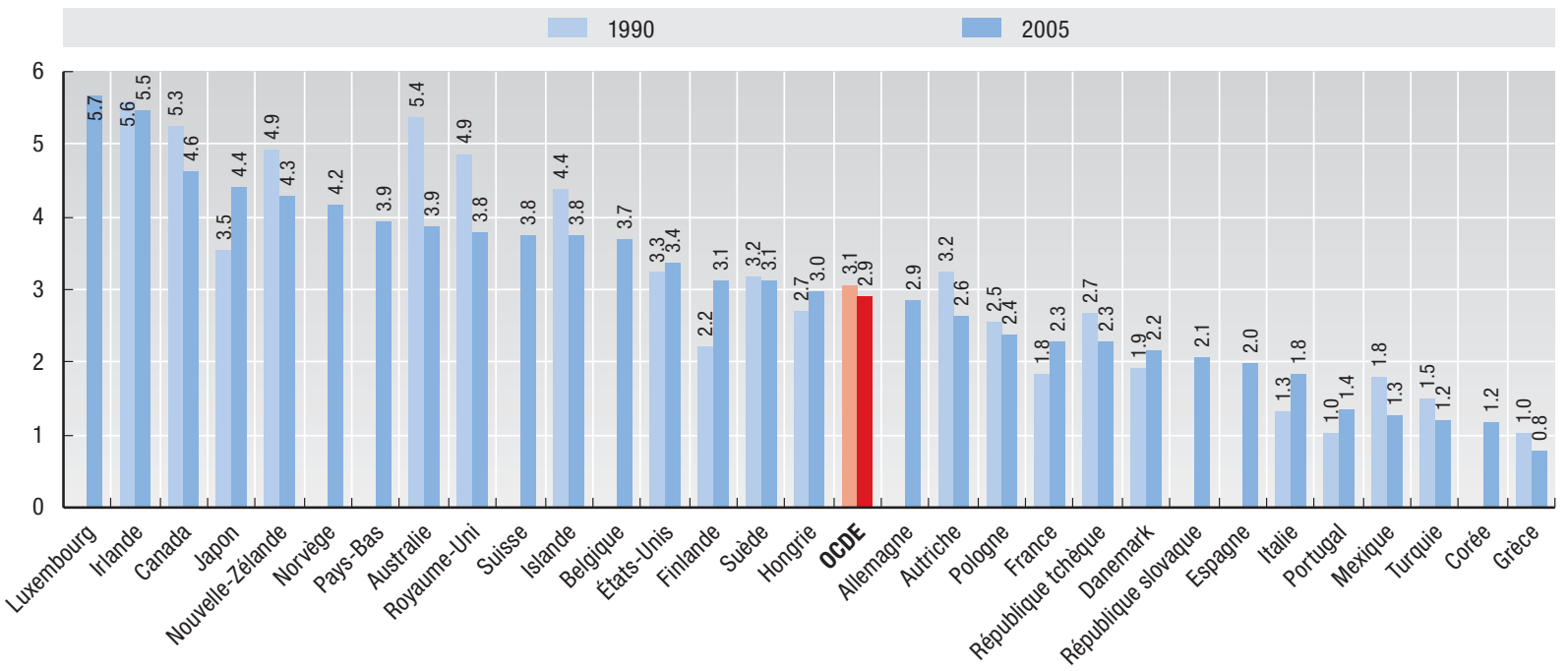

Source : Eco-Santé OCDE 2007. 


\subsection{RÉMUNÉRATION DES PROFESSIONNELS DE SANTÉ (MÉDECINS ET INFIRMIËRES)}

Compte tenu de l'importance des coûts de personnel dans les dépenses de santé, les niveaux de rémunération peuvent fournir des indications sur un des facteurs influençant les variations de dépenses de santé entre les pays. Les données comparatives sur les niveaux de rémunération peuvent également fournir aux autorités nationales des données repères aux fins de la négociation des salaires et des honoraires. Les informations sur les niveaux de rémunération peuvent aussi permettre de mieux comprendre les migrations des professionnels de santé (OCDE, 2007c).

Toutefois, il est difficile de recueillir des données comparables sur la rémunération des médecins et des infirmières car les pays ne prennent pas en compte les mêmes types de rémunération ni les mêmes catégories de médecins et d'infirmières. Ils n'utilisent pas non plus les mêmes sources de données et les mêmes méthodes de calcul (voir l'encadré " Définition et écarts " ci-dessous). Il y a donc lieu d'interpréter avec prudence les données relatives à la rémunération.

Le revenu relatif des médecins spécialistes libéraux varie considérablement entre les pays, dans une proportion allant de 2.3 à 8.4 fois le PIB par habitant. Il est relativement élevé aux Pays-Bas, en Belgique et aux États-Unis (graphique 4.4.1, partie gauche). Le revenu relatif des spécialistes salariés tend à être inférieur à celui des spécialistes libéraux et oscille entre 1.6 et 4.8 fois le revenu national moyen. Il est élevé aux États-Unis et au Royaume-Uni, et faible en République tchèque, en Norvège et en Hongrie.

La rémunération des généralistes varie entre 1.7 fois le PIB par habitant pour les salariés en Hongrie et 4.4 fois le revenu national moyen pour les libéraux aux États-Unis (graphique 4.4.1, partie droite). Dans pratiquement tous les pays, elle est inférieure à celle des spécialistes. En Islande toutefois, les généralistes semblent gagner plus que les spécialistes car ceux qui travaillent dans des régions relativement peuplées ont des horaires plus chargés et aussi parce que la rémunération des spécialistes salariés ne prend pas en compte les revenus additionnels qu'ils perçoivent dans bien des cas de pratiques libérales.

En ce qui concerne les infirmières, il apparaît sur la base des données de 16 pays que le revenu relatif des infirmières hospitalières est égal en moyenne à 1.2 fois le PIB par habitant. C'est au Portugal, suivi des États-Unis et de l'Australie, que la rémunération relative des infirmières par rapport au revenu national moyen est la plus élevée (graphique 4.4.3). En République tchèque, en Hongrie et en Norvège, elle est inférieure au revenu national moyen.

De manière générale, une densité moindre d'une certaine catégorie de professionnels de santé tend à être associée à des niveaux plus élevés de rémunération relative. Le graphique 4.4.2 montre la corrélation pour les spécialistes. Pour un niveau donné de spécialistes par habitant, la rémunération de ceux qui sont salariés tend à être inférieure à la rémunération de ceux qui exercent en libéral. De même, pour un nombre donné de spécialistes par habitant, les niveaux de rémunération peuvent varier sensiblement d'un pays à l'autre. Les variations de la rémunération relative ne peuvent donc pas s'expliquer uniquement par le nombre de professionnels de santé en activité. Elles sont également influencées par d'autres facteurs.

\section{Définition et écarts}

Les données sur la rémunération des professionnels de santé mesurent le revenu annuel brut moyen, qui inclut les cotisations de sécurité sociale et l'impôt sur le revenu à la charge du salarié. La rémunération doit normalement inclure tous les paiements formels supplémentaires tels que les primes et paiements pour travail de nuit, la rémunération du travail sur demande et des heures supplémentaires, mais elle ne doit pas inclure les frais professionnels dans le cas des médecins libéraux. Les niveaux de rémunération sont exprimés par rapport au PIB par habitant, qui constitue une mesure approximative du revenu national moyen. Cependant, pour le Luxembourg et l'Islande, le PIB par habitant surestime fortement le revenu national moyen, d'où l'utilisation pour ces deux pays du revenu national brut par habitant.

Plusieurs facteurs conduisent à sous-estimer les niveaux de rémunération. Premièrement, les données sur les rémunérations de certains pays ne prennent pas en compte le paiement des heures supplémentaires et autres paiements liés, par exemple, au travail de nuit et de week-end. Deuxièmement, dans un certain nombre de pays, les données ne représentent pas l'intégralité du revenu; elles ne prennent pas en compte, par exemple, les revenus de leur activité en libéral pour les médecins salariés ou la rémunération d'emplois salariés pour les médecins exerçant essentiellement en libéral. De même, en Grèce et en Hongrie, les paiements informels ne sont pas une source négligeable de revenus pour les professionnels de santé mais les données ne prennent pas en compte ces paiements. Troisièmement, pour plusieurs pays les données sont tirées de déclarations de revenus dans le cadre d'une enquête, ce qui se traduit bien souvent par une sous-estimation.

En outre, pour certains pays les données se rapportent aux effectifs et incluent la rémunération des travailleurs à temps partiel, ce qui conduit à une sous-estimation par rapport à des données établies sur la base d'équivalents pleintemps. De plus, dans un petit nombre de pays comme la République tchèque, le Danemark, la Finlande, la Norvège et le Portugal, la rémunération de certaines catégories de généralistes est englobée dans celle des spécialistes. Enfin, les données de certains pays ne concernent que les salariés du secteur public dont la rémunération tend à être inférieure à celle des personnes travaillant dans le privé.

D'autres facteurs conduisent, en revanche, à surestimer les rémunérations. Pour les généralistes et les spécialistes libéraux, la Belgique inclut dans ses chiffres les dépenses professionnelles. Pour les infirmières hospitalières, certains pays englobent dans leurs statistiques la rémunération (plus élevée) des infirmières gestionnaires. 


\subsection{RÉMUNÉRATION DES PROFESSIONNELS DE SANTÉ (MÉDECINS ET INFIRMIÈRES)}

\subsubsection{Rémunération des médecins par rapport au PIB par habitant, pays de l'OCDE, 2005} (ou dernière année disponible)

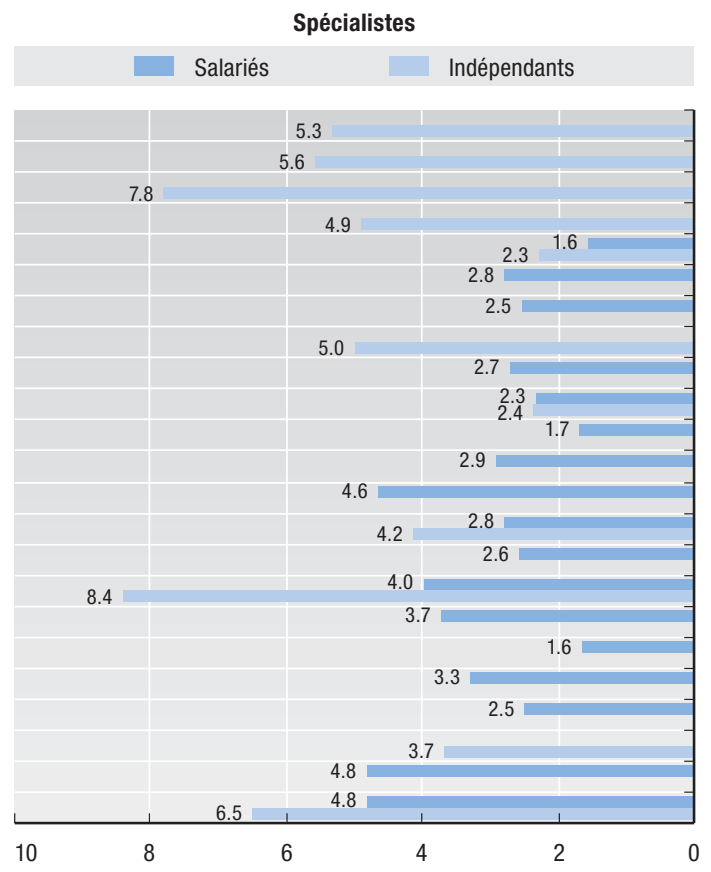

Rapport au PIB par habitant

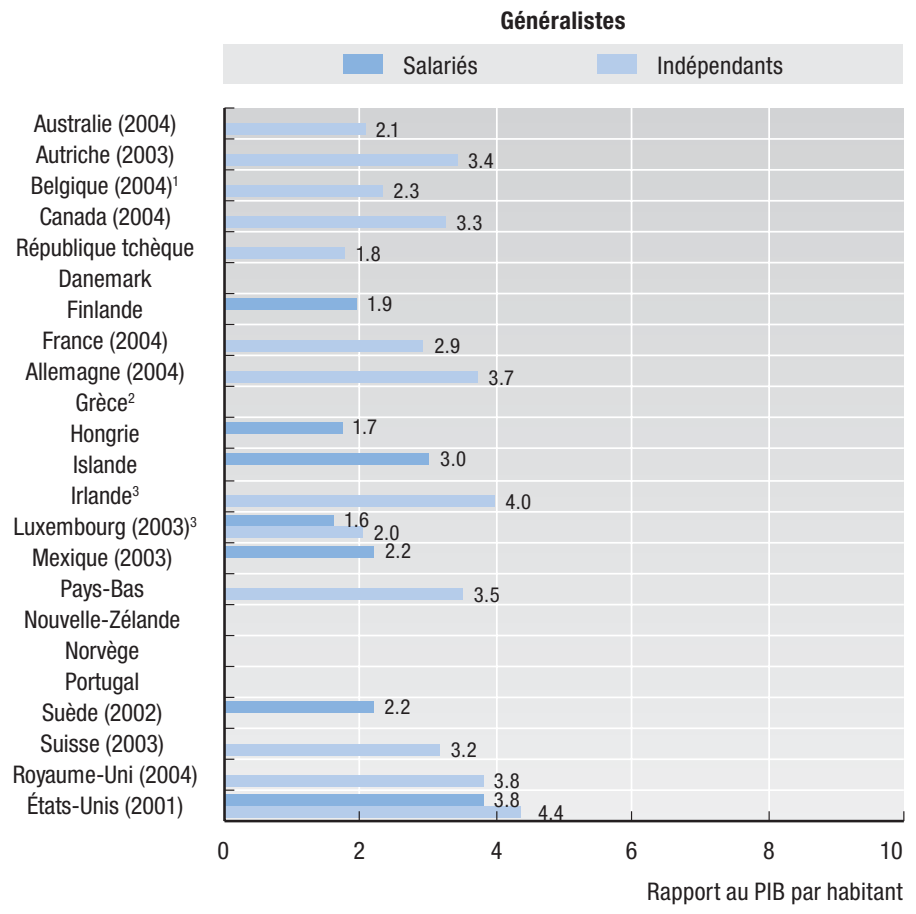

Autriche (2003)

Belgique (2004)

Canada (2004)

Danemark

Finlande

Grese $(2004)$

Islande

Norvège

Portugal

Suède (2002)

Suisse (2003)

Royaume-Uni (2004)

États-Unis (2001)

Rapport au PIB par habitan

1. Les données pour les spécialistes et les généralistes comprennent les dépenses liées à l'activité, entraînant une surestimation.

2. La rémunération des spécialistes salariés se rapporte à 2005, tandis que celle des spécialistes indépendants se rapporte à 2004 .

3. Étant donné que le PIB par habitant excède le revenu moyen, la rémunération est présentée en proportion du revenu national brut. Pour plus d'informations concernant les sources et méthodes, voir les notes de bas de page des tableaux A.4.4a et A.4.4b.

\subsubsection{Rémunération relative et densité des spécialistes pour 1000 habitants, 2005 (ou dernière année disponible)}
Salariés
Indépendants

Rémunération par rapport au PIB par habitant

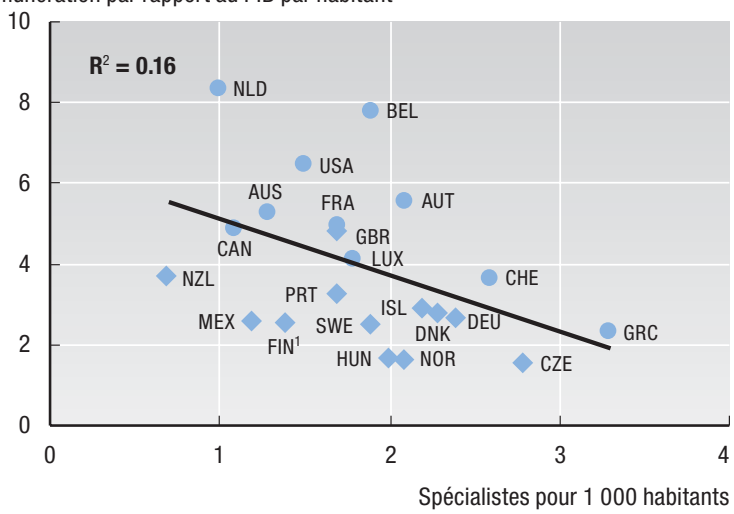

1. Le nombre de spécialistes inclut seulement ceux travaillant dans le secteur public.

\subsubsection{Rémunération des infirmières en hôpital par rapport au PIB par habitant, 2005 (ou dernière année disponible)}

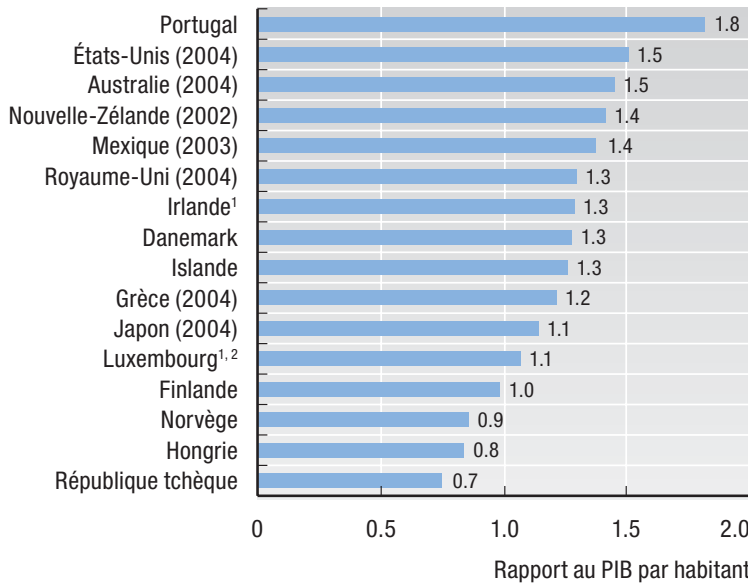

1. Étant donné que le PIB par habitant excède le revenu moyen, la rémunération est présentée en proportion du revenu national brut.

2. Le Luxembourg inclut les aides-soignantes.

Pour plus d'informations concernant les sources et méthodes, voir les notes de bas de pages du tableau A.4.4c. 


\subsection{LITS DE SOINS AIGUS, DISPONIBILITÉ ET TAUX D’OCCUPATION}

Le nombre de lits de soins aigus donne une idée des ressources dont disposent les systèmes de santé pour fournir des soins aigus dans les hôpitaux. Cependant, il ne rend pas compte de la capacité des hôpitaux à dispenser des soins d'urgence ou des interventions programmés dans la journée (sans hospitalisation). De même, cet indicateur ne prend pas en compte les lits alloués pour des soins non aigus (par exemple pour des soins de longue durée).

Le nombre de lits de soins aigus par habitant est très variable dans les pays de l'OCDE. En 2005, c'est au Japon, suivi de la Corée, l'Allemagne et l'Autriche qu'il a été le plus élevé; tous ces pays ont déclaré plus de six lits de soins aigus pour 1000 habitants (graphique 4.5.1). C'est au Mexique, en Turquie et en Suède qu'il a été le plus faible (moins de 2.5 lits pour 1000 habitants). Au Japon et en Corée, on observe un phénomène d'admissions sociales, de nombreux lits de "soins aigus " pouvant être affectés aux soins de longue durée (Jeong et Hurst, 2001; Hurst, 2007).

Dans la plupart des pays de l'OCDE, on observe une tendance à la baisse du nombre de lits de soins aigus. Pour un groupe constant de 24 pays, le nombre de lits de soins aigus dans les hôpitaux est passé de 5.1 pour 1000 habitants en 1990 à 3.9 en 2005. Seule la Corée a enregistré une croissance rapide de son nombre de lits de soins aigus entre 1990 et 2005, qui est passé sur la période de 2.7 à 6.5 pour 1000 habitants. Cela peut s'expliquer par l'utilisation des lits de soins aigus pour des traitements de longue durée, le manque de planification de la capacité de lits d'hôpitaux et les incitations à l'investissement dans le système hospitalier privé (OCDE, 2003b).

Dans la plupart des pays, la réduction du nombre de lits de soins aigus par habitant a été motivée, du moins en partie, par le progrès technologique qui a réduit la nécessité d'hospitaliser les patients et permis de s'orienter vers une chirurgie de jour (OCDE, 2004a). Bien souvent, les réformes du secteur de la santé ont visé également à maîtriser la croissance des coûts d'hospitalisation qui restent le principal poste de dépenses de santé dans pratiquement tous les pays de l'OCDE
(Docteur et Oxley, 2003) (voir l'indicateur 5.3 « Dépenses de santé par fonction"). La baisse du nombre de lits par habitant pourrait avoir été associée à trois changements possibles au niveau de l'activité (Kroneman et Siegers, 2004). Premièrement, le nombre d'admissions et de sorties nécessitant une hospitalisation d'au moins une nuit a légèrement diminué au cours des dix dernières années dans certains pays (voir l'indicateur 4.9 "Sorties d'hôpital "). Deuxièmement, la durée moyenne d'hospitalisation pour soins aigus a beaucoup diminué depuis 1990 dans pratiquement tous les pays (voir l'indicateur 4.10 "Durée moyenne de séjour à l'hôpital "). Troisièmement, dans plusieurs pays, les taux d'occupation des lits ont augmenté à mesure que le nombre de lits de soins aigus par habitant diminuait (graphique 4.5.2).

En 2005, le taux moyen d'occupation des lits de soins aigus dans les pays de l'OCDE était de $75 \%$, soit légèrement supérieur au niveau de 1990. Cette moyenne masquait des disparités considérables entre pays (graphique 4.5.2). Le Canada, la Norvège, la Suisse, l'Irlande et le Royaume-Uni, qui affichent tous un nombre de lits de soins aigus par habitant inférieur à la moyenne de l'OCDE, avaient les taux d'occupation les plus élevés (de l'ordre de $85 \%$ ou plus). Dans tous ces pays, les taux d'occupation ont augmenté et le nombre de lits de soins aigus a diminué au fil du temps. Par ailleurs, le Mexique, les Pays-Bas, la Turquie et le Luxembourg affichaient des taux d'occupation les plus bas, compris entre $61 \%$ et $66 \%$ en 2005 . Au Mexique et en Turquie, pays dans lesquels le nombre de lits de soins aigus par habitant est faible mais stable, les taux d'occupation ont augmenté à mesure qu'augmentait la demande d'admissions dans les hôpitaux. Aux Pays-Bas, en revanche, l'évolution a été différente de celle observée dans la plupart des autres pays de l'OCDE : alors que le nombre de lits de soins aigus par habitant diminuait au fil des ans, les taux d'occupation ont aussi chuté. Ceci peut s'expliquer en partie du moins par le fait que les Pays-Bas et le Luxembourg incluent les lits utilisés pour les soins de jour dans le nombre total de lits de soins aigus alors que les taux d'occupation prennent seulement en compte les séjours nécessitant une hospitalisation d'au moins une nuit.

\section{Définition et écarts}

Les lits de soins aigus incluent, en théorie, uniquement les lits réservés aux "soins curatifs " tels que les définit le Système de comptes de la santé (OCDE, 2000a). Cependant, les fonctions prises en compte dans les " soins aigus " et celles qui en sont exclues (par exemple, le degré d'exclusion des lits affectés aux soins de longue durée, à la réadaptation et aux soins palliatifs) varient d'un pays à l'autre et d'une époque à l'autre, ce qui limite la comparabilité des données. Plusieurs pays (par exemple l'Australie, l'Autriche, le Canada, la Finlande, l'Allemagne, l'Irlande, le Luxembourg, les Pays-Bas, la Pologne, le Portugal, l'Espagne, la Suisse, la Turquie et les États-Unis) déclarent en lits de soins aigus la totalité des lits disponibles dans les hôpitaux " généraux " ou de " soins aigus ». De même, certains lits de soins aigus peuvent être affectés à d'autres usages, tels que les soins de longue durée (par exemple, au Japon et en Corée). En Hongrie et en Irlande, les lits du secteur privé ne sont que partiellement (voire pas du tout) pris en compte. Les chiffres pour la Finlande sont basés sur des estimations (le nombre de nuits à l'hôpital divisé par le nombre de jours durant l'année) qui suppose un taux d'occupation de $100 \%$ des lits ; ceci résulte en une sous-estimation du nombre réel de lits.

Le taux d'occupation des lits de soins aigus correspond au nombre de jours d'occupation des lits de soins aigus divisé par le nombre lits de soins aigus disponibles et multiplié par le nombre de jours (365). 


\section{RESSOURCES EN SANTÉ ET LEUR UTILISATION}

4.5. LITS DE SOINS AIGUS, DISPONIBILITÉ ET TAUX D'OCCUPATION

\subsubsection{Nombre de lits de soins aigus dans les hôpitaux pour 1000 habitants, 1990 et 2005}

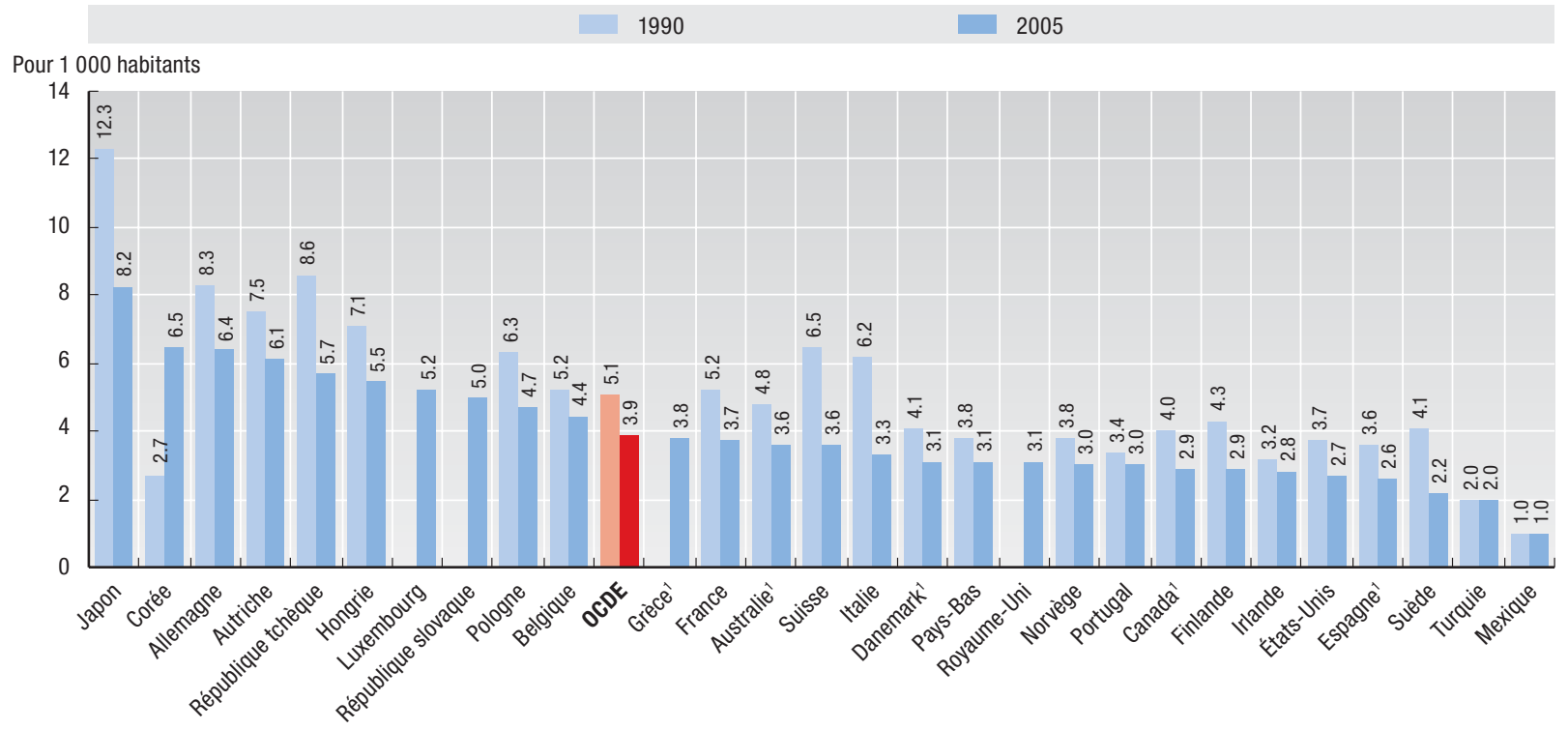

1. 2004.

\subsubsection{Taux d'occupation des lits de soins aigus dans les hôpitaux, 1990 et 2005}

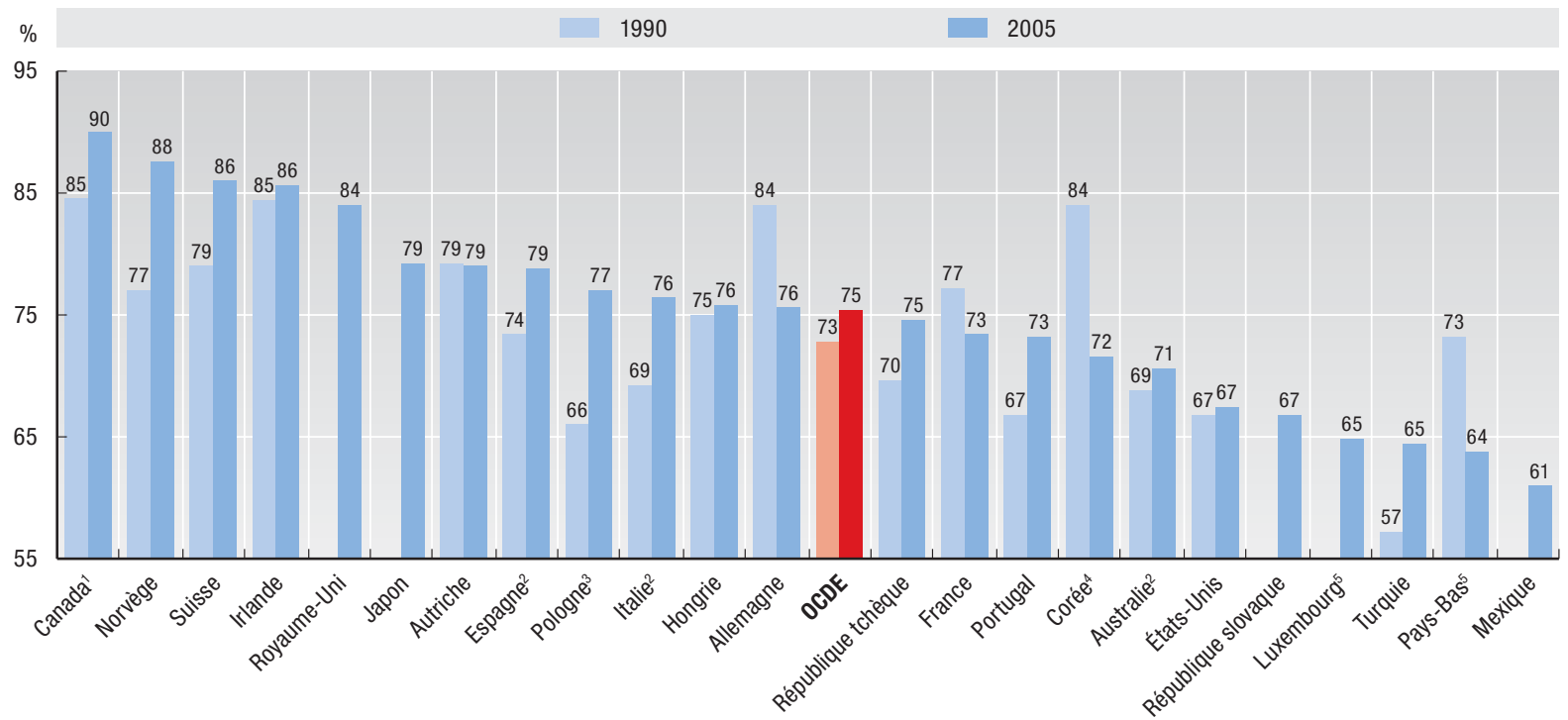

1. 1995-2004. 2. 2004. 3. 2002. 4. 2003.

5. Au Luxembourg et aux Pays-Bas, les taux d'occupation sont légèrement sous-estimés. En effet, alors que le nombre de jours à l'hôpital n'inclut que les patients y passant au moins une nuit, le nombre de lits de soins aigus (le dénominateur) inclut également les lits disponibles pour les soins de jour.

Source : Eco-Santé OCDE 2007. 


\subsection{LITS DE SOINS DE LONGUE DURÉE DANS LES HÔPITAUX ET LES ÉTABLISSEMENTS DE LONG SÉJOUR}

Le nombre de lits de soins de longue durée dans les hôpitaux et les établissements de long séjour fournit une mesure de la capacité physique disponible pour les personnes ayant besoin de soins de santé et d'aides pour les activités de la vie quotidienne sur une base continue.

Les lits de soins de longue durée sont principalement utilisés pour dispenser des soins aux personnes âgées souffrant de maladies chroniques et d'incapacités. Le graphique 4.6.1 représente le nombre de lits de soins de longue durée dans les hôpitaux et les établissements de long séjour par rapport à la population de 65 ans et plus, dans les pays de l'OCDE en 2005. En moyenne, les pays avaient environ 40 lits de soins de longue durée pour 1000 habitants de 65 ans et plus, et ces lits se trouvaient pour la plupart dans des établissements de long séjour. Cependant, le nombre de lits de soins de longue durée varie considérablement d'un pays à l'autre. La Suisse et la Suède comptent environ cinq fois plus de lits dans les établissements de soins de longue durée par personne de 65 ans et plus que l'Italie et le Japon. Cependant, au Japon, plus de la moitié des lits de soins de longue durée se trouvent dans les hôpitaux, même si cette proportion diminue. La Finlande, la République tchèque et l'Irlande ont également une proportion relativement importante de leurs lits de soins de longue durée dans les hôpitaux (ou dans des centres de santé en Finlande).

Au cours de la dernière décennie, de nombreux pays de l'OCDE ont réorganisé leur offre de soins de longue durée (OCDE, 2005c), dans le but de réduire les séjours longs et coûteux en milieu hospitalier, d'encourager si nécessaire l'augmentation du nombre de places dans les établissements de soins de longue durée et, plus généralement, d'appuyer davantage les options de soins à domicile. Le graphique 4.6.2 montre qu'un grand nombre de pays de l'OCDE ont réduit le nombre de lits de soins de longue durée dans les hôpitaux (en proportion du nombre de personnes âgées de 65 ans et plus) sur les cinq à dix dernières années, à l'exception de la République tchèque. Dans certains pays comme l'Islande et l'Irlande, la réduction du nombre de lits de soins de longue durée dans les hôpitaux s'est accompagnée d'une augmentation de leur nombre dans les établissements de long séjour. Dans d'autres comme la Suède et la Finlande, le nombre de lits de soins de longue durée dans les hôpitaux et les établissements de long séjour a diminué par rapport à l'ensemble de la population de personnes âgées. Dans le cas de la Suède, la diminution globale au cours des dix dernières années des lits de soins de longue durée dans les hôpitaux et les établissements de long séjour s'est accompagnée d'une proportion croissante de personnes âgées qui reçoivent des soins à domicile (OCDE, 2007d).

$\mathrm{Au}$ Japon et au Luxembourg, le nombre de lits dans les établissements de soins de longue durée a augmenté rapidement ces dernières années et l'Allemagne a connu une progression analogue entre 1997 et 1999. L'offre accrue de lits dans ces établissements a coïncidé avec la mise en place dans ces trois pays d'un programme universel d'assurance pour les soins de longue durée. On a aussi observé ces dernières années une certaine convergence de la capacité de lits dans les établissements de soins de longue durée entre les pays de l'OCDE, reflétant les efforts pour équilibrer une demande croissante avec le coût des soins dispensés dans différents environnements.

La constitution ou le maintien d'une capacité de lits dans les établissements de soins de longue durée coûte cher. Les dépenses totales par lit de long séjour de ces établissements ont augmenté à un rythme moyen de $3.8 \%$ par an en valeur réelle entre 1995 et 2005, quoique les rythmes de croissance diffèrent d'un pays à l'autre (graphique 4.6.3). L'augmentation des dépenses reflète probablement pour partie une modification de la clientèle des établissements de soins de longue durée, avec une proportion plus importante de résidents sérieusement handicapés nécessitant une plus grande assistance infirmière et médicale. En Suisse, par exemple, la diminution du nombre de lits dans les établissements de long séjour s'est accompagnée d'une augmentation du nombre de personnes âgées recevant des soins à domicile. Dans le même temps, le montant des dépenses réelles par lit de long séjour a régulièrement augmenté, ce qui indique vraisemblablement que les cas les plus graves sont traités dans les établissements de soins de longue durée.

\section{Définition et écarts}

Les lits de soins de longue durée se définissent comme des lits alloués à des personnes ayant besoin d'une assistance continue du fait d'un handicap chronique et d'une autonomie réduite dans les activités de la vie quotidienne. Le nombre total de lits de soins de longue durée est la somme de ces lits dans les hôpitaux et les établissements de long séjour. Les soins dispensés dans chaque cadre institutionnel peuvent combiner des soins de santé et des services sociaux. Un certain nombre de pays (par exemple l'Australie, le Danemark, l'Allemagne, la Norvège et la Suisse), n'indiquent pas le nombre de lits de soins de longue durée de leurs hôpitaux, d'où une sous-estimation de leur nombre total de lits de soins de longue durée. Actuellement, on ne dispose d'aucune information concernant la répartition public-privé des lits de soins de longue durée des hôpitaux et des établissements de long séjour.

Les dépenses courantes au titre des services des établissements de soins de longue durée reflètent la valeur des services de santé dispensés par ces établissements. Dans un grand nombre de cas, on ne dispose d'aucune information sur le coût de l'investissement ou d'autres immobilisations. La comparabilité entre pays s'en trouve donc limitée car la frontière entre dépenses courantes et dépenses d'investissement est floue. 


\section{RESSOURCES EN SANTÉ ET LEUR UTILISATION}

4.6. LITS DE SOINS DE LONGUE DURÉE DANS LES HÔPITAUX ET LES ÉTABLISSEMENTS DE LONG SÉJOUR

4.6.1. Lits de soins de longue durée

dans les hôpitaux et les établissements de long séjour, 2005 (ou dernière année disponible)

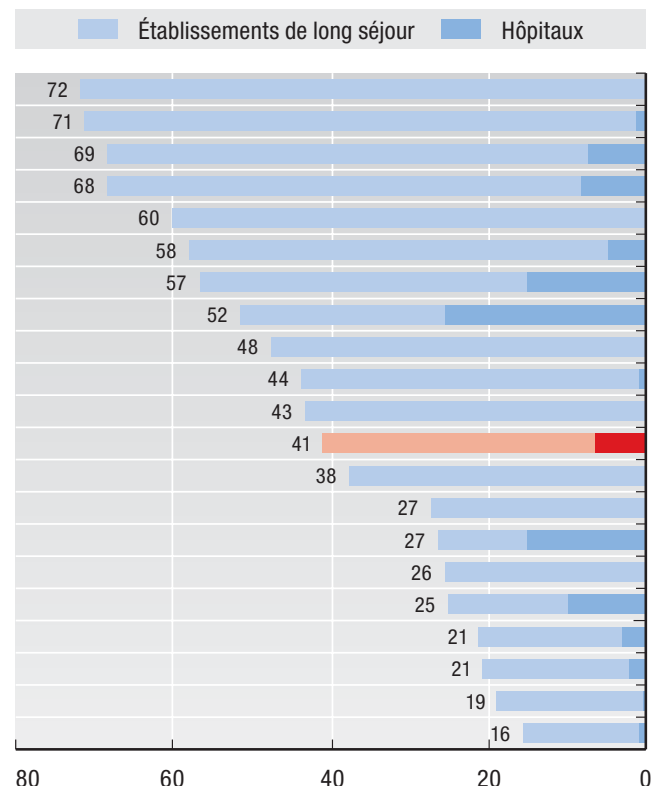

Nombre de lits pour 1000 habitants âgés de 65 ans et plus

\subsubsection{Variation du nombre de lits de soins de longue durée dans les hôpitaux et les établissements de long séjour}

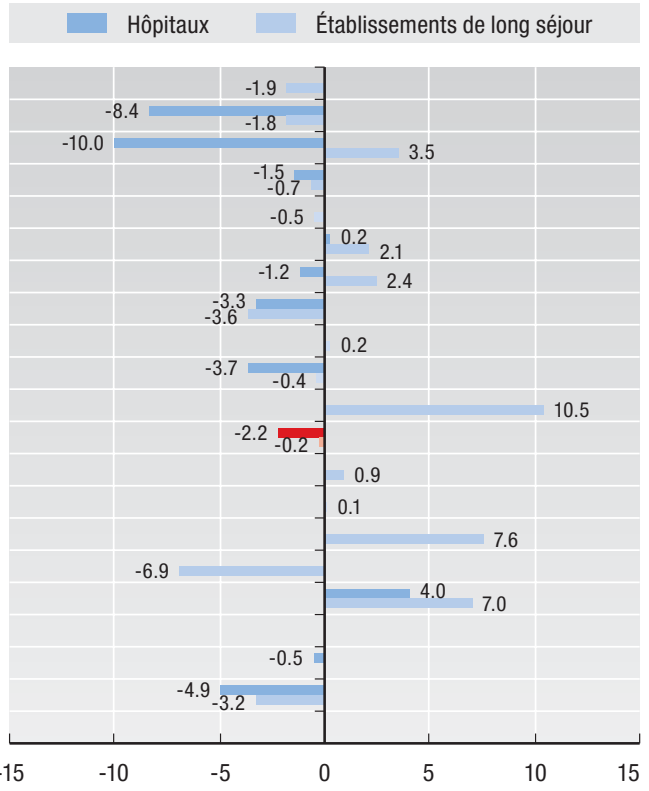

Taux de croissance annuel moyen (\%),

En proportion de la population de 65 ans et plus

Note: Des périodes de temps plus courtes ont été choisies pour certains pays (tels que l'Allemagne, le Luxembourg et la République tchèque) afin d'éviter des ruptures dans les séries résultant de changements de sources ou de méthodologies.

1. Les données sur les lits de soins de longue durée dans les hôpitaux ne sont pas disponibles pour l'Australie, le Danemark, la Norvège et la Suisse.

2. En Islande, les lits de soins de longue durée dans les hôpitaux ne comprennent pas les lits des unités de gériatrie, qui ont augmenté ces dernières années.

3. La moyenne de l'OCDE exclut les pays qui n'ont pas soumis de données complètes.

4. Les données pour le Danemark ne comprennent pas les lits des établissements résidentiels pour personnes âgées, qui sont destinés aux gens souffrant d'une incapacité modérée. Le nombre de lits dans ces établissements a augmenté rapidement ces dernières années.

\subsubsection{Augmentation des dépenses par lit dans les établissements de soins de longue durée, 1995 à 2005}

$$
\text { Pays-Bas } \quad-\quad-\text { États-Unis } \quad-=- \text { Norvège }
$$$$
\text { " " . - Suisse }
$$$$
-\cdots-\text { Australie }
$$

Dépenses en termes réels par lit, en milliers de USD (aux taux de PPA et prix du PIB constants)

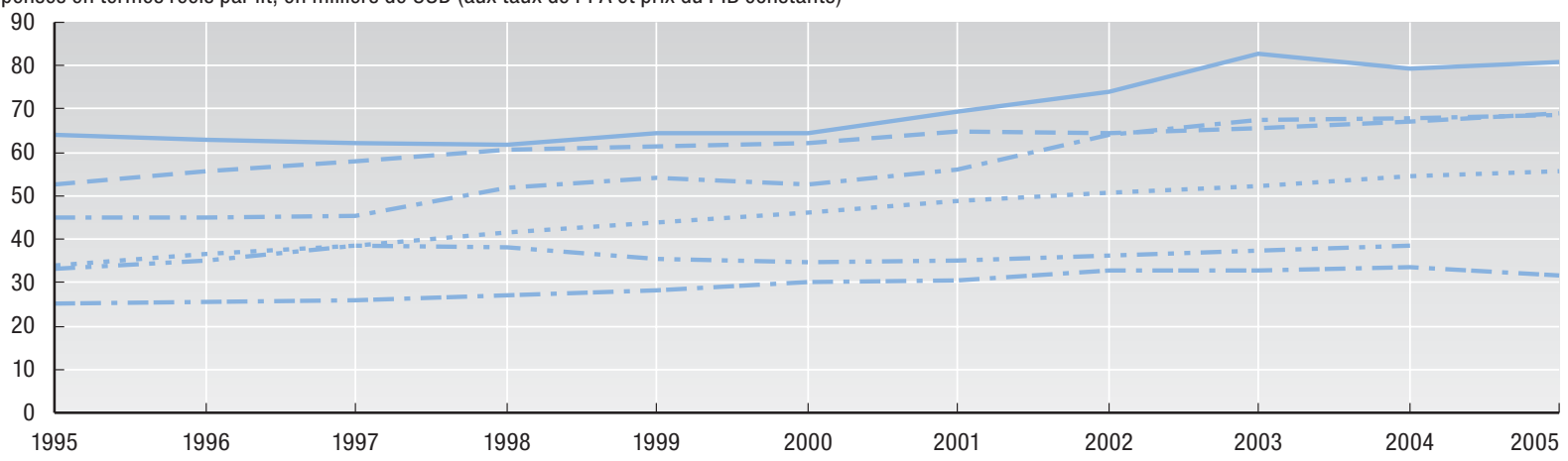

Source : Eco-Santé OCDE 2007. 


\subsection{TECHNOLOGIES MÉDICALES}

La diffusion des technologies médicales modernes est l'une des principales causes de l'augmentation des dépenses de santé dans les pays de l'OCDE. Cette section présente les données sur la disponibilité de trois technologies de diagnostic - la tomodensitométrie, l'imagerie par résonance magnétique (IRM) et la mammographie - et d'une technologie thérapeutique, la radiothérapie. Les données indiquent l'ensemble des équipements disponibles mais ne montrent pas dans quelle mesure ils sont effectivement utilisés.

Les tomodensitomètres (ou CT scans) et les appareils d'IRM facilitent les diagnostics en donnant aux médecins des images transversales de l'intérieur du corps scanné. Contrairement aux techniques traditionnelles (radiographie et CT scanning), les nouvelles techniques d'imagerie médicale utilisées dans les appareils d'IRM n'exposent pas les patients à un rayonnement ionisant. $\mathrm{Au}$ cours des quinze dernières années, le nombre des tomodensitomètres et des appareils d'IRM a augmenté dans la plupart des pays de l'OCDE. Le Japon est, de loin, le pays qui a le plus grand nombre de tomodensitomètres et des appareils d'IRM par habitant (graphiques 4.7.1. et 4.7.2). Certains analystes ont attribué, du moins en partie, leur prolifération rapide au Japon au manque d'évaluation formelle de l'efficience ou de l'efficacité dans les décisions d'achat (Hisashige, 1992). L'adoption précoce et l'application généralisée de ces technologies font du système de santé américain un autre utilisateur intensif de l'IRM et des technologies médicales en général. Par ailleurs, c'est au Mexique, en Hongrie et en Turquie que le nombre de tomodensitomètres et d'appareils d'IRM par habitant est le plus faible, ce qui n'est guère étonnant compte tenu de leur coût élevé.

La mammographie aide à diagnostiquer le cancer du sein, qui est le cancer le plus courant chez la femme. La rapidité de diagnostic et d'intervention accroît sensiblement les taux de survie au cancer du sein (voir l'indicateur 6.4 au chapitre 6). Par exemple, en Suisse, c'est dans les cantons dont le taux de mammographie est le plus élevé que les taux de mortalité sont les plus faibles (OCDE, 2006a). Parmi les 21 pays pour lesquels on dispose de données, c'est en France et en Finlande (graphique 4.7.3) que le nombre des mammographes est le plus élevé. Le Mexique, la Turquie et le Royaume-Uni sont les pays qui déclarent le plus faible nombre de mammographes bien que le nombre de ces appareils ait rapidement augmenté ces dernières années (tableau A.4.7b). La diffusion rapide de ces appareils dans de nombreux pays de l'OCDE a coïncidé avec le développement de programmes organisés de dépistage par mammographie.

La radiothérapie est utilisée dans le traitement de nombreux types de cancers. Plus de la moitié des patients atteints d'un cancer sont soignés par radiothérapie (National Cancer Institute, 2004; DREES, 2005). En 2005, l'Islande était le pays qui affichait le nombre d'appareils de radiothérapie par habitant le plus élevé (ce ratio élevé s'explique toutefois par la faible population du pays, le nombre absolu d'appareils étant en fait très réduit), suivie de la Suisse et de la République slovaque (graphique 4.7.4). On n'observe cependant aucun lien entre le nombre d'unités de radiothérapie et l'incidence des cancers ou la mortalité par cancer dans les pays de l'OCDE (voir l'indicateur 2.5 " Mortalité due au cancer ").

Le revenu national et les dépenses de santé totales sont des facteurs importants pour la diffusion des technologies médicales mais ce ne sont pas les seuls. Une analyse de la diffusion des tomodensitomètres et des appareils d'IRM dans les pays de l'OCDE confirme, une fois neutralisé l'effet de plusieurs autres variables explicatives possibles, que le " pouvoir d'achat " (mesuré en termes de dépenses de santé par habitant) est corrélé positivement à la diffusion de ces technologies (Eun-Hwan Oh et al., 2005). Les pays plus riches adoptent plus vite les nouvelles technologies, même si l'importance du revenu en tant que variable explicative de la disponibilité des technologies médicales dans les pays de l'OCDE décroît généralement au fil du temps (Slade et Anderson, 2001). Ces deux études confirment l'impact de différents types de remboursement, en particulier pour les achats de technologies de diagnostic. Par exemple, des modalités de paiement des hôpitaux fondées sur un remboursement à l'acte ou à la journée sont associées à une diffusion plus large de ces équipements (Eun-Hwan Oh et al., op. cit.).

\section{Définition et écarts}

Les chiffres indiquent le nombre d'appareils par million d'habitants. Les données sur les équipements de radiothérapie englobent les accélérateurs linéaires, les unités de radiothérapie au cobalt 60, les appareils au césium 137 et les appareils de radiothérapie à bas voltage et en orthovoltage (les appareils de curiethérapie sont exclus dans la plupart des pays mais pas tous). En théorie, les données couvrent à la fois le secteur hospitalier et le secteur ambulatoire.

Les données de certains pays peuvent être sous-estimées. Les données sur les tomodensitomètres et les appareils d'IRM à l'extérieur de l'hôpital ne sont souvent pas disponibles. Par exemple, pour l'Allemagne et l'Espagne, elles ne concernent que les équipements des hôpitaux. Pour le Royaume-Uni, les données ne concernent que le secteur public. Pour l'Australie, le nombre d'unités d'IRM (en 1999) et d'unités de mammographie et de radiothérapie (en 2005) ne prend en compte que ceux couverts par l'assurance maladie publique universelle (60\% seulement du total des unités d'IRM en 1999). 
4.7.1. Nombre d'unités d'IRM par million d'habitants, 2005 (ou dernière année disponible)

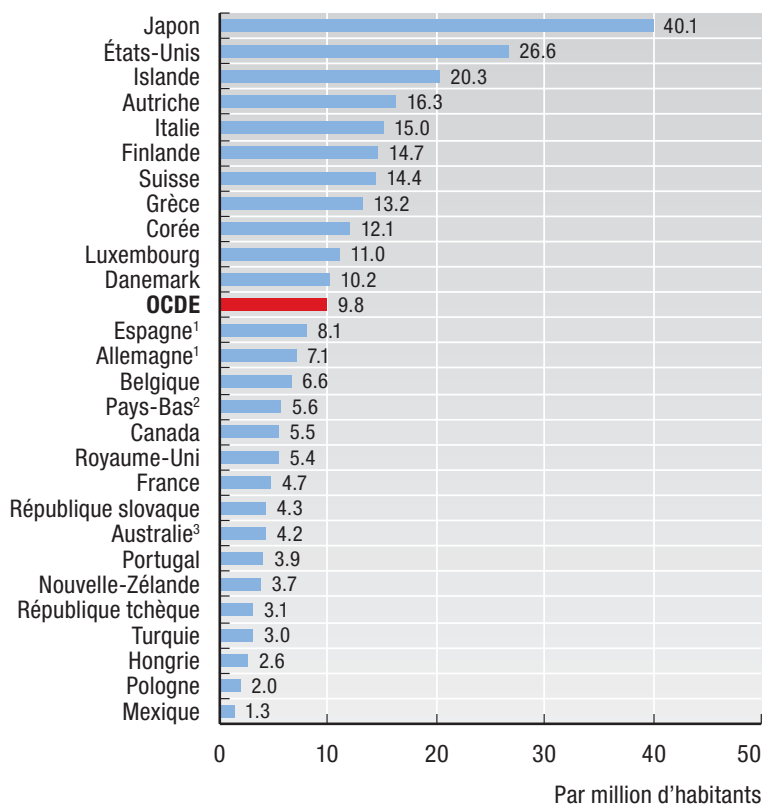

\subsubsection{Nombre de mammographes par million d'habitants, 2005 (ou dernière année disponible)}

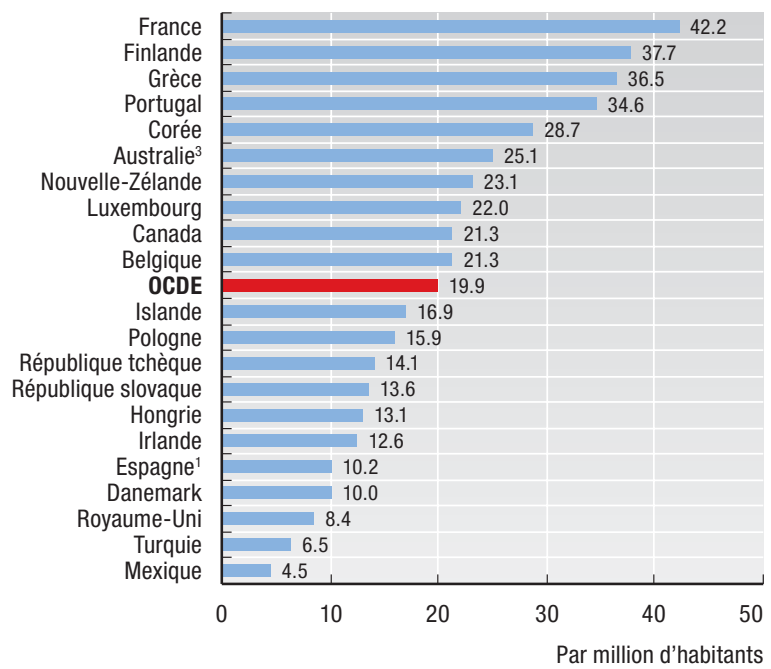

\subsection{TECHNOLOGIES MÉDICALES}

4.7.2. Nombre de tomodensitomètres par million d'habitants, 2005 (ou dernière année disponible)

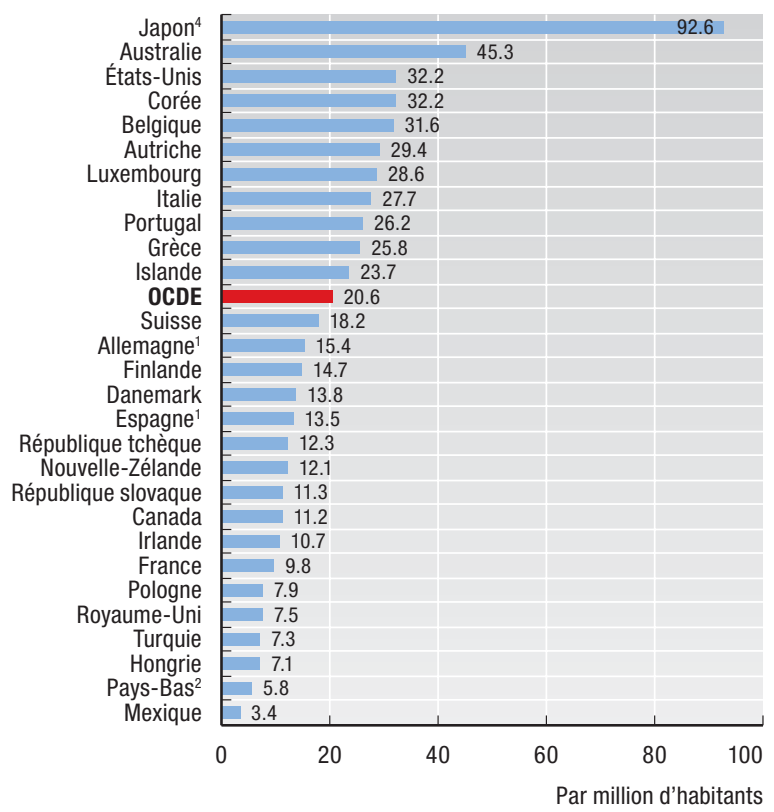

\subsubsection{Nombre d'équipements en radiothérapie par million d'habitants, 2005 (ou dernière année disponible)}

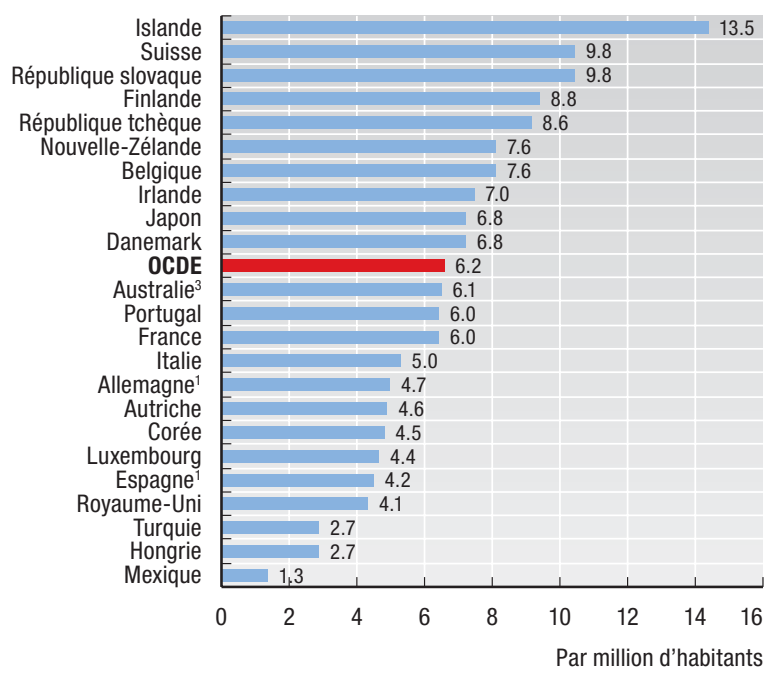

1. Les données pour l'Allemagne et l'Espagne comprennent uniquement les appareils installés dans le secteur hospitalier.

2. Les données pour les Pays-Bas sous-estiment le nombre réel d'appareils, car elles ne concernent que le nombre d'hôpitaux qui déclarent posséder au moins un de ces équipements au lieu du nombre total des équipements dans les hôpitaux et le secteur ambulatoire.

3. En Australie, les données pour les unités d'IRM, mammographes et équipements en radiothérapie concernent uniquement les équipements couverts par l'assurance maladie publique.

4. Au Japon, les données pour les tomodensitomètres se rapportent à 2002 et non à 2005, car les données pour 2005 sont plus restreintes au niveau de la couverture des institutions et des différents types d'appareils.

Source : Eco-Santé OCDE 2007. 


\subsection{CONSULTATIONS DE MÉDECINS}

Les consultations de médecins peuvent avoir lieu dans le cabinet ou la clinique du médecin, dans les services de consultations externes des hôpitaux ou, dans certains cas, au domicile des patients. Dans certains pays (comme l'Australie, le Canada, le Danemark, les Pays-Bas et le Royaume-Uni), les patients sont tenus ou incités à consulter en premier lieu un médecin généraliste qui les orientera, si nécessaire, vers un spécialiste. Dans d'autres (comme la Belgique, le Japon ou la Suisse), les patients peuvent consulter directement les spécialistes.

En 2005, le nombre déclaré de consultations de médecins (tous confondus) par habitant et par an variait considérablement d'un pays de l'OCDE à l'autre, oscillant entre plus de onze au Japon, en Corée, en République tchèque, en Hongrie et en République slovaque, à moins de trois au Mexique et en Suède (graphique 4.8.1). La moyenne de l'OCDE était de près de sept. S'il est probable que des situations sanitaires différentes et des facteurs économiques, comme des différences en termes de paiements directs des patients, de densité médicale et de mode de rémunération des médecins, peuvent expliquer en partie ces variations, il semble probable que le rôle des facteurs culturels soit également important. Le Japon et la Hongrie sont parmi les pays qui ont les taux de consultation les plus élevés; pourtant, l'état de santé déclaré de leur population est très différent et la densité de médecins y est très différente. Bien que certains signes indiquent que les pays dans lesquels les médecins sont principalement payés à l'acte tendent à avoir des taux de consultation supérieurs à la moyenne et ceux dans lesquels ils sont principalement salariés ou payés à la capitation tendent à avoir des taux de consultation inférieurs à la moyenne, certains pays comme la Suisse et les États-Unis où les médecins sont principalement payés à l'acte ont des taux inférieurs à la moyenne.

Les taux de consultation ont augmenté dans plusieurs pays durant la période 1990-2005. Ils ont toutefois baissé légèrement en Belgique, au Canada, aux Pays-Bas et au Royaume-Uni (graphique 4.8.2). La Turquie a déclaré la plus forte progression du taux de consultations par habitant (près de $7 \%$ par an); ce chiffre est probablement la conséquence d'une augmentation relativement rapide de la densité de médecins (voir l'indicateur 4.2 « Médecins en activité "), d'une forte augmentation des dépenses publiques au titre des soins de santé sur une partie de la période (voir l'indicateur 5.1 " Dépenses de santé par habitant ") et d'un meilleur accès aux soins pour les patients à faibles revenus avec le système de la carte verte (Savas et al., 2002). L'augmentation annuelle moyenne était de $0.7 \%$ pour tous les pays de l'OCDE ayant fourni des données.

L'information sur les consultations peut être utilisée pour estimer le nombre de consultations annuel par médecin dans les pays de l'OCDE. Le graphique 4.8.3 montre la variation de cette statistique dans les pays de l'OCDE en 2005. Cette information ne doit pas être utilisée comme mesure de la productivité des médecins, du fait en partie que la durée et l'efficacité des consultations peuvent varier et du fait que cette statistique ne prend pas en compte certains aspects du travail des médecins (patients hospitalisés, travaux administratifs et de recherche). Par ailleurs, elle est sujette aux limites de comparabilité indiquées dans l'encadré "Définition et écarts" ci-dessous. Néanmoins, il est surprenant d'observer que cette statistique varie entre pays de l'OCDE dans une proportion de près de un à neuf. Là encore, on peut penser que certains facteurs culturels jouent un rôle car on trouve ensemble, dans le haut du tableau, les deux pays asiatiques de l'OCDE et les pays d'Europe centrale et orientale. En moyenne, on enregistre environ 2500 consultations par médecin et par an dans la zone OCDE, soit environ dix consultations par jour ouvrable.

Le nombre des consultations par médecin a chuté entre 1990 et 2005 dans la plupart des pays de l'OCDE pour lesquels des données sont disponibles, car le nombre de médecins a augmenté plus vite que le nombre de consultations (graphique 4.8.4). En moyenne, le nombre de consultations par médecin a baissé de $0.9 \%$ par an dans les pays de l'OCDE ayant fourni des données. Cette baisse du nombre des consultations par médecin a coïncidé avec l'augmentation des dépenses par médecin dans un grand nombre de pays (voir l'indicateur 4.2 "Médecins en activité ") et avec des indications d'une probable amélioration de la qualité des soins pour une sélection de conditions (voir le chapitre 6 sur la qualité des soins).

\section{Définition et écarts}

Les consultations de médecins correspondent au nombre de contacts avec des généralistes et des spécialistes. Les consultations peuvent avoir lieu dans le cabinet ou la clinique du médecin, dans les services de consultations externes des hôpitaux ou, dans certains cas, au domicile des patients.

Les estimations qui figurent dans Eco-Santé OCDE proviennent de sources administratives pour la plupart des pays, mais pour certains (l'Italie, les Pays-Bas, la Nouvelle-Zélande, l'Espagne, la Suisse et le Royaume-Uni pour les consultations de généralistes), elles proviennent d'enquêtes sur la santé auprès des ménages et sont alors autodéclarées. Les chiffres des estimations provenant de sources administratives tendent à être plus élevés que ceux obtenus à partir d'enquêtes sur la santé à cause du biais de rappel et du taux de non-réponses dans les enquêtes.

Les chiffres des Pays-Bas ne prennent pas en compte les consultations pour les soins maternels et infantiles. Les chiffres pour le Portugal et la Turquie excluent les visites aux médecins libéraux et celles du Royaume-Uni n'incluent pas les consultations privées des spécialistes. 


\section{RESSOURCES EN SANTÉ ET LEUR UTILISATION}

4.8.1. Nombre de consultations de médecins par habitant, 2005 (ou dernière année disponible)

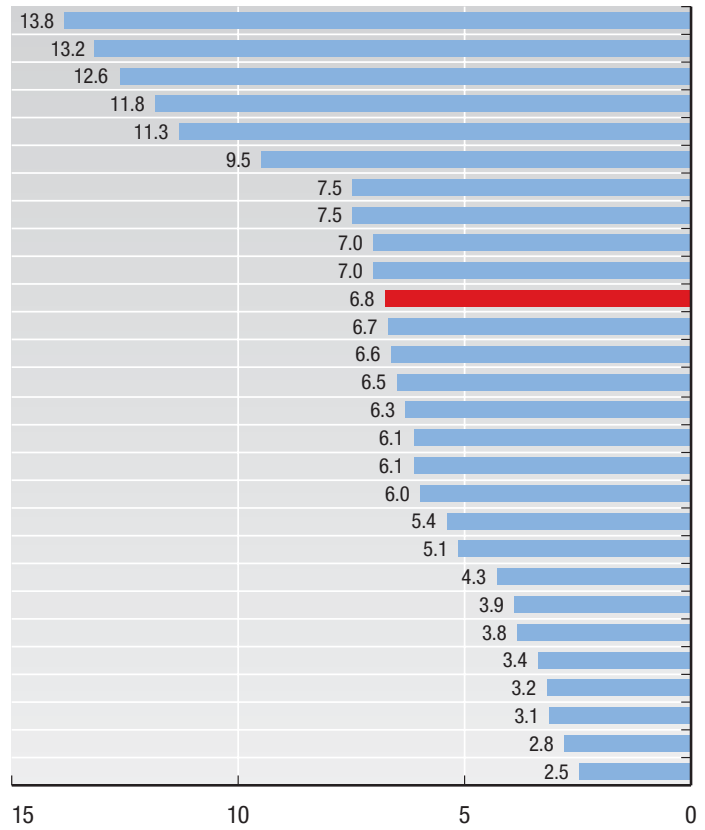

Par habitant

\subsection{CONSULTATIONS DE MÉDECINS}

4.8.2. Taux de croissance annuel moyen du nombre de consultations de médecins par habitant, 1990 à 2005

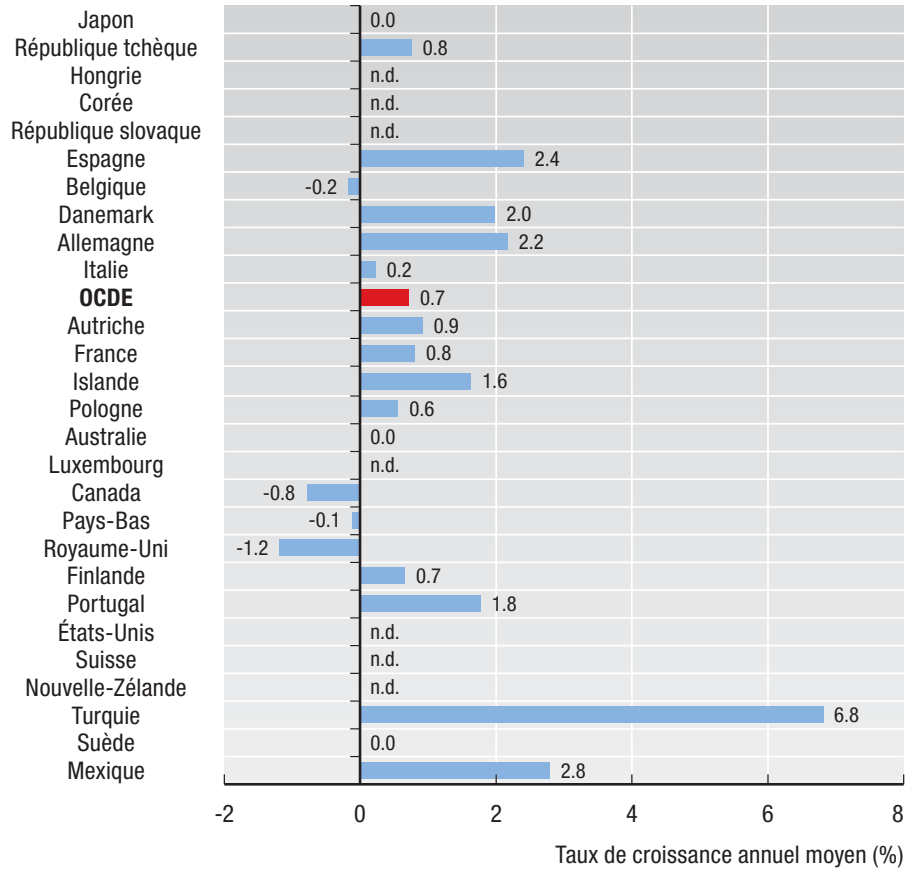

4.8.3. Nombre de consultations par médecin, 2005 (ou dernière année disponible)

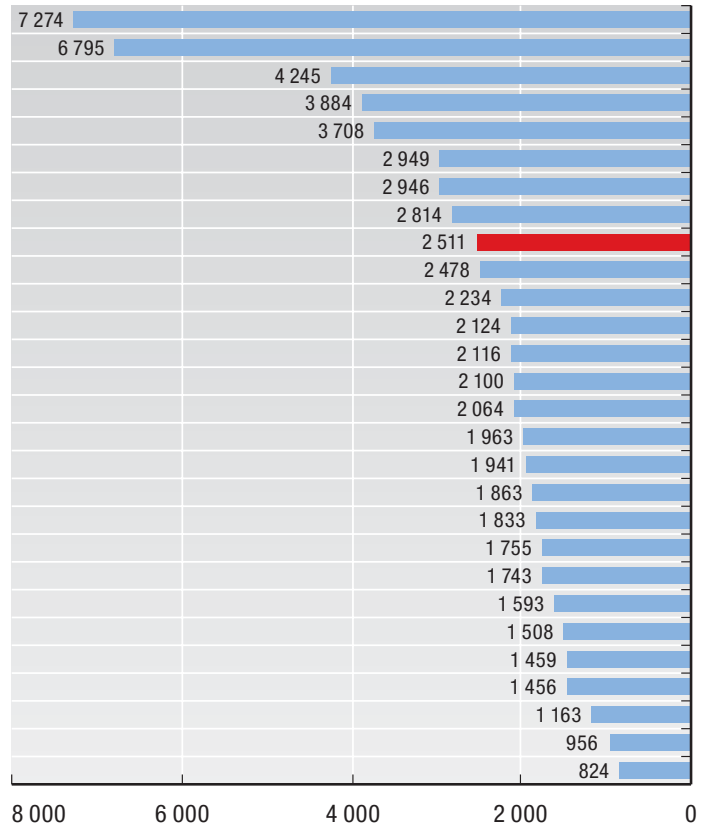

Par médecin

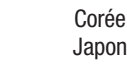

Japon

épublique slovaque République tchèque

Espagne

Pologne

Canada

OCDE

Luxembourg

Australie

Royaume-Uni

Turquie

Danemark

Allemagne

France

Autriche

Belgique

Italie

Finlande

Islande

États-Unis

Mexique

Nouvelle-Zélande

Pays-Bas

Portugal

Suisse

Suède
4.8.4. Taux de croissance annuel moyen du nombre de consultations par médecin, 1990 à 2005

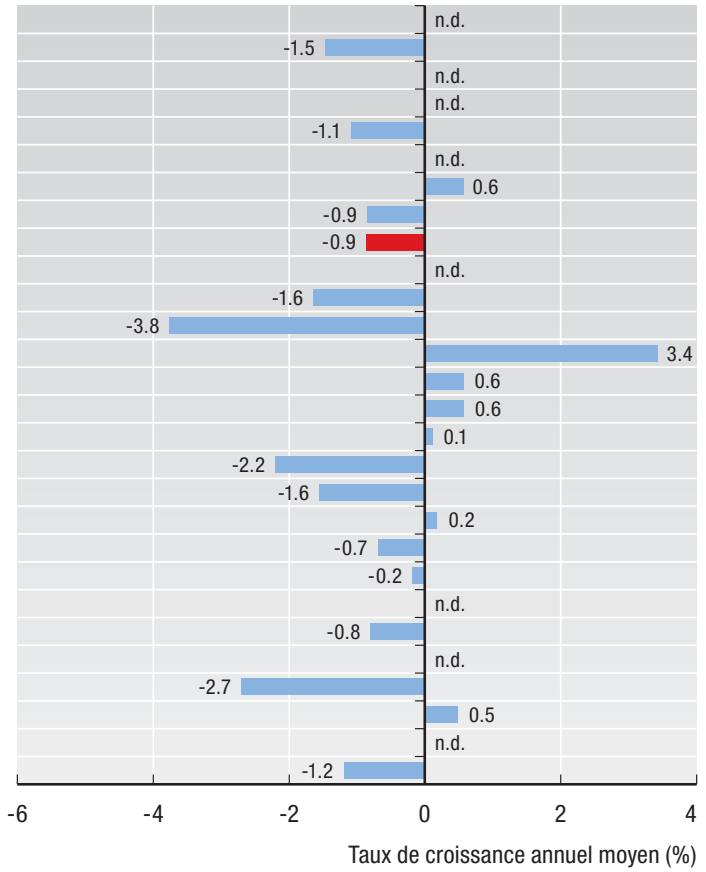

Source : Eco-Santé OCDE 2007. 


\subsection{SORTIES D'HÔPITAL}

Les taux de sortie constituent une mesure importante de l'activité hospitalière. Toutefois, les limites à la comparabilité des données rendent difficile une analyse des variations d'un pays à l'autre. Certains pays englobent dans ce chiffre les traitements ne nécessitant pas de passer la nuit à l'hôpital (sorties le jour de l'admission) et d'autres incluent dans ces données les transferts entre services hospitaliers (voir l'encadré "Définition et écarts" ci-dessous). De plus, la comparaison des taux de sortie ne prend pas en compte les différences de "case mix " (c'est-à-dire du profil global des pathologies ayant conduit à l'hospitalisation).

En 2005, c'est en Autriche que les taux de sortie étaient les plus élevés (graphique 4.9.1). Quatre des cinq pays arrivant derrière incluaient dans leurs données les sorties le jour de l'admission (la France, la Finlande et le Royaume-Uni) ou les transferts vers d'autres unités de soins au sein du même établissement (la République tchèque). Les taux de sortie étaient également élevés en Hongrie et en Allemagne. C'est au Mexique et en Turquie qu'ils étaient les plus bas.

Les taux de sortie ont augmenté au fil du temps dans tous les pays qui comptabilisent les sorties le jour de l'admission (graphique 4.9.2 et tableau A.4.9a). Dans plus de la moitié des pays qui ne comptabilisent pas les sorties le jour de l'admission, les taux de sortie ont augmenté au moins modérément entre 1995 et 2005 . L'augmentation a été particulièrement forte en Corée et en Turquie qui partaient de niveaux relativement bas en 1995. Elle a été également relativement forte en Norvège et en Allemagne. Les taux de sortie sont demeurés relativement stables au Portugal, au Luxembourg et aux Pays-Bas, tandis qu'ils ont baissé au Canada, en Irlande, en Suède, en Islande et en Australie.

L'évolution du nombre des sorties d'hôpital reflète plusieurs facteurs qui ne sont pas faciles à démêler. La demande d'hospitalisations peut évoluer avec le vieillissement démographique. Dans tous les pays, les populations âgées représentent un pourcentage disproportionné des sorties d'hôpital; aux États-Unis, par exemple, 24 \% des sorties d'hôpital déclarées en 2004 ont été le fait de personnes de 75 ans et plus, soit une progression de $18 \%$ par rapport à 1990 (NCHS, 2006). Une étude récente indique que le vieillissement pourrait accroître la demande de services aux personnes hospitalisées de près de $1 \%$ par an aux États-Unis dans les dix prochaines années (Strunk et al., 2006). Cependant, le vieillissement démographique est peut-être un facteur moins important que l'évolution des pratiques médicales qui sont influencées par les progrès technologiques. Par exemple, le nombre de séjours à l'hôpital de personnes de 75 ans et plus sur lesquelles a été pratiqué au moins une angioplastie coronaire est passé, aux États-Unis, de 3.7 à 8.3 pour 1000 habitants entre 1991-92 et 2001-02 (NCHS, 2006). L'interprétation de l'évolution des taux de sortie requiert toutefois une certaine prudence. Le développement et la diffusion d'une nouvelle technologie peuvent entraîner une augmentation mais aussi une diminution des hospitalisations s'il en résulte un passage de procédures avec hospitalisation à des procédures avec sortie le même jour (Nallamothu et al., 2007). Il n'est pas possible de dire comment aurait évolué le nombre des hospitalisations en l'absence de ces nouveaux traitements.

Les principales pathologies ayant fait l'objet de sorties dans les pays de l'OCDE en 2005 étaient les maladies du système circulatoire, les grossesses et accouchements, les maladies de l'appareil digestif, les causes externes (tels que les accidents, la violence et les empoisonnements) et les cancers (graphique 4.9.3 et tableau A.4.9b). Pour la principale catégorie de diagnostic, les maladies du système circulatoire, les taux de sortie ont augmenté depuis 1995 dans plusieurs pays (graphique 4.9.4). Cela peut s'expliquer en partie par le développement des chirurgies cardio-vasculaires (voir l'indicateur 4.11 " Chirurgies cardio-vasculaires »).

\section{Définition et écarts}

Une sortie comptabilise un patient qui quitte un hôpital après une admission. Elle inclut aussi normalement les décès survenus à l'hôpital. Les sorties le jour même de l'admission ne sont normalement pas incluses excepté dans les pays suivants qui les prennent en compte : l'Autriche (pour la période 1989-2002), la République tchèque (avant 1995), la Finlande, la France, la Hongrie (avant 2004), l’Italie (après 2004), le Royaume-Uni et les États-Unis. Les transferts dans d'autres unités de soins du même établissement sont généralement exclus, sauf en République tchèque et au Japon où ils sont inclus.

Quelques autres facteurs limitent la comparabilité des données sur les sorties d'hôpital. Certains pays ne prennent pas en compte la totalité des services de santé. Par exemple, les données fournies pour le Danemark, l'Irlande, le Mexique, la Pologne et le Royaume-Uni ne concernent que les hôpitaux publics ou financés par des fonds publics. Celles fournies pour le Portugal ne concernent que les hôpitaux de la partie continentale (à l'exclusion des îles des Açores et de Madère) et celles fournies pour l'Espagne ne couvrent que 85 \% des hôpitaux. L'Irlande n'englobe pas dans ce chiffre les sorties liées à la grossesse et à l'accouchement ainsi que certaines pathologies survenant pendant la période périnatale. Certains pays (par exemple le Canada, l'Allemagne avant 2004 et les États-Unis) ne comptabilisent pas (ou que partiellement) dans ce chiffre les enfants nés à l'hôpital en bonne santé. La source d'informations peut également varier mais, dans la plupart des cas, il s'agit des registres administratifs des hôpitaux. 


\section{RESSOURCES EN SANTÉ ET LEUR UTILISATION}

4.9. SORTIES D'HÔPITAL

\subsubsection{Taux de sortie d'hôpital pour $\mathbf{1} \mathbf{0 0 0}$ habitants,} 2005 (ou dernière année disponible)

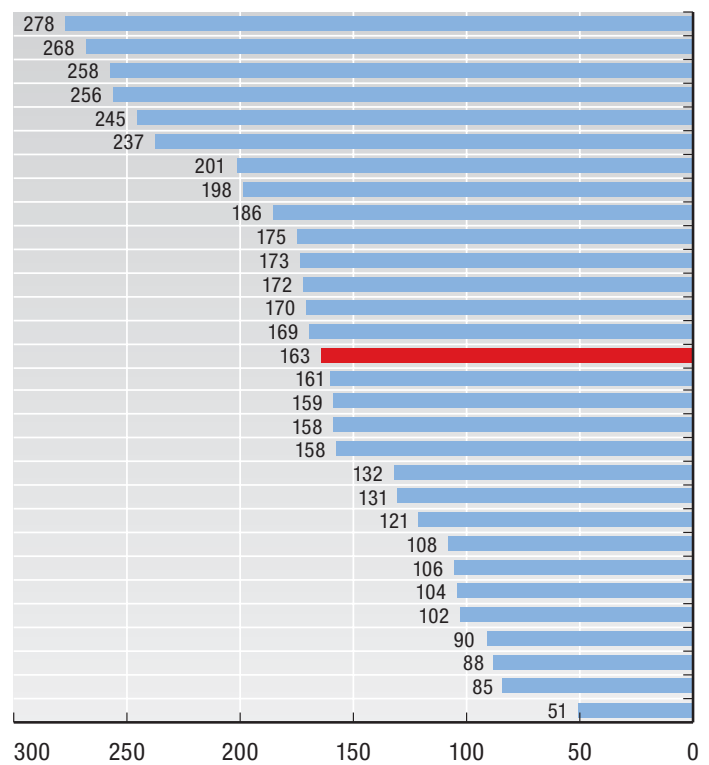

Pour 1000 habitants

1. Inclut les sorties le jour même.

2. Ne comprend pas les sorties d'hôpital des nouveaux-nés en bonne santé.

3. Inclut les transferts d'une unité à une autre au sein d'un hôpital.

4.9.3. Sorties d'hôpital selon les causes d'entrée des patients, OCDE, 2005

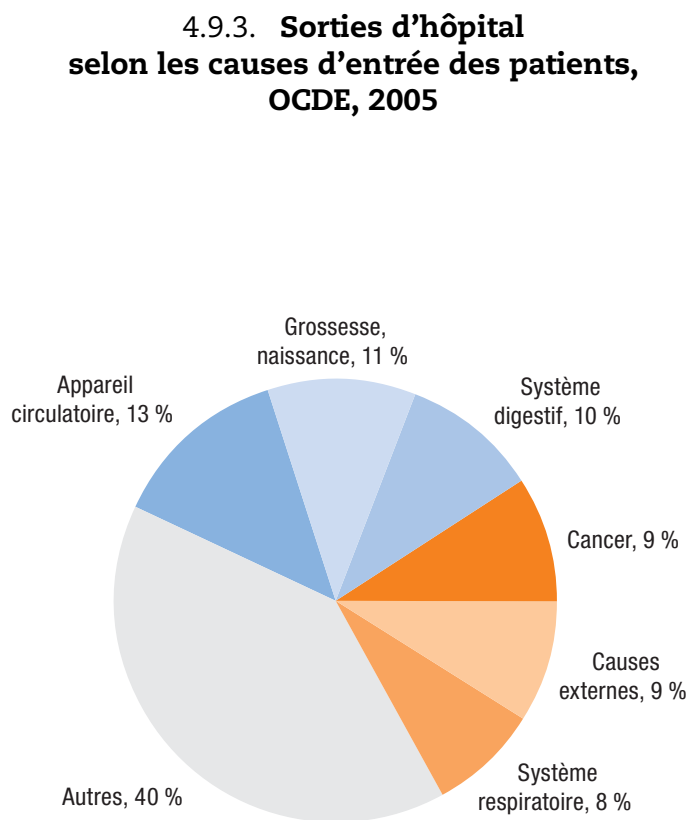

\subsubsection{Variation en pourcentage des taux de sortie} d'hôpital pour 1000 habitants, 1995 à 2005

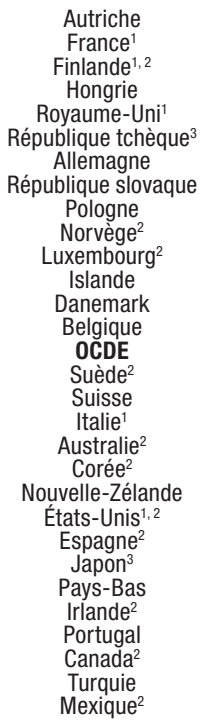

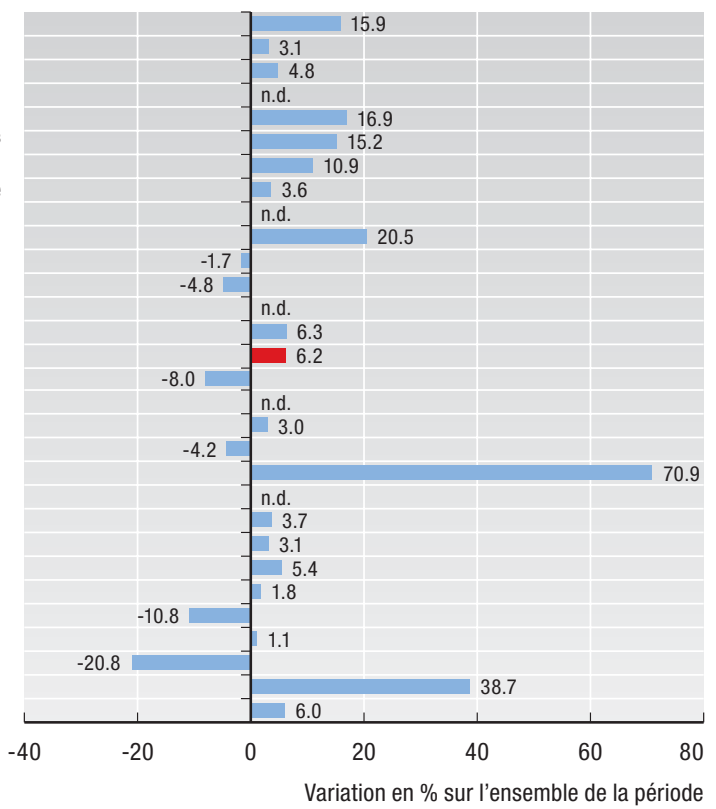

4.9.4. Sorties d'hôpital pour les maladies de l'appareil circulatoire, pour 1000 habitants, 1995 et 2005

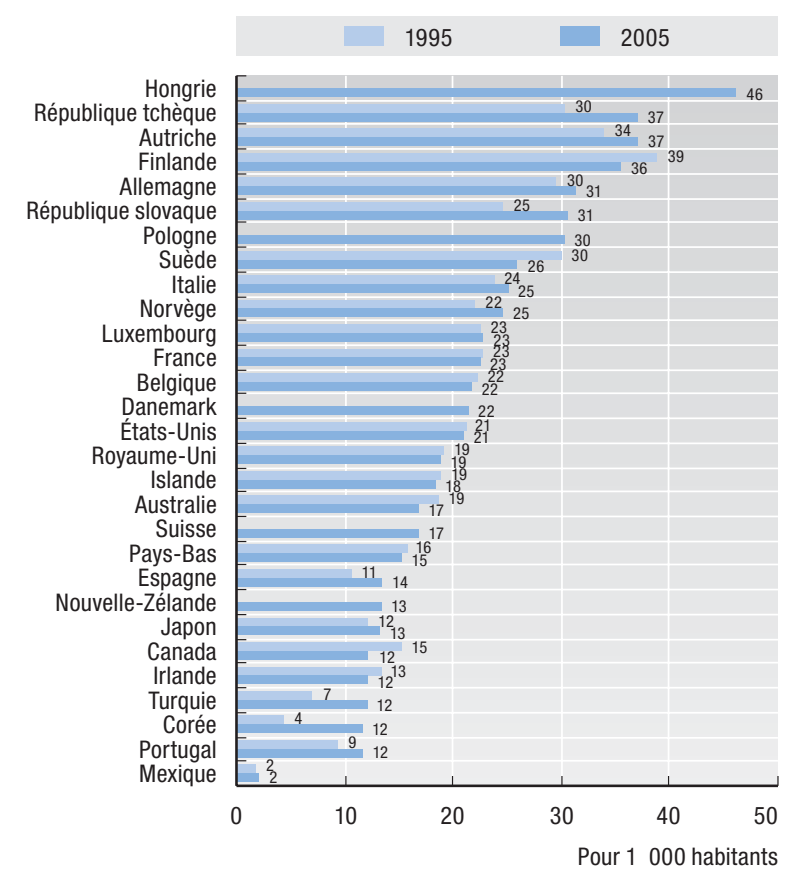

Source: Eco-Santé OCDE 2007. 


\subsection{DURÉE MOYENNE DE SÉJOUR À L'HÔPITAL}

La durée moyenne de séjour à l'hôpital (DMS) est fréquemment utilisée comme indicateur de l'efficience. Toutes choses égales par ailleurs, un séjour plus court diminue le coût par sortie et déplace les soins aux patients hospitalisés vers le cadre moins onéreux des soins postaigus. Toutefois, un séjour plus court exige une intensité de services plus élevée et coûte plus cher par journée d'hospitalisation. De même, un séjour trop court peut compromettre l'efficacité du traitement et s'avérer préjudiciable au confort du patient ou à son rétablissement. Si cela se traduit par une augmentation des taux de réadmission, les coûts ne diminueront que modérément ou risquent même d'augmenter.

En 2005, on continuait d'observer des variations importantes entre pays de l'OCDE en termes de DMS pour soins aigus. Cette durée était relativement courte (moins de cinq jours) dans certains pays nordiques (le Danemark, la Finlande, la Suède) et au Mexique, et relativement longue (plus de huit jours) au Japon, en Corée, en Allemagne et en Suisse (graphique 4.10.1). Plusieurs facteurs peuvent expliquer ces disparités. En Finlande, des temps d'hospitalisation courts sont liés, du moins en partie, à la disponibilité de lits pour les patients convalescents dans les centres de santé (OCDE, 2005a). À l'inverse, en Corée, une DMS élevée pour les soins aigus peut s'expliquer en partie par l'affectation des lits de soins aigus aux patients souffrant de maladies chroniques (OCDE, 2003b). Au Japon (Jeong et al., 1994), il se pourrait que l'offre abondante de lits ait incité les hôpitaux à garder les patients plus longtemps (voir l'indicateur 4.5 « Lits de soins aigus »). Des incitations financières inhérentes aux méthodes de paiement des hôpitaux peuvent également influer sur la durée de séjour. En Suisse, par exemple, des paiements s'effectuant principalement sur une base de jours/lit ont encouragé des durées de séjour importantes (OCDE, 2006a).

La durée moyenne de séjours pour les soins aigus a diminué dans la quasi-totalité des pays de l'OCDE, tombant de 8.7 jours en 1990 à 6.3 jours en 2005 pour les 25 pays pour lesquels on dispose de données cohérentes sur la période (graphique 4.10.1). Le recul de la DMS a été particulièrement rapide dans les pays qui partaient de niveaux relativement élevés en 1990 (l'Allemagne, la Pologne, la Suisse et la République tchèque). Plusieurs facteurs expliquent cette baisse, en particulier des interventions chirurgicales moins invasives, une évolution des méthodes de paiement des hôpitaux vers des systèmes de tarification prospectifs et le développement des programmes de sorties précoces qui permettent le suivi des patients à domicile.

Si l'on se concentre sur la DMS pour des maladies ou des états spécifiques, on peut atténuer le problème de l'hétérogénéité entre les pays (" case mix ") résultant de la nature et de la gravité potentiellement différentes des situations réclamant des soins aigus. Le graphique 4.10.3 montre que la DMS après un accouchement normal varie entre un maximum de deux jours au Mexique, au Royaume-Uni, en Turquie, au Canada et aux États-Unis, à cinq jours voire plus en Hongrie, en République slovaque, en Suisse et en République tchèque. Dans tous les pays de l'OCDE, la DMS pour un accouchement normal a diminué au cours des dix dernières années, tombant de 4.2 jours en 1995 à 3.3 jours en 2005, en moyenne (tableau A.4.10b). Les sorties prématurées de maternité sont devenues un sujet de préoccupation dans certains pays.

La durée d'hospitalisation après un infarctus aigu du myocarde (IAM) a également diminué sur les dix dernières années. En 2005, c'est dans les pays nordiques (la Norvège, le Danemark et la Suède) et aux États-Unis qu'elle était la plus faible (moins de six jours) tandis qu'en Finlande et en Irlande elle était supérieure à dix jours (graphique 4.10.2). Une certaine prudence s'impose toutefois dans les comparaisons entre pays; en Finlande, par exemple, la DMS peut prendre en compte des patients admis à l'origine pour un IAM mais qui ne reçoivent plus de soins aigus et pourraient donc être considérés comme des patients en soins de longue durée (Moïse et al., 2003a).

\section{Définition et écarts}

La durée moyenne de séjour (DMS) en soins aigus est le nombre moyen de jours (avec séjour d'au moins une nuit) que les patients passent dans un établissement de soins aigus. Elle est généralement obtenue en divisant le nombre total de journées passées par l'ensemble des patients dans des établissements de soins aigus au cours d'une année par le nombre des admissions ou des sorties.

La définition proposée pour les "soins aigus " inclut toutes les fonctions de soins relevant des " soins curatifs " tels que les définit le Système des comptes de la santé (OCDE, 2000a). Cependant, les fonctions englobées dans les " soins aigus " et celles qui en sont exclues (par exemple, la prise en compte ou non des lits affectés aux soins de longue durée, à la réadaptation et aux soins palliatifs) varient d'un pays à l'autre, ce qui limite la comparabilité des données.

De plus, dans le calcul des DMS, les jours et les sorties des bébés nés à l'hôpital en bonne santé sont exclues complètement (ou presque complètement) dans un certain nombre de pays (par exemple, l'Allemagne avant 2004, le Canada et les États-Unis). La prise en compte des bébés nés à l'hôpital en bonne santé réduiraient la DMS dans ces pays (d'environ la moitié d'un jour au Canada).

Les résultats des comparaisons entre pays doivent donc s'interpréter avec prudence. 


\section{RESSOURCES EN SANTÉ ET LEUR UTILISATION}

4.10. DURÉE MOYENNE DE SÉJOUR À L'HÔPITAL

4.10.1. Durée moyenne de séjour en soins aigus, 1990 et 2005 (ou année la plus proche)

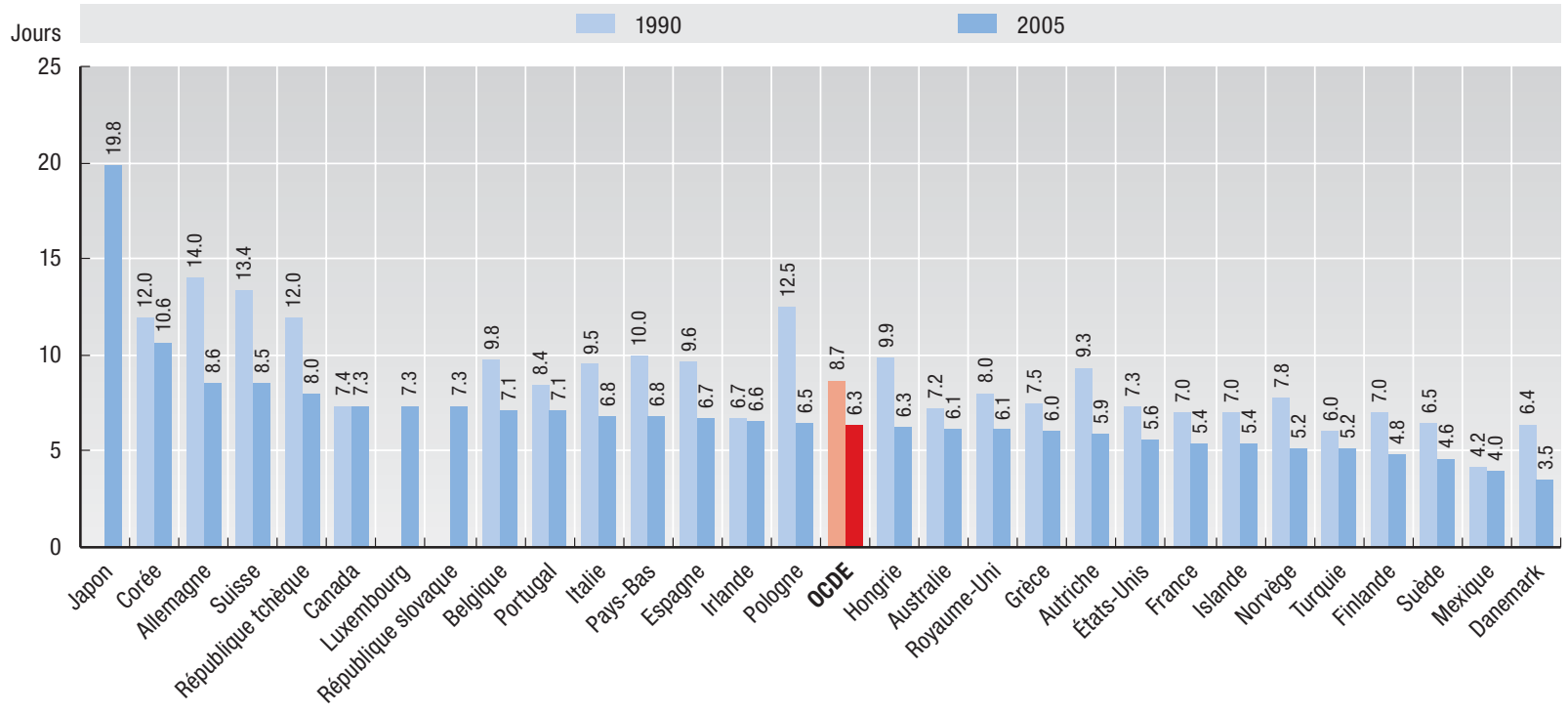

4.10.2. Durée moyenne de séjour suite à un infarctus aigu du myocarde, 2005 (ou année la plus proche)

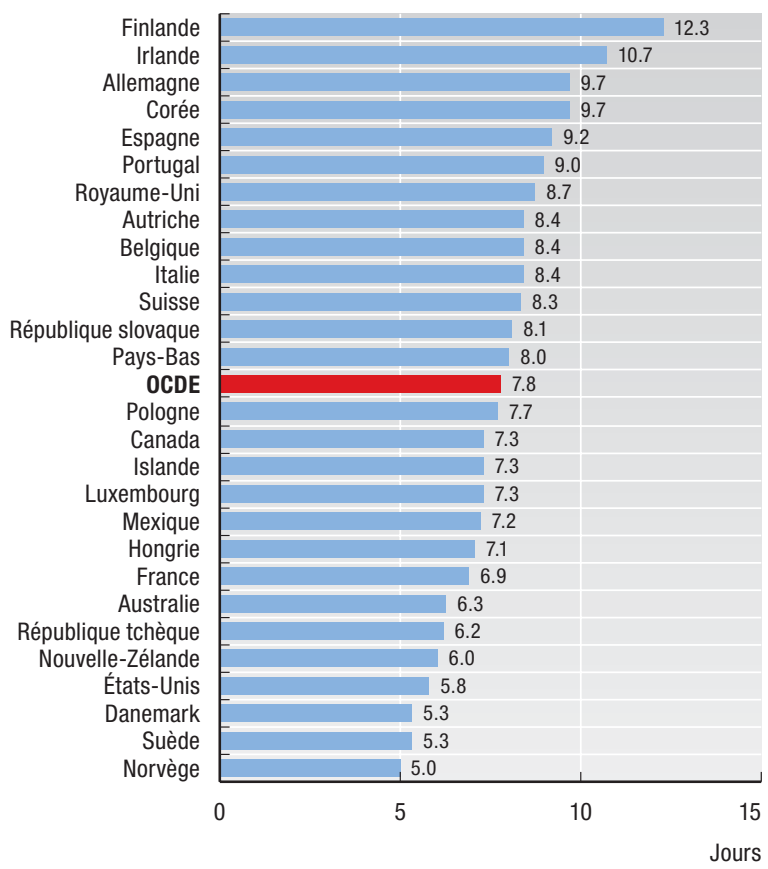

Source : Eco-Santé OCDE 2007.

\subsubsection{Durée moyenne de séjour pour un accouchement normal, 2005 (ou année la plus proche)}

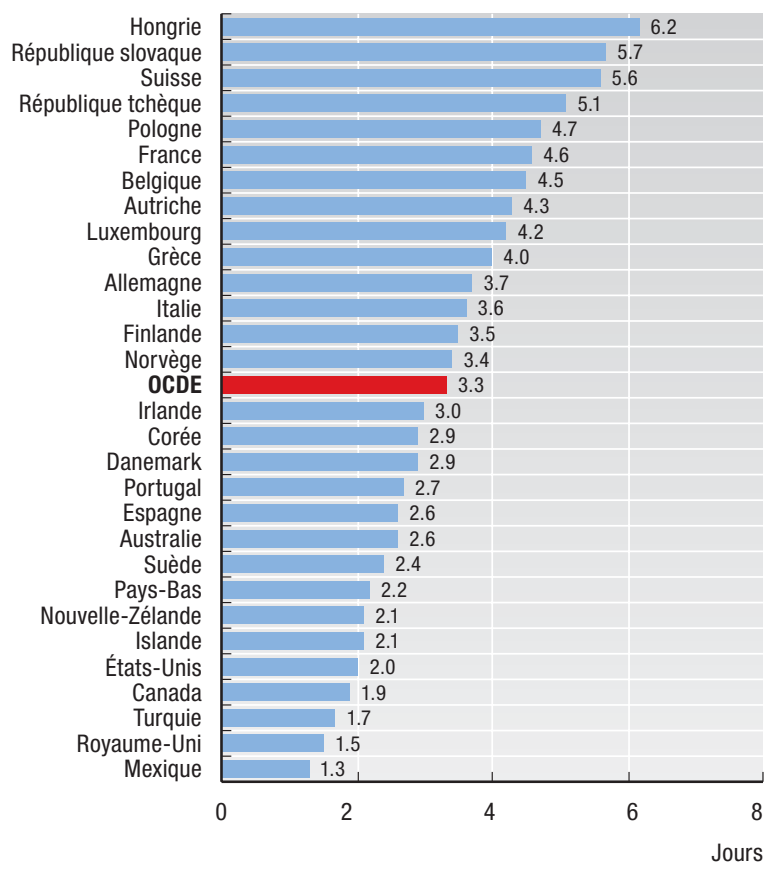

StatLink AाISL http://dx.doi.org/10.1787/128774521683 


\subsection{CHIRURGIES CARDIO-VASCULAIRES (PONTAGES ET ANGIOPLASTIES)}

Les maladies cardiaques sont une cause majeure d'hospitalisation et de décès dans les pays de l'OCDE (voir l'indicateur 2.4 " Mortalité due aux maladies cardiaques et aux accidents vasculaires cérébraux »). Le pontage coronarien et l'angioplastie coronaire sont deux procédures de revascularisation qui ont révolutionné le traitement des maladies cardiaques au cours des dernières décennies.

L'utilisation de ces procédures est très variable selon les pays (graphique 4.11.1). C'est aux États-Unis, suivis de près par la Belgique, que les angioplasties coronaires sont les plus répandues : 433 pour 100000 habitants y ont été pratiquées en 2004. Ces deux pays se classent également en tête du classement pour le nombre de pontages coronariens par habitant; en 2004, 152 pontages coronariens pour 100000 habitants ont été effectués en Belgique contre 145 aux États-Unis. À l'autre extrémité de l'échelle, on n'a recensé en 2004 que deux pontages coronariens et deux angioplasties coronaires pour 100000 habitants au Mexique, ce qui est nettement inférieur aux chiffres du Portugal, pays se classant avant-dernier.

Comme le montre le graphique 4.11.1, la composition des procédures de revascularisation varie considérablement d'un pays à l'autre. Dans la plupart des pays, les angioplasties coronaires représentent entre $65 \%$ et $80 \%$ du total des revascularisations. Au Canada et en Nouvelle-Zélande, les angioplasties coronaires représentant $60 \%$ et $56 \%$ respectivement des revascularisations pour les patients hospitalisés. Il est possible toutefois que dans ces deux pays, une proportion plus forte des angioplasties s'effectue en chirurgie de jour.

L'utilisation de l'angioplastie coronaire s'est développée rapidement au cours des dix dernières années dans la plupart des pays de l'OCDE, supplantant le pontage pour devenir, vers le milieu des années 90, la technique de revascularisation préférée (graphique 4.11.2). C'est à peu près au même moment que les premières publications des essais sur l'efficacité du stenting coronaire ont fait leur apparition (Moïse, 2003a). Le phénomène s'est accéléré ces dernières années avec la mise sur le marché de stents diffusant des médicaments et un recul de l'utilisation du pontage coronarien dans la plupart des pays de l'OCDE. Ces données laissent à penser qu'au fil du temps l'angioplastie coronaire va se substituer au pontage coronarien. Cutler et Huckman (2003) ont estimé qu'entre $25 \%$ et $35 \%$ des angioplasties sont des procédures de substitution de la chirurgie du pontage; ce sont toutefois des procédures de substitution imparfaites, le pontage demeurant la procédure préférée de traitement des obstructions multiples de vaisseaux.

Les déterminants de l'utilisation de procédures de revascularisation ne sont pas faciles à démêler. Utilisant les taux de mortalité dus aux cardiopathies ischémiques comme mesure indirecte, Moïse (2003a) a montré que le PIB par habitant est un déterminant plus fort du taux d'utilisation de ces procédures que le nombre sous-jacent de maladies cardiaques. Toutefois, la relation entre ces deux déterminants et les taux d'utilisation des procédures de revascularisation change lorsque la revascularisation est séparée en ses deux composantes. D'une part, le PIB par habitant n'est pas un déterminant important de l'utilisation de la chirurgie du pontage coronarien alors qu'il est un déterminant important de l'utilisation de l'angioplastie coronaire. De l'autre, le niveau de la cardiopathie sous-jacente est un facteur explicatif important de l'utilisation du pontage coronarien mais pas de l'utilisation de l'angioplastie coronaire (Moïse, 2003b).

\section{Définition et écarts}

Le pontage coronarien consiste à greffer des veines et/ou des artères pour court-circuiter une artère coronaire bouchée. Un pontage peut contourner l'obstruction d'une seule artère coronaire, mais il est plus courant de procéder à des pontages multiples. En angioplastie coronaire, on introduit dans le système artériel un cathéter à l'extrémité duquel est fixé un ballonnet, en entrant habituellement par l'artère fémorale de la jambe et en rejoignant l'artère coronaire endommagée. Le gonflement du ballonnet distend l'artère coronaire au point d'obstruction. La majorité des angioplasties s'accompagnent de la mise en place d'un stent (treillis métallique se présentant comme un petit ressort et destiné à maintenir l'artère ouverte). Les stents éluants (systèmes mis au point récemment qui libèrent des médicaments de manière progressive dans le milieu environnant) sont de plus en plus utilisés pour contenir la croissance de tissu cicatriciel autour du stent.

Les données concernent uniquement le nombre des procédures sur patients hospitalisés, comptabilisant normalement toutes les interventions effectuées. Toutefois, certains pays ne déclarent que l'intervention principale ou le nombre de patients subissant une ou plusieurs interventions. Les données n'incluent pas les angioplasties coronaires effectuées en ambulatoire, qui représentent une part croissante de l'activité globale dans de nombreux pays. 


\section{RESSOURCES EN SANTÉ ET LEUR UTILISATION}

4.11. CHIRURGIES CARDIO-VASCULAIRES (PONTAGES ET ANGIOPLASTIES)

\subsubsection{Interventions de revascularisation, pour 100000 habitants, 2004}

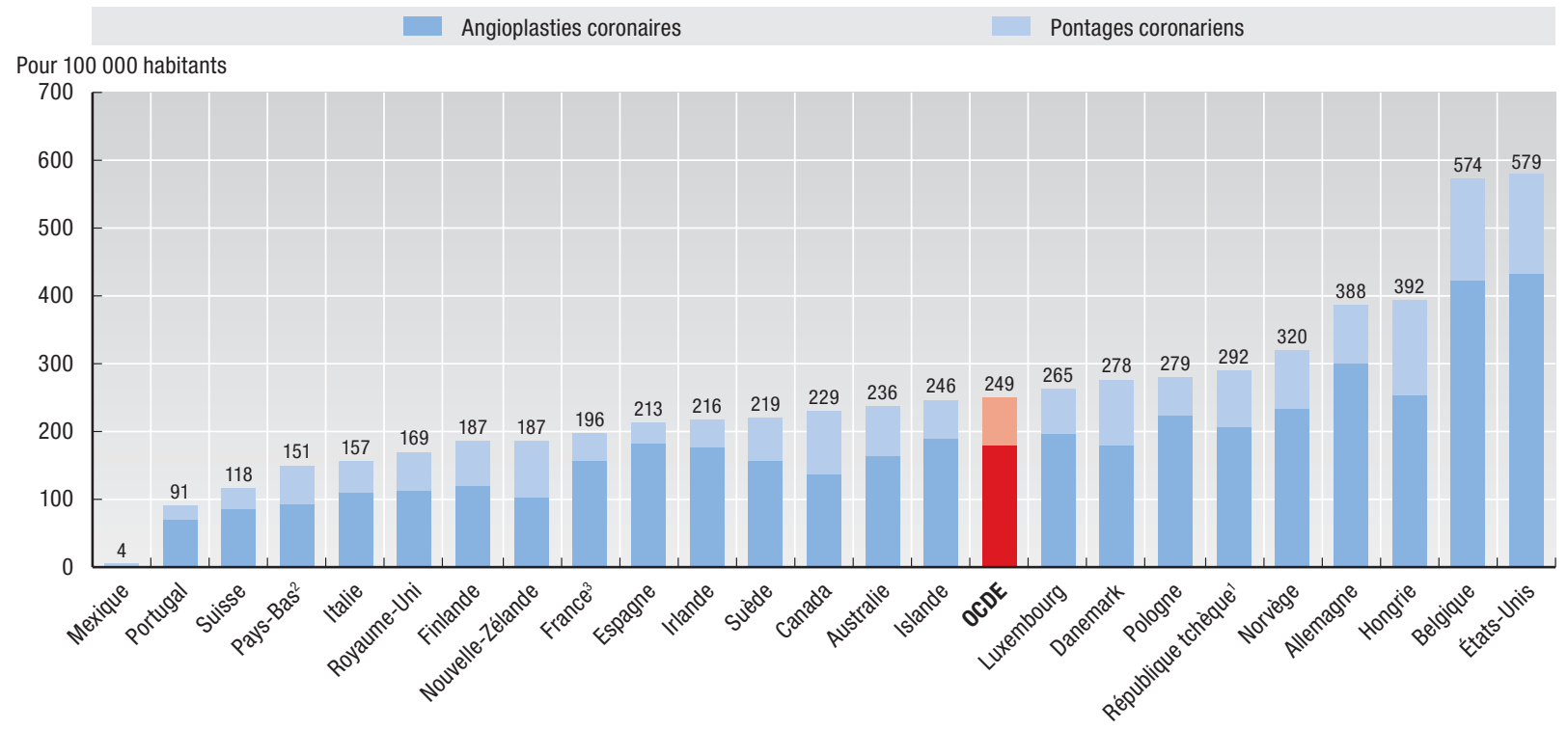

1. 2005. 2. 2003. 3. 2001.

\subsubsection{Angioplasties coronaires, en pourcentage du nombre total d'interventions de revascularisation, 1990-2004}

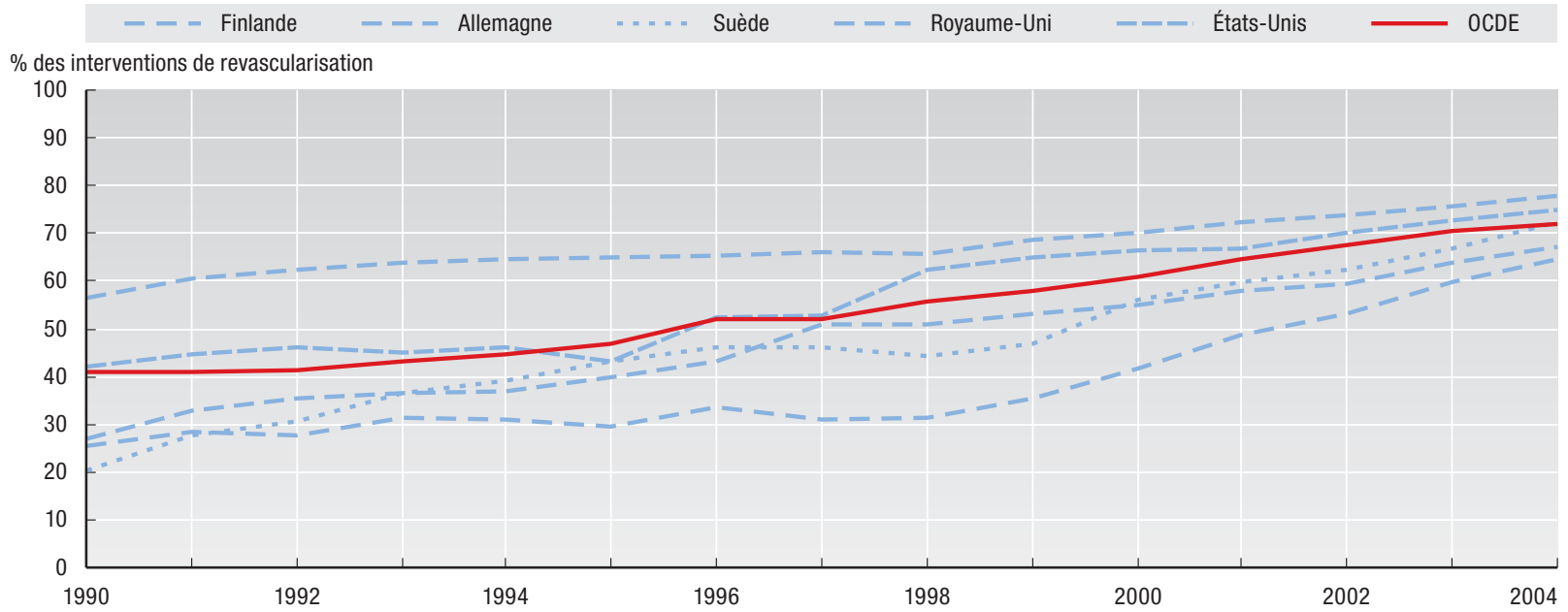

Source : Eco-Santé OCDE 2007. 


\subsection{TRAITEMENT DES INSUFFISANCES RÉNALES (DIALYSES ET TRANSPLANTATIONS RÉNALES)}

L'insuffisance rénale chronique est une pathologie dans laquelle les reins irrémédiablement endommagés ne peuvent plus fonctionner normalement. Parmi les principaux facteurs de risque de l'insuffisance rénale chronique figurent le diabète et l'hypertension, deux pathologies dont la prévalence s'accroît dans les pays de l'OCDE. Aux États-Unis, ces deux pathologies sont responsables de $60 \%$ (36\% pour le diabète et $24 \%$ pour l'hypertension) des diagnostics primaires d'insuffisance rénale chronique (USRDS, 2006). Au stade ultime de la maladie, les patients doivent être traités par dialyse ou transplantation. Le traitement par dialyse est plus onéreux et plus contraignant pour les patients en termes de qualité de vie (du fait de son caractère récurrent et pénible) qu'une transplantation réussie.

Si l'on considère les deux types de traitement, la proportion de personnes traitées pour une insuffisance rénale chronique a augmenté de plus de $6 \%$ par an en moyenne dans les pays de l'OCDE sur les vingt dernières années (graphique 4.12.2). Ceci s'est traduit, en 2005, par un taux de prévalence du traitement de l'insuffisance rénale plus de trois fois supérieur à celui de 1985. En 2005, le Japon et les États-Unis ont enregistré les taux de traitement d'insuffisance rénale chronique les plus élevés, avec plus de 160 patients pour 100000 habitants (graphique 4.12.1), suivis du Portugal qui a enregistré le plus fort taux de croissance depuis 1985 . On ne sait pas très bien pourquoi ces pays déclarent des taux de traitement de l'insuffisance rénale aussi élevés mais cela ne semble pas être seulement ou principalement lié à une plus grande prévalence du diabète, laquelle n'est pas particulièrement plus importante dans ces pays que dans d'autres pays de l'OCDE (IDF, 2006).

Dans la plupart des pays de l'OCDE, la majorité des patients traités pour une insuffisance rénale sont sous dialyse. Cela peut être attribué au fait que la prévalence des personnes souffrant d'insuffisance rénale terminale a fortement augmenté dans de nombreux pays mais que le nombre des transplantations demeure limité par le manque de donneurs. Les exceptions sont la Finlande, l'Islande et les Pays-Bas, où le nombre total de patients traités pour une insuffisance rénale est cependant relativement faible.
Si l'on se concentre sur le principal type de traitement actuellement dispensé aux personnes souffrant d'insuffisance rénale, la proportion des dialysés est beaucoup plus grande au Japon et, dans une moindre mesure, aux États-Unis, que dans les autres pays (graphique 4.12.3). Au Japon, le phénomène est en partie lié au fait que les taux de transplantation rénale sont parmi les plus faibles de la zone OCDE, ce qui signifie que pratiquement tous les patients souffrant d'insuffisance rénale sont sous dialyse. Dans tous les pays, le nombre des personnes sous dialyse a considérablement augmenté au cours des vingt dernières années.

Étant donné la pénurie de donneurs, les transplantations de reins sont normalement opérées sur des patients qui ne peuvent vivre qu'au prix de sessions de dialyse longues et difficiles. Lorsqu'elle est réussie, la transplantation permet à la personne de vivre à nouveau à peu près normalement, sans régime alimentaire strict ni limitation de l'activité. Les progrès des techniques chirurgicales et le développement de nouveaux médicaments anti-rejet ont permis d'effectuer davantage de transplantations, et d'améliorer leur taux de succès par rapport à il y a 20 ans. La prévalence de personnes vivant avec un rein greffé fonctionnant bien a augmenté de façon régulière depuis 1985 dans tous les pays pour lesquels on dispose de données. La moyenne de l'OCDE est passée de 8 à 32 personnes (vivant avec un rein greffé qui fonctionne bien) pour 100000 habitants entre 1985 et 2005 (graphique 4.12.4). En 2005, ce sont les États-Unis, suivis de l'Autriche, la Finlande, l'Espagne et la France, qui ont déclaré le taux le plus élevé de personnes greffées (plus de 40 pour 100000 habitants). À l'autre extrémité de l'échelle, c'est au Japon, suivi de la Corée et de la République slovaque, que la proportion de personnes ayant reçu un rein a été la plus faible.

Le principal frein à une augmentation du nombre des transplantations reste le nombre de donneurs. Dans bien des pays, les listes d'attente des candidats à une greffe s'allongent, la demande étant bien supérieure à l'offre. Les facteurs culturels et les traditions ont un impact sur les taux de transplantation et la transplantation reste peut-être moins bien acceptée dans certains pays comme le Japon.

\section{Définition et écarts}

Le nombre de patients traités pour une insuffisance rénale terminale est le nombre de patients à la fin de chaque année qui reçoivent différentes formes de thérapie de remplacement du rein : hémodialyse/hémofiltration, dialyse péritonéale intermittente, dialyse péritonéale continue ambulatoire, dialyse péritonéale continue cyclique, ou qui ont reçu un rein en bon état de fonctionnement. 


\section{RESSOURCES EN SANTÉ ET LEUR UTILISATION}

\subsection{TRAITEMENT DES INSUFFISANCES RÉNALES (DIALYSES ET TRANSPLANTATIONS RÉNALES)}

\subsubsection{Patients traités pour insuffisance rénale} chronique, par type de traitement, 2005 (ou dernière année disponible)

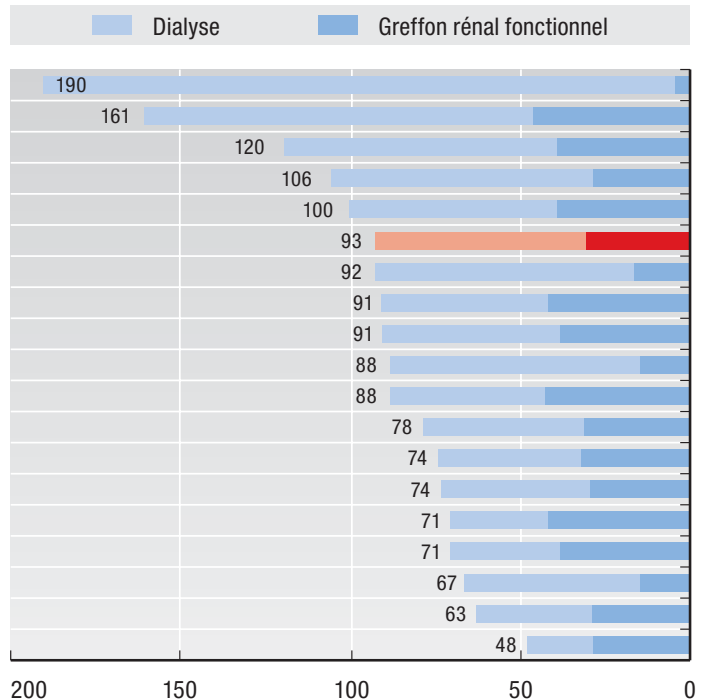

Nombre de patients pour 100000 habitants

\subsubsection{Prévalence des patients dialysés, 1985 à 2005 (ou année la plus proche)}

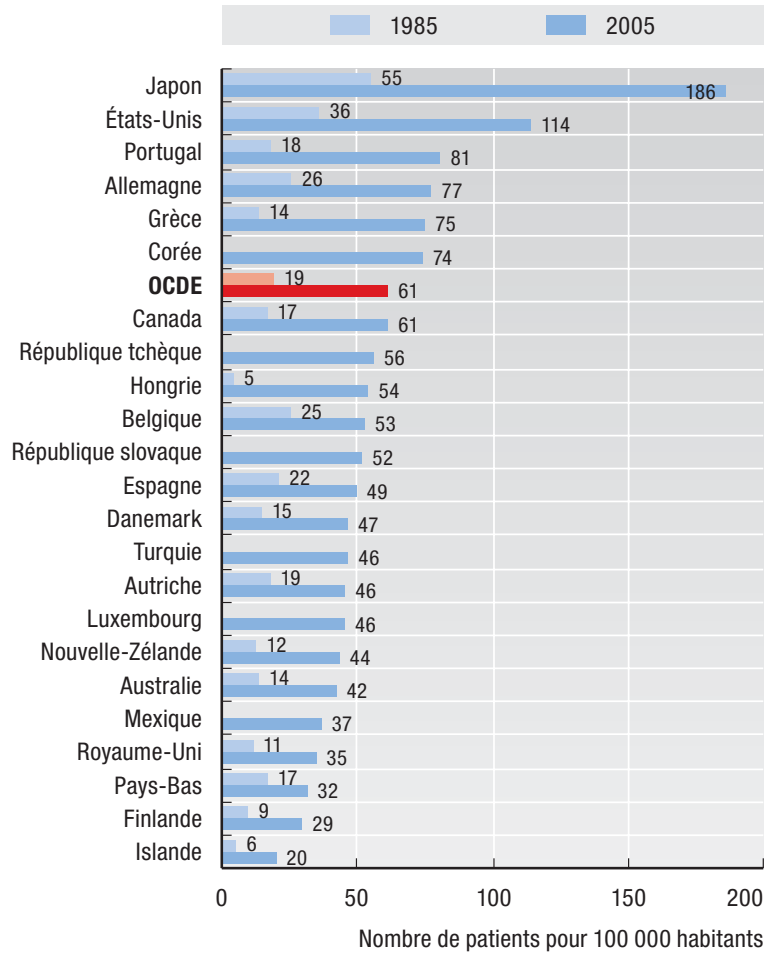

Source : Eco-Santé OCDE 2007.

\subsubsection{Augmentation de la prévalence}

des personnes traitées pour insuffisance rénale chronique, 1985 à 2005

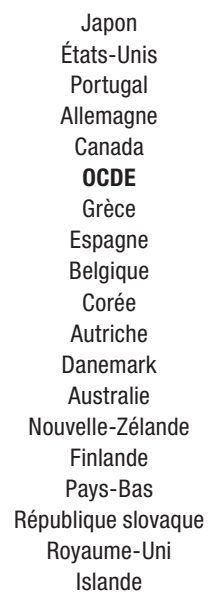

Islande

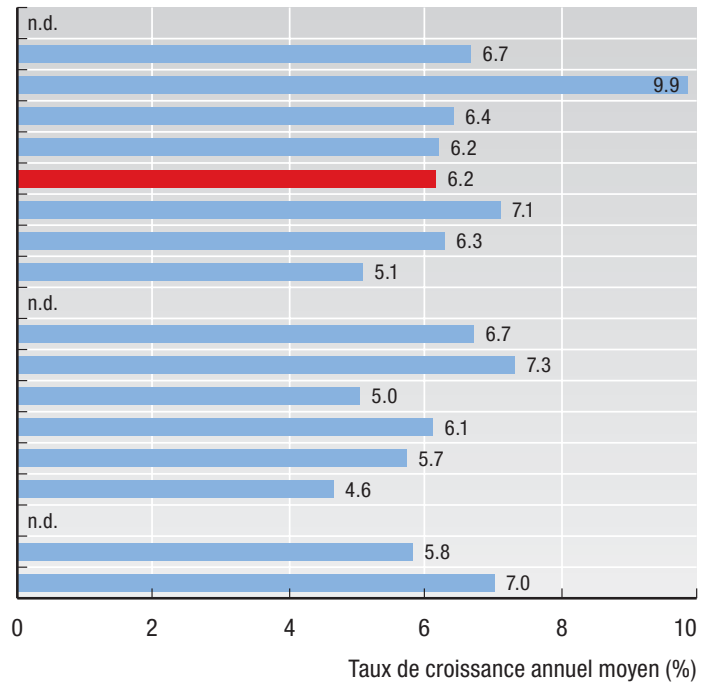

4.12.4. Prévalence des patients vivant avec un greffon rénal fonctionnel, 1985 à 2005 (ou année la plus proche)

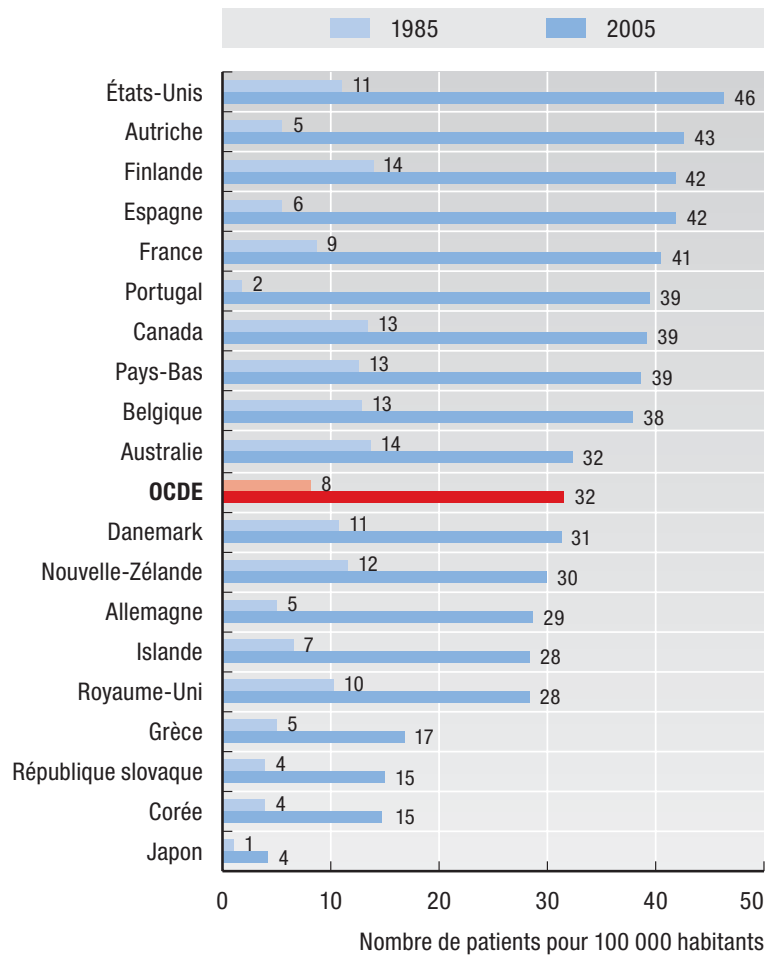

StatLink AाISL $h t t p: / / d x . d o i . o r g / 10.1787 / 130010560714$ 


\subsection{CÉSARIENNES}

$\mathrm{Au}$ cours des dernières décennies, les taux de césariennes en pourcentage de l'ensemble des naissances vivantes ont augmenté dans tous les pays de l'OCDE. Cette augmentation reflète non seulement des facteurs de risque médical (comme l'âge de la mère, les naissances multiples, le fait pour la mère d'avoir déjà eu une césarienne et une période de gestation allant au-delà du terme normal) mais également une évolution des pratiques des professionnels de santé et des préférences des mères. Ce phénomène conduit à se demander si le coût - financier et pour la santé de la mère et de l'enfant - de certaines de ces interventions n'excède pas les avantages.

En 2004, le taux de césariennes en pourcentage de l'ensemble des naissances vivantes était très variable dans les pays de l'OCDE (graphique 4.13.1). Il s'échelonnait entre moins de $20 \%$ aux Pays-Bas, en République tchèque, dans les pays nordiques, en France, en Belgique et en République slovaque, à plus de $33 \%$ en Corée, en Italie et au Mexique. Plusieurs facteurs expliquent les taux élevés enregistrés dans ce deuxième groupe de pays. En Corée, les prix plus élevés facturés pour les césariennes par rapport à des accouchements normaux ont encouragé les médecins à en pratiquer plus (OCDE, 2003b). De même, au Mexique, les médecins opérant dans le secteur privé sont davantage incités financièrement à pratiquer des césariennes programmées (Secretaria de Salud, 2003). En Italie, les taux élevés de césariennes pourraient refléter une préférence des patientes (reposant en partie sur la croyance que les césariennes sont généralement plus sûres que les accouchements normaux) combinée à une évolution des pratiques des médecins qui préfèrent les césariennes en raison de la plus grande rapidité de l'intervention et de la possibilité de la planifier à l'avance.
Les césariennes ont augmenté dans tous les pays de l'OCDE et, dans certains cas, l'augmentation a été relativement rapide (graphique 4.13.2). En moyenne pour un groupe de 20 pays, le taux de césariennes était de $14 \%$ de l'ensemble des naissances en 1990; en 2004, ce taux était passé à $22 \%$. La croissance de cette pratique depuis 1990 a été particulièrement rapide en Irlande, en République slovaque et en République tchèque. En revanche, elle a été beaucoup plus lente dans les pays nordiques.

Il se pourrait que toutes ces interventions n'aient pas été effectuées sur indication médicale. Declercq et al. (2005) ont analysé la hausse du taux de césariennes aux États-Unis entre 1996 et 2001, en neutralisant les facteurs de risque les plus importants. Leur étude a montré que la proportion des césariennes "sans mention de risque " (définies comme s'appliquant à des mères qui accouchent à terme d'un enfant unique, qui n'ont pas subi de césarienne antérieure, qui ne présentent aucun facteur de risque médical déclaré et pour lesquelles le certificat de naissance ne fait mention d'aucune complication lors du travail ou de l'accouchement) est passée de $3.7 \%$ des naissances en 1996 à $5.5 \%$ en 2001.

Le débat sur les avantages relatifs d'un accouchement par voie basse par rapport à une césarienne en cas de grossesse normale sans complications n'est pas encore tranché. Les césariennes comportent moins de risque dans les pays développés que dans les pays en développement (OMS, 2005) mais elles coûtent plus chers que des accouchements normaux et les interventions inutiles tendent à entraîner des problèmes de santé plus nombreux pour la mère et l'enfant (Bewley et Cockburn, 2002; Victora et Barros, 2006). Les césariennes non justifiées impliquent donc que des interventions coûteuses sont pratiquées avec un niveau de risque accru.

\section{Définition et écarts}

Le taux de césariennes est le nombre de césariennes pratiquées pour 100 naissances vivantes. Au Portugal, le dénominateur se rapporte uniquement au nombre de naissances vivantes enregistrées dans les hôpitaux publics du continent (d'où une surestimation du taux de césariennes). 


\section{RESSOURCES EN SANTÉ ET LEUR UTILISATION}

4.13. CÉSARIENNES

\subsubsection{Césariennes pour $\mathbf{1 0 0}$ naissances vivantes, 2004}

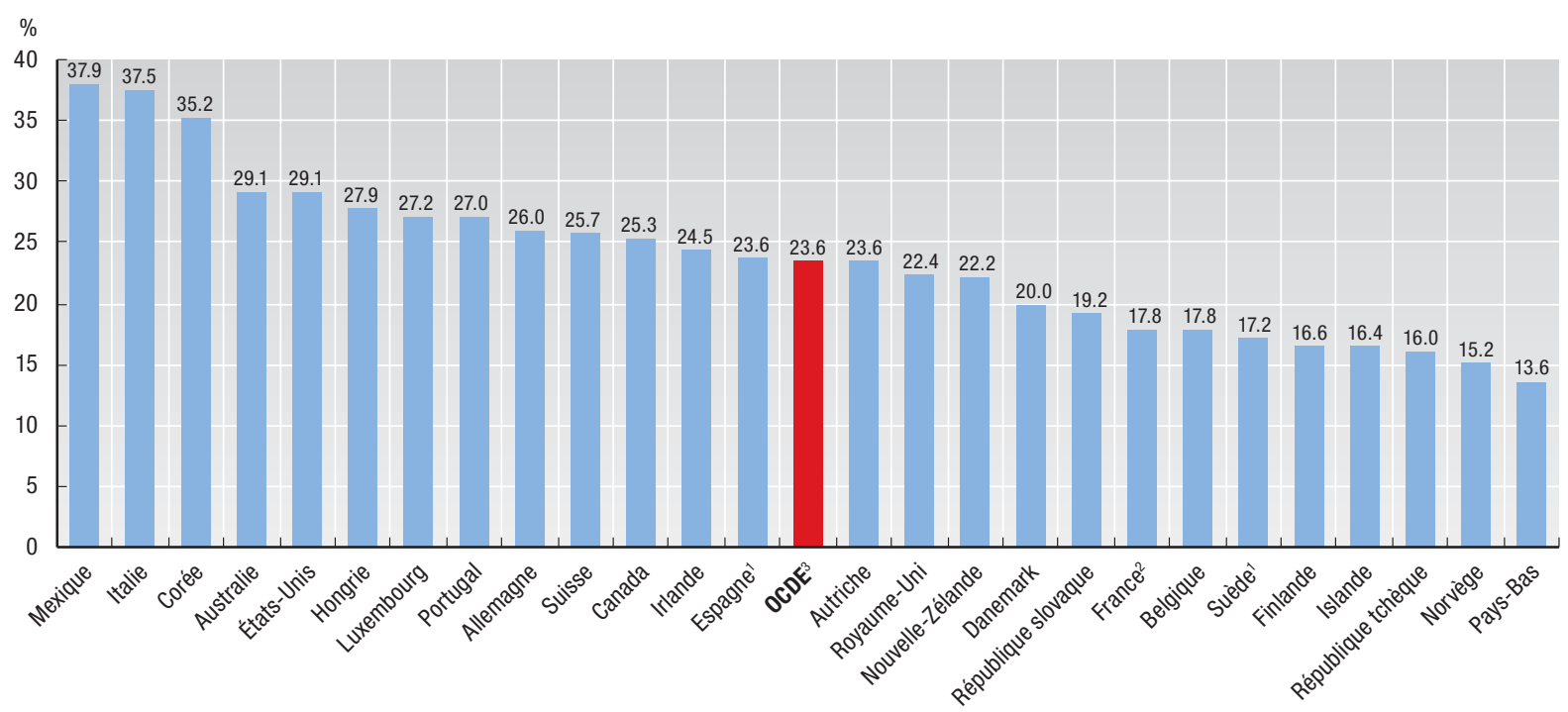

1. 2003. 2. 2001.

3. La moyenne de l'OCDE comprend les dernières données disponibles pour 26 pays.

\subsubsection{Augmentation des césariennes pour 100 naissances vivantes, 1990 à 2004}

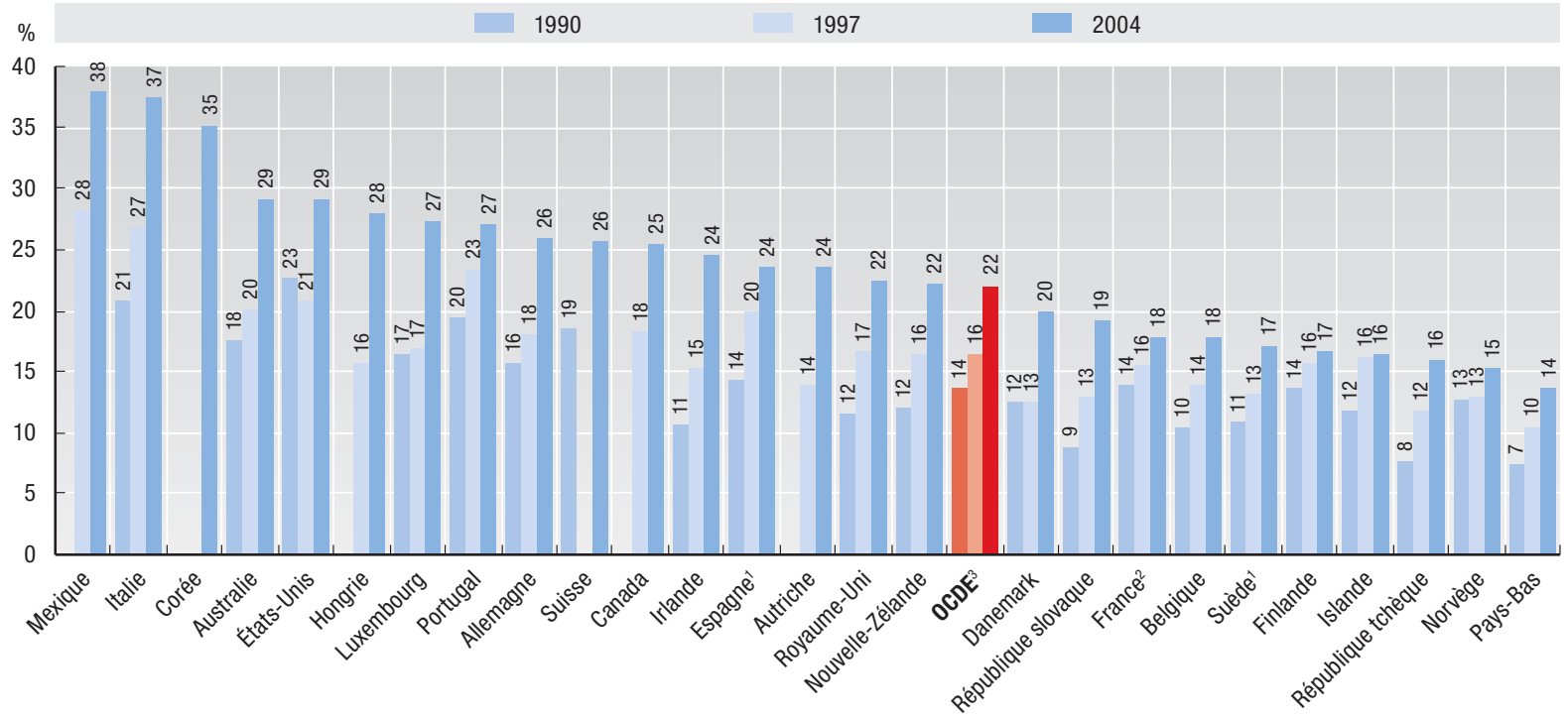

1. 2003. 2. 2001.

3. La moyenne de l'OCDE est la moyenne cohérente d'un groupe commun de 20 pays.

Source : Eco-Santé OCDE 2007. 


\subsection{OPÉRATIONS DE LA CATARACTE, EN AMBULATOIRE ET AVEC HOSPITALISATION}

$\mathrm{Au}$ cours des vingt dernières années, le nombre d'actes réalisés en chirurgie ambulatoire (également appelée chirurgie de jour) a régulièrement augmenté dans tous les pays de l'OCDE grâce aux progrès des technologies médicales, en particulier au développement d'interventions chirurgicales moins invasives, et à l'amélioration des produits d'anesthésie. Ces innovations ont accru l'efficacité des interventions et généré des avantages considérables en termes de sécurité des patients. Elles peuvent également réduire le coût unitaire des interventions en raccourcissant la durée de séjour. Toutefois, leur incidence sur le coût global de la santé dépendra de l'importance relative de l'évolution des coûts unitaires et des volumes d'interventions, de l'impact sur la réduction du nombre de lits d'hôpitaux mais aussi du coût des soins aigus postintervention et des services de santé communautaires.

L'opération de la cataracte est devenue l'intervention chirurgicale la plus pratiquée dans la plupart des pays de l'OCDE. Il s'agit d'un bon exemple d'intervention chirurgicale courante, désormais pratiquée principalement en ambulatoire dans la plupart des pays de l'OCDE.

En 2004 (ou la dernière année disponible), le nombre d'opérations de la cataracte par habitant était très variable parmi les 19 pays fournissant des données sur les interventions avec hospitalisation comme sur les interventions en ambulatoire (graphique 4.14.1). Le taux s'échelonnait entre 51 opérations pour 100000 habitants à 1600 opérations pour 100000 habitants en Belgique. Ces variations entre pays peuvent s'expliquer à la fois par des facteurs de "demande" (par exemple, le vieillissement démographique) et des facteurs " d'offre " (par exemple, la capacité d'effectuer l'intervention en ambulatoire ou avec hospitalisation). Des pratiques différentes d'enregistrement des opérations de la cataracte limitent toutefois la comparabilité des données (voir l'encadré « Définition et écarts » ci-dessous).

Ces dernières années, le nombre des opérations de la cataracte a augmenté dans la plupart des pays de l'OCDE (graphique 4.14.1). Il est probable que cette tendance a été encouragée par une évolution de la demande sous-jacente liée au vieillissement de la population mais la réussite certaine, la sécurité et le bon rapport coût/efficacité des interventions pratiquées en ambulatoire ont été probablement des facteurs plus déterminants (Fedorowicz et al., 2004). En Belgique, au Danemark, en Finlande, en Irlande, en Italie, aux Pays-Bas et au Royaume-Uni (tableau A.4.14), l'augmentation du nombre d'interventions pratiquées en ambulatoire a plus que compensé, au cours des sept dernières années, la baisse du nombre des opérations de la cataracte nécessitant un séjour d'au moins une nuit à l'hôpital, mettant en évidence à la fois un effet "de substitution " (remplacement des opérations avec hospitalisation) et un effet « d'expansion » (augmentation du volume total).

Dans la plupart des pays de l'OCDE, les opérations de la cataracte sont désormais pratiquées principalement en ambulatoire. En effet, la chirurgie de jour représente $90 \%$ ou plus de l'ensemble des opérations de la cataracte dans la moitié des pays pour lesquels on dispose de données (le Canada, la Suède, le Danemark, la Finlande, le Royaume-Uni, l'Australie et la Nouvelle-Zélande) (graphique 4.14.2). Mais, dans d'autres pays, cette pratique est encore relativement peu répandue, ce qui pourrait résulter entre autres de conditions plus avantageuses de remboursement des hospitalisations, des réglementations nationales et d'obstacles à l'évolution des pratiques individuelles des chirurgiens et des anesthésistes (Castoro et al., 2007). La part des opérations de la cataracte effectuées avec hospitalisation du patient continue d'être de l'ordre de $50 \%$ ou plus au Luxembourg, en France, en Irlande et au Portugal.

En France, on a estimé qu'entre $77 \%$ et $90 \%$ des opérations de la cataracte pratiquées en 1999 auraient pu être effectuées en ambulatoire si tous les patients sans contre-indication médicale avaient été opérés de la sorte (Sourty Le Gellec, 2001). Bien qu'en France le pourcentage d'opérations de la cataracte effectuées en chirurgie de jour soit passé de $27 \%$ en 1999 à $36 \%$ en 2001, il restait un potentiel considérable de développement de ce mode plus efficace de traitement de la cataracte.

\section{Définition et écarts}

L'opération de la cataracte consiste à extraire le cristallin de l'œil (à cause de la présence de cataractes qui le rendent partiellement ou complètement opaque) et à le remplacer par un cristallin artificiel. L'intervention peut être effectuée en chirurgie de jour ou avec hospitalisation. La chirurgie de jour (ou ambulatoire) concerne des patients qui sont opérés dans une unité chirurgicale spécialisée (dans un hôpital ou une clinique) et qui sortent le jour de l'intervention. Les termes de chirurgie de jour et de chirurgie sans hospitalisation sont des termes équivalents utilisés dans certains pays. La chirurgie avec hospitalisation concerne des patients qui reçoivent un traitement chirurgical et passent au moins une nuit à l'hôpital.

Les comparaisons de données entre pays exigent une certaine prudence. À l'heure actuelle, les systèmes d'information sur la santé de plusieurs pays ne couvrent qu'imparfaitement les interventions chirurgicales de jour, en particulier celles pratiquées dans des établissements de soins ambulatoires en dehors de l'hôpital ou dans le secteur privé (par exemple, dans des cliniques privées). Ainsi, les chiffres pour l'Espagne par exemple ne comprennent pas les interventions pratiquées dans le secteur privé. Par ailleurs, les modalités d'enregistrement des opérations de la cataracte peuvent varier entre les pays (par exemple, elles peuvent être enregistrées comme une intervention unique comportant au moins deux étapes (l'extraction du cristallin et la pose d'un cristallin artificiel) ou comme deux interventions séparées. 


\section{RESSOURCES EN SANTÉ ET LEUR UTILISATION}

4.14. OPÉRATIONS DE LA CATARACTE, EN AMBULATOIRE ET AVEC HOSPITALISATION

4.14.1. Nombre de chirurgies de la cataracte avec hospitalisation et en ambulatoire, pour 100000 habitants, 1997 et 2004

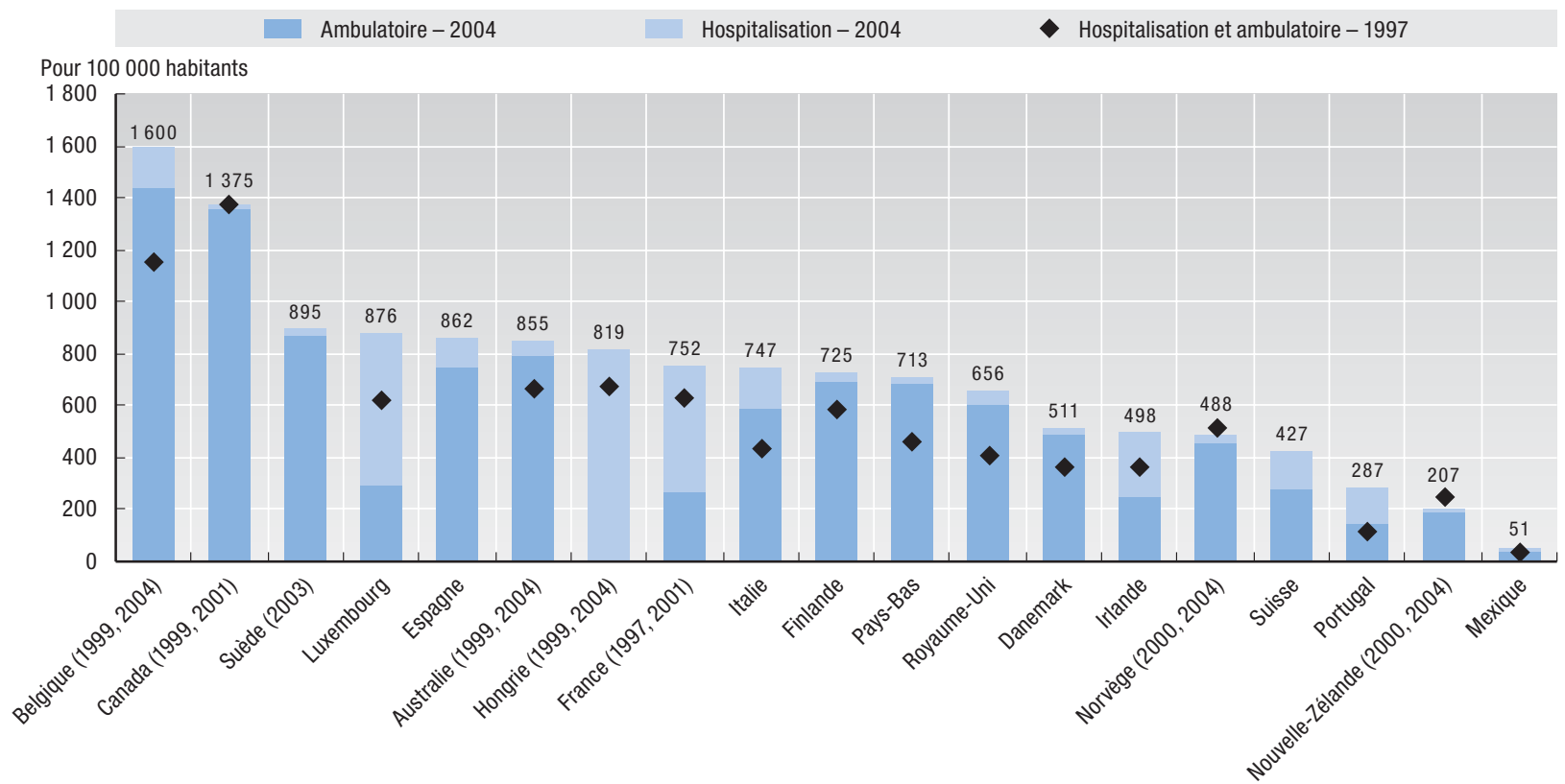

4.14.2. Pourcentage de chirurgies de la cataracte effectuées en soins ambulatoires, 1997 et 2004

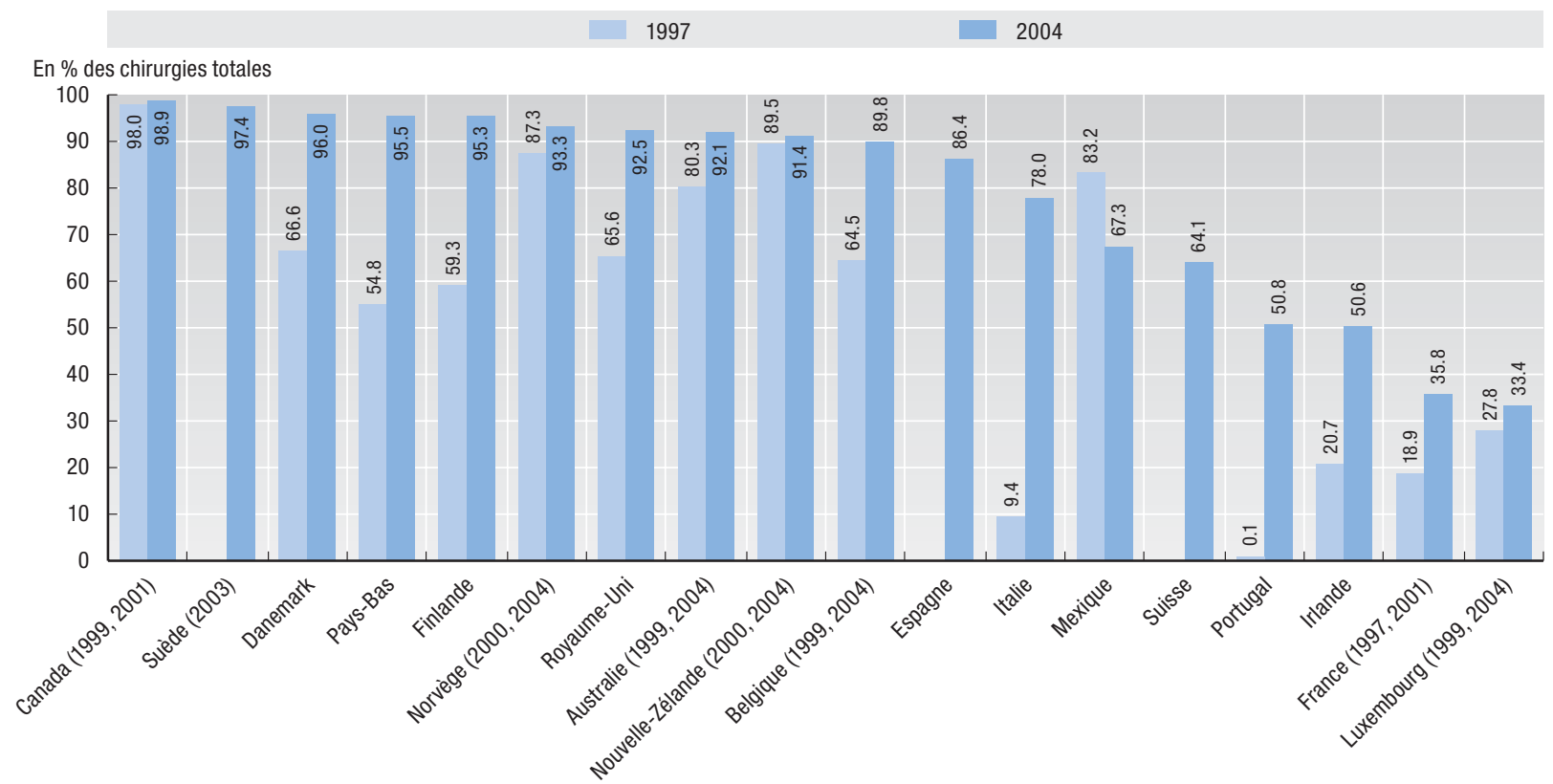

Source : Eco-Santé OCDE 2007. 


\subsection{CONSOMMATION DE MÉDICAMENTS}

La consommation de médicaments augmente dans les pays de l'OCDE non seulement en termes de dépenses (voir l'indicateur 5.4 " Dépenses pharmaceutiques") mais également en termes de volume (ou de quantité) de médicaments consommés. L'un des facteurs qui contribue à l'accroissement de la consommation de médicaments est le vieillissement de la population dans la plupart des pays de l'OCDE, qui s'accompagne d'une augmentation de la demande d'un certain nombre de médicaments pour traiter ou du moins maîtriser les différentes maladies liées au vieillissement. Mais on observe également cette tendance à une augmentation de la consommation de médicaments même dans des pays où le processus de vieillissement de la population est moins avancé, ce qui indique que d'autres facteurs y contribuent comme la mise sur le marché de nouveaux médicaments.

Cette section fournit des informations sur le niveau actuel, et l'évolution au cours des cinq dernières années, de la consommation de quatre des vingt-huit catégories de médicaments pour lesquels on dispose de données dans Eco-Santé OCDE : les antidiabétiques, les antidépresseurs, les produits anti-cholestérol et les antibiotiques. Le volume de consommation de ces médicaments est évalué de manière comparable entre pays grâce à l'utilisation de l'unité dite de "dose quotidienne définie » recommandée par le Collaborating Center for Drug Statistics de l'OMS (voir l'encadré « Définition et écarts » ci-dessous).

Commençant par les médicaments utilisés dans le traitement du diabète, le volume actuel de consommation de ces médicaments par habitant varie considérablement entre les pays, la consommation étant deux fois moindre en Islande et au Danemark qu'en Grèce et en Finlande (graphique 4.15.1). Entre 2000 et 2005, la consommation de produits antidiabétiques a augmenté cependant dans tous les pays. L'augmentation a été particulièrement forte en République slovaque (où la consommation a augmenté de près de $30 \%$ par an mais partait d'un niveau relativement bas en 2000), en Grèce (14 \% par an) et au Luxembourg (environ $10 \%$ par an). L'augmentation de la consommation peut être attribuée à la prévalence grandissante du diabète mais aussi à une augmentation de la proportion de personnes traitées et des doses moyennes administrées dans le cadre des traitements (Melander et al., 2006).

La consommation d'antidépresseurs varie, elle aussi, considérablement d'un pays de l'OCDE à l'autre. C'est l'Islande qui affiche la consommation la plus forte, suivie de l'Australie et des autres pays nordiques (graphique 4.15.2). C'est dans les pays d'Europe centrale et orientale (la République slovaque, la Hongrie et la République tchèque) que la consommation d'antidépresseurs est la plus faible, même si elle a augmenté rapidement au cours des cinq dernières années.

La consommation de produits anti-cholestérol oscille entre un point haut de 182 doses quotidiennes définies par jour pour 1000 habitants en Australie et un point bas de 65 en Allemagne (graphique 4.15.3). Si ces disparités peuvent refléter, du moins en partie, des différences de prévalence dans la population de ces pays de niveaux élevés de mauvais cholestérol, elles peuvent également être attribuées à des différences au niveau des directives cliniques de lutte contre un niveau élevé de cholestérol. En Australie, par exemple, ces directives sont ciblées sur un niveau de mauvais cholestérol inférieur aux niveaux établis par les pays européens, mais il existe également des différences entre pays européens (National Heart Foundation of Australia et al., 2005; Hockley et Gemmill, 2007). La croissance très rapide de la consommation de produits anti-cholestérol, ces dernières années, dans tous les pays de l'OCDE pour lesquels on dispose de données s'explique également par le contexte épidémiologique (par exemple, l'augmentation de l'obésité) ainsi que par la progression du dépistage et des traitements. Entre 2000 et 2005, cette consommation s'est accrue à un rythme annuel de $13 \%$ en Suède, et atteignait presque les $30 \%$ au Portugal et en République tchèque.

La consommation d'antibiotiques varie aussi très largement dans les pays de l'OCDE, entre un point bas de 11 doses quotidiennes définies pour 1000 habitants par jour aux Pays-Bas et un plus haut de 35 en Grèce (graphique 4.15.4). Conscients qu'une consommation excessive d'antibiotiques génère une résistance bactérienne, de nombreux pays ont lancé, ces dernières années, des campagnes d'information ciblées sur les médecins et/ou les patients afin de réduire la consommation des antibiotiques. À la suite de ces campagnes, la consommation s'est stabilisée dans de nombreux pays et elle a même diminué dans d'autres (en France et en Pologne). En revanche, certains pays (le Portugal et la Grèce) ont enregistré une progression importante de leur consommation d'antibiotiques entre 2000 et 2005 .

\section{Définition et écarts}

La dose quotidienne définie (DQD) se définit comme la dose quotidienne moyenne supposée d'un médicament utilisé dans son indication principale pour un adulte. Des DQD sont attribuées par un groupe d'experts internationaux à chaque principe actif ou combinaison de principes actifs d'une catégorie thérapeutique donnée. Par exemple, la DQD de l'aspirine orale est de 3 g, ce qui est la dose quotidienne supposée pour traiter les douleurs chez l'adulte. Les DQD ne reflètent pas nécessairement la dose quotidienne moyenne effectivement utilisée dans un pays donné mais il s'agit d'une unité type qui permet de mesurer la consommation de médicaments. Les DQD peuvent être regroupées à l'intérieur et entre les catégories thérapeutiques de la Classification anatomique thérapeutique (ATC). Pour plus de détails, voir www.whocc.no/atcddd.

Les données concernent généralement la consommation en dehors de l'hôpital, excepté pour la République tchèque, le Danemark, la Finlande, la Hongrie et la Suède, où elles englobent la consommation des hôpitaux. Les données n'englobent pas les médicaments non couverts par l'assurance maladie en Belgique, en Allemagne, en Irlande, aux Pays-Bas et au Portugal. Toutefois, cela ne devrait pas affecter les comparaisons à l'intérieur de ces classes thérapeutiques dans la mesure où la plupart des produits sont généralement couverts par l'assurance maladie. Les données de la Grèce peuvent englober les exportations parallèles. 


\section{RESSOURCES EN SANTÉ ET LEUR UTILISATION}

4.15.1. Consommation d'antidiabétiques, DQD* pour 1000 habitants par jour, 2000 et 2005

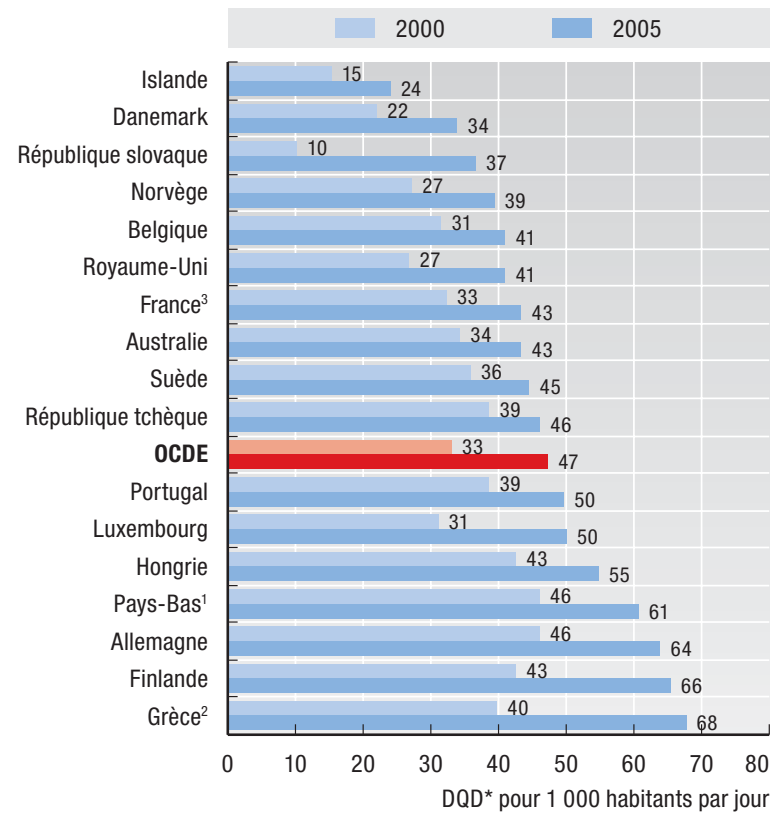

1. 2001-05. 2. 2000-04.

3. Représente seulement $88 \%$ de la consommation.

4.15.3. Consommation d'hypolipidémiants, DQD* pour 1000 habitants par jour, 2000 et 2005

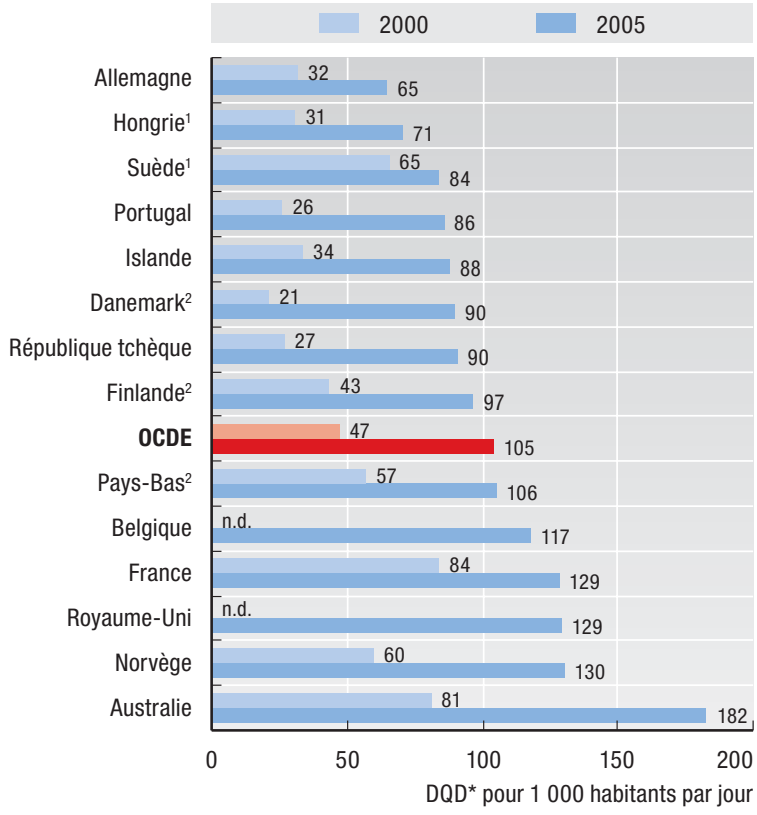

1. 2003-05. 2. 2001-05.

* DQD : Dose quotidienne définie.

Source : Eco-Santé OCDE 2007.

\subsection{CONSOMMATION DE MÉDICAMENTS}

4.15.2. Consommation d'antidépresseurs, DQD* pour 1000 habitants par jour, 2000 et 2005

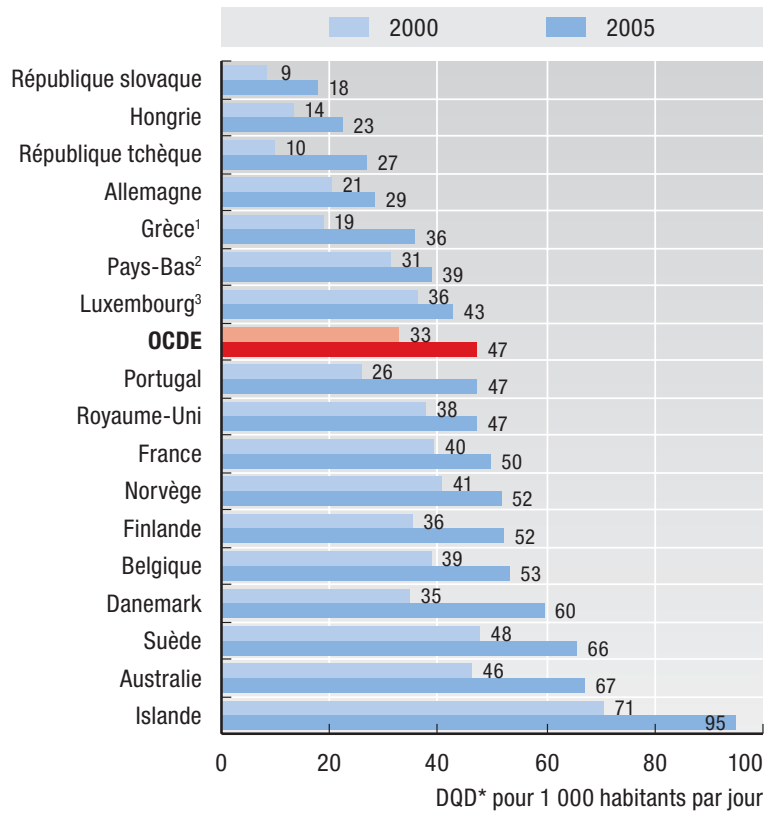

1. 2000-04. 2. 2001-05. 3. 2003-05.

\subsubsection{Consommation d'antibiotiques, $D Q D^{*}$} pour 1000 habitants par jour, 2000 et 2005

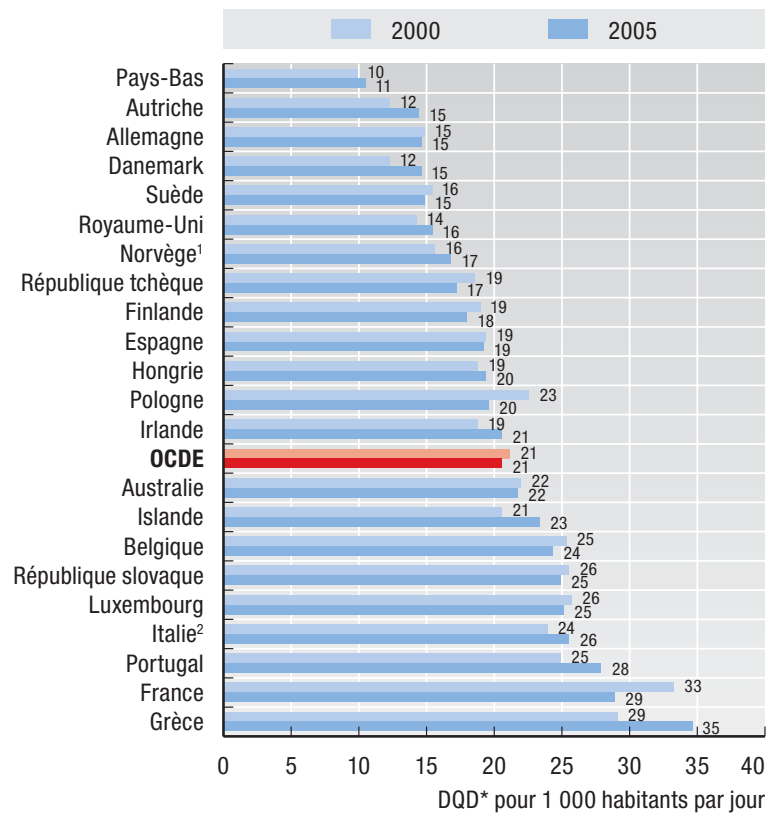

1. $2001-04$. 2. $2000-03$

StatLink Niाsस http://dx.doi.org/10.1787/130113326557 



\section{DÉPENSES DE SANTÉ ET FINANCEMENT}

5.1. DÉPENSES DE SANTÉ PAR HABITANT . . . . . . . . . . . 86

5.2. DÉPENSES DE SANTÉ PAR RAPPORT AU PRODUIT

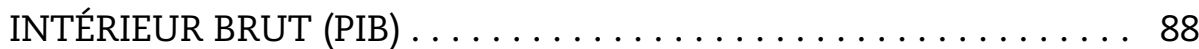

5.3. DÉPENSES DE SANTÉ PAR FONCTION. . . . . . . . . . . . 90

5.4. DÉPENSES PHARMACEUTIQUES. . . . . . . . . . . . . 92

5.5. FINANCEMENT DES DÉPENSES DE SANTÉ ............... 94

5.6. COUVERTURE DE L'ASSURANCE MALADIE

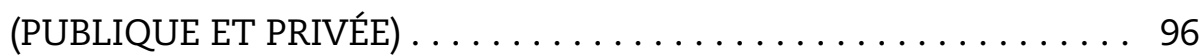




\subsection{DÉPENSES DE SANTÉ PAR HABITANT}

Le niveau des dépenses totales de santé par habitant varie considérablement d'un pays de l'OCDE à l'autre, reflétant un large éventail de facteurs sociaux et de marché mais aussi des structures différentes de financement et d'organisation du système de santé dans chaque pays.

Parmi les pays de l'OCDE qui dépensent le plus pour la santé, les États-Unis arrivent en tête avec un montant de 6401 USD PPA (voir ci-dessous l'encadré « Définition et écarts ") par habitant en 2005 (graphique 5.1.1 et tableau A.5.1), soit plus de 2.25 fois la moyenne des pays de l'OCDE. Le Luxembourg (dont les chiffres incluent les dépenses des non résidents) arrive juste derrière, suivi de la Norvège et de la Suisse avec des dépenses de santé par habitant égales environ aux deux tiers des dépenses des États-Unis mais néanmoins supérieures de plus de $50 \%$ à la moyenne de l'OCDE. Environ la moitié des pays de l'OCDE ont des dépenses comprises entre 2500 et 3500 USD PPA qui représente entre $90 \%$ et $125 \%$ de la moyenne de l'OCDE. À l'autre extrémité de l'échelle, on trouve un groupe de cinq pays (la Turquie, le Mexique, la Pologne, la République slovaque et la Corée) qui ont un niveau de dépenses de santé par habitant inférieur à la moitié de la moyenne de l'OCDE.

Le graphique 5.1.1 ventile le montant total des dépenses de santé en dépenses publiques et dépenses privées (voir également l'indicateur 5.5 « Financement des dépenses de santé "). L'ordre de grandeur de la variation des dépenses publiques de santé est analogue à celui observé pour les dépenses totales. Les pays dans lesquels la proportion des dépenses publiques est importante, comme le Danemark, la Suède et le Royaume-Uni, ont tendance à être classés plus haut en termes de dépenses publiques par habitant. Mais la proportion des dépenses publiques ne détermine pas en soi ce classement. Ainsi, bien que le système de santé américain soit principalement financé par des fonds privés, les États-Unis arrivent en troisième position, derrière la Norvège et le Luxembourg, pour le niveau de leurs dépenses publiques par habitant en 2005, qui est supérieur de plus de $40 \%$ à la moyenne de l'OCDE.

Entre 1995 et 2005, on estime que les dépenses de santé par habitant en moyenne dans les pays de l'OCDE ont augmenté d'environ $4 \%$ sur une base annuelle (graphique 5.1.2 et tableau A.5.1c). Ce chiffre est supérieur à la croissance économique moyenne de 2.5 \% sur la même période, aboutissant à une augmentation de la part du PIB consacré à la santé (voir l'indicateur 5.2 « Dépenses de santé par rapport au PIB »). Mais cette moyenne masque des disparités importantes entre les pays.

De manière générale, les pays qui ont enregistré la plus forte croissance des dépenses de santé par habitant sur cette période, comme la Corée et l'Irlande, sont ceux qui avaient au départ (dans le milieu des années 90) des niveaux relativement moins élevés. Dans ces deux pays (mais également au Luxembourg), la croissance des dépenses de santé a été près de deux fois supérieure à la moyenne de l'OCDE sur la période. En revanche, des pays comme l'Allemagne et la France ont enregistré une croissance modérée de leurs dépenses de santé par habitant de l'ordre de $2 \%$ par an entre 1995 et 2005, qui a résulté en partie des mesures de maîtrise des coûts et d'une faible croissance économique sur la période. En conséquence, en 2005, les dépenses de santé par habitant en Allemagne et en France n'était supérieure que d'environ $20 \%$ en valeur réelle aux niveaux de 1995, alors que la moyenne de l'OCDE était supérieure de près de $50 \%$.

Le graphique 5.1.3 montre les différents taux de croissance des dépenses de santé par rapport à la croissance économique globale sur la dernière décennie. Dans pratiquement tous les pays de l'OCDE (y compris l'Allemagne et la France), la croissance des dépenses de santé a été supérieure à celle de l'activité économique, entraînant une augmentation du ratio dépenses de santé par rapport au PIB (voir l'indicateur 5.2 " Dépenses de santé par rapport au PIB »).

\section{Définition et écarts}

Les dépenses totales de santé mesurent la consommation finale de produits et de services de santé (les dépenses courantes de santé) plus les dépenses d'investissement en infrastructures de soins de santé. Ce chiffre englobe les dépenses effectuées par des agents publics et privés pour les produits et services médicaux (dépenses au titre des services de santé individuels), ainsi que les dépenses liées aux programmes de santé publique et de prévention, et l'administration (dépenses au titre des services collectifs). Sont exclues les dépenses connexes comme la formation, la recherche et la santé environnementale.

Pour réaliser des comparaisons internationales, il faut convertir les dépenses de santé par habitant en une monnaie commune (le dollar américain) et les ajuster pour prendre en compte la différence de pouvoir d'achat (PPA) des monnaies nationales de chaque pays. Les PPA à l'échelle de l'économie (PIB) sont les taux de conversion les plus fiables dont on dispose. Pour plus d'informations sur la définition des dépenses de santé et les comparaisons internationales des dépenses de santé, voir l'annexe B.

Les taux de croissance indiqués dans les graphiques 5.1.2 et 5.1.3 (et les tableaux A.5.1c à A.5.1e) ont été ajustés pour prendre en compte les nombreuses ruptures de séries que présentent les séries de dépenses de santé, dues la plupart du temps aux changements méthodologiques résultant de la mise en œuvre du Système de comptes de la santé (voir l'annexe B). La révision de la frontière du secteur de la santé résulte habituellement en une hausse du niveau des dépenses de santé au moment de la mise en œuvre. Afin de supprimer cet effet, on a supposé que la croissance réelle de l'année de la rupture de série était égale à la croissance moyenne de l'année précédente et de l'année suivante. 


\subsubsection{Dépenses de santé par habitant, publiques et privées, 2005}

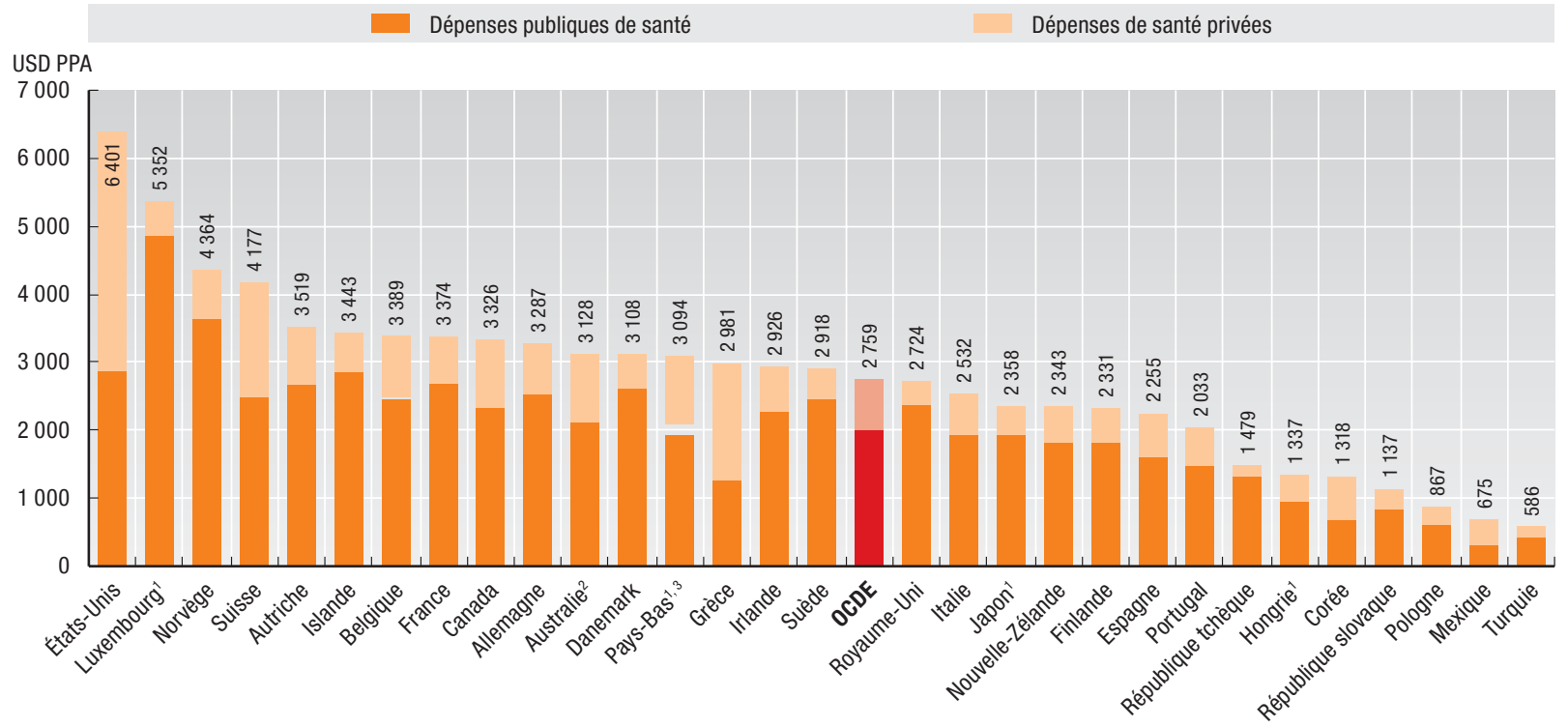

1. 2004. 2. 2004-05.

3. Les dépenses publiques et privées correspondent aux dépenses courantes (n'incluant pas les investissements).

\subsubsection{Taux de croissance annuel moyen des dépenses de santé par habitant, en termes réels, 1995-2005}

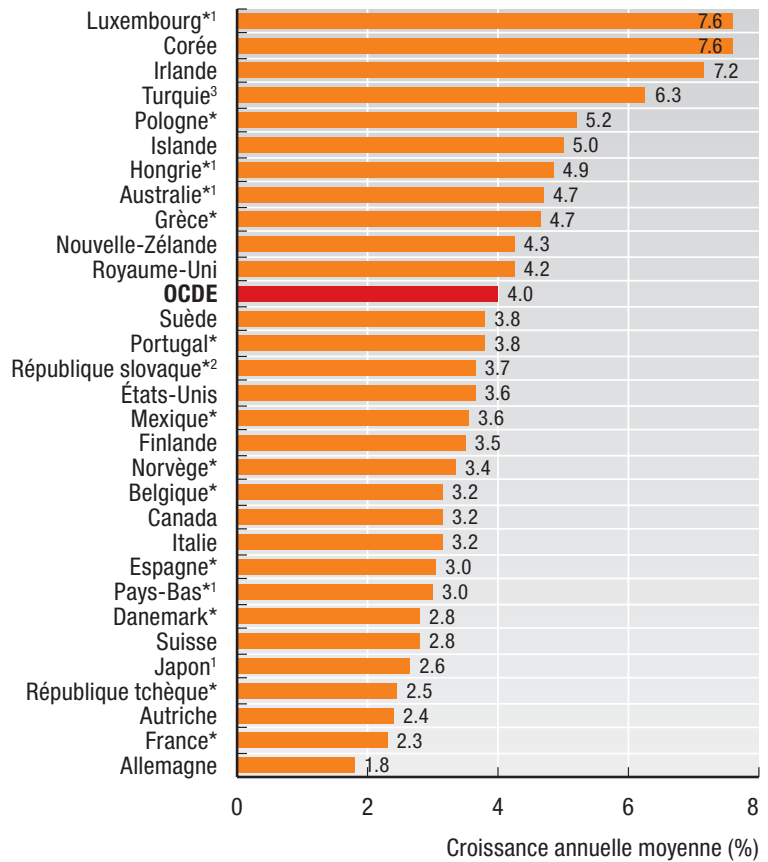

5.1.3. Croissance annuelle moyenne des dépenses de santé par habitant et du PIB, en termes réels, 1995-2005

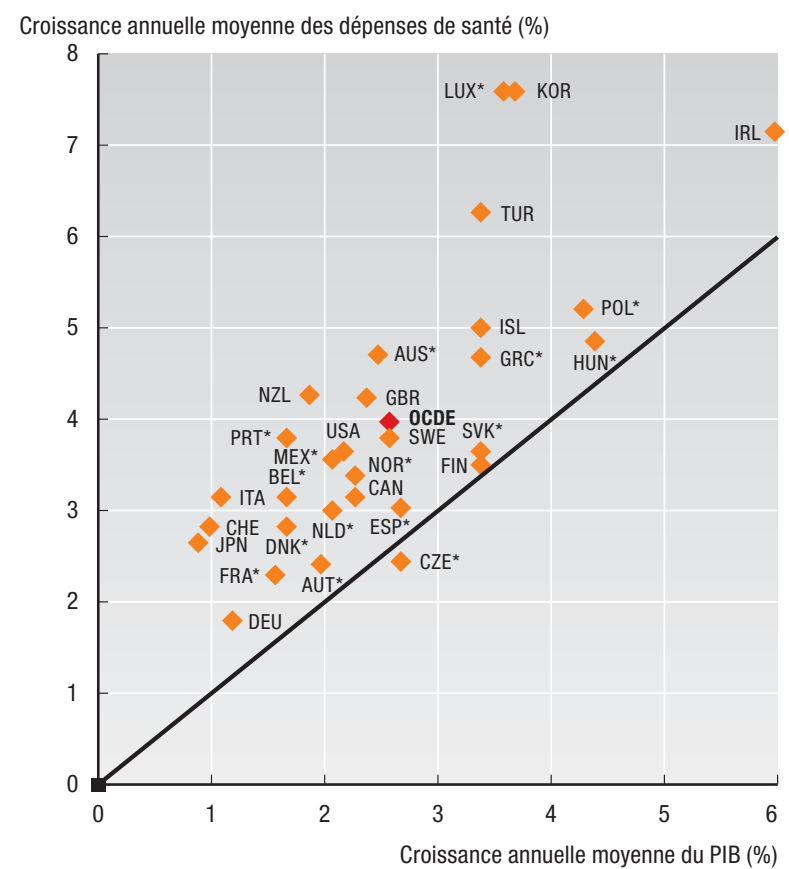

* Taux de croissance ajustés. Consulter l'encadré « Définition et écarts ".

1. 1995-2004. 2. 1997-2005. 3.1998-2005.

Source : Eco-Santé OCDE 2007. 


\subsection{DÉPENSES DE SANTÉ PAR RAPPORT AU PRODUIT INTÉRIEUR BRUT (PIB)}

En 2005 , les pays de l'OCDE ont consacré, en moyenne, $9 \%$ de leur PIB aux dépenses de santé. Cette proportion varie toutefois considérablement d'un pays à l'autre, allant d'environ $6 \%$ en Corée, en Pologne et au Mexique, à $15.3 \%$ aux États-Unis (graphique 5.2.1 et tableau A.5.2a). En 2005, huit pays ont dépensé plus de $10 \%$ de leur PIB au titre des produits et services de santé, contre quatre en 2000 et trois seulement en 1995. Concernant la part des dépenses publiques rapportée au $\mathrm{PIB}$, la différence a été pratiquement de un à trois entre les pays dans lesquels cette part a été la plus élevée et ceux dans lesquels elle a été la plus faible. En France, les dépenses publiques de santé ont représenté $8.9 \%$ du PIB en 2005 alors qu'en Corée, où les dépenses de santé se ventilent de manière égale entre financement public et financement privé, elles ont représenté 3.2\% du PIB.

Pour faire une évaluation plus exhaustive des dépenses de santé d'un pays, il faut considérer ensemble le ratio dépenses de santé/PIB et les dépenses de santé par habitant (voir l'indicateur 5.1 " Dépenses de santé par habitant "). Des pays ayant un ratio dépenses de santé/PIB relativement élevé peuvent avoir des dépenses de santé par habitant relativement faibles et inversement des pays ayant un ratio dépenses de santé/PIB relativement faible peuvent avoir des dépenses de santé par habitant relativement élevées. L'Autriche et le Portugal, par exemple, ont consacré à la santé un peu plus de $10 \%$ de leur PIB mais les dépenses de santé par habitant de l'Autriche (en USD PPA) sont supérieures de plus de $70 \%$. De même, la Grèce et l'Irlande ont affiché, en 2005, des dépenses de santé par habitant analogues mais en Grèce ces dépenses ont représenté plus de $10 \%$ du PIB alors qu'en Irlande elles n'ont représenté que $7.5 \%$ du PIB (graphique 5.2.1).

L'évolution dans le temps du ratio dépenses de santé/PIB reflète l'effet conjugué de l'évolution du PIB et des dépenses de santé (graphique 5.2.3). Sur les dix dernières années, pratiquement tous les pays de l'OCDE ont enregistré une augmentation de la proportion de leur revenu national consacrée à la santé. Aux États-Unis, au Canada et en Suisse, la croissance des dépenses de santé a été nettement supérieure à la croissance économique globale entre 2000 et 2003. En revanche, l'accroissement sur les dix dernières années de la part du PIB consacrée à la santé a été plus modeste en Allemagne et au Japon où une croissance faible des dépenses de santé a été associée à une croissance économique globale faible.

Le graphique 5.2.4 montre l'association positive entre le PIB par habitant et les dépenses de santé par habitant dans les différents pays de l'OCDE. Si, globalement, les pays dont le PIB est plus élevé tendent à dépenser plus pour la santé, on observe entre eux une grande disparité, reflétant le fait que le PIB n'est pas le seul facteur influençant le niveau des dépenses de santé. Cette association est plus forte parmi les pays de l'OCDE à revenu faible que parmi les pays à revenu élevé. Pour des pays ayant des niveaux de revenus analogues, on observe des dépenses de santé très différentes pour un niveau donné de PIB. Par exemple, en dépit d'un PIB par habitant identique, le Japon et l'Allemagne ont des niveaux très différents de dépenses de santé par habitant, le Japon consacrant à la santé moins de $75 \%$ des sommes que lui consacre l'Allemagne.

Les dépenses totales de santé mesurent la consommation finale de produits et de services de santé plus les dépenses d'investissement en infrastructures de soins de santé. Une autre mesure consiste à présenter la part des produits et des services de santé (c'est-à-dire les dépenses courantes de santé, excluant les investissements) par rapport à tous les produits et services consommés par ou pour le compte des particuliers. Ce ratio est supérieur au ratio dépenses de santé/PIB pour tous les pays de l'OCDE (graphique 5.2.2). La part moyenne de la consommation finale effective affectée à la santé dans les pays de l'OCDE est de près de $13 \%$, et près des deux tiers des pays de l'OCDE consacrent à la santé plus de $12 \%$ de leur consommation finale.

\section{Définition et écarts}

Par définition, $\mathrm{PIB}=$ consommation finale + formation brute de capital + exportations nettes. La consommation finale effective des ménages englobe les produits et les services utilisés par les ménages pour satisfaire leurs besoins individuels ou par la collectivité pour satisfaire les besoins sociaux. (Les dépenses de consommation finale effective englobent les dépenses liées à la consommation finale des ménages, de l'administration générale et des institutions à but non lucratif au service des ménages.)

Les différences de positions relatives des pays entre le ratio dépenses totales de santé/PIB et celui dépenses courantes de santé/dépenses de consommation finale effective sont dues à des niveaux différents d'investissement (dans l'ensemble de l'économie et dans le secteur de la santé) et à des différences de solde des échanges extérieurs entre les pays. Cet écart est particulièrement prononcé pour des pays comme le Luxembourg et l'Irlande. 


\subsection{DÉPENSES DE SANTÉ PAR RAPPORT AU PRODUIT INTÉRIEUR BRUT (PIB)}

\subsubsection{Dépenses de santé totales en pourcentage} du PIB, 2005

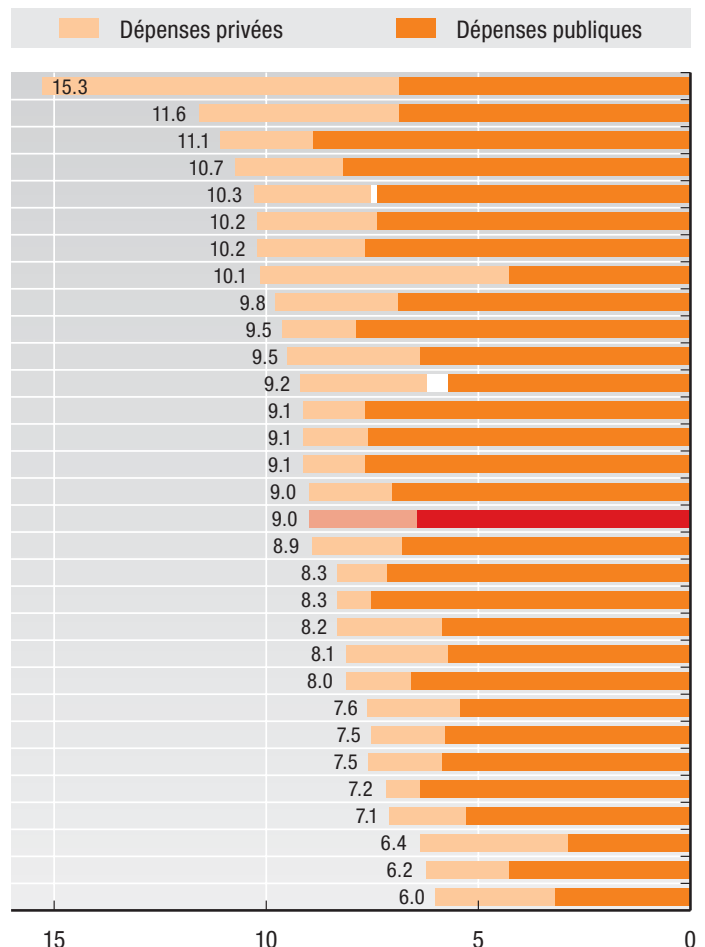

$\%$ PIB

1. 2004. 2. 2004-05

3. Dépenses de santé totales pour les deux graphiques.

4. Les dépenses publiques et privées sont des dépenses courantes (non compris les investissements)
5.2.3. Dépenses de santé totales en pourcentage du PIB, pour quelques pays de l'OCDE, 1995-2005

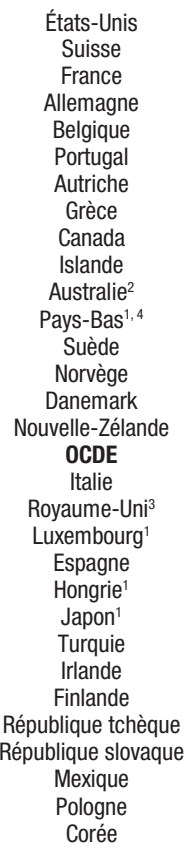

Coree

\subsubsection{Dépenses de santé courantes en pourcentage de la consommation finale effective, 2005}

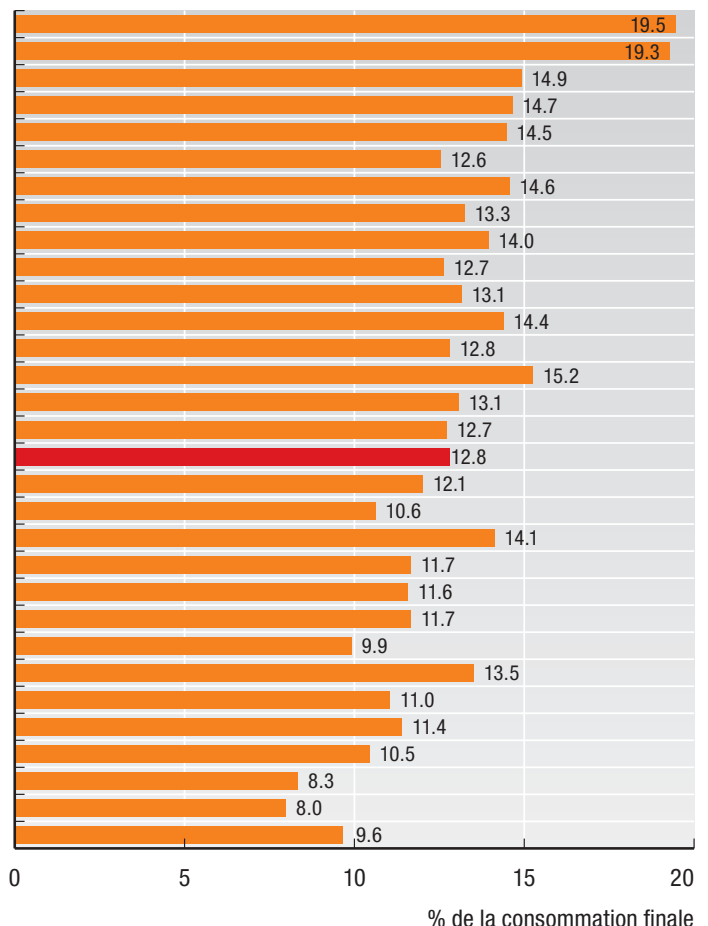

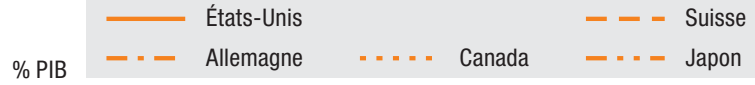

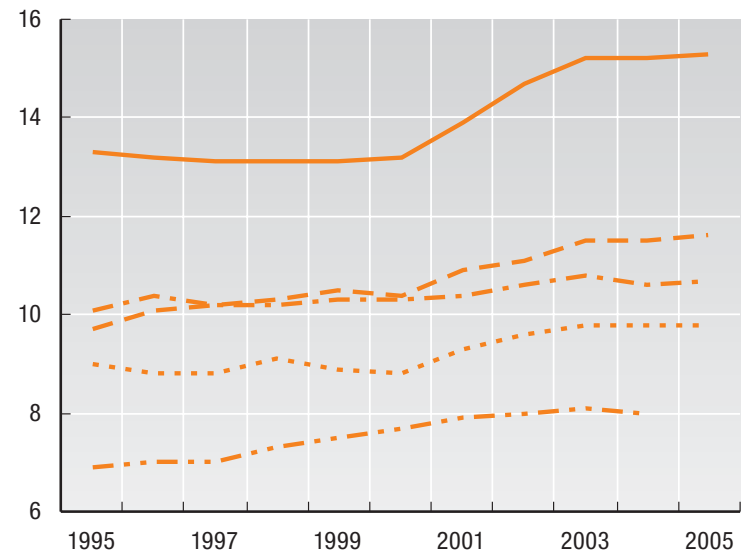

5.2.4. Dépenses de santé par habitant et PIB par habitant, 2005

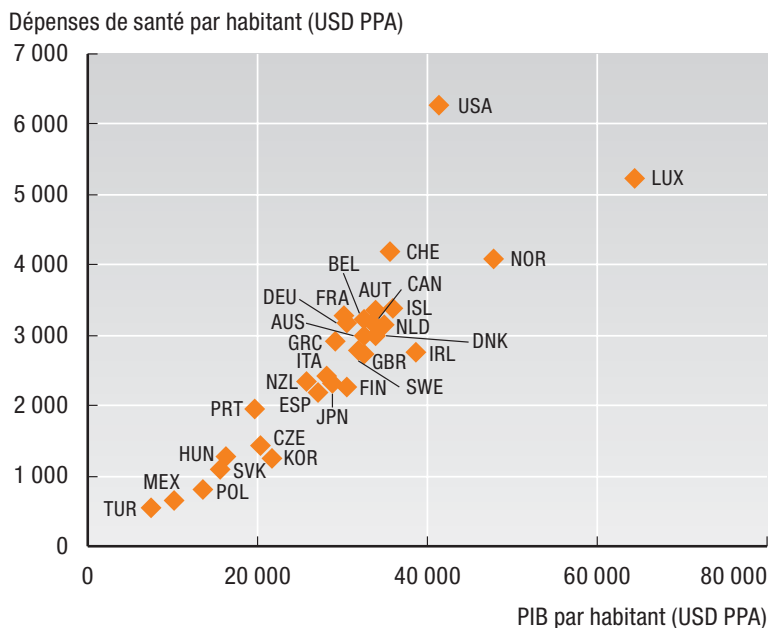

Source : Eco-Santé OCDE 2007. 


\subsection{DÉPENSES DE SANTÉ PAR FONCTION}

La part des dépenses de santé affectée aux différents types de services et de produits médicaux varie considérablement d'un pays de l'OCDE à l'autre. Elle est influencée par un certain nombre de facteurs tels que des différences de capacité (nombre de lits d'hôpitaux et de médecins), d'incitations financières pour les prestataires et de nature des maladies.

En 2005, les services médicaux personnels, qui couvrent les soins curatifs et de rééducation, les soins de longue durée et les services auxiliaires (aux patients externes) ont représenté, en moyenne, plus de $70 \%$ des dépenses courantes de santé des pays de l'OCDE (graphique 5.3.1 et tableau A.5.3). Cette part a oscillé entre 50-60\% dans les pays d'Europe centrale et orientale et plus de $80 \%$ en Norvège, au Danemark, en Islande et en Suisse. Les soins curatifs et de rééducation, qui constituent l'essentiel de ces services, ont représenté en moyenne $57 \%$ des dépenses de santé dans les pays de l'OCDE. La part des soins de longue durée a été de $11 \%$ en moyenne et celle des services auxiliaires (englobant notamment les examens de laboratoire et l'imagerie de diagnostic) a été de $4 \%$. Le solde, soit un peu plus de $20 \%$, a été dépensé au titre des produits médicaux (voir l'indicateur 5.4 « Dépenses pharmaceutiques") s'échelonnant entre un minimum de 11 à $14 \%$ au Luxembourg, aux États-Unis et en Suisse, à un maximum de l'ordre de $35-40 \%$ des dépenses totales de santé en Hongrie et en République slovaque. Les " dépenses collectives ", qui couvrent les dépenses au titre de la santé publique et de la prévention et aussi les dépenses de l'administration de la santé et de l'assurance maladie, ont représenté les $7 \%$ restants.

Les soins curatifs et de rééducation recouvrent les services médicaux fournis non seulement dans le cadre d'une hospitalisation mais également dans le cadre de l'hôpital de jour, des services de consultations externes des hôpitaux, dans le secteur ambulatoire ou au domicile des patients. L'évolution des pratiques médicales et les innovations en termes de technologies médicales sont autant d'éléments qui peuvent affecter l'équilibre entre ces différents types d'offre de soins. Par exemple, on a observé pour certains services de santé une évolution consistant à dispenser en ambulatoire et à domicile des soins qui étaient jusque là dispensés à des patients hospitalisés (graphique 5.3.2). Un certain nombre de pays sont toujours incapables de quantifier séparément les dépenses au titre des soins de jour dispensés à l'hôpital ou dans d'autres établissements, et les englobent dans les soins aux patients hospitalisés; lorsqu'ils sont indiqués, ces soins peuvent représenter jusqu'à 6 ou $7 \%$ des soins curatifs et de rééducation, comme cela est le cas au Canada et en Norvège, par exemple. Les services aux patients externes dispensés dans les hôpitaux et dans le secteur ambulatoire représentent en moyenne plus d'un tiers des dépenses au titre des soins curatifs dans les pays de l'OCDE et les soins dentaires représentent $10 \%$ (plus de $15 \%$ en Hongrie et au Canada) des dépenses totales au titre des soins curatifs. Enfin, les soins à domicile représentent 1 à $2 \%$ des soins curatifs, bien qu'il soit souvent difficile de distinguer ces soins des soins de longue durée dispensés sur le lieu de résidence des patients.

Le graphique 5.3.3 montre la part des dépenses publiques affectée aux activités de santé publique et de prévention. En moyenne, les pays de l'OCDE ont affecté à peine plus de $3 \%$ de leurs dépenses publiques de santé à un large éventail d'activités tels que des programmes de vaccination et des campagnes de santé publique sur l'abus d'alcool et de tabac. La grande disparité reflète, dans une large mesure, l'organisation nationale des campagnes de prévention. Lorsque ces initiatives sont menées au niveau des soins primaires, comme c'est le cas en Espagne, la fonction de prévention n'est pas saisie séparément et elle est plus généralement incluse dans les dépenses au titre des soins curatifs. D'autres pays adoptant une approche plus centralisée des campagnes de santé publique et de prévention peuvent mieux identifier les dépenses au titre de ces programmes.

\section{Définition et écarts}

Le Système de comptes de la santé propose une approche fonctionnelle cohérente pour définir les frontières du système de santé et examiner l'affectation des ressources. Suivant ce système, les dépenses totales de santé englobent les dépenses courantes de santé et l'investissement. Les dépenses courantes de santé englobent les services personnels de santé et les produits fournis directement aux individus ainsi que les services collectifs, couvrant les missions de santé publique comme les services de promotion de la santé et de prévention des maladies et l'administration de la santé. Les services personnels de santé englobent les services de soins curatifs, de rééducation et les soins de longue durée, les services auxiliaires aux soins de santé et les produits médicaux fournis aux malades externes. Les fonctions de base des soins (curatifs, de rééducation, de longue durée) peuvent également être classées par mode de production (soins des personnes hospitalisées, soins ambulatoires et soins à domicile).

Le principal facteur limitant la comparabilité des dépenses de santé par fonction est le mode différent d'estimation des dépenses liées aux soins de longue durée. Un autre problème important est le fait que dans certains pays les dépenses liées aux soins des personnes hospitalisées demeurent rattachées aux dépenses des hôpitaux (c'est-à-dire qu'elles englobent d'autres services tels que les soins ambulatoires dispensés à l'hôpital). Pour des raisons analogues, les services auxiliaires peuvent être englobés dans les dépenses liées aux soins des personnes hospitalisées ou dans les dépenses liées aux soins ambulatoires. (Pour une discussion plus détaillée des questions méthodologiques, voir Orosz et Morgan, 2004.) 


\subsection{DÉPENSES DE SANTÉ PAR FONCTION}

\subsubsection{Dépenses courantes de santé par fonction, 2005}

Les pays sont classés selon la part des services médicaux dans les dépenses courantes de santé

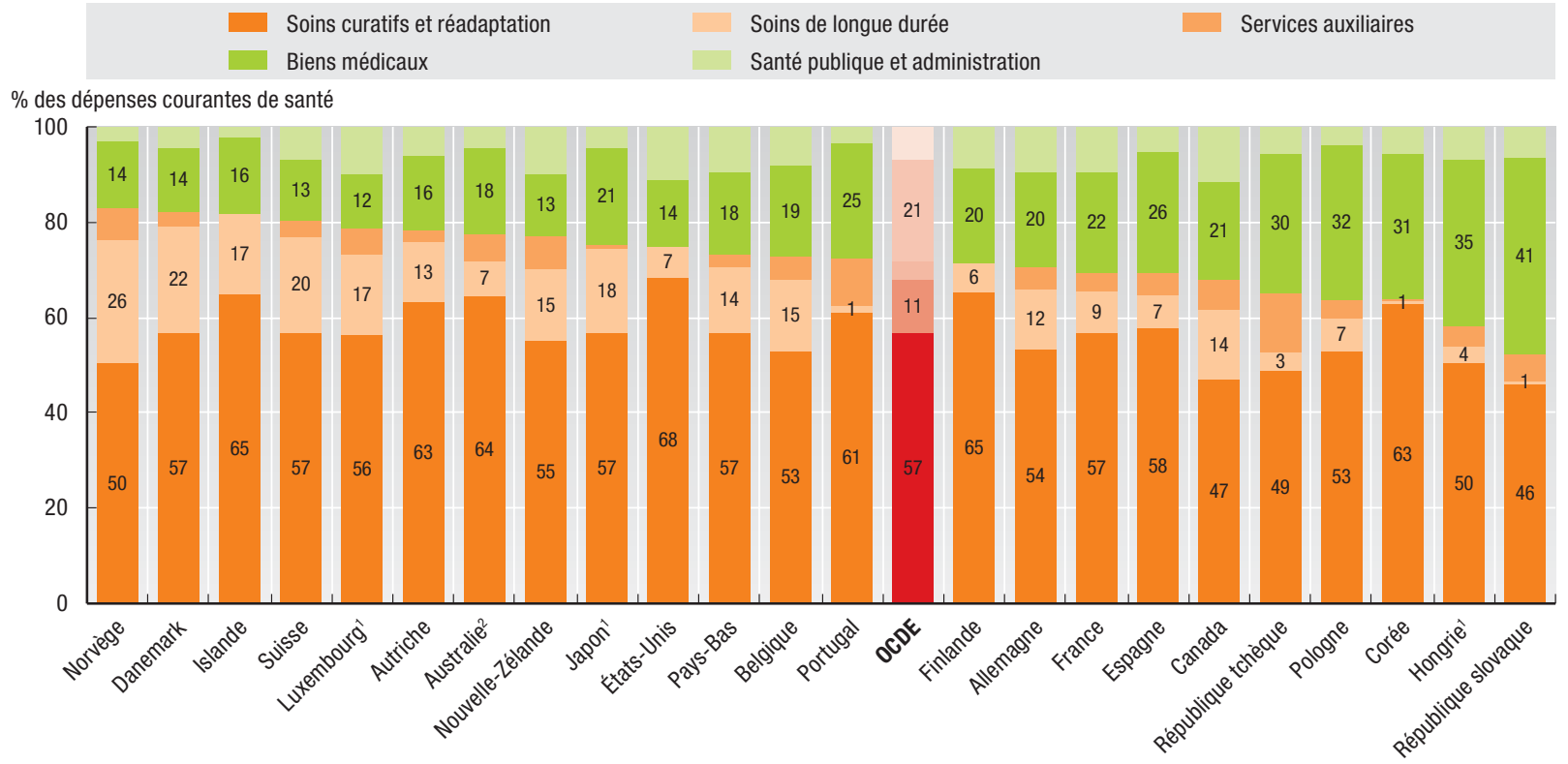

1. 2004 . 2. $2004-05$.

\subsubsection{Dépenses de soins curatifs et de réadaptation par mode de production, 2005}

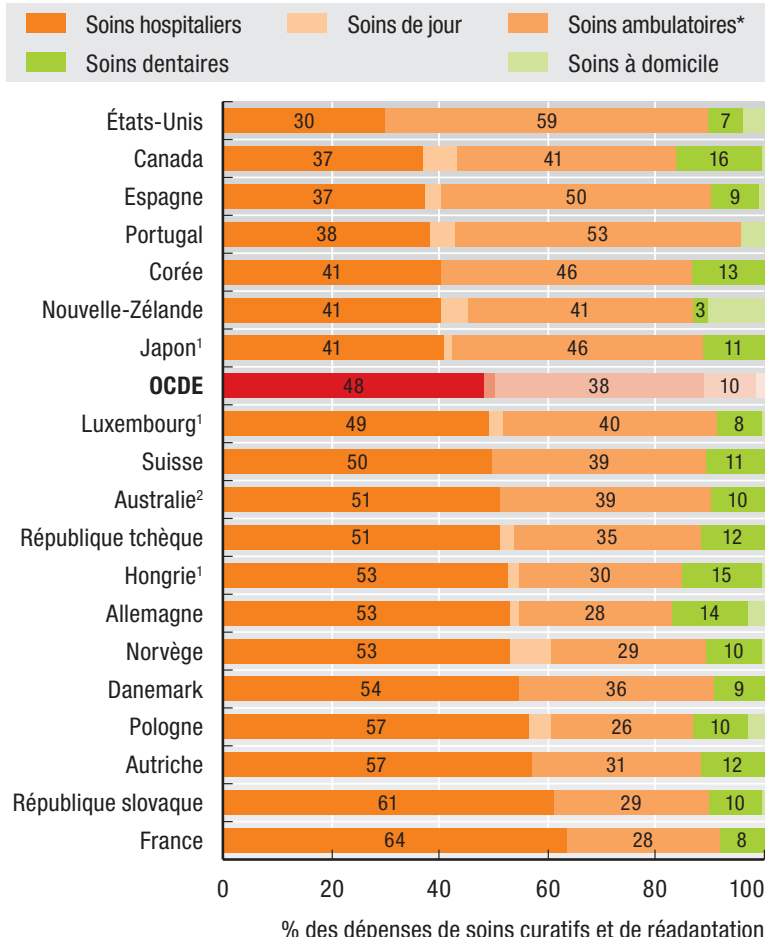

* Excluant les soins dentaires.

1. 2004. 2. 2004-05.

Source : Eco-Santé OCDE 2007.

\subsubsection{Part des dépenses publiques allouées} à la santé publique et la prévention, 2005

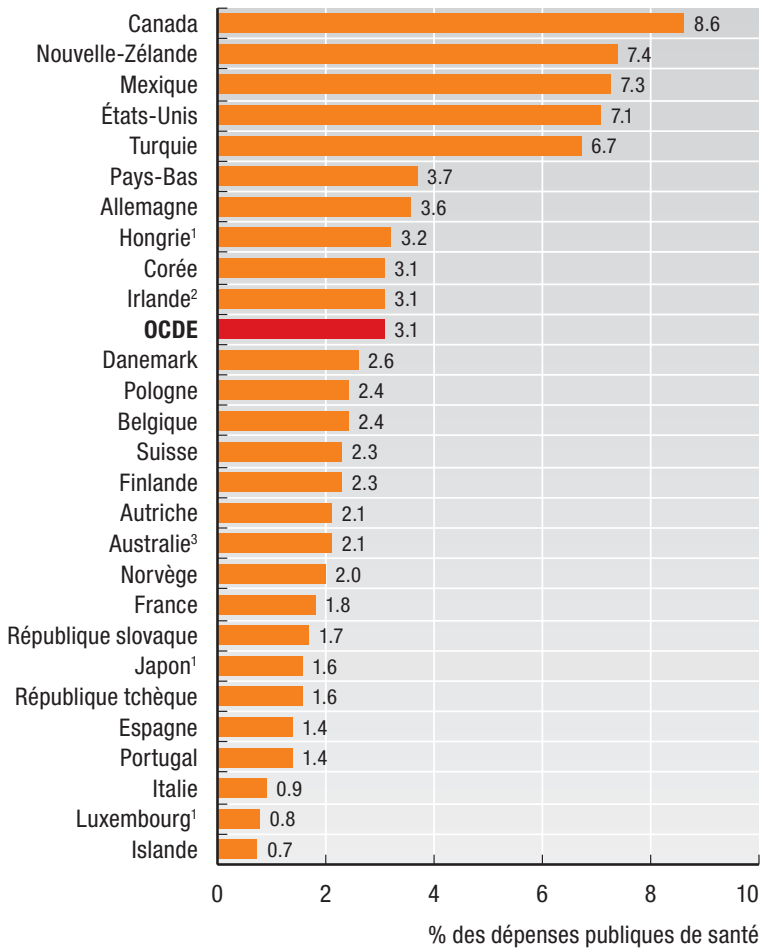

1. 2004 . 2. 2003 . 3. $2004-05$. 


\subsection{DÉPENSES PHARMACEUTIQUES}

Dans la plupart des pays de l'OCDE, les dépenses au titre des produits médicaux, et en particulier des produits pharmaceutiques, ont enregistré une progression rapide et représentent une part de plus en plus importante des dépenses totales de santé. La consommation accrue de médicaments due au vieillissement démographique, à la mise sur le marché et à la diffusion de nouveaux médicaments (voir l'indicateur 4.15 "Consommation de médicaments ") a été, ces dernières années, l'une des principales causes de l'augmentation des dépenses pharmaceutiques et donc des dépenses globales de santé. Toutefois, la relation est complexe en ce sens qu'une augmentation accrue des dépenses pharmaceutiques pour soigner des maladies qui autrement nécessiteraient une hospitalisation et une intervention coûteuses, peut conduire à une réduction des dépenses globales de santé.

On estime qu'en 2005, le montant des dépenses pharmaceutiques de l'ensemble des pays de l'OCDE a été supérieur à 550 milliards d'USD. En moyenne, le montant des dépenses pharmaceutiques par habitant a augmenté de plus de $50 \%$, en valeur réelle, depuis 1995. On observe toutefois dans la zone OCDE des différences considérables de niveaux de dépenses pharmaceutiques entre les pays, qui reflètent des différences de volume, de structure de la consommation et de niveau de prix (graphique 5.4.1 et tableau A.5.4). En 2005, les États-Unis ont eu la consommation de médicaments par habitant la plus forte du monde : ils ont dépensé $86 \%$ de plus que la moyenne de l'OCDE, devant le Canada, la France, l'Espagne et l'Italie. À l'autre extrémité de l'échelle, le Mexique n'a dépensé qu'environ un tiers et la Pologne et le Danemark environ $60 \%$ de la moyenne de l'OCDE. Les produits pharmaceutiques consomment en moyenne environ $17 \%$ des dépenses globales de santé. Rapportée au PIB, la moyenne des dépenses allouées aux médicaments dans les pays de l'OCDE a été de $1.5 \%$, s'échelonnant entre moins de $1 \%$ dans des pays comme la Norvège, le Danemark et l'Irlande et plus de $2 \%$ au Portugal, en République slovaque et en Hongrie (graphique 5.4.2 et tableau A.5.4b).

Depuis 1995, la progression des dépenses pharmaceutiques, en valeur réelle, a été en moyenne de $4.6 \%$ par an, ce qui est supérieur au chiffre de $4 \%$ enregistré pour l'augmentation annuelle des dépenses globales totales de santé sur la période (graphique 5.4.3). De fait, la plupart des pays de l'OCDE ont enregistré une progression de leurs dépenses pharmaceutiques supérieure à celle de leurs dépenses totales de santé sur la période. Parmi les plus gros consommateurs actuels de produits pharmaceutiques, les États-Unis, le Canada et l'Espagne ont enregistré une augmentation de leurs dépenses pharmaceutiques sensiblement supérieure à la moyenne des pays de l'OCDE, même si aux États-Unis la progression des dépenses a ralenti au cours des dernières années. Sur les cinq à dix dernières années, les dépenses pharmaceutiques ont également fortement augmenté en Hongrie et en République slovaque. Bien que d'autres gros consommateurs de médicaments, comme la France et l'Allemagne, aient enregistré une progression de leurs dépenses pharmaceutiques inférieure à la moyenne de l'OCDE, cette progression a été néanmoins bien supérieure à la progression globale des dépenses de santé.

En moyenne dans les pays de l'OCDE, $60 \%$ des dépenses pharmaceutiques sont financées sur fonds publics (graphique 5.4.1), le financement des $40 \%$ restants provenant des paiements directs et, dans une moindre mesure, de l'assurance maladie privée. Toutefois, cette moyenne masque de très grandes disparités, allant de $11 \%$ au Mexique et $24 \%$ aux États-Unis, contre plus de $80 \%$ en Irlande et au Luxembourg (voir l'indicateur 5.5 "Financement des dépenses de santé »).

\section{Définition et écarts}

Les dépenses pharmaceutiques incluent les dépenses liées à l'achat de médicaments délivrés sur ordonnance et à l'automédication, souvent désignée sous le terme d'achat de médicaments en vente libre, mais aussi à l'achat d'autres produits médicaux non durables. Elles incluent également la rémunération des pharmaciens lorsque celle-ci est séparée du prix des médicaments. Les produits pharmaceutiques consommés dans les hôpitaux sont exclus. Les dépenses finales au titre des produits pharmaceutiques incluent les marges des grossistes et des détaillants et la taxe sur la valeur ajoutée. 


\subsubsection{Dépenses de produits pharmaceutiques par habitant, 2005}

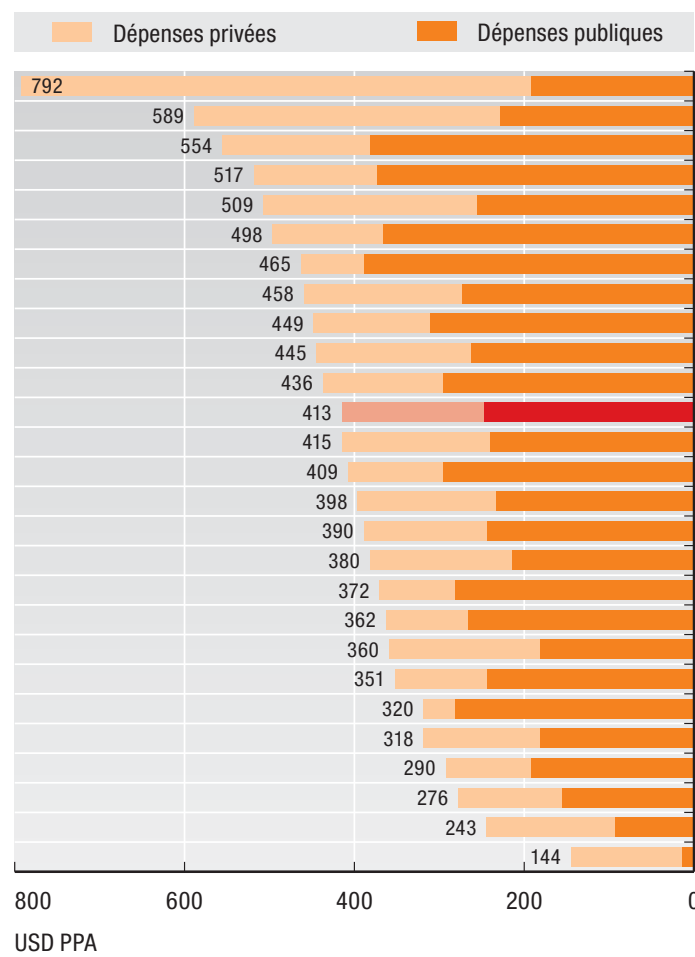

\subsection{DÉPENSES PHARMACEUTIQUES}

\subsubsection{Dépenses de produits pharmaceutiques en pourcentage du PIB, 2005}

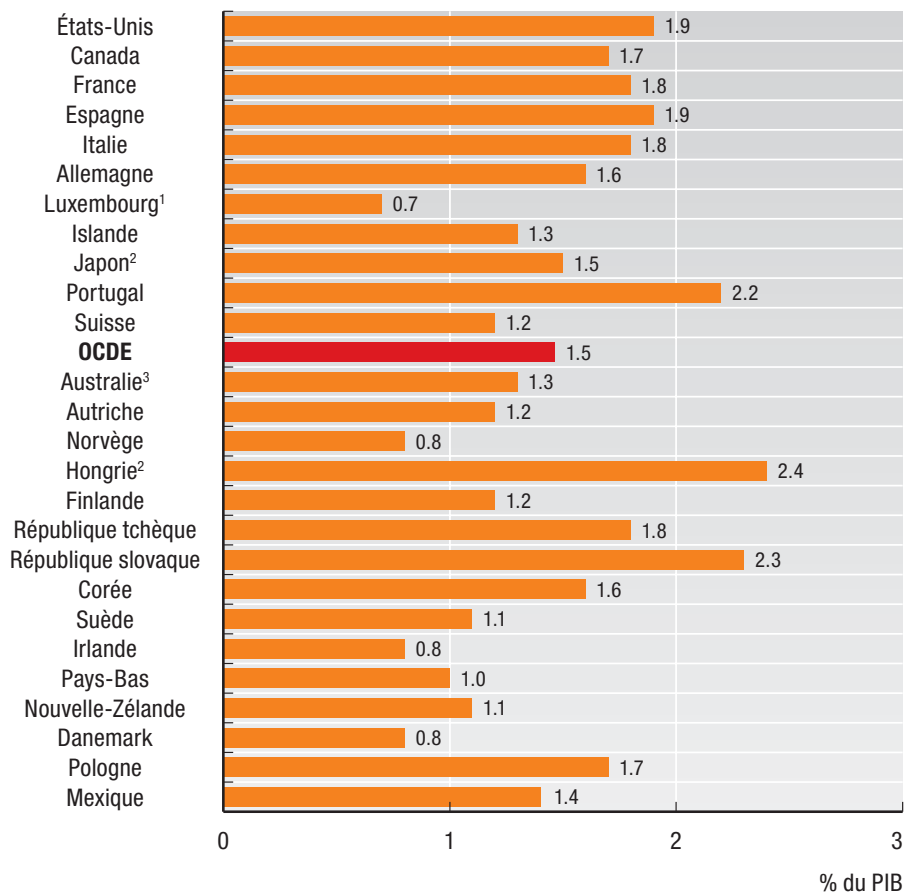

1. Les données ne concernent que les médicaments prescrits.

2. 2004. 3. 2004-05.

\subsubsection{Croissance annuelle réelle des dépenses pharmaceutiques et des dépenses totales de santé par habitant, 1995-2005}

Taux de croissance annuel moyen (\%)

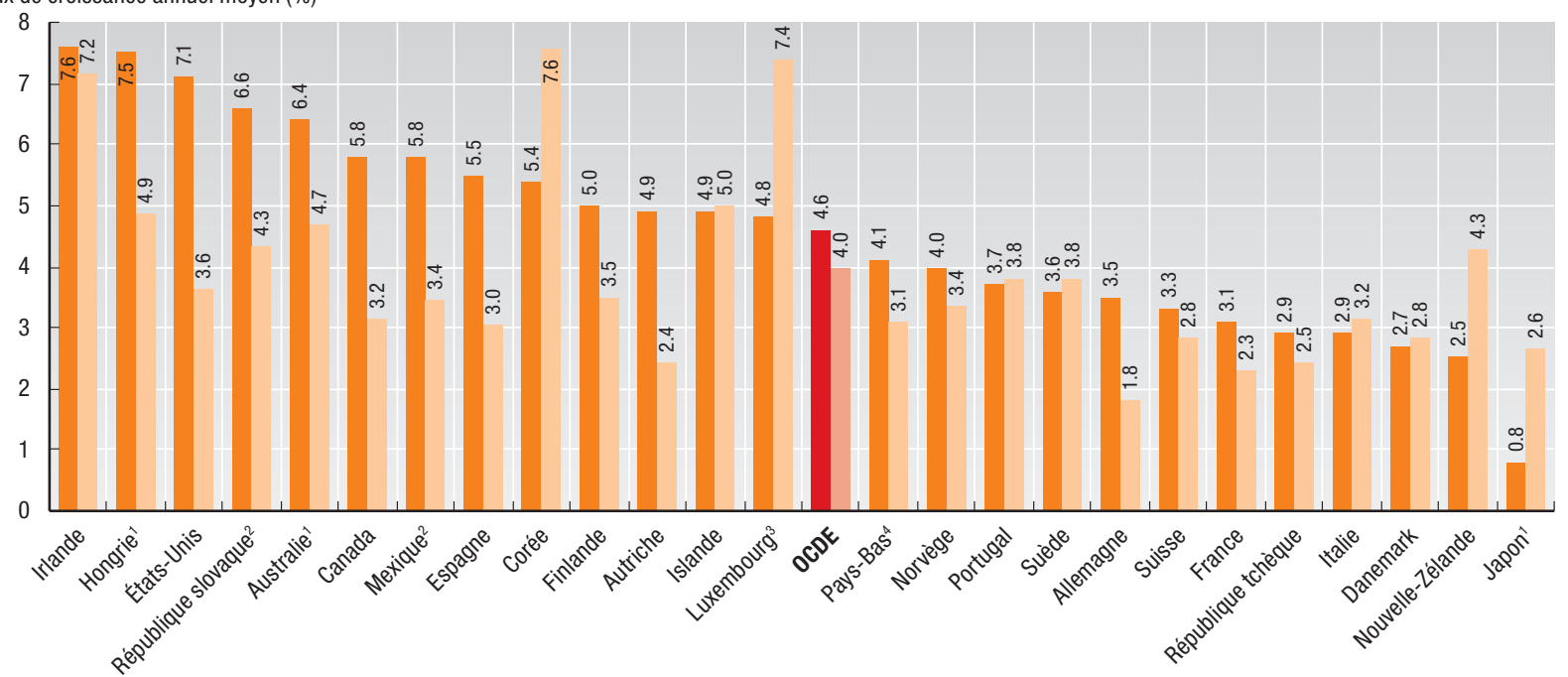

1. $1995-2004$. 2. $1999-2005$. 3. $1995-2003$. 4. 1995-2002.

Source : Eco-Santé OCDE 2007. 


\subsection{FINANCEMENT DES DÉPENSES DE SANTÉ}

Des méthodes différentes de financement des soins de santé peuvent affecter le niveau et la répartition des dépenses de santé, et l'accès des populations aux services de santé. Dans les pays de l'OCDE, les soins de santé sont financés à la fois par des sources publiques et des sources privées. Le financement public peut se limiter aux recettes publiques dans les pays où la responsabilité directe du financement des services de santé incombe au gouvernement central et/ou aux administrations locales (en Espagne et en Norvège, par exemple), ou regrouper les recettes publiques générales et les cotisations sociales dans les pays où le financement repose sur un système d'assurance sociale (en France et en Allemagne, par exemple). Le financement privé englobe les paiements directs des ménages, les mécanismes de tiers-payant qui peuvent prendre des formes diverses d'assurance maladie privée (financée par les employeurs et subventionnée dans certains pays), les services de santé directement assurés par l'employeur (comme la médecine du travail), et autres prestations directes émanant d'organismes caritatifs et assimilés.

Le graphique 5.5.1 montre la part du financement public de la santé dans les différents pays de l'OCDE, en 2005. Le secteur public a continué à être la principale source de financement de la santé dans tous les pays de l'OCDE excepté le Mexique, les États-Unis et la Grèce. En moyenne, la part du secteur public dans les dépenses de santé a été de $73 \%$ en 2005. Au Luxembourg, en République tchèque, dans de nombreux pays nordiques, au Royaume-Uni et au Japon, le financement public a représenté plus de $80 \%$ de toutes les dépenses de santé. De manière générale, il y a eu une certaine convergence des taux de prise en charge publique des dépenses de santé parmi les pays de l'OCDE au cours des dernières décennies. De nombreux pays (la Pologne et la Hongrie, par exemple) dans lesquels la part des dépenses publiques était relativement élevée au début des années 90 , ont enregistré une diminution de cette part, tandis que d'autres (le Portugal et la Turquie, par exemple) qui avaient un niveau relativement faible de dépenses publiques, ont enregistré une augmentation de cette part, reflétant à la fois les réformes des systèmes de santé et l'élargissement de la couverture publique.

Le fait que, pour la plupart des pays, l'ensemble des dépenses de santé soit principalement financé sur fonds publics n'implique pas que le secteur public soit l'acteur dominant dans chacun des domaines des soins de santé. Le graphique 5.5.2 présente la part publique du financement séparément pour les services médicaux et pour les produits médicaux. Dans la plupart des pays, le secteur public continue à jouer un rôle dominant dans le paiement des services médicaux, bien qu'une nouvelle subdivision des services médicaux montre le rôle de plus en plus important du financement privé dans le secteur des services aux patients non hospitalisés (Orosz et Morgan, 2004). Par ailleurs, dans le financement des produits médicaux (principalement constitués par les produits pharmaceutiques), les paiements privés sont plus importants et représentent en fait une part bien supérieure à celle des paiements publics dans un certain nombre de pays dont le Mexique, le Canada et les États-Unis.

L'importance et la composition du financement privé pour les produits et services de santé diffèrent considérablement d'un pays à l'autre (Colombo et Morgan, 2006). En moyenne, les paiements directs (y compris la part des ménages dans un dispositif de participation aux coûts) représentent environ les deux tiers des $27 \%$ des paiements privés pour la santé. Dans certains pays d'Europe centrale et orientale, le niveau des paiements directs est probablement sous-estimé en raison de la pratique des paiements complémentaires non officiels. Alors que dans les pays de l'OCDE l'assurance maladie privée ne représente en moyenne que 6 à $7 \%$ des dépenses totales de santé (graphique 5.5.3), dans certains pays elle finance une partie importante de ces dépenses. Elle assure une couverture de base à certaines catégories de population en Allemagne et à une proportion importante de la population autre que les personnes âgées aux États-Unis où l'assurance maladie privée représente $37 \%$ des dépenses de santé. Dans des pays comme la France et le Canada, l'assurance maladie privée finance 12 à $13 \%$ des dépenses globales mais fournit respectivement une couverture complémentaire et supplémentaire dans un régime public de portée universelle (voir l'indicateur 5.6 "Couverture de l'assurance maladie »). En général, dans les pays de l'OCDE, il n'existe pas de schéma de substitution clairement établi entre les paiements directs et le financement par une assurance maladie privée (OCDE, 2004c; Colombo et Tapay, 2004).

L'impact global des dépenses financées directement par les ménages peut également être mesuré par la part de ces dépenses dans la consommation finale des ménages. Dans plusieurs pays, dont les Pays-Bas et la France, les services de santé financés directement par les patients ont représenté, en 2005, moins de $2 \%$ de la consommation totale des ménages tandis qu'en Suisse et en Grèce ces dépenses ont représenté plus de $6 \%$ de la consommation totale des ménages. Les États-Unis, qui consacrent près de $3 \%$ de leur consommation au financement direct des services de santé par les ménages, sont proches de la moyenne.

\section{Définition et écarts}

Le financement des soins de santé comporte trois éléments importants qui sont les sources ultimes de financement (les ménages, les employeurs et l'État), les accords de financement (par exemple l'assurance obligatoire ou l'assurance volontaire, etc.) et les agents de financement (organismes gérant les régimes de financement). Le terme "Financement " désigne ici les mécanismes de financement tels qu'ils sont définis dans le Système de comptes de la santé. Les sources publiques incluent les recettes publiques générales et les fonds de la sécurité sociale. Les sources privées recouvrent les paiements directs des ménages, les assurances-maladie privées et autres fonds privés tels que les fonds des organisations non gouvernementales, et le financement de la médecine du travail par les entreprises privées.

Les paiements directs sont les dépenses supportées directement par un patient ne bénéficiant d'aucune assurance. Ils incluent la participation aux frais et, dans certains pays, les estimations des paiements non officiels aux prestataires de soins. 


\subsubsection{Part publique des dépenses totales de santé, 2005}

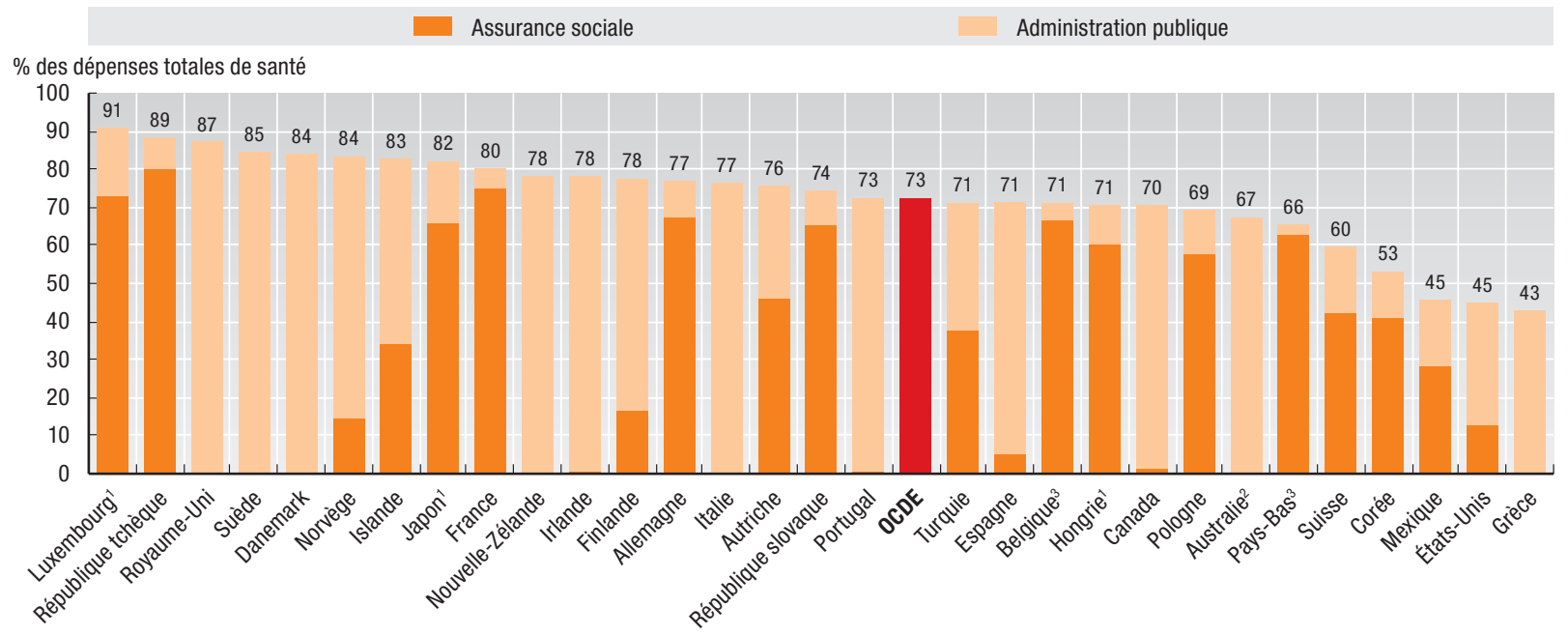

1. 2004. 2. 2004-05.

3. Part des dépenses courantes (c'est-à-dire excluant les investissements).

\subsubsection{Part publique des dépenses de services médicaux \\ et produits pharmaceutiques, 2005}
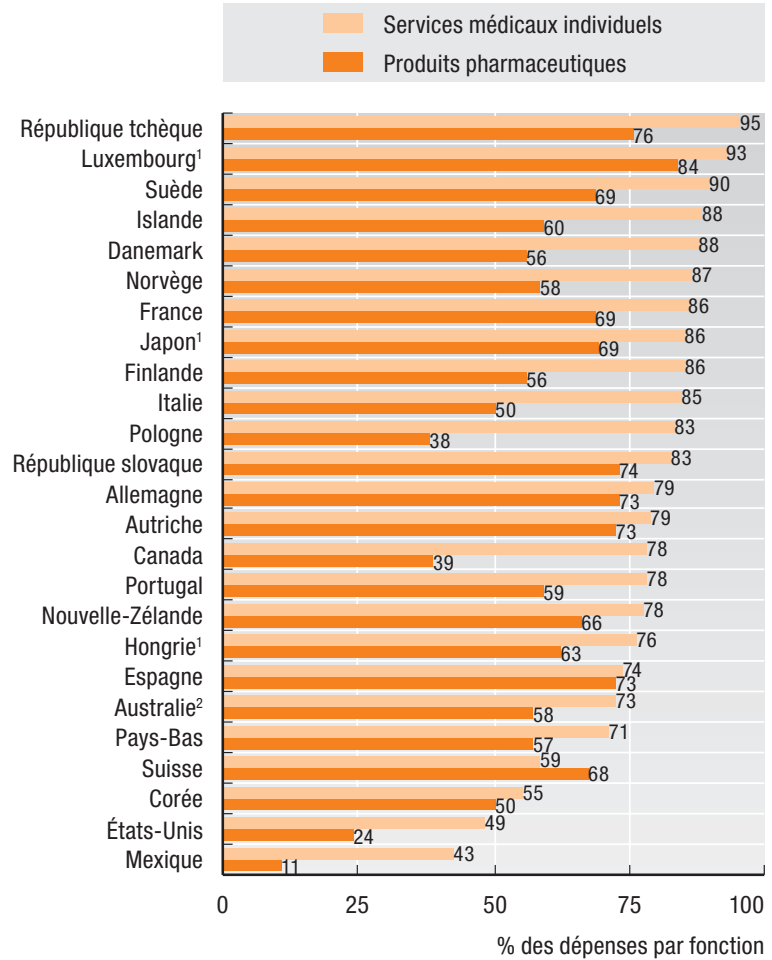

1. 2004 . 2. $2004-05$

Source : Eco-Santé OCDE 2007.

\subsubsection{Versements nets des ménages et dépenses d'assurance maladie privée en pourcentage des dépenses totales de santé, 2005}

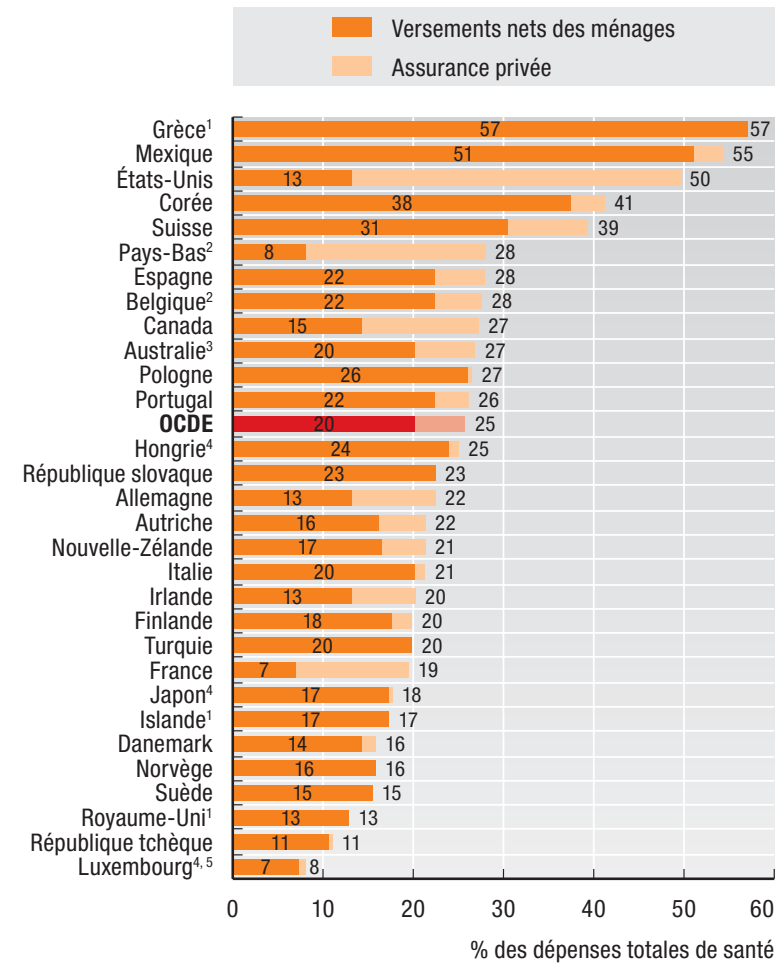

1. La part de l'assurance maladie privée n'est pas connue.

2. Part des dépenses courantes.

3. 2004-05. 4. 2004 .

5. Les versements nets des ménages incluent seulement les co-paiements. 


\subsection{COUVERTURE DE L'ASSURANCE MALADIE (PUBLIQUE ET PRIVÉE)}

La couverture maladie est un élément important de l'accès aux produits et services médicaux. Elle permet de se couvrir contre les aléas financiers d'un accident ou d'une maladie grave; elle permet également un meilleur accès aux traitements innovants et aux mesures de prévention (OCDE, 2004a). La couverture (publique et privée) de l'ensemble de la population constitue toutefois un indicateur imparfait de l'accessibilité aux soins car celle-ci dépend également du " panier » de produits et de services de santé inclus dans la couverture et du degré de participation au coût de ces services.

En 2005, la plupart des pays de l'OCDE avaient mis en place une couverture universelle ou quasi universelle des soins de santé pour un ensemble de services clés (graphique 5.6.1). Une grande majorité a mis en place un système d'accès universel à des services financés sur fonds publics (tableau A.5.6). En Allemagne, la couverture universelle est assurée par un système associant une assurance financée sur fonds publics pour $90 \%$ de la population et une assurance maladie privée pour les catégories à haut revenu (10\% de la population) qui ont choisi de sortir du système public. Aux Pays-Bas, les catégories à haut revenu n'étaient pas admises à bénéficier de l'assurance sociale jusqu'en 2005 et pratiquement toutes avaient souscrit une assurance privée (36\% de la population). En 2006, le gouvernement néerlandais a mis en place un système obligatoire d'assurance maladie universelle et réglementé la concurrence entre les multiples compagnies d'assurances privées, éliminant ainsi la division entre assurance publique et assurance privée pour la couverture de base de la population. De même, la Suisse impose depuis 1996 à l'ensemble de la population résidente de souscrire une assurance maladie de base (OCDE, 2006a).

Trois pays de l'OCDE n'ont pas encore atteint le stade de la couverture maladie universelle ou quasi universelle. Aux États-Unis, seules les personnes âgées, pauvres et handicapés, qui représentent $27 \%$ de la population, bénéficient d'une couverture financée sur fonds publics. À ces $27 \%$ s'ajoutaient $59 \%$ qui bénéficiaient, en 2005 , d'une assurance maladie privée primaire; les $14 \%$ restants n'avaient aucune couverture maladie. La moitié de la population mexicaine n'est pas affiliée à un régime de sécurité sociale; cette population "non assurée» dépend des services médicaux dispensés par les prestataires publics de santé (OCDE, 2005b). En Turquie, les deux tiers seulement de la population bénéficiaient, en 2003, d'une couverture publique.

La proportion de la population couverte par une assurance maladie privée varie considérablement d'un pays de l'OCDE à l'autre (graphique 5.6.2). Sur les 23 pays pour lesquels on dispose de données, cinq seulement (les Pays-Bas, la France, les États-Unis, le Canada et l'Irlande) font état d'une couverture maladie privée pour plus de la moitié de leur population en 2005. En Belgique et en Australie plus de $40 \%$ de la population a souscrit une assurance maladie privée; en Nouvelle-Zélande et en Suisse, la proportion est de près d'un tiers. Dans plusieurs pays de l'OCDE (par exemple la Turquie, la République tchèque, la Hongrie, la Norvège, la Pologne et la République slovaque), la part de la population bénéficiant d'une couverture maladie privée est négligeable, voire nulle.

L'assurance maladie privée joue des rôles divers selon les pays (graphique 5.6.3 et tableau A.5.6b). Outre le fait qu'elle constitue une couverture primaire pour certains groupes de population aux États-Unis, aux Pays-Bas et en Allemagne, elle est pour $87 \%$ de la population française une assurance complémentaire qui couvre la participation aux coûts appliquée au système de sécurité sociale. Le Canada a le plus important marché de l'assurance supplémentaire (66\% de la population), via lequel l'assurance privée prend en charge le coût des médicaments vendus sur ordonnance et des soins dentaires qui ne sont pas remboursés par le régime public. C'est en Irlande (52\%), en Australie (43\%) et en Nouvelle-Zélande (33\%) que l'assurance maladie parallèle (ou duplicative) est la plus développée, offrant (via le secteur privé) un accès plus rapide aux services médicaux en cas de files d'attente dans le secteur public.

L'importance de l'assurance maladie privée n'est pas liée au niveau de développement économique d'un pays. D'autres facteurs sont plus importants pour expliquer le développement de ce marché, notamment la noncouverture de certains produits et services par l'assurance publique, la possibilité d'avoir plus de choix et de rapidité d'accès aux services, le mode de financement des prestataires privés, les interventions des pouvoirs publics orientées vers les marchés de l'assurance maladie privée et l'évolution historique (OCDE, 2004c; Colombo et Tapay, 2004). Il existe une corrélation positive entre le niveau de population couvert par une assurance maladie privée et la part des dépenses totales de santé que représente l'assurance maladie privée.

\section{Définition et écarts}

La couverture de la population est définie comme la proportion de la population éligible à un ensemble de produits et de services de santé en vertu des programmes publics et de l'assurance maladie privée. Les données incluent les personnes couvertes en leur nom propre et les personnes à charge. On entend par couverture publique à la fois les programmes publics, généralement finances par l’impôt, et la sécurité sociale, généralement financée par les cotisations sociales. La souscription d'une assurance privée est souvent volontaire, bien qu'elle puisse être imposée par la loi ou obligatoire pour les salariés dans le cadre de leur contrat de travail. Le montant des primes n'est généralement pas lié aux revenus bien que la souscription d'une couverture privée puisse être subventionnée par les pouvoirs publics.

Les données sur la couverture doivent s'interpréter avec prudence. La frontière entre couverture publique et couverture privée est parfois difficile à établir. La couverture privée mélange des types d'assurances qui ont des fonctions différentes par rapport aux systèmes publics et elle ne montre pas si une personne a plusieurs couvertures. Dans certains pays (la Belgique, le Portugal, l'Islande et la France, par exemple), l'assurance maladie privée joue plusieurs rôles même si les données sont attribuées au rôle prépondérant. Certains pays dans lesquels le marché de l'assurance privée est limité (le Japon, le Luxembourg et la Suède, par exemple) ne publient aucune donnée. 
5.6. COUVERTURE DE L'ASSURANCE MALADIE (PUBLIQUE ET PRIVÉE)

\subsubsection{Couverture de l'assurance maladie pour un ensemble de services, 2005 (ou dernière année disponible)}

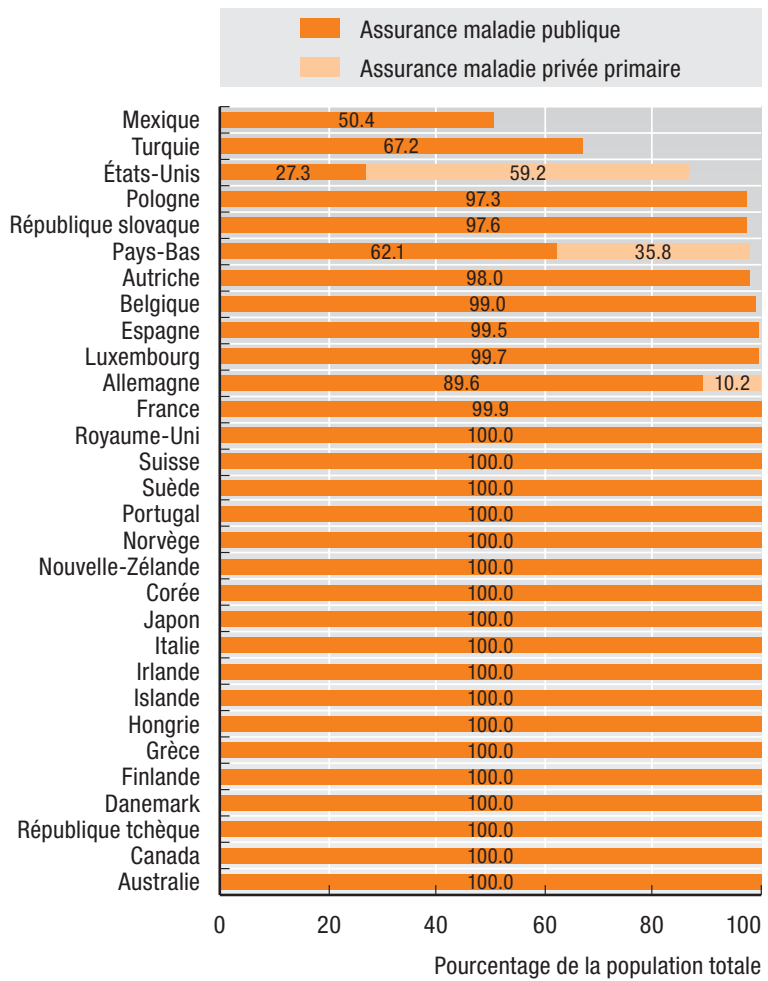

5.6.2. Population couverte par une assurance maladie privée, 2005 (ou dernière année disponible)

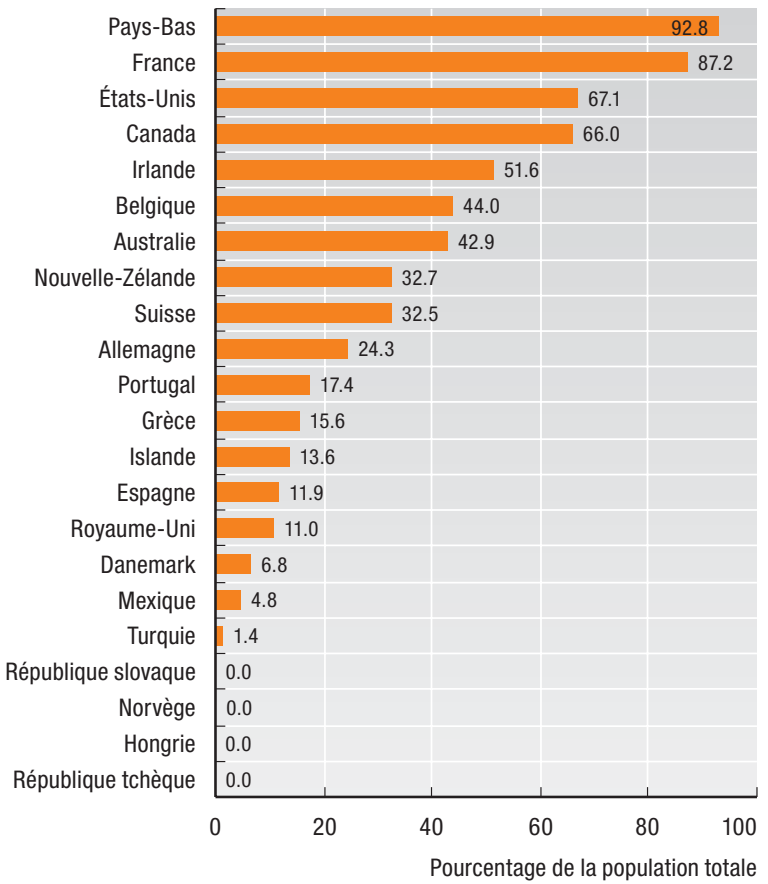

\subsubsection{Couverture selon le type d'assurance maladie privée dans certains pays de l'OCDE, 2005} (ou dernière année disponible)*

Pourcentage de la population totale

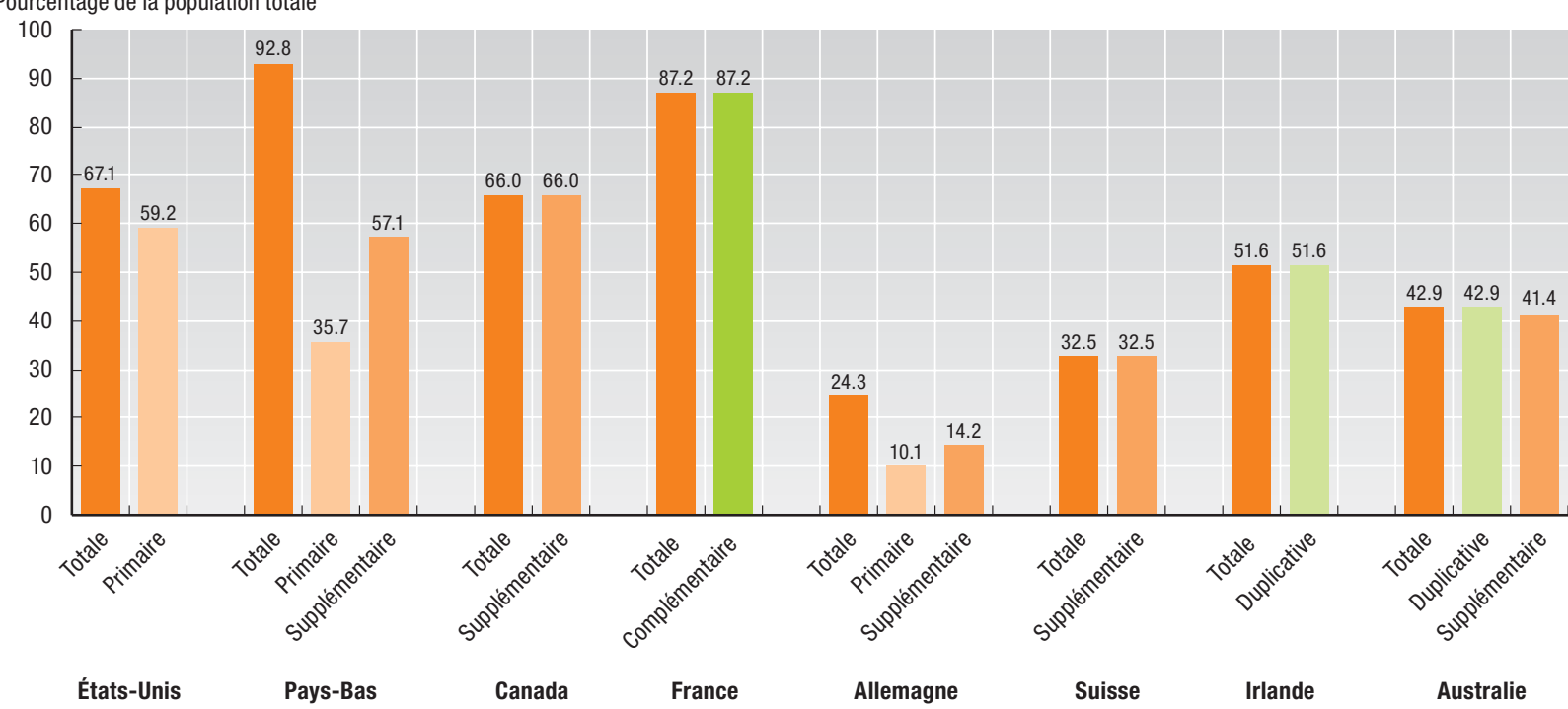

* Pays dans lesquels l'assurance maladie privée compte pour plus de $6 \%$ des dépenses totales de santé. Les pays sont classés selon un ordre décroissant de la part des assurances maladie privées dans le total des dépenses de santé.

Source : Eco-Santé OCDE 2007. 


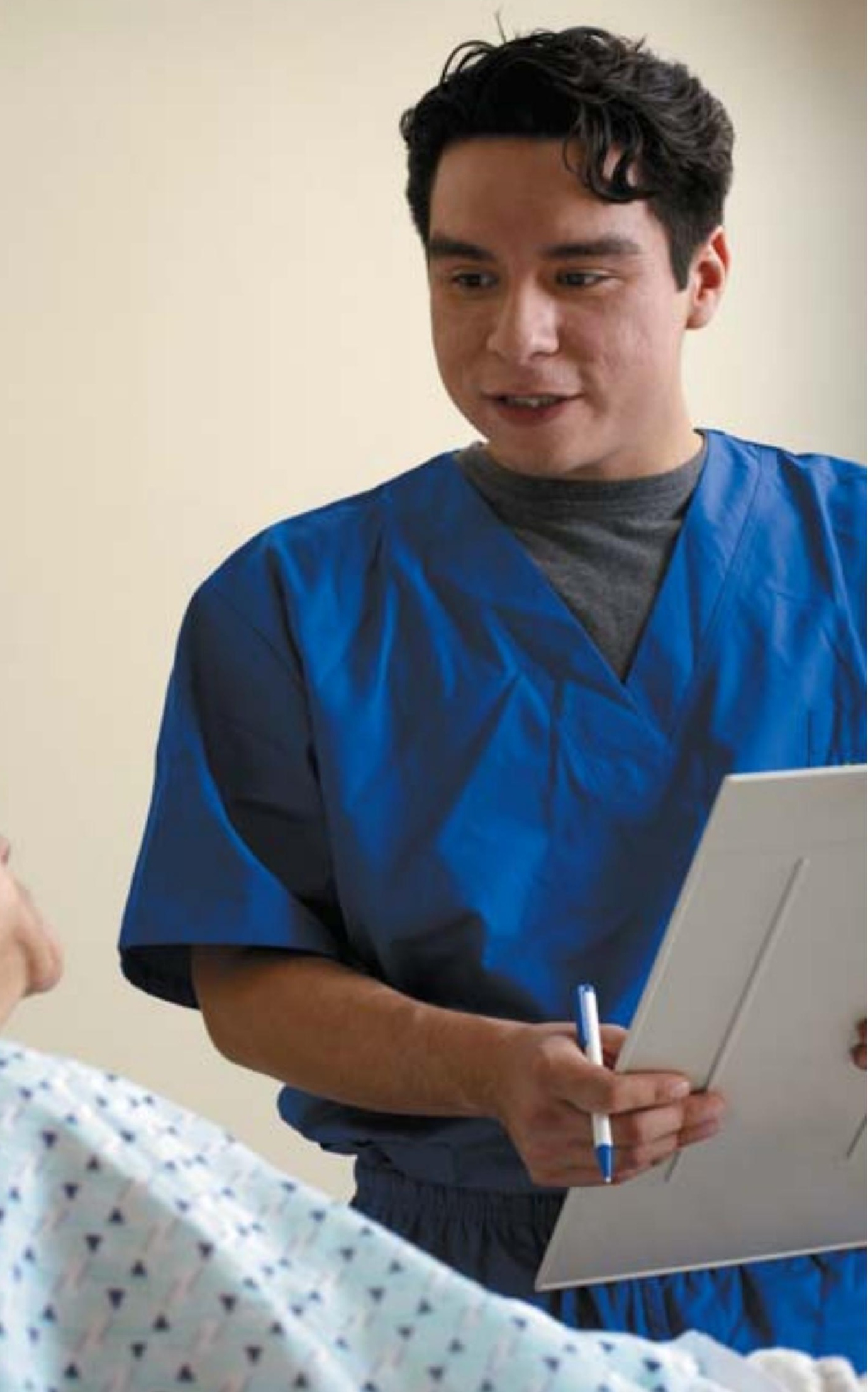

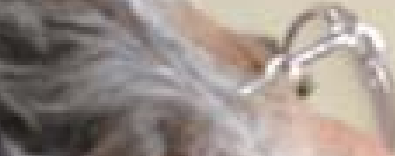
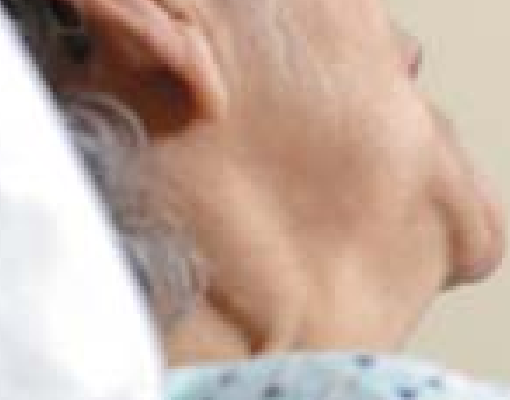

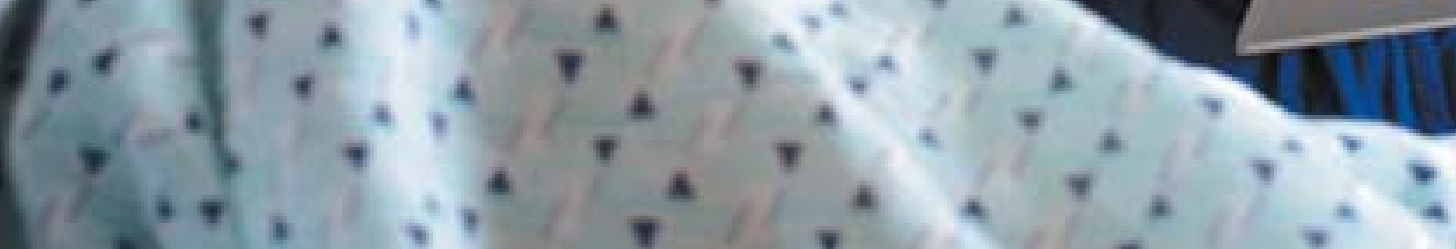

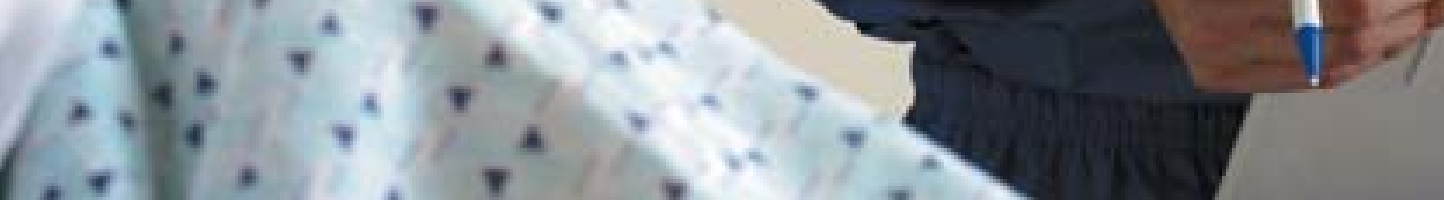

$+$

$+$

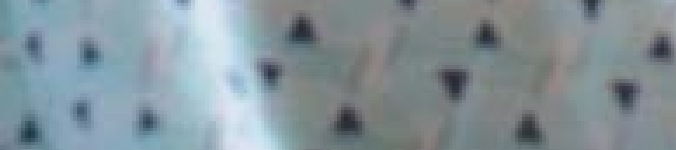

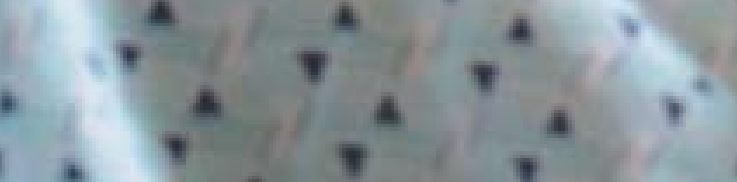

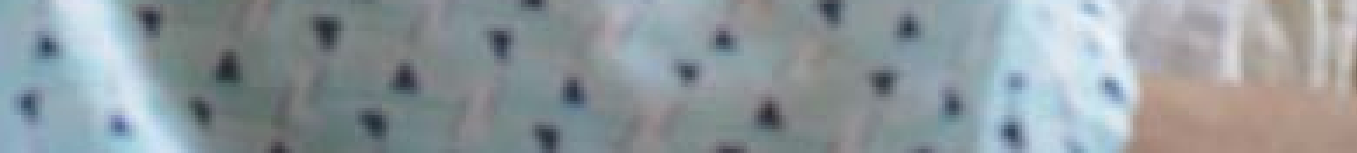




\section{QUALITÉ DES SOINS}

INTRODUCTION 100

\section{LES AFFECTIONS AIGUËS}

6.1. MORTALITÉ À L'HÔPITAL SUITE À UN INFARCTUS AIGU DU MYOCARDE. . . . . . . . . . . . . . . . . . . . . . . 104

6.2. MORTALITÉ À L'HÔPITAL SUITE À UN ACCIDENT VASCULAIRE CÉRÉBRAL ........................ 106

\section{LE CANCER}

6.3. SURVIE AU CANCER COLORECTAL . .................. 108

6.4. SURVIE AU CANCER DU SEIN ET DÉPISTAGE. . . . . . . . . . 110

6.5. SURVIE AU CANCER DU COL DE L'UTÉRUS ET DÉPISTAGE . . . . 112

\section{LES AFFECTIONS CHRONIQUES}

6.6. ADMISSIONS À L'HÔPITAL ET MORTALITÉ ÉVITABLES DUES À L'ASTHME . . . . . . . . . . . . . . . . . . . . . . . . . . . . 114

6.7. EXAMENS ANNUELS DE LA RÉTINE POUR LES DIABÉTIQUES . . 116

\section{LES MALADIES TRANSMISSIBLES}

6.8. VACCINATION DES PERSONNES ÂGÉES CONTRE LA GRIPPE. . . 118

6.9. PROGRAMMES DE VACCINATION DES ENFANTS . . . . . . . . 120 


\section{QUALITÉ DES SOINS}

\section{Introduction}

Pour la première fois, cette publication comporte un chapitre sur la qualité des soins médicaux. Évaluer et améliorer la qualité des soins est, dans de nombreux pays, une priorité de premier ordre qui est liée à la question de savoir si la progression des dépenses de santé est justifiée par des améliorations de l'état de santé des populations.

Le cadre conceptuel adopté dans le projet de l'OCDE sur les indicateurs de la qualité des soins de santé reconnaît que les services de soins ne sont qu'un déterminant parmi d'autres de l'état de santé (Kelley et Hurst, 2006; Lalonde, 1973). Néanmoins, de nombreuses études montrent la contribution forte et croissante des services médicaux à la santé (Mackenbach, 1996), et la grande variabilité de la qualité des soins entre pays et à l'intérieur d'un même pays (Wennberg et Wennberg, 2003; Mc Glynn et al., 2003). Afin d'apporter un complément d'éclairage sur la variabilité de la qualité des soins entre les pays, on s'est efforcé au cours des cinq dernières années, dans le cadre du projet de l'OCDE sur les indicateurs de la qualité des soins de santé, d'élaborer et de mettre en œuvre une série d'indicateurs de la qualité des soins susceptibles de signaler des différences potentielles de qualité (Mattke et al., 2006a).

La qualité des soins peut être évaluée sous plusieurs angles (IOM, 2001). Jusqu'ici, le projet de l'OCDE s'est intéressé essentiellement à la qualité technique des soins. La qualité technique peut être exprimée par des mesures de structure, des processus ou des résultats. Les mesures de structure représentent les caractéristiques du système de soins de santé ou l'adéquation des facteurs de production des services, telles que le nombre de lits d'hôpitaux et les effectifs*. Les mesures des processus montrent si des pratiques universellement admises et reposant sur des données probantes sont suivies, par exemple si les enfants sont immunisés de manière appropriée ou si la tension artérielle des patients est régulièrement contrôlée. Les mesures des résultats, comme le taux des infections nosocomiales ou le taux de survie à une crise cardiaque après un an, rendent compte d'une amélioration (ou d'une dégradation) de la santé liée aux soins médicaux. Avec la réactivité aux besoins et aux attentes des patients, la qualité technique reflète la valeur produite par les systèmes de soins de santé. Associées aux données sur l'utilisation des ressources, les mesures de la qualité technique peuvent être utilisées pour obtenir des indicateurs de l'efficience d'un système de soins de santé ou de son efficacité économique. Avant de pouvoir tirer de ces indicateurs des conclusions fermes, il convient toutefois de s'attaquer à plusieurs problèmes, notamment de mettre en place un ensemble plus global et plus équilibré de mesures, de procéder, lorsque cela est nécessaire et faisable, à des ajustements pour prendre en compte les différences de profil de risques entre pays, et dans la mesure du possible, de tenir compte des facteurs non médicaux qui influent également sur les résultats en termes de santé.

Jusqu'ici, on ne dispose pour les comparaisons internationales que d'un ensemble limité d'indicateurs de la qualité des soins de santé, qui doivent de plus être utilisés à la lumière des mises en garde quant aux limitations persistantes de la comparabilité. Ces indicateurs couvrent toutefois un large spectre de services, depuis les services préventifs

\footnotetext{
* Puisque des mesures de structure sont d'ores et déjà disponibles au niveau international, par exemple dans Eco-Santé OCDE (comme le montre le chapitre 4 de cette publication), et qu'elles représentent des conditions nécessaires mais pas suffisantes pour des soins de qualité, le projet de l'OCDE sur les indicateurs de la qualité des soins de santé se concentre sur les mesures des processus et des résultats.
} 
(comme la vaccination et le dépistage) jusqu'aux services de soins aigus et aux soins liés à la gestion des maladies chroniques. Ces indicateurs s'efforcent de couvrir la qualité des soins liés à des maladies importantes (par exemple, les maladies cardio-vasculaires et les cancers) et des soins dispensés à différents groupes d'âge (par exemple, la vaccination des enfants et la vaccination contre la grippe des personnes âgées). Ils couvrent également les processus et les résultats des soins pour différentes affections.

Dans ce chapitre, les indicateurs sont présentés dans quatre sections qui montrent les résultats initiaux de la collecte de données portant respectivement sur: la qualité des soins pour certaines affections aiguës (infarctus aigu du myocarde et accident vasculaire cérébral); la qualité des soins pour certains cancers (colorectal, du sein et du col de l'utérus), la qualité des soins liés aux affections chroniques (asthme, diabète) et la prévention des maladies transmissibles (vaccination et incidence des maladies pouvant être prévenues par un vaccin).

\begin{tabular}{|c|c|c|}
\hline & Mesure des processus & Mesure des résultats \\
\hline Soins pour affections aiguës & & $\begin{array}{l}\text { Taux de mortalité après un infarctus aigu } \\
\text { du myocarde } \\
\text { Taux de mortalité après un AVC ischémique } \\
\text { Taux de mortalité après un AVC hémorragique }\end{array}$ \\
\hline Soins pour cancers & $\begin{array}{l}\text { Taux de mammographie } \\
\text { Taux de dépistage du cancer du col } \\
\text { de l'utérus }\end{array}$ & $\begin{array}{l}\text { Taux de survie relative au cancer du sein } \\
\text { Taux de survie relative au cancer du col de l'utérus } \\
\text { Taux de survie relative au cancer colorectal }\end{array}$ \\
\hline Soins pour affections chroniques & $\begin{array}{l}\text { Examen annuel de la rétine } \\
\text { pour les diabétiques }\end{array}$ & $\begin{array}{l}\text { Taux de mortalité due à l'asthme } \\
\text { Taux d'admission à l'hôpital d'adultes asthmatiques }\end{array}$ \\
\hline $\begin{array}{l}\text { Soins pour la prévention des maladies } \\
\text { transmissibles }\end{array}$ & $\begin{array}{l}\text { Couverture du programme de vaccination } \\
\text { infantile de base (deux indicateurs) } \\
\text { Couverture du programme de vaccination } \\
\text { contre la grippe des plus de } 65 \text { ans }\end{array}$ & $\begin{array}{l}\text { Incidence des maladies pouvant être prévenues } \\
\text { par un vaccin (deux indicateurs) }\end{array}$ \\
\hline
\end{tabular}

\section{Les affections aiguës (voir indicateurs 6.1 et 6.2)}

Les progrès réalisés en matière de diagnostic et de traitement ont conduit à des améliorations spectaculaires du pronostic vital des patients souffrant d'affections aiguës graves telles que des blessures traumatiques, des infections et des accidents cardiovasculaires aigus. La capacité d'acheminer rapidement les patients vers un hôpital et de leur dispenser en route des soins de réanimation, les progrès dans le domaine des interventions médicales et chirurgicales et, en particulier, la médecine moderne de soins intensifs ont considérablement réduit le risque de décès et d'incapacités. Les prestataires de soins de santé, les chercheurs et les responsables des politiques continuent néanmoins de chercher à améliorer encore la prise en charge de ces affections; ils cherchent notamment à raccourcir le temps s'écoulant entre le diagnostic et le traitement mais aussi à accroître la fiabilité des prestations recommandées. La qualité des soins pour affections aiguës est mesurée dans cette section par le taux de décès après une admission à l'hôpital pour un infarctus aigu du myocarde (IAM) ou un accident vasculaire cérébral (AVC), qui sont deux des causes les plus courantes de décès et d'incapacité dans les pays de l'OCDE.

\section{Le cancer (voir indicateurs 6.3, 6.4 et 6.5)}

Les progrès de la médecine au cours des dernières décennies ont considérablement amélioré le pronostic vital des personnes atteintes de cancers. Si à ce jour « gagner la guerre 


\section{QUALITÉ DES SOINS}

contre le cancer " demeure un objectif fuyant, l'amélioration des techniques de dépistage associée à des campagnes de sensibilisation a accru les chances de détection à un stade précoce, et donc guérissable, de plusieurs types de cancers. Les avancées en matière de techniques chirurgicales, de radiothérapie et de chimiothérapie offrent de nouvelles possibilités de traitement. Dans certains domaines, les progrès ont été spectaculaires : pour la leucémie aiguë chez les enfants ou le cancer des testicules, par exemple, le taux de guérison est désormais de plus de $90 \%$, même lorsque le diagnostic de cancer est fait à un stade avancé. Dans d'autres, comme le cancer du pancréas ou de l'œsophage, le taux de survie demeure faible. Cette section présente des informations sur les taux de survie pour trois types courants de cancer : le cancer colorectal, le cancer du sein et le cancer du col de l'utérus. Dans les trois cas, il existe des tests de dépistage qui permettent un diagnostic précoce et des traitements qui améliorent les chances de survie, même à un stade avancé.

\section{Les affections chroniques (voir indicateurs 6.6 et 6.7)}

La prévention et le traitement des affections chroniques demeurent, pour les responsables des politiques de santé des pays de l'OCDE, un problème délicat car des affections comme les défaillances cardiaques et le diabète représentent une part toujours plus grande des coûts de santé et que les soins dispensés pour ces affections ne sont souvent pas optimaux. Une raison importante de ce déficit de qualité vient de ce qu'une grande partie des systèmes de santé actuels ont été conçus à une époque où l'on se préoccupait essentiellement des affections aiguës, en particulier des maladies infectieuses, qui requièrent un traitement court mais intense. Les systèmes mis en place, qui dispensent des soins dans la période suivant immédiatement le contact entre le médecin et le patient, se sont avérés très efficaces pour soigner les affections aiguës mais sont moins bien adaptés aux besoins de personnes souffrant d'affections chroniques qui requièrent surveillance, éducation et conseils. Pour combler ce déficit, un grand nombre de pays expérimentent actuellement des formules innovantes. L'Allemagne et les États-Unis, par exemple, testent des gestions thérapeutiques. D'autres pays, comme le Royaume-Uni et la Suède, cherchent à mettre en œuvre des modèles efficaces de soins primaires. Tous ont en commun d'espérer qu'une meilleure gestion des affections chroniques pourrait réduire les coûts (ou du moins leur rythme de progression) et améliorer la qualité des soins et l'état de santé. Les indicateurs de la qualité des soins pour affections chroniques présentés dans cette section concernent l'asthme, qui est la maladie chronique la plus courante chez l'enfant et le diabète, qui est l'une des affections les plus courantes chez l'adulte.

\section{Les maladies transmissibles (voir indicateurs 6.8 et 6.9)}

Si des améliorations au niveau de l'hygiène, de la nutrition et des traitements ont réduit de façon spectaculaire le poids des maladies infectieuses dans les pays industrialisés, le contrôle des maladies transmissibles demeure une responsabilité importante des politiques de santé, en particulier au vu d'épidémies modernes telles que le HIV/SIDA, du risque de nouvelles épidémies comme la grippe aviaire et de la menace bioterroriste. Il demeure également un sujet épineux pour les responsables des politiques de santé car il suppose habituellement de trouver un juste équilibre entre droits individuels et intérêt public. Le contrôle des maladies transmissibles requiert la collaboration de différentes parties du système de santé publique et du système de soins, depuis les départements du ministère de la Santé jusqu'aux prestataires de soins préventifs comme les généralistes, les pédiatres et les médecins de famille et jusqu'aux 
prestataires de soins en établissement, comme les hôpitaux et les établissements de soins de longue durée. Pour organiser ces services et concilier les contraintes des exigences légales et des campagnes de sensibilisation, les pays adoptent des approches différentes. La tendance actuelle consiste à s'en remettre largement au secteur des soins médicaux pour l'offre de services et à celui de la santé publique pour la surveillance et la sensibilisation. Plusieurs mesures actuelles du projet de l'OCDE sur les indicateurs de la qualité des soins de santé saisissent le degré de prévention de la diffusion des maladies transmissibles. Ces mesures sont le taux de vaccination contre la grippe des personnes de 65 ans et plus, et le taux de vaccination des enfants (mesures de processus), et les taux d'incidence des maladies pouvant être prévenues par la vaccination (mesures de résultat).

\section{Interprétation et utilisation des données}

Il est important de souligner qu'à ce stade précoce de leur développement, les indicateurs présentés dans ce chapitre doivent être considérés comme expérimentaux. Bien qu'ils se fondent sur des études empiriques rigoureuses et qu'ils aient été utilisés pour des travaux de recherche et d'analyse à l'intérieur des pays, on ne comprend pas encore très bien comment ils sont liés aux résultats en termes de santé ou pourquoi ils varient entre pays. Même si l'on s'est efforcé de réunir des données aussi comparables que possible entre pays, on note que des différences subsistent en termes de définitions, de sources et de méthodes, comme c'est le cas pour d'autres indicateurs de Eco-Santé OCDE présentés dans d'autres chapitres de cette publication. Un problème important concerne l'absence de normalisation selon l'âge pour plusieurs mesures des résultats. À ce stade, seul un nombre limité de pays ont été à même de fournir des taux normalisés selon l'âge par rapport à la population de référence de l'OCDE. Bien que les différences rapportées par ces pays entre les taux bruts et les taux normalisés selon l'âge ne soient pas substantielles, l'incidence sur la comparabilité entre les pays n'est pas encore bien établie; des travaux sont en cours pour calculer des taux normalisés selon l'âge pour un plus grand nombre de pays.

Ces indicateurs doivent donc être considérés comme posant des questions sur la qualité des soins dans les différents pays plutôt que comme apportant des réponses définitives. Les informations fournies doivent être considérées comme un point de départ permettant de mieux comprendre les variations de la qualité des soins, d'encourager une analyse plus approfondie et de tirer des enseignements des différentes expériences nationales.

\section{Priorités pour le développement d'autres indicateurs de la qualité des soins}

Avec la collaboration continue des experts nationaux, le projet de l'OCDE sur les indicateurs de la qualité des soins de santé vise à améliorer et à élargir l'ensemble actuel d'indicateurs de manière à permettre une évaluation plus complète de la performance des systèmes de soins de santé des pays de l'OCDE. Pour l'heure, les priorités de l'élaboration de nouveaux indicateurs concernent les soins pour les maladies mentales, la sécurité des patients, la prévention et les soins primaires, et la réactivité (ou expérience des patients). L'élaboration d'indicateurs dans ces domaines prioritaires devrait permettre d'enrichir ce chapitre dans les éditions futures du Panorama de la santé. 


\subsection{MORTALITÉ À L'HÔPITAL SUITE À UN INFARCTUS AIGU DU MYOCARDE}

\section{Importance de l'indicateur}

Si les maladies coronaires demeurent la principale cause de décès dans les pays industrialisés, les taux de mortalité due à ces maladies sont en baisse depuis les années 70 (Weisfeldt et Zieman, 2007). Cette diminution peut être attribuée, dans une large mesure, à une baisse de la mortalité due à un infarctus aigu du myocarde (IAM) suite à une amélioration du traitement en phase aiguë (Capewell et al., 2000; McGovern et al., 2001). Les soins dispensés en cas d'IAM ont évolué de façon spectaculaire dans les dernières décennies avec l'apparition des unités de soins coronariens dans les années 60, puis l'apparition, dans les années 80 , de traitements visant à rétablir rapidement la circulation sanguine (Gil et al., 1999). Les succès obtenus en termes de réduction de la mortalité sont d'autant plus remarquables que l'incidence de l'IAM ne semble pas avoir diminué durant cette période (Goldberg et al., 1999). Néanmoins, une proportion considérable de patients victimes d'un IAM ne reçoivent pas à temps les soins appropriés, ce qui donne à penser que l'on peut encore mieux faire (McGlynn et al., 2003). L'IAM représente environ la moitié des décès par maladie coronaire, alors que le coût des soins administrés pour les maladies coronaires représente jusqu'à $10 \%$ des dépenses de santé des pays industrialisés (OCDE, 2003a).

\section{Validité scientifique de l'indicateur}

Un grand nombre d'études établissent un lien entre les processus de soins visant à rétablir la circulation sanguine, comme la thrombolyse et l'administration précoce d'aspirine, ou réduisant les risques de complications, comme l'administration d'agents bétabloquants, et l'amélioration du taux de survie à un IAM (Davies et al., 2001). Étant donné l'éventail des services et des dispositifs systémiques à mobiliser pour soigner cette maladie, le taux de décès à l'hôpital de personnes admises suite à un IAM est considéré être une bonne mesure (de résultat) de la qualité des soins aigus. Les taux de mortalité après un IAM ont été utilisés par la US Agency for Healthcare Research and Quality (Davies et al., 2001) pour le benchmarking des hôpitaux par le National Health Service britannique et par toute une série d'associations hospitalières et de groupes de contrôle qualité aux États-Unis. Ces taux ont été également utilisés pour des comparaisons internationales dans le projet de l'OCDE sur les maladies liées au vieillissement (OCDE, 2003a) et dans le projet MONICA de l'OMS (Tunstall-Pedoe, 2003).

\section{Constats}

Le taux de mortalité moyen à l'hôpital dans les 30 jours suivant une admission pour IAM est désormais de l'ordre de $10 \%$ parmi les pays ayant fait état de données (graphique 6.1.1). Il s'agit là d'une amélioration sensible car, dans les années 80 , ce taux était habituellement de l'ordre de 20 \% (Weisfeldt et Zieman, 2007). Bien que les données tendancielles présentées dans le graphique 6.1 .2 soient à interpréter avec prudence compte tenu des périodes différentes qu'elles recouvrent, la tendance observée pour la plupart des pays est favorable.

En 2005 (ou dernière année disponible), l'Australie et la Nouvelle-Zélande affichaient des taux particulièrement bas de mortalité à l'hôpital suite à une admission pour IAM. Les pays scandinaves, à l'exception de la Finlande, affichaient également des taux bas. Parmi les pays de l'OCDE, une grande disparité subsiste, les taux s'échelonnant entre $5.4 \%$ en Nouvelle-Zélande et $24.5 \%$ au Mexique. Même pour des pays voisins ayant un niveau de développement économique similaire et des systèmes de santé analogues, on peut observer des différences importantes. En Autriche, par exemple, le taux de mortalité à l'hôpital est supérieur environ de moitié à celui observé en France (12.0\% contre $7.6 \%)$. Il convient, toutefois, de garder à l'esprit que les données n'ont pas été corrigées pour prendre en compte les différences de risque ou de structure d'âge des patients entre les pays. On ne peut donc pas dire dans quelle mesure les différences de taux de mortalité sont la conséquence de différences de soins ou sont dues à des différences de gravité des cas ou d'âge des patients.

Les différences de taux de mortalité à l'hôpital dans les 30 jours suivant une admission pour IAM ne semblent pas être liées au degré d'utilisation de procédures de revascularisation comme les angioplasties et les pontages coronariens (graphiques 6.1.3 et 6.1.4). Conjuguées au fait que les interventions médicales généralement reconnues comme bénéfiques sont souvent sous-utilisées dans les soins dispensés aux victimes d'IAM (McGlynn et al., 2003), les données suggèrent que les taux de survie à un IAM pourraient être améliorés par une offre plus fiable de traitements simples et d'un bon rapport coût/efficacité.

\section{Définition et écarts}

Le taux de mortalité mesure la proportion de patients présentant un diagnostic donné, ici un infarctus aigu du myocarde (IAM), qui décèdent dans un délai spécifié, qui est ici de 30 jours. Idéalement, ce taux serait établi sur la base de patients suivis individuellement pendant au minimum 30 jours. Toutefois, comme la plupart des pays n'ont pas d'identifiant unique des patients et qu'ils n'ont pas non plus la capacité de suivre les patients après leur sortie de l'hôpital, l'indicateur est établi sur la base d'admissions uniques à l'hôpital et se limite à la mortalité à l'hôpital. Des différences de pratiques pour les sorties et les transferts de patients peuvent donc influer sur les résultats. Les définitions de l'IAM dans la classification CIM-10 sont également légèrement différentes de celles de la version antérieure (CIM-9), ce qui peut limiter les comparaisons entre pays utilisant des versions différentes de la classification. 


\section{QUALITÉ DES SOINS • LES AFFECTIONS AIGUËS}

\subsection{MORTALITÉ À L'HÔPITAL SUITE À UN INFARCTUS AIGU DU MYOCARDE}

\subsubsection{Taux de mortalité à l'hôpital \\ à 30 jours après admission pour un infarctus aigu du myocarde, 2005}

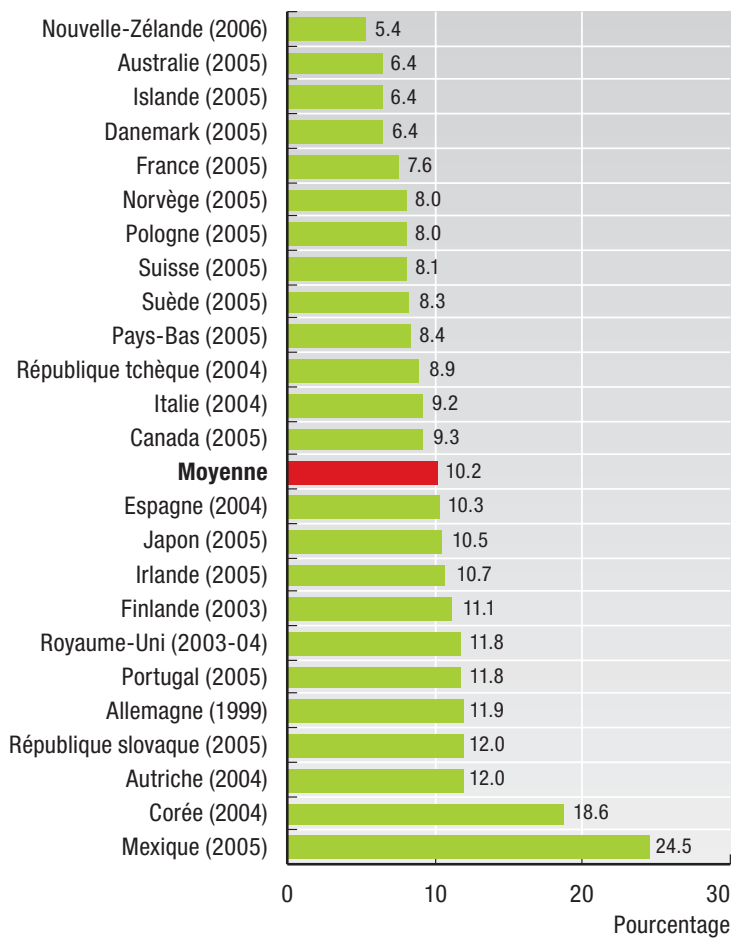

\subsubsection{Variation du taux de mortalité à l'hôpital à 30 jours après admission pour un infarctus aigu du myocarde}

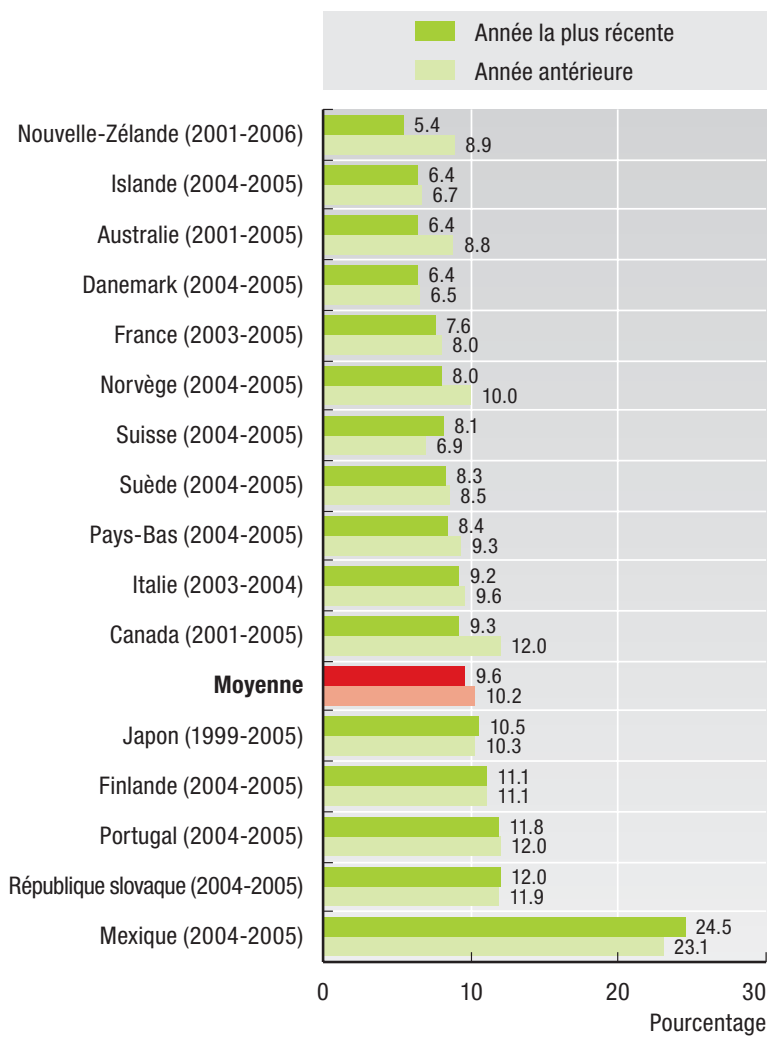

Source : Projet sur les indicateurs de la qualité des soins de santé, OCDE 2007.

\subsubsection{Taux de mortalité à l'hôpital à $\mathbf{3 0}$ jours après admission pour un infarctus aigu du myocarde et taux d'angioplasties, 2005}

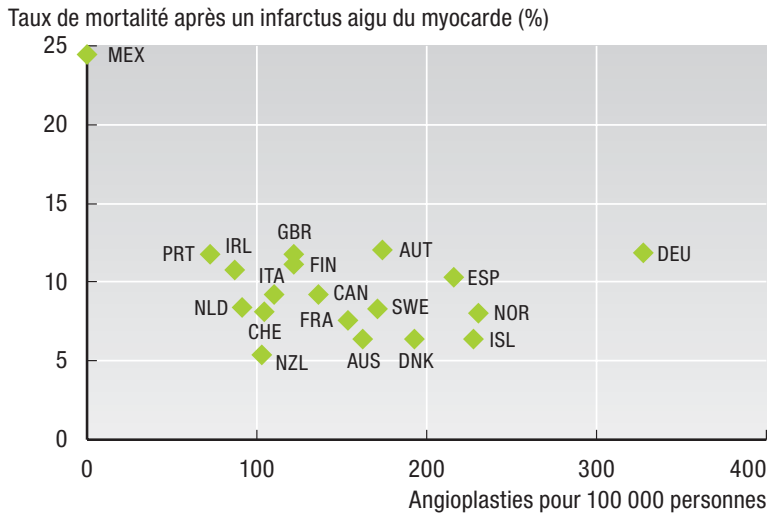

\subsubsection{Taux de mortalité à l'hôpital à 30 jours après admission pour un infarctus aigu du myocarde et taux de pontages coronariens, 2005}

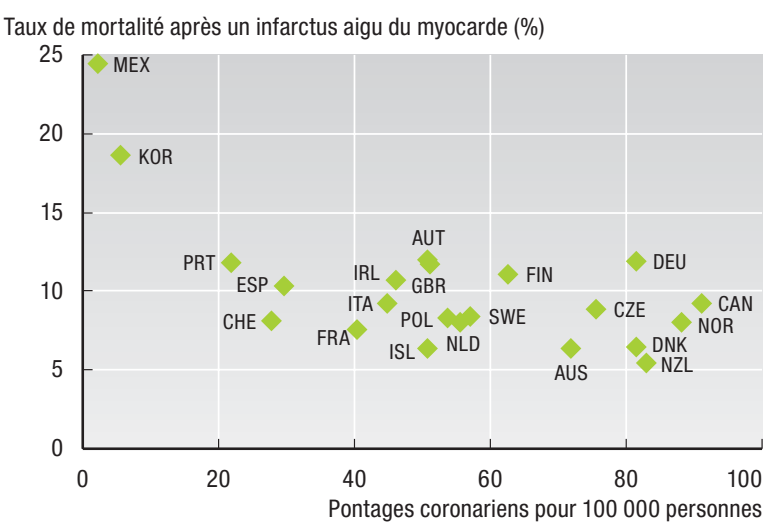

Note : Les taux d'angioplasties et de pontages coronariens se rapportent à 2005, sauf pour l'Australie, le Canada, l'Irlande, l'Italie, la Nouvelle-Zélande et la Norvège qui réfèrent à 2004; les Pays-Bas à 2003; la France à 2001; l'Autriche à 2000.

Source : Projet sur les indicateurs de la qualité des soins de santé, OCDE 2007. Eco-Santé OCDE 2007 (angioplasties et pontages coronariens). 


\subsection{MORTALITÉ À L'HÔPITAL SUITE À UN ACCIDENT VASCULAIRE CÉRÉBRAL}

\section{Importance de l'indicateur}

L'accident vasculaire cérébral (AVC) est la troisième grande cause de décès et d'incapacité dans les pays industrialisés (OMS, 2002b). L'AVC représente 2 à $4 \%$ des dépenses de santé mais également des coûts importants en dehors du système de santé du fait de son impact sur l'incapacité (OCDE, 2003b). Il convient de distinguer deux types d'AVC. Dans l'AVC ischémique, qui représente environ $85 \%$ des cas, l'irrigation sanguine d'une partie du cerveau est interrompue, ce qui endommage gravement le tissu cérébral de la zone concernée. Dans l'AVC hémorragique, la rupture d'un vaisseau sanguin cause une hémorragie à l'intérieur du cerveau; les symptômes sont analogues à ceux de l'AVC ischémique mais, en général, les dommages causés sont plus étendus.

$\mathrm{Au}$ cours de la dernière décennie, le traitement des AVC ischémiques a évolué de façon spectaculaire. Jusque dans les années 90 , on pensait communément que les dommages causés au cerveau étaient irréversibles; en conséquence, les traitements se focalisaient sur la prévention des complications et la réadaptation fonctionnelle. Mais après les améliorations spectaculaires du taux de survie à un IAM qu'a permis d'obtenir une thrombolyse précoce dissolvant les caillots de sang, des essais cliniques lancés au Japon au début des années 90 ont montré les effets bénéfiques du traitement thrombolytique des AVC ischémiques (Mori et al., 1992). Des unités de soins dédiées, sur le modèle des unités de soins cardiaques, ont été mises en place dans de nombreux pays, en particulier dans les pays scandinaves, permettant une thérapie agressive et rapide des victimes d'AVC. Le résultat est que les taux de mortalité par AVC ischémique ont chuté dans un grand nombre de pays (Sarti et al., 2003).

\section{Validité scientifique de l'indicateur}

De nombreuses études montrent l'existence d'un lien entre les services de soins de santé et les résultats suite à un AVC. Dans plusieurs pays européens, on a établi une relation entre l'existence dans les hôpitaux d'unités dédiées au traitement des AVC et l'amélioration des résultats (Stroke Unit Trialists Collaboration, 1997). Aux États-Unis (par exemple, NINDS, 1995) et en Europe (par exemple, Hacke et al., 1995) des essais cliniques de grande envergure ont clairement démontré l'impact du traitement thrombolytique des AVC ischémiques sur le taux de survie et l'incapacité. Toutefois, l'adoption de cette pratique se heurte à une certaine résistance due à des facteurs liés à l'organisation des services de santé (Wardlaw et al., 2003). Les taux de mortalité due à un AVC ont été utilisés pour comparer les performances des hôpitaux à l'intérieur des pays et entre pays (Davies et al., 2001; OCDE, 2003a; et Sarti et al., 2003).

\section{Constats}

Les données confirment le caractère plus grave de l'AVC hémorragique, avec un taux de mortalité moyen 30 jours après l'admission à l'hôpital de $25.1 \%$, contre $10.1 \%$ pour l'AVC ischémique (graphiques 6.2.1 et 6.2.2). On note une grande disparité entre pays des taux de mortalité à l'hôpital, en particulier pour l'AVC ischémique. Le Mexique, par exemple, fait état d'un taux sept fois supérieur à celui du Japon. Même entre pays voisins ayant des niveaux analogues de développement économique on observe des différences importantes $(11.3 \%$ en Irlande contre $5.5 \%$ au Royaume-Uni soit plus de deux fois plus). Bien qu'il faille être vigilant dans l'interprétation des données tendancielles compte tenu des périodes différentes qu'elles concernent, la tendance globale semble favorable avec, toutefois, quelques exceptions comme le Mexique. Une certaine prudence s'impose toutefois car les données n'ont pas été corrigées des différences de risque ou de structure d'âge des patients entre les pays. On ne peut donc pas dire dans quelle mesure les différences de taux de mortalité sont la conséquence de différences de soins, de gravité des cas ou d'âge des patients.

Comme le montre le graphique 6.2.3, les taux de mortalité pour l'AVC ischémique et l'AVC hémorragique sont étroitement liés en ce sens que les pays affichant un meilleur taux de survie à un type d'AVC affichent également de bons résultats pour l'autre. Cela est tout à fait plausible, car les phases de soins initiales (notamment diagnostic précoce, transfert rapide à l'hôpital et accès immédiat à la tomographie assistée par ordinateur pour guider les décisions de traitements aigus) sont identiques dans les deux cas. Le simple fait que la technologie soit disponible ne semble cependant pas avoir d'impact sur la survie; le nombre de tomodensitomètres par million d'habitants n'est pas lié au taux de mortalité (graphique 6.2.4). Cela conduit à penser que l'organisation des services de soins de santé est plus importante que l'infrastructure disponible.

Les faibles taux de mortalité enregistrés dans les pays scandinaves, en particulier en Islande et en Finlande, sont une indication supplémentaire de l'importance de l'organisation des services. En effet, ces pays ont été parmi les premiers à ouvrir dans leurs hôpitaux des unités de soins dédiées aux AVC, pratique qui a prouvé sa capacité à améliorer la survie et le fonctionnement neurologique (Stroke Unit Trialists Collaboration, 2001). Dans ces pays, environ $70 \%$ des victimes d'AVC étaient traitées dans des unités dédiées dès 1998 (OCDE, 2003a).

\section{Définition et écarts}

Le taux de mortalité mesure la proportion de patients présentant un diagnostic donné, ici un AVC, qui meurent dans un délai spécifié, qui est ici de 30 jours. Idéalement, ce taux serait établi sur la base de patients suivis individuellement pendant au minimum 30 jours. Toutefois, comme la plupart des pays n'ont pas d'identifiant unique des patients et qu'ils n'ont pas non plus la capacité de suivre les patients après leur sortie de l'hôpital, l'indicateur est établi sur la base d'admissions uniques à l'hôpital et se limite à la mortalité à l'hôpital. Des différences de pratiques pour les sorties et les transferts de patients peuvent donc influer sur les résultats. Les définitions de l'AVC dans la classification CIM-10 sont légèrement différentes de celles de la version antérieure (CIM-9), ce qui peut limiter les comparaisons entre pays utilisant des versions différentes de la classification. 


\section{QUALITÉ DES SOINS • LES AFFECTIONS AIGUËS}

\subsection{MORTALITÉ À L'HÔPITAL SUITE À UN ACCIDENT VASCULAIRE CÉRÉBRAL}

\subsubsection{Taux de mortalité à l'hôpital à 30 jours après admission pour un accident vasculaire cérébral ischémique, 2005 et année antérieure}
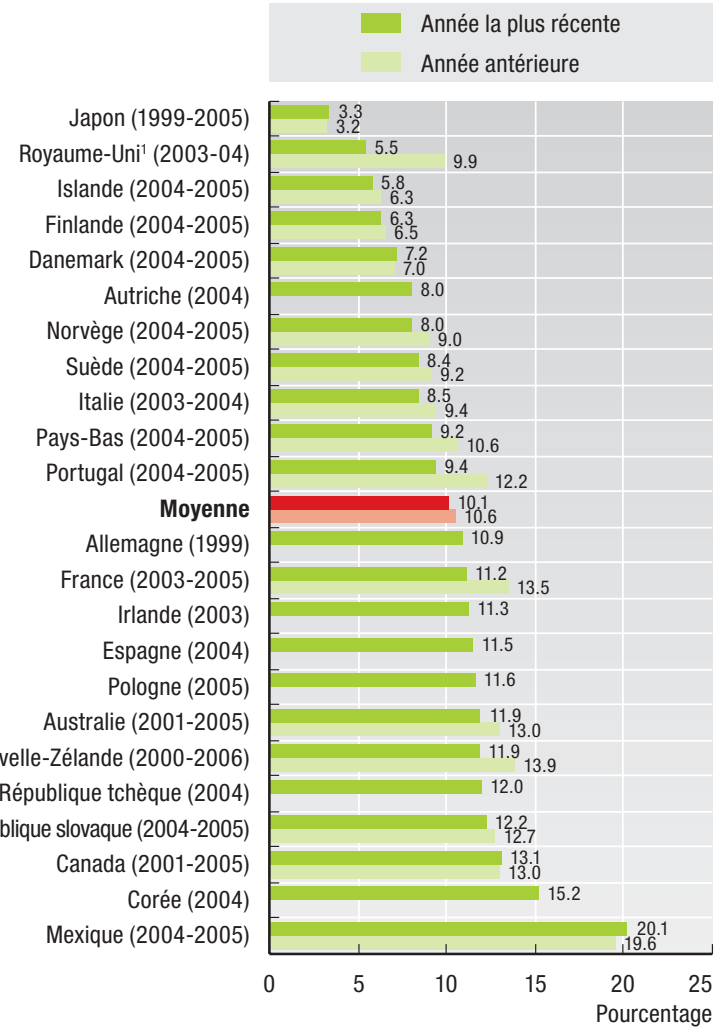

\subsubsection{Taux de mortalité à l'hôpital à 30 jours après admission pour un accident vasculaire cérébral hémorragique, 2005 et année antérieure}

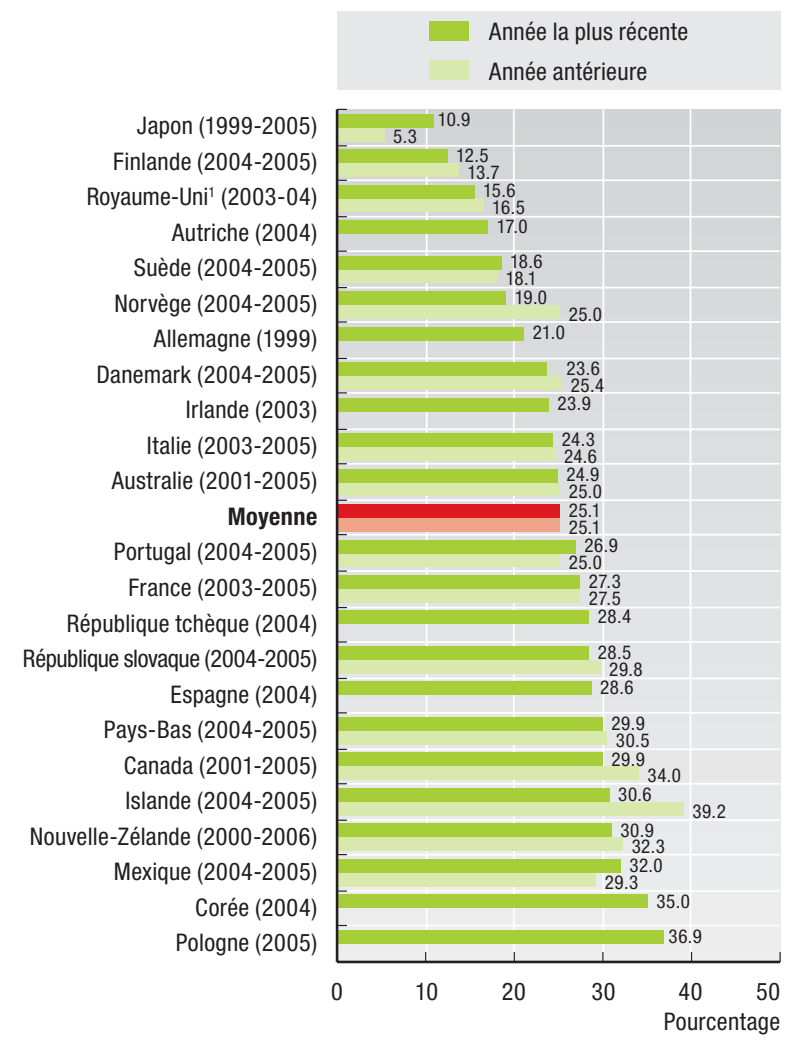

1. $2002-03 / 2003-04$.

Source : Projet sur les indicateurs de la qualité des soins de santé, OCDE 2007.

\subsubsection{Corrélation des taux de mortalité à l'hôpital à 30 jours après admission pour un accident vasculaire cérébral ischémique ou hémorragique, 2005}

Taux de mortalité après un accident vasculaire cérébral hémorragique (\%)

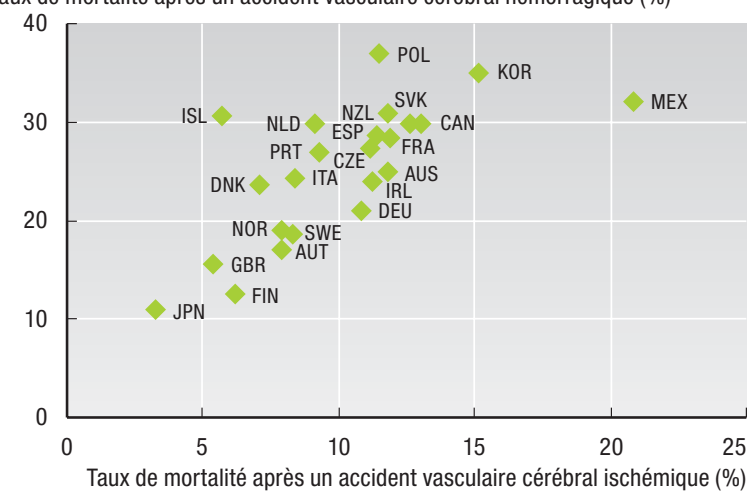

6.2.4. Taux de mortalité à l'hôpital à 30 jours après admission pour un accident vasculaire cérébral et nombre de tomodensitomètres, 2005

Taux de mortalité après un accident vasculaire cérébral ischémique (\%)

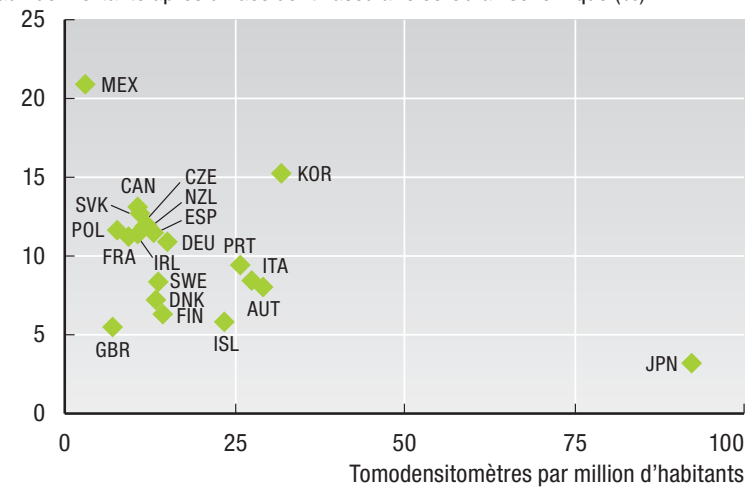

Note : Voir les deux graphiques précédents pour les années auxquelles se rapportent les taux de mortalité suite à un accident vasculaire cérébral. Les chiffres concernant les tomodensitomètres se rapportent à 2005 sauf pour la Nouvelle-Zélande (2004) et la Suède (1999). Source : Projet sur les indicateurs de la qualité des soins de santé, OCDE 2007. Eco-Santé OCDE 2007 (tomodensitomètres). 


\subsection{SURVIE AU CANCER COLORECTAL}

\section{Importance de l'indicateur}

Le cancer colorectal est le troisième type de cancer le plus répandu chez la femme (après le cancer du sein et celui du poumon) comme chez l'homme (après le cancer de la prostate et celui du poumon). On estime à environ USD 8.4 milliards le montant dépensé chaque année aux États-Unis pour le traitement du cancer colorectal (Brown et al., 2002). Au cours des dernières décennies, les progrès en matière de diagnostic et de traitement ont accru le taux de survie. L'amélioration des techniques de dépistage (recherche de sang dans les selles) et, plus récemment, la pratique régulière de coloscopies ont accru le nombre de tumeurs diagnostiquées à un stade pré-cancéreux ou à tout le moins précoce (Midgley et Kerr, 1999), même si dans un grand nombre de pays, les méthodes de dépistage offrant le meilleur rapport coût/efficacité font toujours débat. Les progrès en matière d'anesthésie et de techniques chirurgicales permettent la résection d'un plus grand nombre de tumeurs, même à un stade avancé. L'amélioration des traitements de radiothérapie et des protocoles de chimiothérapie et, plus récemment, la découverte d'agents biologiques et cytotoxiques qui attaquent spécifiquement les cellules cancéreuses offrent de nouvelles possibilités de traitement (Natarajan et Shuster, 2006). Les données historiques de la France laissent penser qu'entre 1976 et 1988 le taux de survie est passé de $33 \%$ à $55 \%$ et que cette amélioration a été obtenue grâce à une augmentation du taux de résection, à une diminution de la mortalité post-opératoire, à un diagnostic plus précoce et au recours accru à la chimiothérapie (Faivre-Finn et al., 2002). Ces constats sont cohérents avec les résultats d'autres pays européens (Gatta et al., 1998a) et des États-Unis (SEER, 2006).

\section{Validité scientifique de l'indicateur}

Des études solides démontrent l'intérêt du dépistage par la pratique routinière de coloscopies et par la recherche de sang occulte dans les selles (USPSTF, 2002) mais aussi l'intérêt de divers modes de traitement comme la chirurgie (Govindarajan et al., 2006) et la chimiothérapie (CCCG, 2000), même à des stades avancés. Les mêmes études donnent également à penser que les différentes options en matière de dépistage et de traitement sont insuffisamment utilisées. Les taux de survie au cancer colorectal ont été utilisés pour les comparaisons entre pays européens dans l'étude Eurocare (Quinn et al., 1998), pour les comparaisons entre pays européens et États-Unis (Gatta et al., 2000), et dans les rapports nationaux de nombreux pays.

\section{Constats}

Comme le montre le graphique 6.3.1, les taux de survie relatifs à cinq ans, qui mesurent la mortalité pouvant être attribuée à un diagnostic de cancer colorectal, se sont légèrement améliorés au fil du temps dans la plupart des pays. Des taux particulièrement élevés de $64.4 \%$ sont rapportés pour les États-Unis. Si les données laissent penser qu'il existe des différences importantes entre les pays ayant fourni des informations, la comparabilité de ces données est parfois limitée par le fait qu'elles concernent des périodes différentes. Les taux de survie en France et en Allemagne apparaissent inférieurs à la moyenne des pays ayant fourni des informations mais il faut garder à l'esprit que les données de ces deux pays sont antérieures de cinq à huit ans à celles de la majorité des pays ayant fourni des informations.

\section{Définition et écarts}

Le temps séparant un diagnostic de cancer d'un décès par cancer se compte habituellement en années, alors que pour d'autres maladies (par exemple, un AVC ou un infarctus aigu du myocarde) il peut se compter en jours ou, pire encore, le diagnostic et le décès peuvent être simultanés. Les taux de survie relatifs au cancer reflètent la proportion de patients atteints d'un certain type de cancer qui sont encore en vie au terme d'une période spécifiée (habituellement de cinq ans) par rapport à la proportion observée dans un groupe de référence de sujets non malades ayant une structure d'âge analogue. Cela signifie que les taux de survie relatifs saisissent la surmortalité pouvant être attribuée à un diagnostic de cancer. À titre d'illustration, un taux de survie relative de $80 \%$ ne signifie pas que $80 \%$ des personnes souffrant d'un cancer sont encore en vie au bout de cinq ans mais que $80 \%$ des patients qui devaient être en vie au terme des cinq ans, compte tenu de leur âge à l'époque du diagnostic, sont effectivement encore en vie. Les pays utilisent des méthodes différentes pour calculer ces taux de survie relatifs, ce qui est susceptible d'affecter les résultats. Ils font aussi état de données correspondant à des périodes différentes, ce qui peut influer sur les taux car la survie au cancer tend à s'améliorer au fil des ans. Les taux de survie ne sont pas corrigés pour prendre en compte le stade auquel la tumeur est diagnostiquée; il est donc impossible de distinguer l'impact relatif d'une détection précoce et d'un traitement plus performant. Enfin, on note de légères différences dans la manière dont les pays traitent les patients dont la trace a été perdue dans la période de suivi. 
6.3. SURVIE AU CANCER COLORECTAL

\subsubsection{Variation dans le temps du taux de survie relatif à cinq ans au cancer colorectal, population totale}

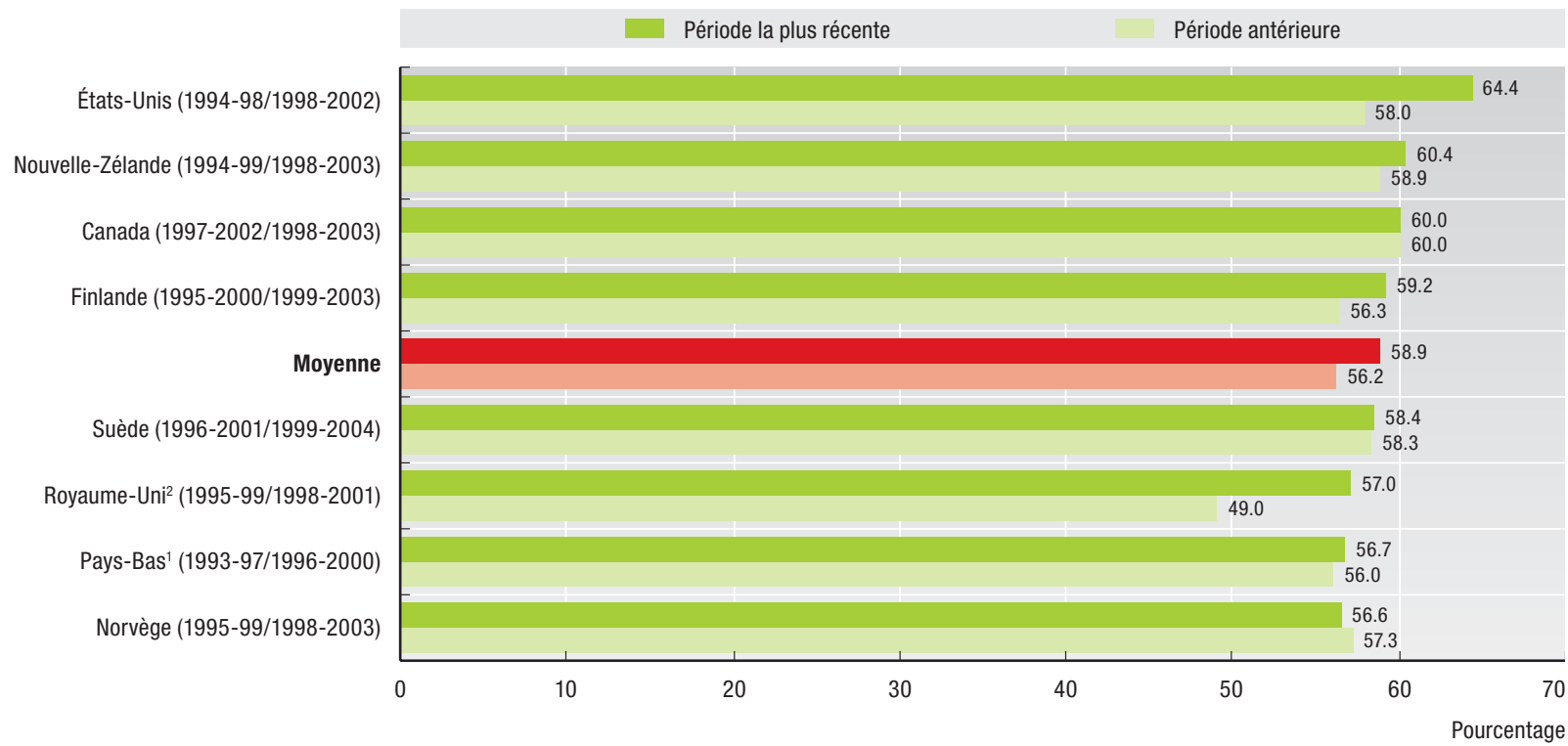

\subsubsection{Taux de survie relatif à cinq ans au cancer colorectal, par sexe, dernière période disponible}
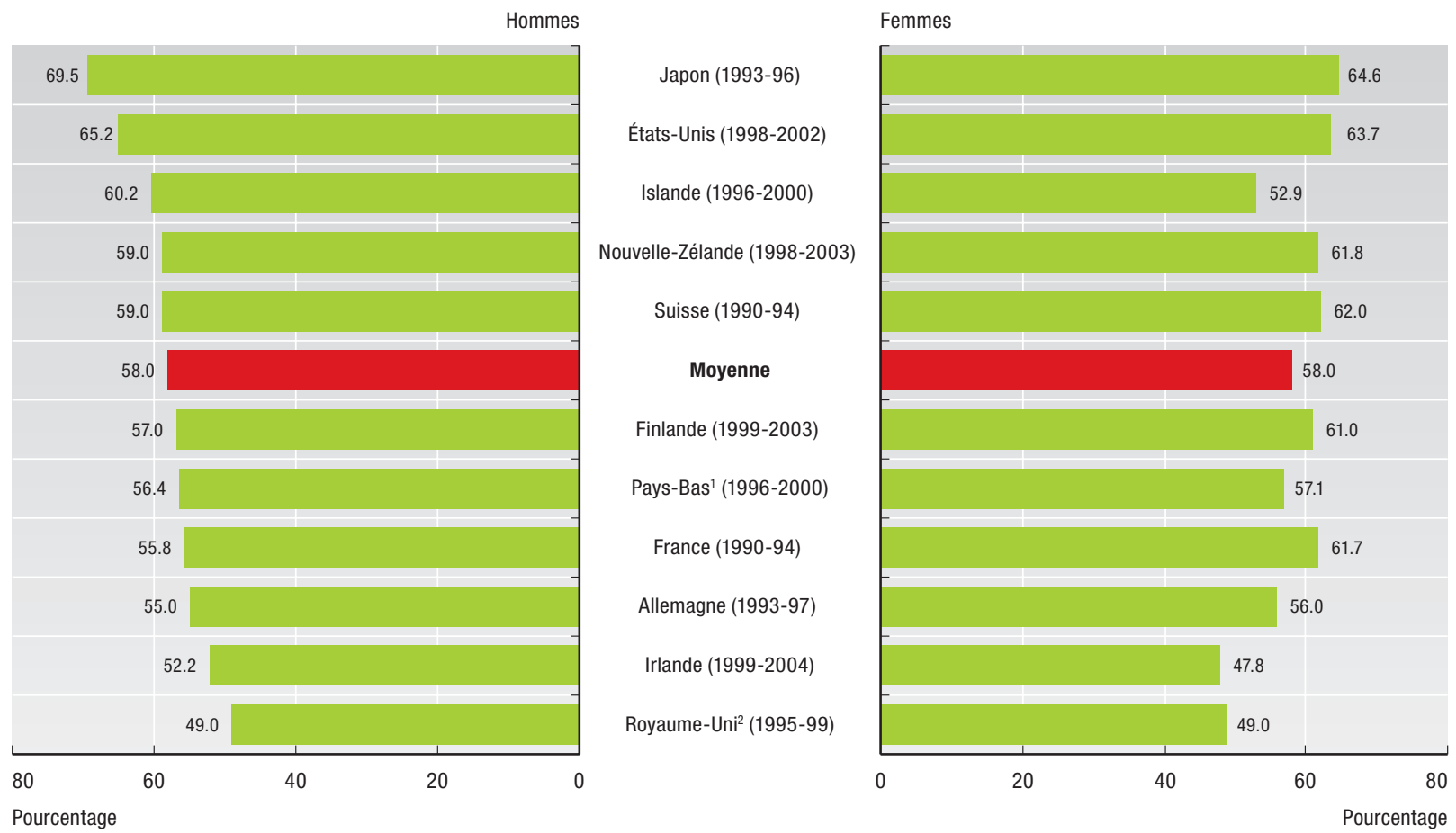

Note : Pour les deux graphiques, il s'agit des taux bruts, sauf pour la France qui a fourni des taux normalisés par âge selon la population Eurocare-3. Les différences entre taux bruts et normalisés pour un pays peuvent varier entre 2 et 4 points.

1. Aux Pays-Bas, les données pour 1993-97 concernent le cancer rectal. Pour la même période le taux de survie au cancer du colon est de $60 \%$. Pour la période 1996-2000 le taux de survie concerne le cancer colorectal.

2. Au Royaume-Uni, les chiffres concernent le cancer du colon.

Source: Projet sur les indicateurs de la qualité des soins de santé, OCDE 2007. 


\subsection{SURVIE AU CANCER DU SEIN ET DÉPISTAGE}

\section{Importance des indicateurs}

Le cancer du sein est la forme de cancer la plus fréquente chez la femme, avec une probabilité d'avoir un cancer du sein au cours de sa vie de l'ordre de $11 \%$ et une probabilité d'en mourir de l'ordre de $3 \%$ (Feuer et al., 2003). Autrement dit, une femme sur neuf contractera un cancer du sein à un moment ou à un autre de sa vie et une sur trente en mourra. Le montant global dépensé au titre des soins du cancer du sein représente habituellement environ 0.5 à $0.6 \%$ des dépenses totales de santé (OCDE, 2003a).

Le cancer du sein est un cancer pour lequel l'action des pouvoirs publics conjuguée aux progrès des techniques médicales a permis d'améliorer sensiblement le taux de survie. Une plus grande sensibilisation à la maladie et l'encouragement du dépistage par autopalpation et mammographie ont permis de détecter les tumeurs à un stade plus précoce. Si le débat sur le rapport coût-efficacité de mammographies régulières n'est pas totalement clos (Goetzsche et Nielsen, 2006), de nombreux pays ont choisi de généraliser le dépistage. Les avancées technologiques, comme l'association d'une chirurgie conservatrice à un traitement de radiothérapie et celle d'un traitement adjuvant de routine à un traitement au tamoxifène ou à une chimiothérapie, ont considérablement amélioré les taux de survie mais aussi la qualité de vie des patientes qui survivent (Sant et al., 2001).

\section{Validité scientifique des indicateurs}

Un grand nombre d'études cliniques ont démontré l'efficacité, en termes de survie, du dépistage et du traitement du cancer du sein. Mais on sait également que les schémas de soins et les ressources qui leur sont affectées varient considérablement entre pays de l'OCDE (OCDE, 2003). Les taux de survie au cancer du sein ont été utilisés dans l'étude Eurocare (Quinn et al., 1998) pour les comparaisons entre pays européens et États-Unis (Gatta et al., 2000) et dans les rapports nationaux de nombreux pays.

\section{Constats}

Comme le montre le graphique 6.4.1, des différences importantes subsistent dans les taux de survie relatifs à cinq ans, qui saisissent la surmortalité pouvant être attribuée à un diagnostic de cancer du sein dans les pays de l'OCDE. En Islande, par exemple, la proportion de femmes ayant un cancer du sein qui vivront aussi longtemps que des femmes non affectées par cette pathologie, est supérieure de 10 points de pourcentage à celle observée en Irlande. D'autre part, les taux de survie au cancer du sein ont augmenté dans pratiquement tous les pays ayant des données tendancielles (graphique 6.4.2).

Le graphique 6.4.3 montre que, dans la plupart des pays, plus de $50 \%$ des femmes passent chaque année une mammographie. Les résultats de l'étude Eurocare indiquent que des taux de survie plus élevés sont observés dans les pays où la détection est plus précoce (Sant et al., 2003). Les séries temporelles sur les taux de dépistage par mammographie étant limitées, il n'est pas possible, avec les données actuellement disponibles, d'évaluer la robustesse de la relation entre taux de dépistage et taux de survie. La disponibilité de matériel de radiothérapie et de mammographie n'est pas étroitement associée au taux de survie à cinq ans, ce qui laisse penser que l'impact des processus de soins est probablement plus grand que celui de la seule infrastructure (graphique 6.4.4).

Les taux de survie ne semblent pas non plus liés à des différences nationales en termes de pratiques chirurgicales. Comme le montre le tableau A.6.4 de l'annexe A, dans la plupart des pays, les admissions à l'hôpital pour une chirurgie conservatrice du sein sont environ deux fois plus nombreuses que les admissions pour une mastectomie radicale, sans que le lien avec le taux de survie soit clairement établi. Les États-Unis sont une exception, mais le nombre très faible des admissions à l'hôpital pour une chirurgie conservatrice du sein reflète simplement le fait que, dans ce pays, ces interventions sont essentiellement pratiquées en clinique externe (AHRQ 2006).

\section{Définition et écarts}

Voir la définition du taux de survie relatif et les limitations générales à la comparabilité des données sous l'indicateur 6.3, «Survie au cancer colorectal ».

Les taux de dépistage par mammographie mesurent la proportion de femmes d'un âge donné (ici, généralement de 50 à 69 ans) ayant eu une mammographie au cours de l'année écoulée. Comme les politiques relatives au groupe d'âge cible et à la périodicité du dépistage diffèrent d'un pays à l'autre, les taux peuvent se fonder sur la politique spécifique de chaque pays. Le fait que certains pays mesurent le taux de dépistage sur la base de données d'enquête et d'autres sur la base de données de programme peut influer sur les résultats. Si un pays a un programme organisé de dépistage mais que des femmes sont dépistées en dehors de ce programme, les taux peuvent être sous-évalués. Parallèlement, les enquêtes peuvent sous-estimer les taux du fait du biais de rappel. 
6.4.1. Taux de survie relatif à cinq ans au cancer du sein, dernière période disponible

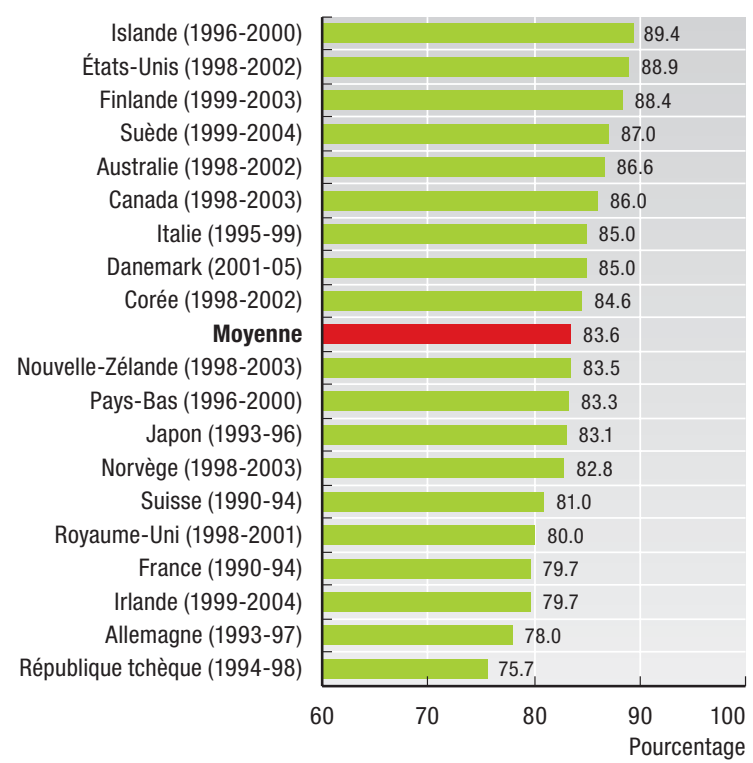

\subsection{SURVIE AU CANCER DU SEIN ET DÉPISTAGE}

\subsubsection{Variation dans le temps du taux de survie relatif à cinq ans au cancer du sein}

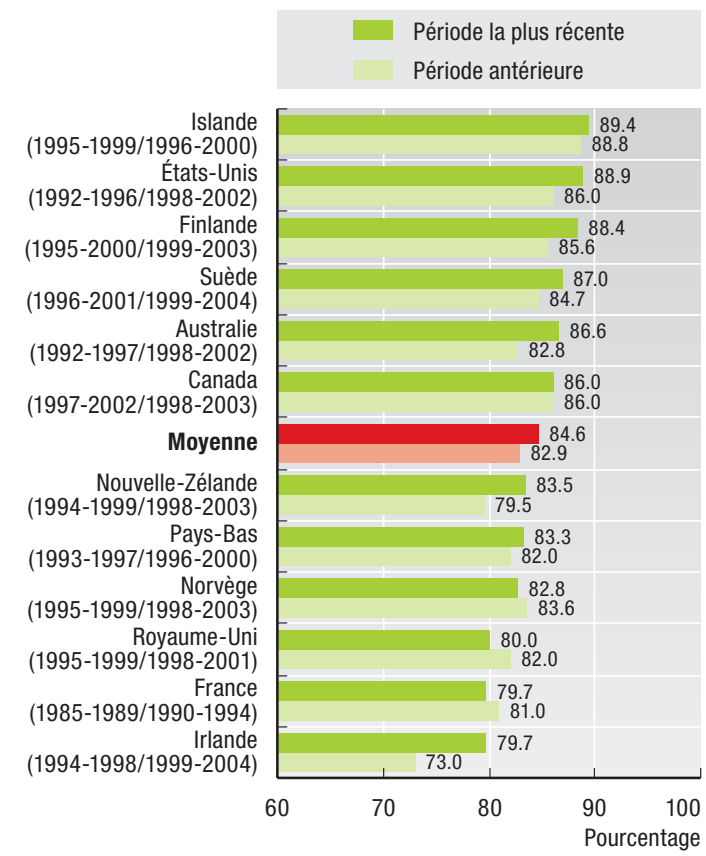

Note : Les taux de survie relatifs au cancer du sein sont des taux bruts, sauf pour la France qui a fourni des taux normalisés par âge selon la population Eurocare-3. Les différences entre taux bruts et taux normalisés pour un pays peuvent varier entre 2 et 4 points.

\subsubsection{Dépistage par mammographie, pourcentage de femmes âgées de 50 à 69 ans, 2005}

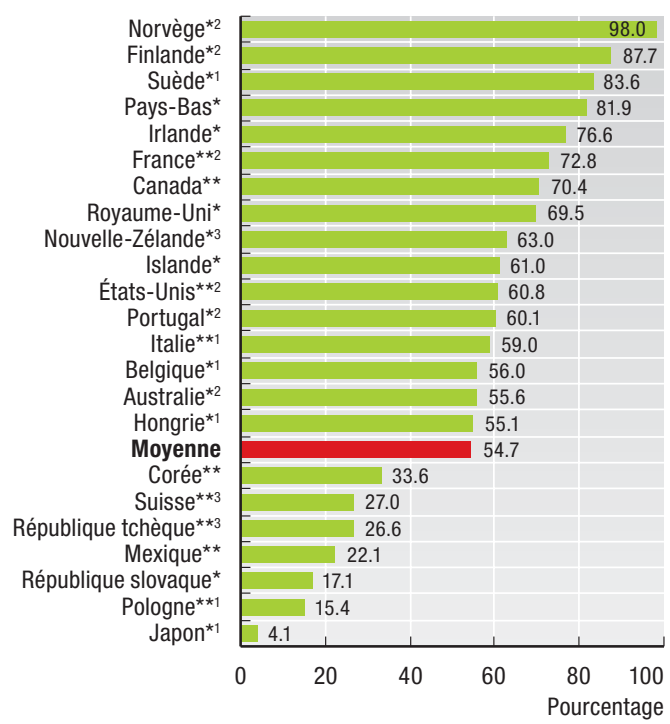

* Données découlant de programmes nationaux.

** Données d'enquêtes.

1. 2004. 2. 2003. 3. 2002.

\subsubsection{Taux de survie relatif à cinq ans au cancer du sein et nombre d'équipements en radiothérapie (évalué deux ans auparavant)}

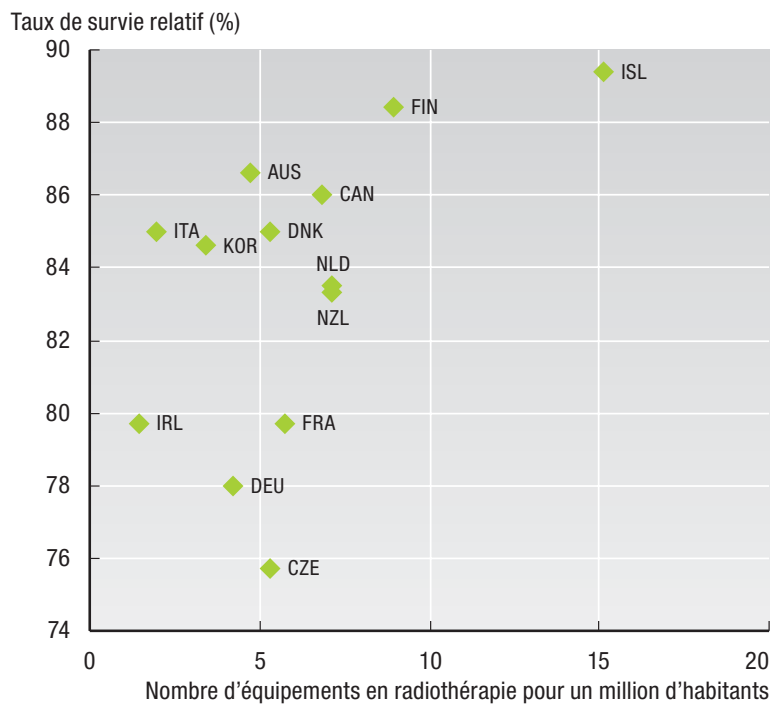

Note : Les données pour les unités de radiothérapie se réfèrent pour l'Irlande à 1981, la France à 1988, la République tchèque et l'Allemagne à 1991, l'Islande et l'Italie à 1993, les Pays-Bas à 1994, l'Australie, le Canada, la Finlande, la Corée et la Nouvelle-Zélande à 1996 et le Danemark à 2000.

Source : Projet sur les indicateurs de la qualité des soins de santé, OCDE 2007. Eco-Santé OCDE 2007 (dépistage par mammographie et équipements en radiothérapie). 


\subsection{SURVIE AU CANCER DU COL DE L'UTÉRUS ET DÉPISTAGE}

\section{Importance des indicateurs}

$\mathrm{Si}$, dans les pays industrialisés, le cancer du col de l'utérus n'est plus une des formes de cancer les plus répandues ou responsables d'un grand nombre de décès parmi les femmes, il est néanmoins d'un grand intérêt pour les décideurs car il peut être largement prévenu. Des examens pelviens réguliers et la pratique du frottis cervicovaginal permettent d'identifier les lésions précancéreuses, qui peuvent être traitées efficacement. Un dépistage régulier accroît également la probabilité de diagnostiquer les tumeurs malignes à un stade précoce, ce qui améliore la survie et peut permettre un traitement curatif sans ablation totale de l'utérus (Gatta et al., 1998b). Les pays de l'OCDE ont mis en place des programmes de dépistage, mais la périodicité du dépistage et les groupes cibles varient. De plus, la découverte du fait que le cancer du col de l'utérus est dû à certaines formes du virus HPV (Human Papilloma Virus) a permis le développement de vaccins prometteurs (Harper, 2006). Cela devrait avoir des implications importantes, en particulier lorsque le manque de ressources rend difficile le maintien de programmes globaux de dépistage.

\section{Validité scientifique des indicateurs}

De nombreuses études cliniques ont clairement démontré l'efficacité du dépistage et du traitement du cancer du col de l'utérus avec l'amélioration du taux de survie qui en résulte. Mais on sait également que les schémas de soins et les ressources qui leur sont affectées varient considérablement entre pays de l'OCDE (OCDE, 2003a). L'évaluation et la comparaison des taux de survie donnent donc une idée de l'efficacité des différents systèmes de santé. Les taux de survie au cancer du col de l'utérus ont été utilisés pour les comparaisons entre pays européens dans l'étude Eurocare (Gatta et al., 1998b), pour les comparaisons entre pays européens et États-Unis (Gatta et al., 2000), et dans les rapports nationaux de nombreux pays.

\section{Constats}

Comme le montre le graphique 6.5.1, les taux de survie relatifs à cinq ans, qui saisissent la surmortalité pouvant être attribuée à un diagnostic de cancer du col de l'utérus, révèlent des différences importantes dans les pays ayant fourni des informations.

Les données longitudinales de l'Australie (Taylor et al., 2001) et du Royaume-Uni (Peto et al., 2004) ont démontré l'impact considérable des programmes de dépistage sur la survie au cancer du col de l'utérus. Le graphique 6.5.3 montre que, dans la plupart des pays de l'OCDE, une majorité de femmes ont eu un test de dépistage du cancer du col de l'utérus sur les trois dernières années. Des programmes de dépistage efficaces peuvent toutefois paraître réduire les taux de survie dans la mesure où le dépistage permet une détection précoce et un traitement de lésions précancéreuses qui ne seront peutêtre pas comptabilisées dans les statistiques des cancers. Les cas qui sont rapportés représentent essentiellement des formes plus agressives de cancer du col de l'utérus, ce qui peut conduire à un apparent recul des taux de survie.

Alors que les pays où le taux de survie est le plus élevé (par exemple, la Nouvelle-Zélande et l'Islande) tendent à avoir des taux de dépistages élevés, le Japon qui affiche le taux de dépistage le plus bas (23.7 \%) et les États-Unis qui affichent le taux de dépistage le plus élevé (82.6\%) ont des taux de survie analogues.

\section{Définition et écarts}

Voir la définition du taux de survie relatif et les limitations générales à la comparabilité des données sous l'indicateur 6.3, "Survie au cancer colorectal ».

Les taux de dépistage du cancer du col de l'utérus reflètent la proportion de femmes d'un âge donné (ici, généralement de 20 à 69 ans) ayant eu un test de dépistage au cours d'une période donnée (généralement de trois ans). Toutefois, comme les politiques relatives à la périodicité du dépistage et aux groupes d'âge diffèrent d'un pays à l'autre, les taux peuvent se fonder sur la politique spécifique de chaque pays. Le fait que certains pays mesurent le taux de dépistage sur la base de données d'enquête et d'autres sur la base de données de programme peut influer sur les résultats. Si un pays a un programme organisé de dépistage mais que des femmes sont dépistées en dehors de ce programme, les taux peuvent être sous-évalués. Parallèlement, les enquêtes peuvent sous-estimer les taux du fait du biais de rappel. 
6.5. SURVIE AU CANCER DU COL DE L'UTÉRUS ET DÉPISTAGE

\subsubsection{Taux de survie relatif à cinq ans du cancer du col de l'utérus, dernière période disponible}

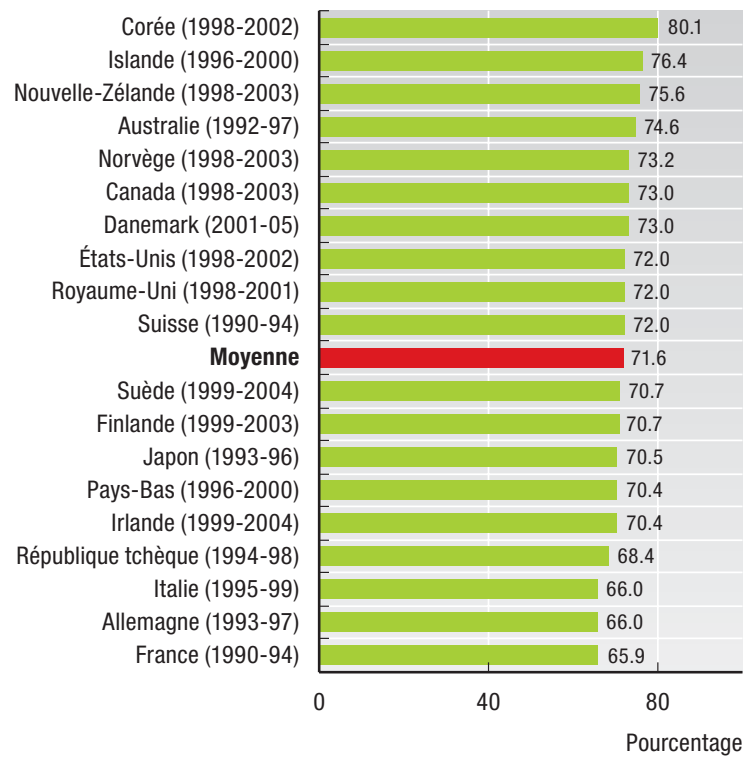

\subsubsection{Variation dans le temps du taux de survie relatif à cinq ans du cancer du col de l'utérus}

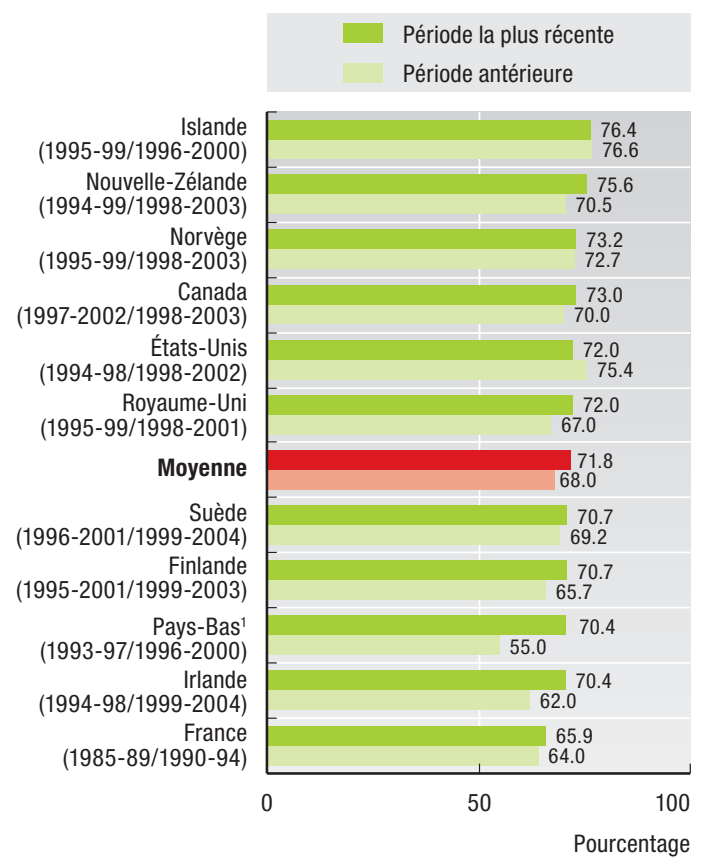

1. Aux Pays-Bas, le taux de survie pour la période 1993-97 fait référence aux femmes de plus de 60 ans. Le taux de survie relatif pour les femmes de moins de 60 ans est de $76 \%$.

Note : Les taux de survie du cancer du col de l'utérus sont des taux bruts, sauf pour la France qui a fourni des taux normalisés par âge selon la population Eurocare-3. Les différences entre taux bruts et taux normalisés pour un pays peuvent varier entre 2 et 4 points.

\subsubsection{Dépistage du cancer du col de l'utérus pour les femmes âgées de 20 à 69 ans, 2005}

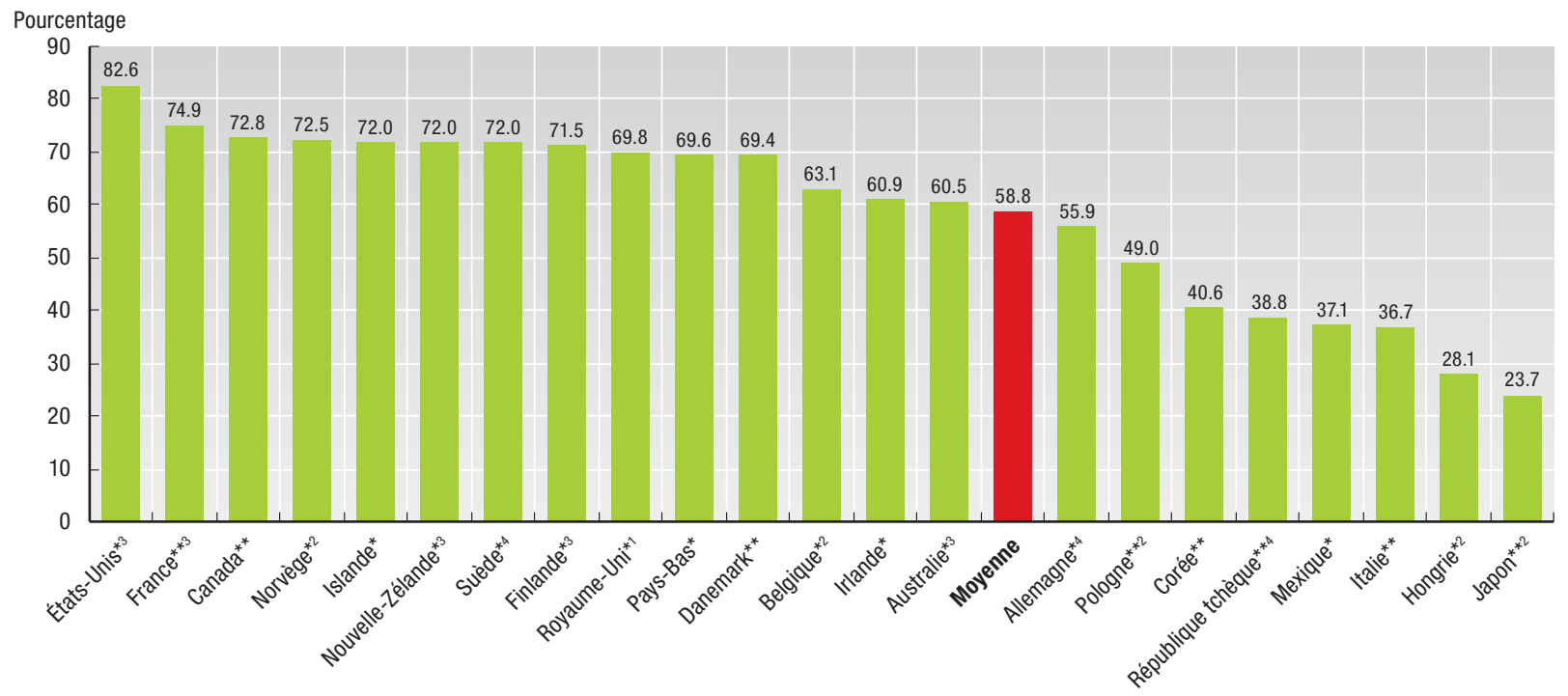

* Données découlant de programmes nationaux.

** Données d'enquêtes.

1. 2006. 2. 2004. 3. 2003. 4. 2002.

Source: Projet sur les indicateurs de la qualité des soins de santé, OCDE 2007. Eco-Santé OCDE 2007 (Dépistage du cancer du col de l'utérus). StatLink Ains http://dx.doi.org/10.1787/130333422085 


\subsection{ADMISSIONS À L'HÔPITAL ET MORTALITÉ ÉVITABLES DUES À L'ASTHME}

\section{Importance des indicateurs}

L'asthme est la maladie chronique la plus courante chez l'enfant et sa prévalence s'est accrue au cours des dernières décennies. Les recherches donnent à penser que l'asthme pourrait être en fait un ensemble de maladies différentes présentant des symptômes analogues (Wenzel, 2006). L'asthme est intrinsèquement une maladie qui se traite par des soins médicaux appropriés.

En 2002, le coût annuel estimé du traitement de l'asthme aux États-Unis était de USD 14 milliards et les soins dispensés à l'hôpital représentaient près d'un tiers des coûts directs (NHLBI, 2002). Les estimations pour l'Union européenne suggèrent un montant annuel de coûts médicaux de EUR 17.7 milliards et des pertes de productivité de EUR 9.8 milliards (ERS, 2003). Au Japon, le coût du traitement de l'asthme représente près de $2 \%$ des dépenses annuelles de soins médicaux (Tanihara et Kobayashi, 2004).

\section{Validité scientifique des indicateurs}

Les soins primaires devraient permettre de traiter efficacement les adultes et les enfants souffrant d'asthme. Le traitement au moyen d'agents anti-inflammatoires, comme les corticostéroïdes inhalés et les inhibiteurs de leukotriène, sont largement à même d'éviter une aggravation et, si elle se produit, les corticostéroïdes et les bronchodilatateurs devraient permettre d'éviter le recours à l'hospitalisation. Si les directives et les protocoles actuels donnent des consignes claires pour le traitement de l'asthme, les études laissent penser que, bien souvent, ces traitements ne sont pas suffisamment utilisés en pratique (Mattke et al., 2006; Halterman, 2001; et AAFA, 2005).

Conséquence de cette insuffisance de traitement, les patients souffrant d'asthme peuvent devoir être hospitalisés. Les taux d'admission pour asthme et les taux de mortalité due à l'asthme ont été utilisés pour évaluer la qualité des soins. Au Royaume-Uni, par exemple, le service national de la santé (NHS) a désigné l'admission pour asthme comme étant un indicateur de performance de haut niveau et, aux États-Unis, le rapport national sur la qualité des soins de santé indique les taux d'admission pour asthme dans les services de pédiatrie et les services pour adultes (AHRQ, 2006). Les taux de mortalité due à l'asthme ont été utilisés comme indicateurs pour évaluer la qualité des soins dans les comparaisons des systèmes de santé de la Communauté européenne, du Royaume-Uni, de l'Australie et de plusieurs autres pays (Charlton et al., 1983; Holland et al., 1997; Manuel et Mao, 2002; AIHW, 2003).

\section{Constats}

Le graphique 6.6.1 montre que les taux d'admission à l'hôpital pour asthme varient fortement entre pays de l'OCDE. Si, dans une année, une moyenne de six adultes sur 10000 sont admis pour un problème d'asthme dans un hôpital, on note une grande disparité entre les pays. La Finlande (13) et les États-Unis (12) font état de taux d'admission relativement élevés tandis que le Mexique (2) et la Suède (3) font état des taux les plus bas.

Le graphique 6.6.2 montre une variation considérable entre les pays des taux de mortalité due à l'asthme. C'est au Royaume-Uni et en Australie que ces taux sont le plus élevés, en Islande, en Finlande et en Suisse qu'ils sont le plus faibles. Les chiffres sont cohérents avec les données rapportées par le projet GINA, qui montrent une forte prévalence de l'asthme en Amérique du Nord, au Royaume-Uni, en Irlande, en Australie et en Nouvelle-Zélande (Massoli et al., 2004). Les données tendancielles montrent une amélioration générale, excepté au Canada et en Suède où les taux de mortalité demeurent toutefois relativement bas.

Les taux d'admission à l'hôpital pour asthme sont généralement liés aux taux de mortalité due à l'asthme, comme le montre le graphique 6.6.3.

\section{Définition et écarts}

Pour calculer le taux de mortalité due à l'asthme, on prend le nombre annuel de décès pour 100000 habitants dans la classe d'âge 5-39 ans. Dans les pays à faible population, une certaine prudence s'impose car les décès liés à l'asthme étant relativement rares, cela peut conduire à une instabilité des taux d'une année sur l'autre. Comme les causes des décès sont tirées des certificats de décès, des inexactitudes de codage peuvent affecter les résultats. Toutefois, les analyses initiales n'ont révélé aucun indice de différences systématiques de codage entre les pays ayant fourni des informations (Mattke et al., 2006). Bien que des différences de prévalence de la maladie peuvent influer sur les taux de mortalité, l'hypothèse sur laquelle repose cet indicateur est que, compte tenu des possibilités actuelles de traitement, aucun décès dû à l'asthme ne doit survenir à un âge très jeune.

Le taux d'admission à l'hôpital pour asthme se définit comme le nombre annuel d'admissions à l'hôpital de personnes de 18 ans et plus pour une population de 10000 personnes appartenant à cette classe d'âge. La comparabilité peut être limitée par des pratiques de codage différentes entre pays. Bien que des différences de prévalence de la maladie peuvent influer sur les taux d'admission pour asthme, l'hypothèse sur laquelle repose cet indicateur est que, compte tenu des possibilités actuelles de traitement pour prévenir les crises aiguës, aucune admission à l'hôpital ne devrait être nécessaire. 


\section{QUALITÉ DES SOINS • LES AFFECTIONS CHRONIQUES}

\subsection{ADMISSIONS À L'HÔPITAL ET MORTALITÉ ÉVITABLES DUES À L'ASTHME}

6.6.1. Taux de mortalité pour cause d'asthme pour 100000 habitants âgés de 5 à 39 ans, 2005

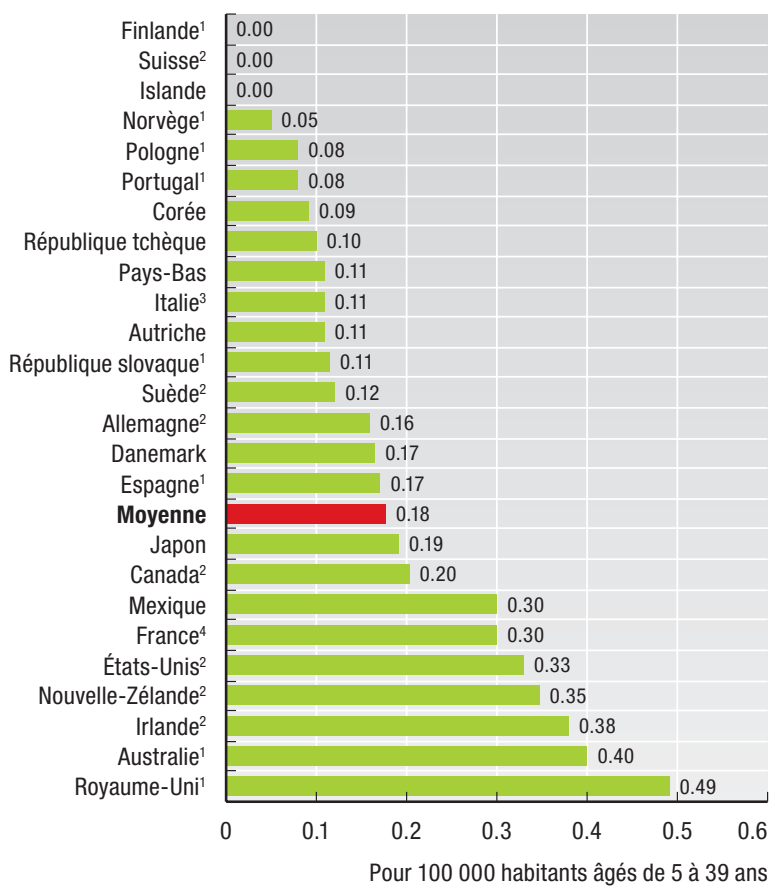

1. 2004. 2. 2003. 3. 2002. 4. 2001.
6.6.2. Taux d'hospitalisation pour cause d'asthme pour 10000 habitants âgés de 18 ans et plus, 2005

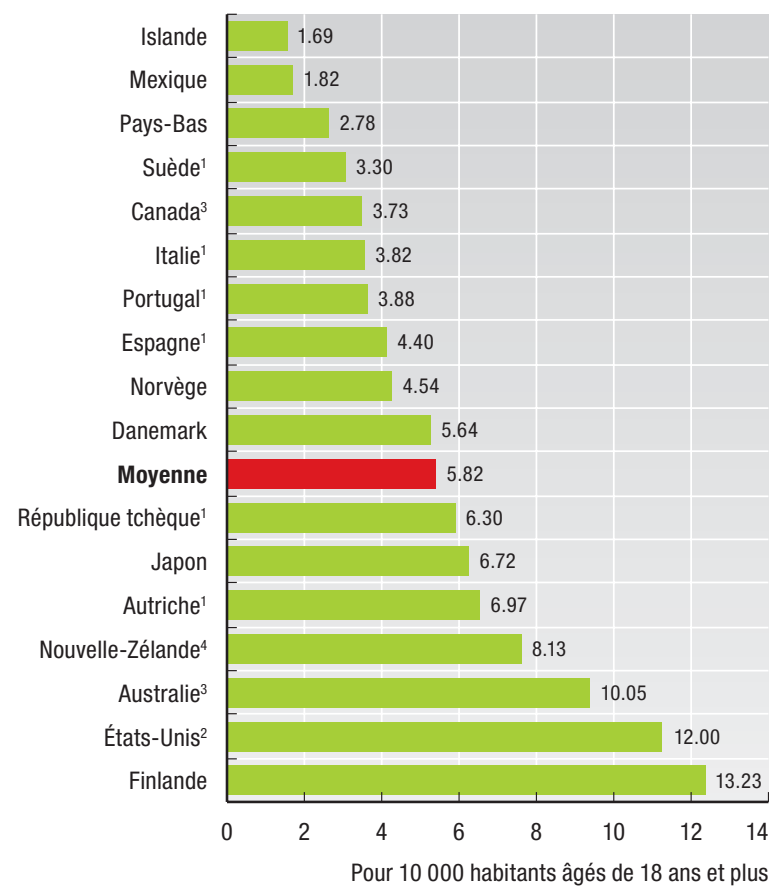

1. 2004. 2. 2002. 3. 2004-05. 4. 2005-06.

\subsubsection{Taux de mortalité pour cause d'asthme, population âgée de 5 à 39 ans et taux d'hospitalisation}

pour cause d'asthme, population âgée de 18 ans et plus, 2005

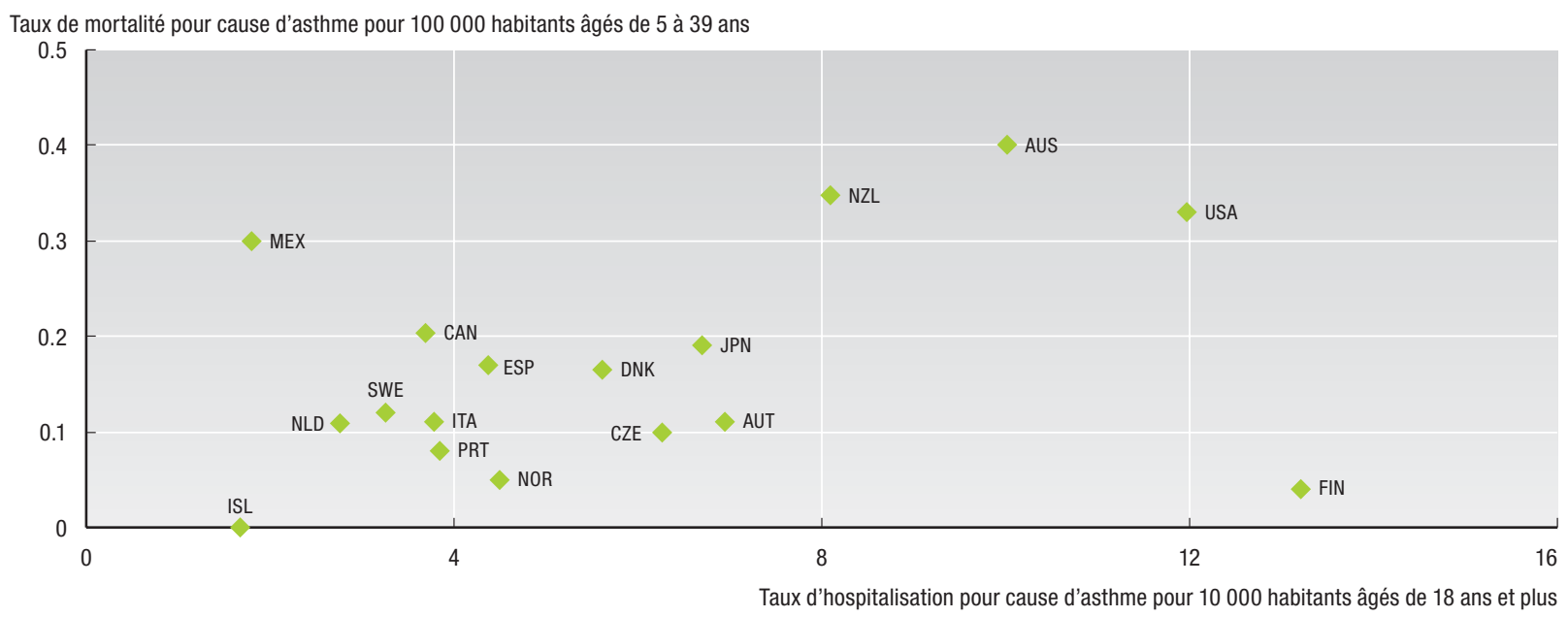

Note: Voir les deux graphiques précédents pour les années auxquelles se rapportent chacun des deux taux.

Source: Projet sur les indicateurs de la qualité des soins de santé, OCDE 2007. 


\subsection{EXAMENS ANNUELS DE LA RÉTINE POUR LES DIABÉTIQUES}

\section{Importance de l'indicateur}

Le diabète est devenu l'un des principaux problèmes de santé publique du $\mathrm{XXI}^{\mathrm{e}}$ siècle. Plus de 150 millions d'adultes dans le monde sont touchés par cette maladie et ce chiffre devrait doubler dans les 25 prochaines années (King et al., 1998; Zimmet et al., 2001). Cette progression de la maladie est due, dans une large mesure, à la progression de l'obésité (voir l'indicateur 3.3 "Surcharge pondérale et obésité »). Le caractère épidémique du diabète requiert de consacrer des ressources au traitement de cette maladie et à ses complications. Le diabète est la première cause de cécité dans les pays industrialisés (Ghafour et al., 1983) et la cause la plus répandue de maladies rénales en phase terminale aux États-Unis, en Europe et au Japon (voir l'indicateur 4.12 "Traitement des insuffisances rénales »). Les individus souffrant d'un diabète de type II ont un risque de maladie cardio-vasculaire deux à quatre fois plus élevé que celui des non-diabétiques (Haffner, 2000). Les amputations non traumatiques sont quinze fois plus fréquentes parmi les patients diabétiques que pour l'ensemble de la population (Ollendorf et al., 1998). Alors que les avancées récentes de la médecine ont conduit à une réduction de la mortalité due aux maladies cardiovasculaires dans les pays de l'OCDE, cette évolution positive n'a pas été constatée pour les patients diabétiques, ce qui laisse penser que ces avancées sont probablement moins efficaces pour les sujets diabétiques (Gu et al., 1999).

En 2002, le coût du diabète aux États-Unis était estimé à USD 92 milliards au titre des dépenses médicales et USD 40 milliards au titre des pertes de productivité (ADA, 2003). Selon les projections de la Fédération internationale du diabète, à l'horizon 2025, les pays consacreront au traitement de la maladie 7 à $13 \%$ de leurs budgets de santé (FID, 2003).

Le poids du diabète dans les dépenses de santé pourrait être largement réduit si les connaissances médicales actuelles se traduisaient mieux en traitements et prévention secondaire. Des résultats empiriques solides indiquent qu'une modification des modes de vie (perte de poids et plus grande activité physique) peut éviter la survenue du diabète chez les personnes à haut risque (Tuomilehto et al., 2001). Il est bien établi qu'un meilleur contrôle de la glycémie réduit les lésions organiques et les complications vasculaires (Diabetes Control and Complications Trial Research Group, 1996). Des études donnent également à penser que ces mesures peuvent réduire les coûts en l'espace de un à deux ans (Wagner et al., 2001). Or, des données empiriques révèlent que ces pratiques sont sous-utilisées (McGlynn et al., 2003).

\section{Validité scientifique de l'indicateur}

Aux États-Unis, le développement, la spécification et l'expérimentation de mesures pour évaluer la qualité des soins aux diabétiques ont fait des progrès considérables (Fleming et al., 2001; NDQIA, 2005). Des efforts ont été menés parallèlement en Italie (Pellegrini et al., 2003) et par le groupe d'investigation de CODE-2 dans huit pays européens (Jonsson, 2002). Étant donné la fréquence des complications ophtalmologiques chez les diabétiques, des examens annuels de l'œil sont l'une des pratiques de soins aux diabétiques les plus simples et les plus universellement admises. Cet examen est donc l'un des indicateurs de processus sur la qualité des soins recommandés pour la comparaison des traitements du diabète au niveau international (Greenfield et al., 2004).

\section{Constats}

Le graphique 6.7.1 montre que dans les pays ayant fourni des informations, un peu plus de la moitié des patients diabétiques font des examens annuels de la rétine mais les disparités entre pays sont grandes. Même au Royaume-Uni, pays le plus performant pour cette mesure, près d'un diabétique sur six ne fait pas ce test simple et utile. Comme le montre le graphique 6.7.2, il n'existe pas de corrélation forte entre les taux de test et les estimations de la prévalence. En Allemagne par exemple, pays qui fait état de la plus forte prévalence du diabète (environ $10 \%$ ), le taux des examens de la rétine est proche du taux moyen.

\section{Définition et écarts}

Le taux d'examens annuels de la rétine de l'œil exprime la proportion de patients diabétiques (types I et II) bénéficiant d'un examen dans une année donnée. Le fait que certains pays mesurent ce taux d'examens sur la base de données d'enquête et d'autres sur la base de données établies lors des contacts avec le système de santé peut influer sur les résultats. Les pays utilisent aussi des méthodes différentes pour déterminer le nombre de diabétiques éligibles à cet examen. Certains utilisent des tests en laboratoire d'échantillons représentatifs, d'autres des données d'enquête, d'autres encore des informations sur les diagnostics enregistrées d'après des contacts avec le système de soins de santé. Ces méthodes différentes peuvent influer sur le dénominateur de l'indicateur et donc sur les résultats. Enfin, dans certains pays n'ayant pas utilisé d'échantillons représentatifs au niveau national pour calculer cet indicateur, le taux national effectif peut être différent du taux indiqué. 


\section{QUALITÉ DES SOINS • LES AFFECTIONS CHRONIQUES}

\subsection{EXAMENS ANNUELS DE LA RÉTINE POUR LES DIABÉTIQUES}

\subsubsection{Taux d'examens de la rétine parmi les personnes diabétiques de 18 à 75 ans, 2005}

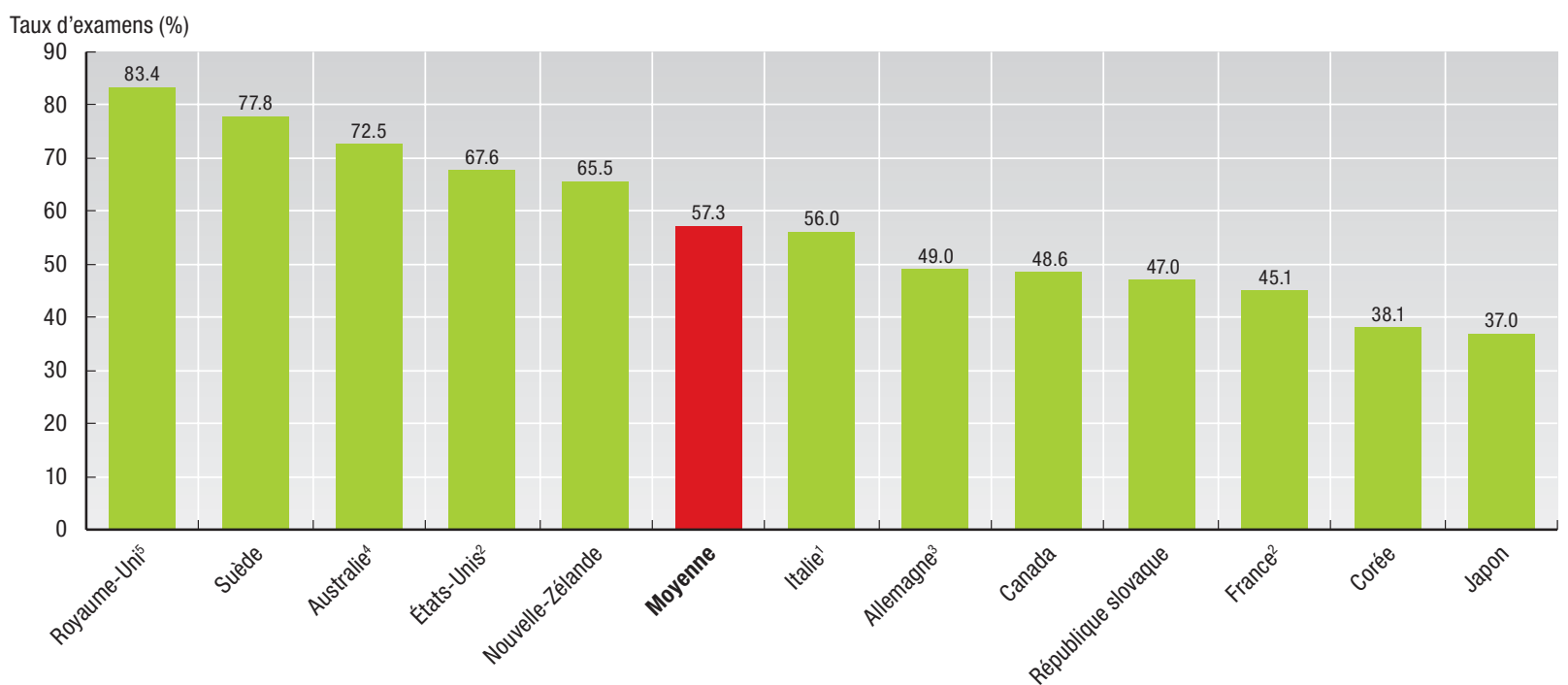

Note : Le taux pour l'Italie se rapporte aux patients diabétiques qui sont suivis dans des cliniques spécialisées (estimés à $60 \%$ de la population totale diabétique).

1. 2003. 2. 2002 . 3. 1998 . 4. $1999-2000$. 5. $2004-05$.

\subsubsection{Taux d'examens de la rétine parmi les personnes diabétiques de 18 à 75 ans, 2005 (ou dernière année disponible) et taux de prévalence du diabète, 2003}

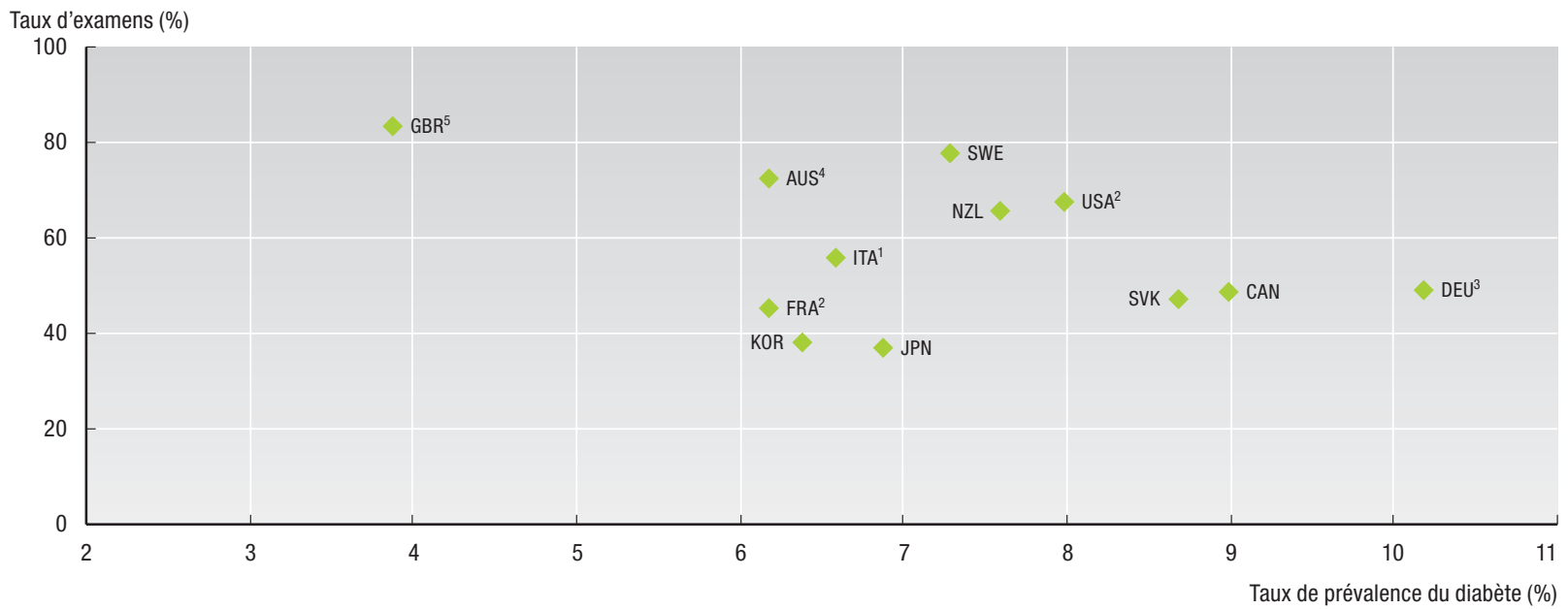

Note pour le taux d'examens de la rétine : Le taux pour l'Italie se rapporte aux patients diabétiques qui sont suivis dans des cliniques spécialisées (estimés à $60 \%$ de la population totale diabétique).

1. 2003. 2. 2002 . 3. 1998 . 4. 1999-2000. 5. 2004-05.

Source : Projet sur les indicateurs de la qualité des soins de santé, OCDE 2007. Les estimations des taux de prévalence du diabète proviennent de la Fédération internationale du diabète (2003), Diabetes Atlas, $2^{\mathrm{e}}$ édition.

StatLink ints http://dx.doi.org/10.1787/130360243841 


\subsection{VACCINATION DES PERSONNES ÂGÉES CONTRE LA GRIPPE}

\section{Importance de l'indicateur}

La grippe est une maladie infectieuse courante qui affecte des personnes de tous âges. On estime que le nombre de cas de grippe enregistrés chaque année aux États-Unis est de 20 à 30 millions (CDC, 2006). La plupart des personnes qui contractent la maladie se rétablissent rapidement mais les personnes âgées et les personnes souffrant d'affections chroniques sont plus exposées au risque de complications, voire de décès. Entre 1979 et 2001, la grippe a entraîné entre 54000 et 430000 hospitalisations par épidémie et une moyenne de 36000 décès par an aux États-Unis (CDC, 2006). L'impact de la grippe sur la population active est considérable même si la morbidité et la mortalité due à la grippe touchent principalement les personnes âgées et les personnes souffrant d'affections chroniques (Keech et al., 1998). Des souches particulièrement virulentes du virus, semblables au sous-type H5N1 de la grippe aviaire, pourraient occasionner des pandémies dont l'impact serait considérable.

\section{Validité scientifique de l'indicateur}

Dans les dix dernières années, l'immunisation contre la grippe des personnes âgées s'est considérablement développée dans les pays de l'OCDE afin d'éviter la contraction de la maladie, l'hospitalisation et la mortalité de cette catégorie de population davantage exposée au risque de complications graves. La vaccination contre la grippe des personnes âgées et des patients souffrant d'affections chroniques est fortement recommandée en Europe, aux États-Unis et dans d'autres pays (Nicholson, 1995).

\section{Constats}

$\mathrm{Au}$ cours des dix dernières années, la vaccination contre la grippe des personnes âgées est devenue une pratique plus courante : le taux moyen de vaccination pour un groupe cohérent de pays est passé de $50 \%$ en 1996 à environ $60 \%$ en 2005 (graphique 6.8.1). Les taux de vaccination sur cette période ont augmenté dans tous les pays ayant fourni des informations, à l'exception de la République slovaque. Au Danemark et en Finlande, où ces taux étaient généralement bas, ils ont été multipliés par deux entre 2001/2002 et 2005. Un certain nombre de facteurs ont contribué à l'augmentation des taux d'immunisation contre la grippe dans les pays de l'OCDE : une plus large acceptation par les patients et les praticiens des services de prévention, une meilleure prise en charge de ces vaccins par la sécurité sociale, et une offre accrue de ce service par des prestataires de soins autres que les médecins (Singleton et al., 2000). En Finlande, l'un des principaux facteurs expliquant la forte progression de la vaccination ces dernières années a été un élargissement des personnes éligibles à la vaccination gratuite : autrefois limitée aux personnes souffrant d'affections chroniques, la gratuité est désormais étendue à toutes les personnes de plus de 65 ans.

Un certain nombre d'obstacles restent néanmoins à lever, si l'on veut accroître la couverture vaccinale. Aux États-Unis, par exemple, les raisons le plus souvent invoquées par les personnes âgées pour ne pas se faire vacciner contre la grippe sont en premier lieu l'ignorance du fait que la vaccination est nécessaire et ensuite la crainte de contracter la maladie ou des effets secondaires (CDC, 2004). La pénurie ou la non-disponibilité du vaccin peut être également une cause importante de nonvaccination aux États-Unis et dans d'autres pays, au cours d'une année donnée.

\section{Définition et écarts}

Le taux d'immunisation contre la grippe fait référence au nombre de personnes de 65 ans et plus qui ont été vaccinées contre la grippe au cours d'une année, divisé par le nombre total de personnes de plus de 65 ans. La principale limitation en termes de comparabilité des données tient à l'utilisation de sources de données différentes susceptibles de conduire à différents types d'erreurs et de biais. Dans un grand nombre de pays, les données proviennent d'enquêtes auprès de la population et peuvent être faussées par un taux de rappel incorrect. Dans certains pays, les données provenant d'enquêtes peuvent également exclure les personnes placées dans des établissements. Dans d'autres pays, les données proviennent de sources administratives, lesquelles ne saisissent probablement que les vaccinations effectuées dans le cadre du système de paiement couvert par les données. On ne sait pas dans quelle mesure l'utilisation de données administratives dans certains pays risque de sous-estimer (ou surestimer) les chiffres par rapport aux pays qui utilisent des données d'enquête. 


\section{QUALITÉ DES SOINS • LES MALADIES TRANSMISSIBLES}

6.8. VACCINATION DES PERSONNES ÂGÉES CONTRE LA GRIPPE

6.8.1. Taux de vaccination contre la grippe parmi les personnes âgées de 65 ans et plus, 2005

Couverture de la population âgée de 65 ans et plus (\%)

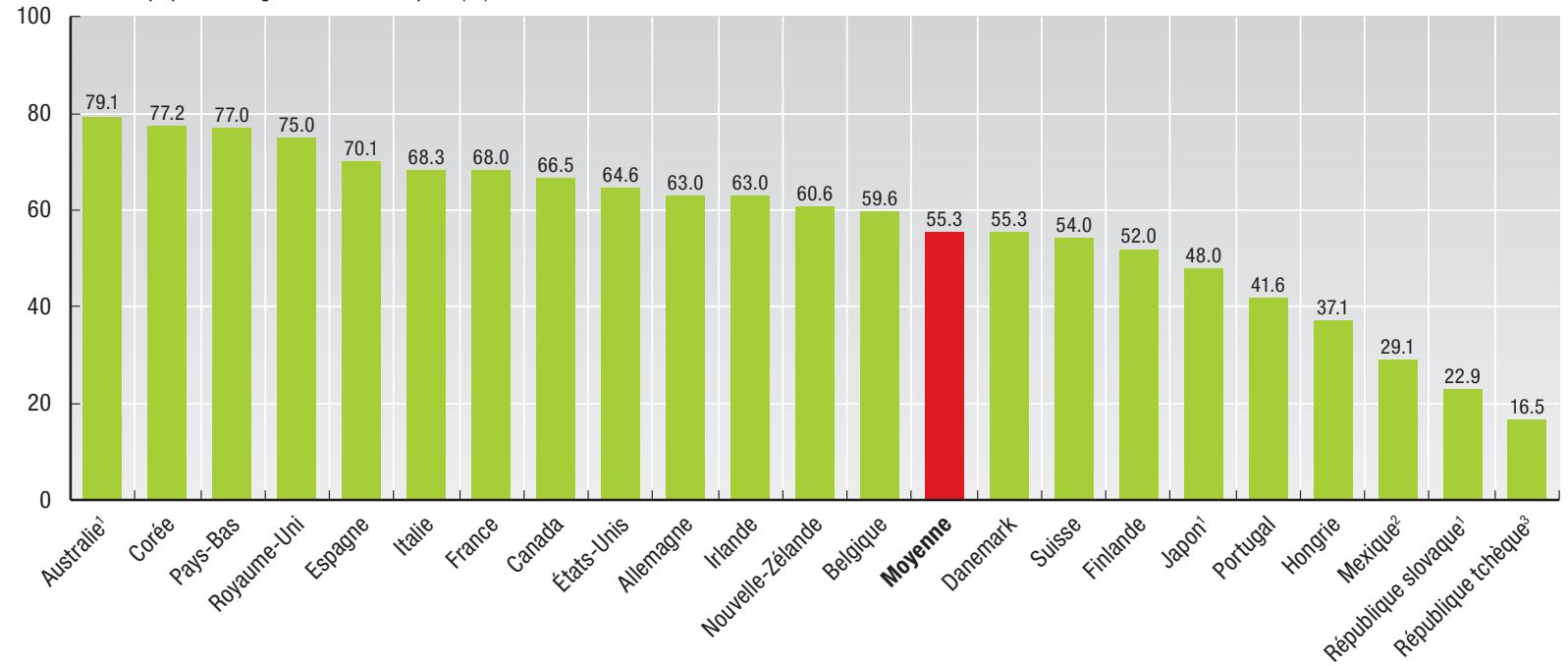

1. 2004. 2. 2003 . 3. 2002 .

6.8.2. Augmentation du taux de vaccination contre la grippe parmi les personnes âgées de 65 ans et plus, 1996 à 2005

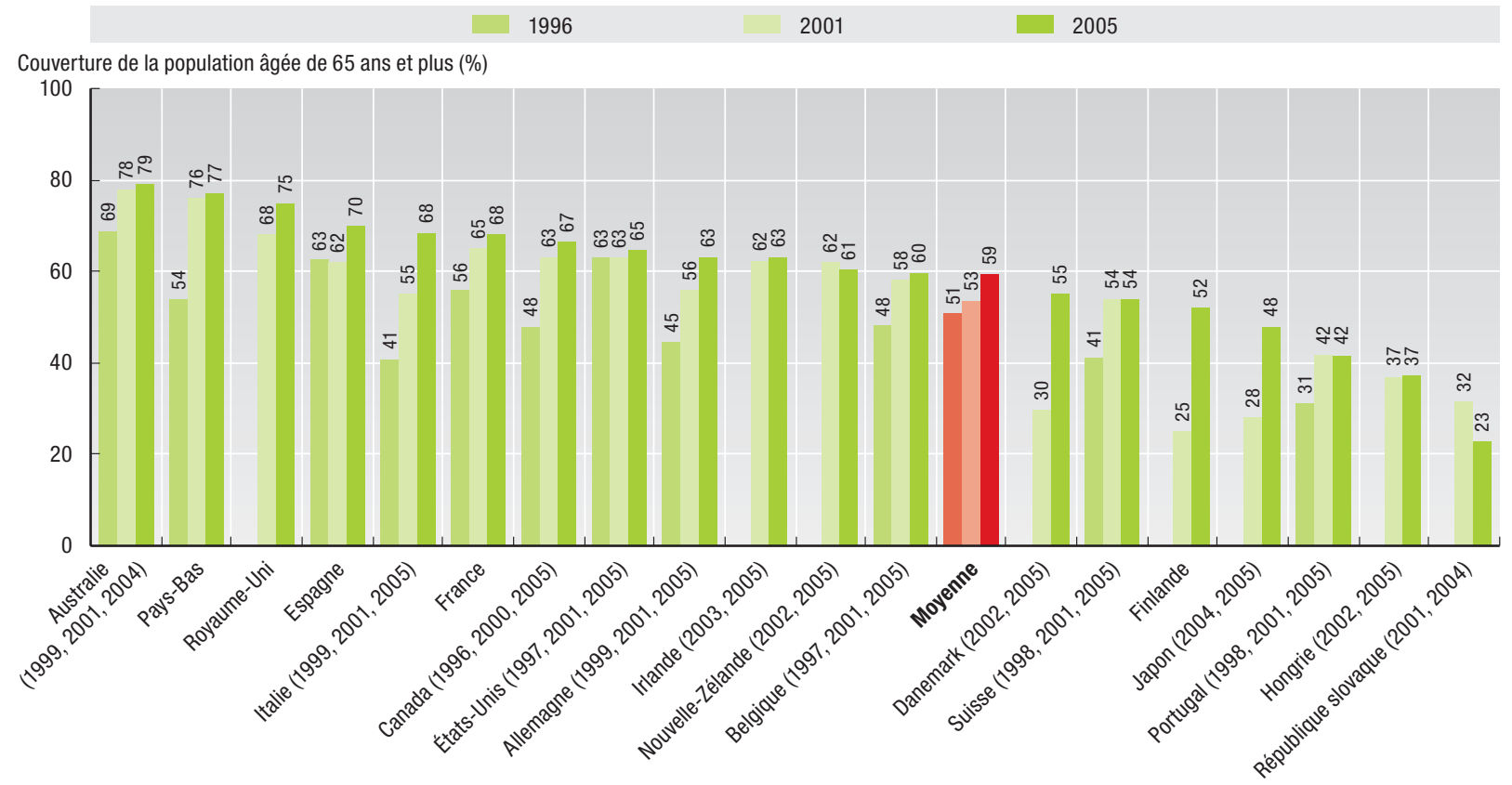

Source : Eco-Santé OCDE 2007. 


\subsection{PROGRAMMES DE VACCINATION DES ENFANTS}

\section{Importance des indicateurs}

La vaccination des enfants demeure l'une des interventions de santé publique offrant le meilleur rapport coût/efficacité. Tous les pays de l'OCDE ont mis en place des programmes de vaccination globale qu'ils continuent d'étendre. Grâce à la vaccination systématique, la polio et la diphtérie ont pu être pratiquement éradiquées dans les pays de l'OCDE. Dans l'ensemble de l'Europe, la mise en place progressive de la vaccination contre la rougeole a permis de diviser par dix environ l'incidence de la maladie par rapport au début des années 90 .

\section{Validité scientifique des indicateurs}

Tous les pays de l'OCDE ou, dans certains cas, les juridictions infranationales ont mis en place des programmes de vaccination qui s'appuient sur leur interprétation des risques et des avantages de chaque vaccin. Pratiquement tous les programmes prévoient une vaccination contre la coqueluche (souvent administrée en association avec la vaccination contre la diphtérie et le tétanos) et contre la rougeole. L'examen des études empiriques montre l'efficacité de la vaccination contre ces maladies.

\section{Constats}

Les taux de vaccination des enfants contre la coqueluche et la rougeole sont globalement élevés dans les pays de l'OCDE (graphiques 6.9.1 et 6.9.2, partie gauche). En moyenne, plus de $80 \%$ des enfants reçoivent les vaccins recommandés et les taux de vaccination sont généralement bien supérieurs à $90 \%$, excepté au Canada où le taux de vaccination contre la coqueluche était inférieur à $80 \%$ en 2004, et en Autriche où les trois quarts seulement des enfants étaient vaccinés contre la rougeole en 2004 .

De façon générale, les taux de vaccination contre la rougeole tendent à être moins élevés que les taux de vaccination contre la coqueluche dans un grand nombre de pays. Cela est probablement lié à la crainte d'un lien entre la vaccination contre la rougeole et l'autisme, même si l'on n'a pas de preuve de l'existence d'un tel lien (Demicheli et al., 2005). En 2006, la faiblesse des taux de vaccination du fait de cette crainte a entraîné la poussée de rougeole la plus sévère qu'ait connu le Royaume-Uni dans les 20 dernières années, avec le premier décès en 14 ans (BBC, 2006). Comme le montre le graphique 6.9.2 (partie droite), d'autres pays comme l'Irlande, la France, le Japon, l'Italie et la Suisse ont connu des poussées similaires, ce qui est très préoccupant car, de toutes les maladies de l'enfance, la rougeole est la plus dangereuse et la plus contagieuse. Cette recrudescence montre probablement la nécessité d'insister à nouveau sur l'importance de la vaccination contre la rougeole dans ces pays.

Néanmoins, le succès des programmes de vaccination mis en place a permis d'éliminer très largement les maladies ciblées dans les pays de l'OCDE, comme en témoignent les graphiques 6.9.1 et 6.9.2 (partie droite). Dans la plupart de ces pays, la coqueluche est devenue une maladie rare mais la disparité des taux indiqués est beaucoup plus grande que pour la rougeole. L'incidence de la coqueluche ne semble cependant pas être étroitement liée aux taux de vaccination puisque, par exemple, en dépit d'un taux de vaccination élevé (supérieur à $90 \%$ ), la Norvège a fait état de 120 cas de coqueluche pour 100000 habitants.

\section{Définition et écarts}

Les taux de vaccination reflètent le pourcentage d'enfants qui reçoit le vaccin considéré durant la période recommandée. Les politiques de vaccination des enfants diffèrent légèrement d'un pays à l'autre. Cet indicateur est donc établi sur la base de la politique effective dans un pays (ou une juridiction infranationale) donné(e). Certains pays contrôlent les vaccinations sur la base d'enquêtes, tandis que d'autres le font sur la base de données administratives, ce qui peut influer sur les résultats.

L'incidence des maladies pouvant être prévenues par la vaccination (coqueluche et rougeole) mesure le nombre de cas rapportés pour 100000 personnes. Dans les pays à faible population, une certaine prudence s'impose étant donné que la rareté des cas peut conduire à une instabilité des taux d'une année sur l'autre. De plus, les pratiques en matière de déclaration diffèrent entre les pays selon que la déclaration est obligatoire ou volontaire et selon que l'on doit déclarer également les cas suspectés ou seulement les cas confirmés. Toutefois, les premières analyses laissent penser que ces différences de pratiques en matière de déclaration entre les pays n'entraînent pas de différences systématiques dans l'incidence des maladies pouvant être prévenues par la vaccination, même si des recherches complémentaires sont nécessaires (Mattke et al., 2006). 


\section{QUALITÉ DES SOINS • LES MALADIES TRANSMISSIBLES}

\subsection{PROGRAMMES DE VACCINATION DES ENFANTS}

\subsubsection{Taux de vaccination contre la coqueluche des enfants âgés de deux ans et incidence de la coqueluche}

parmi la population totale, 2005

Couverture

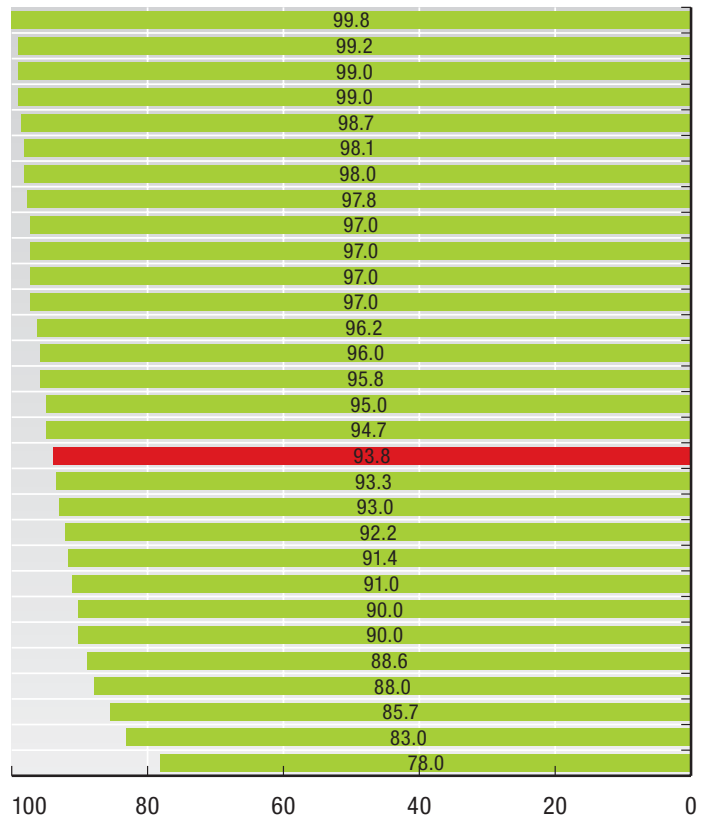

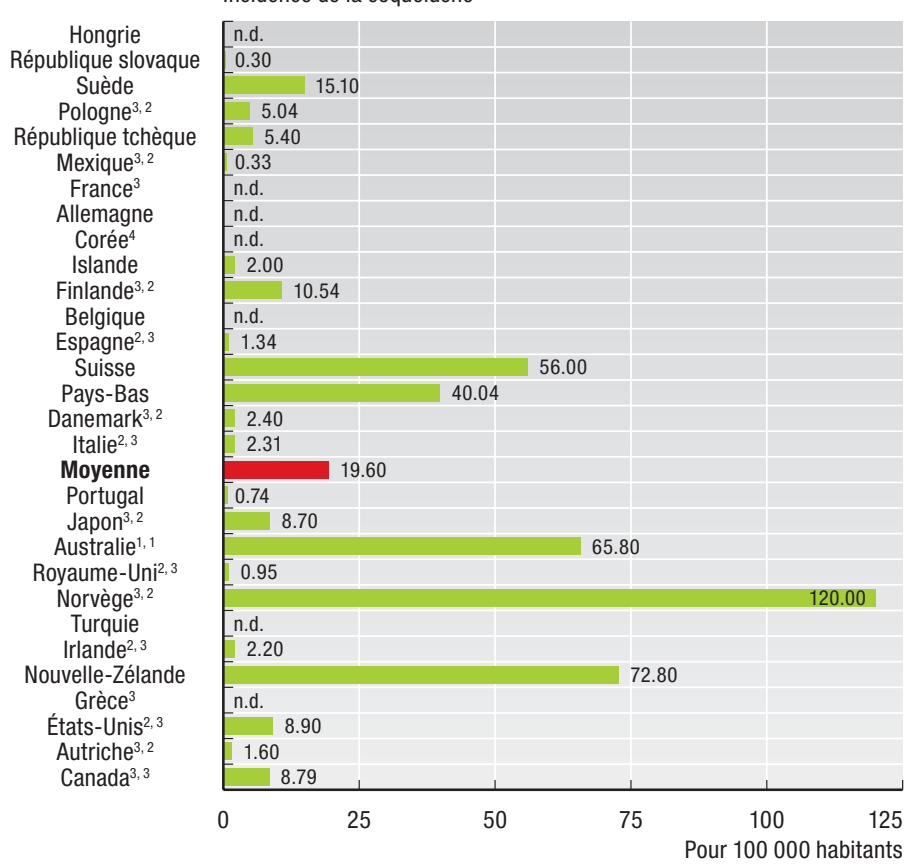

Note : La première note se rapporte au graphique de gauche et la seconde au graphique de droite.

1. 2006. 2. 2005. 3. 2004. 4. 2003.

\subsubsection{Taux de vaccination contre la rougeole des enfants âgés de deux ans et incidence de la rougeole} parmi la population totale, 2005

Couverture

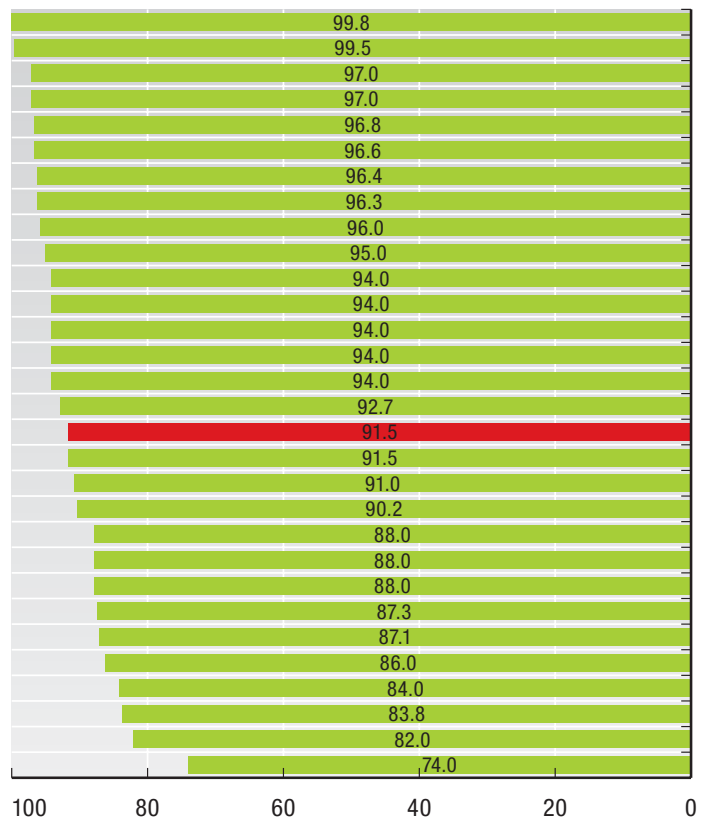

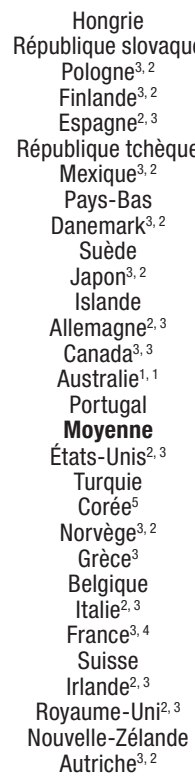

Incidence de la coqueluche

Pourcentage

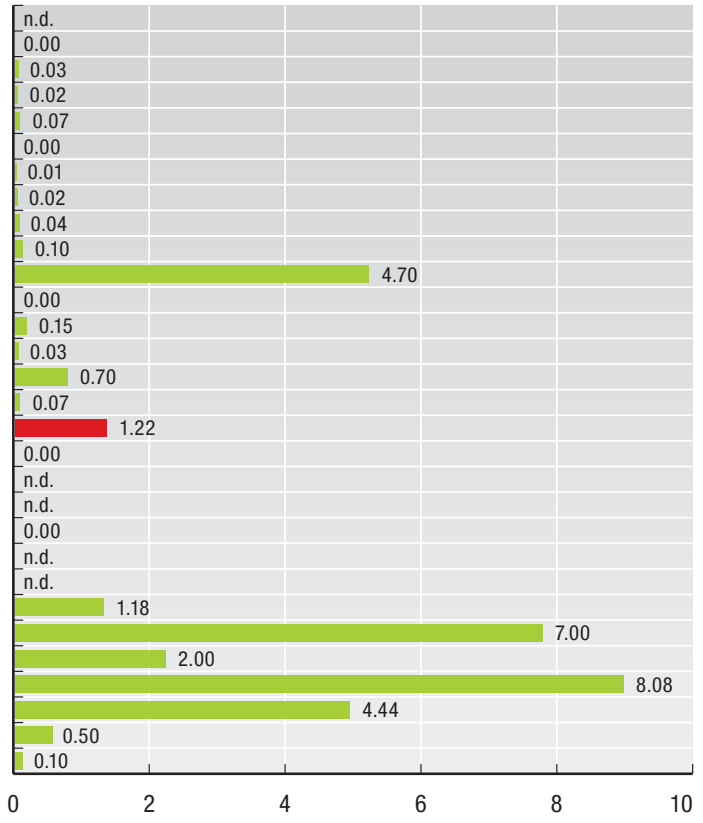

Pour 100000 habitants

Note: La première note se rapporte au graphique de gauche et la seconde au graphique de droite.

1. 2006. 2. 2005. 3. 2004.4 4. 2003. 5. 1999.

Source: Projet sur les indicateurs de la qualité des soins de santé, OCDE 2007. Eco-Santé OCDE 2007 (couverture vaccinale).

StatLink ताIs/ $h t t p: / / d x . d o i . o r g / 10.1787 / 130441316604$ 



\section{Bibliographie}

AAFA - Asthma and Allergy Foundation of America (2006), Asthma Facts and Figures, disponible sur www.aafa.org (consultation du 6 décembre 2006).

AHRQ - Agency for Healthcare Research and Quality (2006), Hospital and Ambulatory Surgery Care for Women's Cancers: HCUP Highlight, $\mathrm{n}^{\circ} 2$, Publication AHRQ $\mathrm{n}^{\circ} 06-0038$, Rockville, MD.

AIHW - Australian Institute of Health and Welfare (2004), Australia's Health 2004, AIHW Cat. $n^{\circ}$ AUS 44 Canberra.

American Diabetes Association (ADA) (2003), "Economic Costs of Diabetes in the US in 2002 ", Diabetes Care, vol. 26, pp. 917-932.

Banque mondiale (1999), Curbing the Epidemic: Governments and the Economics of Tobacco Control, Prabhat Jha (dir. pub.), Washington.

BBC (2006), «UK "in grip of measles outbreak" ", disponible sur http://news.bbc.co.uk/2/hi/uk_news/ england/5081286.stm (consultation du 14 juin 2006).

Bennett, J. (2003), « Investment in Population Health in Five OECD Countries ", document de travail de l'OCDE sur la santé, $n^{\circ} 2, O C D E$, Paris.

Bewley, S. et J. Cockburn (2002), "The Unfacts of "Request" Caesarean Section ", BJOG: An International Journal of Obstetrics \& Gynaecology, vol. 109, pp. 597-605.

Bousquet, J., I.J. Ansotegui, R. van Ree, P.G. Burney, T. Zuberbier et P. van Cauwenberge (2004), "European Union meets the Challenge of the Growing Importance of Allergy and Asthma in Europe ", Allergy, vol. 59, n 1, pp. 1-4.

Brown, M.L., G.F. Riley, N. Schussler et R.D. Etzioni (2002), «Estimating Health Care Costs Related to Cancer Treatment from SEER-Medicare Data », Medical Care, vol. 40, nº 8, pp. IV-104-117.

Capewell, S., B.M. Livingston et K. Macintyre (2000), "Trends in Case-Fatality in 117718 Patients Admitted with Acute Myocardial Infarction in Scotland ", European Heart Journal, vol. 21, pp. 1833-1840.

Castoro, C., L. Bertinato, U. Baccaglini, C.A. Drace et M. McKee (2007), Policy Brief - Day Surgery: Making it Happen, Organisation mondiale de la santé pour le compte du l'Observatoire européen sur les systèmes et politiques de santé, Copenhague.

CDC - Center for Disease Control and Prevention (2000), "Recommendations of the Advisory Committee on Immunisation Practices (ACIP) ", Morbidity and Mortality Weekly Report (MMWR), vol. $49, \mathrm{n}^{\circ} \mathrm{RR}-3$, pp. 1-38.

CDC (2001), "Mortality from Coronary Heart Disease and Acute Myocardial Infarction - United States 1998 ", Mortality and Morbidity Weekly Report, vol. 50, pp. 90-93.

CDC (2003), National Vital Statistics Report, vol. 52, nº 10, US National Center for Health Statistics, pp. 87-88.

CDC (2004), «Influenza Vaccination and Self-reported Reasons for Not Receiving Influenza Vaccination Among Medicare Beneficiaries Aged 65 Years and Older - United States, 1991-2002 ", Morbidity and Mortality Weekly Report (MMWR), vol. 53, n² 43, pp. 1012-1015.

CDC (2006), « Behavioral Risk Factor Surveillance System, Prevalence Data 2005 », disponible sur http://apps.nccd.cdc.gou/brfss/index.asp (consultation du 30 octobre 2006).

CEMT - Conférence européenne des ministres du Transport (2007), Évolution des transports 1970-2005, OCDE, Paris.

Charlton, J.R., R.M. Hartley, R. Silver et W.W. Holland (1983), « Geographical Variation in Mortality from Conditions Amenable to Medical Intervention in England and Wales ", The Lancet, vol. 1, n⿳ 8236, pp. 691-696. 
Colombo, F. et N. Tapay (2004), " Private Health Insurance in OECD Countries: The Benefits and Costs for Individuals and Health Systems », document de travail de l'OCDE sur la santé, $n^{\circ} 15, \mathrm{OCDE}$ Paris.

Colombo, F. et D. Morgan (2006), "Évolution des dépenses de santé dans les pays de l'OCDE ", Revue française des affaires sociales, avril-septembre.

Colorectal Cancer Collaborative Group (2000), " Palliative Chemotherapy for Advanced Colorectal Cancer: Systematic Review and Meta-analysis ", British Medical Journal, vol. 321, pp. 531-535.

Cutler, D. et E.L. Glaeser (2006), «Why do Europeans Smoke More than Americans? », National Bureau of Economic Research, Working Paper $n^{\circ} 12124$, mars.

Cutler, D. et R. Huckman (2003), « Technological Development and Medical Productivity: The Diffusion of Angioplasty in New York State ", Journal of Health Economics, vol. 22, n² 2, pp. 187-217, mars.

D'Addio, A.C. et M. Mira d'Ercole (2005), " Trends and Determinants of Fertility Rates in OECD Countries: The Role of Policies ", document de travail de l'OCDE sur les affaires sociales, l'emploi et les migrations, $n^{\circ} 27$, OCDE, Paris.

Davies, S.M., J. Geppert, M. McClellan, K.M. McDonald, P.S. Romano et K.J. Shojania (2001), « Refinement of the HCUP Quality Indicators ", Publication AHRQ n 01-0035.

Declercq. E., F. Menacker et M. Macdorman (2005), « Rise in "No Indicated Risk" Primary Caesareans in the United States, 1991-2001: Cross Sectional Analysis ", British Medical Journal, vol. 330, pp. 71-72.

Demicheli, V., T. Jefferson, A. Rivetti et D. Price (2005), " Vaccines for Measles, Mumps and Rubella in Children ", The Cochrane Database of Systematic Reviews.

Diabetes Control and Complications Trial Research Group (1996), "Lifetime Benefits and Costs of Intensive Therapy as Practiced in the Diabetes Control and Complications Trial ", Journal of the American Medical Association (JAMA), vol. 276, pp. 725-734.

Docteur, E. et H. Oxley (2003), « Health Care Systems: Lessons from the Reform Experience », document de travail de l'OCDE sur la santé, $n^{\circ}$ 9, OCDE, Paris.

Docteur, E. et al. (2003), "The US Health System: An Assessment and Prospective Directions for Reform ", document de travail du Département des affaires économiques de l'OCDE, $n^{\circ} 350, O C D E$, Paris.

Donabedian, A. (1990), "The Quality of Care. How it Can Be Assessed ", Journal of the American Medical Association (JAMA), vol. 260, $\mathrm{n}^{\circ} 12$, pp. 1743-1748.

DREES - Direction de la recherche, des études, de l'évaluation et des statistiques (2005), Études et Résultats, No. 387, ministère de la Solidarité, de la Santé et de la Famille, Paris, mars.

Durst, M., L. Gissmann, H. Ikenberg et H. zur Hausen (1983), « A Papillomavirus DNA from a Cervical Carcinoma and its Prevalence in Cancer Biopsy Samples from Different Geographic Regions ", Proceedings, National Academy of Sciences, vol. 80, pp. 3812-3815.

Eder, W., M. Ege et E. von Mutius (2006), "The Asthma Epidemic », New England Journal of Medicine, vol. 355, n² 21 , pp. 2226-2235.

ERS - European Respiratory Society (2003), Asthma, European Respiratory Monograph $n^{\circ} 23$, ERS, $^{2}$ Suisse.

Eun-Hwan Oh, Y. Imanaka et E. Evans (2005), "Determinants of the Diffusion of Computed Tomography and Magnetic Resonance Imaging ", International Society for Technology Assessment in Health Care, vol. 21, n 1.

Faivre-Finn, C., A.M. Bouvier-Benhamiche, J.M. Phelip, S. Manfredi, V. Dancourt et J. Faivre (2002), "Colon Cancer in France: Evidence for Improvement in Management and Survival ", Gut, vol. 51, $\mathrm{n}^{\circ} 1$, pp. 60-64.

Fedorowicz, Z., D. Lawrence et P. Gutierrez (2004), « Day Care versus In-patient Surgery for Age-related Cataract ", Cochrane Database of Systematic Reviews, vol. 25, n CD004242.

Feuer, E.J., L.M. Wun, C.C. Boring, W.D. Flanders, J. Marilyt et T.T. Timmel (2003), The Lifetime Risk of Developing Breast Cancer, disponible sur http://srab.cancer.gov/devcan/report1.pdf (consultation du 19 août 2003).

Fleming, B.B., S. Greenfield, M.M. Engelgau, L.M. Pogach, S.B. Clauser et M.A. Parrott (2001), « The Diabetes Quality Improvement Project: Moving Science into Health Policy to Gain an Edge on the Diabetes Epidemic », Diabetes Care, vol. 24, n 10 , pp. 1815-1820. 
Förster, M. et M. Mira d'Ercole (2005), « Income Distribution and Poverty in OECD Countries in the Second Half of the 1990s ", document de travail de l'OCDE sur les affaires sociales, l'emploi et les migrations, $\mathrm{n}^{\circ} 22$, OCDE, Paris.

Gatta, G., J. Faivre, R. Capocaccio et M. Ponz de Leon (1998a), "Survival of Colorectal Cancer Patient in Europe during the Period 1978-1989 ", European Journal of Cancer, vol. 34, pp. 2176-2183.

Gatta, G., M.B. Lasota, A. Verdecchia et le Groupe de travail EUROCARE (1998b), "Survival of European women with gynaecological tumours, during the period 1978-1989 ", European Journal of Cancer, vol. 34, n 14 , pp. 2218-2225.

Gatta G., R. Capocaccia, M.P. Coleman, L.A. Gloeckler Ries, T. Hakulinen, A. Micheli, M. Sant, A. Verdecchia et F. Berrino (2000), " Toward a Comparison of Survival in American and European Cancer Patients ", Cancer, vol. 89, n 4, pp. 893-900.

Gaziano, T.A. (2007), "Reducing the Growing Burden of Cardiovascular Disease in the Developing World ", Health Affairs (Millwood), vol. 26, n 1, pp. 13-24.

Ghafour, I.M., D. Allan et W.S. Foulds (1983), " Common Causes of Blindness and Visual Handicap in the West of Scotland », British Journal of Ophthalmology, vol. 67, n 4, pp. 209-213.

Gil, M., J. Marrugat et J. Sala (1999), « Relationship of Therapeutic Improvements and 28-day Case Fatality in Patients Hospitalized with Acute Myocardial Infarction between 1978 and 1993 in the REGICOR Study, Gerona, Spain », Circulation, vol. 99, pp. 1767-1773.

Gøtzsche P.C. et M. Nielsen (2006), "Screening for Breast Cancer with Mammography », Cochrane Database of Systematic Reviews, $\mathrm{n}^{\circ} 4$, Art. $\mathrm{n}^{\circ} \mathrm{CD} 001877$.

Goldberg, R.J., J. Yaerzebski et D. Lessard (1999), «A Two-decades (1975 to 1995) Long Experience in the Incidence, In-hospital and Long-term Case-fatality Rates of Acute Myocardial Infarction: A Community-wide Perspective ", Journal of the American College of Cardiology, vol. 33, pp. 1533-1539.

Govindarajan, A., N.G. Coburn, A. Kiss, L. Rabeneck, A.J. Smith et C.H. Law (2006), " Population-based Assessment of the Surgical Management of Locally Advanced Colorectal Cancer ", Journal of the National Cancer Institute, vol. 98, pp. 1474-1481.

Gu, K., C.C. Cowie et M.I. Harris (1999), « Diabetes and Decline in Heart Disease Mortality in US adults », Journal of the American Medical Association (JAMA), vol. 14, n 281, pp. 1291-1297.

Hacke, W., M. Kaste, C. Fieschi, D. Toni, E. Lesaffre, R. von Kummer, G. Boysen, E. Bluhmki, G. Hoxter et M.H. Mahagne (1995), "Intravenous Thrombolysis with Recombinant Tissue Plasminogen Activator for Acute Hemispheric Stroke. The European Cooperative Acute Stroke Study (ECASS) ", Journal of the American Medical Association (JAMA), vol. 274, $\mathrm{n}^{0} 13$, pp. 1017-1025.

Haffner, S.M. (2000), “Coronary Heart Disease in Patients with Diabetes », New England Journal of Medicine, vol. 342, pp. 1040-1042.

Halterman, J.S., H.L. Yoos, K. Sidora, H. Kitzman et A. McMullen (2001), « Medication Use and Health Care Contacts among Symptomatic Children with Asthma ", Ambulatory Pediatrics, vol. 1, $\mathrm{n}^{\circ} 5$, pp. 275-279.

Harper, D.M., E.L. Franco, C.M. Wheeler, A.B. Moscicki, B. Romanowski, C.M. Roteli-Martins, D. Jenkins, A. Schuind, S.A. Costa Clemens, G. Dubin et le Groupe d'étude HPV Vaccine (2006), "Sustained Efficacy up to 4-5 Years of a Bivalent L1 Virus-like Particle Vaccine against Human Papillomavirus Types 16 and 18: Follow-up from Randomised Control Trial ", The Lancet, vol. 367, pp. 1247-1255.

Hisashige, A. (1992), "The Introduction and Évaluation of MRI in Japan ", International Society for Technology Assessment in Health Care, vol. 3, nº 126.

Hockley, T. et M. Gemmill (2007), European Cholesterol Guidelines Report, Policy Analysis Centre, London School of Economics, Londres.

Holland, W.W. et le Groupe de travail de la CE sur les services de santé (1997), " Avoidable Death ", European Community Atlas of Avoidable Death 1985-1989, $3^{e}$ édition, Oxford University Press, Oxford.

Hurst, J. (2007), « Towards a Sustainable Health and Long-Term Care Policy ", chapitre 4 in Facing the Future: Korea's Family, Pension and Health Policy Challenges, OCDE, Paris.

IARC - International Agency for Research on Cancer (2004), GLOBOCAN 2002: Cancer Incidence, Mortality and Prevalence Worldwide, IARC CancerBase $\mathrm{n}^{\circ}$ 5, Version 2.0, IARC Press, Lyon.

IDF - International Diabetes Federation (2003), International Diabetes Federation Diabetes Atlas 3rd Edition, D. Gan (dir. pub.), International Diabetes Federation, Bruxelles. 
Institute of Alcohol Studies (2007), IAS Fact Sheet: Binge Drinking, Nature, Prevalence and Causes, disponible sur www.ias.org.uk/resources/factsheets/binge_drinking.pdf (consultation du 11 juin 2007).

Institute of Cancer Research (2003), Prostate Cancer, Fact Sheet, disponible sur www.icr.ac.uk/everyman/ about/prostate.html (consultation du 10 mai 2005).

International Association for the Study of Obesity (2007), International Obesity Taskforce Database, disponible sur www.iotf.org/documents/Europeandatatable_000.pdf (consultation du 11 juin 2007).

IOM - Institute of Medicine Committee on Quality Health Care in America (2001), Crossing the Quality Chasm: A New Health System for the 21st Century, National Academy Press, Washington, D.C.

Jeong, H.S. et A. Gunji (1994), « The Influence of System Factors upon the Macro-economic Efficiency of Health Care: Implications for the Health Policies of Developing and Developed Countries », Health Policy, vol. 27.

Jeong, H.S. et J. Hurst (2001), " An Assessment of the Performance of the Japanese Health Care System ", Document de l'OCDE sur le marché du travail et les affaires sociales, $n^{\circ} 56$, OCDE, Paris.

Johnson, L.A. (2007), « Merck Suspends Lobbying for Vaccine ", The Washington Post, 12 février.

Jonsson, B. (2002), « Revealing the Cost of Type II Diabetes in Europe », Diabetologia, vol. 45, n 7 , pp. S5-12.

Kalra, L., A. Evans, I. Perez, M. Knapp, C. Swift et N. Donaldson (2005), « A Randomised Controlled Comparison of Alternative Strategies in Stroke Care ", Health Technology Assessment, vol. 9, $n^{\circ}$ 18, pp. 1-94.

Keech, M., A.J. Scott et P.J. Ryan (1998), «The Impact of Influenza and Influenza-like Illness on Productivity and Healthcare Resource Utilization in a Working Population ", Occupational Medicine, vol. 49, pp. 85-90

Kelley, E. et J. Hurst (2006), " Health Care Quality Indicators Project: Conceptual Framework Paper ", document de travail de l'OCDE sur la santé, n⿳0 23, OCDE, Paris.

Khush, K.K., E. Rapaport et D. Waters (2005), « The History of the Coronary Care Unit », Canadian.Journal of Cardiology, vol. 21, pp. 1041-1045.

Kiely, J., K. Brett, S. Yu et D. Rowley (1995), " Low Birth Weight and Intrauterine Growth Retardation ", in L. Wilcox et J. Marks (dir. pub.), From Data to Action: CDC's Public Health Surveillance for Women, Infants, and Children, USDHHS, Centers for Disease Control and Preventions (CDC), Atlanta, pp. 185-202.

King, H., R.E. Aubert et W.H. Herman (1998), "Global Burden of Diabetes, 1995-2025: Prevalence, Numerical Estimates, and Projections ", Diabetes Care, vol. 21, nº 9, pp. 1414-1431.

Kroneman, M. et J. Siegers (2004), " The Effect of Hospital Bed Reduction on the Use of Beds: A Comparative Study of 10 European Countries », Social Science and Medicine, vol. 59, pp. 1731-1740.

Lafortune, G., G. Balestat et al. (2007), « Trends in Severe Disability among Elderly People: Assessing the Evidence in 12 OECD Countries and Future Implications ", document de travail de l'OCDE sur la santé, $\mathrm{n}^{\circ} 26$, OCDE, Paris.

Lalonde, M.A. (1973), " Nouvelle perspectives de la santé des Canadiens ", Gouvernement canadien, ministère de la Santé nationale et du Bien-être social.

Langhorne P., B.O. Williams, W. Gilchrist et K. Howie (1993), « Do Stroke Units Save Lives? », The Lancet, vol. 342, nº 8868, pp. 395-398.

Launois, R., M. Giroud, A.C. Megnigbeto, K. Le Lay, G. Presente, M.H. Mahagne, I. Durand et A.F. Gaudin (2004), " Estimating the Cost-effectiveness of Stroke Units in France Compared with Conventional Care ", Stroke, vol. 35, n 3, pp. 770-775.

Laws P.J., N. Grayson et E.A. Sullivan (2006), Australia's Mothers and Babies 2004, Perinatal Statistics Series $n^{\circ} 8$, AIHW Cat. $n^{\circ}$ PER 34, AIHW National Perinatal Statistics Unit, Sydney.

Loddenkemper, R., G.J. Gibson et Y. Sibille (2003), European Lung White Book, European Respiratory Society, Sheffield.

Mackenbach, J.P. (1996), « The Contribution of Medical Care to Mortality Decline: Mc Keown Revisited », Journal of Clinical Epidemiology, vol. 49, pp. 1207-1213.

Manuel, D.G. et Y. Mao (2002), " Avoidable Mortality in the United States and Canada, 1980-1996", American Journal of Public Health, vol. 92, nº 9, pp. 1481-1484. 
Masoli, M., D. Fabian, S. Holt et R. Beasley (2004), "Global Burden of Asthma », Global Initiative for Asthma, disponble sur www.ginasthma.com/ReportItem.asp?l1=2\&l2=2\&intId=94.

Mathers, C. et al. (2005), " Counting the Dead and What They Died From: An Assessment of the Global Status of Cause of Death Data ", Bulletin of the World Health Organisation, vol. 83, n 3, pp. 171-177, mars.

Mattke, S., E. Kelley, P. Scherer, J. Hurst et M.L. Gil Lapetra (2006), « Health Care Quality Indicators Project Initial Indicators Report », document de travail de l'OCDE sur la santé, $n^{\circ} 22$, OCDE, Paris.

Mattke, S., F. Martorell, P. Sharma, L.K. Morse, N. Lurie et M. Lara (2006), « Estimating the Impact of Improving Asthma Treatment. A Review and Synthesis of the Literature ", RAND Working Paper $\mathrm{n}^{\circ}$ WR-448-MCAN.

McGlynn, E.A., S.M. Aschs, J. Adams, J. Keesey, J. Hicks, A. de Christofar et E.A. Kerr (2003), « The Quality of Health Care Delivered to Adults in the United States ", New England Journal of Medicine, vol. 348, $\mathrm{n}^{\circ} 26$, pp. 2635-2645.

McGovern, P.G., D.R. Jacobs et E. Shahar (2001), "Trends in Acute Heart Disease Mortality, Morbidity, and Medical Care from 1985 through 1997: The Minnesota Heart Survey ", Circulation, vol. 104, pp. 19-24.

Melander et al. (2006), « Utilisation of Antihyperglycaemic Drugs in Ten European Countries: Different Developments and Different Levels », Diabetologia, vol. 49, pp. 2024-2029.

Midgley, R. et D. Kerr (1999), “ Colorectal Cancer », The Lancet, vol. 353, nº 9150, pp. 391-399.

Miilunpalo, S. et al. (1997), "Self-rated Health Status as a Health Measure: The Predictive Value of Selfreported Health Status on the Use of Physician Services and on Mortality in the Working-age Population », Journal of Clinical Epidemiology, vol. 50, n 5, pp. 517-528, mai.

Mitry, E., A. Bouvier, M. Esteve et J. Faivre (2005), «Improvement in Colorectal Cancer Survival: A Population-based Study ", European Journal of Cancer, vol. 41, n 15, pp. 2297-2303.

MMWR - Mortality and Morbidity Weekly Report (2001), " Mortality from Coronary Heart Disease and Acute Myocardial Infarction - United States 1998 », vol. 50, pp. 90-93.

Moïse, P. (2003a), « The Heart of the Health Care System: Summary of the Ischaemic Heart Disease Part of the OECD Ageing-related Diseases Study ", A Disease-based Comparison of Health Systems: What is Best and at What Cost?, OCDE, Paris.

Moïse, P. (2003b), «The Technology-Health Expenditure Link: A Perspective from the Ageing-related Diseases Study ", A Disease-based Comparison of Health Systems: What is Best and at What Cost?, OCDE, Paris.

Moïse, P. et al. (2003), " OECD Study of Cross-national Differences in the Treatment, Costs and Outcomes for Ischaemic Heart Disease », document de travail de l'OCDE sur la santé, $n^{\circ} 3, O_{C D E}$ Paris.

Mokdad, A.H., E.S. Ford, B.A. Bowman, W.H. Dietz, F. Vinicor, V.S. Bales et J.S. Marks (2003), « Prevalence of Obesity, Diabetes, and Obesity-related Health Risk Factors, 2001 ", Journal of the American Medical Association (JAMA), vol. 289, $\mathrm{n}^{\circ} 1$, pp. 76-79.

Moon, L., P. Moïse et S. Jacobzone (2003), « Stroke Care in OECD Countries: A Comparison of Treatment, Costs and Outcomes in 17 OECD Countries ", document de travail de l'OCDE sur la santé, $n^{\circ} 5$, OCDE, Paris.

Mori, E., Y. Yoneda, M. Tabuchi, T. Yoshida, S. Ohkawa, Y. Ohsumi, K. Kitano, A. Tsutsumi et A. Yamadori (1992), "Intravenous Recombinant Tissue Plasminogen Activator in Acute Carotid Artery Territory Stroke ", Neurology, vol. 42, n 5, pp. 976-982.

Nallamothu, B.K., J. Young, H.S. Gurm, G. Pickens et K. Safavi (2007), "Recent Trends in Hospital Utilization for Acute Myocardial Infarction and Coronary Revascularization in the United States ", American Journal of Cardiology, $\mathrm{n}^{\circ}$ 99, pp. 749-753.

Natarajan, N. et T.D. Shuster (2006), « New Agents, Combinations, and Opportunities in the Treatment of Advanced and Early-stage Colon Cancer ", The Surgical Clinics of North America, vol. 86, $n^{\circ} 4$, pp. 1023-1043.

National Cancer Institute (2004), « Radiation Therapy for Cancer: Questions and Answers ", Cancer Facts, vol. 7.1. 
National Diabetes Quality Improvement Alliance (2005), « National Diabetes Quality Improvement Alliance Performance Measurement Set for Adult Diabetes », disponible sur http:// nationaldiabetesalliance.org/measures.html (consultation de août 2005).

National Heart Foundation of Australia and the Cardiac Society of Australia and New-Zealand (2005), Position Statement on Lipid Management - 2005.

HCHS - National Centre for Health Statistics (2006), Health, United States, 2006, with Chartbook on Trends in the Health of Americans, Hyattsville, Maryland.

NHLBI - National, Heart, Lung, and Blood Institute (2002), « Morbidity \& Mortality: 2002 Chart Book on Cardiovascular, Lung and Blood Diseases ", disponible sur www.nhlbi.nih.gov/resources/docs/ 02_chtbk.pdf (consultation du 6 décembre 2006).

Nicholson, K.G., R. Snacken et A.M. Palache (1995), "Influenza Immunization Policies in Europe and the United States », Vaccine, vol. 13, n 4, pp. 365-369.

NINDS - National Institute of Neurological Disorders and Strokert-PA Stroke Study Group (1995), "Tissue Plasminogen Activator for Acute Ischemic Stroke ", New England Journal of Medicine, vol. $333, n^{\circ} 24$, pp. 1581-1587.

NOMESCO (2007), Health Statistics in the Nordic Countries 2005, Copenhague.

OCDE (2000a), Système de comptes de la santé, OCDE, Paris.

OCDE (2000b), Comptes nationaux, OCDE, Paris.

OCDE (2003a), A Disease-based Comparison of Health Systems: What is Best and at What Cost?, OCDE, Paris.

OCDE (2003b), Examens de l'OCDE des systèmes de santé - Corée, OCDE, Paris.

OCDE (2004a), Vers des systèmes de santé plus performants, OCDE, Paris.

OCDE (2004b), Vers des systèmes de santé plus performants : études thématiques, OCDE, Paris.

OCDE (2004c), L'assurance maladie privée dans les pays de l'OCDE, OCDE, Paris.

OCDE (2005a), Examens de l'OCDE des systèmes de santé - Finlande, OCDE, Paris.

OCDE (2005b), Examens de l'OCDE des systèmes de santé - Mexique, OCDE, Paris.

OCDE (2005c), Les soins de longue durée pour les personnes âgées, OCDE, Paris.

OCDE (2005d), Accroître les chances de chacun : pour une politique sociale active au bénéfice de tous, OCDE, Paris.

OCDE (2006a), Examens de l'OCDE des systèmes de santé - Suisse, OCDE, Paris.

OCDE (2006b), « Projecting OECD Health and Long-Term Care Expenditures: What Are the Main Drivers? », document de travail du Département des affaires économiques de l'OCDE, $\mathrm{n}^{\circ} 477$, OCDE, Paris.

OCDE (2007a), Eco-Santé OCDE - Statistiques et indicateurs pour 30 pays, CD-Rom et version en ligne, OCDE, Paris.

OCDE (2007b), Understanding National Accounts, par F. Lequiller et D. Blades (dir. pub.), OCDE, Paris.

OCDE (2007c), Perspectives des migrations internationales, OCDE, Paris.

OCDE (2007d), Panorama de la société - Les indicateurs sociaux de l'OCDE, Paris.

OCDE (2007e), Les pensions dans les pays de l'OCDE - Panorama des politiques publiques, OCDE, Paris.

Ohmi, H., K. Hirooka, A. Hata et Y. Mochizuki (2001), « Recent Trend of Increase in Proportion of Low Birth Weight Infants in Japan ", International Journal of Epidemiology, vol. 30, pp. 1269-1271.

Oliveira Martins, J., F. Gonand, P. Antolin, C. de la Maisonneuve et K-Y. Yoo. (2005), « The Impact of Ageing on Demand, Factor Markets and Growth ", document de travail du Département des affaires économiques de l'OCDE, $\mathrm{n}^{\circ} 420$, OCDE, Paris.

Ollendorf, D.A., J.G. Kotsanos, W.J. Wishner, M. Friedman, T. Cooper, M. Bittoni et G. Oster (1998), "Potential Economic Benefits of Lower-extremity Amputation Prevention Strategies in Diabetes ", Diabetes Care, vol. 21, n 8, pp. 1240-1245.

OMS - Organisation mondiale de la santé (1997), Obesity: Preventing and Managing the Global Epidemic, OMS, Genève.

OMS (2002a), The Tobacco Atlas, Judith Mackay, OMS, Genève.

OMS (2002b), Rapport sur la santé dans le monde 2002, OMS, Genève. 
OMS (2003), The World Oral Health Report 2003: Continuous Improvement of Oral Health in the 21st Century - The Approach of the WHO Global Oral Health Programme, P.E. Petersen (dir. pub.), OMS, Genève.

OMS (2005), The World Health Report 2005 - Make Every Mother and Child Count, OMS, Genève.

OMS (2007), « Measles », Fact Sheet n 286, révision de janvier 2007, OMS, Genève.

Orosz, E. et D. Morgan (2004), "SHA-Based National Health Accounts in Thirteen OECD Countries: A Comparative Analysis ", document de travail de l'OCDE sur la santé, n 16, OCDE, Paris.

Park, J. et al. (2003), "Secular Trends of Suicide Mortality in Korea ", Korean Journal of Epidemiology, vol. 25, pp. 84-91.

Peden, M. et al. (2004), World Report on Road Traffic Injury Prevention, Organisation mondiale de la santé, Genève.

Pellegrini, F., M. Belfiglio, G. De Beradis, M. Franciosi, B. Di Nardo, S. Greenfield, S.H. Kaplan, M. Sacco, G. Tognoni, M. Valentini, D. Corrado, A. D'Ettorre, A. Nicolucci et le Groupe d'étude QuED (2003), "Role of Organisational Factors in Poor Blood Pressure Control in Patients with Type 2 Diabetes: The QuED Study Group - Quality of Care and Outcomes in Type 2 Diabetes ", Archives of Internal Medicine, vol. 163, $\mathrm{n}^{\circ} 4$, pp. 473-480.

Peto J., C. Gilham, O. Fletcher et F.E. Matthews (2004), " The Cervical Cancer Epidemic that Screening Has Prevented in the UK ", The Lancet, vol. 364, pp. 249-256.

Quinn, M.J., C. Martinez-Garcia, F. Berrino et le Groupe de travail EUROCARE (1998), "Variations in Survival from Breast Cancer in Europe by Age and Country, 1978-1989 ", European Journal of Cancer, vol. $34, \mathrm{n}^{\circ} 14$, pp. 2204-2211.

$\mathrm{Ra}$, H. et al. (2006), " Factors Influencing the Impulse of Suicide in Adolescence ", Journal of the Korean Academy of Family Medicine, vol. 27, pp. 988-997.

Retzlaff-Roberts, D., C. Chang et R. Rubin (2004), "Technical Efficiency in the Use of Health Care Resources: A Comparison of OECD Countries ", Health Policy, vol. 69, pp. 55-72.

Ritz, E., I. Rychlik, F. Locatelli et S. Halimi (1999), "End-stage Renal Failure in Type 2 Diabetes: A Medical Catastrophe of Worldwide Dimensions ", American Journal of Kidney Disease, vol. 34, $n^{\circ} 5$, pp. 795-808.

Sachs, B.P. et al. (1995), " The Impact of Extreme Prematurity and Congenital Anomalies on the Interpretation of International Comparisons of Infant Mortality ", Obstetrics \& Gynaecology, vol. 85, pp. 941-946.

Sant, M., C. Allemani, F. Berrino, M.P. Coleman, T. Aareleid, G. Chaplain, J.W. Coebergh, M. Colonna, P. Crosignani, A. Danzon, M. Federico, L. Gafa, P. Grosclaude, G. Hedelin, J. Mace-Lesech, C.M. Garcia, H. Moller, E. Paci, N. Raverdy, B. Tretarre, E.M. Williams et le Groupe de travail EUROCARE (2004), "Breast Carcinoma Survival in Europe and the United States: A Populationbased Study ", Cancer, vol. 100, pp. 715-722.

Sant, M., C. Allemani, R. Capocaccia, T. Hakulinen, T. Aareleid, M.P. Coleman, P. Grosclaude, C. Martinez-Garcia, C.M.J. Bell, J. Youngson, F. Berri et le Groupe de travail EUROCARE (2003), « Stage at Diagnosis is a Key Explanation of Differences in Breast Cancer Survival across Europe », International Journal of Cancer, vol. 106, pp. 416-422.

Sant, M., R. Capocaccia, M.P. Coleman, F. Berrino, G. Gatta, A. Micheli, A. Verdecchia, J. Faivre, T. Hakulinen, J.W. Coebergh, C. Martinez-Garcia, D. Forman, A. Zappone et le Groupe de travail EUROCARE (2001), "Cancer Survival Increases in Europe, but International Differences Remain Wide ", European Journal of Cancer, vol. 1, nº 37, pp. 1659-1667.

Sarti, C., B. Stebmayr, H. Tolonen, M. Mahonen, J. Tuomilehto, K. Asplund et le Projet WHO MONICA (2003), "Are Changes in Mortality from Stroke Cause by Changes in Stroke Event Rates or Case Fatality? Results from the WHO MONICA Project ", Stroke, vol. 34, pp. 1833-1840.

Savas, B. et al. (2002), Health Care Systems in Transition: Turkey, European Observatory on Health Care Systems, Copenhague.

Secretaria de Salud (2003), Nacimientos por cesarea en Mexico, Sintesis Ejecutiva, Mexique.

SEER - Surveillance, Epidemiology, and End Results Program (2006), SEER*Stat Database: Incidence - SEER 17 Regs Public-Use, Nov 2005 Sub (1973-2003 varying), National Cancer Institute, DCCPS, Surveillance Research Program, Cancer Statistics Branch, disponible sur www.seer.cancer.gou.

Simoens, S. et J. Hurst (2006), "The Supply of Physician Services in OECD Countries », document de travail de l'OCDE sur la santé, $n^{\circ} 21$, OCDE, Paris. 
Simoens, S., M. Villeneuve et J. Hurst (2005), "Tackling Nurse Shortages in OECD Countries ", document de travail de l'OCDE sur la santé, n 19, OCDE, Paris.

Singleton, J.A., S.M. Greby, K.G. Wooten, F.J. Walker et R. Strikas (2000), «Influenza, Pneumococcal, and Tetanus Toxoid Vaccination of Adults - United States, 1993-1997 ", CDC Surveillance Summaries, Morbidity and Mortality Weekly Report, vol. 49, n SS-9, pp. 39-63.

Slade, E.P. et G.F. Anderson (2001), « The Relationship between Per Capita Income and Diffusion of Medical Technologies ", Health Policy, $\mathrm{n}^{\circ}$ 58, vol. 1, pp. 1-14.

Sourty Le-Guellec, M. (2001), Le potentiel de développement de la chirurgie ambulatoire de la cataracte en France, en 1999, Rapport $n^{\circ} 1357$, Centre de recherche d'étude et de documentation en économie de la santé (CREDES), septembre.

Starfield, B. et L. Shi (2002), " Policy Relevant Determinants of Health: An International Perspective ", Health Policy, vol. 60, pp. 201-218.

Starfield, B., L. Shi et J. Mackino (2005), « Contribution of Primary Care to Health Systems and Health », The Milbank Quarterly, vol. 83, $\mathrm{n}^{\circ} 3$, pp. 457-502.

Statistique Canada et Centers for Disease Control and Prevention (2004), Enquête conjointe Canada/ États-Unis sur la santé, 2002-03, Statistique Canada Cat. 82M0022-XIE, Ottawa.

Stegmayr, B. et al. (1997), "Stroke Incidence and Mortality Correlated to Stroke Risk Factors in the WHO MONICA Project ", Stroke, vol. 28, nº 7, pp. 1367-1374.

Stroke Unit Trialists' Collaboration (1997), " How Do Stroke Units Improve Patient Outcomes? A Collaborative Systematic Review of the Randomized Trials ", Stroke, vol. 28, n 11, pp. 2139-2144.

Stroke Unit Trialists' Collaboration (2001), "Organised Inpatient (Stroke Unit) Care for Stroke ", Cochrane Database of Systematic Reviews, n³ Art. n CD000197.

Strunk, B.C., P.B. Ginsburg et M.I. Banker (2006), " The Effect of Population Ageing on Future Hospital Demand ", Health Affairs, vol. 25, n³ 3, pp. w141-w149.

Sturm, R. (2002), "The Effects of Obesity, Smoking and Drinking on Medical Problems and Costs ", Health Affairs, vol. 21, $n^{\circ} 2$, pp. 245-253, mars/avril.

Tanihara, S. et Y. Kobayashi (2004), "Sequential Evaluation of the National Medical Expenditures for Asthma Care in Japan ", Journal of Epidemiology, vol. 14, pp. 100-103.

Taylor R.J., S.L. Morrell, H.A. Mamoon et G.V. Wain (2001), « Effects of Screening on Cervical Cancer Incidence and Mortality in New South Wales Implied by Influences of Period of Diagnosis and Birth Cohort ", Journal of Epidemiology and Community Health, vol. 55, $\mathrm{n}^{\circ}$ 11, pp. 782-788.

Thompson, D. et A.M. Wolf (2001), «The Medical-care Burden of Obesity », Obesity Reviews, $n^{\circ} 2$, International Association for the Study of Obesity, pp. 189-197.

Tunstall-Pedoe, H. (2003), « MONICA's quarter century », European Journal of Cardiovascular Prevention and Rehabilitation, vol. 10, $\mathrm{n}^{\circ} 6$, pp. 409-410.

Tuomilehto, J., J. Lindstrom, J.G. Eriksson, T.T. Valle, H. Hamalainen, P. Ilanne-Parikka, S. KeinanenKiukaanniemi, M. Laakso, A. Louheranta, M. Rastas, V. Salminen, M. Uusitupa et le Groupe d'étude Finnish Diabetes Prevention (2001), « Prevention of Type 2 Diabetes Mellitus by Changes in Lifestyle among Subjects with Impaired Glucose Tolerance ", New England Journal of Medicine, vol. 344, pp. 1343-1350.

UNAIDS (2006), AIDS Epidemic Update: December 2006, Genève.

UNICEF et OMS (2004), Low Birthweight: Country, Regional and Global Estimates, UNICEF, New York.

USPSTF - US Preventive Services Task Force (2002), « Screening for Colorectal Cancer: Recommendations and Rationale ", Annals of Internal Medicine, vol. 137, n² 2, pp. 129-131.

USRDS - US Renal Data System (2006), 2006 Annual Data Report: Atlas of End-Stage Renal Disease in the United States, National Institutes of Health, National Institute of Diabetes and Digestive and Kidney Diseases, Bethesda, MD.

Victora, C.G. et F.C. Barros (2006), « Beware: Unnecessary Caesarean Sections May Be Hazardous », The Lancet, vol. 367, n 9525, pp. 1796-1797, 3-9 juin.

Wagner, E.H., N. Sandhu, K.M. Newton, D.K. McCulloch, S.D. Ramsey et L.C. Grothaus (2001), « Effect of Improved Glycemic Control on Health Care Costs and Utilization ", Journal of the American Medical Association (JAMA), vol. 285, pp. 182-189. 
Wardlaw, J.M., P.A.G. Sandercock et E. Berge (2003), « Thrombolytic Therapy with Recombinant Tissue Plasminogen Activator for Acute Ischemic Stroke. Where Do We Go From Here? A Cumulative Meta-Analysis ", Stroke, vol. 34, pp. 1437-1443.

Weisfeldt, M.L. et S.J. Zieman (2007), « Advances in the Prevention and Treatment of Cardiovascular Disease ", Health Affairs, vol. 26, pp. 25-37.

Wennberg, D.E. et J.E. Wennberg (2003), « Addressing Variations: Is There Hope for the Future? », Health Affairs (Millwood), Supplément, n ${ }^{\circ}$ W3-614-7.

Wenzel, S.E. (2006), «Asthma: Defining of the Persistent Adult Phenotypes », The Lancet, vol. 368, $\mathrm{n}^{\circ}$ 9537, pp. 804-813.

Wilkinson, R.G. (1996), Unhealthy Societies: The Afflictions of Inequality, Routledge, Londres.

Wilkinson, R.G. (2000), " Putting the Picture Together: Prosperity, Redistribution, Health and Welfare ", in M. Marmot et R. Wilkinson (dir. pub.), Social Determinants of Health, Oxford University Press, Oxford.

Zimmet, P., K.G. Alberti et J. Shaw (2001), " Global and Societal Implications of the Diabetes Epidemic », Nature, vol. 414, nº 6865, pp. 782-787. 



\section{Annexe statistique}

Tableau A.1.1a. Population totale en milieu d'année, en milliers, 1960 à 2005

\begin{tabular}{|c|c|c|c|c|c|c|}
\hline & 1960 & 1970 & 1980 & 1990 & 2000 & 2005 \\
\hline Allemagne $^{a}$ & 55585 & 60651 & 61566 & 63254 & 82160 & 82466 \\
\hline Australie & 10275 & 12507 & 14695 & 17065 & 19153 & 20329 \\
\hline Autriche & 7047 & 7467 & 7549 & 7718 & 8110 & 8233 \\
\hline Belgique & 9153 & 9656 & 9859 & 9967 & 10251 & 10479 \\
\hline Canada & 17870 & 21297 & 24516 & 27698 & 30689 & 32271 \\
\hline Corée & 25012 & 32241 & 38124 & 42869 & 47008 & 48294 \\
\hline Danemark & 4581 & 4929 & 5123 & 5141 & 5340 & 5416 \\
\hline Espagne & 30256 & 33859 & 37527 & 38851 & 40264 & 43398 \\
\hline États-Unis & 180671 & 205052 & 227225 & 249623 & 282194 & 296410 \\
\hline Finlande & 4430 & 4606 & 4779 & 4986 & 5176 & 5246 \\
\hline France & 45684 & 50772 & 53880 & 56709 & 59013 & 60873 \\
\hline Grèce & 8327 & 8793 & 9642 & 10089 & 10917 & 11104 \\
\hline Hongrie & 9984 & 10338 & 10711 & 10374 & 10211 & 10087 \\
\hline Irlande & 2834 & 2950 & 3401 & 3503 & 3790 & 4131 \\
\hline Islande & 176 & 205 & 228 & 255 & 281 & 296 \\
\hline Italie & 48967 & 52771 & 55657 & 56737 & 57189 & 58135 \\
\hline Japon & 94302 & 104665 & 117060 & 123611 & 126926 & 127757 \\
\hline Luxembourg & 315 & 340 & 365 & 384 & 436 & 455 \\
\hline Mexique & . & 48225 & 66847 & 81250 & 98658 & 106203 \\
\hline Norvège & 3585 & 3879 & 4086 & 4241 & 4491 & 4623 \\
\hline Nouvelle-Zélande & 2377 & 2820 & 3144 & 3363 & 3858 & 4099 \\
\hline Pays-Bas & 11486 & 13039 & 14150 & 14951 & 15926 & 16320 \\
\hline Pologne & 29561 & 32526 & 35578 & 38119 & 38256 & 38161 \\
\hline Portugal & 9077 & 8663 & 9819 & 9873 & 10229 & 10563 \\
\hline République slovaque & 3994 & 4528 & 4984 & 5298 & 5401 & 5387 \\
\hline République tchèque & 9660 & 9805 & 10327 & 10362 & 10272 & 10221 \\
\hline Royaume-Uni & 52373 & 55632 & 56330 & 57237 & 58886 & 60227 \\
\hline Suède & 7480 & 8043 & 8311 & 8559 & 8872 & 9030 \\
\hline Suisse & 5328 & 6181 & 6319 & 6712 & 7184 & 7437 \\
\hline Turquie & 27506 & 35321 & 44439 & 56156 & 67420 & 72064 \\
\hline Total OCDE & 717896 & 851761 & 946241 & 1024955 & 1128561 & 1169715 \\
\hline
\end{tabular}

a) Les chiffres de la population allemande concernent l'Allemagne de l'Ouest avant 1991

Source : Eco-Santé OCDE 2007. 
Tableau A.1.1b. Pourcentage de la population âgée de 65 ans et plus, 1960 à 2005

\begin{tabular}{|c|c|c|c|c|c|c|}
\hline & 1960 & 1970 & 1980 & 1990 & 2000 & 2005 \\
\hline Allemagne & 10.8 & 13.2 & 15.5 & 15.3 & 17.2 & 19.2 \\
\hline Australie & 8.5 & 8.3 & 9.6 & 11.1 & 12.4 & 13.1 \\
\hline Autriche & 12.2 & 14.1 & 15.4 & 15.1 & 15.5 & 16.3 \\
\hline Belgique & 12.0 & 13.4 & 14.3 & 14.9 & 16.8 & 17.02002 \\
\hline Canada & 7.6 & 8.0 & 9.4 & 11.3 & 12.6 & 13.1 \\
\hline Corée & 2.9 & 3.1 & 3.8 & 5.1 & 7.2 & 9.1 \\
\hline Danemark & 10.6 & 12.3 & 14.4 & 15.6 & 14.8 & 15.1 \\
\hline Espagne & 8.2 & 9.6 & 11.2 & 13.6 & 16.8 & 16.7 \\
\hline États-Unis & 9.2 & 9.8 & 11.3 & 12.5 & 12.4 & 12.4 \\
\hline Finlande & 7.3 & 9.1 & 12.0 & 13.4 & 14.9 & 15.9 \\
\hline France & 11.6 & 12.9 & 13.9 & 14.1 & 16.1 & 16.4 \\
\hline Grèce & 8.1 & 11.1 & 13.1 & 14.0 & 16.6 & 17.72003 \\
\hline Hongrie & 9.0 & 11.5 & 13.4 & 13.4 & 15.1 & 15.7 \\
\hline Irlande & 10.9 & 11.2 & 10.7 & 11.4 & 11.2 & 11.2 \\
\hline Islande & 8.1 & 8.9 & 9.9 & 10.6 & 11.6 & 11.7 \\
\hline Italie & 9.0 & 10.5 & 12.9 & 14.6 & 17.7 & 19.3 \\
\hline Japon & 5.7 & 7.1 & 9.1 & 12.0 & 17.3 & 20.0 \\
\hline Luxembourg & 10.8 & 12.6 & 13.6 & 13.4 & 14.1 & 14.3 \\
\hline Mexique & .. & 3.7 & 3.8 & 4.2 & 5.3 & 5.92004 \\
\hline Norvège & 10.9 & 12.9 & 14.8 & 16.3 & 15.2 & 14.7 \\
\hline Nouvelle-Zélande & 8.7 & 8.4 & 9.7 & 11.1 & 11.8 & 12.1 \\
\hline Pays-Bas & 9.0 & 10.2 & 11.5 & 12.8 & 13.6 & 13.82003 \\
\hline Pologne & 5.8 & 8.2 & 10.1 & 10.1 & 12.2 & 13.2 \\
\hline Portugal & 7.7 & 9.5 & 11.4 & 13.6 & 16.4 & 17.0 \\
\hline République slovaque & 6.9 & 9.2 & 10.5 & 10.3 & 11.4 & 11.7 \\
\hline République tchèque & 9.6 & 12.1 & 13.5 & 12.5 & 13.8 & 14.0 \\
\hline Royaume-Uni & 11.7 & 13.0 & 15.0 & 15.7 & 15.8 & 16.02004 \\
\hline Suède & 11.8 & 13.7 & 16.3 & 17.8 & 17.3 & 17.3 \\
\hline Suisse & 10.7 & 11.8 & 14.3 & 15.0 & 15.8 & 16.22004 \\
\hline Turquie & 3.5 & 4.4 & 4.7 & 4.5 & 5.4 & 5.9 \\
\hline Moyenne la plus récente ${ }^{a}$ & . & . & . & . & . & 14.4 \\
\hline Moyenne cohérente $(29)^{b}$ & 8.9 & 10.3 & 11.9 & 12.8 & 14.1 & 14.7 \\
\hline
\end{tabular}

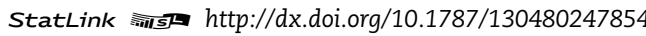

a) La moyenne comprend les dernières données disponibles pour les 30 pays de l'OCDE.

b) Exclut le Mexique.

Source: Eco-Santé OCDE 2007. 
Tableau A.1.2. Taux de fécondité en nombre d'enfants par femme âgée de 15 à 49 ans, 1960 à 2005

\begin{tabular}{|c|c|c|c|c|c|c|}
\hline & 1960 & 1970 & 1980 & 1990 & 2000 & 2005 \\
\hline Allemagne & 2.4 & 2.0 & 1.6 & 1.5 & 1.4 & 1.3 \\
\hline Australie & 3.5 & 2.9 & 1.9 & 1.9 & 1.8 & 1.8 \\
\hline Autriche & 2.7 & 2.3 & 1.6 & 1.5 & 1.3 & 1.4 \\
\hline Belgique & 2.6 & 2.3 & 1.7 & 1.6 & 1.7 & 1.7 \\
\hline Canada & 3.9 & 2.3 & 1.7 & 1.7 & 1.5 & 1.5 \\
\hline Corée & 6.0 & 4.5 & 2.8 & 1.6 & 1.5 & 1.1 \\
\hline Danemark & 2.6 & 2.0 & 1.6 & 1.7 & 1.8 & 1.8 \\
\hline Espagne & 2.9 & 2.9 & 2.2 & 1.4 & 1.2 & 1.3 \\
\hline États-Unis & 3.7 & 2.5 & 1.8 & 2.1 & 2.1 & 2.1 \\
\hline Finlande & 2.7 & 1.8 & 1.6 & 1.8 & 1.7 & 1.8 \\
\hline France & 2.7 & 2.5 & 2.0 & 1.8 & 1.9 & 1.9 \\
\hline Grèce & 2.3 & 2.4 & 2.2 & 1.4 & 1.3 & 1.3 \\
\hline Hongrie & 2.0 & 2.0 & 1.9 & 1.8 & 1.3 & 1.3 \\
\hline Irlande & 3.8 & 3.9 & 3.3 & 2.1 & 1.9 & 1.9 \\
\hline Islande & 4.2 & 2.8 & 2.5 & 2.3 & 2.1 & 2.1 \\
\hline Italie & 2.4 & 2.4 & 1.6 & 1.3 & 1.2 & 1.3 \\
\hline Japon & 2.0 & 2.1 & 1.8 & 1.5 & 1.4 & 1.3 \\
\hline Luxembourg & 2.3 & 2.0 & 1.5 & 1.6 & 1.8 & 1.7 \\
\hline Mexique & 7.3 & 6.8 & 4.7 & 3.4 & 2.7 & 2.2 \\
\hline Norvège & 2.9 & 2.5 & 1.7 & 1.9 & 1.9 & 1.8 \\
\hline Nouvelle-Zélande & 4.2 & 3.3 & 2.1 & 2.1 & 2.0 & 2.0 \\
\hline Pays-Bas & 3.1 & 2.6 & 1.6 & 1.6 & 1.7 & 1.7 \\
\hline Pologne & 3.0 & 2.2 & 2.3 & 2.0 & 1.3 & 1.2 \\
\hline Portugal & 3.1 & 2.8 & 2.2 & 1.6 & 1.6 & 1.4 \\
\hline République slovaque & 3.1 & 2.4 & 2.3 & 2.1 & 1.3 & 1.3 \\
\hline République tchèque & 2.1 & 1.9 & 2.1 & 1.9 & 1.1 & 1.3 \\
\hline Royaume-Uni & 2.7 & 2.4 & 1.9 & 1.8 & 1.7 & 1.8 \\
\hline Suède & 2.2 & 1.9 & 1.7 & 2.1 & 1.5 & 1.8 \\
\hline Suisse & 2.4 & 2.1 & 1.6 & 1.6 & 1.5 & 1.4 \\
\hline Turquie & 6.4 & 5.0 & 4.6 & 3.1 & 2.3 & 2.2 \\
\hline Moyenne (30) & 3.2 & 2.7 & 2.1 & 1.9 & 1.6 & 1.6 \\
\hline
\end{tabular}

Source: Eco-Santé OCDE 2007. 
Tableau A.1.3. Produit intérieur brut (PIB) par habitant, 2005 et taux de croissance annuel moyen, 1970 à 2005

\begin{tabular}{|c|c|c|c|c|c|}
\hline & \multirow{2}{*}{$\begin{array}{c}\text { PIB par habitant, USD PPA } \\
2005\end{array}$} & \multicolumn{4}{|c|}{ Taux de croissance annuel moyen } \\
\hline & & $1970-80$ & $1980-90$ & $1990-2000$ & $2000-05$ \\
\hline Allemagne & 30776 & 2.7 & 2.1 & 0.3 & 0.6 \\
\hline Australie & 34484 & 1.3 & 1.4 & 2.4 & 2.1 \\
\hline Autriche & 34394 & 3.5 & 2.0 & 2.0 & 1.1 \\
\hline Belgique & 33021 & 3.2 & 1.9 & 1.9 & 1.0 \\
\hline Canada & 34057 & 2.6 & 1.6 & 1.9 & 1.5 \\
\hline Corée & 22098 & 5.4 & 7.5 & 5.1 & 3.9 \\
\hline Danemark & 34110 & 1.9 & 2.0 & 2.2 & 1.1 \\
\hline Espagne & 27400 & 2.6 & 2.6 & 2.4 & 1.7 \\
\hline États-Unis & 41827 & 2.2 & 2.3 & 2.0 & 1.4 \\
\hline Finlande & 30911 & 3.3 & 2.6 & 1.6 & 2.3 \\
\hline France & 30350 & 3.0 & 1.9 & 1.5 & 1.0 \\
\hline Grèce & 29578 & 3.7 & 0.2 & 1.5 & 4.0 \\
\hline Hongrie & 17484 & . & . & . & 4.6 \\
\hline Irlande & 39019 & 3.3 & 3.3 & 6.3 & 3.4 \\
\hline Islande & 36183 & 5.3 & 1.6 & 1.5 & 3.1 \\
\hline Italie & 28401 & 3.3 & 2.2 & 1.5 & 0.4 \\
\hline Japon & 30777 & 3.3 & 3.4 & 1.0 & 1.2 \\
\hline Luxembourg & 70600 & 1.9 & 4.4 & 3.7 & 2.2 \\
\hline Mexique & 10537 & 3.3 & 0.1 & 1.5 & 0.3 \\
\hline Norvège & 48162 & 4.1 & 2.1 & 3.1 & 1.6 \\
\hline Nouvelle-Zélande & 25963 & 0.7 & 1.2 & 1.4 & 2.2 \\
\hline Pays-Bas & 35112 & 2.1 & 1.7 & 2.5 & 0.7 \\
\hline Pologne & 13915 & . & . & 3.7 & 3.1 \\
\hline Portugal & 20030 & 3.5 & 3.2 & 2.5 & 0.1 \\
\hline République slovaque & 15983 & . & . & . & 4.6 \\
\hline République tchèque & 20633 & . & . & 0.3 & 3.7 \\
\hline Royaume-Uni & 32896 & 1.8 & 2.5 & 2.1 & 2.0 \\
\hline Suède & 32111 & 1.6 & 1.9 & 1.6 & 2.0 \\
\hline Suisse & 35956 & 1.0 & 1.6 & 0.4 & 0.4 \\
\hline Turquie & 7711 & .. & . & 1.7 & 2.9 \\
\hline Moyenne & 30149 & 2.8 & 2.3 & 2.2 & 1.7 \\
\hline
\end{tabular}

StatLink नintst http://dx.doi.org/10.1787/130542265406

Source : Eco-Santé OCDE 2007 
Tableau A.2.1a. Espérance de vie à la naissance, population totale, 1960 à 2005

\begin{tabular}{|c|c|c|c|c|c|c|}
\hline & 1960 & 1970 & 1980 & 1990 & 2000 & 2005 \\
\hline Allemagne & 69.6 & 70.4 & 72.9 & 75.2 & 78.0 & 79.0 \\
\hline Australie & 70.9 & 70.8 & 74.6 & 77.0 & 79.3 & 80.9 \\
\hline Autriche & 68.7 & 70.0 & 72.6 & 75.5 & 78.1 & 79.5 \\
\hline Belgique & 70.6 & 71.0 & 73.4 & 76.1 & 77.8 & 78.7 \\
\hline Canada & 71.31961 & 72.9 & 75.3 & 77.6 & 79.3 & 80.22004 \\
\hline Corée & 52.4 & 62.2 & 65.9 & 71.4 & 76.0 & 78.5 \\
\hline Danemark & 72.4 & 73.3 & 74.3 & 74.9 & 76.9 & 77.9 \\
\hline Espagne & 69.8 & 72.0 & 75.6 & 76.8 & 79.2 & 80.7 \\
\hline États-Unis & 69.9 & 70.9 & 73.7 & 75.3 & 76.8 & 77.82004 \\
\hline Finlande & 69.0 & 70.8 & 73.4 & 74.9 & 77.6 & 78.9 \\
\hline France & 70.3 & 72.2 & 74.3 & 76.9 & 79.0 & 80.3 \\
\hline Grèce & 69.9 & 72.0 & 74.5 & 77.1 & 78.0 & 79.3 \\
\hline Hongrie & 68.0 & 69.2 & 69.1 & 69.4 & 71.7 & 72.8 \\
\hline Irlande & 70.0 & 71.2 & 72.9 & 74.9 & 76.5 & 79.5 \\
\hline Islande & 72.9 & 74.3 & 76.7 & 78.0 & 80.1 & 81.2 \\
\hline Italie & 69.81961 & 72.01971 & 74.0 & 76.9 & 79.6 & 80.4 \\
\hline Japon & 67.8 & 72.0 & 76.1 & 78.9 & 81.2 & 82.1 \\
\hline Luxembourg & 69.4 & 70.3 & 72.5 & 75.4 & 78.0 & 79.3 \\
\hline Mexique & 57.5 & 60.9 & 67.2 & 71.2 & 74.1 & 75.5 \\
\hline Norvège & 73.6 & 74.2 & 75.8 & 76.6 & 78.7 & 80.1 \\
\hline Nouvelle-Zélande & 71.3 & 71.5 & 73.2 & 75.4 & 78.7 & 79.6 \\
\hline Pays-Bas & 73.5 & 73.7 & 75.9 & 77.0 & 78.0 & 79.4 \\
\hline Pologne & 67.8 & 70.0 & 70.2 & 70.7 & 73.9 & 75.1 \\
\hline Portugal & 64.0 & 67.5 & 71.5 & 73.9 & 76.6 & 78.2 \\
\hline République slovaque & 70.6 & 69.8 & 70.6 & 71.0 & 73.3 & 74.0 \\
\hline République tchèque & 70.7 & 69.6 & 70.3 & 71.5 & 75.0 & 76.0 \\
\hline Royaume-Uni & 70.8 & 71.9 & 73.2 & 75.7 & 77.8 & 79.0 \\
\hline Suède & 73.1 & 74.7 & 75.8 & 77.6 & 79.7 & 80.6 \\
\hline Suisse & 71.6 & 73.8 & 76.2 & 77.4 & 79.8 & 81.3 \\
\hline Turquie & 48.3 & 54.2 & 58.1 & 66.1 & 70.5 & 71.4 \\
\hline Moyenne (30) & 68.5 & 70.3 & 72.7 & 74.9 & 77.3 & 78.6 \\
\hline
\end{tabular}

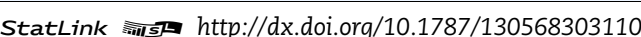
Note : La méthodologie utilisée pour calculer l'espérance de vie peut varier d'un pays à l'autre, affectant la comparabilité des données (en fonction de la méthode, l'espérance de vie d'un pays peut varier d'une fraction d'année). L'espérance de vie à la naissance pour la population totale est estimée par le Secrétariat de l'OCDE pour tous les pays, en prenant une moyenne non pondérée de l'espérance de vie pour les hommes et les femmes.

Source : Eco-Santé OCDE 2007. (Pour les 22 pays européens, la base de données Eurostat NewCronos est la principale source de données à partir de 1985.) 
Tableau A.2.1b. Espérance de vie des femmes à la naissance, 1960 à 2005

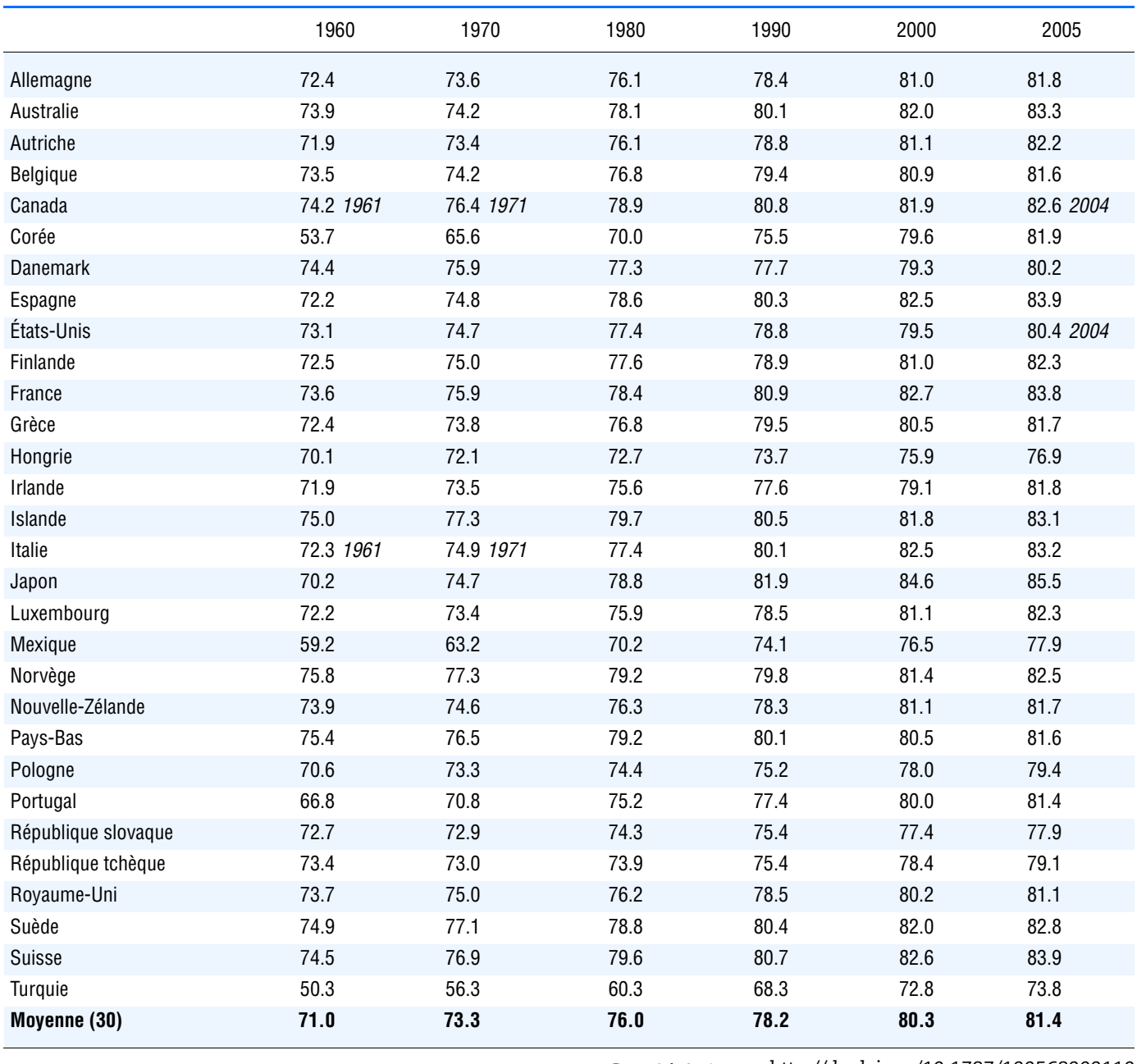

StatLink intsL http://dx.doi.org/10.1787/130568303110 Note : La méthodologie utilisée pour calculer l'espérance de vie peut varier d'un pays à l'autre, affectant la comparabilité des données (en fonction de la méthode, l'espérance de vie d'un pays peut varier d'une fraction d'année).

Source : Eco-Santé OCDE 2007. (Pour les 22 pays européens, la base de données Eurostat NewCronos est la principale source de données à partir de 1985.) 
Tableau A.2.1c. Espérance de vie des hommes à la naissance, 1960 à 2005

\begin{tabular}{|c|c|c|c|c|c|c|}
\hline & 1960 & 1970 & 1980 & 1990 & 2000 & 2005 \\
\hline Allemagne & 66.9 & 67.2 & 69.6 & 72.0 & 75.0 & 76.2 \\
\hline Australie & 67.9 & 67.4 & 71.0 & 73.9 & 76.6 & 78.5 \\
\hline Autriche & 65.4 & 66.5 & 69.0 & 72.2 & 75.1 & 76.7 \\
\hline Belgique & 67.7 & 67.8 & 70.0 & 72.7 & 74.6 & 75.8 \\
\hline Canada & 68.41961 & 69.31971 & 71.7 & 74.4 & 76.7 & 77.82004 \\
\hline Corée & 51.1 & 58.7 & 61.8 & 67.3 & 72.3 & 75.1 \\
\hline Danemark & 70.4 & 70.7 & 71.2 & 72.0 & 74.5 & 75.6 \\
\hline Espagne & 67.4 & 69.2 & 72.5 & 73.3 & 75.8 & 77.4 \\
\hline États-Unis & 66.6 & 67.1 & 70.0 & 71.8 & 74.1 & 75.22004 \\
\hline Finlande & 65.5 & 66.5 & 69.2 & 70.9 & 74.2 & 75.5 \\
\hline France & 67.0 & 68.4 & 70.2 & 72.8 & 75.3 & 76.7 \\
\hline Grèce & 67.3 & 70.1 & 72.2 & 74.6 & 75.5 & 76.8 \\
\hline Hongrie & 65.9 & 66.3 & 65.5 & 65.1 & 67.4 & 68.6 \\
\hline Irlande & 68.1 & 68.8 & 70.1 & 72.1 & 73.9 & 77.1 \\
\hline Islande & 70.7 & 71.2 & 73.7 & 75.4 & 78.4 & 79.2 \\
\hline Italie & 67.21961 & 69.01971 & 70.6 & 73.6 & 76.6 & 77.6 \\
\hline Japon & 65.3 & 69.3 & 73.4 & 75.9 & 77.7 & 78.6 \\
\hline Luxembourg & 66.5 & 67.1 & 69.1 & 72.3 & 74.8 & 76.2 \\
\hline Mexique & 55.8 & 58.5 & 64.1 & 68.3 & 71.6 & 73.0 \\
\hline Norvège & 71.3 & 71.0 & 72.3 & 73.4 & 76.0 & 77.7 \\
\hline Nouvelle-Zélande & 68.7 & 68.3 & 70.0 & 72.4 & 76.3 & 77.5 \\
\hline Pays-Bas & 71.5 & 70.8 & 72.5 & 73.8 & 75.5 & 77.2 \\
\hline Pologne & 64.9 & 66.6 & 66.0 & 66.2 & 69.7 & 70.8 \\
\hline Portugal & 61.2 & 64.2 & 67.7 & 70.4 & 73.2 & 74.9 \\
\hline République slovaque & 68.4 & 66.7 & 66.8 & 66.6 & 69.1 & 70.1 \\
\hline République tchèque & 67.9 & 66.1 & 66.8 & 67.6 & 71.6 & 72.9 \\
\hline Royaume-Uni & 67.9 & 68.7 & 70.2 & 72.9 & 75.4 & 76.9 \\
\hline Suède & 71.2 & 72.2 & 72.8 & 74.8 & 77.4 & 78.4 \\
\hline Suisse & 68.7 & 70.7 & 72.8 & 74.0 & 76.9 & 78.7 \\
\hline Turquie & 46.3 & 52.0 & 55.8 & 63.8 & 68.1 & 68.9 \\
\hline Moyenne (30) & 66.0 & 67.2 & 69.3 & 71.6 & 74.3 & 75.7 \\
\hline
\end{tabular}

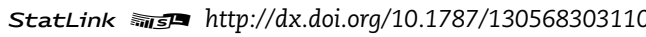
Note : La méthodologie utilisée pour calculer l'espérance de vie peut varier d'un pays à l'autre, affectant la comparabilité des données (en fonction de la méthode, l'espérance de vie d'un pays peut varier d'une fraction d'année).

Source : Eco-Santé OCDE 2007. (Pour les 22 pays européens, la base de données Eurostat NewCronos est la principale source de données à partir de 1985.) 
Tableau A.2.2a. Espérance de vie des femmes à 65 ans, 1960 à 2005

\begin{tabular}{|c|c|c|c|c|c|c|}
\hline & 1960 & 1970 & 1980 & 1990 & 2000 & 2005 \\
\hline Allemagne & 14.6 & 15.0 & 16.7 & 17.6 & 19.4 & 19.62003 \\
\hline Australie & 15.6 & 15.6 & 17.9 & 19.0 & 20.4 & 21.4 \\
\hline Autriche & 14.7 & 14.9 & 16.3 & 17.8 & 19.4 & 20.3 \\
\hline Belgique & 14.8 & 15.3 & 16.9 & 18.5 & 19.6 & 20.0 \\
\hline Canada & 16.11961 & 17.51971 & 18.9 & 19.9 & 20.4 & 21.02004 \\
\hline Corée & $\ldots$ & 14.6 & 15.1 & 16.3 & 18.2 & 19.9 \\
\hline Danemark & 15.3 & 16.7 & 17.6 & 17.8 & 18.3 & 18.62003 \\
\hline Espagne & 15.3 & 16.0 & 17.9 & 19.0 & 20.4 & 20.72002 \\
\hline États-Unis & 15.8 & 17.0 & 18.3 & 18.9 & 19.2 & 20.02004 \\
\hline Finlande & 13.7 & 14.41971 & 16.5 & 17.7 & 19.6 & 21.1 \\
\hline France & 15.6 & 16.8 & 18.2 & 19.8 & 21.2 & 21.42002 \\
\hline Grèce & 14.6 & 15.2 & 16.8 & 18.0 & 18.3 & 19.4 \\
\hline Hongrie & 13.8 & 14.3 & 14.6 & 15.3 & 16.5 & 16.9 \\
\hline Irlande & 14.4 & 15.0 & 15.7 & 16.9 & 17.8 & 18.92003 \\
\hline Islande & 16.81963 & 17.81973 & 19.1 & 19.5 & 19.7 & 20.7 \\
\hline Italie & 15.31961 & 16.21971 & 17.1 & 18.8 & 20.4 & . \\
\hline Japon & 14.1 & 15.3 & 17.7 & 20.0 & 22.4 & 23.2 \\
\hline Luxembourg & 14.5 & 14.9 & 16.0 & 18.2 & 19.7 & 19.02003 \\
\hline Mexique & 14.6 & 15.6 & 17.0 & 18.0 & 18.3 & 18.7 \\
\hline Norvège & 16.0 & 16.7 & 18.0 & 18.5 & 19.7 & 20.12003 \\
\hline Nouvelle-Zélande & 15.6 & 16.0 & 17.0 & 18.3 & 20.0 & 20.4 \\
\hline Pays-Bas & 15.3 & 16.1 & 18.0 & 18.9 & 19.2 & 20.0 \\
\hline Pologne & 14.9 & 15.3 & 15.5 & 16.1 & 17.5 & 18.6 \\
\hline Portugal & 15.3 & 15.0 & 16.5 & 17.0 & 18.7 & 18.92003 \\
\hline République slovaque & 14.6 & 14.5 & 15.4 & 15.7 & 16.5 & 16.9 \\
\hline République tchèque & 14.5 & 14.2 & 14.3 & 15.2 & 17.1 & 17.32003 \\
\hline Royaume-Uni & 15.1 & 16.0 & 16.6 & 17.9 & 18.9 & 19.12002 \\
\hline Suède & 15.3 & 16.8 & 17.9 & 19.0 & 20.0 & 20.6 \\
\hline Suisse & $\ldots$ & . & 18.31982 & 19.4 & 20.7 & 21.02003 \\
\hline Turquie & 12.1 & 12.6 & 12.8 & 13.9 & 14.6 & 15.0 \\
\hline Moyenne cohérente (28) & 14.9 & 15.6 & 16.8 & 17.9 & 19.0 & 19.6 \\
\hline
\end{tabular}

StatLink 完15 $h$ ttp://dx.doi.org/10.1787/130573778055 Note : La méthodologie utilisée pour calculer l'espérance de vie peut varier d'un pays à l'autre, affectant la comparabilité des données (en fonction de la méthode, l'espérance de vie d'un pays peut varier d'une fraction d'année).

a) Exclut la Corée et la Suisse. La moyenne OCDE de 2005 inclut les dernières données disponibles pour l'Italie (2001). Source: Eco-Santé OCDE 2007. (Pour les 22 pays européens, la base de données Eurostat NewCronos est la principale source de données à partir de 1985.) 
Tableau A.2.2b. Espérance de vie des hommes à 65 ans, 1960 à 2005

\begin{tabular}{|c|c|c|c|c|c|c|}
\hline & 1960 & 1970 & 1980 & 1990 & 2000 & 2005 \\
\hline Allemagne & 12.4 & 12.0 & 13.0 & 14.0 & 15.7 & 16.12003 \\
\hline Australie & 12.5 & 11.9 & 13.7 & 15.2 & 16.9 & 18.1 \\
\hline Autriche & 12.0 & 11.7 & 12.9 & 14.3 & 16.0 & 17.0 \\
\hline Belgique & 12.4 & 12.1 & 13.0 & 14.3 & 15.5 & 16.3 \\
\hline Canada & 13.51961 & 13.71971 & 14.5 & 15.7 & 16.8 & 17.72004 \\
\hline Corée & . . & 10.2 & 10.5 & 12.4 & 14.3 & 15.8 \\
\hline Danemark & 13.7 & 13.7 & 13.6 & 14.0 & 15.2 & 15.52003 \\
\hline Espagne & 13.1 & 13.3 & 14.8 & 15.4 & 16.6 & 16.82002 \\
\hline États-Unis & 12.8 & 13.1 & 14.1 & 15.1 & 16.3 & 17.12004 \\
\hline Finlande & 11.5 & 11.41971 & 12.5 & 13.7 & 15.6 & 17.0 \\
\hline France & 12.5 & 13.0 & 13.6 & 15.5 & 16.7 & 17.12002 \\
\hline Grèce & 13.4 & 13.9 & 14.6 & 15.7 & 16.2 & 17.2 \\
\hline Hongrie & 12.3 & 12.0 & 11.6 & 12.0 & 12.7 & 13.1 \\
\hline Irlande & 12.6 & 12.4 & 12.6 & 13.3 & 14.6 & 15.72003 \\
\hline Islande & 15.01963 & 15.01973 & 15.8 & 16.2 & 18.1 & 18.0 \\
\hline Italie & 13.41961 & 13.31971 & 13.3 & 15.1 & 16.5 & .. \\
\hline Japon & 11.6 & 12.5 & 14.6 & 16.2 & 17.5 & 18.1 \\
\hline Luxembourg & 12.5 & 12.1 & 12.3 & 14.2 & 15.5 & 15.52003 \\
\hline Mexique & 14.2 & 14.8 & 15.4 & 16.2 & 16.8 & 17.1 \\
\hline Norvège & 14.5 & 13.8 & 14.3 & 14.6 & 16.0 & 16.72003 \\
\hline Nouvelle-Zélande & 13.0 & 12.4 & 13.2 & 14.7 & 16.7 & 17.5 \\
\hline Pays-Bas & 13.9 & 13.3 & 13.7 & 14.4 & 15.3 & 16.4 \\
\hline Pologne & 12.7 & 12.5 & 12.0 & 12.4 & 13.6 & 14.4 \\
\hline Portugal & 13.0 & 12.2 & 12.9 & 13.9 & 15.3 & 15.62003 \\
\hline République slovaque & 13.2 & 12.3 & 12.3 & 12.2 & 12.9 & 13.2 \\
\hline République tchèque & 12.5 & 11.1 & 11.2 & 11.6 & 13.7 & 13.92003 \\
\hline Royaume-Uni & 11.9 & 12.0 & 12.6 & 14.0 & 15.7 & 16.12002 \\
\hline Suède & 13.7 & 14.2 & 14.3 & 15.3 & 16.7 & 17.4 \\
\hline Suisse & . & .. & 14.61982 & 15.3 & 16.9 & 17.52003 \\
\hline Turquie & 11.2 & 11.5 & 11.7 & 12.4 & 12.9 & 13.1 \\
\hline Moyenne cohérente (28) ${ }^{a}$ & 12.9 & 12.8 & 13.4 & 14.3 & 15.6 & 16.2 \\
\hline
\end{tabular}

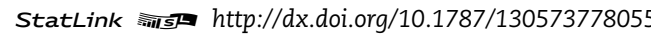
Note : La méthodologie utilisée pour calculer l'espérance de vie peut varier d'un pays à l'autre, affectant la comparabilité des données (en fonction de la méthode, l'espérance de vie d'un pays peut varier d'une fraction d'année).

a) Exclut la Corée et la Suisse. La moyenne OCDE de 2005 inclut la dernière donnée disponible pour l'Italie (2001). Source: Eco-Santé OCDE 2007. (Pour les 22 pays européens, la base de données Eurostat NewCronos est la principale source de données à partir de 1985.) 
Tableau A.2.3a. Années potentielles de vie perdues (APVP) des femmes, toutes causes confondues, 1970 à 2004

\begin{tabular}{|c|c|c|c|c|c|}
\hline & 1970 & 1980 & 1990 & 2000 & 2004 \\
\hline Allemagne & 6673 & 4702 & 3539 & 2606 & 2351 \\
\hline Australie & 6311 & 4267 & 3294 & 2605 & 23622003 \\
\hline Autriche & 6803 & 4950 & 3456 & 2638 & 2386 \\
\hline Belgique & 6176 & 4807 & 3574 & 30531987 & . \\
\hline Canada & 5646 & 4385 & 3317 & 2676 & 26692002 \\
\hline Corée & .. & . & 3980 & 2940 & 2466 \\
\hline Danemark & 5169 & 4523 & 3989 & 3055 & 30812001 \\
\hline Espagne & 63501969 & 3933 & 3184 & 2325 & 2079 \\
\hline États-Unis & 6679 & 5125 & 4338 & 3772 & 37192002 \\
\hline Finlande & 5177 & 3363 & 3312 & 2603 & 2511 \\
\hline France & 5358 & 4206 & 3210 & 2609 & 24672003 \\
\hline Grèce & 6189 & 4411 & 3215 & 2352 & 2160 \\
\hline Hongrie & 8019 & 6908 & 6262 & 4772 & 43102003 \\
\hline Irlande & 6321 & 4780 & 3731 & 3085 & 2471 \\
\hline Islande & 4029 & 2948 & 2938 & 2117 & 2241 \\
\hline Italie & 6867 & 4324 & 3022 & 2307 & 21792002 \\
\hline Japon & 5555 & 3382 & 2490 & 2068 & 1906 \\
\hline Luxembourg & 7389 & 5261 & 3814 & 2747 & 2087 \\
\hline Mexique & 17634 & 105771981 & 8029 & .. & . \\
\hline Norvège & 4110 & 3289 & 3101 & 2561 & 2434 \\
\hline Nouvelle-Zélande & 6275 & 5518 & 4206 & 3108 & 30692001 \\
\hline Pays-Bas & 4680 & 3579 & 3163 & 2842 & 2500 \\
\hline Pologne & 7682 & 5996 & 5227 & 3705 & 3306 \\
\hline Portugal & 11811 & 6308 & 4399 & 3166 & 28582003 \\
\hline République slovaque & . & . & 45391992 & 3587 & 36382002 \\
\hline République tchèque & .. & . & 4415 & 3019 & 2697 \\
\hline Royaume-Uni & 5756 & 4667 & 3559 & 29471999 & 2713 \\
\hline Suède & 4345 & 3429 & 2937 & 2191 & 21412002 \\
\hline Suisse & 4910 & 3704 & 3016 & 2399 & 2133 \\
\hline Turquie & . & . & . & . & . \\
\hline Moyenne la plus récente ${ }^{a}$ & . & . & 3836 & 2852 & 2627 \\
\hline Moyenne cohérente $(24)^{b}$ & 6171 & 4498 & 3613 & 2802 & 2589 \\
\hline
\end{tabular}

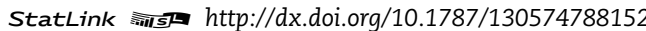

a) La moyenne comprend tous les pays pour lesquels des données récentes sont disponibles.

b) Exclut la Belgique, la Corée, le Mexique, la République slovaque, la République tchèque et la Turquie.

Source: Eco-Santé OCDE 2007. Les statistiques de mortalité sont extraites de la base de données sur la mortalité de l'OMS (avril 2007). 
Tableau A.2.3b. Années potentielles de vie perdues (APVP) des hommes, toutes causes confondues, 1970 à 2004

\begin{tabular}{|c|c|c|c|c|c|}
\hline & 1970 & 1980 & 1990 & 2000 & 2004 \\
\hline Allemagne & 11385 & 8600 & 6809 & 4984 & 4354 \\
\hline Australie & 10869 & 7946 & 6016 & 4618 & 40822003 \\
\hline Autriche & 12301 & 9771 & 6836 & 5139 & 4619 \\
\hline Belgique & 10566 & 8592 & 6505 & 55761987 & . \\
\hline Canada & 9830 & 8130 & 6122 & 4456 & 42962002 \\
\hline Corée & .. & & 8706 & 6388 & 5219 \\
\hline Danemark & 8207 & 7380 & 6537 & 4953 & 47232001 \\
\hline Espagne & 100441969 & 7265 & 6884 & 5142 & 4528 \\
\hline États-Unis & 11937 & 9516 & 8262 & 6478 & 64182002 \\
\hline Finlande & 11697 & 8465 & 7714 & 5682 & 5417 \\
\hline France & 9929 & 8717 & 7184 & 5608 & 52342003 \\
\hline Grèce & 9257 & 7241 & 5728 & 5140 & 4622 \\
\hline Hongrie & 12881 & 12766 & 13288 & 10520 & 94832003 \\
\hline Irlande & 9209 & 7914 & 6151 & 5505 & 4246 \\
\hline Islande & 9318 & 7133 & 5607 & 4526 & 3114 \\
\hline Italie & 10816 & 7942 & 6029 & 4368 & 41982002 \\
\hline Japon & 9012 & 5913 & 4602 & 3932 & 3606 \\
\hline Luxembourg & 12534 & 7829 & 7013 & 5211 & 4737 \\
\hline Mexique & 22909 & 173831981 & 12916 & .. & . \\
\hline Norvège & 8086 & 6799 & 5940 & 4711 & 3961 \\
\hline Nouvelle-Zélande & 10395 & 8495 & 7361 & 5208 & 50352001 \\
\hline Pays-Bas & 7938 & 6298 & 5231 & 4256 & 3693 \\
\hline Pologne & 13026 & 12717 & 11969 & 8698 & 8075 \\
\hline Portugal & 17404 & 11987 & 9064 & 7130 & 60242003 \\
\hline République slovaque & . & . & 106831992 & 8739 & 81172002 \\
\hline République tchèque & .. & . & 9690 & 6581 & 6012 \\
\hline Royaume-Uni & 9208 & 7502 & 5934 & 48201999 & 4390 \\
\hline Suède & 7178 & 6258 & 5041 & 3661 & 34912002 \\
\hline Suisse & 8966 & 7074 & 5991 & 4400 & 3769 \\
\hline Turquie & .. & . & . & . & . \\
\hline Moyenne la plus récente ${ }^{a}$ & . & . & 7442 & 5587 & 5017 \\
\hline Moyenne cohérente $(24)^{b}$ & 10476 & 8319 & 6971 & 5381 & 4838 \\
\hline
\end{tabular}

StatLink न्ताsh http://dx.doi.org/10.1787/130574788152

a) La moyenne comprend tous les pays pour lesquels des données récentes sont disponibles.

b) Exclut la Belgique, la Corée, le Mexique, la République slovaque, la République tchèque et la Turquie.

Source: Eco-Santé OCDE 2007. Les statistiques de mortalité sont extraites de la base de données sur la mortalité de l'OMS (avril 2007). 
Tableau A.2.4. Taux de mortalité due à une crise cardiaque ou à un accident vasculaire cérébral, taux pour 100000 habitants normalisés par rapport à l'âge, 1980 et 2004

\begin{tabular}{|c|c|c|c|c|c|c|c|c|c|c|c|c|}
\hline & \multicolumn{6}{|c|}{ Crise cardiaque } & \multicolumn{6}{|c|}{ Accident vasculaire cérébral } \\
\hline & \multicolumn{3}{|c|}{1980} & \multicolumn{3}{|c|}{2004} & \multicolumn{3}{|c|}{1980} & \multicolumn{3}{|c|}{2004} \\
\hline & Femmes & Hommes & Total & Femmes & Hommes & Total & Femmes & Hommes & Total & Femmes & Hommes & Total \\
\hline Allemagne & 107.6 & 249.7 & 162.2 & 75.7 & 142.9 & 104.2 & 115.3 & 145.9 & 126.8 & 41.3 & 49.9 & 45.4 \\
\hline Australie & 164.9 & 341.5 & 242.5 & 65.4 & 122.3 & 91.32003 & 104.5 & 116.0 & 110.7 & 39.5 & 43.7 & 41.72003 \\
\hline Autriche & 100.5 & 219.3 & 147.1 & 82.7 & 144.5 & 108.3 & 133.1 & 168.7 & 146.7 & 36.1 & 45.0 & 40.1 \\
\hline Belgique & 80.4 & 186.7 & 126.0 & .. & . & . & 89.8 & 111.5 & 98.7 & .. & .. & .. \\
\hline Canada & 159.1 & 322.0 & 231.8 & 67.7 & 134.8 & 97.22002 & 64.1 & 78.1 & 70.2 & 32.3 & 39.0 & 35.32002 \\
\hline Corée & . & . & .. & 27.1 & 45.6 & 34.9 & .. & . & .. & 81.3 & 118.2 & 95.8 \\
\hline Danemark & 179.6 & 366.1 & 261.2 & 75.3 & 148.6 & 106.42001 & 68.6 & 88.7 & 77.0 & 50.8 & 64.9 & 56.92001 \\
\hline Espagne & 49.1 & 108.7 & 75.1 & 34.8 & 79.1 & 54.5 & 121.2 & 142.4 & 130.3 & 39.1 & 49.6 & 44.0 \\
\hline États-Unis & 168.5 & 330.2 & 237.1 & 94.3 & 170.3 & 127.62002 & 65.1 & 76.7 & 70.0 & 38.3 & 41.4 & 39.92002 \\
\hline Finlande & 168.9 & 411.4 & 265.2 & 91.0 & 201.3 & 137.2 & 100.4 & 122.1 & 108.9 & 47.7 & 61.2 & 53.7 \\
\hline France & 47.3 & 108.0 & 73.5 & 26.3 & 64.2 & 42.52003 & 78.2 & 111.7 & 91.8 & 29.5 & 41.1 & 34.52003 \\
\hline Grèce & 44.2 & 114.5 & 76.3 & 53.9 & 115.8 & 82.9 & 151.8 & 143.9 & 148.9 & 99.3 & 96.0 & 98.5 \\
\hline Hongrie & 156.1 & 298.0 & 217.0 & 169.6 & 292.4 & 219.72003 & 189.1 & 243.0 & 211.7 & 108.6 & 160.8 & 129.52003 \\
\hline Irlande & 177.2 & 367.3 & 264.9 & 80.3 & 164.4 & 118.4 & 127.3 & 132.0 & 129.7 & 41.2 & 45.9 & 43.7 \\
\hline Islande & 136.7 & 325.1 & 224.5 & 61.6 & 160.8 & 106.0 & 67.0 & 67.0 & 67.0 & 37.1 & 46.5 & 41.1 \\
\hline Italie & 86.9 & 169.6 & 123.2 & 46.9 & 97.7 & 68.52002 & 104.0 & 133.9 & 116.7 & 49.4 & 64.1 & 55.42002 \\
\hline Japon & 39.5 & 65.7 & 50.9 & 19.5 & 42.0 & 29.5 & 148.1 & 203.8 & 172.3 & 39.6 & 65.7 & 50.7 \\
\hline Luxembourg & 96.5 & 191.0 & 137.7 & 47.1 & 110.0 & 72.5 & 159.5 & 203.8 & 177.0 & 50.4 & 57.2 & 53.4 \\
\hline Mexique & . & & & . & & . & . & & & . & . & \\
\hline Norvège & 125.2 & 293.4 & 200.6 & 56.6 & 120.7 & 84.6 & 86.7 & 106.6 & 95.4 & 41.2 & 50.8 & 45.4 \\
\hline Nouvelle-Zélande & 193.3 & 386.3 & 277.2 & 97.1 & 179.4 & 133.42001 & 116.0 & 126.0 & 120.2 & 54.2 & 54.7 & 55.12001 \\
\hline Pays-Bas & 106.1 & 246.2 & 167.2 & 40.8 & 89.6 & 61.5 & 73.6 & 89.5 & 80.4 & 41.6 & 49.5 & 45.2 \\
\hline Pologne & 57.5 & 160.7 & 101.5 & 76.1 & 160.1 & 110.9 & 69.3 & 82.1 & 75.1 & 77.2 & 102.5 & 88.1 \\
\hline Portugal & 64.2 & 124.3 & 89.6 & 43.8 & 78.8 & 59.42003 & 250.1 & 306.2 & 273.9 & 99.6 & 125.9 & 111.22003 \\
\hline République slovaque & .. & . & .. & 215.9 & 341.3 & 266.82002 & .. & .. & . & 70.9 & 105.6 & 84.92002 \\
\hline République tchèque & . & . & .. & 117.3 & 209.4 & 155.6 & . & .. & . & 95.8 & 121.5 & 106.5 \\
\hline Royaume-Uni & 162.0 & 366.6 & 247.7 & 73.1 & 153.6 & 108.7 & 107.4 & 122.8 & 114.1 & 53.1 & 58.3 & 55.9 \\
\hline Suède & 187.7 & 388.5 & 276.8 & 75.7 & 159.1 & 112.02002 & 69.6 & 83.4 & 75.9 & 48.7 & 58.2 & 53.12002 \\
\hline Suisse & 71.5 & 175.7 & 115.6 & 47.3 & 95.2 & 67.5 & 80.3 & 98.1 & 87.4 & 26.2 & 33.2 & 29.2 \\
\hline Turquie & .. & . & . & .. & .. & .. & .. & . & . & .. & .. & .. \\
\hline Moyenne la plus récente ${ }^{a}$ & . & . & . & 72.7 & 141.6 & 102.3 & . & . & . & 54.4 & 68.5 & 60.5 \\
\hline Moyenne cohérente (24) & 118.8 & 255.4 & 177.8 & 67.0 & 134.8 & 96.3 & 110.4 & 133.0 & 119.9 & 50.9 & 62.7 & 56.1 \\
\hline
\end{tabular}

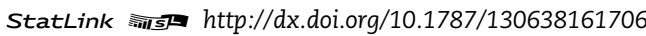
Note : Les taux de mortalité sont normalisés par rapport à l'âge en fonction de la structure de la population de l'ensemble des pays de l'OCDE de 1980.

a) La moyenne comprend tous les pays pour lesquels des données récentes sont disponibles (2001+).

b) Exclut la Belgique, la Corée, le Mexique, la République slovaque, la République tchèque et la Turquie.

Source: Eco-Santé OCDE 2007. Les statistiques de mortalité sont extraites de la base de données sur la mortalité de l'OMS (avril 2007). 
Tableau A.2.5a. Taux de mortalité due au cancer, taux pour 100000 habitants normalisés par rapport à l'âge, 1960, 1980 et 2004

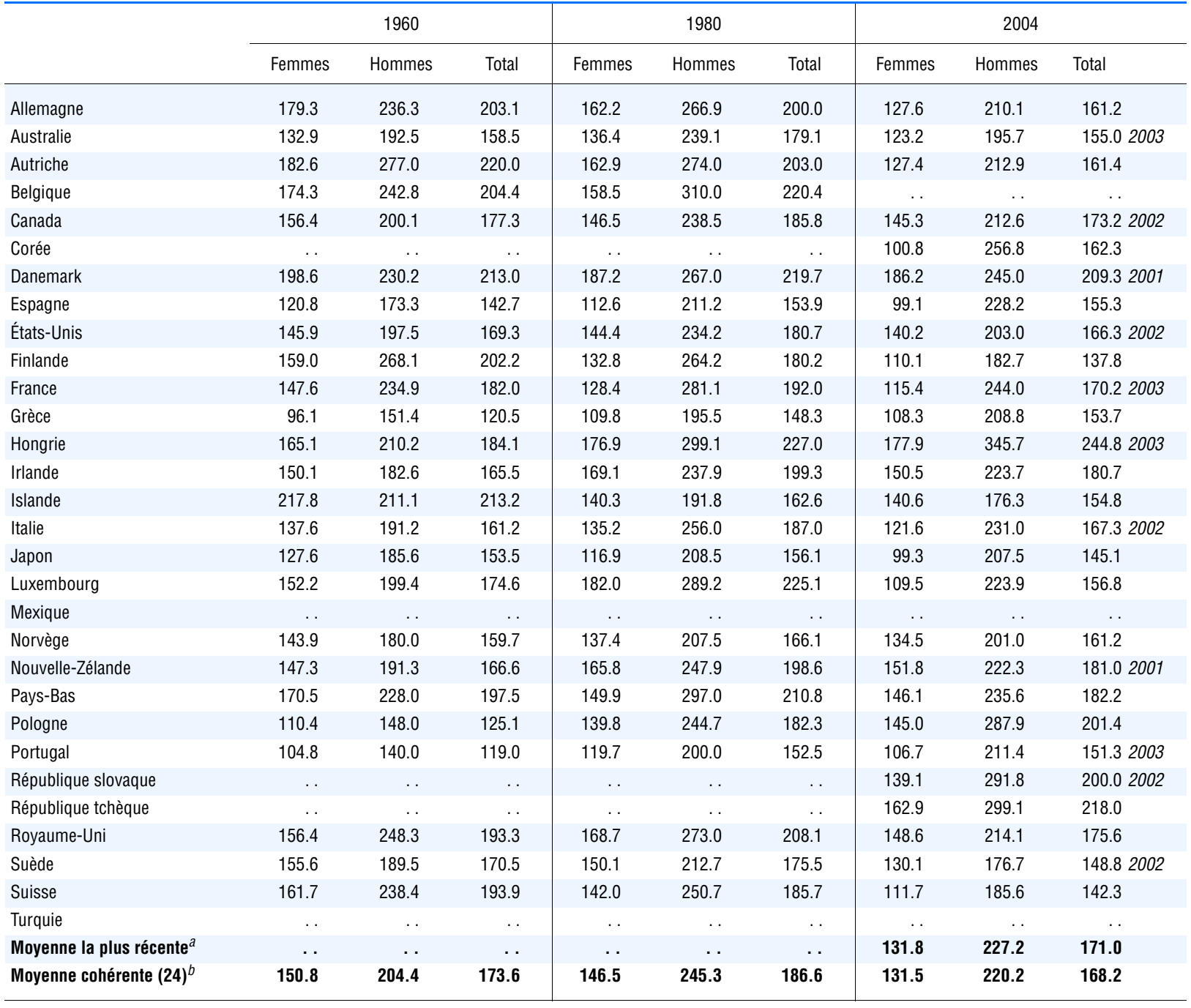

StatLink नilst $h t t p: / / d x . d o i . o r g / 10.1787 / 130647115052$ Note : Les taux de mortalité sont normalisés par rapport à l'âge en fonction de la structure de la population de l'ensemble des pays de l'OCDE de 1980.

a) La moyenne comprend tous les pays pour lesquels des données récentes sont disponibles (2001+).

b) Exclut la Belgique, la Corée, le Mexique, la République slovaque, la République tchèque et la Turquie.

Source: Eco-Santé OCDE 2007. Les statistiques de mortalité sont extraites de la base de données sur la mortalité de l'OMS (avril 2007). 
Tableau A.2.5b. Taux de mortalité due au cancer du poumon, taux pour 100000 habitants normalisés par rapport à l'âge, 1960, 1980 et 2004

\begin{tabular}{|c|c|c|c|c|c|c|c|c|c|}
\hline & \multicolumn{3}{|c|}{1960} & \multicolumn{3}{|c|}{1980} & \multicolumn{3}{|c|}{2004} \\
\hline & Femmes & Hommes & Total & Femmes & Hommes & Total & Femmes & Hommes & Total \\
\hline Allemagne & 6.0 & 45.2 & 22.7 & 8.1 & 70.8 & 31.9 & 15.7 & 53.5 & 31.9 \\
\hline Australie & 4.6 & 35.9 & 19.0 & 13.0 & 70.8 & 38.2 & 19.5 & 42.5 & 30.02003 \\
\hline Autriche & 7.3 & 65.5 & 31.2 & 10.4 & 72.5 & 34.0 & 16.3 & 48.5 & 30.0 \\
\hline Belgique & 5.1 & 48.5 & 24.8 & 8.4 & 110.6 & 51.7 & . & $\ldots$ & $\ldots$ \\
\hline Canada & 4.8 & 33.9 & 19.2 & 17.2 & 75.0 & 42.9 & 35.1 & 63.5 & 47.42002 \\
\hline Corée & $\ldots$ & . & . & . & $\ldots$ & . & 15.3 & 66.3 & 35.1 \\
\hline Danemark & 8.1 & 38.2 & 22.3 & 18.8 & 75.2 & 43.5 & 39.0 & 62.9 & 49.02001 \\
\hline Espagne & 5.1 & 22.5 & 12.7 & 5.4 & 46.1 & 22.9 & 7.5 & 63.4 & 32.5 \\
\hline États-Unis & 6.0 & 40.7 & 22.4 & 22.1 & 77.2 & 45.5 & 37.3 & 65.0 & 49.32002 \\
\hline Finlande & 3.8 & 76.1 & 33.0 & 8.7 & 94.2 & 41.1 & 11.3 & 45.1 & 25.1 \\
\hline France & 4.5 & 28.2 & 14.6 & 5.2 & 57.1 & 27.6 & 11.5 & 59.1 & 32.72003 \\
\hline Grèce & 6.1 & 32.6 & 18.11961 & 8.3 & 59.7 & 31.7 & 10.3 & 67.7 & 36.6 \\
\hline Hongrie & 8.5 & 35.6 & 20.5 & 14.0 & 79.5 & 41.7 & 29.5 & 105.5 & 60.52003 \\
\hline Irlande & 7.3 & 32.0 & 19.5 & 19.8 & 62.9 & 39.7 & 27.3 & 51.8 & 38.1 \\
\hline Islande & 16.5 & 12.6 & 15.1 & 29.7 & 24.5 & 27.0 & 38.5 & 37.3 & 37.8 \\
\hline Italie & 5.2 & 26.4 & 14.9 & 8.2 & 71.8 & 36.3 & 12.5 & 66.4 & 35.72002 \\
\hline Japon & 4.6 & 13.4 & 8.6 & 10.2 & 35.7 & 21.1 & 11.8 & 46.9 & 26.6 \\
\hline Luxembourg & . & . & . & 10.6 & 96.5 & 46.8 & 14.3 & 59.6 & 33.5 \\
\hline Mexique & $\ldots$ & . & . & 7.0 & 18.4 & 12.21981 & . & $\ldots$ & $\ldots$ \\
\hline Norvège & 3.6 & 16.2 & 9.5 & 7.6 & 33.8 & 19.3 & 23.7 & 44.3 & 32.7 \\
\hline Nouvelle-Zélande & 6.1 & 37.6 & 20.7 & 17.6 & 71.6 & 41.1 & 26.6 & 45.2 & 34.62001 \\
\hline Pays-Bas & 4.6 & 53.9 & 28.0 & 8.1 & 113.0 & 52.9 & 26.0 & 72.2 & 45.4 \\
\hline Pologne & 4.2 & 22.5 & 11.8 & 9.5 & 72.6 & 36.0 & 17.8 & 91.0 & 47.8 \\
\hline Portugal & 2.9 & 11.4 & 6.4 & 5.0 & 28.7 & 15.0 & 7.0 & 40.7 & 21.82003 \\
\hline République slovaque & . & . & . & . & $\cdots$ & . & 9.8 & 73.0 & 35.52002 \\
\hline République tchèque & . & . & . & . & . & . & 17.4 & 77.5 & 42.7 \\
\hline Royaume-Uni & 10.4 & 82.0 & 40.9 & 23.8 & 103.7 & 56.1 & 28.7 & 52.4 & 39.0 \\
\hline Suède & 4.9 & 19.1 & 11.6 & 9.9 & 35.8 & 21.4 & 19.2 & 29.9 & 23.82002 \\
\hline Suisse & 4.2 & 41.5 & 20.6 & 7.4 & 66.3 & 32.6 & 15.5 & 43.4 & 27.6 \\
\hline Turquie & . & . & . & . & . & . & . & . & $\cdots$ \\
\hline Moyenne la plus récente ${ }^{a}$ & . & $\ldots$ & $\ldots$ & . & . & . & 20.2 & 58.3 & 36.4 \\
\hline Moyenne cohérente $(23)^{b}$ & 6.1 & 35.8 & 19.3 & 12.5 & 65.2 & 34.8 & 21.2 & 56.4 & 36.3 \\
\hline
\end{tabular}

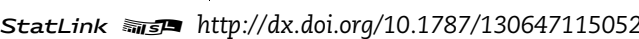
Note : Les taux de mortalité sont normalisés par rapport à l'âge en fonction de la structure de la population de l'ensemble des pays de l'OCDE de 1980.

a) La moyenne comprend tous les pays pour lesquels des données récentes sont disponibles (2001+).

b) Exclut la Belgique, la Corée, le Luxembourg, le Mexique, la République slovaque, la République tchèque et la Turquie.

Source : Eco-Santé OCDE 2007. Les statistiques de mortalité sont extraites de la base de données sur la mortalité de l'OMS (avril 2007). 
Tableau A.2.5c. Taux de mortalité due aux cancers du sein et de la prostate, taux pour 100000 habitants normalisés par rapport à l’âge, 1960, 1980, 2004

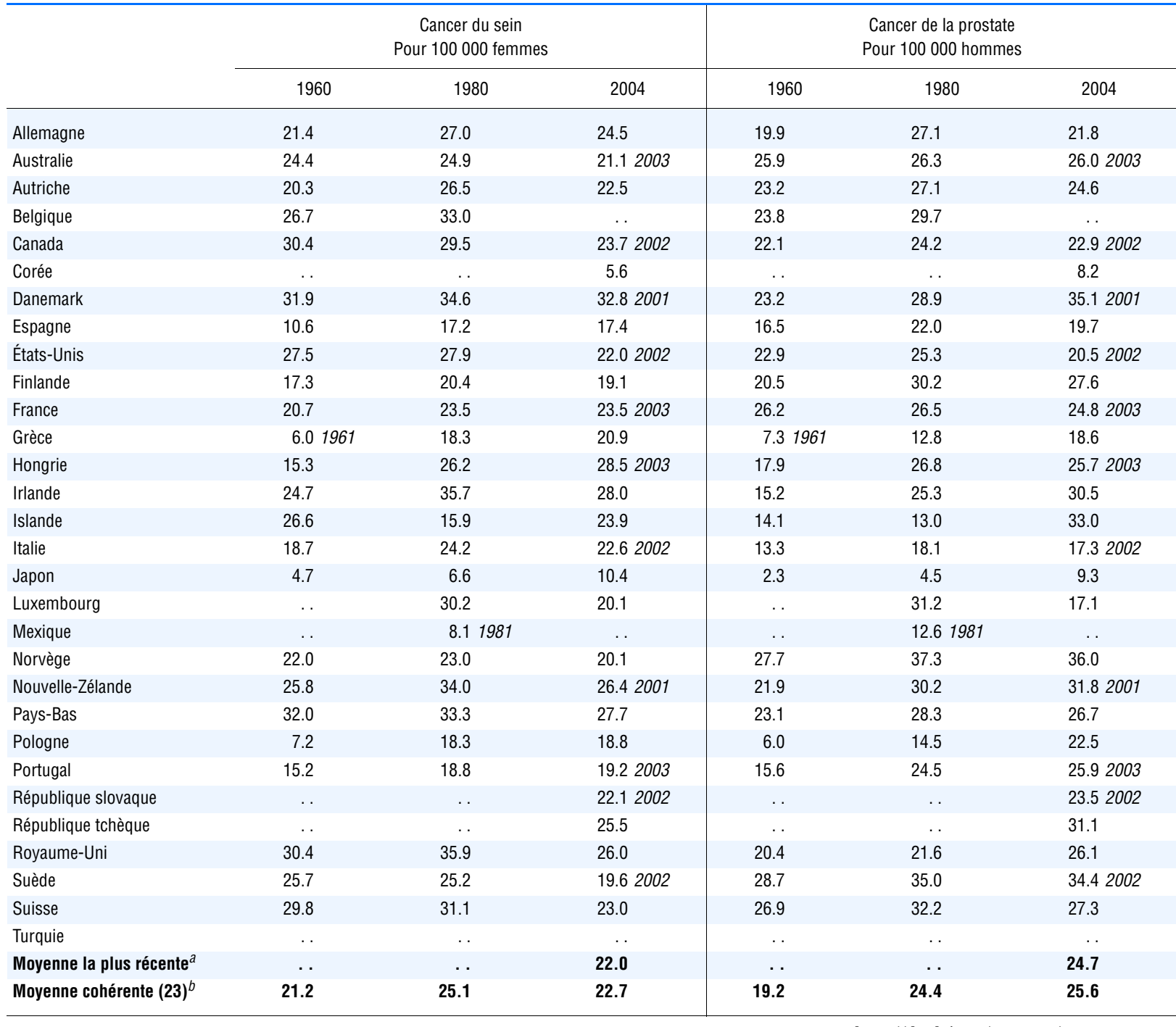

StatLink intst http://dx.doi.org/10.1787/130647115052 Note : Les taux de mortalité sont normalisés par rapport à l'âge en fonction de la structure de la population de l'ensemble des pays de l'OCDE de 1980.

a) La moyenne comprend tous les pays pour lesquels des données récentes sont disponibles (2001+).

b) Exclut la Belgique, la Corée, le Luxembourg, le Mexique, la République slovaque, la République tchèque et la Turquie.

Source: Eco-Santé OCDE 2007. Les statistiques de mortalité sont extraites de la base de données sur la mortalité de l'OMS (avril 2007). 
Tableau A.2.6. Taux de mortalité due à un accident de la route, taux pour 100000 habitants normalisés par rapport à l'âge, 1960 à 2004

\begin{tabular}{|c|c|c|c|c|c|c|}
\hline & 1960 & 1970 & 1980 & 1990 & 2000 & 2004 \\
\hline Allemagne & 25.6 & 32.2 & 20.3 & 13.6 & 9.5 & 7.1 \\
\hline Australie & 28.1 & 32.8 & 24.6 & 14.4 & 10.2 & 8.42003 \\
\hline Autriche & 27.6 & 33.3 & 25.5 & 18.3 & 11.2 & 9.8 \\
\hline Belgique & 19.2 & 29.8 & 24.9 & 18.1 & $\ldots$ & . \\
\hline Canada & 21.8 & 25.0 & 22.1 & 13.9 & 9.6 & 9.32002 \\
\hline Corée & .. & . & . & 36.1 & 27.0 & 17.7 \\
\hline Danemark & 17.1 & 24.2 & 13.5 & 11.2 & 9.1 & 8.22001 \\
\hline Espagne & 8.5 & 13.61969 & 17.1 & 19.9 & 14.6 & 11.2 \\
\hline États-Unis & 22.6 & 27.0 & 22.4 & 18.5 & 15.8 & 15.92002 \\
\hline Finlande & 18.1 & 23.7 & 11.4 & 13.8 & 7.5 & 7.6 \\
\hline France & 18.1 & 23.2 & 20.4 & 17.5 & 12.5 & 9.72003 \\
\hline Grèce & 4.8 & 11.6 & 17.1 & 21.2 & 18.2 & 16.4 \\
\hline Hongrie & 6.7 & 16.3 & 19.1 & 26.9 & 13.3 & 14.12003 \\
\hline Irlande & 9.0 & 16.6 & 17.9 & 13.9 & 10.5 & 6.2 \\
\hline Islande & 5.3 & 16.6 & 10.1 & 11.1 & 11.7 & 7.1 \\
\hline Italie & 17.8 & 24.3 & 19.2 & 14.9 & 12.2 & 12.02002 \\
\hline Japon & 15.9 & 22.5 & 11.4 & 11.9 & 8.5 & 6.6 \\
\hline Luxembourg & 23.8 & 41.6 & 29.6 & 18.7 & 18.7 & 11.9 \\
\hline Mexique & . & 9.3 & 33.81981 & 21.7 & . & . . \\
\hline Norvège & 8.6 & 15.0 & 9.1 & 8.1 & 8.6 & 6.7 \\
\hline Nouvelle-Zélande & 16.5 & 24.2 & 19.8 & 22.3 & 13.4 & 12.82001 \\
\hline Pays-Bas & 17.6 & 24.8 & 13.7 & 8.8 & 7.1 & 5.2 \\
\hline Pologne & 4.7 & 12.1 & 19.91983 & 24.8 & 16.7 & 14.9 \\
\hline Portugal & 9.1 & 22.5 & 29.4 & 28.0 & 13.0 & 17.42003 \\
\hline République slovaque & . & . & $\cdots$ & 20.61992 & 14.4 & 13.02002 \\
\hline République tchèque & . & . & . & 14.5 & 14.1 & 11.8 \\
\hline Royaume-Uni & 14.3 & 14.2 & 12.1 & 9.8 & 5.81999 & 5.8 \\
\hline Suède & 14.4 & 16.5 & 10.5 & 8.8 & 6.3 & 5.72002 \\
\hline Suisse & 22.0 & 26.1 & 18.5 & 13.1 & 8.2 & 6.7 \\
\hline Turquie & . & . & $\cdots$ & . & . & $\cdots$ \\
\hline Moyenne la plus récente ${ }^{a}$ & $\cdots$ & $\cdots$ & $\cdots$ & $\cdots$ & $\cdots$ & 10.3 \\
\hline Moyenne cohérente $(24)^{b}$ & 15.8 & 22.5 & 18.1 & 16.0 & 11.3 & 9.9 \\
\hline
\end{tabular}

StatLink 完药 http://dx.doi.org/10.1787/130711210518 Note : Les taux de mortalité sont normalisés par rapport à l'âge en fonction de la structure de la population de l'ensemble des pays de l'OCDE de 1980.

a) La moyenne comprend tous les pays pour lesquels des données récentes sont disponibles (2001+).

b) Exclut la Belgique, la Corée, le Mexique, la République slovaque, la République tchèque et la Turquie. Source: Eco-Santé OCDE 2007. Les statistiques de mortalité sont extraites de la base de données sur la mortalité de l'OMS (avril 2007). 
Tableau A.2.7. Taux de mortalité due à un suicide, taux pour 100000 habitants normalisés par rapport à l'âge, 1960 à 2004

\begin{tabular}{|c|c|c|c|c|c|c|}
\hline & 1960 & 1970 & 1980 & 1990 & 2000 & 2004 \\
\hline Allemagne & 17.5 & 20.2 & 18.5 & 14.5 & 11.0 & 10.3 \\
\hline Australie & 11.3 & 13.3 & 11.2 & 12.5 & 11.8 & 10.22003 \\
\hline Autriche & 21.2 & 23.0 & 23.7 & 20.5 & 16.5 & 14.5 \\
\hline Belgique & 13.3 & 15.1 & 20.2 & 16.6 & . & $\ldots$ \\
\hline Canada & 8.8 & 12.4 & 13.9 & 12.0 & 10.8 & 10.62002 \\
\hline Corée & . & .. & .. & 7.9 & 14.1 & 24.2 \\
\hline Danemark & 19.7 & 20.4 & 29.2 & 20.5 & 11.4 & 11.32001 \\
\hline Espagne & 6.0 & 4.61969 & 4.4 & 6.8 & 6.9 & 6.6 \\
\hline États-Unis & 11.4 & 12.3 & 11.6 & 11.9 & 9.8 & $10.2(2002$ \\
\hline Finlande & 21.6 & 21.4 & 24.1 & 27.8 & 20.4 & 18.4 \\
\hline France & 15.0 & 14.7 & 17.9 & 17.7 & 15.6 & 15.32003 \\
\hline Grèce & 4.1 & 3.1 & 3.1 & 3.1 & 3.0 & 2.6 \\
\hline Hongrie & 25.6 & 32.9 & 41.4 & 35.3 & 26.8 & 22.62003 \\
\hline Irlande & 3.0 & 1.9 & 7.1 & 10.1 & 11.8 & 10.0 \\
\hline Islande & 9.5 & 14.9 & 11.4 & 15.9 & 18.0 & 11.7 \\
\hline Italie & 6.2 & 5.6 & 6.7 & 6.5 & 5.8 & 5.62002 \\
\hline Japon & 25.1 & 17.4 & 17.9 & 14.5 & 19.1 & 19.1 \\
\hline Luxembourg & 8.7 & 13.6 & 11.6 & 16.0 & 12.8 & 12.5 \\
\hline Mexique & .. & 1.7 & 2.31981 & 3.0 & . & .. \\
\hline Norvège & 6.2 & 8.1 & 11.9 & 14.4 & 11.6 & 10.9 \\
\hline Nouvelle-Zélande & 10.7 & 10.7 & 11.3 & 13.4 & 12.0 & 13.02001 \\
\hline Pays-Bas & 7.3 & 8.5 & 9.9 & 8.7 & 8.2 & 7.9 \\
\hline Pologne & 8.9 & 11.7 & 12.61979 & 12.9 & 13.8 & 14.0 \\
\hline Portugal & 9.8 & 8.9 & 7.6 & 8.0 & 4.1 & 8.72003 \\
\hline République slovaque & .. & .. & .. & 14.61992 & 12.4 & 11.92002 \\
\hline République tchèque & .. & .. & . & 17.8 & 13.8 & 13.0 \\
\hline Royaume-Uni & 9.7 & 7.3 & 8.1 & 7.4 & 6.91999 & 6.3 \\
\hline Suède & 15.9 & 20.4 & 17.7 & 15.0 & 10.9 & 11.42002 \\
\hline Suisse & 18.6 & 18.2 & 23.8 & 19.1 & 16.2 & 14.0 \\
\hline Turquie & .. & . & .. & . & . & . \\
\hline Moyenne la plus récente ${ }^{a}$ & . & . & . & . & . & 12.1 \\
\hline Moyenne cohérente $(24)^{b}$ & 12.6 & 13.6 & 14.9 & 14.4 & 12.3 & 11.6 \\
\hline
\end{tabular}

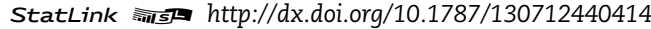
Note : Les taux de mortalité sont normalisés par rapport à l'âge en fonction de la structure de la population de l'ensemble des pays de l'OCDE de 1980.

a) La moyenne comprend tous les pays pour lesquels des données récentes sont disponibles (2001+).

b) Exclut la Belgique, la Corée, le Mexique, la République slovaque, la République tchèque et la Turquie. Source : Eco-Santé OCDE 2007. Les statistiques de mortalité sont extraites de la base de données sur la mortalité de l'OMS (avril 2007). 
Tableau A.2.8. Taux de mortalité infantile en nombre de décès pour 1000 naissances vivantes, 1970 à 2005

\begin{tabular}{|c|c|c|c|c|c|}
\hline & 1970 & 1980 & 1990 & 2000 & 2005 \\
\hline Allemagne & 22.5 & 12.4 & 7.0 & 4.4 & 3.9 \\
\hline Australie & 17.9 & 10.7 & 8.2 & 5.2 & 5.0 \\
\hline Autriche & 25.9 & 14.3 & 7.8 & 4.8 & 4.2 \\
\hline Belgique & 21.1 & 12.1 & 6.5 & 4.8 & 3.7 \\
\hline Canada & 18.8 & 10.4 & 6.8 & 5.3 & 5.32004 \\
\hline Corée & 45.0 & 17.01981 & 12.01989 & 6.21999 & 5.32002 \\
\hline Danemark & 14.2 & 8.4 & 7.5 & 5.3 & 4.4 \\
\hline Espagne & 28.1 & 12.3 & 7.6 & 4.4 & 4.1 \\
\hline États-Unis & 20.0 & 12.6 & 9.2 & 6.9 & 6.82004 \\
\hline Finlande & 13.2 & 7.6 & 5.6 & 3.8 & 3.0 \\
\hline France & 18.2 & 10.0 & 7.3 & 4.4 & 3.6 \\
\hline Grèce & 29.6 & 17.9 & 9.7 & 5.4 & 3.8 \\
\hline Hongrie & 35.9 & 23.2 & 14.8 & 9.2 & 6.2 \\
\hline Irlande & 19.5 & 11.1 & 8.2 & 6.2 & 4.0 \\
\hline Islande & 13.3 & 7.8 & 5.8 & 3.0 & 2.3 \\
\hline Italie & 29.0 & 14.6 & 8.2 & 4.5 & 4.7 \\
\hline Japon & 13.1 & 7.5 & 4.6 & 3.2 & 2.8 \\
\hline Luxembourg & 25.0 & 11.4 & 7.3 & 5.1 & 2.6 \\
\hline Mexique & 79.4 & 51.0 & 36.2 & 23.3 & 18.8 \\
\hline Norvège & 12.7 & 8.1 & 6.9 & 3.8 & 3.1 \\
\hline Nouvelle-Zélande & 16.7 & 13.0 & 8.4 & 6.1 & 5.1 \\
\hline Pays-Bas & 12.7 & 8.6 & 7.1 & 5.1 & 4.9 \\
\hline Pologne & 36.7 & 25.5 & 19.3 & 8.1 & 6.4 \\
\hline Portugal & 55.5 & 24.2 & 11.0 & 5.5 & 3.5 \\
\hline République slovaque & 25.7 & 20.9 & 12.0 & 8.6 & 7.2 \\
\hline République tchèque & 20.2 & 16.9 & 10.8 & 4.1 & 3.4 \\
\hline Royaume-Uni & 18.5 & 12.1 & 7.9 & 5.6 & 5.1 \\
\hline Suède & 11.0 & 6.9 & 6.0 & 3.4 & 2.4 \\
\hline Suisse & 15.1 & 9.1 & 6.8 & 4.9 & 4.2 \\
\hline Turquie & 145.0 & 117.5 & 55.4 & 28.9 & 23.6 \\
\hline Moyenne (30) & 28.7 & 17.8 & 11.1 & 6.7 & 5.4 \\
\hline
\end{tabular}

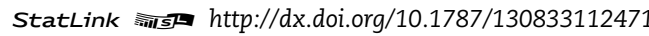
Note : Au Canada, aux États-Unis, au Japon et dans certains pays nordiques, les grands prématurés avec une faible chance de survie sont enregistrés comme naissances vivantes, ce qui augmente le taux de mortalité en comparaison aux pays qui ne les enregistrent pas comme naissances vivantes.

Source: Eco-Santé OCDE 2007. 
Tableau A.2.9. Nourissons ayant un faible poids à la naissance en pourcentage des naissances vivantes, 1980 à 2005

\begin{tabular}{|c|c|c|c|c|}
\hline & 1980 & 1990 & 2000 & 2005 \\
\hline Allemagne & 5.5 & 5.7 & 6.4 & 6.8 \\
\hline Autriche & 5.7 & 5.6 & 6.3 & 6.8 \\
\hline Belgique & 5.61982 & 6.1 & 7.3 & 7.82004 \\
\hline Danemark & 5.8 & 5.2 & 4.7 & 4.9 \\
\hline Espagne & 2.81982 & 4.5 & 6.5 & 7.12004 \\
\hline États-Unis & 6.8 & 7.2 & 7.6 & 8.12004 \\
\hline Finlande & 3.9 & 3.6 & 4.3 & 4.1 \\
\hline Irlande & . & 4.2 & 4.8 & 4.92004 \\
\hline Islande & 3.4 & 2.9 & 3.9 & 3.9 \\
\hline Italie & 5.6 & 5.6 & 6.7 & 6.72004 \\
\hline Japon & 5.2 & 6.3 & 8.6 & 9.5 \\
\hline Luxembourg & 6.3 & 5.4 & 4.32001 & 4.92003 \\
\hline Mexique & $\ldots$ & . & 9.7 & 8.8 \\
\hline Norvège & 3.8 & 4.6 & 5.0 & 4.82004 \\
\hline Nouvelle-Zélande & 5.8 & 6.2 & 6.4 & 6.12004 \\
\hline Pays-Bas & 4.01979 & 4.8 & 5.2 & 6.2 \\
\hline Suède & 4.2 & 4.5 & 4.4 & 4.22004 \\
\hline Suisse & 5.1 & 5.5 & 6.3 & 7.0 \\
\hline Turquie & . & . & $\ldots$ & 11.32003 \\
\hline Moyenne la plus récente ${ }^{a}$ & $\cdots$ & $\cdots$ & . & 6.6 \\
\hline Moyenne cohérente $(\mathbf{2 6})^{b}$ & 5.5 & 5.7 & 6.2 & 6.5 \\
\hline
\end{tabular}

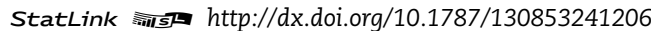

a) La moyenne comprend tous les pays pour lesquels des données récentes sont disponibles (2003+).

b) Exclut la Corée, l'Irlande, le Mexique et la Turquie.

Source : Eco-Santé OCDE 2007. 
Tableau A.2.10. Nombre moyen de dents cariées, absentes ou obturées chez les enfants de 12 ans, 1980 à 2003

\begin{tabular}{|c|c|c|c|c|c|c|}
\hline & 1980 & 1985 & 1990 & 1995 & 2000 & 2003 \\
\hline Allemagne & 6.4 & 6.31986 & 4.1 & 2.3 & 1.2 & 0.72005 \\
\hline Australie & 3.6 & 2.1 & 1.4 & 1.0 & 0.8 & 1.02001 \\
\hline Autriche & 3.0 & 4.3 & 4.2 & 3.01993 & 1.02002 & 1.02002 \\
\hline Belgique & $\ldots$ & 3.21986 & 2.7 & 1.91994 & 1.12001 & 1.12001 \\
\hline Canada & 3.21982 & $\ldots$ & $\ldots$ & $\ldots$ & $\ldots$ & $\ldots$ \\
\hline Corée & . & $\ldots$ & . & 3.1 & 3.3 & $\ldots$ \\
\hline Danemark & . & $\ldots$ & 1.31991 & 1.2 & 1.0 & 0.9 \\
\hline Espagne & . & 4.2 & 3.51989 & 2.31994 & 1.1 & $\ldots$ \\
\hline États-Unis & 2.6 & 1.81986 & 1.31991 & 1.31996 & 1.2 & 1.32004 \\
\hline Finlande & 5.21979 & 2.8 & 1.21991 & 1.21994 & 1.2 & 1.2 \\
\hline France & $\ldots$ & 4.21987 & 3.0 & 2.11993 & 1.91998 & 1.22006 \\
\hline Grèce & . & 4.7 & 4.4 & 2.5 & 2.71998 & . \\
\hline Hongrie & 6.6 & 5.0 & 4.31991 & 3.81996 & 3.32001 & 3.32001 \\
\hline Irlande & 5.4 & 2.9 & 2.7 & 1.51996 & 1.12002 & 1.12002 \\
\hline Islande & 7.81983 & 6.61986 & 3.41991 & 1.51996 & . & 2.12005 \\
\hline Italie & 5.5 & 4.0 & 4.0 & 2.11996 & . & 1.2 \\
\hline Japon & 5.41981 & 4.91987 & 3.61993 & 3.61993 & 2.41999 & 1.72005 \\
\hline Luxembourg & 4.01982 & 3.3 & 2.7 & 2.31994 & 1.22001 & 0.9 \\
\hline Mexique & $\ldots$ & $\ldots$ & . & $\ldots$ & $\ldots$ & $\ldots$ \\
\hline Norvège & 3.31982 & 3.4 & 2.4 & 1.9 & 1.5 & 1.7 \\
\hline Nouvelle-Zélande & 5.1 & 3.2 & 2.0 & 1.4 & 1.6 & 1.6 \\
\hline Pays-Bas & 4.8 & 2.4 & 1.5 & 0.71996 & 1.1 & 0.82002 \\
\hline Pologne & 7.0 & 4.4 & 5.11991 & 4.01998 & 3.8 & 3.82000 \\
\hline Portugal & 4.61979 & 3.8 & 3.2 & . & 3.0 & 3.02000 \\
\hline République slovaque & $\ldots$ & . & . & $\ldots$ & 3.22001 & 2.8 \\
\hline République tchèque & $\ldots$ & 3.31987 & . & 3.11994 & 3.1 & 3.0 \\
\hline Royaume-Uni & 3.11983 & 3.11983 & 1.61988 & 1.11996 & 0.9 & 0.8 \\
\hline Suède & 3.21982 & 3.1 & 2.0 & 1.4 & 1.0 & 1.12002 \\
\hline Suisse & 3.0 & 2.21984 & 1.61988 & 0.81996 & 0.9 & 0.92005 \\
\hline Turquie & $\ldots$ & 2.71987 & 2.7 & . & . & 1.92004 \\
\hline Moyenne la plus récente ${ }^{a}$ & $\ldots$ & . & . & . & $\ldots$ & 1.6 \\
\hline Moyenne cohérente $(\mathbf{1 6})^{b}$ & 4.5 & 3.5 & 2.6 & 2.0 & 1.5 & 1.4 \\
\hline
\end{tabular}

StatLink त्ताजम http://dx.doi.org/10.1787/131017368861

a) La moyenne comprend tous les pays pour lesquels des données récentes sont disponibles (2000+).

b) Exclut la Belgique, le Canada, la Corée, le Danemark, l'Espagne, la France, la Grèce, l'Islande, l'Italie, le Mexique, le Portugal, la République slovaque, la République tchèque et la Turquie.

Source : Eco-Santé OCDE 2007. 
Tableau A.2.12. Taux d'incidence du SIDA, par million d'habitants, 1985 à 2005

\begin{tabular}{|c|c|c|c|c|c|}
\hline & 1985 & 1990 & 1995 & 2000 & 2005 \\
\hline Allemagne & 4.0 & 23.0 & 22.9 & 9.6 & 8.1 \\
\hline Australie & 8.0 & 39.0 & 45.0 & 14.0 & 13.0 \\
\hline Autriche & 3.0 & 21.4 & 26.2 & 10.5 & 12.5 \\
\hline Belgique & 7.0 & 21.0 & 24.5 & 13.6 & 15.5 \\
\hline Canada & 15.5 & 52.8 & 56.2 & 16.2 & 9.8 \\
\hline Corée & 0.0 & 0.1 & 0.3 & 0.7 & 1.4 \\
\hline Danemark & 7.5 & 38.3 & 40.7 & 10.9 & 8.9 \\
\hline Espagne & 4.3 & 96.8 & 178.1 & 69.3 & 36.0 \\
\hline États-Unis ${ }^{a}$ & 34.0 & 167.0 & 270.0 & 143.0 & 137.0 \\
\hline Finlande & 1.0 & 3.0 & 8.0 & 3.3 & 5.4 \\
\hline France & 10.1 & 74.0 & 88.6 & 28.4 & 22.02004 \\
\hline Grèce & 1.4 & 14.1 & 20.7 & 11.8 & 9.1 \\
\hline Hongrie & 0.1 & 1.8 & 3.0 & 2.6 & 3.3 \\
\hline Irlande & 1.4 & 17.4 & 14.7 & 3.4 & 11.2 \\
\hline Islande & 4.1 & 11.8 & 15.0 & 3.6 & 3.4 \\
\hline Italie & 3.0 & 52.0 & 98.8 & 33.8 & 25.4 \\
\hline Japon & 0.1 & 0.3 & 1.4 & 2.6 & 2.9 \\
\hline Luxembourg & 8.0 & 24.0 & 36.6 & 23.0 & 17.4 \\
\hline Mexique & 4.6 & 32.5 & 46.4 & 46.2 & 45.32003 \\
\hline Norvège & 3.0 & 14.0 & 15.4 & 8.4 & 8.52003 \\
\hline Nouvelle-Zélande & 3.7 & 21.0 & 16.9 & 6.5 & 8.3 \\
\hline Pays-Bas & 4.6 & 28.0 & 34.5 & 15.6 & 12.1 \\
\hline Pologne & 0.1 & 0.6 & 3.0 & 3.2 & 4.3 \\
\hline Portugal & 2.9 & 25.5 & 79.4 & 97.2 & 79.5 \\
\hline République slovaque & 0.0 & 0.0 & 0.4 & 0.9 & 0.6 \\
\hline République tchèque & 0.1 & 0.5 & 1.3 & 1.4 & 1.1 \\
\hline Royaume-Uni & 4.0 & 22.0 & 30.1 & 14.1 & 13.3 \\
\hline Suède & 3.0 & 15.2 & 22.2 & 6.8 & 5.1 \\
\hline Suisse & 13.0 & 97.0 & 85.3 & 28.7 & 37.1 \\
\hline Turquie & 0.0 & 0.3 & 0.4 & 0.7 & 5.0 \\
\hline Moyenne (30) & 5.1 & 30.5 & 42.9 & 21.0 & 18.8 \\
\hline
\end{tabular}

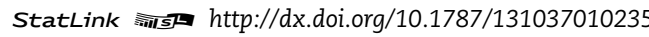
Note : Les données concernant les pays européens sont extraites du Centre européen pour la surveillance épidémiologique du SIDA.

a) Les États-Unis ont étendu la définition et la surveillance des cas du SIDA en 1993, ce qui implique des taux d'incidence plus élevés.

Source : Eco-Santé OCDE 2007. 
Tableau A.3.1. Pourcentage de la population adulte qui déclare fumer quotidiennement, 1980 à 2005

\begin{tabular}{|c|c|c|c|c|c|c|c|c|c|}
\hline & \multicolumn{3}{|c|}{1980} & \multicolumn{3}{|c|}{1990} & \multicolumn{3}{|c|}{2005} \\
\hline & Femmes & Hommes & Total & Femmes & Hommes & Total & Femmes & Hommes & Total \\
\hline Allemagne & 21.2 & 48.4 & 34.81978 & 22.2 & 38.0 & 31.21989 & 19.1 & 29.8 & 24.32003 \\
\hline Australie & 31.1 & 41.1 & 36.0 & 27.0 & 30.2 & 28.61989 & 16.5 & 18.9 & 17.72004 \\
\hline Autriche & . & . & $\ldots$ & $\ldots$ & . & $\ldots$ & . & $\ldots$ & $\ldots$ \\
\hline Belgique & 28.4 & 52.6 & 40.51982 & 26.0 & 38.0 & 32.0 & 16.0 & 23.0 & 20.0 \\
\hline Canada & 30.1 & 38.6 & 34.41979 & 26.7 & 29.8 & 28.2 & 15.5 & 19.1 & 17.3 \\
\hline Corée & . & $\ldots$ & $\ldots$ & 6.4 & 65.7 & 34.61989 & 4.6 & 46.6 & 25.3 \\
\hline Danemark & 44.0 & 57.0 & 50.5 & 42.0 & 47.0 & 44.5 & 23.0 & 29.0 & 26.02004 \\
\hline Espagne & . & $\ldots$ & . & 21.4 & 51.5 & 35.91989 & 22.4 & 34.2 & 28.12003 \\
\hline États-Unis & 29.3 & 37.6 & 33.5 & 22.8 & 28.4 & 25.6 & 14.9 & 19.1 & 16.9 \\
\hline Finlande & 16.6 & 35.2 & 26.1 & 20.0 & 32.4 & 25.9 & 18.2 & 26.0 & 21.8 \\
\hline France & 16.0 & 44.0 & 30.0 & 20.0 & 38.0 & 30.0 & 19.0 & 28.0 & 23.02004 \\
\hline Grèce & . & $\ldots$ & . & 26.0 & 51.0 & 38.5 & 31.3 & 46.0 & 38.62004 \\
\hline Hongrie & . & $\ldots$ & $\ldots$ & $\ldots$ & . & . & 24.6 & 36.9 & 30.42003 \\
\hline Irlande & . & . & . & 29.0 & 31.0 & 30.0 & 26.0 & 28.0 & 27.02002 \\
\hline Islande & . & . & . & 29.9 & 30.8 & 30.3 & 19.5 & 19.5 & 19.5 \\
\hline Italie & 16.7 & 54.3 & 35.5 & 17.8 & 37.8 & 27.8 & 16.4 & 28.7 & 22.3 \\
\hline Japon & 14.4 & 70.2 & 42.3 & 14.3 & 60.5 & 37.4 & 13.8 & 45.8 & 29.2 \\
\hline Luxembourg & . & . & . & 26.0 & 40.0 & 33.01992 & 19.0 & 27.0 & 23.0 \\
\hline Mexique & . & . & . & 14.4 & 38.3 & 25.81988 & 16.1 & 39.1 & 26.42002 \\
\hline Norvège & 30.0 & 42.0 & 36.0 & 33.0 & 36.0 & 35.0 & 24.0 & 26.0 & 25.0 \\
\hline Nouvelle-Zélande & 29.0 & 35.0 & 32.01981 & 27.0 & 28.0 & 28.0 & 22.5 & 22.5 & 22.5 \\
\hline Pays-Bas & 34.0 & 52.0 & 43.0 & 32.0 & 43.0 & 37.0 & 26.0 & 35.0 & 31.0 \\
\hline Pologne & . & $\ldots$ & . & 28.0 & 55.0 & 41.51992 & 19.3 & 33.9 & 26.32004 \\
\hline Portugal & . & $\ldots$ & . & 5.1 & 33.6 & 19.01987 & 9.0 & 26.0 & 17.0 \\
\hline République slovaque & . & . & $\ldots$ & $\ldots$ & . & $\ldots$ & 22.5 & 25.5 & 24.3 \\
\hline République tchèque & . & . & . & 21.0 & 31.9 & 26.11993 & 19.4 & 29.6 & 24.3 \\
\hline Royaume-Uni & 37.0 & 42.0 & 39.0 & 30.0 & 31.0 & 30.0 & 23.0 & 25.0 & 24.0 \\
\hline Suède & 28.7 & 36.3 & 32.4 & 25.9 & 25.8 & 25.8 & 18.0 & 13.9 & 15.9 \\
\hline Suisse & . & . & . & 22.8 & 33.9 & 28.21992 & 22.8 & 31.0 & 26.8 \\
\hline Turquie & . & . & . & 24.3 & 62.8 & 43.61989 & 17.8 & 51.1 & 32.12003 \\
\hline Moyenne la plus récente ${ }^{a}$ & . & . & . & . & . & . & 19.3 & 29.8 & 24.3 \\
\hline Moyenne cohérente $(15)^{b}$ & 27.1 & 45.8 & 36.4 & 25.8 & 36.3 & 31.1 & 19.1 & 26.0 & 22.5 \\
\hline
\end{tabular}

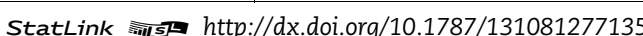

a) La moyenne comprend tous les pays de l'OCDE pour lesquels des données récentes sont disponibles (2002+).

b) Exclut l'Autriche, la Corée, l'Espagne, la Grèce, la Hongrie, l'Irlande, l'Islande, le Luxembourg, le Mexique, la Pologne, le Portugal, la République slovaque, la République tchèque, la Suisse et la Turquie.

Source : Eco-Santé OCDE 2007. 
Tableau A.3.2. Consommation d'alcool en litres par habitant, population âgée de 15 ans et plus, 1960 à 2005

\begin{tabular}{|c|c|c|c|c|c|c|}
\hline & 1960 & 1970 & 1980 & 1990 & 2000 & 2005 \\
\hline Allemagne & 7.5 & 13.4 & 14.21982 & 13.8 & 10.5 & 10.0 \\
\hline Australie & 9.4 & 11.6 & 12.9 & 10.5 & 9.8 & 9.82004 \\
\hline Autriche & 10.9 & 13.9 & 13.8 & 12.6 & 11.1 & 11.12003 \\
\hline Belgique & 8.9 & 11.7 & 13.5 & 12.1 & 10.3 & 10.72003 \\
\hline Canada & 7.0 & 8.8 & 10.7 & 7.4 & 7.7 & 7.92004 \\
\hline Corée & .. & .. & . & 9.1 & 8.9 & 8.1 \\
\hline Danemark & 5.5 & 8.6 & 11.7 & 11.7 & 11.5 & 11.3 \\
\hline Espagne & 14.61962 & 16.1 & 18.4 & 13.5 & 11.5 & 11.72003 \\
\hline États-Unis & 7.8 & 9.5 & 10.5 & 9.3 & 8.3 & 8.42004 \\
\hline Finlande & 2.7 & 5.8 & 7.9 & 9.5 & 8.6 & 10.0 \\
\hline France & .. & 20.4 & 19.5 & 15.9 & 14.0 & 13.02004 \\
\hline Grèce & .. & .. & 13.2 & 10.6 & 9.5 & 9.02003 \\
\hline Hongrie & 8.2 & 11.5 & 14.9 & 13.9 & 12.0 & 13.22004 \\
\hline Irlande & 4.9 & 7.0 & 9.6 & 11.2 & 14.2 & 13.5 \\
\hline Islande & 2.51961 & 3.8 & 4.3 & 5.2 & 6.1 & 7.1 \\
\hline Italie & 16.6 & 17.8 & 16.3 & 11.0 & 9.0 & 8.12003 \\
\hline Japon & 5.01963 & 6.1 & 7.1 & 8.0 & 7.6 & 7.72004 \\
\hline Luxembourg $^{a}$ & 13.1 & 12.8 & 13.4 & 14.8 & 15.4 & 15.52003 \\
\hline Mexique & . & . & 3.3 & 4.9 & 4.8 & 4.62003 \\
\hline Norvège & 3.4 & 4.7 & 5.3 & 5.0 & 5.7 & 6.4 \\
\hline Nouvelle-Zélande & 5.3 & 9.8 & 11.8 & 10.1 & 8.9 & 9.4 \\
\hline Pays-Bas & 3.7 & 7.8 & 11.5 & 9.9 & 10.1 & 9.72003 \\
\hline Pologne & 6.31961 & 8.01971 & 8.71981 & 8.3 & 8.3 & 8.12003 \\
\hline Portugal & 17.21961 & 17.81969 & 14.8 & 16.1 & 12.9 & 11.42003 \\
\hline République slovaque & 6.9 & 12.8 & 14.5 & 13.4 & 8.9 & 9.3 \\
\hline République tchèque & .. & . & 11.7 & 11.3 & 11.8 & 12.0 \\
\hline Royaume-Uni & . & 7.1 & 9.4 & 9.8 & 10.4 & 11.3 \\
\hline Suède & 4.8 & 7.2 & 6.7 & 6.4 & 6.2 & 6.6 \\
\hline Suisse & 12.1 & 14.2 & 13.5 & 12.9 & 11.2 & 10.1 \\
\hline Turquie & 0.9 & 1.1 & 1.8 & 1.4 & 1.5 & 1.3 \\
\hline Moyenne la plus récente ${ }^{b}$ & . & . & . & . & . & 9.5 \\
\hline Moyenne cohérente $(24)^{C}$ & 7.7 & 10.1 & 11.2 & 10.3 & 9.5 & 9.5 \\
\hline
\end{tabular}

StatLink तiाst http://dx.doi.org/10.1787/131113876036

a) Au Luxembourg, les ventes nationales ne reflètent pas de manière précise la consommation des résidents, du fait du niveau élevé de la consommation des touristes et du trafic transfrontalier de boissons alcoolisées.

b) La moyenne comprend les dernières données disponibles pour tous les pays de l'OCDE.

c) Exclut la Corée, la France, la Grèce, le Mexique, la République tchèque et le Royaume-Uni.

Source : Eco-Santé OCDE 2007. 
Tableau A.3.3. Pourcentage de la population adulte en surpoids ou obèse, dernière année disponible

\begin{tabular}{|c|c|c|c|c|c|c|c|c|c|c|}
\hline & & \multicolumn{3}{|c|}{ Population en surpoids $25<I M C<30$} & \multicolumn{3}{|c|}{ Population obèse IMC $\geq 30$} & \multicolumn{3}{|c|}{ Population en surpoids/obèse IMC > 25} \\
\hline & & Femmes & Hommes & Total & Femmes & Hommes & Total & Femmes & Hommes & Total \\
\hline Allemagne & 2005 & 28.7 & 43.5 & 36.0 & 12.8 & 14.4 & 13.6 & 41.5 & 57.9 & 49.6 \\
\hline Australie $^{a}$ & 1999 & 28.2 & 45.3 & 36.7 & 21.4 & 21.9 & 21.7 & 49.6 & 67.2 & 58.4 \\
\hline Autriche & 1999 & 21.3 & 54.3 & 37.0 & 9.1 & 9.1 & 9.1 & 30.4 & 63.4 & 46.1 \\
\hline Belgique & 2004 & 24.4 & 38.7 & 31.4 & 13.4 & 11.9 & 12.7 & 37.8 & 50.6 & 44.1 \\
\hline Canada & 2005 & 24.7 & 39.3 & 31.9 & 19.0 & 17.0 & 18.0 & 43.7 & 56.3 & 49.9 \\
\hline Corée & 2005 & 23.7 & 30.3 & 27.0 & 3.3 & 3.7 & 3.5 & 27.0 & 34.0 & 30.5 \\
\hline Danemark & 2005 & 26.4 & 40.9 & 33.2 & 11.8 & 11.0 & 11.4 & 38.2 & 51.9 & 44.6 \\
\hline Espagne & 2003 & 27.6 & 43.5 & 35.3 & 13.4 & 12.9 & 13.1 & 40.9 & 56.3 & 48.4 \\
\hline États-Unis ${ }^{a}$ & 2004 & 28.6 & 39.7 & 34.1 & 33.2 & 31.1 & 32.2 & 61.8 & 70.8 & 66.3 \\
\hline Finlande & 2005 & 26.6 & 44.8 & 35.0 & 13.5 & 14.9 & 14.1 & 40.1 & 59.7 & 49.2 \\
\hline France & 2004 & 19.6 & 31.1 & 25.1 & 9.3 & 9.8 & 9.5 & 29.0 & 40.5 & 34.6 \\
\hline Grèce & 2003 & 29.9 & 41.1 & 35.2 & 18.2 & 26.0 & 21.9 & 48.1 & 67.1 & 57.1 \\
\hline Hongrie & 2003 & 29.8 & 38.7 & 34.0 & 18.0 & 19.6 & 18.8 & 47.8 & 58.3 & 52.8 \\
\hline Irlande & 2002 & 25.0 & 41.0 & 34.0 & 12.0 & 14.0 & 13.0 & 37.0 & 55.0 & 47.0 \\
\hline Islande & 2002 & 28.0 & 44.6 & 35.9 & 12.4 & 12.4 & 12.4 & 40.4 & 57.0 & 48.3 \\
\hline Italie & 2005 & 26.2 & 43.9 & 34.7 & 9.7 & 10.2 & 9.9 & 35.9 & 54.0 & 44.6 \\
\hline Japon & 2004 & 16.9 & 24.5 & 20.3 & 3.2 & 2.8 & 3.0 & 20.1 & 27.3 & 23.3 \\
\hline Luxembourg $^{a}$ & 2005 & 25.4 & 41.1 & 34.6 & 18.5 & 18.8 & 18.6 & 43.9 & 59.9 & 53.3 \\
\hline Mexique & 2005 & 36.6 & 42.6 & 39.1 & 34.7 & 23.7 & 30.2 & 71.3 & 66.4 & 69.2 \\
\hline Norvège & 2005 & 26.0 & 43.0 & 34.0 & 8.0 & 9.0 & 9.0 & 34.0 & 52.0 & 43.0 \\
\hline Nouvelle-Zélande ${ }^{a}$ & 2003 & 28.4 & 42.1 & 35.2 & 21.7 & 20.1 & 20.9 & 50.2 & 62.2 & 56.2 \\
\hline Pays-Bas & 2005 & 28.2 & 40.5 & 34.2 & 11.4 & 9.9 & 10.7 & 39.6 & 50.4 & 44.9 \\
\hline Pologne & 2004 & 26.6 & 39.5 & 32.8 & 12.5 & 12.6 & 12.5 & 39.1 & 52.1 & 45.3 \\
\hline Portugal & 1999 & 31.8 & 42.3 & 36.8 & 14.0 & 11.4 & 12.8 & 45.8 & 53.7 & 49.6 \\
\hline République slovaque & 2003 & 24.9 & 42.0 & 32.2 & 15.6 & 15.2 & 15.4 & 40.5 & 57.2 & 47.6 \\
\hline République tchèque $^{a}$ & 2005 & 29.0 & 42.0 & 35.0 & 17.0 & 18.0 & 17.0 & 46.0 & 60.0 & 52.0 \\
\hline Royaume-Uni ${ }^{a}$ & 2005 & 32.1 & 42.6 & 37.0 & 24.2 & 22.1 & 23.0 & 56.3 & 64.7 & 60.0 \\
\hline Suède & 2005 & 25.9 & 40.7 & 33.3 & 10.3 & 11.1 & 10.7 & 36.2 & 51.8 & 44.0 \\
\hline Suisse & 2002 & 21.8 & 37.5 & 29.4 & 7.5 & 7.9 & 7.7 & 29.3 & 45.4 & 37.1 \\
\hline Turquie & 2003 & 28.9 & 33.6 & 31.6 & 14.5 & 9.7 & 12.0 & 43.4 & 43.3 & 43.4 \\
\hline
\end{tabular}

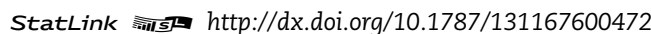

Note : Les taux d'obésité correspondent au pourcentage de la population ayant un indice de masse corporelle (IMC) supérieur ou égal à 30. Les taux de surpoids correspondent au pourcentage de la population ayant un IMC compris entre 25 et 30 . L'indice de masse corporelle est un chiffre rapportant le poids d'un individu à sa taille (poids/taille ${ }^{2}$, le poids étant exprimé en kilogrammes et la taille en mètres).

a) Pour l'Australie, les États-Unis, le Luxembourg, la Nouvelle-Zélande, la République tchèque et le Royaume-Uni, les données sont basées sur des examens de santé, plutôt que sur de simples déclarations des répondants. Les estimations provenant d'examens sont généralement supérieures et plus fiables que celles provenant de déclarations, puisqu'elles excluent la possibilité de fausses déclarations, mais seulement quelques pays mènent régulièrement des enquêtes par examen.

Source : Eco-Santé OCDE 2007. 
Tableau A.4.1a. Nombre de diplômés en médecine pour 1000 médecins, 1985 à 2005

\begin{tabular}{|c|c|c|c|c|c|}
\hline & 1985 & 1990 & 1995 & 2000 & 2005 \\
\hline Allemagne & . & 44.01991 & 50.4 & 30.7 & . \\
\hline Australie & 46.2 & 28.6 & 30.8 & 29.6 & 32.12004 \\
\hline Autriche & 106.3 & 82.6 & 48.6 & 59.9 & 58.32003 \\
\hline Belgique & . & . & 49.3 & 45.1 & 51.02003 \\
\hline Canada & 35.3 & 29.2 & 28.1 & 24.5 & 26.9 \\
\hline Corée & . & . & .. & 58.0 & 55.7 \\
\hline Danemark & 41.2 & 32.8 & 20.1 & 25.2 & 40.02004 \\
\hline Espagne & . & .. & 47.1 & 33.2 & 29.62003 \\
\hline États-Unis & . & 31.01993 & 30.5 & 27.8 & 26.52004 \\
\hline Finlande & . & 46.4 & 67.4 & 36.9 & 41.2 \\
\hline France & 57.9 & 30.8 & 24.9 & 20.3 & 17.52004 \\
\hline Grèce & 29.2 & . & 34.4 & 29.02001 & . \\
\hline Hongrie & 37.4 & 30.8 & 32.8 & 29.5 & 38.4 \\
\hline Irlande & . & 61.01992 & 61.4 & 64.7 & 51.9 \\
\hline Islande & 83.1 & 52.3 & 40.8 & 34.1 & 39.9 \\
\hline Italie & . & 35.91993 & 30.8 & 27.6 & 28.9 \\
\hline Japon & 45.0 & 40.3 & 36.91994 & 30.5 & 29.12004 \\
\hline Luxembourg & . & .. & . & .. & . \\
\hline Mexique & . & .. & . & $\ldots$ & . \\
\hline Norvège & 34.9 & 25.31991 & 26.3 & 31.8 & 27.3 \\
\hline Nouvelle-Zélande & 45.2 & 45.1 & 36.5 & 37.5 & 38.02004 \\
\hline Pays-Bas & 45.5 & 38.7 & 33.51998 & 27.8 & 28.8 \\
\hline Pologne & . & .. & . & . & . \\
\hline Portugal & 35.6 & 18.5 & 14.3 & 19.0 & 20.8 \\
\hline République slovaque & . & . & . & 34.3 & 34.62004 \\
\hline République tchèque & 52.7 & 33.2 & 45.0 & 23.3 & 22.8 \\
\hline Royaume-Uni & 54.41987 & 39.3 & 37.4 & 38.5 & 35.8 \\
\hline Suède & 38.9 & 27.5 & 29.2 & 28.3 & 25.12004 \\
\hline Suisse & 51.5 & 40.8 & 30.6 & 30.0 & 22.1 \\
\hline Turquie & 59.4 & 88.7 & 60.8 & 60.3 & 42.72004 \\
\hline Moyenne la plus récente ${ }^{a}$ & . & . & . & . & 34.6 \\
\hline Moyenne cohérente $(21)^{b}$ & . & 40.9 & 36.5 & 33.7 & 33.1 \\
\hline
\end{tabular}

StatLink न्ता1s/ $h t t p: / / d x . d o i . o r g / 10.1787 / 131220486154$

a) La moyenne comprend tous les pays pour lesquels des données récentes sont disponibles (2003+).

b) Exclut l'Allemagne, la Belgique, la Corée, l'Espagne, la Grèce, le Luxembourg, le Mexique, la Pologne et la République slovaque.

Source : Eco-Santé OCDE 2007 


\section{Tableau A.4.1b. Nombre de diplômés en études d'infirmière} pour 1000 infirmières, 1985 à 2005

\begin{tabular}{|c|c|c|c|c|c|}
\hline & 1985 & 1990 & 1995 & 2000 & 2005 \\
\hline Allemagne & $\ldots$ & $\ldots$ & $\ldots$ & $\ldots$ & . \\
\hline Australie & 22.41986 & 19.3 & 29.5 & 22.8 & 28.72004 \\
\hline Autriche & 42.5 & 36.5 & 39.5 & 36.9 & 38.12004 \\
\hline Belgique & $\ldots$ & $\ldots$ & . & . & $\ldots$ \\
\hline Canada $^{a}$ & 43.3 & 36.7 & 30.3 & 20.2 & 31.72004 \\
\hline Corée & $\ldots$ & $\ldots$ & $\ldots$ & 165.6 & 152.3 \\
\hline Danemark & 58.7 & 64.4 & 49.7 & 50.0 & 45.42004 \\
\hline Espagne & . & . & . & . & 49.02003 \\
\hline États-Unis ${ }^{b}$ & 77.4 & 56.7 & 66.8 & 56.71998 & . \\
\hline Finlande & $\ldots$ & 136.0 & 233.8 & 113.3 & 70.42003 \\
\hline France & 57.0 & 41.9 & 39.7 & 36.3 & 44.62004 \\
\hline Grèce & $\ldots$ & $\ldots$ & 21.6 & 25.8 & 36.82004 \\
\hline Hongrie & . & 65.7 & 67.4 & 57.1 & 47.6 \\
\hline Irlande & . & 39.8 & 32.3 & 17.92001 & 22.7 \\
\hline Islande & . & . & . & . & 37.3 \\
\hline Italie & . & . & . & 18.32002 & 22.3 \\
\hline Japon $^{C}$ & . & . & . & . & 40.1 \\
\hline Luxembourg & . & $\ldots$ & $\ldots$ & 17.92003 & 11.0 \\
\hline Mexique & $\ldots$ & $\ldots$ & $\ldots$ & $\ldots$ & . \\
\hline Norvège & . & . & 66.91997 & 68.0 & 51.3 \\
\hline Nouvelle-Zélande & $\cdots$ & . & 40.91996 & 32.0 & 29.02003 \\
\hline Pays-Bas & . & . & 28.01997 & 21.8 & 23.5 \\
\hline Pologne & $\cdots$ & . & . & . & . \\
\hline Portugal & 38.9 & 35.2 & 55.4 & 35.1 & 52.4 \\
\hline République slovaque & $\cdots$ & $\ldots$ & 118.6 & 72.5 & 96.62004 \\
\hline République tchèque & 60.5 & 62.4 & 85.2 & 55.81999 & 58.1 \\
\hline Royaume-Uni & . & . & . & 33.22003 & 38.5 \\
\hline Suède & 49.0 & 45.7 & 18.8 & 32.6 & 40.02004 \\
\hline Suisse & $\cdots$ & . & $\cdots$ & 44.6 & 44.72004 \\
\hline Turquie & . & . & $\cdots$ & 22.5 & 30.72004 \\
\hline Moyenne la plus récente ${ }^{d}$ & $\cdots$ & $\cdots$ & . & $\cdots$ & 45.7 \\
\hline Moyenne cohérente $(16)^{e}$ & . & . & 59.9 & 43.6 & 44.8 \\
\hline
\end{tabular}

StatLink त्राजम http://dx.doi.org/10.1787/131220486154

a) Le faible taux de diplômés en 2000 pour le Canada peut s'expliquer par une faible participation à l'enquête qui permet de collecter les données auprès des écoles d'infirmières.

b) Aux États-Unis, les données sont seulement disponibles jusqu'en 1998, car la collecte des données concernant certaines catégories d'infirmières diplômées s'est arrêtée par la suite.

c) Au Japon, le nombre de diplômés en études d'infirmière se rapporte à 2005 alors que le nombre d'infirmières se rapporte à 2004 .

d) La moyenne comprend tous les pays pour lesquels des données récentes sont disponibles (2003+).

e) Exclut l'Allemagne, la Belgique, la Corée, l'Espagne, les États-Unis, l'Islande, l'Italie, le Japon, le Luxembourg, le Mexique, la Pologne, le Royaume-Uni, la Suisse et la Turquie.

Source : Eco-Santé OCDE 2007. 
Tableau A.4.2. Médecins en activité pour 1000 habitants, 1970 à 2005

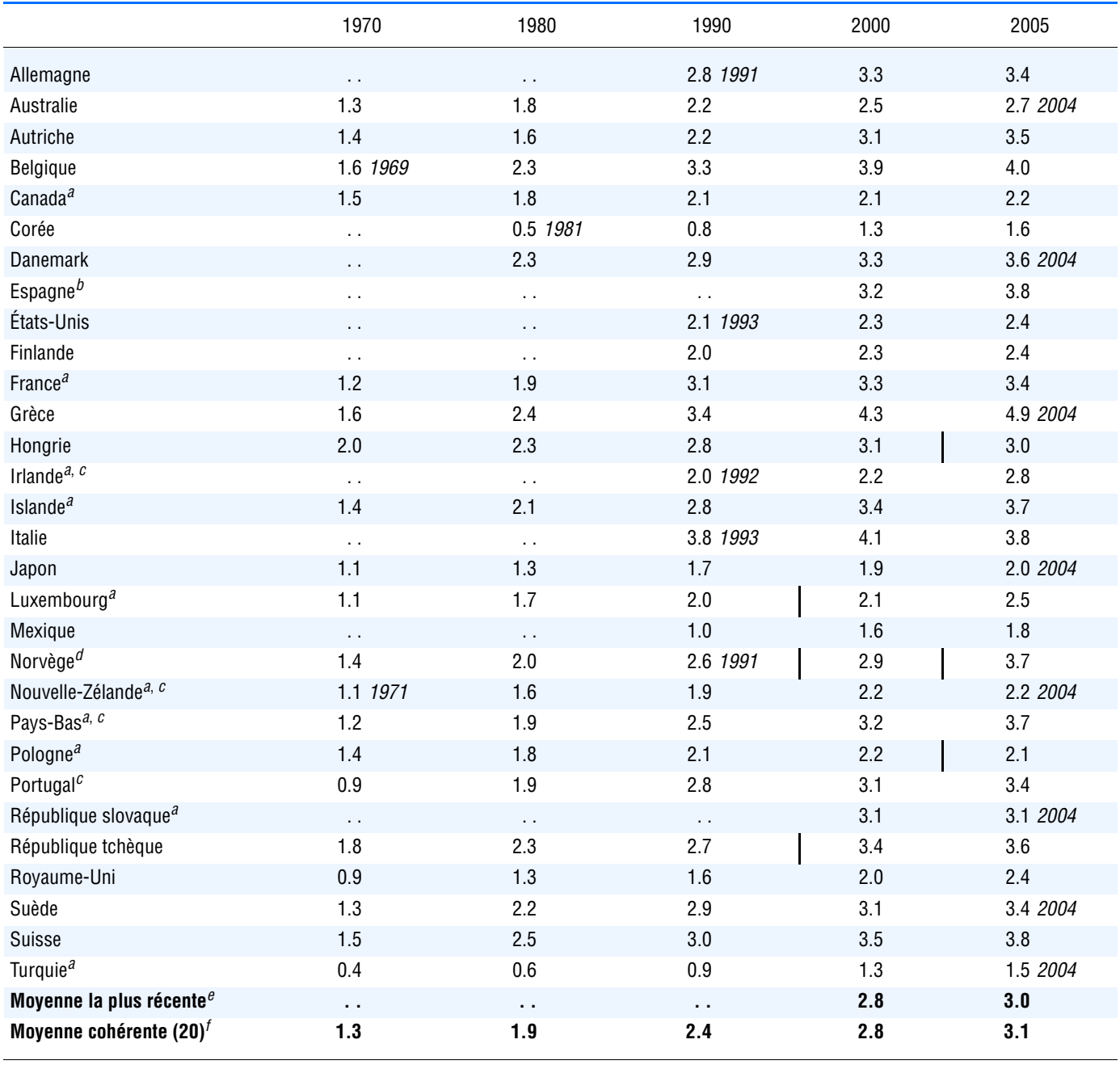

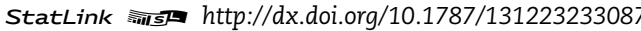

a) Les données pour le Canada, la France, l'Irlande, l'Islande, le Luxembourg (avant 2000), la Nouvelle-Zélande, les Pays-Bas, la Pologne (avant 2004), la République slovaque et la Turquie incluent les médecins qui travaillent dans l'industrie, l'administration et la recherche (et pas seulement ceux qui sont en contact avec les patients).

b) L’Espagne inclut les dentistes et stomatologistes dans le nombre de médecins.

c) L'Irlande, la Nouvelle-Zélande, les Pays-Bas et le Portugal communiquent le nombre de médecins autorisés à exercer plutôt que le nombre de médecins en activité.

d) En Norvège, les données de 1997 à 2001 font référence aux équivalents plein-temps, alors que les autres années font référence au nombre d'individus.

e) La moyenne comprend tous les pays de l'OCDE.

f) Exclut l'Allemagne, la Corée, le Danemark, l'Espagne, les États-Unis, la Finlande, l'Irlande, l'Italie, le Mexique et la République slovaque.

Source : Eco-Santé OCDE 2007. 
Tableau A.4.3. Personnel infirmier en activité pour 1000 habitants, 1970 à 2005

\begin{tabular}{|c|c|c|c|c|c|}
\hline & 1970 & 1980 & 1990 & 2000 & 2005 \\
\hline Allemagne & .. & . & .. & 9.4 & 9.7 \\
\hline Australie & 6.7 & 10.3 & 11.6 & 10.5 & 10.42004 \\
\hline Autriche $^{a}$ & 3.4 & 5.4 & 7.2 & 9.2 & 9.4 \\
\hline Belgique & . & .. & . & . & 14.8 \\
\hline Canada & 7.0 & 9.6 & 11.1 & 10.1 & 10.0 \\
\hline Corée & .. & .. & .. & 1.4 & 1.9 \\
\hline Danemark & . & 4.9 & 5.7 & 7.5 & 7.72004 \\
\hline Espagne & .. & . & . & 6.4 & 7.4 \\
\hline États-Unis & 3.7 & 5.6 & 7.2 & 8.0 & 7.92002 \\
\hline Finlande & . & . & 4.4 & 6.1 & 7.62004 \\
\hline France & 3.11971 & 4.7 & 5.6 & 6.7 & 7.7 \\
\hline Grèce & 1.4 & 1.9 & 3.4 & 3.2 & 3.82004 \\
\hline Hongrie & . & .. & 7.5 & 7.8 & 8.8 \\
\hline Irlande & .. & .. & 11.3 & 14.0 & 15.2 \\
\hline Islande & 4.2 & 8.9 & 12.5 & 13.3 & 14.0 \\
\hline Italie & .. & .. & 5.01993 & 5.2 & 7.0 \\
\hline Japon & 2.5 & 4.1 & 5.8 & 7.6 & 9.02004 \\
\hline Luxembourg $^{b}$ & .. & .. & $\ldots$ & 12.32003 & 13.9 \\
\hline Mexique & . & .. & 1.8 & 2.2 & 2.2 \\
\hline Norvège & .. & $\ldots$ & . & 10.3 & 15.4 \\
\hline Nouvelle-Zélande & .. & 6.1 & 9.3 & 9.6 & 9.52004 \\
\hline Pays-Bas ${ }^{C}$ & .. & . & . & 13.4 & 14.5 \\
\hline Pologne & 3.0 & 4.4 & 5.5 & 5.0 & 5.1 \\
\hline Portugal & 1.81971 & 2.3 & 2.8 & 3.7 & 4.6 \\
\hline République slovaque & . & .. & .. & 7.4 & 6.32004 \\
\hline République tchèque & 5.2 & 5.9 & 7.2 & 7.6 & 8.1 \\
\hline Royaume-Uni & . & .. & 7.9 & 8.4 & 9.1 \\
\hline Suède & 4.3 & 7.0 & 9.2 & 9.9 & 10.62004 \\
\hline Suisse & $\ldots$ & . & .. & 12.9 & 14.12004 \\
\hline Turquie & . & 1.0 & 1.3 & 1.7 & 1.82004 \\
\hline Moyenne la plus récente ${ }^{d}$ & . & . & . & $\ldots$ & 8.9 \\
\hline Moyenne cohérente $(15)^{e}$ & . & 5.5 & 7.0 & 7.6 & 8.0 \\
\hline
\end{tabular}

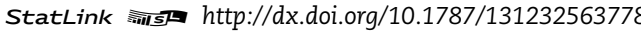

a) L'Autriche ne prend en compte que les infirmières employées dans les hôpitaux.

b) Le Luxembourg inclut les aides-soignantes.

c) Les Pays-Bas communiquent le nombre d'infirmières autorisées à exercer plutôt que celui des infirmières effectivement en activité.

d) La moyenne comprend tous les pays de l'OCDE.

e) Exclut l'Allemagne, la Belgique, la Corée, l'Espagne, la Finlande, la Hongrie, l'Irlande, l'Italie, le Luxembourg, le Mexique, la Norvège, les Pays-Bas, la République slovaque, le Royaume-Uni et la Suisse.

Source : Eco-Santé OCDE 2007. 


\section{Tableau A.4.4a. Rémunération des spécialistes, salariés et indépendants par rapport au PIB par habitant}

\begin{tabular}{|c|c|c|c|c|c|c|c|c|c|c|c|c|}
\hline & \multicolumn{6}{|c|}{ Salariés } & \multicolumn{6}{|c|}{ Indépendants } \\
\hline & 2000 & 2001 & 2002 & 2003 & 2004 & 2005 & 2000 & 2001 & 2002 & 2003 & 2004 & 2005 \\
\hline Allemagne $^{a}$ & & & & & 2.68 & & & & & & & \\
\hline Australie $^{b}$ & & & & & & & 5.20 & 5.21 & 5.37 & 5.31 & 5.30 & \\
\hline Autriche $^{c}$ & & & & & & & 5.44 & 5.37 & 5.27 & 5.57 & & \\
\hline Belgique $^{d}$ & & & & & & & 7.44 & 7.61 & 7.23 & 7.43 & 7.81 & \\
\hline Canada $^{e}$ & & & & & & & & 5.15 & 5.12 & 5.03 & 4.89 & \\
\hline Danemark $^{f}$ & 2.65 & 2.71 & 2.77 & 2.86 & 2.90 & 2.79 & & & & & & \\
\hline États-Unis & & 4.82 & & & & & & 6.49 & & & & \\
\hline Finlande $\mathrm{e}^{g}$ & & 2.36 & 2.59 & 2.62 & 2.53 & 2.54 & & & & & & \\
\hline France $^{h}$ & & & & & & & 4.59 & 4.65 & 4.79 & 4.92 & 4.98 & \\
\hline Grèce $^{i}$ & & & & & & 2.30 & & & 2.60 & 2.66 & 2.36 & \\
\hline Hongrie $^{j}$ & & & & 1.74 & 1.68 & 1.69 & & & & & & \\
\hline Irlande ${ }^{k}$ & & & & & 4.65 & 4.65 & & & & & & \\
\hline Islande' & & & & & & 2.89 & & & & & & \\
\hline Luxembourg $^{m}$ & & & & 2.77 & & & & & & 4.15 & & \\
\hline Mexique $^{n}$ & & & & 2.58 & & & & & & & & \\
\hline Norvège $^{0}$ & & 1.69 & 1.77 & 2.00 & 1.81 & 1.65 & & & & & & \\
\hline
\end{tabular}

a) Les données ne concernent que le secteur public. On ne dispose d'aucune donnée sur la rémunération moyenne des heures supplémentaires. Les revenus provenant de la clientèle privée ne sont pas inclus.

b) Comprend les médecins pratiquant à temps partiel. Le Secrétariat de l'OCDE a déduit les dépenses professionnelles en appliquant la moyenne des pays de l'OCDE ( $30 \%$ de la rémunération brute). Les salaires et les rémunérations à la vacation ne sont pas inclus.

c) Comprend les médecins pratiquant à temps partiel.

d) Comprend les médecins pratiquant à temps partiel. Les données incluent au moins une partie des dépenses professionnelles.

e) Données établies sur la base d'une rémunération à l'acte pour les spécialistes ayant facturé aux régimes provinciaux de soins de santé un minimum de 60000 CAD. Elles n'incluent pas les revenus provenant d'autres modes de paiement.

f) Les données incluent les médecins ayant une spécialisation en médecine générale. Elles n'incluent pas les revenus provenant de la clientèle privée.

g) Les données incluent les spécialistes en médecine générale et les directeurs d’hôpitaux ou de centres de santé. La rémunération est établie sur la base du salaire et des paiements additionnels pour ceux qui travaillent plus de $90 \%$ du temps indiqué dans la convention collective.

h) Les données ne prennent pas en compte la rémunération des médecins qui ouvrent ou qui ferment leur cabinet dans l'année en question et ceux qui ont plus de 65 ans. Elles sont établies sur la base d'une rémunération à l'acte et n'incluent pas les salaires. Elles comprennent la rémunération des stomatologues.

i) Pour les spécialistes salariés, les données concernent des spécialistes situés aux niveaux intermédiaires de la hiérarchie de l'hôpital pour enfants "Agia (Sainte) Sofia ». On considère que ces données sont représentatives de l'ensemble des spécialistes salariés du secteur public. Pour les libéraux, elles correspondent au revenu déclaré. Les paiements informels ne sont pas inclus dans les données, pour les spécialistes salariés comme pour les libéraux ; ils sont plus fréquents pour les spécialistes salariés.

j) Les données ne concernent que le secteur public. Elles n'incluent pas les gratifications, les paiements informels et les revenus provenant de la clientèle privée.

k) La rémunération est établie sur la base du salaire horaire brut pour une durée hebdomadaire clinique de 33 heures. Les chiffres ne prennent pas en compte les indemnités de rappel pour urgences et les paiements pour travail à la demande à l'exception du paiement annuel d'un montant minimum uniforme de 3500 EUR. Les données ne concernent que le secteur public; elles n'incluent pas les revenus provenant de la clientèle privée. Étant donné que le PIB par habitant excède le revenu moyen, la rémunération est présentée en proportion du revenu national brut.

l) Les données ne concernent que le secteur public. Elles n'incluent pas les revenus provenant de la clientèle privée.

m) Les chiffres n'incluent pas les médecins dont le revenu annuel est inférieur au salaire minimum social (16 425 EUR en 2003$)$. Les spécialistes ayant ouvert ou fermé leur cabinet au cours de l'exercice ne sont pas inclus. Étant donné que le PIB par habitant excède le revenu moyen, la rémunération est présentée en proportion du revenu national brut.

n) Les données ne concernent que le secteur public.

o) Les données englobent les généralistes. Elles ne concernent que le secteur public. Elles n'incluent pas les indemnités de jours fériés. 


\section{Tableau A.4.4a. Rémunération des spécialistes, salariés et indépendants} par rapport au PIB par habitant (suite)

\begin{tabular}{|c|c|c|c|c|c|c|c|c|c|c|c|c|}
\hline & \multicolumn{6}{|c|}{ Salariés } & \multicolumn{6}{|c|}{ Indépendants } \\
\hline & 2000 & 2001 & 2002 & 2003 & 2004 & 2005 & 2000 & 2001 & 2002 & 2003 & 2004 & 2005 \\
\hline Nouvelle-Zélande ${ }^{p}$ & 3.87 & 3.77 & 3.78 & 3.74 & 3.60 & 3.70 & & & & & & \\
\hline Pays-Bas ${ }^{q}$ & & & & 4.11 & 4.02 & 3.96 & & & & 7.94 & 8.64 & 8.38 \\
\hline Portugal $^{r}$ & 3.51 & 3.47 & 3.48 & 3.42 & 3.31 & 3.28 & & & & & & \\
\hline République tchèque $^{S}$ & 1.39 & 1.46 & 1.60 & 1.65 & 1.57 & 1.56 & 2.22 & 2.49 & 2.44 & 2.42 & 2.21 & 2.28 \\
\hline Royaume-Uni ${ }^{t}$ & 4.44 & & 4.73 & & 4.82 & & & & & & & \\
\hline Suède ${ }^{u}$ & & & 2.50 & & & & & & & & & \\
\hline Suisse ${ }^{v}$ & & & & & & & & 3.74 & 3.80 & 3.65 & & \\
\hline
\end{tabular}

p) Les données couvrent les spécialistes travaillant pour le District Health Board mais ne couvrent pas les spécialistes employés par les Boards dans le cadre de contrats de travail individuels. Elles excluent le remboursement des frais professionnels et des dépenses de formation continue, le paiement des heures travaillées au-delà des 40 heures hebdomadaires, l'indemnité versée aux médecins autorisés pour leurs activités en dehors des heures de travail ou tout paiement exceptionnel (par exemple les frais de recrutement et de fidélisation) mais incluent les congés payés. Elles n'incluent pas les revenus provenant de la clientèle privée.

q) Les spécialistes travaillant dans les hôpitaux généraux et les hôpitaux universitaires sont inclus dans les spécialistes salariés. Les données sur la rémunération des salariés des hôpitaux généraux n'incluent pas les primes, le paiement des heures supplémentaires et autres paiements additionnels.

r) Les données incluent toutes les catégories de généralistes et de spécialistes travaillant dans le service national de santé. Elles n'incluent pas les revenus additionnels tels que les paiements pour travail de nuit et de week-end, heures supplémentaires et primes mais incluent la prime de Noël et les congés payés. Les données n'incluent pas les revenus provenant de la clientèle privée.

s) Les généralistes salariés, les médecins (généralistes et spécialistes) en formation et les dentistes sont inclus dans les spécialistes salariés. Les salaires et rémunérations en nature ainsi que les revenus provenant de la clientèle privée ne sont pas inclus. Pour ceux qui exercent en libéral, le salaire reçu d'un hôpital n'est pas inclus.

t) Les données concernent uniquement le secteur public et n'incluent pas les revenus provenant de la clientèle privée. Elles concernent uniquement l'Angleterre.

u) Les paiements au titre des heures supplémentaires ne sont pas inclus. Les données concernent uniquement le secteur public et n'incluent pas les revenus provenant de la clientèle privée.

v) Comprend les médecins pratiquant à temps partiel. Les données n'incluent pas les rémunérations des médecins de plus de 65 ans Source: Eco-Santé OCDE 2007. 


\section{Tableau A.4.4b. Rémunération des généralistes, salariés et indépendants par rapport au PIB par habitant}

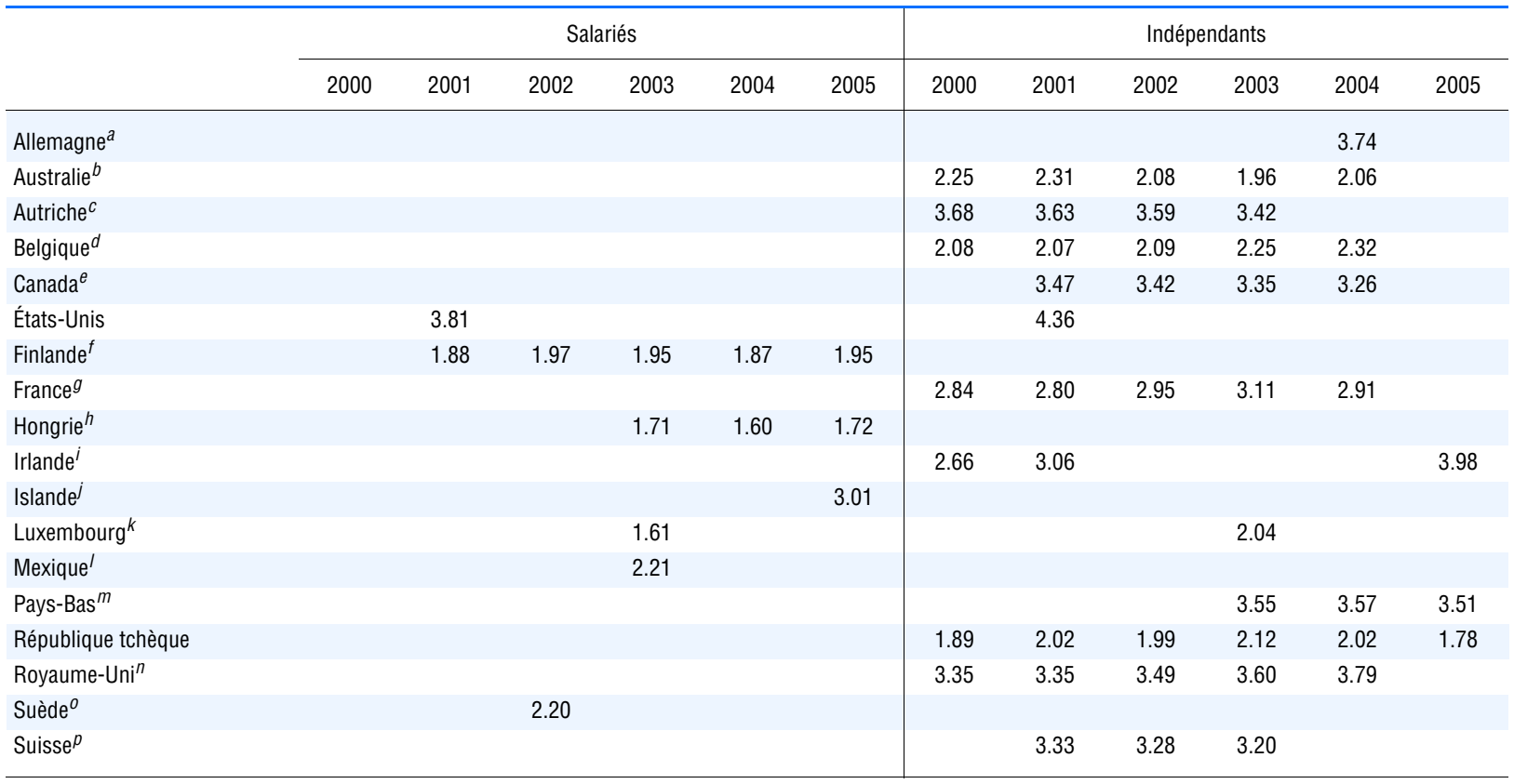

StatLink नiाIst http://dx.doi.org/10.1787/131260665140

a) Les données font référence aux paiements de l'assurance maladie et aux revenus provenant de la clientèle privée.

b) Comprend les médecins pratiquant à temps partiel. Le Secrétariat de l'OCDE a déduit les dépenses professionnelles en appliquant la moyenne des pays de l'OCDE (30\% de la rémunération brute). Les salaires et les rémunérations à la vacation ne sont pas inclus.

c) Comprend les médecins pratiquant à temps partiel.

d) Comprend les médecins pratiquant à temps partiel. Les données incluent au moins une partie des dépenses professionnelles.

e) Les données sont établies sur la base d'une rémunération à l'acte pour les généralistes ayant facturé aux régimes provinciaux de soins de santé un minimum de 60000 CAD. Elles n'incluent pas les revenus provenant d'autres modes de paiement.

f) La rémunération est établie sur la base du salaire et des paiements additionnels pour ceux qui travaillent plus de $90 \%$ du temps indiqué dans la convention collective.

g) Les données couvrent les généralistes et les médecins généralistes à exercice particulier (MEP). Cette catégorie recouvre les médecins exerçant des activités spécifiques comme les acupuncteurs et les homéopathes. Les données n'incluent pas la rémunération des médecins qui ouvrent ou qui ferment leur cabinet dans l'année en question et ceux qui ont plus de 65 ans. Elles sont établies sur la base d'une rémunération à l'acte et n'incluent pas les salaires.

h) Les données ne concernent que le secteur public. Elles n'incluent pas les gratifications, les paiements informels et les revenus tirés de la clientèle privée.

i) Comprend les médecins pratiquant à temps partiel. Le Secrétariat de l'OCDE a déduit les dépenses professionnelles en appliquant la moyenne des pays de l"OCDE (30\% de la rémunération brute). Les paiements émanant des Primary Community and Continuing Care (PCCC) Local Health Areas ne sont pas inclus. Étant donné que le PIB par habitant excède le revenu moyen, la rémunération est présentée en proportion du revenu national brut.

j) Les données ne concernent que le secteur public. Elles ne prennent pas en compte la rémunération des généralistes travaillant dans des centres de santé gérés par des municipalités ou dans dans des centres de santé privés.

k) Les chiffres n'incluent pas les médecins dont le revenu annuel est inférieur au salaire social minimum (16 425 EUR en 2003). Ils ne prennent pas en compte les généralistes qui ouvrent ou qui ferment leur cabinet dans l'année en question. Étant donné que le PIB par habitant excède le revenu moyen, la rémunération est présentée en proportion du revenu national brut.

l) Les données ne concernent que le secteur public.

m) Les données sont établies sur la base de la rémunération correspondant à la norme (2 350 patients).

n) Comprend les médecins pratiquant à temps partiel. Les chiffres correspondent à la rémunération des généralistes sous contrat GMS (General Medical Services) et ne prennent pas en compte les " GP registrars », les généralistes salariés, les assistants et les généralistes sous contrat PMS (Personal Medical Services). Les données ne concernent que le secteur public. Elles n'incluent pas les revenus provenant de la clientèle privée et les revenus additionnels du travail à l'hôpital. Elles concernent la Grande-Bretagne.

o) Les données ne concernent que le secteur public. Elles ne prennent pas en compte les paiements pour travail à la demande et la rémunération des heures supplémentaires.

p) Comprend les médecins pratiquant à temps partiel. Les rémunérations des médecins de plus de 65 ans sont exclues.

Source : Eco-Santé OCDE 2007. 


\section{Tableau A.4.4c. Rémunération des infirmières hospitalières salariées} par rapport au PIB par habitant

\begin{tabular}{|c|c|c|c|c|c|c|}
\hline & 2000 & 2001 & 2002 & 2003 & 2004 & 2005 \\
\hline Australie $^{a}$ & 1.46 & 1.48 & 1.49 & 1.47 & 1.45 & \\
\hline Danemark $^{b}$ & 1.29 & 1.30 & 1.32 & 1.33 & 1.33 & 1.29 \\
\hline États-Unis ${ }^{c}$ & 1.38 & & & & 1.51 & \\
\hline Finlande ${ }^{d}$ & & 0.94 & 0.96 & 0.96 & 0.96 & 0.99 \\
\hline Grèce $^{e}$ & & & & & 1.22 & \\
\hline Hongrie $^{f}$ & & & & 0.88 & 0.84 & 0.84 \\
\hline Irlande ${ }^{g}$ & & & & & 1.32 & 1.30 \\
\hline Islande ${ }^{h}$ & & & & & & 1.26 \\
\hline Japon $^{i}$ & & & 1.17 & & 1.14 & \\
\hline Luxembourg ${ }^{j}$ & & & & & 1.11 & 1.07 \\
\hline Mexique $^{k}$ & & & & 1.38 & & \\
\hline Norvège ${ }^{l}$ & & 0.88 & 0.96 & 0.96 & 0.92 & 0.86 \\
\hline Nouvelle-Zélande ${ }^{m}$ & & & 1.41 & & & \\
\hline Portugal $^{n}$ & 1.72 & 1.70 & 1.87 & 1.84 & 1.77 & 1.82 \\
\hline République tchèque ${ }^{0}$ & 0.67 & 0.73 & 0.79 & 0.82 & 0.77 & 0.75 \\
\hline Royaume-Uni ${ }^{p}$ & 1.40 & & 1.39 & & 1.34 & \\
\hline
\end{tabular}

StatLink त्राजा http://dx.doi.org/10.1787/131260665140

a) Les données couvrent tous les niveaux d'infirmières autorisées employées dans des hôpitaux de soins aigus et des hôpitaux psychiatriques.

b) Les données couvrent tous les niveaux d'infirmières autorisées.

c) Les données n'incluent pas les licensed practical nurses (LPN) et les licensed vocational nurses (LVN) mais incluent les infirmières gestionnaires.

d) Les infirmières praticiennes ne sont pas incluses. La rémunération est établie sur la base du salaire et des paiements additionnels pour ceux qui travaillent plus de $90 \%$ du temps indiqué dans la convention collective.

e) Les données concernent les infirmières hospitalières salariées aux niveaux intermédiaires de la hiérarchie de l'hôpital pour enfants "Agia (Sainte) Sofia ". On considère que ces données sont représentatives de l'ensemble des infirmières hospitalières salariées du secteur public.

f) Les données ne concernent que le secteur public. Elles n'incluent pas les gratifications provenant de la clientèle privée.

g) Les données ne concernent que le secteur public. La rémunération est indiquée sur la base d'une semaine de travail de 39 heures et n'inclut pas le paiement des heures supplémentaires. Étant donné que le PIB par habitant excède le revenu moyen, la rémunération est présentée en proportion du revenu national brut.

h) Les données ne concernent que les hôpitaux généraux publics et les centres médicaux publics.

i) Les données ont été calculées par l'OCDE sur la base des données sur le salaire mensuel et les revenus additionnels, et des statistiques fournies par le ministère de la Santé, du Travail et de la Protection sociale concernant le nombre d'infirmières et d'infirmières adjointes. Les chiffres sont une moyenne pondérée de la rémunération annuelle des infirmières et des infirmières adjointes.

j) Les données incluent les infirmières adjointes mais ne couvrent que les infirmières travaillant dans des unités de soins aigus. Elles ont été obtenues en divisant le salaire brut annuel total par le nombre d'infirmières équivalents plein-temps. Étant donné que le PIB par habitant excède le revenu moyen, la rémunération est présentée en proportion du revenu national brut.

k) Les données ne concernent que le secteur public.

l) Les données ne concernent que le secteur public. Elles n'incluent pas les compléments pour congés payés.

m) Les données ne concernent que le secteur public. Elles ont été calculées en divisant le montant total des coûts de personnel par le nombre total d'infirmières équivalents plein-temps.

n) Les données ne concernent que le secteur public. Elles n'incluent pas les revenus additionnels tels que les paiements pour travail de nuit et de week-end, heures supplémentaires et primes mais incluent la prime de Noël et les congés payés.

o) Les données prennent en compte les infirmières et les sages-femmes travaillant dans des hôpitaux généraux et dans des instituts thérapeutiques spécialisés (à l'exclusion des instituts balnéaires). Les salaires et rémunérations en nature ne sont pas inclus.

p) Les données ne concernent que le secteur public en Angleterre. Elles ont été obtenues en divisant le total des paiements au titre des infirmières par le nombre total d'infirmières équivalents plein-temps.

Source : Eco-Santé OCDE 2007. 
Tableau A.4.5a. Nombre de lits de soins aigus pour 1000 habitants, 1980 à 2005

\begin{tabular}{|c|c|c|c|c|c|c|}
\hline & 1980 & 1985 & 1990 & 1995 & 2000 & 2005 \\
\hline Allemagne & .. & .. & 8.31991 & 7.5 & 6.8 & 6.4 \\
\hline Australie & 6.4 & 5.3 & 4.81989 & 4.1 & 3.6 & 3.62004 \\
\hline Autriche & .. & 8.3 & 7.5 & 6.8 & 6.3 & 6.1 \\
\hline Belgique & . & $\ldots$ & 5.2 & 5.0 & 4.7 & 4.4 \\
\hline Canada & 4.6 & 4.4 & 4.0 & 4.1 & 3.2 & 2.92004 \\
\hline Corée & .. & . & 2.7 & 3.8 & 5.2 & 6.5 \\
\hline Danemark & 5.3 & 4.7 & 4.1 & 3.9 & 3.5 & 3.12004 \\
\hline Espagne & 3.8 & 3.7 & 3.6 & 3.5 & 2.8 & 2.62004 \\
\hline États-Unis & 4.4 & 4.2 & 3.7 & 3.4 & 2.9 & 2.7 \\
\hline Finlande & 4.9 & 4.8 & 4.3 & 4.0 & 3.2 & 2.9 \\
\hline France & 6.2 & 5.7 & 5.2 & 4.6 & 4.1 & 3.7 \\
\hline Grèce & 4.9 & 4.3 & .. & 3.9 & 3.8 & 3.82004 \\
\hline Hongrie & 6.6 & 6.8 & 7.1 & 6.5 & 5.8 & 5.5 \\
\hline Irlande & 4.3 & 4.1 & 3.2 & 3.1 & 2.8 & 2.8 \\
\hline Islande & .. & . & 4.3 & 3.8 & .. & .. \\
\hline Italie & 8.0 & 7.0 & 6.2 & 5.6 & 4.1 & 3.3 \\
\hline Japon & .. & .. & 12.31993 & 12.0 & 9.6 & 8.2 \\
\hline Luxembourg & .. & .. & . & 5.81998 & 5.7 & 5.2 \\
\hline Mexique & .. & .. & 1.0 & 1.1 & 1.0 & 1.0 \\
\hline Norvège & 5.2 & 4.7 & 3.8 & 3.3 & 3.1 & 3.0 \\
\hline Nouvelle-Zélande & .. & . & . & . & . & . \\
\hline Pays-Bas & .. & .. & 3.8 & 3.4 & 3.1 & 3.1 \\
\hline Pologne & 5.6 & 5.7 & 6.3 & 5.8 & 5.2 & 4.7 \\
\hline Portugal & 4.1 & 3.5 & 3.4 & 3.3 & 3.2 & 3.0 \\
\hline République slovaque & .. & .. & . & 6.31996 & 5.8 & 5.0 \\
\hline République tchèque & 8.7 & 8.8 & 8.6 & 7.3 & 6.1 & 5.7 \\
\hline Royaume-Uni & .. & . & .. & 4.1 & 3.3 & 3.1 \\
\hline Suède & 5.1 & 4.6 & 4.1 & 3.0 & 2.4 & 2.2 \\
\hline Suisse & 7.2 & 6.8 & 6.5 & 5.5 & 4.1 & 3.6 \\
\hline Turquie & 1.5 & 1.6 & 2.0 & 2.1 & 2.2 & 2.0 \\
\hline Moyenne la plus récente ${ }^{a}$ & . & . & . & . & $\ldots$ & 3.9 \\
\hline Moyenne cohérente (24) ${ }^{b}$ & . & . & 5.1 & 4.7 & 4.1 & 3.9 \\
\hline
\end{tabular}

StatLink न्ताst $h t t p: / / d x . d o i . o r g / 10.1787 / 131318030641$ Note : La définition des lits de "soins aigus " peut varier d'un pays à l'autre. Par conséquent, les variations entre les pays doivent être interprétées avec précaution.

a) La moyenne comprend tous les pays pour lesquels des données récentes sont disponibles (2004+).

b) Exclut la Grèce, l'Islande, le Luxembourg, la Nouvelle-Zélande, la République slovaque et le Royaume-Uni.

Source : Eco-Santé OCDE 2007. 
Tableau A.4.5b. Taux d'occupation des lits de soins aigus dans les hôpitaux, en pourcentage, 1980 à 2005

\begin{tabular}{|c|c|c|c|c|c|c|}
\hline & 1980 & 1985 & 1990 & 1995 & 2000 & 2005 \\
\hline Allemagne & $\ldots$ & . & 84.11991 & 82.1 & 81.9 & 75.6 \\
\hline Australie & 66.3 & 69.0 & 68.81989 & 69.5 & 70.4 & 70.62004 \\
\hline Autriche & 80.81982 & 79.9 & 79.3 & 76.9 & 77.3 & 79.0 \\
\hline Belgique & 77.7 & 83.31986 & 81.9 & 79.7 & 79.91998 & $\ldots$ \\
\hline Canada & 80.4 & 83.4 & 78.6 & 84.6 & 91.2 & 90.02004 \\
\hline Corée & 60.81981 & 61.0 & 83.9 & 66.3 & 67.2 & 71.62003 \\
\hline Danemark & 75.3 & 78.9 & 78.5 & 78.6 & 85.0 & $\ldots$ \\
\hline Espagne & . & 72.2 & 73.5 & 76.4 & 77.1 & 78.82004 \\
\hline États-Unis & 75.4 & 64.8 & 66.8 & 62.8 & 63.9 & 67.4 \\
\hline Finlande & .. & 76.2 & 74.2 & 74.0 & .. & .. \\
\hline France & 79.0 & 79.1 & 77.3 & 76.0 & 75.0 & 73.4 \\
\hline Grèce & 66.0 & 66.0 & 63.2 & 66.4 & 78.1 & . \\
\hline Hongrie & 83.3 & 80.6 & 74.9 & 72.6 & 73.2 & 75.7 \\
\hline Irlande & 82.2 & 75.9 & 84.5 & 82.5 & 84.5 & 85.6 \\
\hline Islande & . & .. & . & .. & . & .. \\
\hline Italie & 69.0 & 67.9 & 69.3 & 70.7 & 75.6 & 76.42004 \\
\hline Japon & . & .. & .. & 81.6 & 81.8 & 79.2 \\
\hline Luxembourg $^{a}$ & . & .. & .. & 67.91998 & 66.5 & 64.7 \\
\hline Mexique & . & .. & 48.41993 & 50.1 & 57.4 & 61.0 \\
\hline Norvège & 79.3 & 82.0 & 77.0 & 79.4 & 85.2 & 87.6 \\
\hline Nouvelle-Zélande & . & .. & . & .. & . & . \\
\hline Pays-Bas ${ }^{a}$ & 83.5 & 79.1 & 73.3 & 73.3 & 65.7 & 63.9 \\
\hline Pologne & 85.0 & 77.0 & 66.0 & 67.3 & 74.0 & 77.02002 \\
\hline Portugal & . & 67.7 & 66.7 & 72.6 & 71.3 & 73.2 \\
\hline République slovaque & . & .. & .. & 79.61996 & 70.6 & 66.7 \\
\hline République tchèque & 81.8 & 80.8 & 69.6 & 72.6 & 70.5 & 74.6 \\
\hline Royaume-Uni & . & 76.1 & . & 77.1 & 82.2 & 83.9 \\
\hline Suède & 72.1 & 75.3 & 72.2 & 75.9 & . & . \\
\hline Suisse & 77.9 & 80.0 & 79.0 & 77.71994 & 84.8 & 86.1 \\
\hline Turquie & 44.01979 & 52.1 & 57.2 & 55.4 & 58.7 & 64.5 \\
\hline Moyenne la plus récente ${ }^{b}$ & . & . & . & . & . & 75.1 \\
\hline Moyenne cohérente $(19)^{C}$ & . & . & 72.5 & 72.0 & 73.9 & 75.4 \\
\hline
\end{tabular}

StatLink 尚Ist $h t t p: / / d x . d o i . o r g / 10.1787 / 131318030641$

a) Aux Pays-Bas et au Luxembourg, les taux d'occupation sont légèrement sous-estimés. En effet, alors que le nombre de jours à l'hôpital n'inclut que les patients y passant au moins une nuit, le nombre de lits de soins aigus (le dénominateur) inclut également les lits disponibles pour les soins de jour.

b) La moyenne comprend tous les pays pour lesquels des données récentes sont disponibles (2002+).

c) Exclut la Belgique, le Danemark, la Finlande, la Grèce, l'Islande, le Japon, le Luxembourg, la Nouvelle-Zélande, la République slovaque, le Royaume-Uni et la Suède.

Source : Eco-Santé OCDE 2007. 


\section{Tableau A.4.6. Nombre de lits de soins de longue durée dans les hôpitaux et les établissements de long séjour, pour 1000 habitants âgés de 65 ans et plus, 1995, 2000 et 2005}

\begin{tabular}{|c|c|c|c|c|c|c|}
\hline & \multicolumn{3}{|c|}{ Hôpitaux } & \multicolumn{3}{|c|}{ Établissements de long séjour } \\
\hline & 1995 & 2000 & 2005 & 1995 & 2000 & 2005 \\
\hline Allemagne & . & . & . & 22.9 & 46.71999 & 47.8 \\
\hline Australie & . & .. & .. & 34.7 & 35.9 & 37.52004 \\
\hline Autriche & 8.4 & 7.6 & 2.8 & . & .. & . \\
\hline Belgique & 1.1 & 1.1 & 1.22002 & .. & . & . \\
\hline Canada & 2.7 & 1.7 & 1.62004 & . & . & .. \\
\hline Corée & . & 2.12003 & 5.8 & .. & .. & $\ldots$ \\
\hline Danemark $^{a}$ & . & .. & . & 52.2 & 42.9 & 25.5 \\
\hline Espagne & 2.1 & 1.9 & 2.0 & .. & . & 18.9 \\
\hline États-Unis & 1.01998 & 0.9 & 0.82004 & 44.11998 & 43.6 & 43.12004 \\
\hline Finlande & 34.71996 & 28.9 & 25.6 & 36.31996 & 31.2 & 26.1 \\
\hline France & 9.01996 & 8.8 & 8.12003 & 63.41996 & .. & 60.32003 \\
\hline Grèce & 6.7 & 5.3 & 5.02003 & .. & .. & . \\
\hline Hongrie & 4.7 & 4.5 & 4.8 & 43.3 & 48.4 & 53.1 \\
\hline Irlande & 17.2 & 16.6 & 15.2 & 32.6 & 37.8 & 41.4 \\
\hline Islande ${ }^{b}$ & 17.41996 & 12.7 & 7.5 & 46.41996 & 51.4 & 61.0 \\
\hline Italie & . & 1.02001 & 0.92003 & . & 13.02001 & 14.82003 \\
\hline Japon & . & 12.0 & 15.0 & 5.6 & 10.6 & 11.6 \\
\hline Luxembourg & . & 1.2 & 0.0 & . & 26.4 & 43.4 \\
\hline Mexique & . & . & . & . & . & . \\
\hline Norvège & . & . & . & 63.2 & 63.0 & 60.3 \\
\hline Nouvelle-Zélande & . & . & . & . & .. & . \\
\hline Pays-Bas & . & .. & 0.02003 & 27.1 & 26.9 & 27.42003 \\
\hline Pologne & . & 3.22003 & 3.0 & .. & 18.22003 & 18.3 \\
\hline Portugal & . & . & . & .. & . & . \\
\hline République slovaque & 9.81996 & 10.0 & 8.1 & . & . & . \\
\hline République tchèque & 4.9 & 8.2 & 10.0 & 8.3 & 10.6 & 14.9 \\
\hline Royaume-Uni & 0.61997 & 0.5 & 0.42004 & 24.31997 & 22.0 & 18.72004 \\
\hline Suède & 3.6 & 2.1 & 1.5 & 84.2 & 82.7 & 69.9 \\
\hline Suisse & . & . & . & 81.81997 & 74.1 & 71.72004 \\
\hline Turquie & . & .. & 5.1 & . & .. & . \\
\hline Moyenne la plus récente ${ }^{c}$ & . & . & 5.7 & . & 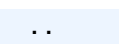 & 38.3 \\
\hline
\end{tabular}

StatLink त्ञाI $h$ ttp://dx.doi.org/10.1787/131341500670

a) Les données pour le Danemark ne comprennent pas les lits des établissements résidentiels pour personnes âgées, qui sont destinés aux gens souffrant d'une incapacité modérée. Le nombre de lits dans ces établissements a augmenté rapidement ces dernières années.

b) En Islande, depuis 2000 les lits de soins de longue durée dans les hôpitaux ne comprennent pas les lits des unités de gériatrie, qui ont augmenté ces dernières années.

c) La moyenne comprend tous les pays pour lesquels des données récentes sont disponibles.

Source : Eco-Santé OCDE 2007. 
Tableau A.4.7a. Technologie médicale : nombre de tomodensitomètres et d'unités d'imagerie par résonance magnétique (IRM) par million d'habitants, 1990 à 2005

\begin{tabular}{|c|c|c|c|c|c|c|c|c|}
\hline & \multicolumn{4}{|c|}{ Tomodensitomètres } & \multicolumn{4}{|c|}{ Unités d'IRM } \\
\hline & 1990 & 1995 & 2000 & 2005 & 1990 & 1995 & 2000 & 2005 \\
\hline Allemagne $^{a}$ & 6.41991 & 9.0 & 12.7 & 15.4 & 1.11991 & 2.3 & 4.9 & 7.1 \\
\hline Australie $^{b}$ & 13.8 & 20.5 & 26.1 & 45.32004 & 0.6 & 2.9 & 3.5 & 4.2 \\
\hline Autriche & 11.7 & 23.91996 & 25.8 & 29.4 & 1.21989 & 7.41996 & 10.9 & 16.3 \\
\hline Belgique & 16.1 & 16.71994 & 21.8 & 31.62004 & 2.0 & 3.3 & 6.0 & 6.6 \\
\hline Canada & 7.1 & 8.0 & 9.82001 & 11.2 & 0.7 & 1.4 & 2.5 & 5.5 \\
\hline Corée & 12.21993 & 15.5 & 28.4 & 32.2 & 1.41992 & 3.9 & 5.4 & 12.1 \\
\hline Danemark & 4.3 & 7.3 & 11.4 & 13.8 & 2.5 & $\ldots$ & 5.4 & 10.22004 \\
\hline Espagne $^{a}$ & 4.41988 & 8.3 & 12.0 & 13.5 & 0.71988 & 2.7 & 4.8 & 8.1 \\
\hline États-Unis & . & 24.11997 & 25.11999 & 32.22004 & 11.51993 & 12.3 & 15.41999 & 26.62004 \\
\hline Finlande & 9.8 & 11.7 & 13.5 & 14.7 & 1.8 & 4.3 & 9.9 & 14.7 \\
\hline France & 6.7 & 9.2 & 9.5 & 9.8 & 0.8 & 2.1 & 2.6 & 4.7 \\
\hline Grèce $^{c}$ & 6.5 & 13.51997 & 17.12002 & 25.8 & 0.4 & 1.91997 & 1.91998 & 13.2 \\
\hline Hongrie & 1.9 & 4.6 & 5.7 & 7.1 & 0.1 & 1.0 & 1.8 & 2.6 \\
\hline Irlande & 4.3 & $\ldots$ & $\ldots$ & 10.7 & . & $\ldots$ & .. & .. \\
\hline Islande & 11.8 & 18.7 & 21.3 & 23.7 & 3.9 & 7.5 & 10.7 & 20.3 \\
\hline Italie & 6.0 & 12.11994 & 21.0 & 27.7 & 1.3 & 2.61994 & 7.7 & 15.0 \\
\hline Japon $^{d}$ & 55.2 & 74.71996 & 84.41999 & 92.62002 & 6.1 & 18.81996 & 23.21999 & 40.1 \\
\hline Luxembourg & 5.2 & 26.6 & 25.2 & 28.6 & 2.6 & 2.4 & 2.3 & 11.0 \\
\hline Mexique & . & . & 2.52001 & 3.4 & . & . & 1.12001 & 1.3 \\
\hline Norvège & . & . & . & . & .. & .. & .. & .. \\
\hline Nouvelle-Zélande & 3.6 & 7.51996 & 8.8 & 12.12004 & . & 2.71996 & 2.61998 & 3.72003 \\
\hline Pays-Bas ${ }^{e}$ & 7.3 & 9.01993 & . & 5.8 & 0.9 & 3.9 & .. & 5.6 \\
\hline Pologne & . & 3.51998 & 4.4 & 7.9 & . & . & 0.92002 & 2.0 \\
\hline Portugal $^{c}$ & 4.6 & 12.41997 & 12.82003 & 26.2 & 0.8 & 2.81997 & 3.92003 & 3.92003 \\
\hline République slovaque & . & . & 8.72003 & 11.3 & . & . & 2.02003 & 4.3 \\
\hline République tchèque & 2.11991 & 6.7 & 9.6 & 12.3 & 0.21991 & 1.0 & 1.7 & 3.1 \\
\hline Royaume-Uni ${ }^{f}$ & . & . & 4.5 & 7.5 & . & .. & 4.7 & 5.4 \\
\hline Suède & 10.5 & 13.81993 & 14.21999 & .. & 1.5 & 6.8 & 7.91999 & . \\
\hline Suisse & . & 18.31997 & 18.5 & 18.2 & . & 12.41997 & 12.9 & 14.4 \\
\hline Turquie & 1.6 & 2.91994 & 7.11999 & 7.32003 & . & 0.61996 & 3.01998 & 3.02004 \\
\hline Moyenne la plus récente ${ }^{g}$ & . & . & . & 20.6 & . & . & . & 9.8 \\
\hline $\begin{array}{l}\text { Moyenne cohérente } \\
(\mathbf{2 0}, \mathbf{1 8})^{h}\end{array}$ & 9.6 & 15.5 & 19.2 & 24.0 & 2.1 & 4.5 & 6.6 & 12.0 \\
\hline
\end{tabular}

StatLink ailst http://dx.doi.org/10.1787/131351710247

a) Les données pour l'Allemagne et l'Espagne comprennent uniquement les appareils installés dans le secteur hospitalier.

b) En Australie, à partir de 2000 les données sur les IRM concernent uniquement les équipements couverts par l'assurance maladie publique (60 \% du total en 1999).

c) Les données pour la Grèce et le Portugal prennent en compte le secteur privé depuis 2005.

d) Avant 2002, les données sur les IRM au Japon se référent aux hôpitaux ; après 2002 elles se réfèrent aux hôpitaux et aux cliniques générales.

e) Les données en 2005 concernant les Pays-Bas sous-estiment le nombre réel d'appareils, car elles ne concernent que le nombre d'hôpitaux qui déclarent posséder au moins un de ces équipements au lieu du nombre total des équipements dans les hôpitaux et le secteur ambulatoire.

f) Les données brutes de l'Angleterre et du Pays de Galles ont été augmentées au prorata pour fournir des estimations pour le RoyaumeUni. Le secteur privé n'est pas inclus.

g) La moyenne comprend tous les pays pour lesquels des données récentes sont disponibles (2002+).

h) La moyenne des tomodensitomètres exclut les États-Unis, l'Irlande, le Mexique, la Norvège, les Pays-Bas, la Pologne, la République slovaque, le Royaume-Uni, la Suède et la Suisse. La moyenne des unités d'IRM exclut le Danemark, l'Irlande, le Mexique, la Nouvelle-Zélande, la Norvège, les Pays-Bas, la Pologne, la République slovaque, le Royaume-Uni, la Suède, la Suisse et la Turquie.

Source : Eco-Santé OCDE 2007. 


\section{Tableau A.4.7b. Technologie médicale: nombre de mammographes et d'équipements} en radiothérapie, par million d'habitants, 1990 à 2005

\begin{tabular}{|c|c|c|c|c|c|c|c|c|}
\hline & \multicolumn{4}{|c|}{ Mammographes } & \multicolumn{4}{|c|}{ Équipements en radiothérapie } \\
\hline & 1990 & 1995 & 2000 & 2005 & 1990 & 1995 & 2000 & 2005 \\
\hline Allemagne $^{a}$ & .. & .. & . & .. & 4.31991 & 4.5 & 4.8 & 4.7 \\
\hline Australie $^{b}$ & 15.91989 & . & . & 25.1 & 2.9 & 4.4 & 5.2 & 6.1 \\
\hline Autriche & . & .. & . & . & . & 3.21996 & 4.2 & 4.6 \\
\hline Belgique & . & . & 20.52002 & 21.32004 & 6.11991 & 6.1 & 6.41997 & 7.6 \\
\hline Canada & . & . & 19.32001 & 21.3 & 5.61993 & 6.9 & 7.11997 & . \\
\hline Corée & . & . & 13.3 & 28.7 & 4.51992 & 4.1 & 5.3 & 4.5 \\
\hline Danemark & $\ldots$ & . & . & 10.0 & . & . & 5.4 & 6.8 \\
\hline Espagne $^{a}$ & . & . & 10.02003 & 10.22004 & 2.91988 & 3.3 & 3.7 & 4.2 \\
\hline États-Unis & . & . & . & . & . & .. & . & . \\
\hline Finlande & 29.3 & 37.6 & 36.41999 & 37.7 & 10.0 & 8.6 & 8.7 & 8.8 \\
\hline France & 23.8 & 42.0 & 42.6 & 42.22002 & 6.0 & 6.2 & 6.1 & 6.02002 \\
\hline Grèce $^{c}$ & . & .. & 27.92002 & 36.5 & 5.4 & 5.7 & 4.01999 & .. \\
\hline Hongrie & . & 6.81997 & 9.4 & 13.1 & 1.5 & 1.71992 & 2.3 & 2.7 \\
\hline Irlande & $\ldots$ & .. & . & 12.6 & $\ldots$ & . & $\ldots$ & 7.0 \\
\hline Islande & 15.7 & 18.7 & 17.8 & 16.9 & 23.5 & 15.0 & 14.2 & 13.5 \\
\hline Italie & .. & . & . & . & 1.3 & 2.11994 & 3.7 & 5.0 \\
\hline Japon & . & . & .. & . & .. & .. & 6.62002 & 6.8 \\
\hline Luxembourg & 25.31992 & 24.2 & 22.9 & 22.0 & . & . & 4.6 & 4.4 \\
\hline Mexique & . & .. & 2.82001 & 4.5 & .. & . & 1.22001 & 1.3 \\
\hline Norvège & . & .. & . & . & .. & . & .. & . \\
\hline Nouvelle-Zélande & . & 17.21997 & 19.31999 & 23.12004 & . & 7.21996 & 9.9 & 7.62004 \\
\hline Pays-Bas & . & . & . & . & 6.51992 & 7.1 & 7.21997 & . \\
\hline Pologne & $\ldots$ & .. & 11.0 & 15.9 & .. & . & . & . \\
\hline Portugal $^{c}$ & . & 3.61997 & 11.62003 & 34.6 & . & 2.91997 & 3.32003 & 6.0 \\
\hline République slovaque & . & . & 13.02003 & 13.6 & . & . & 7.12003 & 9.8 \\
\hline République tchèque & 3.81991 & 8.4 & 10.6 & 14.1 & 5.41991 & 4.9 & 8.5 & 8.6 \\
\hline Royaume-Uni ${ }^{d}$ & . & 5.0 & 6.1 & 8.4 & $\ldots$ & .. & 3.92002 & 4.1 \\
\hline Suède & . & . & . & . & .. & . & . & . \\
\hline Suisse & .. & . & . & . & . & 11.11997 & 10.4 & 9.8 \\
\hline Turquie & .. & 1.91996 & 4.51999 & 6.52003 & 0.31988 & 0.61996 & 1.41999 & 2.72003 \\
\hline Moyenne la plus récente ${ }^{e}$ & . & . & . & 19.9 & $\ldots$ & . & $\ldots$ & 6.2 \\
\hline $\begin{array}{l}\text { Moyenne cohérente } \\
(\mathbf{1 0 , 1 6})^{f}\end{array}$ & $\ldots$ & 16.5 & 18.1 & 21.9 & $\ldots$ & 5.4 & 6.1 & 6.4 \\
\hline
\end{tabular}

StatLink ailst http://dx.doi.org/10.1787/131351710247

a) Les données pour l'Allemagne et l'Espagne comprennent uniquement les appareils installés dans le secteur hospitalier.

b) En Australie, les données à partir de 2005 concernent uniquement les équipements couverts par l'assurance maladie publique.

c) Les données pour la Grèce (concernant les mammographes) et pour le Portugal prennent en compte le secteur privé depuis 2005.

d) Les données brutes concernant le nombre d'équipements en radiothérapie de l'Angleterre et du Pays de Galles ont été augmentées au prorata pour fournir des estimations pour le Royaume-Uni. Le secteur privé n'est pas inclus.

e) La moyenne comprend tous les pays pour lesquels des données récentes sont disponibles (2002+).

f) La moyenne des mammographes exclut l'Allemagne, l'Australie, l'Autriche, la Belgique, le Canada, la Corée, le Danemark, l'Espagne, les États-Unis, la Grèce, l'Irlande, l'Italie, le Japon, le Mexique, la Norvège, les Pays-Bas, la Pologne, la République slovaque, la Suède et la Suisse. La moyenne des équipements en radiothérapie exclut le Canada, le Danemark, les États-Unis, la Grèce, l'Irlande, le Japon, le Luxembourg, le Mexique, la Norvège, les Pays-Bas, la Pologne, la République slovaque, le Royaume-Uni et la Suède.

Source: Eco-Santé OCDE 2007. 
Tableau A.4.8. Consultations de médecins par habitant, 1980 à 2005

\begin{tabular}{|c|c|c|c|c|}
\hline & 1980 & 1990 & 2000 & 2005 \\
\hline Allemagne $^{a}$ & . & 5.31991 & 7.3 & 7.02004 \\
\hline Australie & 4.0 & 6.1 & 6.4 & 6.1 \\
\hline Autriche & 5.4 & 5.9 & 6.7 & 6.72004 \\
\hline Belgique & 7.1 & 7.7 & 7.9 & 7.5 \\
\hline Canada & 5.6 & 6.7 & 6.3 & 6.02004 \\
\hline Corée & . & $\ldots$ & 8.81999 & 11.8 \\
\hline Danemark $^{b}$ & 5.0 & 5.7 & 6.9 & 7.52004 \\
\hline Espagne $^{c}$ & . & 6.51987 & 8.72001 & 9.52003 \\
\hline États-Unis & . & $\ldots$ & 3.7 & 3.82004 \\
\hline Finlande & 3.2 & 3.9 & 4.3 & 4.3 \\
\hline France & 4.2 & 5.9 & 6.8 & 6.62004 \\
\hline Grèce & 2.6 & 2.5 & 2.51998 & . \\
\hline Hongrie & . & . & 11.1 & 12.6 \\
\hline Irlande & . & $\ldots$ & $\ldots$ & $\ldots$ \\
\hline Islande & . & 5.1 & 5.8 & 6.5 \\
\hline Italie ${ }^{c}$ & . & 6.81991 & 6.1 & 7.0 \\
\hline Japon & . & 13.8 & 14.4 & 13.82004 \\
\hline Luxembourg & . & . & 6.1 & 6.1 \\
\hline Mexique & 1.3 & 1.7 & 2.5 & 2.52004 \\
\hline Norvège & . & $\cdots$ & . & . \\
\hline Nouvelle-Zélande ${ }^{c}$ & 3.7 & $\ldots$ & 4.42001 & 3.22003 \\
\hline Pays-Bas ${ }^{c, d}$ & 4.9 & 5.5 & 5.9 & 5.4 \\
\hline Pologne & 6.5 & 5.8 & 5.4 & 6.3 \\
\hline Portugal $^{e}$ & 3.7 & 3.0 & 3.5 & 3.9 \\
\hline République slovaque & . & . & 15.0 & 11.3 \\
\hline République tchèque & 12.4 & 11.8 & 12.6 & 13.2 \\
\hline Royaume-Uni ${ }^{a, f}$ & 5.2 & 6.1 & 5.3 & 5.1 \\
\hline Suède & 2.6 & 2.8 & 2.8 & 2.8 \\
\hline Suisse ${ }^{c}$ & . & . & 3.42002 & 3.42002 \\
\hline Turquie $^{e}$ & 1.2 & 1.51993 & 2.5 & 3.12004 \\
\hline Moyenne la plus récente ${ }^{g}$ & $\cdots$ & $\cdots$ & $\cdots$ & 6.8 \\
\hline Moyenne cohérente $(20)^{h}$ & $\cdots$ & 5.9 & 6.4 & 6.5 \\
\hline
\end{tabular}

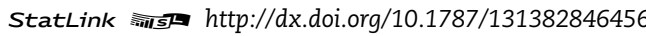

a) Les données pour l'Allemagne représentent le nombre de cas traités par médecin selon les procédures de remboursement de l'assurance sociale. Un cas traité n'est comptabilisé que lors du premier contact en trois mois même si le patient consulte son médecin plus souvent.

b) Le Danemark et le Royaume-Uni comprennent les consultations téléphoniques. Les données ne se limitent donc pas aux visites de médecin.

c) Pour l'Espagne, l'Italie, la Nouvelle-Zélande (2003), les Pays-Bas et la Suisse les données proviennent d'enquêtes de santé avec entretien.

d) Les Pays-Bas excluent les consultations pour les soins maternels et infantiles.

e) Le Portugal et la Turquie excluent les visites chez les médecins libéraux.

f) Le Royaume-Uni exclut les consultations de spécialistes du secteur indépendant ainsi que les consultations des spécialistes hors des services de consultation externe des hôpitaux.

g) La moyenne comprend tous les pays pour lesquels des données récentes sont disponibles (2002+).

h) La moyenne exclut la Corée, les États-Unis, la Grèce, la Hongrie, l'Irlande, le Luxembourg, la Norvège, la Nouvelle-Zélande, la République slovaque et la Suisse.

Source : Eco-Santé OCDE 2007. 
Tableau A.4.9a. Taux de sorties de l'hôpital pour 100000 habitants, toutes causes confondues, 1990 à 2005

\begin{tabular}{|c|c|c|c|c|}
\hline & 1990 & 1995 & 2000 & 2005 \\
\hline Allemagne & . & 18163 & 19559 & 201492004 \\
\hline Australie $^{a}$ & 164831993 & 16482 & 15813 & 157862004 \\
\hline Autriche $^{b}$ & 22704 & 23955 & 28449 & 27765 \\
\hline Belgique & 157201993 & 15884 & 17329 & 168872004 \\
\hline Canada $^{a}$ & 12899 & 11047 & 9401 & 87512004 \\
\hline Corée ${ }^{a}$ & 6536 & 77101994 & 95932001 & 13173 \\
\hline Danemark $^{C}$ & .. & .. & 17220 & 17013 \\
\hline Espagne $^{a}$ & 9501 & 10512 & 11183 & 108382004 \\
\hline États-Unis ${ }^{a, b}$ & 12423 & 11661 & 11380 & 120932004 \\
\hline Finlande $^{a, b}$ & 21745 & 24566 & 26663 & 25751 \\
\hline France $^{b}$ & . & 259671997 & 26802 & 267802004 \\
\hline Grèce $^{a}$ & 12688 & 14078 & 16076 & . \\
\hline Hongrie $^{b}$ & $\cdots$ & & 24071 & 25623 \\
\hline Irlande ${ }^{a}$ & . & 11463 & 10858 & 10227 \\
\hline Islande & 17641 & 18116 & 18190 & 17244 \\
\hline Italie $^{b}$ & . & 153621996 & 15632 & 158222004 \\
\hline Japon $^{d}$ & 96821993 & 100091996 & 104342001 & 10551 \\
\hline Luxembourg $^{a}$ & $\ldots$ & 176181998 & 18075 & 17327 \\
\hline Mexique $^{a, c}$ & .. & 4838 & 5165 & 5129 \\
\hline Norvège ${ }^{a}$ & 145421993 & 14544 & 15408 & 17519 \\
\hline Nouvelle-Zélande & . & & 14058 & 13075 \\
\hline Pays-Bas & 10212 & 10230 & 9265 & 10414 \\
\hline Pologne ${ }^{c}$ & $\ldots$ & . & 174062003 & 18599 \\
\hline Portugal & . & 8903 & 8620 & 9004 \\
\hline République slovaque & . & 19112 & 19607 & 19804 \\
\hline République tchèque ${ }^{d}$ & .. & 20568 & 20953 & 23690 \\
\hline Royaume-Uni ${ }^{b}$ & 17338 & 20971 & 22362 & 24516 \\
\hline Suède ${ }^{a}$ & 17884 & 17457 & 16458 & 16052 \\
\hline Suisse & . & & 152972002 & 15898 \\
\hline Turquie & 5674 & 6092 & 7416 & 84512004 \\
\hline Moyenne la plus récente ${ }^{e}$ & . & . & . & 16342 \\
\hline Moyenne cohérente (24) ${ }^{f}$ & . & 15051 & 15609 & 15988 \\
\hline
\end{tabular}

StatLink न्ताsh http://dx.doi.org/10.1787/131388237608

a) Les données pour l'Australie, le Canada, la Corée, l'Espagne, les États-Unis, la Finlande, la Grèce, l'Irlande, le Luxembourg, le Mexique, la Norvège et la Suède ne comprennent pas les sorties d'hôpital des nouveauxnés en bonne santé.

b) L'Autriche (avant 2003), les États-Unis, la Finlande, la France, la Hongrie (avant 2004), l'Italie (à partir de 2004) et le Royaume-Uni incluent les sorties le jour même, alors que celles-ci sont exclues dans la majorité des pays.

c) Les données fournies par le Danemark, le Mexique et la Pologne ne concernent que les hôpitaux publics.

d) Le Japon et la République tchèque incluent les transferts d'une unité à une autre au sein d'un hôpital, alors que les autres pays les excluent.

e) La moyenne comprend tous les pays pour lesquels des données récentes sont disponibles (2004+).

f) Exclut le Danemark, la Grèce, la Hongrie, la Nouvelle-Zélande, la Pologne et la Suisse.

Source : Eco-Santé OCDE 2007. 
Tableau A.4.9b. Taux de sorties de l'hôpital pour certaines maladies, pour 100000 habitants, 1995 à 2005

\begin{tabular}{|c|c|c|c|c|c|c|}
\hline & \multicolumn{2}{|c|}{ Système circulatoire } & \multicolumn{2}{|c|}{ Système respiratoire } & \multicolumn{2}{|c|}{ Système digestif } \\
\hline & 1995 & 2005 & 1995 & 2005 & 1995 & 2005 \\
\hline Allemagne & 2955 & 31262004 & 1223 & 12312004 & 1766 & 20802004 \\
\hline Australie & 1854 & 16882004 & 1512 & 13712004 & 1590 & 15132004 \\
\hline Autriche $^{a}$ & 3382 & 3698 & 1761 & 1796 & 2164 & 2440 \\
\hline Belgique & 2235 & 21792004 & 1319 & 12772004 & 1768 & 17042004 \\
\hline Canada & 1533 & 12242004 & 1072 & 7782004 & 1282 & 9312004 \\
\hline Corée & 4371994 & 1171 & 6331994 & 956 & 8951994 & 1196 \\
\hline Danemark $^{b}$ & . & 2161 & .. & 1438 & .. & 1372 \\
\hline Espagne & 1055 & 13592004 & 873 & 10532004 & 1152 & 12912004 \\
\hline États-Unis ${ }^{a}$ & 2121 & 21012004 & 1254 & 12132004 & 1138 & 12192004 \\
\hline Finlande $^{a}$ & 3891 & 3552 & 2387 & 1903 & 1817 & 1873 \\
\hline France $^{a}$ & 22861997 & 22512004 & 14961997 & 12422004 & 29971997 & 30802004 \\
\hline Grèce & 2010 & .. & 1169 & .. & 1561 & .. \\
\hline Hongrie $^{a}$ & .. & 4606 & . & 2193 & .. & 1996 \\
\hline Irlande & 1349 & 1224 & 1578 & 1341 & 1311 & 1200 \\
\hline Islande & 19021998 & 1830 & 12891998 & 983 & .. & 1351 \\
\hline Italie $^{a}$ & 23811996 & 25082004 & 11001996 & 11572004 & 17821996 & 14772004 \\
\hline Japon $^{c}$ & 12101996 & 1330 & 8941996 & 956 & 10781996 & 1069 \\
\hline Luxembourg & 22651998 & 2286 & 17791998 & 1443 & 16601998 & 1673 \\
\hline Mexique $^{b}$ & 182 & 211 & 244 & 250 & 369 & 471 \\
\hline Norvège & 2194 & 2467 & 1158 & 1531 & 1006 & 1238 \\
\hline Nouvelle-Zélande ${ }^{a}$ & & 1345 & .. & 1091 & . & 980 \\
\hline Pays-Bas & 1589 & 1528 & 704 & 731 & 890 & 916 \\
\hline Pologne ${ }^{b}$ & .. & 3024 & .. & 1558 & .. & 1766 \\
\hline Portugal & 941 & 1165 & 597 & 927 & 950 & 986 \\
\hline République slovaque & 2464 & 3054 & 1728 & 1660 & 2074 & 1889 \\
\hline République tchèque $^{C}$ & 3039 & 3712 & 1751 & 1559 & 2153 & 2214 \\
\hline Royaume-Uni ${ }^{a}$ & 1907 & 1886 & 1468 & 1458 & 2166 & 2481 \\
\hline Suède & 2994 & 2597 & 1292 & 1047 & 1380 & 1232 \\
\hline Suisse & . & 1680 & .. & 856 & 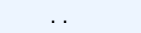 & 1323 \\
\hline Turquie & 695 & 12202004 & 688 & 11132004 & 605 & 8962004 \\
\hline Moyenne la plus récente ${ }^{d}$ & . & 2144 & . & 1245 & . & 1512 \\
\hline Moyenne cohérente (23) ${ }^{e}$ & 1955 & 2067 & 1240 & 1217 & 1478 & 1525 \\
\hline
\end{tabular}

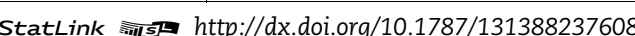

a) L'Autriche (avant 2003), les États-Unis, la Finlande, la France, la Hongrie (avant 2004), l'Italie (à partir de 2004) et le Royaume-Uni incluent les sorties le jour même, alors que celles-ci sont exclues dans la majorité des pays.

b) Les données fournies par le Danemark, le Mexique et la Pologne ne concernent que les hôpitaux publics.

c) Le Japon et la République tchèque incluent les transferts d'une unité à une autre au sein d'un hôpital, alors que les autres pays les excluent.

d) La moyenne comprend tous les pays pour lesquels des données récentes sont disponibles (2004+).

e) Exclut le Danemark, la Grèce, la Hongrie, l'Islande, la Nouvelle-Zélande, la Pologne et la Suisse.

Source: Eco-Santé OCDE 2007. 


\section{Tableau A.4.10a. Durée moyenne de séjour en soins aigus,} toutes causes confondues, en jours, 1990 à 2005

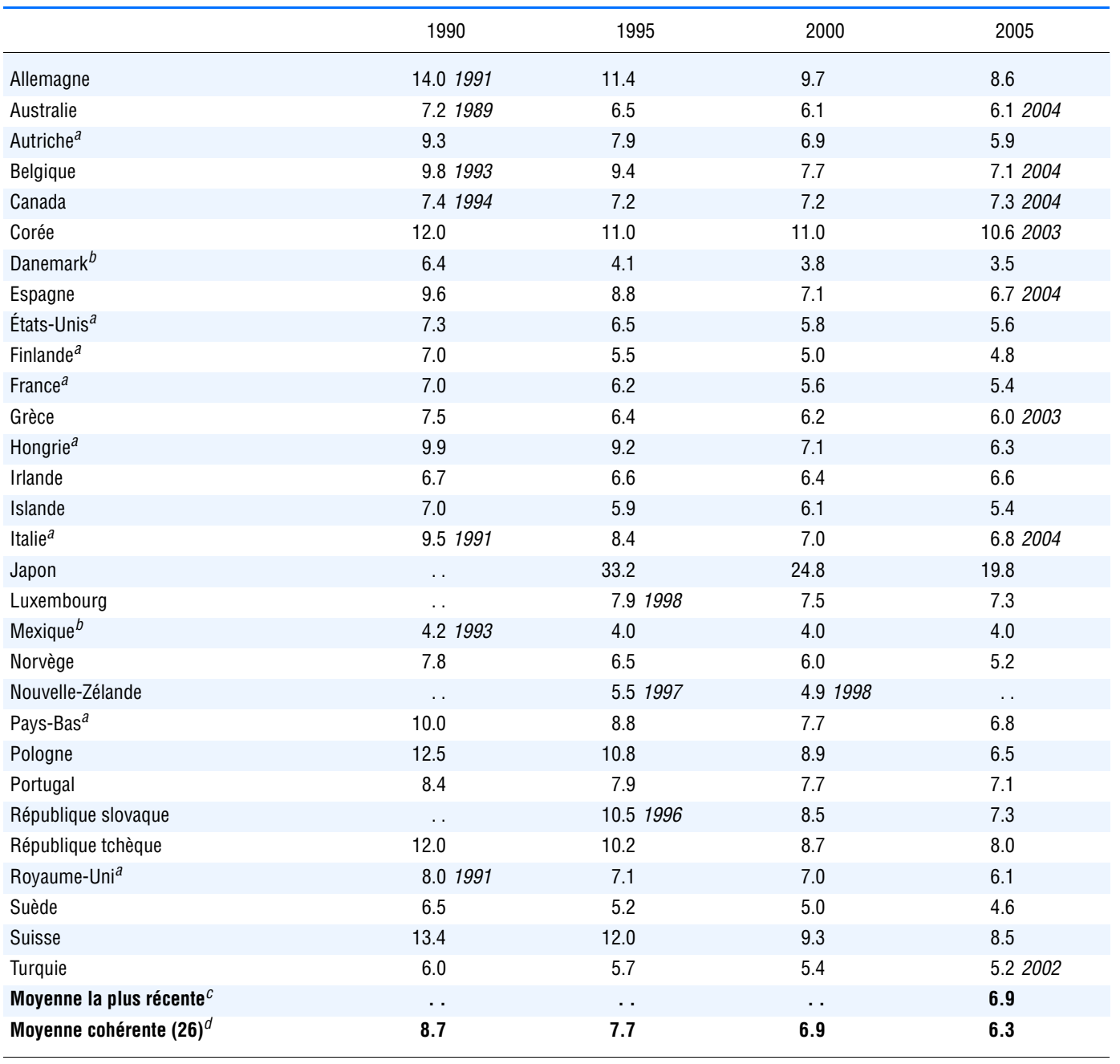

StatLink त्राजम http://dx.doi.org/10.1787/131407655668

a) Dans les données concernant les sorties de l'hôpital, l'Autriche (avant 2003), les États-Unis, la Finlande, la France, la Hongrie (avant 2004), l'Italie (après 2004) et le Royaume-Uni incluent les sorties le jour même, alors qu'elles sont exclues dans la majorité des pays.

b) Les données fournies par le Danemark et le Mexique ne concernent que les hôpitaux publics.

c) La moyenne comprend tous les pays pour lesquels des données récentes sont disponibles (2002+).

d) Exclut la Grèce, le Japon, le Luxembourg, la Nouvelle-Zélande et la République slovaque.

Source : Eco-Santé OCDE 2007. 
Tableau A.4.10b. Durée moyenne de séjour en hôpital pour certaines causes spécifiques, en jours, 1990 à 2005

\begin{tabular}{|c|c|c|c|c|c|c|c|c|}
\hline & \multicolumn{2}{|c|}{ Infarctus aigu du myocarde } & \multicolumn{2}{|c|}{ Accident vasculaire cérébral } & \multicolumn{2}{|c|}{ Pneumonie } & \multicolumn{2}{|c|}{ Accouchement normal } \\
\hline & 1995 & 2005 & 1995 & 2005 & 1995 & 2005 & 1995 & 2005 \\
\hline Allemagne & 17.0 & 9.72004 & 19.0 & 13.32004 & . . & 11.12004 & 5.8 & 3.72004 \\
\hline Australie & 7.7 & 6.32004 & 14.0 & 11.12004 & 7.8 & 6.72004 & 3.5 & 2.62004 \\
\hline Autriche & 16.4 & 8.4 & 17.7 & 14.4 & .. & 9.9 & 6.5 & 4.3 \\
\hline Belgique & 10.7 & 8.42004 & 19.6 & 14.32004 & .. & 11.82004 & 5.4 & 4.52004 \\
\hline Canada & 9.0 & 7.32004 & 20.8 & 14.82004 & 8.1 & 7.62004 & 2.2 & 1.92004 \\
\hline Corée & 12.61996 & 9.7 & 22.71996 & 32.5 & 7.81996 & 9.3 & 3.51995 & 2.9 \\
\hline Danemark $^{a}$ & .. & 5.3 & .. & 9.9 & .. & 7.0 & .. & 2.9 \\
\hline Espagne & 12.4 & 9.22004 & 17.1 & 12.12004 & 11.4 & 9.32004 & 3.6 & 2.62004 \\
\hline États-Unis & 6.6 & 5.82004 & 7.4 & 5.82004 & 6.7 & 5.52004 & 1.5 & 2.02004 \\
\hline Finlande & 17.7 & 12.3 & 43.1 & 37.8 & 36.61996 & 24.2 & 4.1 & 3.5 \\
\hline France & 8.11997 & 6.92004 & 11.71997 & 11.72004 & 10.21997 & 9.82004 & 5.31997 & 4.62004 \\
\hline Grèce & 8.0 & .. & 13.0 & . & .. & .. & 4.0 & 4.02004 \\
\hline Hongrie & 12.7 & 7.1 & .. & 8.1 & .. & 9.3 & 6.2 & 6.2 \\
\hline Irlande & 10.5 & 10.7 & 21.3 & 22.4 & 11.8 & 11.4 & 4.4 & 3.02004 \\
\hline Islande & 9.81994 & 7.3 & 11.81998 & 11.1 & .. & 8.7 & 4.61994 & 2.1 \\
\hline Italie & 13.61994 & 8.42004 & 16.11994 & 13.12004 & .. & 10.42004 & 5.31994 & 3.62004 \\
\hline Japon & $\ldots$ & $\ldots$ & $\ldots$ & .. & $\ldots$ & . & $\ldots$ & . \\
\hline Luxembourg & 10.71998 & 7.3 & 16.11998 & 13.3 & 10.01998 & 9.8 & 4.51998 & 4.2 \\
\hline Mexique $^{a}$ & 7.2 & 7.2 & 7.4 & 7.9 & 6.0 & 6.3 & 1.3 & 1.3 \\
\hline Norvège & 8.3 & 5.0 & 14.9 & 10.3 & . & 7.7 & 4.4 & 3.4 \\
\hline Nouvelle-Zélande & 8.1 & 6.0 & 32.71994 & 8.9 & .. & 4.9 & 2.8 & 2.1 \\
\hline Pays-Bas & . & 8.0 & 22.6 & 12.6 & .. & 10.8 & 3.2 & 2.2 \\
\hline Pologne $^{a}$ & .. & 7.7 & . & 12.8 & .. & 10.8 & . & 4.7 \\
\hline Portugal & 11.0 & 9.0 & 12.5 & 10.4 & 10.6 & 10.6 & 3.1 & 2.7 \\
\hline République slovaque & 15.5 & 8.1 & 16.3 & 11.5 & 13.6 & 10.2 & 7.8 & 5.7 \\
\hline République tchèque & 12.7 & 6.2 & 15.2 & 14.5 & 12.1 & 10.4 & 6.9 & 5.1 \\
\hline Royaume-Uni & 8.7 & 8.7 & 30.2 & 23.4 & .. & 11.6 & 2.4 & 1.5 \\
\hline Suède & 7.6 & 5.3 & 15.1 & 11.8 & 6.61998 & 6.4 & 3.1 & 2.4 \\
\hline Suisse & 12.8 & 8.3 & $\ldots$ & 17.4 & .. & 11.3 & 6.2 & 5.6 \\
\hline Turquie & $\ldots$ & .. & 8.3 & 7.62004 & . & 6.02004 & 1.9 & 1.72003 \\
\hline $\begin{array}{l}\text { Moyenne } \\
\text { la plus récente }\end{array}$ & . & 7.8 & . & 14.1 & . & 9.6 & .. & 3.3 \\
\hline Moyenne cohérente ${ }^{C}$ & 11.1 & 7.9 & 18.1 & 14.4 & 11.4 & 9.8 & 4.2 & 3.3 \\
\hline
\end{tabular}

a) Les données fournies par le Danemark, le Mexique et la Pologne ne concernent que les hôpitaux publics.

b) La moyenne comprend tous les pays pour lesquels des données récentes sont disponibles (2003+).

c) Inclut les pays pour lesquels des données sont disponibles pour 1995 et 2005.

Source: Eco-Santé OCDE 2007. 
Tableau A.4.11. Chirurgies cardio-vasculaires, pour 100000 habitants, 1990 à 2005

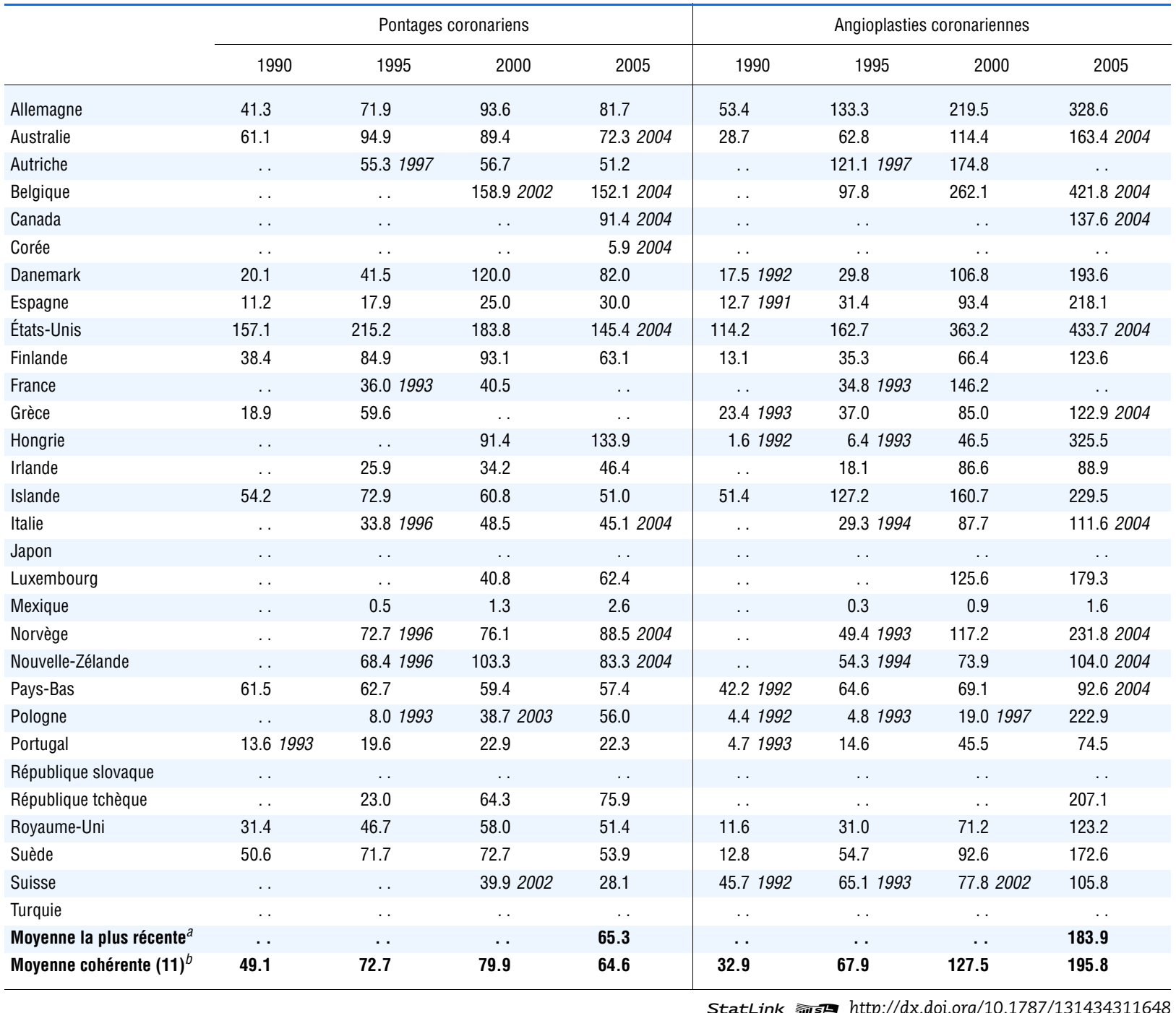

Note : Les données concernent uniquement le nombre d'actes sur patients hospitalisés. Elles n'incluent pas les angioplasties coronariennes effectuées en chirurgie de jour (qui représentent une part croissante de l'activité globale dans de nombreux pays).

a) La moyenne comprend tous les pays pour lesquels des données récentes sont disponibles (2002+).

b) Inclut l'Allemagne, l'Australie, le Danemark, l'Espagne, les États-Unis, la Finlande, l'Islande, les Pays-Bas, le Portugal, le Royaume-Uni et la Suède.

Source : Eco-Santé OCDE 2007. 
Tableau A.4.13. Césariennes pour 100 naissances vivantes, 1990 à 2005

\begin{tabular}{|c|c|c|c|c|}
\hline & 1990 & 1995 & 2000 & 2005 \\
\hline Allemagne & 15.7 & 17.2 & 20.9 & 26.7 \\
\hline Australie & 17.5 & 19.2 & 23.1 & 29.12004 \\
\hline Autriche & . & 12.4 & 17.2 & 24.4 \\
\hline Belgique & 10.5 & 13.5 & 16.3 & 17.82004 \\
\hline Canada & . & 17.5 & 20.9 & 25.32004 \\
\hline Corée & . & . & 39.62001 & 35.22004 \\
\hline Danemark & 12.4 & 12.5 & 14.7 & 19.4 \\
\hline Espagne & 14.2 & 18.8 & 21.5 & 23.62003 \\
\hline États-Unis & 22.7 & 20.8 & 22.9 & 29.12004 \\
\hline Finlande & 13.7 & 15.8 & 16.0 & 16.4 \\
\hline France & 13.9 & 15.21993 & 17.1 & . \\
\hline Grèce & . & . & . & . \\
\hline Hongrie & . & 13.6 & 20.1 & 29.1 \\
\hline Irlande & 10.5 & 13.4 & 20.7 & 24.52004 \\
\hline Islande & 11.8 & 14.1 & 17.7 & 15.6 \\
\hline Italie & 20.8 & 26.1 & 33.3 & 37.52004 \\
\hline Japon & . & . & . & . \\
\hline Luxembourg & 16.5 & 16.4 & 21.8 & 27.5 \\
\hline Mexique & $\ldots$ & 25.3 & 32.0 & 39.3 \\
\hline Norvège & 12.8 & 12.6 & 13.7 & 15.22004 \\
\hline Nouvelle-Zélande & 12.1 & 15.1 & 20.2 & 22.22004 \\
\hline Pays-Bas & 7.4 & 9.7 & 11.9 & 13.62004 \\
\hline Pologne & . & 15.2 & 16.11997 & . \\
\hline Portugal $^{a}$ & 19.51993 & 21.6 & 23.9 & 27.8 \\
\hline République slovaque & 8.7 & 11.5 & 14.7 & 20.7 \\
\hline République tchèque & 7.6 & 11.2 & 12.9 & 17.1 \\
\hline Royaume-Uni & 11.6 & 15.8 & 22.3 & 23.3 \\
\hline Suède & 10.8 & 12.0 & 15.2 & 17.22003 \\
\hline Suisse & 18.6 & $\cdots$ & 24.22002 & 26.7 \\
\hline Turquie & . & 13.61998 & 13.61998 & $\cdots$ \\
\hline Moyenne la plus récente ${ }^{b}$ & $\cdots$ & $\cdots$ & . & 24.2 \\
\hline Moyenne cohérente $(19)^{C}$ & 13.5 & 15.6 & 19.1 & 22.3 \\
\hline
\end{tabular}

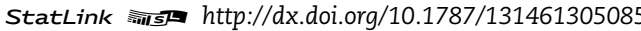

a) Au Portugal, seules les naissances ayant eu lieu dans les hôpitaux publics (sur le continent) sont inclus. Cela entraîne une surestimation des taux de césariennes.

b) La moyenne comprend tous les pays pour lesquels des données récentes sont disponibles (2002+).

c) Exclut l'Autriche, le Canada, la Corée, la France, la Grèce, la Hongrie, le Japon, le Mexique, la Pologne, la Suisse et la Turquie.

Source : Eco-Santé OCDE 2007 
Tableau A.4.14. Nombre de chirurgies de la cataracte avec hospitalisation et en ambulatoire, pour 100000 habitants, 1995 à 2005

\begin{tabular}{|c|c|c|c|c|c|c|c|c|c|}
\hline & \multicolumn{3}{|c|}{1995} & \multicolumn{3}{|c|}{2000} & \multicolumn{3}{|c|}{2005} \\
\hline & Hospitalisation & Ambulatoire & Total & Hospitalisation & Ambulatoire & Total & Hospitalisation & Ambulatoire & Total \\
\hline Allemagne & $\ldots$ & . & . . & .. & . & .. & . & .. & .. \\
\hline Australie & .. & .. & .. & 113.4 & 599.8 & 713.1 & 67.42004 & 787.82004 & 855.32004 \\
\hline Autriche & 465.41997 & . & .. & 571.8 & . & .. & 697.9 & . & .. \\
\hline Belgique & 552.6 & .. & .. & 359.4 & 888.4 & 1247.7 & 163.72004 & 1436.32004 & 1600.02004 \\
\hline Canada & 99.3 & . & . & 22.3 & 1414.4 & 1436.6 & 7.52004 & $\ldots$ & . \\
\hline Corée & .. & .. & .. & .. & .. & .. & .. & .. & 444.62004 \\
\hline Danemark & 133.11996 & 222.21996 & 355.31996 & 85.1 & 327.8 & 412.9 & 19.7 & 502.4 & 522.2 \\
\hline Espagne & 362.81997 & $\ldots$ & . & 235.0 & . & .. & 101.5 & 892.9 & 994.5 \\
\hline États-Unis & 6.8 & .. & .. & 2.7 & .. & .. & 3.32004 & .. & .. \\
\hline Finlande & 304.8 & 152.5 & 457.3 & 116.3 & 529.5 & 645.8 & 29.9 & 764.6 & 794.5 \\
\hline France & 388.21993 & 42.11993 & 430.31993 & 497.5 & 230.6 & 728.2 & . & $\ldots$ & .. \\
\hline Grèce & . & . & 333.51996 & . & .. & 611.9 & . & .. & 878.92003 \\
\hline Hongrie & $\ldots$ & .. & . & . & . & 698.7 & 827.3 & 2.9 & 830.3 \\
\hline Irlande & 316.3 & 38.7 & 355.0 & 303.6 & 128.9 & 432.5 & 111.4 & 139.6 & 251.1 \\
\hline Islande & . & . & .. & 54.41998 & 204.91998 & 259.31998 & .. & . & 740.2 \\
\hline Italie & 339.81996 & 20.51996 & 360.31996 & 415.9 & 250.6 & 666.5 & 164.32004 & 582.92004 & 747.22004 \\
\hline Japon & . & . & . & & . & . & 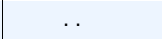 & . & . \\
\hline Luxembourg & $\ldots$ & . & 589.51996 & 505.8 & 209.9 & 715.8 & 635.2 & 314.1 & 949.2 \\
\hline Mexique & 2.5 & 24.0 & 26.4 & 7.2 & 30.7 & 37.8 & 17.5 & 37.3 & 54.7 \\
\hline Norvège & . & . & .. & 65.4 & 448.8 & 514.2 & 32.62004 & 455.12004 & 487.72004 \\
\hline Nouvelle-Zélande & . & .. & .. & 25.6 & 218.4 & 243.9 & 17.72004 & 188.82004 & 206.52004 \\
\hline Pays-Bas & 274.4 & 115.9 & 390.3 & 95.4 & 457.9 & 553.3 & 25.4 & 736.5 & 761.9 \\
\hline Pologne & . & . . & . & 212.12003 & . & .. & 304.4 & .. & . \\
\hline Portugal & 99.5 & 0.1 & 99.6 & 132.4 & 13.2 & 145.6 & 134.6 & 153.3 & 287.9 \\
\hline République slovaque & .. & .. & .. & . & .. & .. & .. & .. & .. \\
\hline République tchèque & & . & . & 331.5 & . & . & 410.6 & .. & . \\
\hline Royaume-Uni & 196.1 & 141.9 & 338.0 & 95.3 & 451.7 & 546.9 & 43.1 & 583.8 & 626.9 \\
\hline Suède & 33.51998 & .. & .. & 25.52001 & 614.92001 & 640.42001 & 22.92003 & 871.62003 & 894.52003 \\
\hline Suisse & . & . & . & 225.52002 & 251.42002 & 476.92002 & 112.2 & 317.0 & 429.1 \\
\hline Turquie & .. & .. & .. & . & .. & .. & . & .. & .. \\
\hline
\end{tabular}

StatLink न्ताst http://dx.doi.org/10.1787/131483788800

Note: Les variations entre pays doivent être interprétées avec précaution car ils enregistrent de façon différente les interventions chirurgicales de la cataracte et n'incluent pas toujours tous les établissements de santé (en particulier les hôpitaux privés et les activités effectuées dans le secteur ambulatoire) dans leurs données.

Source : Eco-Santé OCDE 2007. 
Tableau A.5.1a. Dépenses totales de santé par habitant, USD PPA, 1980 à 2005

\begin{tabular}{|c|c|c|c|c|c|c|c|c|}
\hline & 1980 & 1990 & 2000 & 2001 & 2002 & 2003 & 2004 & 2005 \\
\hline Allemagne & 950 & 19331992 & 2634 & 2754 & 2886 & 3129 & 3169 & 3287 \\
\hline Australie & 697 & 1307 & 2397 & 2541 & 2724 & 2892 & 3128 & $31282004-05$ \\
\hline Autriche & 769 & 1327 & 2825 & 2898 & 3018 & 3236 & 3418 & 3519 \\
\hline Belgique & 636 & 1341 & 2301 & 2452 & 2631 & 3080 & $3290 \mathrm{e}$ & $3389 e$ \\
\hline Canada & 780 & 1738 & 2509 & 2727 & 2867 & 3006 & 3161 & 3326 \\
\hline Corée & 1591983 & 356 & 780 & 932 & 977 & 1051 & 1138 & 1318 \\
\hline Danemark & 883 & 1521 & 2381 & 2561 & 2656 & 2793 & $2972 e$ & $3108 \mathrm{e}$ \\
\hline Espagne & 363 & 872 & 1520 & 1617 & 1723 & 1954 & $2099 \mathrm{e}$ & $2255 \mathrm{e}$ \\
\hline États-Unis & 1068 & 2738 & 4569 & 4917 & 5306 & 5684 & 6037 & 6401 \\
\hline Finlande & 582 & 1392 & 1717 & 1861 & 2012 & 2045 & 2202 & 2331 \\
\hline France & 677 & 1499 & 2487 & 2649 & 2795 & 3011 & 3191 & 3374 \\
\hline Grèce & 486 & 843 & 1950 & 2178 & 2364 & 2616 & 2669 & 2981 \\
\hline Hongrie & . & 6001991 & 857 & 977 & 1115 & $1291 \mathrm{e}$ & $1337 \mathrm{e}$ & $13372004 e$ \\
\hline Irlande & 519 & 796 & 1822 & 2151 & 2368 & 2536 & 2742 & 2926 \\
\hline Islande & 733 & 1619 & 2697 & 2775 & 3036 & 3161 & 3331 & 3443 \\
\hline Italie & . & 1380 & 2078 & 2188 & 2278 & 2281 & 2437 & 2532 \\
\hline Japon & 583 & 1121 & 1967 & 2080 & 2138 & $2243 e$ & $2358 \mathrm{e}$ & $23582004 e$ \\
\hline Luxembourg & 640 & 1532 & 2984 & 3270 & 3729 & 4727 & $5352 e$ & $53522004 e$ \\
\hline Mexique & .. & 306 & 506 & 548 & 578 & 608 & 655 & 675 \\
\hline Norvège & 676 & 1392 & 3082 & 3293 & 3616 & 3872 & 4103 & 4364 \\
\hline Nouvelle-Zélande & 509 & 991 & 1605 & 1709 & 1850 & 1911 & $2148 e$ & $2343 e$ \\
\hline Pays-Bas & 755 & 1434 & 2258 & 2525 & 2775 & $2910 \mathrm{e}$ & $3094 \mathrm{e}$ & $30942004 e$ \\
\hline Pologne & .. & 296 & 590 & 647 & 734 & 754 & 814 & $867 e$ \\
\hline Portugal & 292 & 673 & 1625 & 1685 & 1783 & $1832 \mathrm{e}$ & $1896 \mathrm{e}$ & $2033 e$ \\
\hline République slovaque & .. & .. & 595 & 642 & 716 & 798 & 1061 & 1137 \\
\hline République tchèque & . & 570 & 971 & 1055 & 1199 & 1353 & 1413 & 1479 \\
\hline Royaume-Uni & 482 & 989 & 1859 & 2034 & 2228 & 2328 & 2560 & 2724 \\
\hline Suède & 938 & 1581 & 2272 & 2409 & 2593 & 2760 & 2827 & 2918 \\
\hline Suisse & 1030 & 2028 & 3181 & 3371 & 3650 & 3861 & 4045 & 4177 \\
\hline Turquie & 76 & 168 & 451 & 461 & 484 & 514 & 562 & 586 \\
\hline Moyenne la plus récente ${ }^{a}$ & . & . & $\ldots$ & . & . & . & . & 2759 \\
\hline Moyenne cohérente $(24)^{b}$ & 637 & 1300 & 2245 & 2410 & 2592 & 2798 & 2979 & 3114 \\
\hline
\end{tabular}

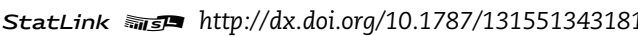

e : Estimation préliminaire.

a) La moyenne comprend les dernières données disponibles pour tous les pays de l'OCDE.

b) Exclut la Hongrie, l'Italie, le Mexique, la Pologne, la République slovaque et la République tchèque.

Source : Eco-Santé OCDE 2007. 
Tableau A.5.1b. Dépenses publiques de santé par habitant, USD PPA, 1980 à 2005

\begin{tabular}{|c|c|c|c|c|c|c|c|c|}
\hline & 1980 & 1990 & 2000 & 2001 & 2002 & 2003 & 2004 & 2005 \\
\hline Allemagne & 747 & 15751992 & 2098 & 2184 & 2286 & 2462 & 2437 & 2527 \\
\hline Australie & 439 & 877 & 1640 & 1715 & 1839 & 1952 & 2110 & $21102004-05$ \\
\hline Autriche & 529 & 976 & 2144 & 2195 & 2276 & 2438 & 2582 & 2665 \\
\hline Belgique & . & . & 1750 & 1878 & 1979 & 2205 & $2404 \mathrm{e}$ & $2451 e$ \\
\hline Canada & 590 & 1296 & 1766 & 1909 & 1994 & 2110 & 2220 & 2337 \\
\hline Corée & 411983 & 130 & 365 & 494 & 504 & 546 & 598 & 698 \\
\hline Danemark & 775 & 1258 & 1963 & 2117 & 2203 & 2353 & $2506 e$ & $2614 e$ \\
\hline Espagne & 290 & 687 & 1089 & 1151 & 1228 & 1373 & $1487 e$ & $1609 e$ \\
\hline États-Unis & 439 & 1080 & 1995 & 2191 & 2372 & 2528 & 2698 & 2884 \\
\hline Finlande & 460 & 1126 & 1290 & 1412 & 1536 & 1558 & 1700 & 1813 \\
\hline France & 542 & 1148 & 1948 & 2075 & 2197 & 2389 & 2534 & 2693 \\
\hline Grèce & 270 & 453 & 862 & 1032 & 1111 & 1214 & 1190 & 1277 \\
\hline Hongrie & . & 5341991 & 606 & 674 & 783 & $921 \mathrm{e}$ & $942 e$ & $9422004 e$ \\
\hline Irlande & 423 & 571 & 1329 & 1584 & 1791 & 1945 & 2143 & 2281 \\
\hline Islande & 647 & 1402 & 2211 & 2273 & 2512 & 2607 & 2746 & 2842 \\
\hline Italie & . & 1097 & 1507 & 1633 & 1697 & 1703 & 1847 & 1938 \\
\hline Japon & 416 & 870 & 1599 & 1699 & 1742 & $1828 \mathrm{e}$ & $1927 e$ & $19272004 e$ \\
\hline Luxembourg & 594 & 1426 & 2665 & 2874 & 3367 & 4281 & $4851 e$ & $48512004 e$ \\
\hline Mexique & . & 124 & 235 & 246 & 254 & 268 & 304 & 307 \\
\hline Norvège & 576 & 1153 & 2542 & 2752 & 3019 & 3241 & 3428 & 3647 \\
\hline Nouvelle-Zélande & 448 & 816 & 1252 & 1306 & 1441 & 1497 & $1665 e$ & $1829 e$ \\
\hline Pays-Bas & 523 & 962 & 1424 & 1586 & 1733 & 17332002 & 17332002 & 17332002 \\
\hline Pologne & $\cdots$ & 271 & 413 & 465 & 522 & 527 & 558 & $601 e$ \\
\hline Portugal & 188 & 441 & 1179 & 1205 & 1288 & $1344 \mathrm{e}$ & $1358 e$ & $1478 \mathrm{e}$ \\
\hline République slovaque & . & . & 532 & 574 & 638 & 705 & 782 & 846 \\
\hline République tchèque & . & 555 & 877 & 947 & 1085 & 1215 & 1259 & 1310 \\
\hline Royaume-Uni & 431 & 827 & 1503 & 1687 & 1857 & 1993 & 2209 & 2371 \\
\hline Suède & 868 & 1421 & 1929 & 2045 & 2207 & 2357 & 2391 & 2469 \\
\hline Suisse & $\cdots$ & 1062 & 1769 & 1926 & 2113 & 2258 & 2367 & 2493 \\
\hline Turquie & 22 & 102 & 284 & 314 & 341 & 368 & 406 & 418 \\
\hline Moyenne la plus récente ${ }^{a}$ & . & . & . & 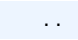 & . & . & . & 1999 \\
\hline Moyenne cohérente $(22)^{b}$ & 466 & 936 & 1594 & 1718 & 1857 & 2005 & 2133 & 2231 \\
\hline
\end{tabular}

StatLink त्ञाजम http://dx.doi.org/10.1787/131551343181

e : Estimation préliminaire.

a) La moyenne comprend les dernières données disponibles pour tous les pays de l'OCDE.

b) Exclut la Belgique, la Hongrie, l'Italie, la République tchèque, le Mexique, la Pologne, la République slovaque et la Suisse.

Source : Eco-Santé OCDE 2007. 


\section{Tableau A.5.1c. Croissance des dépenses totales et publiques de santé par habitant,} comparée à la croissance du PIB par habitant, en termes réels, 1995 à 2005

\begin{tabular}{|c|c|c|c|c|c|c|}
\hline & \multicolumn{2}{|c|}{ Dépenses totales de santé par habitant } & \multicolumn{2}{|c|}{ Dépenses publiques de santé par habitant } & \multicolumn{2}{|c|}{ PIB par habitant } \\
\hline & $\begin{array}{l}\text { Taux de croissance } \\
\text { annuel moyen }\end{array}$ & $\begin{array}{c}\text { Dépenses en termes } \\
\text { réels en } 2005 \\
1995=100\end{array}$ & $\begin{array}{l}\text { Taux de croissance } \\
\text { annuel moyen }\end{array}$ & $\begin{array}{c}\text { Dépenses en termes } \\
\text { réels en } 2005 \\
1995=100\end{array}$ & $\begin{array}{l}\text { Taux de croissance } \\
\text { annuel moyen }\end{array}$ & $\begin{array}{c}\text { PIB en termes réels } \\
\text { en } 2005 \\
1995=100\end{array}$ \\
\hline Allemagne & 1.8 & 119 & 1.2 & 113 & 1.2 & 113 \\
\hline Australie $^{b}(1995-2004)$ & 4.7 & 151 & 4.2 & 145 & 2.5 & 125 \\
\hline Autriche & 2.4 & 127 & 3.0 & 134 & 2.0 & 121 \\
\hline Belgique $^{b}$ & 3.2 & 136 & 2.8 & 132 & 1.7 & 119 \\
\hline Canada $^{b}$ & 3.2 & 136 & 3.0 & 134 & 2.3 & 126 \\
\hline Corée & 7.6 & 208 & 11.9 & 308 & 3.7 & 144 \\
\hline Danemark $^{b}$ & 2.8 & 132 & 2.9 & 133 & 1.7 & 119 \\
\hline Espagne $^{b}$ & 3.0 & 135 & 3.1 & 136 & 2.7 & 130 \\
\hline États-Unis & 3.6 & 143 & 3.6 & 142 & 2.2 & 124 \\
\hline Finlande & 3.5 & 141 & 3.8 & 145 & 3.4 & 139 \\
\hline France $^{b}$ & 2.3 & 126 & 2.4 & 127 & 1.6 & 118 \\
\hline Grèce $^{b}$ & 4.7 & 158 & 5.1 & 165 & 3.4 & 140 \\
\hline Hongrie $^{b}(1995-2004)$ & 4.9 & 153 & 3.6 & 137 & 4.4 & 148 \\
\hline Irlande & 7.2 & 200 & 8.0 & 217 & 6.0 & 178 \\
\hline Islande & 5.0 & 163 & 4.8 & 160 & 3.4 & 140 \\
\hline Italie & 3.2 & 136 & 4.0 & 148 & 1.1 & 111 \\
\hline Japon $^{b}(1995-2004)$ & 2.6 & 126 & 2.5 & 125 & 0.9 & 108 \\
\hline Luxembourg $^{b}(1995-2004)$ & 7.6 & 193 & 7.5 & 191 & 3.6 & 137 \\
\hline Mexique $^{b}$ & 3.6 & 142 & 4.7 & 158 & 2.1 & 123 \\
\hline Norvège $^{b}$ & 3.4 & 139 & 3.7 & 144 & 2.3 & 126 \\
\hline Nouvelle-Zélande & 4.3 & 152 & 4.4 & 154 & 1.9 & 121 \\
\hline Pays-Bas ${ }^{a, b}(1995-2004)$ & 3.0 & 131 & 2.1 & 115 & 2.1 & 122 \\
\hline Pologne ${ }^{b}$ & 5.2 & 166 & 4.8 & 160 & 4.3 & 153 \\
\hline Portugal $^{b}$ & 3.8 & 145 & 4.6 & 156 & 1.7 & 118 \\
\hline $\begin{array}{l}\text { République slovaque } \\
(1997-2005)\end{array}$ & 3.7 & 133 & 3.3 & 129 & 3.4 & 131 \\
\hline République tchèque $^{b}$ & 2.5 & 127 & 2.3 & 125 & 2.7 & 130 \\
\hline Royaume-Uni & 4.2 & 151 & 4.6 & 157 & 2.4 & 127 \\
\hline Suède & 3.8 & 145 & 3.5 & 142 & 2.6 & 129 \\
\hline Suisse & 2.8 & 132 & 3.9 & 147 & 1.0 & 110 \\
\hline Turquie (1999-2005) & 6.3 & 144 & 9.1 & 168 & 3.4 & 122 \\
\hline Moyenne & 4.0 & 146 & 4.3 & 152 & 2.6 & 128 \\
\hline
\end{tabular}

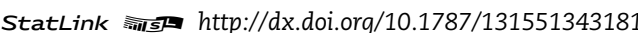

a) Les dépenses publiques pour les Pays-Bas correspondent à la période 1995-2002.

b) Les taux de croissance présentés dans les tableaux A.5.1c à A.5.1e ont été ajustés afin de prendre en compte les nombreuses ruptures de séries. Ces ruptures sont généralement le résultat de changements méthodologiques faisant suite à l'introduction du "Système des comptes de la santé » (annexe B). La révision de la frontière du secteur de la santé résulte de manière générale en un déplacement vers le haut du niveau des dépenses de santé. Pour essayer de supprimer cet effet, la croissance réelle de l'année de rupture a été calculée comme la croissance moyenne de l'année précédente et de l'année suivante.

Source : Eco-Santé OCDE 2007. 
Tableau A.5.1d. Taux de croissance annuel des dépenses totales de santé par habitant, en termes réels, 1995 à 2005

\begin{tabular}{|c|c|c|c|c|c|c|c|c|c|c|}
\hline & $1995-96$ & $1996-97$ & $1997-98$ & 1998-99 & $1999-2000$ & 2000-01 & 2001-02 & $2002-03$ & 2003-04 & 2004-05 \\
\hline Allemagne & 3.6 & 0.0 & 2.1 & 2.7 & 3.0 & 2.5 & 1.7 & 1.4 & -0.8 & 1.8 \\
\hline Australie & 4.8 & 4.3 & 4.9 & $5.3^{*}$ & 5.6 & 4.4 & 4.5 & 3.5 & 5.1 & .. \\
\hline Autriche & 2.5 & 3.8 & 5.6 & 2.8 & 1.0 & 1.2 & 2.0 & 1.9 & 2.6 & 0.8 \\
\hline Belgique & 3.3 & 1.3 & 3.0 & 5.9 & 3.9 & 1.7 & 3.7 & $4.0^{*}$ & 4.2 & 0.7 \\
\hline Canada & -1.8 & 2.8 & 6.4 & 2.8 & 3.4 & 6.1 & 5.0 & 2.6 & 2.3 & 2.1 \\
\hline Corée & 9.7 & 3.0 & -7.3 & 17.4 & 11.2 & 16.7 & 4.3 & 5.4 & 5.2 & 12.5 \\
\hline Danemark & 3.3 & 2.0 & 3.9 & 4.0 & 0.7 & 4.0 & 2.5 & $3.0^{*}$ & 3.5 & 1.4 \\
\hline Espagne & 2.4 & 1.8 & 3.9 & 4.2 & 2.7 & 2.9 & 1.4 & $2.9^{*}$ & 4.3 & 3.9 \\
\hline États-Unis & 2.1 & 2.2 & 3.0 & 3.4 & 3.6 & 5.1 & 6.0 & 4.9 & 3.3 & 2.9 \\
\hline Finlande & 5.3 & 0.9 & -0.7 & 2.2 & 1.9 & 4.6 & 6.0 & 5.7 & 4.1 & 5.1 \\
\hline France & 0.6 & 0.4 & 2.3 & 3.3 & 2.6 & 2.4 & 3.3 & $3.2^{*}$ & 3.0 & 2.0 \\
\hline Grèce & 1.2 & 1.6 & 1.9 & 6.0 & $8.0^{*}$ & 10.0 & 2.4 & 7.8 & 0.3 & 8.0 \\
\hline Hongrie & -2.2 & 1.2 & $3.1^{*}$ & 5.0 & 2.3 & 7.6 & 10.4 & 14.2 & 3.0 & .. \\
\hline Irlande & 4.7 & 8.7 & 4.4 & 10.1 & 8.5 & 15.4 & 6.9 & 5.0 & 5.0 & 3.6 \\
\hline Islande & 3.7 & 3.3 & 11.9 & 11.9 & 1.5 & 0.9 & 7.7 & 5.0 & 3.6 & 1.1 \\
\hline Italie & 2.3 & 5.5 & 2.2 & 2.7 & 7.0 & 3.4 & 1.7 & 0.1 & 5.1 & 1.8 \\
\hline Japon & 5.0 & 0.5 & 1.9 & 3.2 & 4.6 & 3.3 & 0.4 & 2.8 & 2.1 & .. \\
\hline Luxembourg & 2.4 & 3.5 & 6.5 & 9.9 & 7.9 & 10.3 & 9.3 & $9.4^{\star}$ & 9.4 & .. \\
\hline Mexique & -6.1 & 7.9 & $7.3^{*}$ & 6.7 & 4.4 & 5.8 & 2.7 & 3.0 & 4.8 & 0.0 \\
\hline Norvège & 3.9 & $8.2^{*}$ & 12.4 & 2.2 & -7.4 & 6.1 & 12.3 & 2.7 & -0.5 & -4.3 \\
\hline Nouvelle-Zélande & 1.2 & 3.2 & 5.5 & 3.0 & 2.7 & 4.4 & 7.6 & -0.2 & 10.2 & 5.5 \\
\hline Pays-Bas & 1.5 & 0.4 & $2.4^{\star}$ & 4.3 & 1.5 & 5.5 & 6.3 & 2.9 & 2.5 & .. \\
\hline Pologne & 13.9 & 2.1 & 10.6 & 1.4 & 1.4 & 7.4 & $4.9^{*}$ & 2.4 & 4.7 & 3.9 \\
\hline Portugal & 7.0 & 4.2 & 1.4 & 6.9 & $4.0^{*}$ & 1.0 & 2.0 & 6.2 & 1.5 & 4.0 \\
\hline République slovaque & .. & .. & 1.9 & 1.5 & -3.4 & 4.0 & 6.2 & 8.9 & $6.5^{\star}$ & 4.1 \\
\hline République tchèque & -0.2 & -1.3 & -1.2 & 0.4 & $2.8^{\star}$ & 5.1 & 8.2 & $5.0^{\star}$ & 1.8 & 4.4 \\
\hline Royaume-Uni & 3.0 & 0.5 & 3.6 & 6.6 & 5.2 & 5.4 & 4.2 & 3.7 & 6.2 & 4.1 \\
\hline Suède & 4.3 & 0.3 & 5.2 & 5.6 & 4.1 & 5.3 & 6.4 & 3.1 & 1.5 & 2.4 \\
\hline Suisse & 4.4 & 1.9 & 4.2 & 2.1 & 2.9 & 5.1 & 1.6 & 2.0 & 2.2 & 1.9 \\
\hline Turquie & .. & .. & . & . & 8.6 & 3.0 & 5.3 & 7.0 & 9.6 & 4.3 \\
\hline Moyenne & 3.1 & 2.6 & 3.9 & 4.9 & 3.5 & 5.4 & 4.9 & 4.3 & 3.9 & 3.1 \\
\hline
\end{tabular}

* Voir les notes du tableau A.5.1c.

StatLink 凋S $h t t p: / / d x . d o i . o r g / 10.1787 / 131551343181$

Source : Eco-Santé OCDE 2007. 
Tableau A.5.1e. Taux de croissance annuel des dépenses publiques de santé par habitant, en termes réels, 1995 à 2005

\begin{tabular}{|c|c|c|c|c|c|c|c|c|c|c|}
\hline & $1995-96$ & $1996-97$ & $1997-98$ & $1998-99$ & $1999-2000$ & $2000-01$ & 2001-02 & $2002-03$ & 2003-04 & 2004-05 \\
\hline Allemagne & 4.4 & -1.6 & 1.2 & 2.3 & 2.9 & 2.1 & 1.6 & 0.7 & -3.1 & 1.8 \\
\hline Australie & 4.0 & 6.8 & 4.2 & $3.7^{*}$ & 3.1 & 3.0 & 4.5 & 3.4 & 5.1 & .. \\
\hline Autriche & 1.0 & 11.2 & 5.9 & 3.3 & 0.7 & 1.0 & 1.5 & 1.8 & 2.9 & 1.0 \\
\hline Belgique & 4.5 & -2.0 & 2.3 & 5.6 & 3.8 & 2.4 & 1.8 & $4.1^{*}$ & 6.4 & -0.3 \\
\hline Canada & -2.5 & 1.9 & 7.1 & 1.8 & 3.9 & 5.6 & 4.3 & 3.6 & 2.3 & 2.2 \\
\hline Corée & 19.4 & 9.8 & 4.4 & 18.0 & 11.0 & 32.1 & 1.6 & 6.0 & 6.6 & 13.3 \\
\hline Danemark & 3.2 & 1.8 & 3.6 & 4.3 & 0.9 & 4.3 & 2.8 & $3.2^{*}$ & 3.6 & 1.1 \\
\hline Espagne & 2.7 & 2.0 & 3.5 & 3.9 & 2.1 & 2.3 & 1.5 & $3.4^{*}$ & 5.2 & 4.6 \\
\hline États-Unis & 2.2 & 1.5 & 0.6 & 2.2 & 4.0 & 7.2 & 6.4 & 4.4 & 3.8 & 3.7 \\
\hline Finlande & 5.6 & 1.3 & -0.4 & 0.9 & 1.7 & 5.7 & 6.6 & 5.5 & 5.5 & 5.8 \\
\hline France & 0.4 & 0.5 & 2.3 & 2.9 & 2.6 & 2.5 & 3.7 & $3.4^{*}$ & 3.0 & 2.6 \\
\hline Grèce & 3.0 & 1.4 & 0.4 & 8.8 & $13.4^{*}$ & 17.9 & 1.5 & 6.4 & -3.6 & 3.8 \\
\hline Hongrie & -5.0 & 0.8 & $1.3^{*}$ & 1.7 & 0.0 & 4.9 & 12.4 & 16.0 & 1.8 & . \\
\hline Irlande & 3.8 & 12.5 & 4.1 & 8.9 & 8.8 & 16.4 & 9.8 & 6.5 & 6.9 & 3.4 \\
\hline Islande & 2.8 & 1.8 & 10.7 & 14.5 & 0.2 & 0.8 & 8.8 & 4.7 & 3.6 & 1.2 \\
\hline Italie & 2.0 & 5.9 & 1.5 & 3.2 & 9.7 & 6.4 & 1.5 & 0.3 & 6.7 & 2.8 \\
\hline Japon & 4.7 & -1.0 & 1.0 & 3.6 & 4.9 & 3.8 & 0.1 & 2.8 & 2.5 & . \\
\hline Luxembourg & 2.8 & 3.1 & 6.4 & 6.8 & 7.3 & 8.5 & 12.3 & $10.9^{*}$ & 9.5 & . \\
\hline Mexique & -7.7 & 16.5 & $13.7^{\star}$ & 10.8 & 1.7 & 2.0 & 0.4 & 3.6 & 10.1 & -2.0 \\
\hline Norvège & 3.8 & $8.8^{\star}$ & 13.7 & 2.6 & -7.5 & 7.4 & 12.2 & 3.0 & -0.7 & -4.2 \\
\hline Nouvelle-Zélande & 0.6 & 4.0 & 5.1 & 3.6 & 3.4 & 2.3 & 9.6 & 0.4 & 9.1 & 6.2 \\
\hline Pays-Bas & -5.4 & 2.9 & $2.5^{*}$ & 2.0 & 2.1 & 5.0 & 5.7 & 2.9 & 2.5 & .. \\
\hline Pologne & 14.7 & 0.1 & 0.5 & 10.4 & -0.2 & 10.3 & $5.5^{\star}$ & 0.6 & 2.7 & 5.0 \\
\hline Portugal & 11.5 & 4.9 & 3.6 & 7.7 & $3.6^{\star}$ & -0.5 & 3.1 & 7.9 & -0.9 & 5.5 \\
\hline République slovaque & . & .. & 1.8 & -0.6 & -3.7 & 3.9 & 5.9 & 8.0 & $6.5^{\star}$ & 5.0 \\
\hline République tchèque & -0.4 & -1.7 & -1.0 & 0.4 & $2.5^{\star}$ & 4.5 & 9.0 & $5.1^{\star}$ & 1.1 & 3.8 \\
\hline Royaume-Uni & 1.9 & -2.6 & 3.6 & 7.0 & 5.6 & 8.1 & 4.6 & 6.5 & 7.1 & 5.0 \\
\hline Suède & 4.6 & -1.0 & 5.1 & 5.5 & 3.1 & 5.3 & 6.7 & 3.4 & 0.5 & 2.4 \\
\hline Suisse & 6.2 & 2.8 & 3.6 & 2.9 & 3.4 & 7.9 & 3.0 & 3.0 & 2.3 & 4.0 \\
\hline Turquie & . & . & . & .. & 11.8 & 11.7 & 8.7 & 8.8 & 10.6 & 3.1 \\
\hline Moyenne & 3.2 & 3.3 & 3.9 & 5.1 & 3.6 & 6.5 & 5.2 & 4.7 & 4.0 & 3.2 \\
\hline
\end{tabular}

* Voir les notes du tableau A.5.1c.

StatLink न्ता St http://dx.doi.org/10.1787/131551343181

Source : Eco-Santé OCDE 2007. 
Tableau A.5.2a. Dépenses de santé totales en pourcentage du PIB, 1980 à 2005

\begin{tabular}{|c|c|c|c|c|c|c|c|c|}
\hline & 1980 & 1990 & 2000 & 2001 & 2002 & 2003 & 2004 & 2005 \\
\hline Allemagne & 8.4 & 9.61992 & 10.3 & 10.4 & 10.6 & 10.8 & 10.6 & 10.7 \\
\hline Australie & 6.8 & 7.5 & 8.8 & 8.9 & 9.1 & 9.2 & 9.5 & $9.52004-05$ \\
\hline Autriche & 7.5 & 7.0 & 10.0 & 10.0 & 10.1 & 10.2 & 10.3 & 10.2 \\
\hline Belgique & 6.3 & 7.2 & 8.6 & 8.7 & 9.0 & 10.1 & $10.2 \mathrm{e}$ & $10.3 e$ \\
\hline Canada & 7.0 & 8.9 & 8.8 & 9.3 & 9.6 & 9.8 & 9.8 & 9.8 \\
\hline Corée & 4.11983 & 4.3 & 4.8 & 5.4 & 5.3 & 5.4 & 5.5 & 6.0 \\
\hline Danemark & 8.9 & 8.3 & 8.3 & 8.6 & 8.8 & 9.1 & $9.2 \mathrm{e}$ & $9.1 \mathrm{e}$ \\
\hline Espagne & 5.3 & 6.5 & 7.2 & 7.2 & 7.3 & 7.9 & $8.1 \mathrm{e}$ & $8.2 \mathrm{e}$ \\
\hline États-Unis & 8.8 & 11.9 & 13.2 & 13.9 & 14.7 & 15.2 & 15.2 & 15.3 \\
\hline Finlande & 6.3 & 7.7 & 6.6 & 6.7 & 7.0 & 7.3 & 7.4 & 7.5 \\
\hline France & 7.0 & 8.4 & 9.6 & 9.7 & 10.0 & 10.9 & 11.0 & 11.1 \\
\hline Grèce & 5.1 & 5.8 & 9.3 & 9.8 & 9.7 & 10.0 & 9.6 & 10.1 \\
\hline Hongrie & .. & 7.01991 & 6.9 & 7.2 & 7.6 & $8.3 \mathrm{e}$ & $8.1 \mathrm{e}$ & $8.12004 e$ \\
\hline Irlande & 8.3 & 6.1 & 6.3 & 7.0 & 7.2 & 7.3 & 7.5 & 7.5 \\
\hline Islande & 6.3 & 7.8 & 9.3 & 9.2 & 10.0 & 10.3 & 10.0 & 9.5 \\
\hline Italie & . & 7.7 & 8.1 & 8.2 & 8.3 & 8.3 & 8.7 & 8.9 \\
\hline Japon & 6.5 & 6.0 & 7.7 & 7.9 & 8.0 & $8.1 \mathrm{e}$ & $8.0 \mathrm{e}$ & $8.02004 e$ \\
\hline Luxembourg & 5.2 & 5.4 & 5.8 & 6.4 & 6.8 & 7.8 & $8.3 \mathrm{e}$ & $8.32004 e$ \\
\hline Mexique & . & 4.8 & 5.6 & 6.0 & 6.2 & 6.3 & 6.5 & 6.4 \\
\hline Norvège & 7.0 & 7.6 & 8.4 & 8.8 & 9.8 & 10.0 & 9.7 & 9.1 \\
\hline Nouvelle-Zélande & 5.9 & 6.9 & 7.7 & 7.8 & 8.2 & 8.0 & $8.6 \mathrm{e}$ & $9.0 \mathrm{e}$ \\
\hline Pays-Bas & 7.5 & 8.0 & 8.0 & 8.3 & 8.9 & $9.1 \mathrm{e}$ & $9.2 \mathrm{e}$ & $9.22004 e$ \\
\hline Pologne & . & 4.8 & 5.5 & 5.9 & 6.3 & 6.2 & 6.2 & $6.2 \mathrm{e}$ \\
\hline Portugal & 5.3 & 5.9 & 8.8 & 8.8 & 9.0 & $9.7 \mathrm{e}$ & $9.8 \mathrm{e}$ & $10.2 \mathrm{e}$ \\
\hline République slovaque & . & . & 5.5 & 5.5 & 5.6 & 5.9 & 7.2 & 7.1 \\
\hline République tchèque & . & 4.7 & 6.5 & 6.7 & 7.1 & 7.4 & 7.3 & 7.2 \\
\hline Royaume-Uni & 5.6 & 6.0 & 7.3 & 7.5 & 7.7 & 7.8 & 8.1 & 8.3 \\
\hline Suède & 9.0 & 8.3 & 8.4 & 8.7 & 9.1 & 9.3 & 9.1 & 9.1 \\
\hline Suisse & 7.4 & 8.3 & 10.4 & 10.9 & 11.1 & 11.5 & 11.5 & 11.6 \\
\hline Turquie & 3.3 & 3.6 & 6.6 & 7.5 & 7.4 & 7.6 & 7.7 & 7.6 \\
\hline Moyenne la plus récente ${ }^{a}$ & . & . & . & . & . & . & . & 9.0 \\
\hline Moyenne cohérente $(24)^{b}$ & 6.6 & 7.2 & 8.3 & 8.6 & 8.9 & 9.3 & 9.3 & 9.4 \\
\hline
\end{tabular}

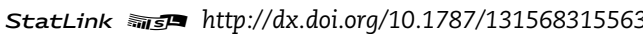

e : Estimation préliminaire.

a) La moyenne comprend les dernières données disponibles pour tous les pays de l'OCDE.

b) Exclut la Hongrie, l'Italie, le Mexique, le Pologne, la République slovaque et la République tchèque.

Source : Eco-Santé OCDE 2007. 
Tableau A.5.2b. Dépenses publiques de santé en pourcentage du PIB, 1980 à 2005

\begin{tabular}{|c|c|c|c|c|c|c|c|c|}
\hline & 1980 & 1990 & 2000 & 2001 & 2002 & 2003 & 2004 & 2005 \\
\hline Allemagne & 6.6 & 7.81992 & 8.2 & 8.3 & 8.4 & 8.5 & 8.1 & 8.2 \\
\hline Australie & 4.3 & 5.1 & 6.0 & 6.0 & 6.2 & 6.2 & 6.4 & $6.42004-05$ \\
\hline Autriche & 5.1 & 5.1 & 7.6 & 7.6 & 7.6 & 7.7 & 7.8 & 7.7 \\
\hline Belgique & .. & .. & 6.6 & 6.7 & 6.7 & 7.2 & $7.5 \mathrm{e}$ & $7.4 \mathrm{e}$ \\
\hline Canada & 5.3 & 6.6 & 6.2 & 6.5 & 6.7 & 6.8 & 6.8 & 6.9 \\
\hline Corée & 1.11983 & 1.6 & 2.2 & 2.9 & 2.7 & 2.8 & 2.9 & 3.2 \\
\hline Danemark & 7.9 & 6.9 & 6.8 & 7.1 & 7.3 & 7.7 & $7.8 \mathrm{e}$ & $7.7 \mathrm{e}$ \\
\hline Espagne & 4.2 & 5.1 & 5.2 & 5.2 & 5.2 & 5.5 & $5.7 \mathrm{e}$ & $5.9 e$ \\
\hline États-Unis & 3.6 & 4.7 & 5.8 & 6.2 & 6.6 & 6.7 & 6.8 & 6.9 \\
\hline Finlande & 5.0 & 6.2 & 4.9 & 5.1 & 5.4 & 5.6 & 5.7 & 5.9 \\
\hline France & 5.6 & 6.4 & 7.5 & 7.6 & 7.9 & 8.6 & 8.7 & 8.9 \\
\hline Grèce & 2.8 & 3.1 & 4.1 & 4.7 & 4.6 & 4.7 & 4.3 & 4.3 \\
\hline Hongrie & .. & 6.31991 & 4.9 & 4.9 & 5.3 & $5.9 \mathrm{e}$ & $5.7 \mathrm{e}$ & $5.72004 e$ \\
\hline Irlande & 6.8 & 4.4 & 4.6 & 5.1 & 5.4 & 5.6 & 5.8 & 5.8 \\
\hline Islande & 5.5 & 6.8 & 7.6 & 7.5 & 8.3 & 8.5 & 8.3 & 7.9 \\
\hline Italie & . & 6.1 & 5.8 & 6.1 & 6.2 & 6.2 & 6.6 & 6.8 \\
\hline Japon & 4.7 & 4.6 & 6.2 & 6.5 & 6.5 & $6.6 \mathrm{e}$ & $6.6 \mathrm{e}$ & $6.62004 e$ \\
\hline Luxembourg & 4.8 & 5.0 & 5.2 & 5.6 & 6.1 & 7.0 & $7.5 \mathrm{e}$ & $7.52004 e$ \\
\hline Mexique & . & 2.0 & 2.6 & 2.7 & 2.7 & 2.8 & 3.0 & 2.9 \\
\hline Norvège & 5.9 & 6.3 & 6.9 & 7.4 & 8.2 & 8.4 & 8.1 & 7.6 \\
\hline Nouvelle-Zélande & 5.1 & 5.7 & 6.0 & 6.0 & 6.4 & 6.3 & $6.7 \mathrm{e}$ & $7.0 \mathrm{e}$ \\
\hline Pays-Bas & 5.2 & 5.4 & 5.0 & 5.2 & 5.5 & 5.52002 & 5.52002 & 5.52002 \\
\hline Pologne & . & 4.4 & 3.9 & 4.2 & 4.5 & 4.4 & 4.3 & $4.3 e$ \\
\hline Portugal & 3.4 & 3.8 & 6.4 & 6.3 & 6.5 & $7.1 \mathrm{e}$ & $7.0 \mathrm{e}$ & $7.4 \mathrm{e}$ \\
\hline République slovaque & . & . & 4.9 & 4.9 & 5.0 & 5.2 & 5.3 & 5.3 \\
\hline République tchèque & . & 4.6 & 5.9 & 6.0 & 6.4 & 6.7 & 6.5 & 6.4 \\
\hline Royaume-Uni & 5.0 & 5.0 & 5.9 & 6.2 & 6.4 & 6.7 & 6.9 & 7.2 \\
\hline Suède & 8.3 & 7.5 & 7.1 & 7.4 & 7.8 & 7.9 & 7.7 & 7.7 \\
\hline Suisse & . & 4.3 & 5.8 & 6.2 & 6.5 & 6.7 & 6.8 & 6.9 \\
\hline Turquie & 1.0 & 2.2 & 4.2 & 5.1 & 5.2 & 5.4 & 5.6 & 5.4 \\
\hline Moyenne la plus récente ${ }^{a}$ & . & . & . & . & . & .. & . & 6.4 \\
\hline Moyenne cohérente $(22)^{b}$ & 4.9 & 5.2 & 5.9 & 6.2 & 6.4 & 6.6 & 6.7 & 6.7 \\
\hline
\end{tabular}

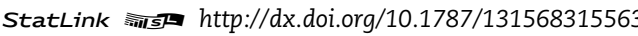

e : Estimation préliminaire.

a) La moyenne comprend les dernières données disponibles pour tous les pays de l'OCDE.

b) Exclut la Belgique, la Hongrie, l'Italie, le Mexique, la Pologne, la République slovaque, la République tchèque et la Suisse.

Source : Eco-Santé OCDE 2007. 
Tableau A.5.3. Dépenses courantes de santé par fonction, 2005

\begin{tabular}{|c|c|c|c|c|c|c|c|c|}
\hline & \multirow{2}{*}{$\begin{array}{c}\text { Services } \\
\text { de santé } \\
\text { individuels }\end{array}$} & \multicolumn{3}{|c|}{ dont: } & \multirow{2}{*}{$\begin{array}{c}\text { Biens } \\
\text { médicaux }\end{array}$} & \multirow{2}{*}{$\begin{array}{c}\text { Santé } \\
\text { collective }\end{array}$} & \multicolumn{2}{|c|}{ dont: } \\
\hline & & $\begin{array}{l}\text { Soins curatifs } \\
\text { et réadaptation }\end{array}$ & $\begin{array}{c}\text { Soins } \\
\text { de longue durée }\end{array}$ & $\begin{array}{l}\text { Services } \\
\text { auxiliaires }\end{array}$ & & & $\begin{array}{c}\text { Prévention } \\
\text { et santé publique }\end{array}$ & $\begin{array}{l}\text { Administration } \\
\text { et assurances }\end{array}$ \\
\hline Allemagne & 71 & 54 & 12 & 5 & 20 & 9 & 3 & 6 \\
\hline Australie (2004-05) & 77 & 64 & 7 & 5 & 18 & 4 & 2 & 3 \\
\hline Autriche & 78 & 63 & 13 & 2 & 16 & 6 & 2 & 4 \\
\hline Belgique & 72 & 53 & 15 & 4 & 19 & 8 & 2 & 6 \\
\hline Canada $^{a}$ & 68 & 47 & 14 & 6 & 21 & 11 & 6 & 4 \\
\hline Corée & 63 & 63 & 1 & 0 & 31 & 6 & 2 & 4 \\
\hline Danemark & 82 & 57 & 22 & 3 & 14 & 4 & 2 & 2 \\
\hline Espagne & 69 & 58 & 7 & 4 & 26 & 5 & 2 & 3 \\
\hline États-Unis & 75 & 68 & 7 & 0 & 14 & 11 & 4 & 8 \\
\hline Finlande $^{b}$ & 72 & 65 & 6 & 0 & 20 & 6 & 4 & 2 \\
\hline France & 69 & 57 & 9 & 4 & 22 & 9 & 2 & 7 \\
\hline Grèce & .. & .. & .. & .. & . & .. & .. & .. \\
\hline Hongrie (2004) & 58 & 50 & 4 & 4 & 35 & 7 & 5 & 1 \\
\hline Irlande & .. & . & $\ldots$ & .. & . & . & . & . \\
\hline Islande & 82 & 65 & 17 & 0 & 16 & 2 & 1 & 2 \\
\hline Italie & 78 & .. & .. & . & 21 & 1 & 1 & 0 \\
\hline Japon (2004) & 75 & 57 & 18 & 1 & 21 & 4 & 2 & 2 \\
\hline Luxembourg (2004) & 79 & 56 & 17 & 6 & 12 & 10 & 1 & 9 \\
\hline Mexique $^{a}$ & 62 & .. & .. & . & 22 & 14 & 3 & 11 \\
\hline Norvège & 83 & 50 & 26 & 7 & 14 & 3 & 2 & 1 \\
\hline Nouvelle-Zélande & 77 & 55 & 15 & 7 & 13 & 10 & 6 & 4 \\
\hline Pays-Bas & 73 & 57 & 14 & 2 & 18 & 9 & 5 & 5 \\
\hline Pologne & 64 & 53 & 7 & 4 & 32 & 4 & 2 & 2 \\
\hline Portugal & 72 & 61 & 1 & 10 & 25 & 3 & 2 & 1 \\
\hline République slovaque & 52 & 46 & 1 & 6 & 41 & 6 & 2 & 4 \\
\hline République tchèque & 65 & 49 & 3 & 12 & 30 & 5 & 2 & 3 \\
\hline Royaume-Uni & . & . & . & .. & . & .. & .. & .. \\
\hline Suède & 83 & . & . & . & 15 & 1 & & 1 \\
\hline Suisse & 80 & 57 & 20 & 3 & 13 & 7 & 2 & 5 \\
\hline Turquie & .. & .. & .. & .. & .. & . & .. & .. \\
\hline Moyenne cohérente $(23)^{C}$ & 72 & 57 & 11 & 4 & 21 & 7 & 3 & 4 \\
\hline
\end{tabular}

StatLink 角实 $h t t p: / / d x . d o i . o r g / 10.1787 / 131571037623$

a) Les dépenses courantes pour le Canada et le Mexique incluent aussi des dépenses non allouées.

b) Les dépenses courantes pour la Finlande incluent aussi des dépenses de santé environnementale.

c) Exclut la Grèce, l'Irlande, l'Italie, le Mexique, le Royaume-Uni, la Suède et la Turquie.

Source: Eco-Santé OCDE 2007. 
Tableau A.5.4a. Dépenses de produits pharmaceutiques par habitant, 2005, et taux de croissance annuel moyen par habitant, en termes réels, 1995 à 2005

\begin{tabular}{|c|c|c|c|c|c|c|}
\hline & \multicolumn{3}{|c|}{ Par habitant (USD PPA), 2005} & \multicolumn{2}{|c|}{$\begin{array}{l}\text { Taux de croissance annuel moyen } \\
\text { par habitant, en termes réels, 1995-2005 }\end{array}$} & \multirow{2}{*}{$\begin{array}{c}\text { Dépenses } \\
\text { pharmaceutiques, } \\
\text { en termes réels } \\
1995=100\end{array}$} \\
\hline & Totales & Publiques & Privées & $\begin{array}{c}\text { Dépenses } \\
\text { pharmaceutiques }\end{array}$ & $\begin{array}{l}\text { Dépenses totales } \\
\text { de santé }\end{array}$ & \\
\hline Allemagne & 498 & 365 & 133 & 3.5 & 1.8 & 141 \\
\hline Australie (2004-05) & 415 & 239 & 176 & 6.4 & 4.7 1995-2004 & 175 \\
\hline Autriche & 409 & 297 & 111 & 4.9 & 2.4 & 161 \\
\hline Belgique & .. & .. & .. & .. & .. & .. \\
\hline Canada & 589 & 228 & 361 & 5.8 & 3.2 & 175 \\
\hline Corée & 360 & 181 & 179 & 5.4 & 7.6 & 169 \\
\hline Danemark & 276 & 154 & 122 & 2.7 & 2.8 & 130 \\
\hline Espagne & 517 & 375 & 142 & 5.5 & 3.0 & 172 \\
\hline États-Unis & 792 & 191 & 601 & 7.1 & 3.6 & 199 \\
\hline Finlande & 380 & 214 & 166 & 5.0 & 3.5 & 163 \\
\hline France & 554 & 382 & 172 & 3.1 & 2.3 & 136 \\
\hline Grèce & .. & .. & .. & .. & .. & .. \\
\hline Hongrie (2004) & 390 & 244 & 145 & 7.5 & $4.91995-2004$ & 192 \\
\hline Irlande & 320 & 281 & 39 & 7.6 & 7.2 & 209 \\
\hline Islande & 458 & 273 & 186 & 4.9 & 5.0 & 161 \\
\hline Italie & 509 & 255 & 254 & 2.9 & 3.2 & 132 \\
\hline Japon (2004) & 449 & 311 & 138 & 0.8 & $2.61995-2004$ & 108 \\
\hline Luxembourg (2003) & 465 & 390 & 74 & 4.8 & 7.4 1995-2003 & 140 \\
\hline Mexique & 144 & 16 & 128 & 5.8 & 3.4 1999-2005 & 140 \\
\hline Norvège & 398 & 232 & 165 & 4.0 & 3.4 & 147 \\
\hline Nouvelle-Zélande & 290 & 192 & 99 & 2.5 & 4.3 & 128 \\
\hline Pays-Bas (2002) & 318 & 182 & 136 & 4.1 & 3.1 1995-2002 & 132 \\
\hline Pologne & 243 & 92 & 151 & .. & . & . \\
\hline Portugal & 445 & 262 & 183 & 3.7 & 3.8 & 144 \\
\hline République slovaque & 362 & 266 & 96 & 6.6 & 4.3 1999-2005 & 147 \\
\hline République tchèque & 372 & 281 & 91 & 2.9 & 2.5 & 134 \\
\hline Royaume-Uni & . & . & . & . & .. & .. \\
\hline Suède & 351 & 243 & 109 & 3.6 & 3.8 & 142 \\
\hline Suisse & 436 & 295 & 141 & 3.3 & 2.8 & 138 \\
\hline Turquie & .. & . & . & . & . & . \\
\hline Moyenne la plus récente ${ }^{a}$ & 413 & 248 & 165 & . & . & . \\
\hline Moyenne cohérente (25) ${ }^{b}$ & 420 & 254 & 166 & 4.6 & 3.9 & 153 \\
\hline
\end{tabular}

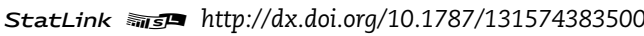

a) La moyenne comprend tous les pays pour lesquels des données récentes sont disponibles.

b) Exclut la Belgique, la Grèce, la Pologne, le Royaume-Uni et la Turquie.

Source : Eco-Santé OCDE 2007. 
Tableau A.5.4b. Dépenses pharmaceutiques en pourcentage des dépenses totales de santé et du PIB, 1980 à 2005

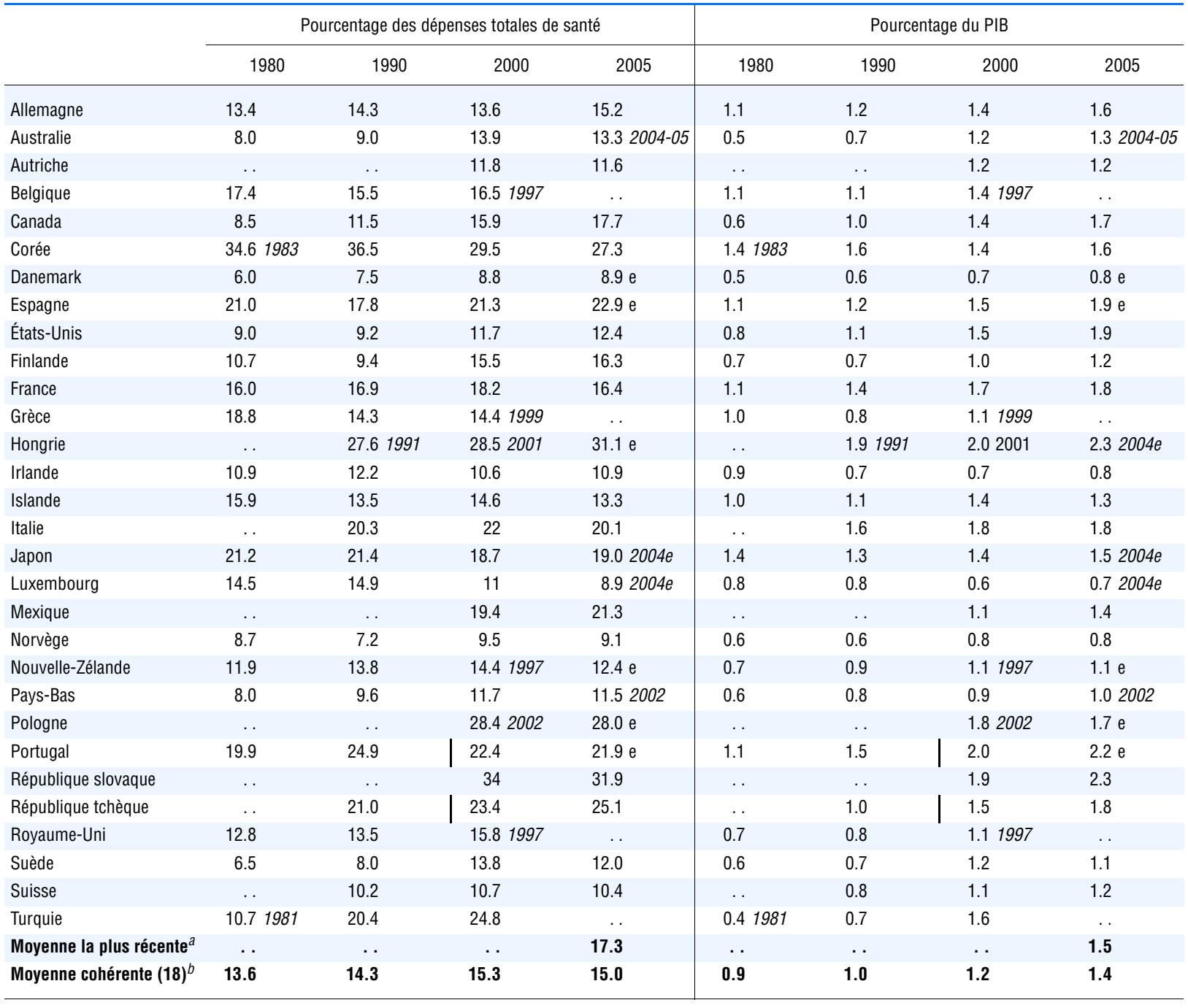

StatLink 䨛 $h$ http://dx.doi.org/10.1787/131574383500

a) La moyenne comprend tous les pays pour lesquels des données récentes sont disponibles.

b) Exclut l'Autriche, la Belgique, la Grèce, la Hongrie, l'Italie, le Mexique, la Pologne, la République tchèque, la République slovaque, le Royaume-Uni, la Suisse et la Turquie.

Source : Eco-Santé OCDE 2007. 
Tableau A.5.5. Dépenses de santé par source de financement, 2005

Dépenses totales de santé $=100$

\begin{tabular}{|c|c|c|c|c|c|c|c|}
\hline & \multirow{2}{*}{$\begin{array}{c}\text { Dépenses } \\
\text { publiques totales }\end{array}$} & \multicolumn{2}{|c|}{ Dont: } & \multirow{2}{*}{$\begin{array}{c}\text { Dépenses } \\
\text { privées totales }\end{array}$} & \multicolumn{3}{|c|}{ Dont : } \\
\hline & & $\begin{array}{c}\text { Administrations } \\
\text { publiques }\end{array}$ & $\begin{array}{l}\text { Sécurité } \\
\text { sociale }\end{array}$ & & $\begin{array}{l}\text { Assurance } \\
\text { privée }\end{array}$ & $\begin{array}{l}\text { Versements nets } \\
\text { des ménages }\end{array}$ & $\begin{array}{l}\text { Autres sources } \\
\text { de financement }\end{array}$ \\
\hline Allemagne & 77 & 10 & 67 & 23 & 9 & 13 & 1 \\
\hline Australie (2004-05) & 67 & 67 & 0 & 33 & 7 & 20 & 6 \\
\hline Autriche & 76 & 30 & 46 & 24 & 5 & 16 & 3 \\
\hline Belgique $^{a}$ & 71 & 4 & 66 & 29 & 5 & 22 & 1 \\
\hline Canada & 70 & 69 & 1 & 30 & 13 & 15 & 2 \\
\hline Corée & 53 & 12 & 41 & 47 & 3 & 38 & 6 \\
\hline Danemark & 84 & 84 & 0 & 16 & 2 & 14 & 0 \\
\hline Espagne & 71 & 66 & 5 & 29 & 6 & 22 & 1 \\
\hline États-Unis & 45 & 32 & 13 & 55 & 37 & 13 & 5 \\
\hline Finlande & 78 & 61 & 17 & 22 & 2 & 18 & 2 \\
\hline France & 80 & 5 & 75 & 20 & 13 & 7 & 1 \\
\hline Grèce $^{b}$ & 43 & 43 & 0 & 57 & & 57 & 0 \\
\hline Hongrie (2004) & 71 & 11 & 60 & 29 & 1 & 24 & 4 \\
\hline Irlande & 78 & 77 & 1 & 22 & 7 & 13 & 2 \\
\hline Islande ${ }^{b}$ & 83 & 49 & 34 & 17 & & 17 & 0 \\
\hline Italie & 77 & 76 & 0 & 23 & 1 & 20 & 2 \\
\hline Japon $(2004)^{b}$ & 82 & 16 & 66 & 18 & & 17 & 1 \\
\hline Luxembourg $^{c}(2004)$ & 91 & 17 & 73 & 9 & 1 & 7 & 1 \\
\hline Mexique & 45 & 17 & 28 & 55 & 3 & 51 & 0 \\
\hline Norvège & 84 & 69 & 15 & 16 & 0 & 16 & 1 \\
\hline Nouvelle-Zélande & 78 & 78 & 0 & 22 & 5 & 17 & 1 \\
\hline Pays-Bas ${ }^{a}$ & 66 & 3 & 63 & 34 & 20 & 8 & 6 \\
\hline Pologne & 69 & 11 & 58 & 31 & 1 & 26 & 4 \\
\hline Portugal & 73 & 72 & 1 & 27 & 4 & 22 & 1 \\
\hline République slovaque & 74 & 9 & 65 & 26 & 0 & 23 & 3 \\
\hline République tchèque & 89 & 9 & 80 & 11 & 0 & 11 & 0 \\
\hline Royaume-Uni ${ }^{b}$ & 87 & 87 & 0 & 13 & & 13 & 0 \\
\hline Suède & 85 & 85 & 0 & 15 & 0 & 15 & 0 \\
\hline Suisse & 60 & 17 & 43 & 40 & 9 & 31 & 1 \\
\hline Turquie & 71 & 34 & 38 & 29 & 0 & 20 & 9 \\
\hline Moyenne OCDE & 73 & 41 & 32 & 27 & 6 & 20 & 2 \\
\hline
\end{tabular}

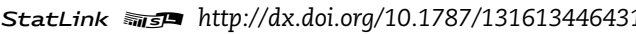

a) Part des dépenses courantes plutôt que celle des dépenses totales (les investissements sont exclus).

b) La part de l'assurance maladie privée n'est pas connue.

c) Les versements nets des ménages au Luxembourg incluent seulement les co-paiements.

d) Comprend le financement des institutions à but non lucratif, des entreprises et le financement externe (reste du monde).

Source : Eco-Santé OCDE 2007. 
Tableau A.5.6a. Couverture de l'assurance maladie publique, pourcentage de la population totale, 1970 à 2005

\begin{tabular}{|c|c|c|c|c|c|}
\hline & 1970 & 1980 & 1990 & 2000 & 2005 \\
\hline Allemagne & 89.2 & 92.3 & 88.8 & 90.7 & 89.6 \\
\hline Australie & 85.0 & 100.0 & 100.0 & 100.0 & 100.0 \\
\hline Autriche & 91.0 & 99.0 & 99.0 & 99.0 & 98.0 \\
\hline Belgique & 97.8 & 99.0 & 97.3 & 99.0 & 99.0 \\
\hline Canada & 100.0 & 100.0 & 100.0 & 100.0 & 100.0 \\
\hline Corée & .. & 29.8 & 100.0 & 100.0 & 100.0 \\
\hline Danemark & 100.0 & 100.0 & 100.0 & 100.0 & 100.0 \\
\hline Espagne & 61.0 & 83.0 & 98.11991 & 98.92001 & 99.52003 \\
\hline États-Unis & .. & . & 24.5 & 24.7 & 27.3 \\
\hline Finlande & 100.0 & 100.0 & 100.0 & 100.0 & 100.0 \\
\hline France & 95.6 & 99.1 & 99.4 & 99.9 & 99.9 \\
\hline Grèce & 55.0 & 88.0 & 100.0 & 100.0 & 100.02004 \\
\hline Hongrie & .. & 100.0 & 100.0 & 100.0 & 100.0 \\
\hline Irlande & 85.0 & 100.0 & 100.0 & 100.0 & 100.0 \\
\hline Islande & 100.0 & 100.0 & 100.0 & 100.0 & 100.0 \\
\hline Italie & 93.0 & 100.0 & 100.0 & 100.0 & 100.0 \\
\hline Japon & 100.0 & 100.0 & 100.0 & 100.0 & 100.0 \\
\hline Luxembourg & 99.6 & 99.8 & 98.81993 & 98.2 & 99.72004 \\
\hline Mexique & . & .. & . & 51.02002 & 50.4 \\
\hline Norvège & 100.0 & 100.0 & 100.0 & 100.0 & 100.0 \\
\hline Nouvelle-Zélande & 100.0 & 100.0 & 100.0 & 100.0 & 100.0 \\
\hline Pays-Bas & 69.0 & 68.3 & 61.4 & 64.5 & 62.1 \\
\hline Pologne & . & .. & . & . & 97.3 \\
\hline Portugal & 40.0 & 100.0 & 100.0 & 100.0 & 100.0 \\
\hline République slovaque & . & . & .. & 98.8 & 97.6 \\
\hline République tchèque & 100.0 & 100.0 & 100.0 & 100.0 & 100.0 \\
\hline Royaume-Uni & 100.0 & 100.0 & 100.0 & 100.0 & 100.0 \\
\hline Suède & 100.0 & 100.0 & 100.0 & 100.0 & 100.0 \\
\hline Suisse & 89.0 & 96.5 & 99.5 & 100.0 & 100.0 \\
\hline Turquie & 26.9 & 38.4 & 55.1 & 66.01997 & 67.22003 \\
\hline Moyenne la plus récente ${ }^{a}$ & . & . & . & . & 92.9 \\
\hline Moyenne cohérente $(27)^{b}$ & . & . & 93.4 & 94.1 & 94.2 \\
\hline
\end{tabular}

StatLink तiाst http://dx.doi.org/10.1787/131625581844

a) La moyenne comprend tout les pays pour lesquels des données récentes sont disponibles.

b) Exclut le Mexique, la Pologne et la République slovaque.

Source : Eco-Santé OCDE 2007. 
Tableau A.5.6b. Assurance maladie privée par type de couverture, pourcentage de la population totale, 2005 (ou dernière année disponible)

\begin{tabular}{|c|c|c|c|c|c|}
\hline & $\begin{array}{l}\text { Couverture totale } \\
\text { de l'assurance maladie } \\
\text { privée }\end{array}$ & $\begin{array}{c}\text { Assurance } \\
\text { primaire }\end{array}$ & $\begin{array}{l}\text { Assurance } \\
\text { duplicative }\end{array}$ & $\begin{array}{c}\text { Assurance } \\
\text { complémentaire }\end{array}$ & $\begin{array}{c}\text { Assurance } \\
\text { supplémentaire }\end{array}$ \\
\hline Australie & 42.9 & 0.0 & 42.9 & 0.0 & 41.4 \\
\hline Allemagne & 24.3 & 10.2 & 0.0 & 0.0 & 14.2 \\
\hline Autriche & . & . & 0.0 & . & . \\
\hline Belgique & 44.0 & .. & 0.0 & 44.0 & .. \\
\hline Canada & 66.0 & 0.0 & 0.0 & 0.0 & 66.0 \\
\hline Corée & . & 0.0 & 0.0 & .. & .. \\
\hline Danemark & 6.8 & 0.0 & 0.0 & 6.8 & 6.8 \\
\hline Espagne & 11.9 & . & . & 0.0 & . \\
\hline États-Unis & 67.1 & 59.2 & 0.0 & . & . \\
\hline Finlande & . & 0.0 & . & . & . \\
\hline France & 87.2 & 0.0 & 0.0 & 87.2 & 0.0 \\
\hline Grèce & 15.6 & 0.0 & 15.6 & 0.0 & 0.0 \\
\hline Hongrie & 0.0 & 0.0 & 0.0 & 0.0 & 0.0 \\
\hline Irlande & 51.6 & 0.0 & 51.6 & 0.0 & 0.0 \\
\hline Islande & 13.6 & 0.0 & 0.0 & 0.0 & 13.6 \\
\hline Italie & . & 0.0 & . & 0.0 & . \\
\hline Japon & . & 0.0 & . & . & .. \\
\hline Luxembourg & . & 0.0 & 0.0 & . & . \\
\hline Mexique & 4.8 & 0.0 & .. & 0.0 & . \\
\hline Norvège & 0.0 & 0.0 & 0.0 & 0.0 & 0.0 \\
\hline Nouvelle-Zélande & 32.7 & 0.0 & 32.7 & 0.0 & 0.0 \\
\hline Pays-Bas & 92.8 & 35.8 & 0.0 & 0.0 & 57.1 \\
\hline Pologne & . & 0.0 & 0.0 & 0.0 & 0.0 \\
\hline Portugal & 17.4 & 0.0 & 17.4 & . & . \\
\hline République slovaque & 0.0 & 0.0 & 0.0 & 0.0 & 0.0 \\
\hline République tchèque & 0.0 & 0.0 & 0.0 & 0.0 & 0.0 \\
\hline Royaume-Uni & 11.0 & 0.0 & 11.0 & 0.0 & 0.0 \\
\hline Suède & . & 0.0 & 0.0 & . & . \\
\hline Suisse & 32.5 & 0.0 & 0.0 & 0.0 & 32.5 \\
\hline Turquie & 1.4 & 0.0 & 0.0 & . & . \\
\hline
\end{tabular}

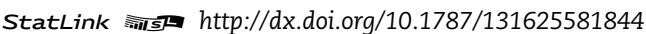

Note: La couverture totale de l'assurance maladie privée montre le pourcentage de la population qui est couverte par au moins un type d'assurance maladie privée. Pour un pays donné, la couverture privée totale n'est pas nécessairement égale à la somme des différents types d'assurance maladie privée, car les individus peuvent avoir plus d'une couverture (par exemple en Australie). Dans certains pays, les assurances maladies privées jouent plusieurs rôles mais les données se réfèrent au type de couverture prédominant (comme en Belgique, France, Portugal et Islande).

Source : Eco-Santé OCDE 2007 
Tableau A.6.4. Taux de survie au cancer du sein et procédures chirurgicales pour 100000 femmes, 2005 (ou dernière année disponible)

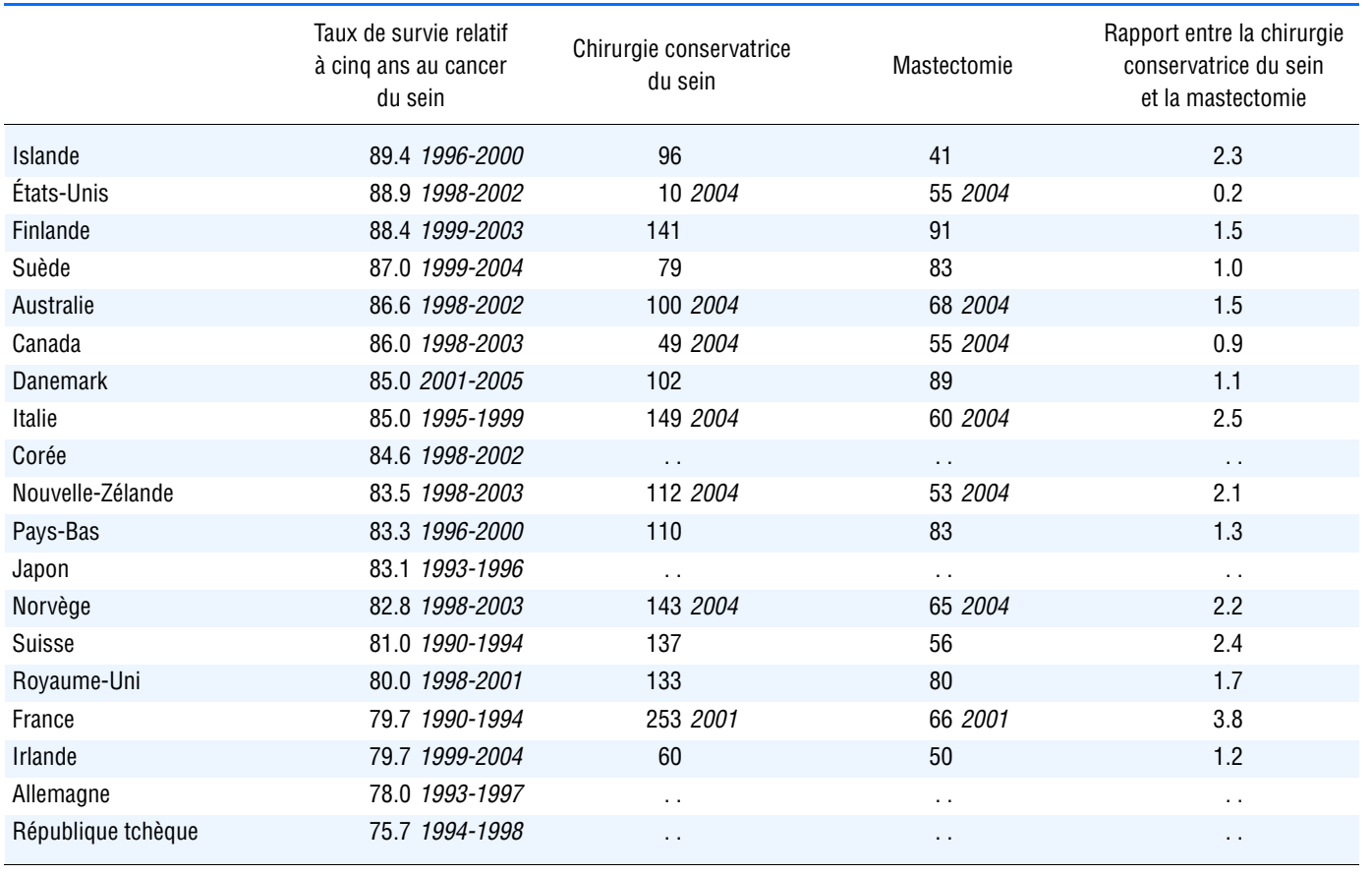

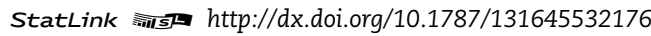
Source : Projet sur les indicateurs de la qualité des soins de santé, OCDE 2007. Eco-Santé OCDE 2007 (chirurgie conservatrice du sein et mastectomie). 


\section{ANNEXE B \\ Définition des dépenses de santé et notes méthodologiques sur la comparabilité des données}

\section{Définition des dépenses de santé}

Les dépenses totales de santé mesurent la consommation finale de produits et de services de santé et l'investissement en capital lié aux infrastructures de soins. Elles incluent les sommes dépensées par les acteurs publics et privés (y compris les ménages) au titre des produits et des services médicaux, des programmes de santé publique et de prévention et de l'administration. Les dépenses connexes comme la formation, la recherche et la santé environnementale sont exclues. Les dépenses totales de santé n'incluent pas aussi l'indemnisation de la perte de revenu due à des problèmes de santé (congés maladie et allocations d'invalidité). Pour une définition plus détaillée, consulter Système de comptes de la santé (OCDE, 2000a).

Le tableau qui suit présente les grandes catégories de dépenses utilisées dans Eco-Santé OCDE 2007 et dans les tableaux de cette publication.

\begin{tabular}{ll}
\hline Code ICHA & Description \\
\hline HC.1; HC. 2 & Services de soins curatifs et de réadaptation (patients hospitalisés et externes, soins à domicile) \\
HC. 3 & Services de soins de longue durée (personnes en institutions et soins à domicile) \\
HC. 4 & Services auxiliaires de soins de santé \\
HC.1-HC. & Services médicaux \\
HC.5 & Produits médicaux administrés à des malades externes \\
HC.1-HC.5 & Dépenses totales au titre de la santé personnelle \\
HC.6 & Services de prévention et de santé publique \\
HC.7 & Administration de la santé et assurance maladie \\
HC.6-HC.7 & Dépenses totales au titre de la santé collective \\
HC.1-HC.7 & Dépenses totales courantes de santé \\
HC.R.1 & Formation de capital (investissements) des établissements de soins de santé \\
HC.1-HC.7+HC.R.1 & DÉPENSES TOTALES DE SANTÉ \\
\hline
\end{tabular}

\section{Comparaison des dépenses de santé entre pays}

Les pays de l'OCDE en sont à des stades variables de présentation de leurs dépenses totales de santé selon les catégories proposées dans le manuel de l'OCDE Système de comptes de la santé. Cela signifie que les données présentées dans Eco-Santé OCDE 2007 ont des niveaux variables de comparabilité. La comparabilité de la ventilation fonctionnelle des dépenses de 
santé dans Eco-Santé OCDE s'est progressivement améliorée au cours des dernières années. Toutefois, elle reste limitée (même entre pays dont les dépenses totales sont relativement comparables) du fait que la collecte des données est liée aux enregistrements administratifs des systèmes de financement. En Australie, au Canada et aux États-Unis, par exemple, les dépenses au titre des patients hospitalisés n'incluent pas la facturation indépendante des honoraires des médecins pour les soins à des patients hospitalisés, alors qu'en Allemagne et aux Pays-Bas elles incluent les soins aux patients externes dispensés dans les hôpitaux. Des pratiques différentes d'inclusion des soins de longue durée dans les dépenses sociales ou de santé affectent également la comparabilité des données.

Concernant la ventilation fonctionnelle des dépenses de santé présentée dans cette publication, les dépenses de soins externes sont prises dans une acception large incluant à la fois les soins aux patients externes dans les hôpitaux, ainsi que les soins dans le secteur ambulatoire. Eco-Santé OCDE 2007 présente une ventilation plus fine (comme le montre le tableau ci-dessus).

Pour plus d'information, consulter la «Note sur la comparabilité générale des dépenses de santé et de leur financement » dans Eco-Santé OCDE 2007.

\section{Ajustement pour prise en compte des différences dues à la monnaie nationale}

Les dépenses de santé exprimées dans les monnaies nationales peuvent être utilisées pour comparer certains indicateurs tels que la part des dépenses de santé dans le PIB et les taux de progression des dépenses de santé dans le temps.

Toutefois, pour effectuer des comparaisons utiles des dépenses de santé entre pays à un instant donné, il est nécessaire de convertir les données exprimées en monnaie nationale en une unité monétaire commune, telle que le dollar américain. Il est également utile de prendre en compte les différences de pouvoir d'achat des monnaies nationales dans chaque pays. Pour calculer le taux de conversion des monnaies nationales en dollar de parité de pouvoir d'achat (PPA), on calcule le prix d'un même panier de biens et de services dans différents pays en l'exprimant dans la monnaie nationale puis on le convertit en dollars. Par exemple, si un panier identique de biens et de services coûte au Canada 140 dollars canadiens et aux ÉtatsUnis 100 USD, la parité de pouvoir d'achat sera de 1.4 CAD pour 1 USD. Les PPA du PIB sont utilisées car ce sont les taux de conversion disponibles les plus fiables. Elles sont calculées à partir d'un large panier de biens et de services choisis pour être représentatifs de l'ensemble de l'activité économique. Ainsi, les variations de dépenses de santé selon les pays reflètent non seulement les variations du niveau de consommation des services de santé mais aussi les différences de prix de ces services par rapport au PIB entre les pays.

Les dépenses de santé converties en USD PPA ne sont pas ajustées de l'inflation des prix ; elles ne conviennent donc pas pour des comparaisons dans le temps des taux de croissance réels.

\section{Correction des données pour prise en compte de l'inflation des prix}

Pour effectuer des comparaisons valables dans le temps des taux réels de croissance, il faut déflater les dépenses de santé nominales par un indice des prix pertinent et les diviser par la population pour obtenir les dépenses réelles par habitant. En raison du manque d'indices de prix fiables dont on dispose dans le domaine de la santé, cette publication utilise l'indice des prix du PIB (niveaux de prix du PIB 2000). Il faut garder à l'esprit que, dans la plupart des pays, le secteur de la santé a habituellement un taux d'inflation supérieur à celui de l'économie dans son ensemble. 


\section{Liste des variables contenues dans Eco-Santé OCDE 2007}

\section{Partie 1. État de santé}

Mortalité

Espérance de vie

Causes de mortalité

Mortalité infantile et maternelle

Années de vie potentielle perdues

Morbidité

Perception de l'état de santé

Santé du nourrisson

Santé buccale

Maladies transmissibles (VIH/SIDA)

Cancer

Accidents

Absentéisme au travail pour cause de maladie

\section{Partie 2. Ressources en santé}

Éducation dans le secteur de la santé

Emploi dans le secteur de la santé

Rémunération des professionnels de santé

Lits

Lits en hôpitaux

Lits pour soins de longue durée

Ratio emploi/lits

Technologie médicale

Partie 3. Utilisation des ressources en santé

Prévention (Vaccination)

Dépistage

Consultations (Docteurs et dentistes)

Hospitalisation

Lits de soins aigus

Taux d'occupation

Taux de renouvellement

Durée moyenne de séjour en hôpitaux

Durée moyenne de séjour par catégories de diagnostic

Taux de sortie

Taux de sortie par catégories de diagnostic

Procédures chirurgicales

Nombre total d'actes chirurgicaux

Procédures chirurgicales par CIM-9-MC

Greffes et dialyses

Partie 4. Dépenses de santé

Dépenses totales de santé

Dépenses courantes de santé

Investissement médical

Dépenses en soins de santé individuels

Dépenses en services médicaux

Dépenses en soins en milieu hospitalier

Dépenses en soins en hospitalisation de jour

Dépenses en soins ambulatoires

Dépenses en soins à domicile

Dépenses en services auxiliaires

Dépenses en biens médicaux

Produits pharma. et autres biens médicaux non durables

Appareils thérapeutiques et autres biens médicaux durables
Dépenses de santé pour la collectivité

Prévention et santé publique

Dép. d'administration de la santé et assurance maladie

Agrégats supplémentaires de dépenses de santé

Soins de santé curatifs et préventifs

Dépenses totales en soins de longue durée

Dépenses liées à la santé

Dépenses courantes de santé par prestataire de soins Dépenses en services fournis par les hôpitaux

Dép. en maisons médicalisées, soins avec hébergement

Dép. en services de prestataires de soins ambulatoires

Dép. en détaillants et autres dist. de biens médicaux

Dép. en services des organisations de santé publique

Dép. en services d'administration générale de santé

Dépenses par classe d'âge et par sexe

Indice de prix

Partie 5. Financement du système de santé

Dépenses de santé par agent de financement/programme Administrations publiques

Administrations de sécurité sociale

Versements nets des ménages

Assurance privée

\section{Partie 6. Protection sociale}

Dépenses sociales

Couverture médicale

Assurance maladie publique/sociale

Assurance maladie privée

Partie 7. Marché pharmaceutique

Activité de l'industrie pharmaceutique

Consommation de médicaments

Quantités consommées par médicaments

Ventes de médicaments

Données de ventes par médicaments

Partie 8. Déterminants non médicaux de la santé

Mode de vie et environnement

Alimentation

Consommation d'alcool

Consommation de tabac

Masse pondérale

Environnement : qualité de l'air

Partie 9. Références démographiques

Données démographiques générales

Population par structure d'âge

Population active

Niveau d'éducation

Partie 10. Références économiques

Références macroéconomiques

Taux de conversion monétaire

Autres tableaux

Bénéficiaires de soins de longue durée à la maison et en institution

Plus d'information sur Eco-Santé OCDE 2007 est disponible sur www.oecd.org/sante/ecosante. 


\section{ANNEXE D}

\section{Catégories de maladies et blessures et codes CIM}

Les causes de décès présentées dans Eco-Santé OCDE 2007 sont répertoriées dans la liste ci-dessous en fonction des codes de la $10^{\mathrm{e}}$ et $9^{\mathrm{e}}$ révisions de la Classification internationale des maladies (CIM).

\begin{tabular}{|c|c|c|}
\hline Catégories de maladies et blessures & CIM-10 & CIM-9 \\
\hline 0. Toutes catégories & A00-R99,V01-Y89 & 001-799,E800-E999 \\
\hline 1. Maladies infectieuses, parasitaires & A00-B99 & $001-139,042-044$ \\
\hline 2. Maladies dues au VIH & B20-B24 & 042-044 \\
\hline 3. Tumeurs malignes (cancer) & $\mathrm{C00-C97}$ & $140-208$ \\
\hline 4. Tumeurs malignes du gros intestin, du rectum et du canal anal & C18-C21 & $153-154$ \\
\hline 5. Tumeur maligne de la trachée, des bronches et du poumon & C33, C34 & 162 \\
\hline 6. Tumeur maligne du sein & C50 & 174 \\
\hline 7. Tumeur maligne du col de l'utérus & C53 & 180 \\
\hline 8. Tumeur maligne de la prostate & C61 & 185 \\
\hline 9. Maladies du sang et des organes hématopoiétiques & D50-D89 & $279-289$ \\
\hline 10. Maladies endocriniennes, nutritionnelles et métaboliques & E00-E89 & $240-279$ \\
\hline 11. Diabète & E10-E14 & 250 \\
\hline 12. Troubles mentaux et du comportement & F01-F99 & $290-319$ \\
\hline 13. Maladies du système nerveux et des organes des sens & G00-H95 & $320-389$ \\
\hline 14. Maladies de l'appareil circulatoire & $100-199$ & $390-459$ \\
\hline 15. Cardiopathies ischémiques & $120-125$ & $410-414$ \\
\hline 16. Infarctus aigu du myocarde & $|21| 22$, & 410 \\
\hline 17. Maladies cérébro-vasculaires & $160-169$ & $430-438$ \\
\hline 18. Maladies de l'appareil respiratoire & J00-J98 & $460-519$ \\
\hline 19. Grippe et pneumonie & J10-J18 & $480-487$ \\
\hline 20. Bronchite, asthme et emphysème & $\mathrm{J} 40-\mathrm{J} 43, \mathrm{~J} 45, \mathrm{~J} 46$ & $490-493$ \\
\hline 21. Maladies de l'appareil digestif & K00-K92 & $520-579$ \\
\hline 22. Hépatite chronique et cirrhose du foie & $\mathrm{K} 70, \mathrm{~K} 73, \mathrm{~K} 74, \mathrm{~K} 76$ & 571 \\
\hline 23. Maladies de la peau et des tissus sous-cutanés & L00-L98 & $680-709$ \\
\hline 24. Maladies du système ostéo-musculaire & M00-M99 & $710-739$ \\
\hline 25. Maladies du système génito-urinaire & N00-N99 & $580-629$ \\
\hline 26. Grossesse, accouchement et puerpéralité & 000-099 & $630-676$ \\
\hline 27. Pathologies périnatales & P00-P96 & $760-779$ \\
\hline 28. Malformations congénitales & Q00-Q99 & $740-759$ \\
\hline 29. Symptômes, signes, états morbides mal définis & R00-R99 & $780-799$ \\
\hline 30. Causes externes de mortalité & V01-Y89 & E800-E999 \\
\hline 31. Accidents de transport & V01-V89 & E810-E829 \\
\hline 32. Chutes accidentelles & W00-W19 & E880-E888 \\
\hline 33. Lésions auto-infligées (suicides) & $\mathrm{X} 60-\mathrm{X} 84$ & E950-E959 \\
\hline 34. Agressions (homicides) & X85-Y09 & E960-969 \\
\hline 35. Effets indésirables des médicaments & Y40-Y59 & E930-E949 \\
\hline 36. Complications dues à un acte médical ou chirurgical & Y60-Y84 & E870-E879 \\
\hline
\end{tabular}



LES ÉDITIONS DE L'OCDE, 2, rue André-Pascal, 75775 PARIS CEDEX 16 IMPRIMÉ EN FRANCE

(81 2007052 P) ISBN 978-92-64-02733-6 - n 558122007 


\section{Panorama de la santé 2007}

\section{LES INDICATEURS DE L'OCDE}

Les progrès accomplis dans la prévention et le traitement des maladies ont contribué à l'amélioration remarquable de l'espérance de vie et de la qualité de vie dans les pays de l'OCDE au cours des dernières décennies. Parallèlement, les dépenses de santé continuent d'augmenter et absorbent une part croissante du revenu national. À l'heure actuelle, elles représentent $9 \%$ du PIB en moyenne dans les pays de l'OCDE, contre un peu plus de $5 \%$ en 1970.

Cette quatrième édition du Panorama de la santé présente les données les plus récentes et les tendances de différents aspects des performances des systèmes de santé des pays de l'OCDE. Elle montre clairement les fortes variations entre pays en ce qui concerne l'état de santé de la population et les risques sanitaires, ainsi que les différences de coûts, d'affectation des ressources et d'activités des systèmes de santé. Pour la première fois, cette édition contient un chapitre qui présente de nouveaux indicateurs comparables sur la qualité des soins, montrant les variations entre pays concernant les taux de survie après une crise cardiaque, un accident vasculaire cérébral et différents types de cancer.

Chaque indicateur est présenté dans un format qui se veut le plus clair possible : des graphiques illustrent les variations entre pays et dans le temps, un commentaire succinct tire les principales conclusions des données présentées, et un encadré méthodologique rappelle la définition de l'indicateur. Par ailleurs, une annexe statistique fournit des précisions supplémentaires sur la plupart des indicateurs, étayées par des séries temporelles qui dans certains cas remontent jusqu'en 1960.

Cette publication s'appuie principalement sur Eco-Santé OCDE 2007, la base de données la plus complète qui existe sur la santé et les systèmes de santé dans les 30 pays membres de l'OCDE. ECo-Santé OCDE 2007 est accessible en ligne à l'adresse www.SourceOECD.org ou peut être commandé en version CD-ROM à la librairie en ligne de l'OCDE (www.oecd.org/bookshop).

\section{www.oecd.org/sante}

Le texte complet de cet ouvrage est disponible en ligne à l'adresse suivante : www.sourceocde.org/questionssociales/9789264027336

Les utilisateurs ayant accès à tous les ouvrages en ligne de l'OCDE peuvent également y accéder via : www.sourceocde.org/9789264027336

SourceOCDE est une bibliothèque en ligne qui a reçu plusieurs récompenses. Elle contient les livres, périodiques et bases de données statistiques de l'OCDE. Pour plus d'informations sur ce service ou pour obtenir un accès temporaire gratuit, veuillez contacter votre bibliothécaire ou SourceOECD@oecd.org. 